\section{Pacific Northwest}

National Laboratory

Operated by Battelle for the

U.S. Department of Energy

\title{
Novel Concepts for Damage-Resistant Alloys in Next Generation Nuclear Power Systems Final Report, Project 99-0280
}

\author{
S. M. Bruemmer \\ * P. L. Andresen \\ E. P. Simonen \\ * L. Young \\ J. Gan \\ ** G. S. Was \\ F. A. Garner \\ ** L. Fournier \\ D. S. Gelles \\ ** B. H. Sencer \\ D. J. Edwards \\ * General Electric Global Research \& Development \\ ** University of Michigan
}

December 2002

Prepared for the U.S. Department of Energy under Contract DE-AC06-76RL01830 


\title{
DISCLAIMER
}

This report was prepared as an account of work sponsored by an agency of the United States Government. Neither the United States Government nor any agency thereof, nor Battelle Memorial Institute, nor any of their employees, makes any warranty, express or implied, or assumes any legal liability or responsibility for the accuracy, completeness, or usefulness of any information, apparatus, product, or process disclosed, or represents that its use would not infringe privately owned rights. Reference herein to any specific commercial product, process, or service by trade name, trademark, manufacturer, or otherwise does not necessarily constitute or imply its endorsement, recommendation, or favoring by the United States Government or any agency thereof, or Battelle Memorial Institute. The views and opinions of authors expressed herein do not necessarily state or reflect those of the United States Government or any agency thereof.

\author{
PACIFIC NORTHWEST NATIONAL LABORATORY \\ operated by \\ BATTELLE \\ for the \\ UNITED STATES DEPARTMENT OF ENERGY \\ under Contract DE-AC06-76RL01830
}

Ty 
NUCLEAR ENERGY RESEARCH INITIATIVE

\section{NOVEL CONCEPTS FOR DAMAGE-RESISTANT ALLOYS IN NEXT GENERATION NUCLEAR POWER SYSTEMS}

Final Report, Phase 3

Project Number 99-0280

\section{Pacific Northwest National Laboratory}

S. M. Bruemmer and E. P. Simonen, Principal Investigators

J. Gan, F. A. Garner, D. S. Gelles and D. J. Edwards

General Electric Global Research \& Development

P. L. Andresen, Principal Investigator

L. Young

\section{University of Michigan}

G. S. Was, Principal Investigator

L. Fournier and B. H. Sencer

December 27, 2002

Prepared for:

U.S. Department of Energy

Oakland Operations Office 1301 Clay Street

Oakland, CA 94612

Attn: Rebecca L. Richardson, NSPD

U.S. Department of Energy

Office of Nuclear Energy, Science and Technology

19901 Germantown Road

Germantown, MD 20874

Attn: Lisa C. Herrera, NE-50

Pacific Northwest National Laboratory

Richland, Washington 99352 


\section{TABLE OF CONTENTS}

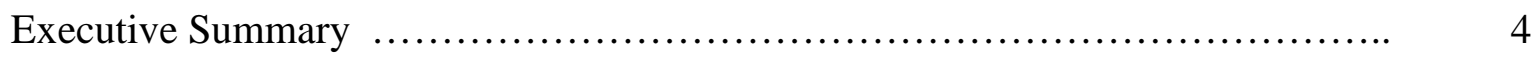

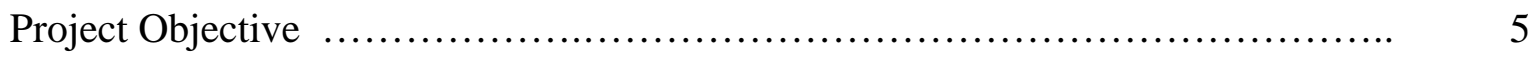

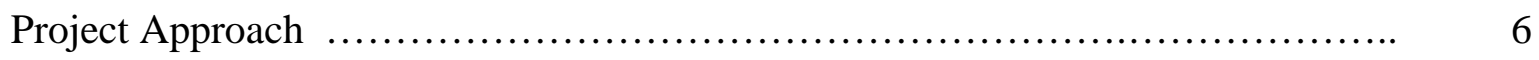

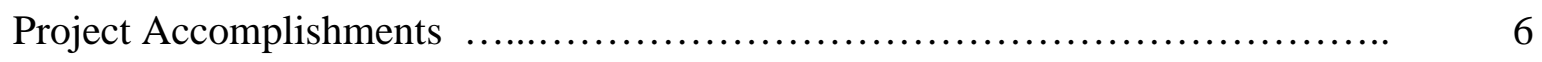

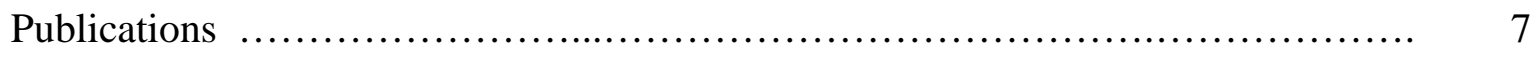

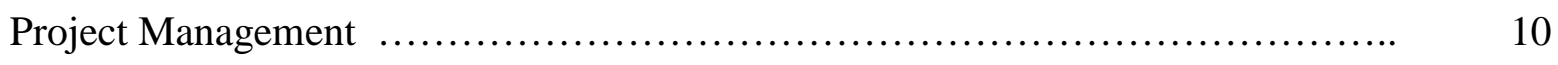

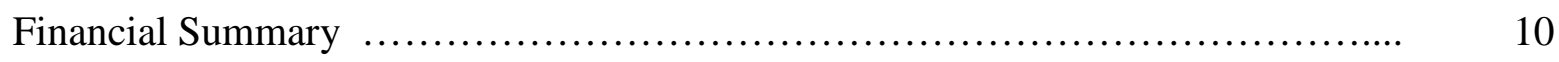

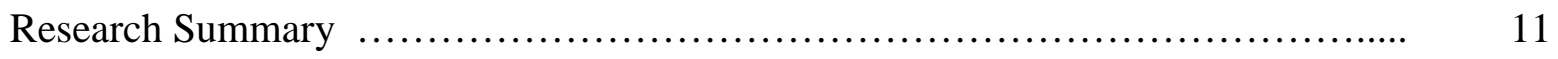

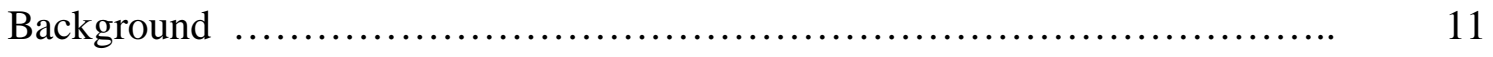

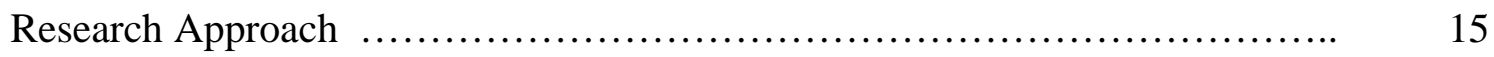

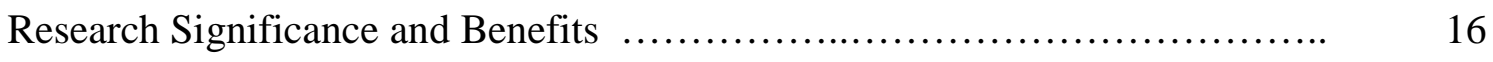

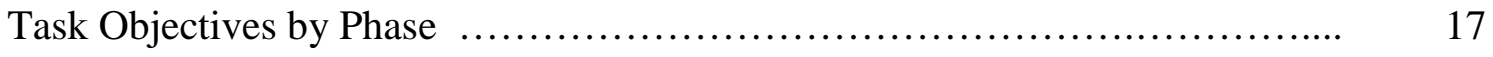

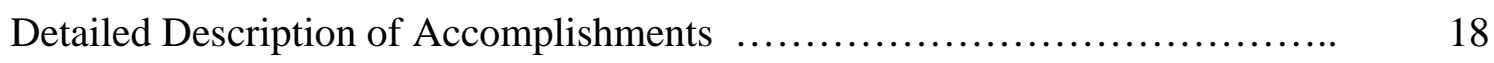

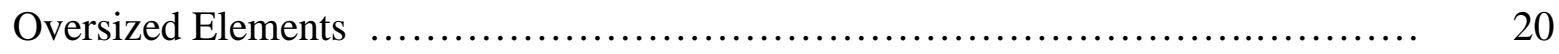

Materials Processing ..................................................... 20

Irradiations ........................................................... 20

Heavy-Ion Irradiation........................................................ 21

Proton Irradiation .............................................................. 21

Neutron Irradiation .................................................... $\quad 24$

Characterization of Radiation-Induced Microstructures.......................... 26

Transmission Electron Microscopy Procedures............................... $\quad 26$

TEM Measurements of Defect Microstructures.............................. $\quad 27$ 
Characterization of Radiation-Induced Grain Boundary Segregation................ 48 Measured Grain Boundary Compositions $-\mathrm{Ni}^{++}$Irradiation..................... 48 Measured Grain Boundary Compositions - Proton Irradiations.................... 54

Discussion of Oversized Element Concept for Radiation-Damage

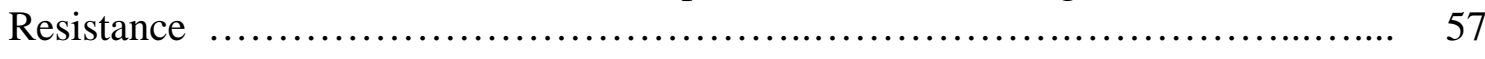

Insights on Current Understanding for Oversized Solute........................ 65

Multiphase Microstructure.................................................. 67

Ni-Base Alloy Concept Evaluation.......................................... 69

Fe-Base Alloy Concept Evaluation........................................... 73

Ferritic/Martensitic Concept Evaluation....................................... 79

Mechanical Behavior and Irradiation-Assisted Stress Corrosion Cracking............. 88

Radiation-Induced Hardening .............................................. 88

Irradiation-Assisted Stress Corrosion Cracking............................... 90

Crack-Growth Rate Evaluation of Stress Corrosion Cracking........................ 97

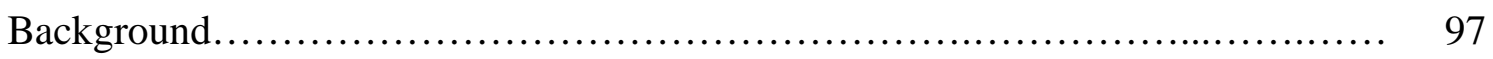

Materials and Experimental Procedures....................................... 98

Crack Growth Test Results and Discussion...................................... 102

Stress Corrosion Cracking of Cold-Worked Stainless Steels.................... 102

Stress Corrosion Cracking of Sensitized and Desensitized Stainless Steels...... 141

Comparisons and Summary of Stress Corrosion Response....................... 157

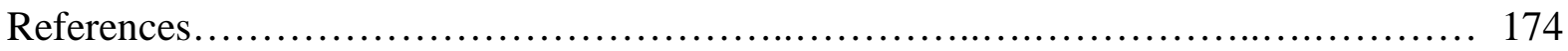




\title{
Executive Summary
}

\section{Novel Concepts for Damage-Resistant Alloys in Next Generation Nuclear Power Systems}

\author{
S. M. Bruemmer and E. P. Simonen \\ Pacific Northwest National Laboratory \\ P. L. Andresen \\ General Electric Global Research \\ G. S. Was \\ University of Michigan
}

December 27, 2002

\begin{abstract}
This project has elucidated approaches for managing damage evolution through control of matrix solute and multiphase structures. Radiation microstructures, microchemistries and properties affected by irradiation were evaluated with the goal of developing damage-resistant alloys for next generation nuclear power systems. Three complementary experimental approaches identified key factors for the development of alloys: $\mathrm{Ni}^{++}$irradiation, proton irradiation and manipulation of simulated irradiation characteristics in non-irradiated alloys. Specifically, alloys processed with oversized solute, i.e., Hf, and multiphase alloys show promise for retarding damage evolution.
\end{abstract}

The discovery of a damage-resistant alloy based on Hf solute additions (oversized solute) to a low-carbon $316 \mathrm{SS}$ is the highlight of the research. This damage resistance is supported by characterization of radiation-induced microstructures and microchemistries along with measurements of environmental cracking. The addition of Hf to a low-carbon 316SS reduced the detrimental impact of radiation by inhibiting the formation of voids and initiation of cracking during slow-strain rate environmental tests. Because cracking susceptibility is associated with several material characteristics, separate effect experiments exploring strength effects using nonirradiated stainless steels were conducted. These crack growth tests suggest that irradiation strength by itself can promote cracking in high-temperature reactor water environments.

The concept of using oversized solutes to promote catalyzed defect recombination was a major thrust of this Nuclear Energy Research Initiative project. The successful demonstration of damage resistance in the optimized Hf-doped alloy demonstrates promise for developing damage-resistant alloys for future generation nuclear reactors. Differences between irradiation responses for Hf-doped and Pt-doped alloys suggest that the influence of the oversized elements has a significant influence on partitioning of point defects in the development of radiation damage. However, current theories describing the effect of oversized elements on mutual recombination do not account for all of the observations. Elimination of void formation to a dose of $50 \mathrm{dpa}$ (during heavy-ion irradiation) is a significant improvement in material performance. Samples from these initial alloys were prepared and included in a major multiyear, neutron-irradiation program as part of collaborative research activities. 
The second concept for developing damage-resistant alloys is the use of multiphase alloys to stabilize the microstructure during irradiation. Three alloys have been tailored for evaluation of precipitate stability influences on damage evolution. The first alloy is a Ni-base alloy (alloy 718) that has been characterized after neutron and Ni-ion irradiations. The radiation-induced dissolution of the nanometer-scale precipitates ( $\gamma^{\prime}$ and $\left.\gamma^{\prime \prime}\right)$ alters damage evolution, grain boundary segregation and decreases alloy strength with increasing dose. Two Fe-base alloys (PH 17-7 and PH 17-4) that also have nanometer-scale precipitate structures were also evaluated after Ni-ion irradiations. Fe-base alloys are more practical in light water reactors (LWRs) nuclear structures because of their lower Ni content and hence lesser transmutation to He. The $\mathrm{PH}$ alloys were much more complex with base multiphase microstructures including delta ferrite and tempered martensite. As for the alloy 718 case, $\gamma^{\prime}$ particles are unstable with irradiation while fine carbides slightly coarsen. In all cases, the complex multiphase structures resulted in damage resistance when compared to the simple base alloy design. In addition to the above experimental studies, a review was conducted to evaluate multiphase ferritic/martensitic alloys with oxide-dispersion strengthening. One final multiphase alloy approach was employed by tailoring precipitate distributions and compositions at grain boundaries in a 300-series stainless steel. Precipitates were found to reduce intergranular SCC crack growth in cold-worked 304SS.

The successful development of next generation nuclear power systems must address and mitigate several materials degradation issues that now strongly impact existing LWRs and that will limit structural materials performance at the more aggressive radiation exposures envisioned for various advanced reactor concepts. Although previous fast reactor and fusion device programs have focused on the development of improved structural materials for their relevant conditions, there has been no comparable effort directed toward the conditions unique to LWRs. In addition, this work directly links material changes at the nanoscale to bulk behavior. Novel, nontraditional approaches are necessary to create durable damage-resistant alloys and establish the foundation for advanced reactor designs.

\section{Project Objective}

The objective of the proposed research is to develop the scientific basis for a new class of radiation-resistant materials to meet the needs for higher performance and extended life in next generation power reactors. New structural materials are being designed to delay or eliminate the detrimental radiation-induced changes that occur in austenitic alloys, i.e., a significant increase in strength and loss in ductility ( $<10 \mathrm{dpa})$, environment-induced cracking ( $<10 \mathrm{dpa})$, swelling $(<50$ dpa) and embrittlement ( $<100 \mathrm{dpa})$. Non-traditional approaches are employed to ameliorate the root causes of materials degradation in current LWR systems. Changes in materials design are based on mechanistic understanding of radiation damage processes and environmental degradation and the extensive experience of the principal investigators with core component response. This work was integrated with fundamental research at Pacific Northwest National Laboratory (PNNL) and with focused international projects at PNNL, General Electric Global Research (GE) and University of Michigan (UM) led by EPRI. This leveraged approach facilitated the revolutionary advances envisioned in NERI by creating a multi-faceted effort into the basic and applied science necessary to drive mechanistic understanding and promote development of next generation materials. The research strategy capitalized on unique national 
laboratory, industry and university capabilities for studies of radiation damage and environmental cracking response.

\section{Project Approach}

This project was accomplished in three distinct tasks over a three-year period as documented in this report. Accomplishments by task of the project are highlighted in the description of Tasks $\mathrm{A}, \mathrm{B}$, and $\mathrm{C}$ as part of the detailed report. In summary, novel alloys were selected on the mechanistic basis for radiation damage resistance. Although neutron irradiation of large specimens for materials characterization and mechanical property testing would be desirable, this three-year program could not accommodate that need. In contrast, the program strategy emphasized irradiation using rapid $\mathrm{Ni}^{++}$ion irradiation for damage response. Proton irradiations were conducted to verify the heavy-ion response and allow selected mechanical properties and SCC behavior studies. To evaluate the effect of the irradiation damage condition on SCC, testing of deformation-hardened stainless steels was included in work scope. These highstrength, non-irradiated samples allowed confirmation that matrix strength similar to that created by irradiation can promote SCC susceptibility and enable tailored microstructural changes to be evaluated. Finally, in partnership with an international program, promising alloys are being neutron irradiated for future evaluation as warranted.

\section{Project Accomplishments}

Project research is highlighted by the discovery of superior damage resistance for an optimized alloy of 316LSS+Hf. The damage resistance was evidenced by no void swelling to high dpa, restricted dislocation loop growth, no radiation-induced segregation after proton irradiation (at low dose) and no evidence of environmental cracking after proton irradiation. This is in contrast to the clear evidence of voids, large diameter loops, radiation-induced segregation, and cracking found in the base 316LSS alloy without Hf. Samples were prepared and included in an international neutron-irradiation program to achieve doses from 5 to $70 \mathrm{dpa}$. In addition, the influence of strength on environmental cracking was quantified. The key implication is that strength alone promotes cracking without the influence of radiation-induced grain boundary segregation, radiation-induced microstructure, hydrogen enhancement, or martensite formation. Precipitate distribution and stability were demonstrated to alter radiation-induced microstructure, segregation, hardening and SCC.

\section{Task A: Oversized Elements}

The damage-resistant alloy based on Hf solute additions (oversized solute) to a low-carbon 316SS showed promise for development of a damage-resistant austenitic stainless steel. Characterization of radiation-induced microstructures and microchemistries along with measurements of environmental cracking supported the promise for this alloy. Radiation induced loop growth was retarded and void formation was suppressed to a high dose of $50 \mathrm{dpa}$.

\section{Task B: Multiphase Microstructures}

Two multiphase microstructures were evaluated with irradiation and microstructural characterization and exhibited no void formation to the highest doses tested. The complex structures inhibited the development of radiation-induced dislocation loops and hence formation 
of voids. The concept of using ferritic/martensitic alloys with oxide-dispersion strengthening was reviewed and strategies for development of these alloys were assessed. Carbide precipitate distributions at grain boundaries were tailored in 304SS and 316SS along with the local segregation and depletion of alloying elements.

\section{Task C: Mechanical Behavior and Stress Corrosion Cracking}

The dependence of IASCC on irradiation and strength was evaluated using proton irradiation and in a separate experiment using cold/warm work on non-irradiated specimens. Cracking susceptibility was demonstrated in the proton-irradiated base 316SS and the 316SS+Pt alloys. Cracking was not observed in the 316SS+Hf optimized alloy. The dependence of crack growth rate on alloy strength in non-irradiated SSs was extensively evaluated. The strength effect was confirmed for contrasting conditions of electrochemical potential, martensite formation and hydrogen contribution. Grain boundary precipitates were found to reduce the crack growth rate by up to a factor of ten for an oxidizing environment.

\section{Publications}

1. S. M. Bruemmer, D. J. Edwards, B. W. Arey and L. A. Charlot, "Microstructural, Microchemical and Hardening Evolution in LWR-Irradiated Austenitic Stainless Steel," 9th International Symposium on Environmental Degradation of Materials in Nuclear Power Systems - Water Reactors, TMS, 1999, p. 1079.

2. D. J. Edwards, E. P. Simonen and S. M. Bruemmer, "Radiation Hardening in Austenitic Stainless Steels Irradiated in LWRs," 9th International Symposium on Environmental Degradation of Materials in Nuclear Power Systems - Water Reactors, TMS, 1999, p. 1007.

3. S. M. Bruemmer, "Heat-to-Heat Variability in Radiation-Induced Microstructure and Microchemistry for Commercial 300-Series Stainless Steels", Minutes of the International Collaborative Group on Environmentally Assisted Cracking, April 2000, Williamsburg, VA, Paper I13.

4. E. P. Simonen, "Transitions in Stainless Steel Radiation-Induced Microstructures from 270 to $370{ }^{\circ} \mathrm{C}$ ", Minutes of the International Collaborative Group on Environmentally Assisted Cracking, April 2000, Williamsburg, VA, Paper I14.

5. J. Gan, G. Was and R. Stoller, "Microstructure Evolution in Austenitic Stainless Steels Irradiated Under Conditions Relevant to Light Water Reactor Core," $20^{\text {th }}$ ASTM International Symposium on Effects of Radiation on Materials, June 2000, Williamsburg, VA.

6. P. L. Andresen, T. M. Angeliu, W. R. Catlin, L. M. Young and R. M. Horn, "Effect of Deformation on SCC of Unsensitized Stainless Steel," Corrosion 2000, NACE, 2000, Paper 203.

7. D. J. Edwards, E. P. Simonen, F. A. Garner and S. M. Bruemmer, "Microstructural Evaluation of a Cold-Worked 316SS Baffle Bolt Irradiated in A Commercial PWR," 10th 
Int. Conf. Environmental Degradation of Materials in Nuclear Power Systems - Water Reactors, NACE, 2002.

8. L. Fournier, J. Gan, E. P. Simonen, S. M. Bruemmer and G. S. Was, "Effect of Oversized Solute Additions on Irradiation-Assisted Stress Corrosion Cracking of Austenitic Stainless Steels," 10th Int. Conf. Environmental Degradation of Materials in Nuclear Power Systems Water Reactors, NACE, 2002.

9. J. Gan, D. J. Edwards, E. P. Simonen, G. S. Was and S. M. Bruemmer, "Microstructural Evolution and Hardening in 300-Series Stainless Steels: Comparisons Between Neutron and Proton Irradiations," 10th Int. Conf. Environmental Degradation of Materials in Nuclear Power Systems - Water Reactors, NACE, 2002.

10. S. M. Bruemmer, "New Issues Concerning Radiation-Induced Material Changes and Irradiation-Assisted Stress Corrosion Cracking," Invited Paper, 10th Int. Conf. Environmental Degradation of Materials in Nuclear Power Systems - Water Reactors, NACE, 2002.

11. S. M. Bruemmer, D. J. Edwards, V. Y. Gertsman and E. P. Simonen, "Grain Boundary Modification During Neutron Irradiation at Intermediate Temperatures," Invited Paper, Microstructural Processes in Irradiated Materials, Materials Research Society (MRS), 2001, p. R2.1.

12. E. P. Simonen and S. M. Bruemmer, "The Influence of Submicroscopic Vacancy Clusters During Neutron Irradiation of Stainless Steel near $300^{\circ} \mathrm{C}$," Microstructural Processes in Irradiated Materials, MRS, 2001, p. R2.5.

13. S. M. Bruemmer and L. E. Thomas, "Insights into Environmental Degradation Mechanisms from High-Resolution Characterization of Crack Tips," Invited Paper, Proc. Symposium on Chemistry and Electrochemistry of Corrosion and Stress Corrosion, The Minerals, Metals and Materials Society (TMS), 2001, p. 321.

14. D. J. Edwards, E. P. Simonen and S. M. Bruemmer, "Nature of the Fine-Scale Defects and Radiation Hardening in Stainless Steel Neutron-Irradiated at $550^{\circ} \mathrm{K}$," Microstructural Processes in Irradiated Materials, MRS, 2001, p. R2.7.

15. L. E. Thomas, B. H. Sencer and S. M. Bruemmer, "Radiation-Induced Phase Instabilities and Their Effects on Hardening and Solute Segregation in Precipitation-Strengthened Alloy 718," Microstructural Processes in Irradiated Materials, MRS, 2001, p. R1.5.

16. E. P. Simonen, "Definitions of Defect Nanostructures Through Post-Irradiation Annealing”, Minutes of the International Collaborative Group on Environmentally Assisted Cracking, April 2001, Kjongju, South Korea, Paper I15. 
17. S. M. Bruemmer, "Linking Radiation-Induced Material Changes to IASCC Susceptibility", Minutes of the International Collaborative Group on Environmentally Assisted Cracking, April 2001, Kjongju, South Korea, Paper I11.

18. T. M. Angeliu, P. L. Andresen, E. Hall, J. A. Sutliff, S. Sitzman, Strain and Microstructure Characterization of Austenitic Stainless Steel Weld HAZs", Corrosion/2000, Paper 00186, NACE, 2000.

19. P. L. Andresen, T. M. Angeliu, L. M. Young, W. R. Catlin, and R. M. Horn, "Mechanisms and Kinetics of SCC in Stainless Steels", Proc. Tenth Int. Symp. on Environmental Degradation of Materials in Nuclear Power Systems - Water Reactors, NACE, 2002.

20. P. L. Andresen, "Perspective and Direction of Stress Corrosion Cracking in Hot Water", Invited Paper, Proc. Tenth Int. Symp. on Environmental Degradation of Materials in Nuclear Power Systems - Water Reactors, NACE, 2002.

21. P. L. Andresen, L. M. Young, W. R. Catlin and R. M. Horn, "Stress Corrosion Crack Growth Rate Behavior of Various Grades of Cold Worked Stainless Steel in High Temperature Water", Corrosion/02, Paper 02511, NACE, 2002.

22. P. L. Andresen, T. M. Angeliu and L. M. Young, "Effect of Martensite \& Hydrogen on SCC of Stainless Steels", Paper \#01228, Corrosion/01, NACE, 2001.

23. P. L. Andresen, "Similarity of Cold Work and Radiation Hardening in Enhancing Yield Strength and SCC Growth of Stainless Steel in Hot Water", Corrosion/02, Paper 02509, NACE, 2002.

24. P. L. Andresen, T. M. Angeliu and L. M. Young, "Immunity, Thresholds, and Other SCC Fiction", Invited Paper, Proc. Staehle Symp. on Chemistry and Electrochemistry of Corrosion and SCC, TMS, Feb. 2001.

25. G. S. Was, J. T. Busby, T. Allen, E. A. Kenik, A. Jensson, S. M. Bruemmer, J. Gan, D. J. Edwards, P. M. Scott, and P. L. Andresen, J. Nucl. Mater. Vol. 300, No. 2-3, pp. 198-216, 2002.

26. J. Gan, E. P. Simonen, S. M. Bruemmer, L. Fournier, B. H. Sencer and G. S. Was, "The Effect of Oversized Solute Additions on the Microstructure of 316SS Irradiated with $5 \mathrm{MeV}$ $\mathrm{Ni}^{++}$Ions of 3.2 MeV Protons," J. Nuclear Materials, in press.

27. D. J. Edwards, E. P. Simonen and S. M. Bruemmer, "Evaluation of Fine-Scale defects in Stainless Steels Neutron-Irradiated at 275C," J. Nucl. Materials, in press.

28. D. J. Edwards, E. P. Simonen, F. A. Garner, B. A. Oliver and S. M. Bruemmer, "Sensitivity of Microstructural Evolution Due To Temperature and Dose Gradients In Neutron-Irradiated 316SS," J. Nucl. Materials, in press. 
29. L. Fournier, B. H. Sencer, G. S. Was, J. Gan, S. M. Bruemmer, E. P. Simonen, E. A. Kenik, Proceedings of Fontevraud 5 - Contribution of Materials Investigation to the Resolution of Problems Encountered in Pressurized Water Reactors, French Nuclear Energy Society, September 2002.

30. G. S. Was, J. T. Busby, M. Hash, R. Dropek, B. H. Sencer, L. Fournier, T. Allen, J. Gan, J. Cole, E. Kenik, E. P. Simonen and S. M. Bruemmer, "Influence of Oversize Solute Additions on IASCC of Proton-Irradiated Austenitic Stainless Steels," $11^{\text {th }}$ Int. Conf. Environmental Degradation of Materials in Nuclear Power Systems - Water Reactors, American Nuclear Society, 2003, to be published.

31. P. L. Andresen, L. Young and R. Horn, "Effects of Yield Strength, Potential, Stress Intensity and Grain Boundary Carbides on Stress Corrosion Cracking," $11^{\text {th }}$ Int. Conf. Environmental Degradation of Materials in Nuclear Power Systems - Water Reactors, American Nuclear Society, 2003, to be published.

\section{Project Management}

All milestones were completed on schedule. Irradiation and characterization were accomplished to $50 \mathrm{dpa}$ using $\mathrm{Ni}^{++}$ions and to $5 \mathrm{dpa}$ using protons utilizing both microstructural and microchemical examinations. Solute addition and multiphase alloys were fabricated for the irradiation experiments. Charged particle irradiations of the solute addition and multiphase alloys were completed and extended to the optimized alloy for the case of Hf addition. Demonstration of matrix strength effects on SCC growth rates was achieved. The thesis for damage modification using misfit solute atoms and multiphase microstructures were confirmed by analyzing high-dose microstructures and by performing SCC tests on selected protonirradiated alloys.

\section{Financial Summary}

Spending occurred at a consistent rate meeting projections throughout this 39 -month project that ended September 30, 2002.

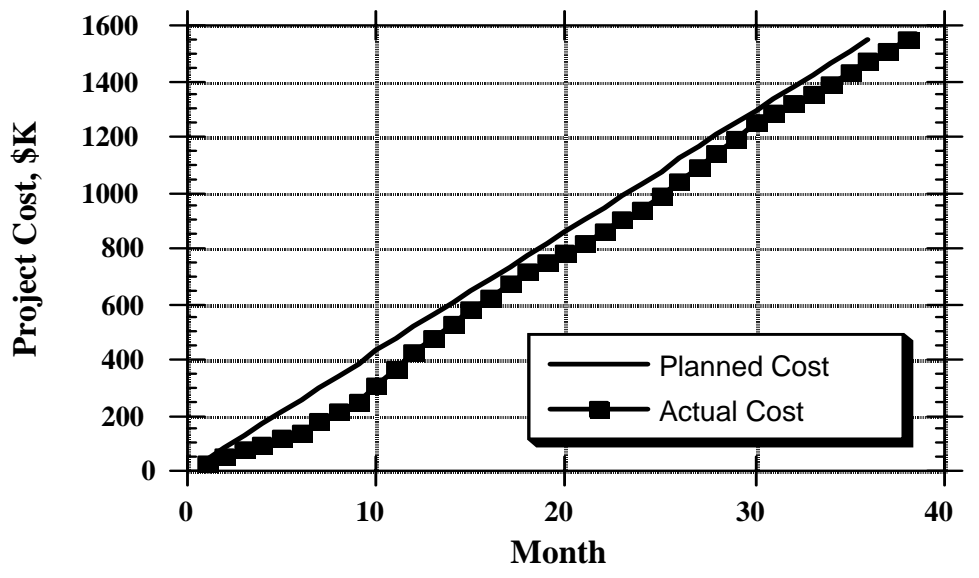




\section{Research Summary}

\section{Background}

Material degradation is a primary limitation for the safe and economical operation of commercial nuclear power reactors. ${ }^{1,2}$ Traditional approaches for alloy development have emphasized minor modifications in alloys (predominantly austenitic stainless steels) that were developed for use in non-irradiation environments. As problems were discovered during service, alloy characteristics or service conditions were adjusted to provide modest improvements in performance and hopefully achieve adequate component lifetimes.

However, it is now clearly recognized that such minor traditional alterations are not sufficient to mitigate radiation-induced property degradation (loss in ductility and toughness, void swelling and embrittlement) and environmental cracking (referred to as irradiation-assisted SCC or IASCC). Therefore, unconventional metallurgical approaches and alloys outside standard stainless steels must be adopted to optimize performance in the radiation and corrosive environments of next generation nuclear reactors.

Radiation-induced degradation is driven by the displacement of atoms from their lattice sites and the subsequent creation of interstitial and vacancy defects as shown in Figure 1. Defect recombination, migration and their annihilation at sinks promotes a wide variety of potentially detrimental material changes including dislocation loop formation that leads to extensive

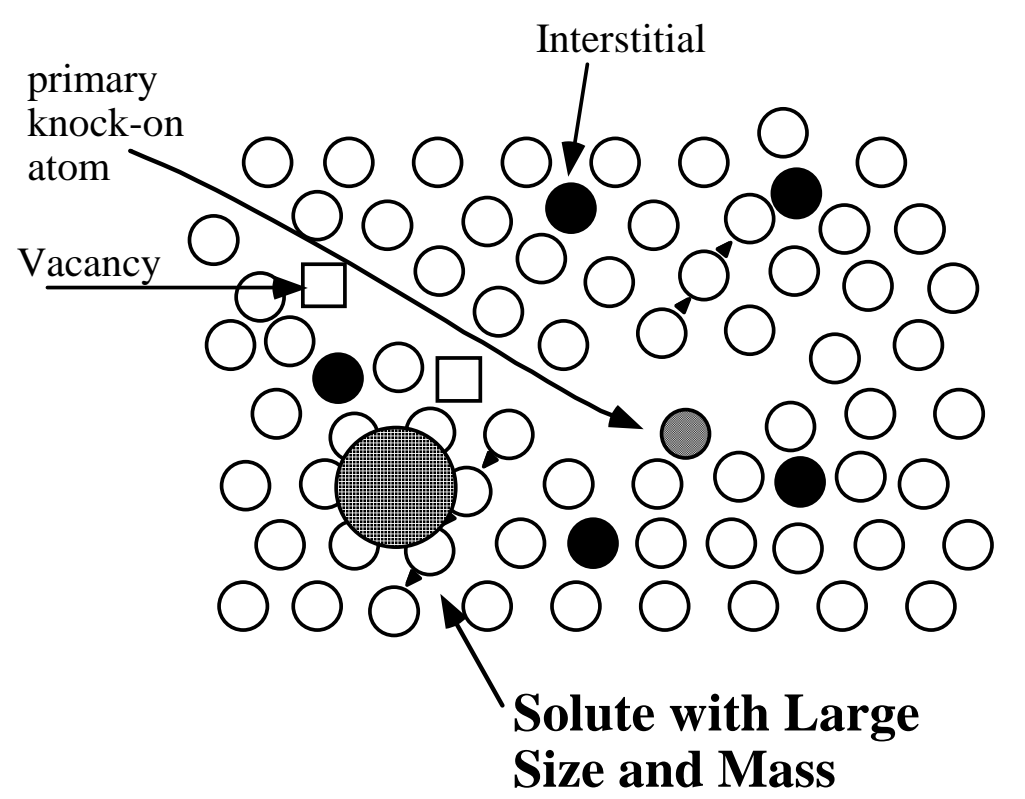

Figure 1. Schematic of the damage process for influence from a large size or large mass solute. The primary knock-on atom can be created with ions, protons or neutrons. Minor additions (3,000 appm) of massive oversized solute are being explored. Large elements in solution perturb the cascade production of defects and subsequent irradiation damage. 
hardening and limited uniform ductility, segregation of alloying and impurity elements to grain boundaries and other sinks, and void swelling. These changes are inevitable in standard stainless steels during LWR irradiation and result in an increasing susceptibility to failure with core component exposure. Displacement of atoms from irradiation cannot be avoided; they are the result of fundamental Newtonian mechanics. The path for aggregation of defects into detrimental microstructures can be avoided. The processes of migration and aggregation of displacement defects are illustrated in Figure 2. Atoms of large size or mass misfit and finescale precipitate distributions interfere with the processes of recombination, migration and aggregation.

Displacement events can be created by ion, proton or neutron particles. Heavy ions and protons have the advantage of producing damage at high rates and therefore are commonly used for fundamental studies of damage mechanisms. Neutrons have the advantage of producing damage in bulk materials and therefore have the advantage for studies of bulk properties that are relevant to assessment of structural integrity. In this research, heavy-ion irradiation is used first as a screening tool to assess damage microstructures in candidate alloys. Protons are used for additional microstructural studies and for studies of mechanical behavior and stress corrosion cracking. Specimens for neutron-induced damage are included in a collaborative research program using the Bor-60 reactor in Russia. The neutron-irradiated samples will not be examined under this NERI program. Damage from ions, protons and neutrons can be related

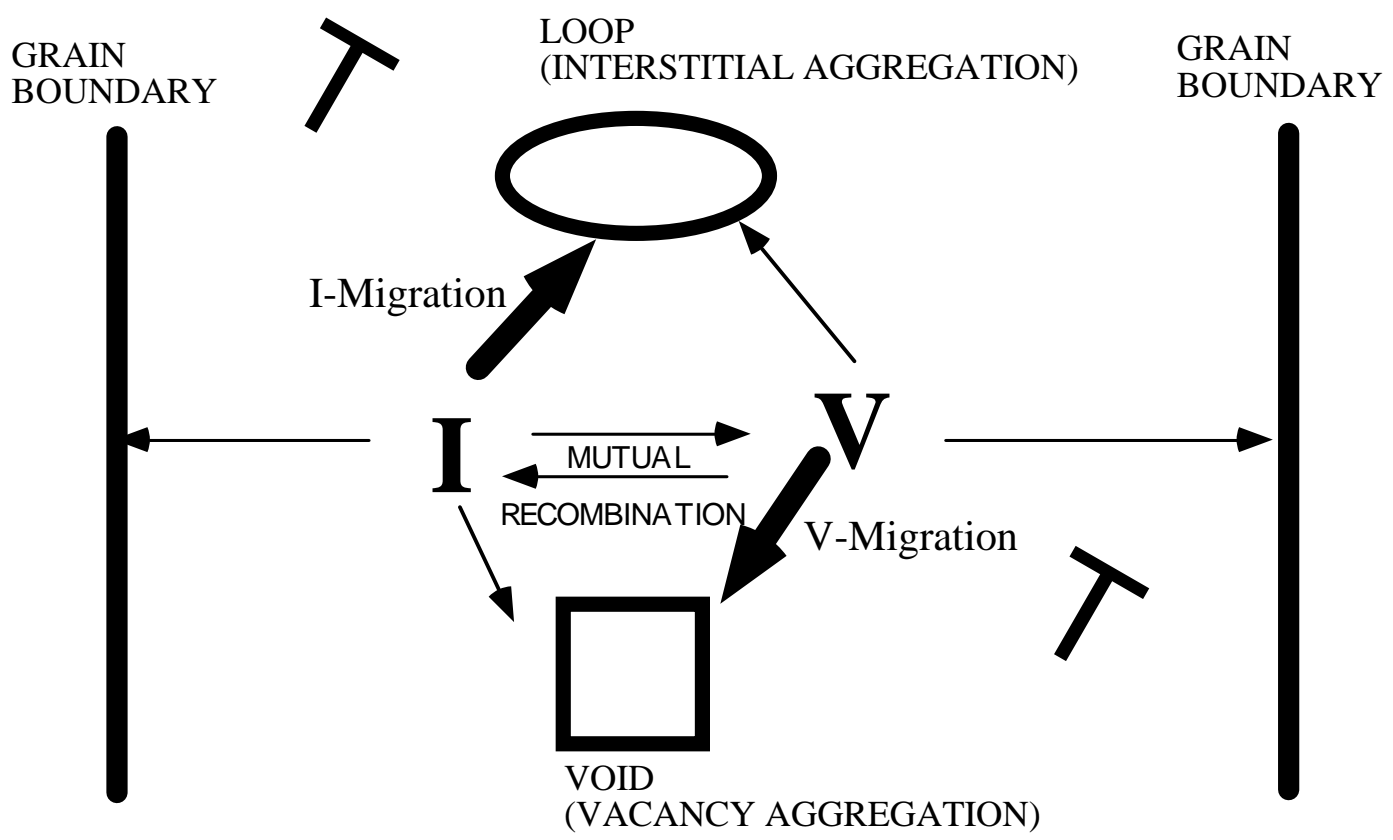

Figure 2. The fundamental products of displacement damage are interstitials (I) and vacancies $(\mathrm{V})$. These point defects migrate and collect in components of radiation damage. Interstitials collect preferentially in interstitial loops and the remaining supersaturation of vacancies collect in voids. Solute atoms of large size or mass misfit and preciptiates disturb the migration and aggregation of these point defects and therefore damage evolution is optimized. Damage can be induced using ions, protons or neutrons and can be characterized using transmission electron microscopy. 


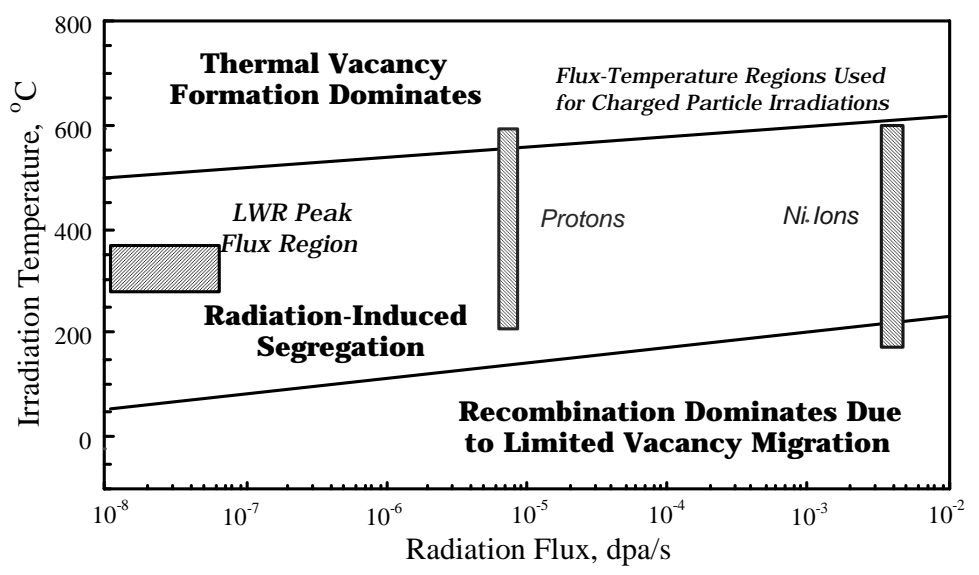

Figure 3. Increasing the rate of damage requires using a higher temperature to obtain equivalent damage microstructures. The lower temperature range is limited by mutual recombination and the upper temperature range is limited by the energy for vacancy formation. Equivalent ranges for neutrons, protons and ions are shown.

using a compensation of higher temperature for higher rate processes. The interdependence of damage rate, temperature and particle type is shown in Figure 3.

The development of radiation damage microstructures has two primary effects on components: (1) swelling and (2) mechanical behavior. Swelling is detrimental because the component dimensions change during service that affect stresses and core component dimensional tolerances. A primary concern in the present research is the affect on mechanical behavior and IGSCC. The effect of displacement damage on interstitial loop development, and on the increase in yield strength, for LWR-irradiated components has been documented. The measured increase in tensile yield strength is shown in Figure 4.

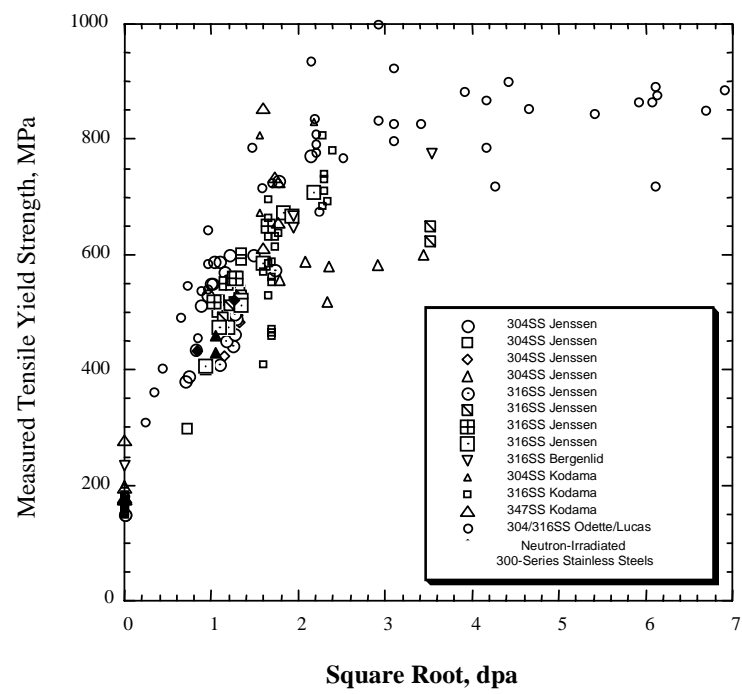

Figure 4. Irradiation Dose Effects on Measured Tensile Yield Strength for Several 300-Series Stainless Steels reported in the literature near $288^{\circ} \mathrm{C}$. Microstructures for several of these alloys were characterized at PNNL. 
A four-fold increase in yield strength occurs over the first few dpa of irradiation. The effect of this large increase in yield strength on IGSCC is a critical issue examined in this research.

The critical links between damage processes, materials characterization and property measurements are highlighted in Figure 5. Although the primary components of irradiation damage (vacancies and interstitials) are simple, their aggregation into the microstructure and influence on deformation, void swelling and fracture are complex processes requiring a complementary mix of experiments being conducted on this NERI program.

The above radiation damage mechanisms affect lifetime for components exposed to high irradiation doses as shown in Figure 6. Recent work has enabled many aspects of IASCC phenomenology to be explained (and predicted) based on the experience with IGSCC of nonirradiated stainless steel in BWR water environments. This continuum approach has successfully accounted for radiation effects on water chemistry and its influence on electrochemical corrosion potential. However, the specific radiation-induced microstructural and microchemical changes that promote IASCC susceptibility are largely unknown. Well-controlled IASCC data from properly irradiated, and properly characterized, materials is sorely lacking due to the affect experimental difficulties and financial limitations related to working with highly activated

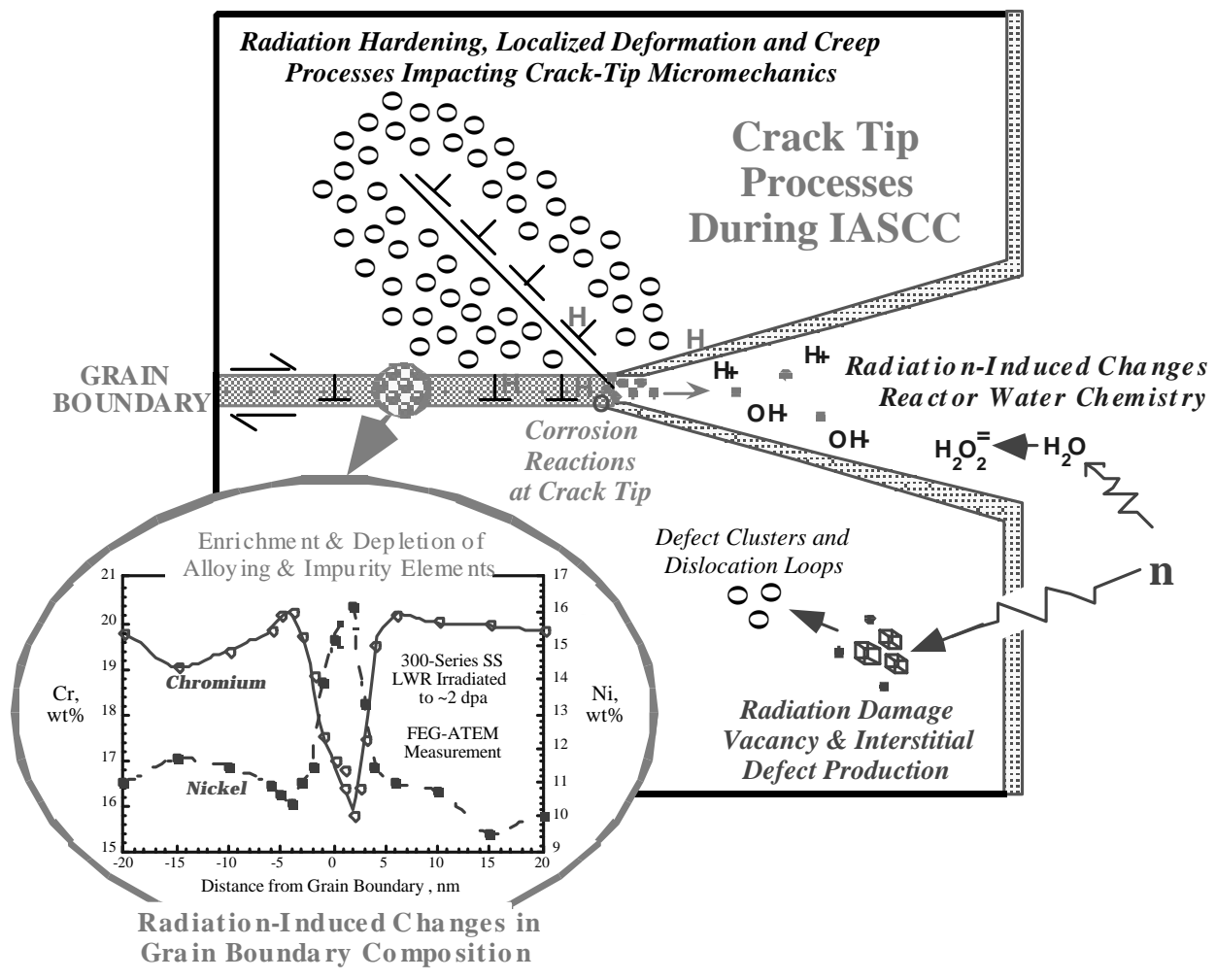

Figure 5. Schematic illustrating mechanistic issues believed to influence crack advance during IASCC. Understanding is advanced through alloy design, irradiation experiments (ion, protons and neutrons, microstructural and microchemical characterization, and measurements of mechanical and stress corrosion cracking properties. Interstitial aggregation results in loops that strength, which in turn affects SCC. Concurrent void production (not shown in the schematic) does not affect IASCC but does affect dimensional stability and component lifetime. 


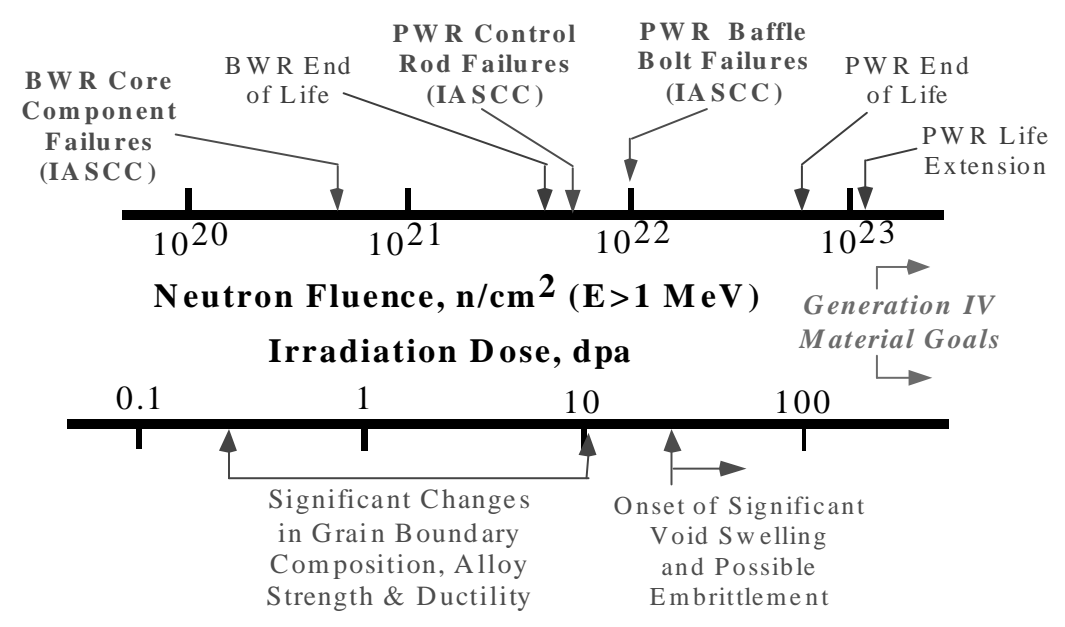

Figure 6. Neutron fluence effects on alloy characteristics and IASCC susceptibility of 304SS in LWR environments. Dose effects on mechanical behavior and swelling are also shown.

materials. The paramount need to understand and mitigate IASCC led to the formation of an internationally funded, Cooperative IASCC Research (CIR) project of which PNNL, GE and UM are participants. Many of the important metallurgical, mechanical and environmental aspects that are believed to play a role in the cracking process are illustrated in Figure 5. Since only persistent material changes are required for IASCC to occur, in-core processes such as radiation creep and radiolysis influence cracking, but are not controlling mechanisms. The current understanding of persistent material changes (dose affects) that are produced in stainless alloys during LWR irradiation is elucidated based on the fundamentals of radiation damage from experimental measurements.

\section{Research Approach}

The response of alloys to irradiation has been evaluated using a variety of irradiation experiments using neutrons, electrons, protons and heavy ions. Each irradiation technique possesses both advantages and disadvantages. Neutron irradiation is the preferred choice based on providing the practical environment of engineering interest. Conversely, neutron irradiation has the disadvantage of requiring expensive resources and many years for materials studies. PNNL has an extensive history in the conduct and interpretation of neutron and charged-particle experiments from mechanistic and alloy development efforts for fast-reactor and fusion-energy programs.

A productive approach for obtaining screening results has been to use charged particle irradiations for establishing the physical basis for phenomenological interpretation and mechanism development. Research conducted over the last ten years at PNNL has demonstrated that quantitative comparisons between $\mathrm{Ni}^{++}$ion and LWR neutron irradiations can be made for radiation-induced microstructural (defect clusters, dislocation loops and voids) and microchemical (grain boundary segregation) evolution. In addition, localized deformation and hardening behavior has been effectively investigated. Within this irradiation strategy, neutron irradiations are used to confirm and calibrate models for mechanistic interpretation and engineering applications. 
Proton irradiation techniques have been developed in parallel with heavy-ion irradiation techniques. Similar advantages and disadvantages occur for both. However, proton penetration depths are greater than for heavy ions $(40 \mu \mathrm{m}$ vs. $1 \mu \mathrm{m})$ making protons a preferred technique for observing "bulk" irradiation effects and mechanical property behavior. Proton PKA spectra are softer than heavy ions and neutrons but none-the-less many experimental correlations have indicated the usefulness of proton irradiation for simulating neutron irradiation effects. Recent work at UM has demonstrated that radiation microstructures (dislocation loops and voids), microchemistries (grain boundary segregation), hardening and IASCC susceptibility can be produced by proton irradiation (at $360^{\circ} \mathrm{C}$ ) in 300 -series stainless steels in a nearly identical fashion as for LWR neutrons (at $280^{\circ} \mathrm{C}$ ).

Standard mechanical property and SCC tests cannot be performed in ion-irradiated specimens because of the limited depth range of ions. Therefore, a strategy has been adopted based on using thermo-mechanical heat treatments of non-irradiated alloys to produce similar microchemistries and mechanical properties as expected in irradiated alloys. A continuum in SCC behavior has been demonstrated using irradiated and non-irradiated specimens of the same alloy type. Cold working on non-irradiated 300 series stainless steels is evaluated to demonstrate if material strengthening can promote enhanced IG crack growth rates without the need for grain boundary microchemical changes.

\section{Research Significance and Benefits}

The present research has the potential to eliminate one of the most significant economic and safety issues facing current and future nuclear power systems. This work integrates fundamental and applied science resources to design advanced core component structural materials with the required resistance to radiation and environmental degradation mechanisms. Thus, the research benefits a wide range of areas from fundamental science to applied alloy development. The research strategy and the combination of national laboratory, industry and university resources enables important NERI objectives to be achieved:

1) Advanced materials concepts are being developed and scientific breakthroughs facilitated to overcome a principal obstacle facing nuclear fission reactors. The focus on next generation LWR concepts will promote relatively near-term (within 10 years) benefits for the safe and economical operation of commercial nuclear power. This is essential considering the current status of the nuclear power industry worldwide.

(2) The effective integration of national laboratory (PNNL), industry (GE) and university (UM) team members focuses their scientific research and development infrastructures on nuclear energy issues and fosters a leveraged approach to address basic and mission-oriented research. Industry partners and collaborators ensure that radiation material science breakthroughs are effectively transferred to advance the state of nuclear technology. International cooperation and U.S. leadership in the research is ensured by the collaborative experimental activities as part of two separate multi-national projects led by EPRI. Principal investigators play an important role in the oversight of these projects and foster continuous interactions to transfer novel materials concepts into engineering application. 
(3) The unique research team and shared activities among national laboratory, industry and university promotes and maintains nuclear science and engineering to meet future technical challenges. The relationship among participants is one of the strengths of this proposal and fosters the development of new radiation materials science expertise through research activities by university students (UM) and junior staff (PNNL and GE) at laboratories.

(4) The objective of this research is to help solve critical materials degradation problems for current and next generation LWRs. The development of new damage-resistant alloys will allow advanced power reactors to be designed and operated for improved performance, efficiency, reliability and economics.

Task Objectives by Phase

The success of this program was achieved in three phases. In Phase 1, tailored alloys were produced to evaluate solute misfit influences on damage evolution at high doses. The progress was enabled through upgrades of irradiation facilities and methods for specimen characterization. Heavy-ion irradiation provided a rapid screening technique to assess damage followed by proton irradiation that allowed property evaluations. Simultaneously, thermo-mechanical treatments were used to fabricate specimens for measurement of crack-growth rates in for alloys having strength levels similar to irradiated steels. In Phase 2 , property evaluation of alloys studied in Phase 1 were conducted and experiments using tailored precipitate microstructures that show superior retention of the desirable pre-irradiation microstructures and properties were initiated. In Phase 3 , concluding studies of the two alloy types, i.e., with solute additions or multiphase precipitates, were finalized and mechanistic understanding was established to rationalize the beneficial on detrimental effects on damage evolution in the tailored alloys.

\section{Phase 1}

Phase 1 was completed and objectives achieved. Materials available from prior or on-going research efforts at PNNL and GE were obtained and samples were prepared for initial charged particle irradiation. In addition, capital improvements to accelerators to allow higher beam currents and dose rates at UM and to provide a new high-flux, high-temperature irradiation chamber at PNNL were completed. Alloys containing Pt additions or Hf additions were cast by GE and processed at PNNL to prepare samples for heavy-ion and proton irradiations. $\mathrm{Ni}^{++}$ion screening experiments were completed at PNNL to assess the influence of these solutes on radiation-induced microstructural and microchemical evolution. Proton irradiation and material characterizations at UM were initiated using the same alloys used at PNNL. Direct comparison were made between the UM results and existing PNNL work on these alloys. Testing at GE was conducted on a commercial 316L SS to quantify strength effects on SCC so that the influence of the misfit solute additions can be documented in Phases 2 and 3. This material is closely integrated into collaborative industrial research efforts on SCC behavior in LWR core component materials.

Phase 2

Heavy-ion irradiation screening experiments were completed and specific alloys were included in the proton-irradiation and SCC crack-growth test matrices. The most promising alloys were entered into the neutron-irradiation experiments as part of the collaborative EPRI-led, 
international research programs. Further work at PNNL was conducted on the oversized solute approach by altering composition to optimize defect recombination. Research at UM (radiation damage and SCC resistance of irradiated microstructures) and GE (SCC resistance of thermomechanically processed alloys to simulate radiation-hardened microstructures) were focused on the most promising alloys based on the screening tests. Examinations of alternative multiphase alloys with optimized metastable second-phase distributions were started at PNNL to indicate dynamic radiation phase stability.

$\underline{\text { Phase } 3}$

PNNL heavy-ion irradiation experiments and materials characterizations were completed. Dosedependent microstructural and microchemical evolution were documented out to $50 \mathrm{dpa}$. Mechanistic underpinning for these two approaches were established and rate theory modeling was used to investigate specific effects during point defect production, migration and annihilation as well as cascade formation and healing. UM proton irradiation, characterization and testing were completed on the optimized alloys to confirm low-dose radiation-induced material changes and effects on IASCC resistance. Specific alloys were selected for neutron irradiation to confirm improved damage resistance of optimized alloys and mechanistic aspects controlling this behavior. Crack-growth experiments at GE focused on second-phase microstructural effects (grain boundary carbides) in austenitic stainless steel. Thermomechanical treatments were used to simulate radiation-induced precipitate microstructures and strength levels to evaluate resistance to SCC.

\section{Detailed Description of Accomplishments}

This research has established that oversized elements affect radiation-induced microstructure and microchemistry. The comparison of behavior in alloys containing Pt addition and $\mathrm{Hf}$ addition reveals mechanistic insights for development of damage-resistant alloys. The experimental findings reveal that solute size, reactivity and distribution are critical characteristics affecting radiation damage. Key findings include (1) reduction in damage evolution for Hf addition but not for Pt addition, (2) radiation-induced segregation (RIS) for Hf addition reduced for protons but not for $\mathrm{Ni}^{++}$ions, (3) resistance to IASCC for proton-irradiated Hf-doped alloy, (4) multiphase alloys promote damage resistance compared to single-phase austenitic alloys of $\mathrm{Ni}$ and $\mathrm{Fe},(5)$ strength in the absence of sensitization promotes crack growth in non-irradiated alloys (favors irradiation strengthening mechanism for IASCC), (6) hydrogen permeation (and implied transmutation hydrogen) does not promote environmental cracking, and (7) grain boundary carbides decrease cracking susceptibility if interfacial composition is controlled.

Mechanistic Conclusions - Damage Analysis

Fundamentally, radiation effects are dominated by the production, recombination and understood in terms of in-cascade recombination, intra-cascade recombination and partitioning of defects annihilation of vacancies and interstitials. The mechanistic influence of oversized solute is into accumulating microstructure. The roles of these mechanisms are elucidated in the present comparison experiments. The following discussion describes mechanistic conclusions relating oversized solute effects.

In-cascade recombination is not significant because the radiation response using heavy $\mathrm{Ni}^{++}$ions 
was observed to be similar to that for light protons. $\mathrm{Ni}^{++}$ions produce point defects mainly in large cascades whereas protons produce damage mainly in isolated pairs, which is a consequence of the bombarding particle mass. If in-cascade recombination is dominant then the damage induced with $\mathrm{Ni}^{++}$would have been much less than that induced with protons. Furthermore, RIS modeling indicates that the necessary reduction in damage efficiencies must be two orders of magnitude less for the doped alloys compared to the base alloy to explain measured segregation results. The assumed low damage efficiency cannot be justified based on independent experiments.

Rate theory calculations of defect production, recombination and annihilation indicate that binding between oversized solute and vacancies likely does not cause reduction in RIS observed with proton irradiation. Model calculations show that the reduced RIS cannot be attributed to recombination cause by slow vacancy mobility. Furthermore, the existence of precipitates in the Hf-doped alloy does not impact intra-cascade recombination. The precipitate sizes and number densities are not high enough to compete with alternative mechanisms. Also, the similar RIS behavior for the Hf-doped alloy and the Pt-doped alloy indicates that Hf precipitates are not important for control of RIS.

Remarkably, specific solute effects on RIS do not parallel effects on microstructural evolution. This implies that solute size alone does not control production of radiation microstructure. Based on size, $\mathrm{Pt}$ is expected to have an intermediate effect on damage when compared to the base 316SS and the Hf-doped alloy. The influence of Pt addition (10\% misfit) on microstructural evolution was not significant in contrast to the dominant influence of Hf addition (26\% misfit). Hf-doped alloys developed smaller interstitial loops and lack of voids when compared to the Pt-doped alloy and the base 316SS alloy. These observations support a mechanism for Hf influence related to suppression of loop growth and suppression of void nucleation. The extreme size misfit for $\mathrm{Hf}$ and the reactive chemical nature of $\mathrm{Hf}$ likely impart the damage resistance observed in this study. The beneficial microstructures impart deformation characteristics that result in a more ductile alloy and greater resistance to IASCC initiation in slow-strain-rate tests.

The multiphase alloys exhibited reduced damage compared to single-phase alloys. This benefit is likely due to the extremely high non-irradiated, matrix sink strength that exists at the throughout the irradiation history. These multiphase Ni-base and Fe-base alloys are sufficiently complex that quantitative analyses of kinetic mechanisms are not practical.

Studies of non-irradiated alloys are useful for interpreting cracking behavior of irradiated alloys. The observation of IGSCC enhancement with cold-work strength suggests that strength per se is more important that the mechanism for strengthening. Details in deformation mode of irradiated alloys may not be important for understanding IGSCC crack growth, but may play a role in crack initiation. Interestingly, precipitates on grain boundaries may improve cracking resistance. Therefore, the design of alloys resistant to IASCC may include options of promoting grain boundary precipitation.

The processing, irradiation, characterization and property measurements that support these conclusions are detailed in the following sections. 


\title{
Oversized Elements
}

\author{
Materials Processing
}

Three high-purity austenitic stainless steel alloys supplied by GE were used in this study. The alloys consisted of a 316L SS and two alloys with the same composition but doped with 1.0 wt.\% Pt (316SS+Pt) or $0.915 \mathrm{wt} . \% \mathrm{Hf}$ (316SS+Hf). The compositions of these three alloys are given in Table 1. As-received alloys were solution annealed at $1200^{\circ} \mathrm{C}$ for 1 hour and water quenched prior to $70 \%$ cold working. Cold working was followed by annealing at $900^{\circ} \mathrm{C}$ for 20 minutes and water quenching to obtain a grain size of about $10 \mu \mathrm{m}$. Hafnium-rich precipitates with a density of $8.6 \times 10^{20} \mathrm{~m}^{-3}$ and a mean size of $25 \mathrm{~nm}$ were observed in the 316SS+Hf alloy annealed at $900^{\circ} \mathrm{C}$ for 20 minutes. In addition to the heat treatment at $900^{\circ} \mathrm{C}$ for 20 minutes, the alloy $316+\mathrm{Hf}$ was also heat treated at $1100^{\circ} \mathrm{C}$ for 30 minutes to put additional $\mathrm{Hf}$ back in solution. This heat treatment resulted in a grain size of about $20 \mu \mathrm{m}$, a decrease in the Hf-rich precipitate density to $1 \times 10^{19} \mathrm{~m}^{-3}$ as well as an increase in mean size to $129 \mathrm{~nm}$. The $1100^{\circ} \mathrm{C}$ heat treatment temperature was chosen based on a balance between increased grain size and reduced precipitate microstructure.

Table 1. Oversized Solute Alloy Compositions in wt $\%$.

\begin{tabular}{lllllllll}
\multicolumn{1}{r}{ Alloy } & $\mathrm{Cr}$ & $\mathrm{Ni}$ & $\mathrm{Mn}$ & $\mathrm{Mo}$ & $\mathrm{Si}$ & $\mathrm{C}$ & Other & Treatment \\
& & & & & & & & \\
316L SS & 17.3 & 14 & 1.2 & 2.2 & 0.08 & 0.020 & Base $316 \mathrm{~L}$ & $900^{\circ} \mathrm{C} / 20 \mathrm{~min}$ \\
316L+Pt & 17.3 & 14 & 1.2 & 2.2 & 0.08 & 0.020 & $+1.0 \% \mathrm{Pt}$ & $900^{\circ} \mathrm{C} / 20 \mathrm{~min}$ \\
316L+Hf & 17.3 & 14 & 1.2 & 2.2 & 0.08 & 0.020 & $+0.915 \% \mathrm{Hf}^{*}$ & $900^{\circ} \mathrm{C} / 20 \mathrm{~min}$ \\
316L+Hf mod. & 17.3 & 14 & 1.2 & 2.2 & 0.08 & 0.020 & $+0.915 \% \mathrm{Hf}^{*}$ & $1100^{\circ} \mathrm{C} / 30 \mathrm{~min}$
\end{tabular}

Photomicrographs of grain structures were obtained using scanning electron microscopy (SEM) and defect microstructures using transmission electron microscopy (TEM) for the non-irradiated material conditions. For the same heat treatment, the Pt-doped alloy has grain sizes smaller than the base alloy and the Hf-doped alloy has the smallest grain sizes. A low density of network dislocations is observed with precipitates in the Hf-doped alloys. Prior to Ni-ion irradiation the samples were wet-polished with $\mathrm{SiC}$ paper to 600-grit, diamond abrasive film to 3-mm and a final polish with $0.3-\mathrm{mm}$ alumina. For the proton irradiation, the specimens were polished to a 4000 -grit $\mathrm{SiC}$ paper finish and then electropolished in a $60 \%$ phosphoric acid and $40 \%$ sulfuric acid solution at $45^{\circ} \mathrm{C}$ for 3 minutes at $30 \mathrm{~V}$.

\section{Irradiations}

Initial irradiation response was mapped using $\mathrm{Ni}$ ions to high dose. These low-to-high dose irradiations explored microstructural and microchemical evolution and the influence of solute addition. Proton irradiations were conducted at lower dose to evaluate radiation effects on environmental cracking and hardness in addition to microstructure and microchemistry. The temperatures for Ni-ion and proton irradiations were chosen to compensate for the damage rate difference in order to produce irradiation damage in materials relevant to LWR cores. ${ }^{2}$ Lastly, 
alloys in this program were included in neutron irradiation experiments for future characterization activities.

\section{Heavy-Ion Irradiation}

Nickel ion irradiation was performed using a 3.4 MeV ion accelerator at PNNL's Environmental and Molecular Sciences Laboratory. This NERI research program is the first to use this accelerator for controlled high-temperature displacement damage experiments. Nickel ions are produced from solid Ni sources using $\mathrm{Cs}^{+}$sputtering in the SNICS II (Source of Negative Ions by Cesium Sputtering) ion source. The negative Ni ions are injected into the accelerator by an injector magnet at the low energy beam line. The low energy beam line is equipped with several other components that include an electrostatic x-y steerer to steer the beam, a beam profile monitor to measure the profile of the ion beam, a Faraday cup for current measurements, and an einzel lens for focusing.

The accelerator is a National Electrostatics Corporation (NEC) model 9SDH-2, 3.4 MV tandem electrostatic accelerator equipped with two Pelletron charging chains capable of carrying 300 $\mathrm{mA}$ charging current to the terminal. The accelerated ions can be focused through the highenergy beam line using a magnetic quadrupole and a y-axis electrostatic steerer, which are attached to the high-energy beam line.

The beam line is equipped with a single slit followed by a matched set of two slits to control the divergence of the ion beam; a quadrupole magnet at the focal position of the switching magnet to enhance the focusing capability of the beam line; $\mathrm{x}$ and $\mathrm{y}$ electromagnetic steerers; a Faraday cup for current measurement; and a beam profile monitor to monitor the beam profile. Differential pumping is included in the beam line and apertures so that the end station can be kept in the low $10^{-10}$ torr pressure range. Turbo pumps are attached to all beam lines and ion sources. Typical base pressure in the low energy beam line is $1 \times 10^{-8}$ torr to $2 \times 10^{-8}$ torr, and the mid-to-high $10^{-9}$ torr range in the high-energy beam lines. When the accelerator is in operation, typical pressures in the high-energy beam lines are in the low-to-mid $10^{-8}$ torr range.

Specifically in this NERI program, $5 \mathrm{MeV} \mathrm{Ni}^{++}$ions are used at a current density of $90 \mathrm{nA}$ on an irradiated area of $7 \times 7 \mathrm{~mm}^{2}$. Using the TRIM 2000 code, ${ }^{4}$ the calculated displacement rate is $4.73 \times 10^{-4} \mathrm{dpa} / \mathrm{s}$ at a depth of $0.5 \mu \mathrm{m}$. The calculated damage depth profile is shown in Figure 7. At this rate, $1 \mathrm{dpa}$ is obtained in 35 minutes. The beam current is increased by a factor of 4 for high dose irradiation, i.e., $50 \mathrm{dpa}$. The dpa calculations are based on the full cascade detailed calculation assuming a displacement energy of $40 \mathrm{eV}$ and an alloy composition for $316 \mathrm{SS}$.

The sample temperature was monitored using a thermocouple and an optical pyrometer. Calibration experiments were performed to ensure a temperature within $10^{\circ} \mathrm{C}$ of the planned irradiation temperature of $500^{\circ} \mathrm{C}$. The beam profile was monitored using slits that indicate the symmetry of the beam and the assurance of a constant beam current.

\section{Proton Irradiation}

Proton irradiations were conducted at the Michigan Ion Beam Laboratory at the University of Michigan to nominal doses of 2.5 and 5.0 dpa were conducted at $400^{\circ} \mathrm{C}$ with $3.2 \mathrm{MeV}$ protons at 


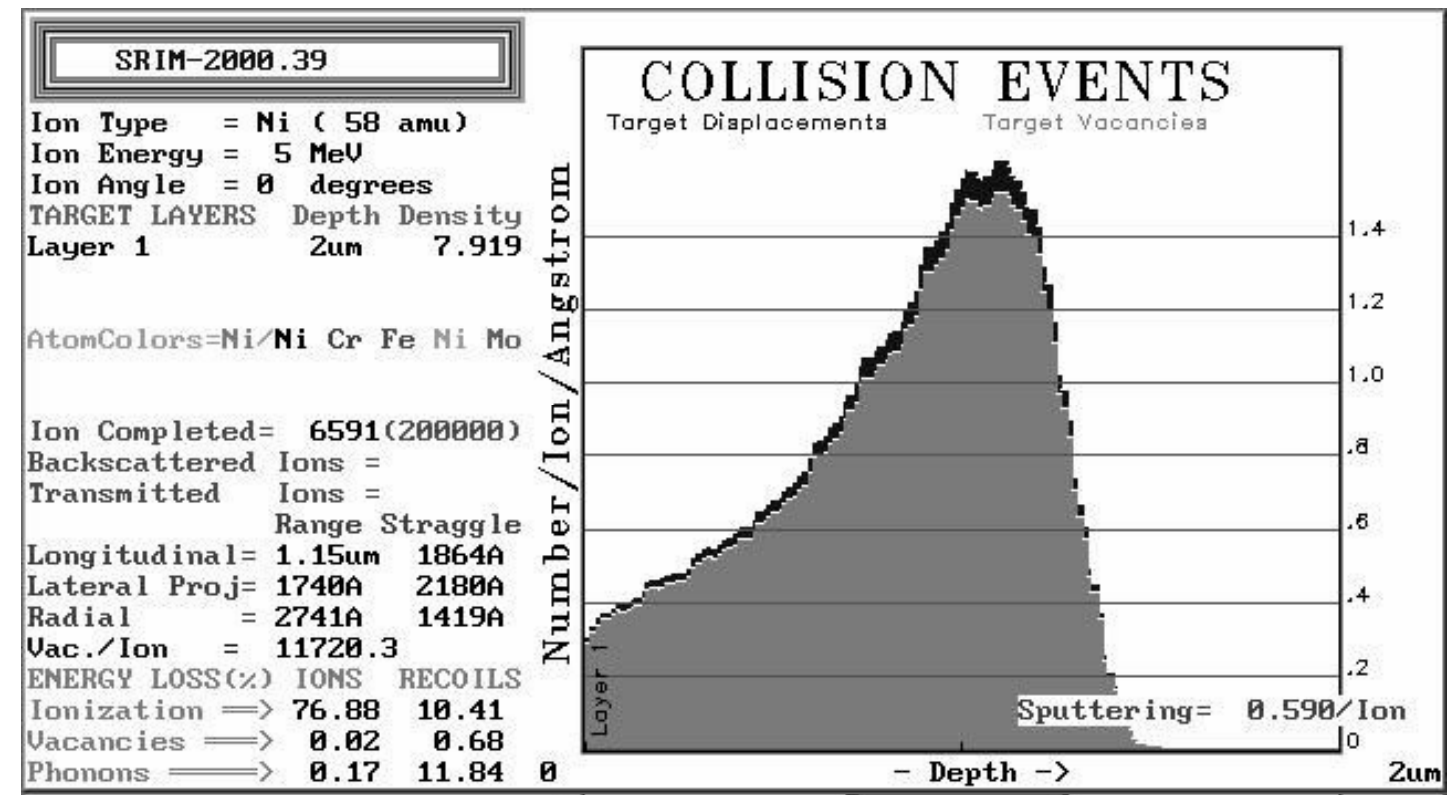

Figure 7. The depth distribution of damage based on the TRIM 2000 code. The ion range is $1.15 \mu \mathrm{m}$. In this study, microstructures were examined at a depth of $0.5 \mu \mathrm{m}$.

a dose rate of approximately $1.7 \times 10^{-5} \mathrm{dpa} / \mathrm{s}$ (based on a displacement energy of $40 \mathrm{eV}$ ), resulting in a nearly uniform damage rate throughout the first $35 \mu \mathrm{m}$ of the proton range $(40 \mu \mathrm{m})$. For proton irradiation, the experimental doses and dose rates were calculated using TRIM97. ${ }^{5}$ The displacement damage profiles for both $3.2 \mathrm{MeV}$ protons and 5.0 MeV nickel ions calculated with TRIM97 are displayed in Figure 8. As recommended in ASTM E 521-89 [3], a displacement energy of $40 \mathrm{eV}$ was used for all doses and dose rates calculations. Two types of samples were used for proton irradiation: TEM bars and SCC specimens. Drawings of sample designs are provided in Figure 9. All samples were fabricated by electric discharge machining. Prior to irradiation, specimens were mechanically wet-polished using SiC paper (300-4000 grit) and then electropolished for 3 minutes in a $60 \%$ phosphoric acid $-40 \%$ sulfuric acid solution. Samples were mounted on a specially designed irradiation stage attached to the main target chamber of a General Ionex Tandetron accelerator. Temperature control was achieved by mounting the samples on a copper block with an indium liquid metal coupling to facilitate heat conduction between the samples and the stage. A schematic of the irradiation stage is given in Figure 10.

In order to reach the higher dose requirements of this research program, a TORVIS ion source from National Electrostatics Corporation was installed. The new ion source provides greater beam current and higher reliability than the older duoplasmatron source. The system was installed during July 2000 with significant assistance of several NERI projects at UM. The source specification called for $\mathrm{H}^{+}$currents of $300 \mu \mathrm{A}$ in the low energy section of the accelerator. Given the transmission character of the accelerator tube, a maximum of $100 \mu \mathrm{A}$ to be transmitted to the target end was expected. This is well beyond the value needed to conduct high dose rate irradiations. Thus far, up to $50 \mu \mathrm{A}$ has been delivered to the samples, which represents a $3 x$ increase over what was achievable with the old source. The TORVIS source could support a $5 x$ increase but prompt radiation fields heat removal from the irradiation stage are limiting. 


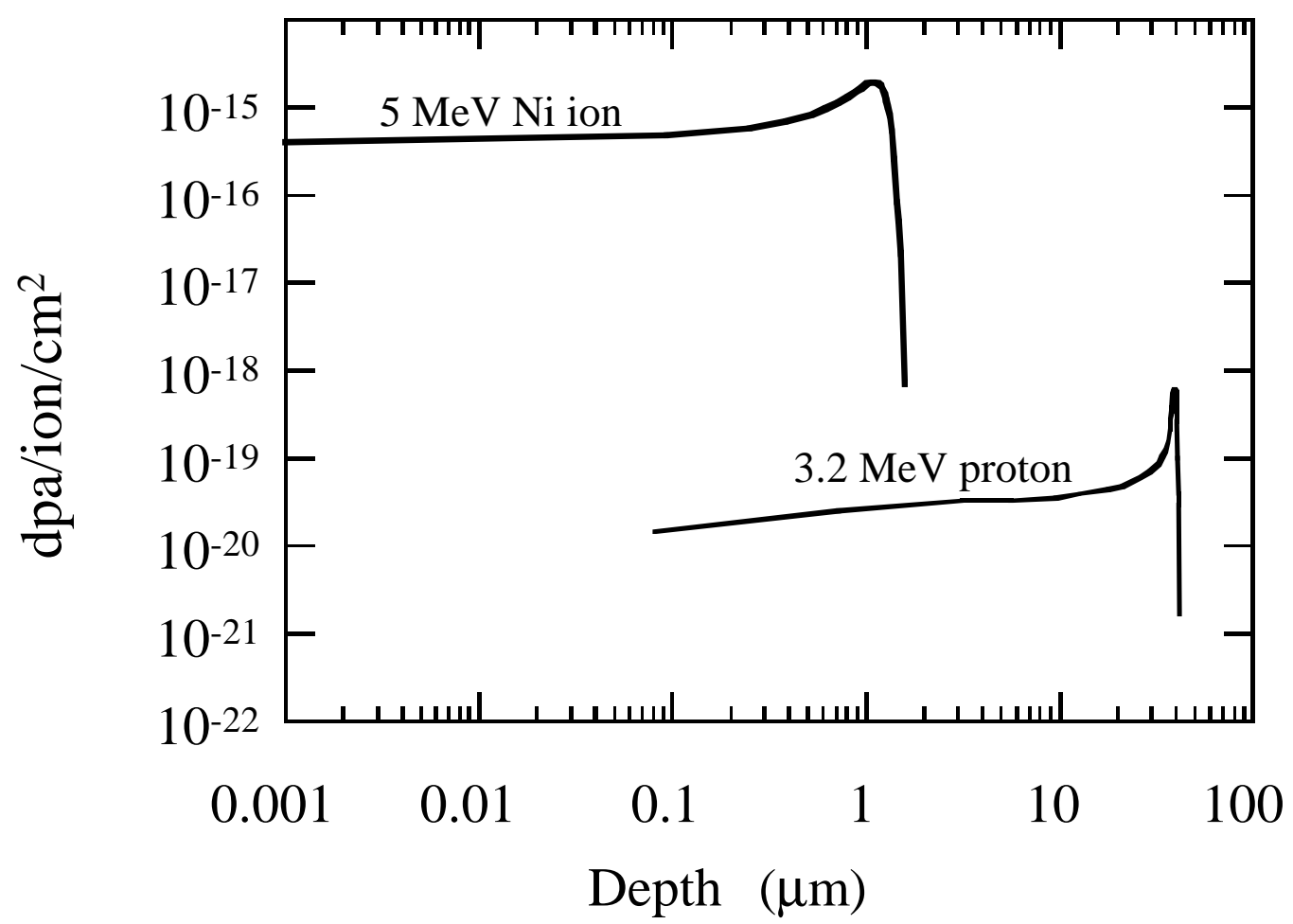

Figure 8. Damage profile comparison between $5 \mathrm{MeV} \mathrm{Ni}$ ions and 3.2 $\mathrm{MeV}$ protons.

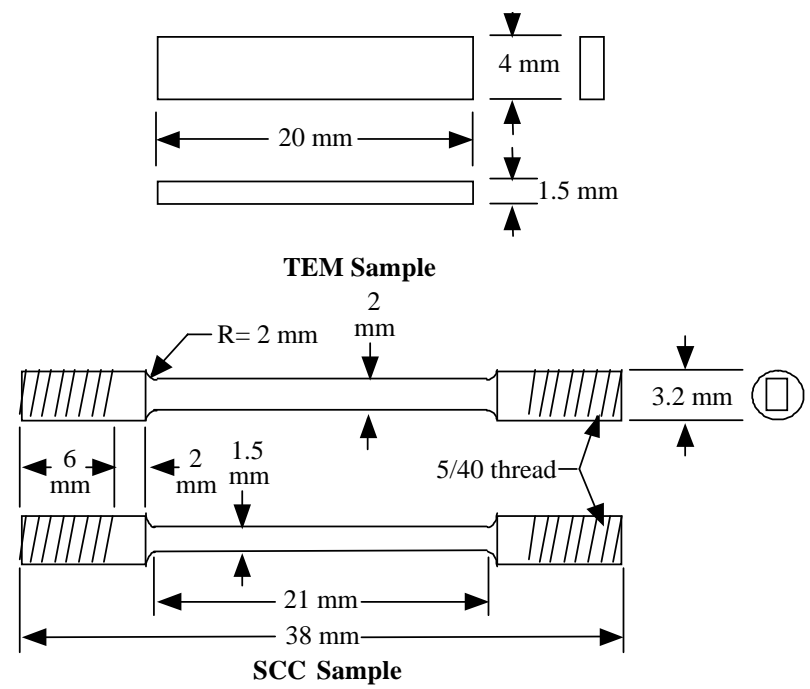

Figure 9. Geometries of samples irradiated for TEM examination and for SCC testing. 
The irradiation stage was electrically isolated from the beam line and four rectangular tantalum apertures were used to define the area on the sample bars that was irradiated with the proton beam. The approximately 3-mm-diameter proton beam was rastered across the stage so that about half the total beam current was deposited on the samples and half on the apertures. This rastering ensured that samples at any position on the stage received the same dose. Additionally, balancing the amount of current on each of the apertures centered the proton beam.

The irradiation stage was designed to control the sample temperature by controlling the stage temperature. The stage was heated using a resistive cartridge heater and cooled using room temperature air flowing through cooling lines that penetrated the back of the stage. The stage surface is made of copper to provide good heat conduction away from the samples. To provide effective thermal contact between the sample bars and the stage, a thin layer of indium was placed between the samples and the stage surface. Typically, three to five thermocouples were attached to the samples during any one irradiation. A separate thermocouple monitored the temperature at the back of the stage. In addition to the thermocouples, a calibrated infrared pyrometer monitored the surface temperature of the samples during irradiation. The pyrometer was also controlled remotely to scan the surface of the specimens to insure a uniform temperature. The pyrometer was calibrated prior to irradiation by heating the samples with the cartridge heater to the set point temperature and adjusting the pyrometer's emissivity setting so that the pyrometer reading matched that of the thermocouples. During irradiation, the sample temperature was controlled to $\pm 10^{\circ} \mathrm{C}$ of the set point temperature $\left(400^{\circ} \mathrm{C}\right)$ by controlling the amount of heating and/or cooling provided to the stage. By providing a large fraction of the total heat input to the samples from the cartridge heater, temperature fluctuations due to fluctuations in beam current were minimized.

Experimental parameters were tracked continuously during irradiation using a PC-based monitoring system. The monitoring software recorded the stage current, current for each of the apertures, pyrometer temperature and up to five thermocouple temperatures. This system allowed the operator to continuously monitor experimental parameters while also providing a comprehensive history of each irradiation. Alarms were installed to alert the operator when experimental parameters moved outside acceptable limits.

After irradiation, the samples were allowed to "cool" for 3-7 days to allow for the short-lived isotopes to decay. The majority of the residual radioactivity was in the form of $\gamma$-decay and was measured using a gas proportional detector. The activity of each sample was normalized to the sample area and compared the activity of other specimens from the irradiation batch to determine the dose uniformity. Measurements were also compared against a database of measurements and the resulting empirical model to determine that the total dose was correct.

\section{Neutron Irradiation}

A critical aspect in the development of new radiation-resistant, reactor structural alloys is obviously establishing alloy performance after high-dose neutron irradiations. Time and cost made it impractical to include work on such materials as part of this NERI program. The long lead times for neutron irradiations and high cost for handling, preparation and testing in hot cells make their examination in a short-term (3-year) program of limited resources impossible. 

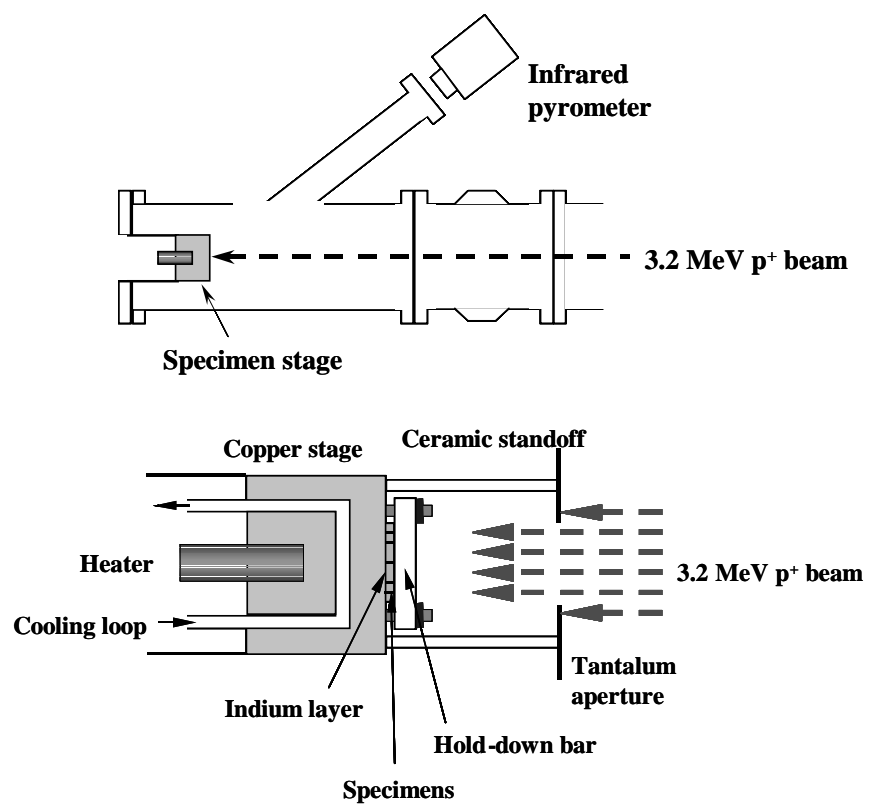

Figure 10. Detailed diagram of the proton irradiation technique.

However, it was imperative that neutron irradiation activities be started. To that end, negotiations were carried out with two international research initiatives, the Cooperative IASCC Research (CIR) project and the Joint Owners Baffle Bolt (JoBB) project. Both of these projects focus on LWR core component issues and have been organized by EPRI. Collaborations were established enabling access to fast reactor irradiations at the BOR-60 fast reactor located at the Research Institute of Atomic Reactors in Dimitrovgrad, Russia. Samples were placed in the BORIS irradiation vehicle, a high-flow rate, insulated, heat pipe subassembly in the BOR-60 reactor. Such a design yields very low temperature rises through the assembly, allowing a nearly isothermal distribution throughout the subassembly. Oversized solute alloys investigated and optimized in this program have been inserted in three successive BORIS neutron irradiations for possible future study. It is anticipated that promising results from this program on charged particle irradiated alloys will guide critical characterizations on neutron-irradiated materials during a future project.

The first irradiation phase, BORIS 5, was planned in 1999 just as this NERI project was starting. In collaboration with our DOE Basic Energy Science (BES) project on IASCC, disc samples were prepared, encapsulated and submitted for irradiation in February 2000. Two capsules were inserted into BORIS 5 for irradiation to $20 \mathrm{dpa}$ at $330^{\circ} \mathrm{C}$ and will provide a reference for correlating the present high-dose ion irradiations with neutron irradiation. Each capsule contained 104 3-mm discs for subsequent microstructural/microchemical characterizations (TEM, density and transmutation) and mechanical property examinations (hardness and shear punch). Six commercial and 16 high-purity laboratory heats were included in the alloy matrix focused on single-element additions. Important to this NERI project were oversized element additions of $\mathrm{Hf}, \mathrm{Pt}$ and $\mathrm{Ti}$ as well as isolating effects of C, N, P and $\mathrm{Si}$ in 300-series stainless steels. One of the two sample capsules was removed from reactor in April 2001 (sample dose of $\sim 20 \mathrm{dpa}$ ), while the second capsule was included in the next irradiation cycle, Boris 6 . The intent is to continue the irradiation of this second capsule through the following Boris 7 
irradiation and reach a final dose of approximately $70 \mathrm{dpa}$. As part of the EPRI-international projects, the 20-dpa capsule irradiated in BORIS 5 has been shipped to Westinghouse and should reach PNNL by December 2002.

The second irradiation phase, BORIS 6, required that samples be prepared by February 2001. At that time the NERI project heats, 316L SS, 316L SS+Pt and 316L SS+Hf, were available. These materials were prepared and encapsulated in February 2001 for irradiation at doses of 5, 10 and 20 dpa. In addition, single-element addition alloys (304LSS plus additions of Ti, Nb, C, P and $\mathrm{Si}$ ) were included in this matrix. Once again, 3-mm disc samples were put into reactor with two capsules being removed after 5, 10 and 20 dpa. The low-dose condition was removed in late 2001, the intermediate dose in mid-2002 and the highest dose (20 dpa) should be removed by March 2003. Current plans are to continue one of the two 20 dpa capsules through the next irradiation cycle reaching a dose of $40 \mathrm{dpa}$. Disposition of the neutron-irradiated disc samples (about 500 in 5 capsules) is uncertain at present, since the majority of these samples are tailored to meet needs for the EPRI-CIR project. It is anticipated that these samples will be available in early 2004. We have an agreement to receive all samples related to our NERI (and BES) project interests and will need to arrange shipment at that time.

The final neutron irradiation phase, BORIS 7, is scheduled to start in mid 2003 and be completed by the end of 2004. Because of the promising results on the 316L SS+Hf alloy within this NERI project, the EPRI CIR project was willing to add two compact-tension (CT) SCC crack-growth samples and six tensile samples into the irradiation matrix. The tensile samples will be tested at the Research Institute of Atomic Reactors, while the disposition of the CTs has yet to be decided by the EPRI-CIR sponsor group. In addition, a new series of oversized element alloys were included as 3-mm discs with a focus on multiple conditions for the radiation-resistant 316L SS+Hf alloy. It is anticipated that this final irradiation will be completed in late 2004 and be ready for shipment in 2005. At this time, the 70-dpa capsule started in BORIS 5 and the 40-dpa capsule started in BORIS 6 will be available along with new materials at 20 dpa started in BORIS 7.

\section{Characterization of Radiation-Induced Microstructures}

Radiation-induced microstructure evolution in both Ni-ion irradiated specimens and protonirradiated specimens was characterized using transmission electron microscopy. The dominant microstructural features observed for all specimens were faulted dislocation loops and voids. Voids were imaged in bright field mode while faulted loops were imaged by the rel-rod technique. Rel-rods are associated with thin planar defects and Frank loops can be imaged by the rel-rod technique, down to very small sizes $\leq 1 \mathrm{~nm}$. Smaller faulted Frank loops are either not visible in the rel-rod image or cannot be conclusively identified. ${ }^{6}$

\section{Transmission Electron Microscopy Procedures}

Ni Irradiation at PNNL

Damage resistance was characterized using JEOL 2010F high-resolution analytical TEM and a JEOL 2000 TEM. A particular challenge for examining ion-irradiated samples is the preparation of near-surface regions to obtain well-defined dose levels. The depth of penetration of $5 \mathrm{MeV}$ $\mathrm{Ni}^{++}$ions into stainless steel is limited to about $1.5 \mu \mathrm{m}$ as shown in Figure 7. The optimum 
depth for examination is about $0.5 \mu \mathrm{m}$. At depths near $1 \mu \mathrm{m}$, the damage gradient is steep and therefore determination of the local dose is difficult. At depths less than $0.3 \mu \mathrm{m}$, the near presence of the surface influences the local damage causing it to be atypical of bulk radiation damage.

Prior to examination of irradiated samples, a technique was developed for precise thinning. The procedure included variations in polishing electrolyte composition, electric potential and geometry of the sample relative to the jet-polishing stream. After each variation in condition, the measured depth of material removed was established using a computerized Zygo interferometer. The interferometer displays the surface topology in a three-dimensional color format. An example of the polished crater is shown in Figure 11.

The projection enables precise evaluation of the depth and uniformity of polishing craters on the alloy surface. Once a large uniform area of depth is established, samples are thinned from the backside to perforation. This creates a significant area of electron transparent material for TEM examination.

After each irradiation, the sample was electropolished using a single-jet tenupol and a solution of $2 \%$ perchloric acid $+15 \%$ ethylene glycol $+83 \%$ methanol held at $-60^{\circ} \mathrm{C}$ and $25 \mathrm{~V}$ for $5 \mathrm{~s}$. This removed a $0.5-\mu \mathrm{m}$ layer from the irradiated surface and was followed by back-thinning from the non-irradiated side at $-55^{\circ} \mathrm{C}$ and $45 \mathrm{~V}$ till perforation.

Proton Irradiation at UM

Proton irradiated bar samples were wet polished from the nonirradiated side using 320 grit SiC paper to obtain a thickness of about $300 \mu \mathrm{m}$. TEM disks were cut from the thinned bar and further thinned to about $120 \mu \mathrm{m}$ using 400-2400 grit SiC polishing paper. Final thinning to TEM transparency was performed using a jet-polish solution of 5 vol. $\%$ perchloric acid and 95 vol $\%$ methanol at $-65^{\circ} \mathrm{C}$ for an applied current between $15-18 \mathrm{~mA}$. After thinning, an acetone and methanol rinse removed any chemical residue on the sample surface. Characterization was performed using a JEOL 2000FX TEM and direct correlations were established between measurements at UM and PNNL.

\section{TEM Measurements of Defect Microstructures}

The generally expected sequence of radiation-induced microstructural evolution was observed for these alloys with solute additions. The nucleation and growth of interstitial loops followed by the nucleation and growth of voids with increasing dose is supported by numerous observations in the literature. The thesis of the present research was confirmed indicating that a low concentration of large misfit atoms can alter the microstructural evolution path. Remarkably, void swelling was completely suppressed to a dose of 50 dpa in the 316+Hf alloy with the modified heat treatment.

The Hf-containing alloy exhibited a high density of precipitates after the low-temperature $\left(900^{\circ} \mathrm{C}\right)$ heat treatment. This fine-scale distribution transformed to a coarse-scale distribution and a much lower density after high-temperature $\left(1100^{\circ} \mathrm{C}\right)$ heat treatment. The heat treatment clearly affected the damage resistance of this alloy as will be demonstrated. 

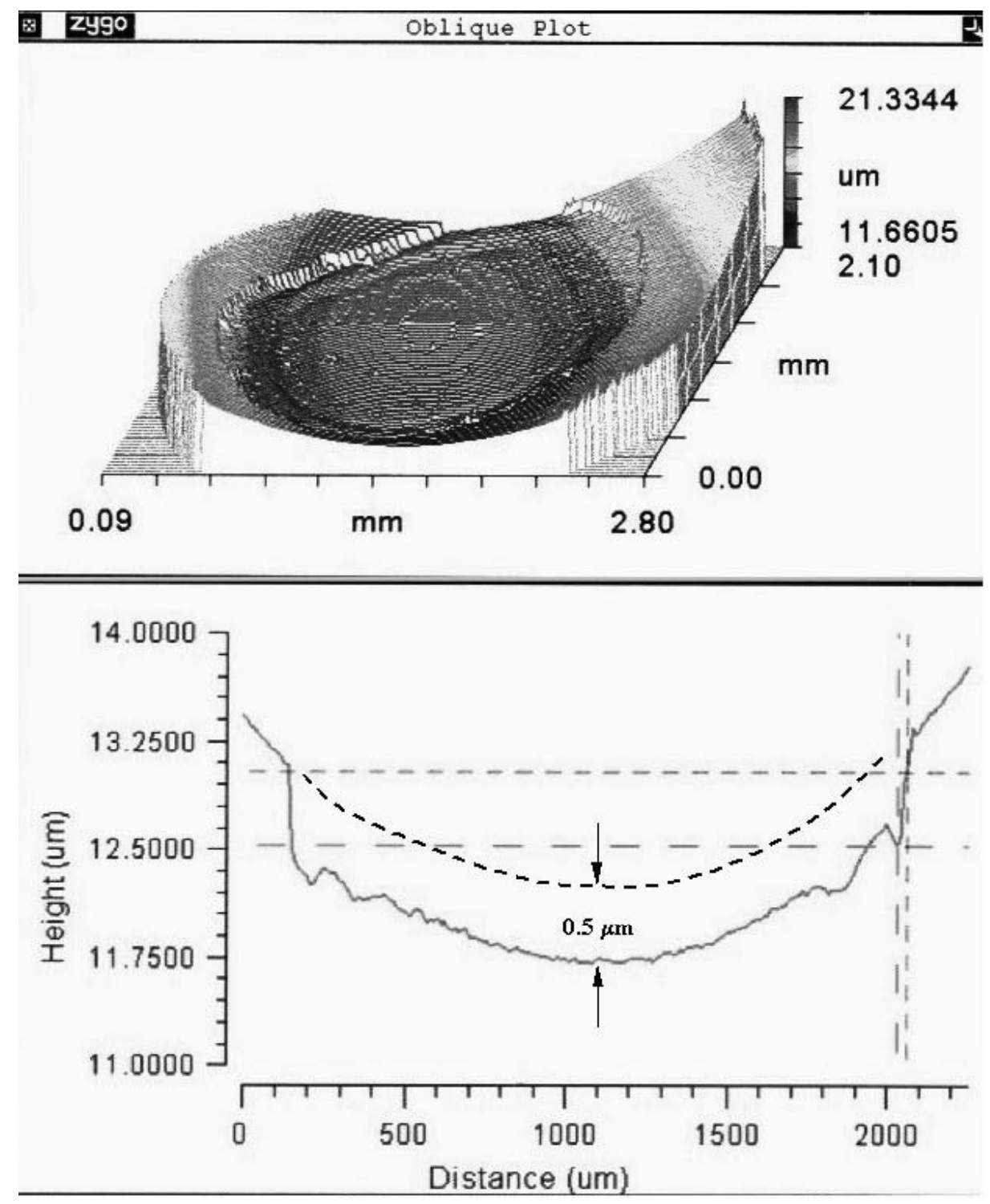

Figure 11. Profile of polishing crater showing uniform removal of a surface layer for precise examination of the ion-induced microstructure. Note that the vertical scale is about one thousand times smaller that the lateral scale.

$\mathrm{Ni}^{++}$Irradiations at PNNL

The three alloys were characterized at doses of 0.5, 2.0, 10, 30 and 50 dpa. The non-irradiated alloy characterization confirmed the small grain size and the initial low density of network dislocations as shown in Figure 12. Irradiated alloys exhibited unique trends in the development of loop and void microstructures. Most interestingly for the Pt-doped alloy, the loop volume fraction progressed through a maximum near $2 \mathrm{dpa}$ and decreased significantly from 2 to $50 \mathrm{dpa}$. The base alloy and Hf-doped alloy showed no such maximum. In the case of the alloy containing $\mathrm{Hf}$, the $900^{\circ} \mathrm{C}(20 \mathrm{~min})$ heat-treated samples completely suppressed the swelling up to 


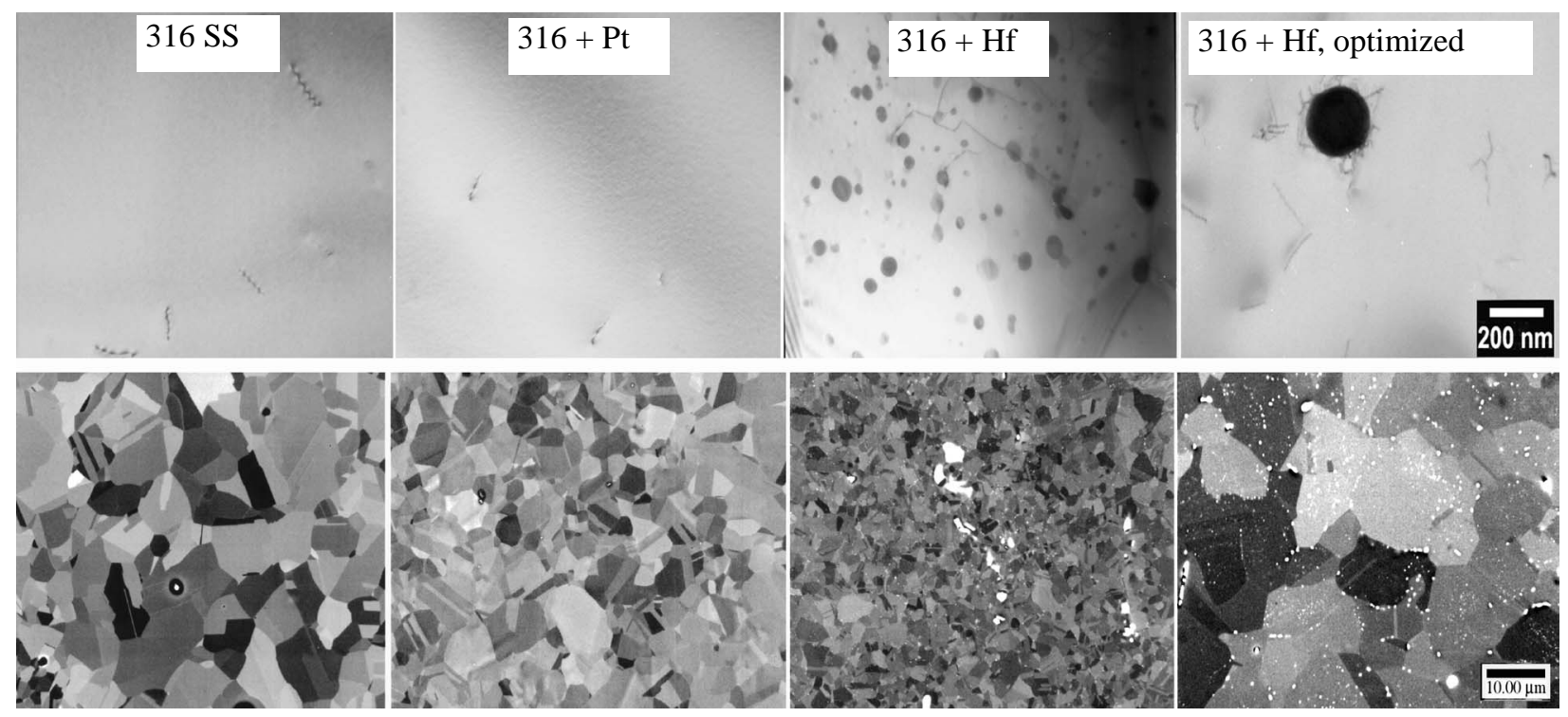

Figure 12. Micrographs show condition prior to irradiation. A low density of network dislocations is observed with precipitates only for the Hf alloy. The marker is $200 \mathrm{~nm}$ for TEM images (top) and $10 \mathrm{~mm}$ for SEM images of grain structure (bottom).

a dose of $10 \mathrm{dpa}$ while the samples heat treated at $1100^{\circ} \mathrm{C}(30 \mathrm{~min})$ completely suppressed swelling at a dose $50 \mathrm{dpa}$. This indicates that 0.3 at $\% \mathrm{Hf}$ addition is very effective in altering the irradiated microstructure and the initial heat treatment condition is important.

In the non-irradiated condition, the base alloy and the alloy with Pt addition revealed clean grain interiors with a low density of line dislocations. In contrast, the alloy with $\mathrm{Hf}$ addition revealed a fine distribution of Hf-rich precipitates as presented in Figure 12. It is expected that the Hf-rich phase precipitated during cooling from the solution-anneal temperature and did not dissolve during the $900^{\circ} \mathrm{C} / 20 \mathrm{~min}$ heat treatment. A modified heat treatment at $1100^{\circ} \mathrm{C} / 30 \mathrm{~min}$ substantially reduced the precipitate density by two orders of magnitudes.

The irradiated samples were imaged using a JEOL 2000. Three approaches were followed: (1) bright-field loop imaging, (2) rel-rod loop imaging and (3) bright-field void imaging. Examples of bright-field loop images are shown in Figure 13 for the three alloys irradiated to 0.5 to 50 dpa with $5 \mathrm{MeV}$ Ni-ions at $500{ }^{\circ} \mathrm{C}$. Similarly, rel-rod dark-field images and bright-field void images are shown in Figures 14 and 15, respectively.

Quantitative microstructure measurements are included in Tables 2-4 for doses ranging from 0.5 to $50 \mathrm{dpa}$. The loop size and number density as a function of dose is shown in Figures 16 and 17. The characteristic feature of the dose dependence of the loop number density is the rapid increase to a saturation number density at low dose for the alloys not containing Hf. The loop size dependence on dose followed a trend of rapid increase in size at low dose followed by saturation in the size at high dose. An exception was the Pt-containing alloy, which exhibited a maximum in size and number density at $0.5 \mathrm{dpa}$. The interstitial volume fraction shown in 
Figure 18 reflects differences in aggregating interstitials into loops and differences in incorporation large loops into the network dislocation density for each of the three alloys. The alloys with elemental additions show a striking difference from the base alloy in that the
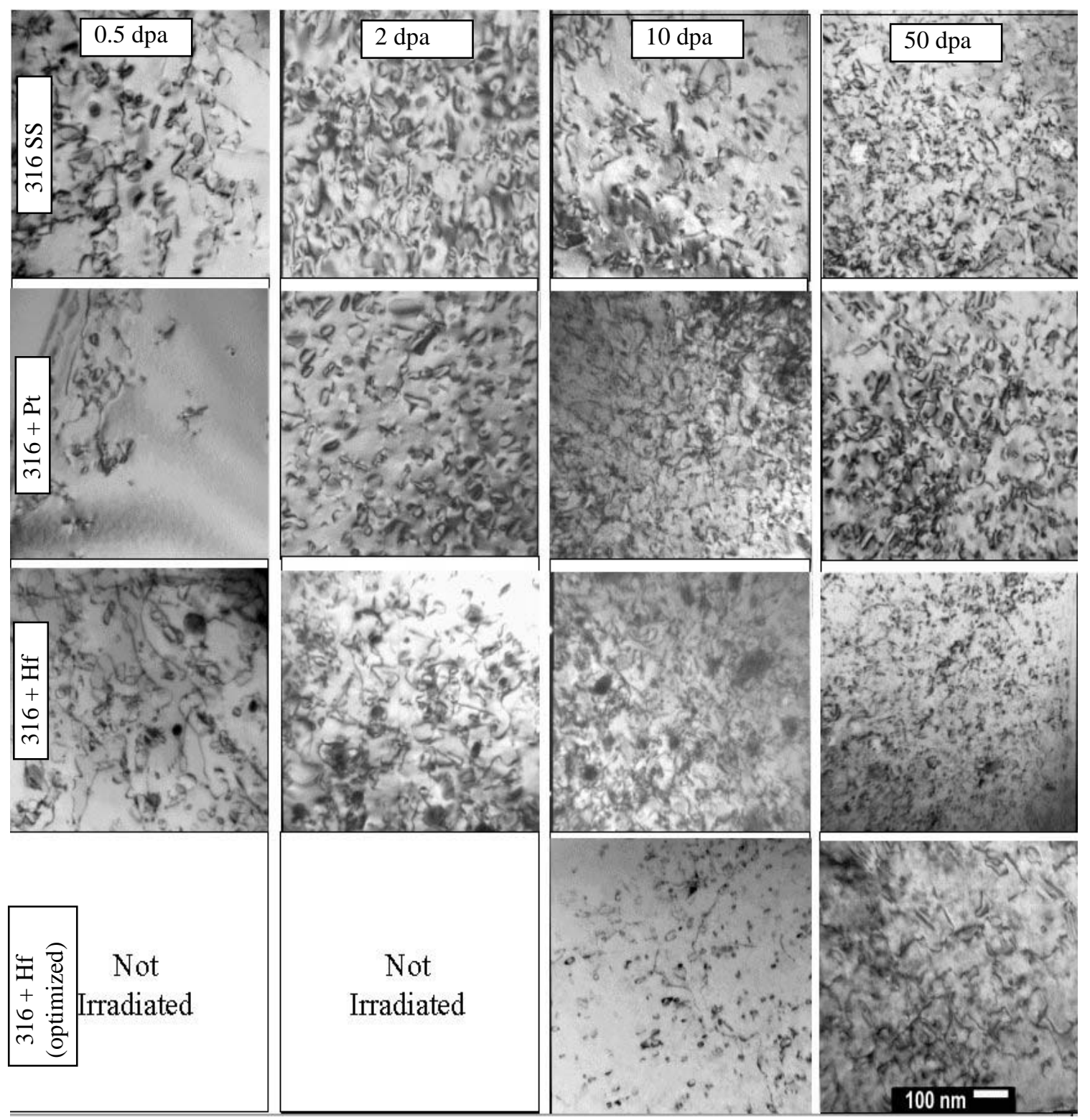

Figure 13. Bright-field micrographs of loop images for the three alloys irradiated to the indicated dose. Both faulted and unfaulted loops are imaged. Note the very high density of dislocation line length compared to the non-irradiated case. Larger loops of irregular geometry are unfaulted loops. The very dense image makes loop characterization difficult using bright-field techniques. The marker in the inset indicates $100 \mathrm{~nm}$. 


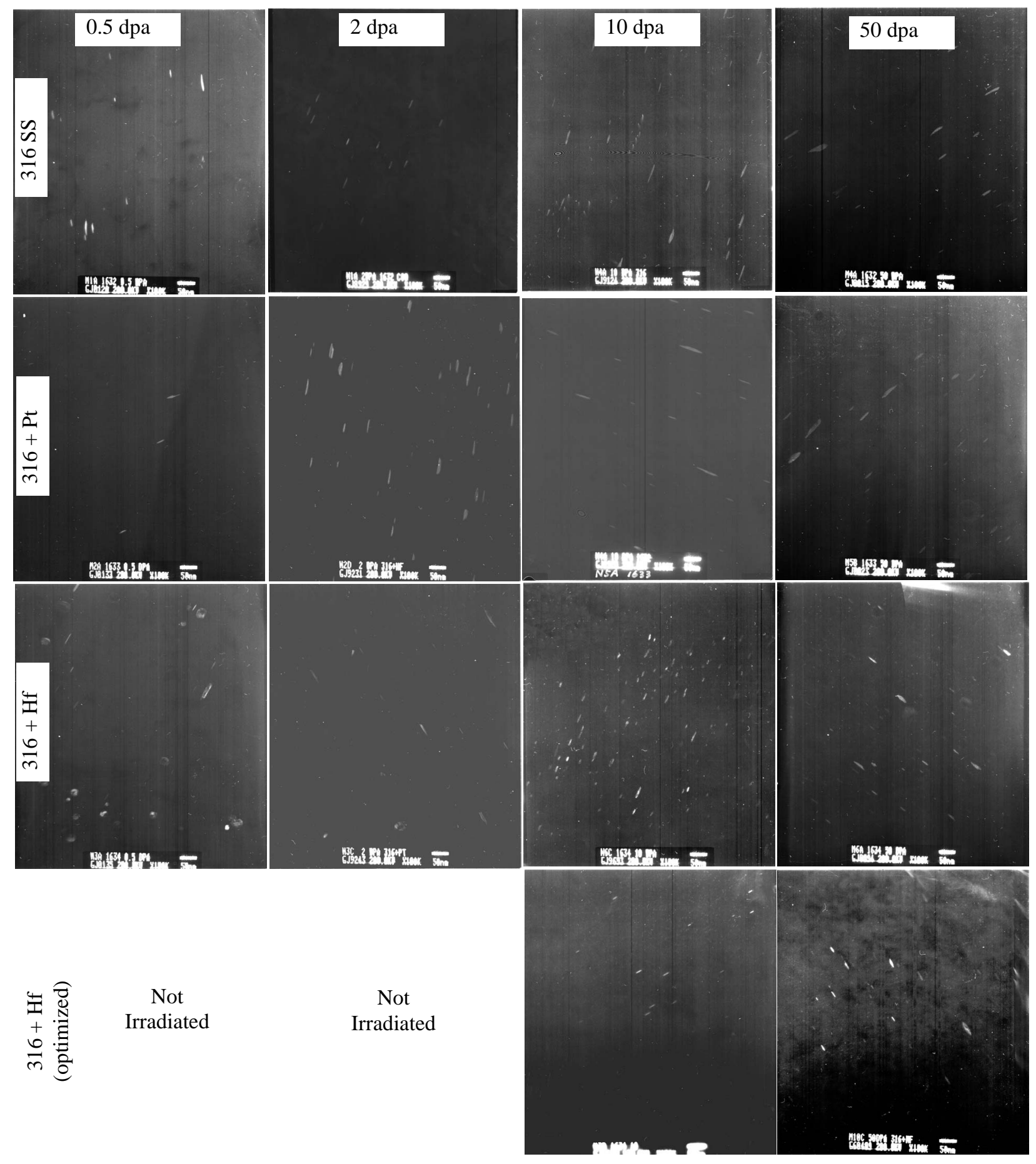

Figure 14. Rel-rod micrographs of loop images for the three alloys irradiated to the indicated doses. The short line segments are loop images. The rel-rod technique only images faulted dislocation loops. Because of diffraction conditions, only $1 / 4$ of the loops can be imaged for one specimen orientation. This technique provides better visibility for identifying individual loops. The marker in the inset indicates $50 \mathrm{~nm}$. 
interstitial fraction in loops has a maximum at $2 \mathrm{dpa}$ for alloy the $316+\mathrm{Pt}$ alloy but has a continuous increase with dose for the $316+$ Hf alloys. This indicates that the elements are strongly affecting the loop formation and loop absorption into the network dislocations at doses up to about 20 dpa.
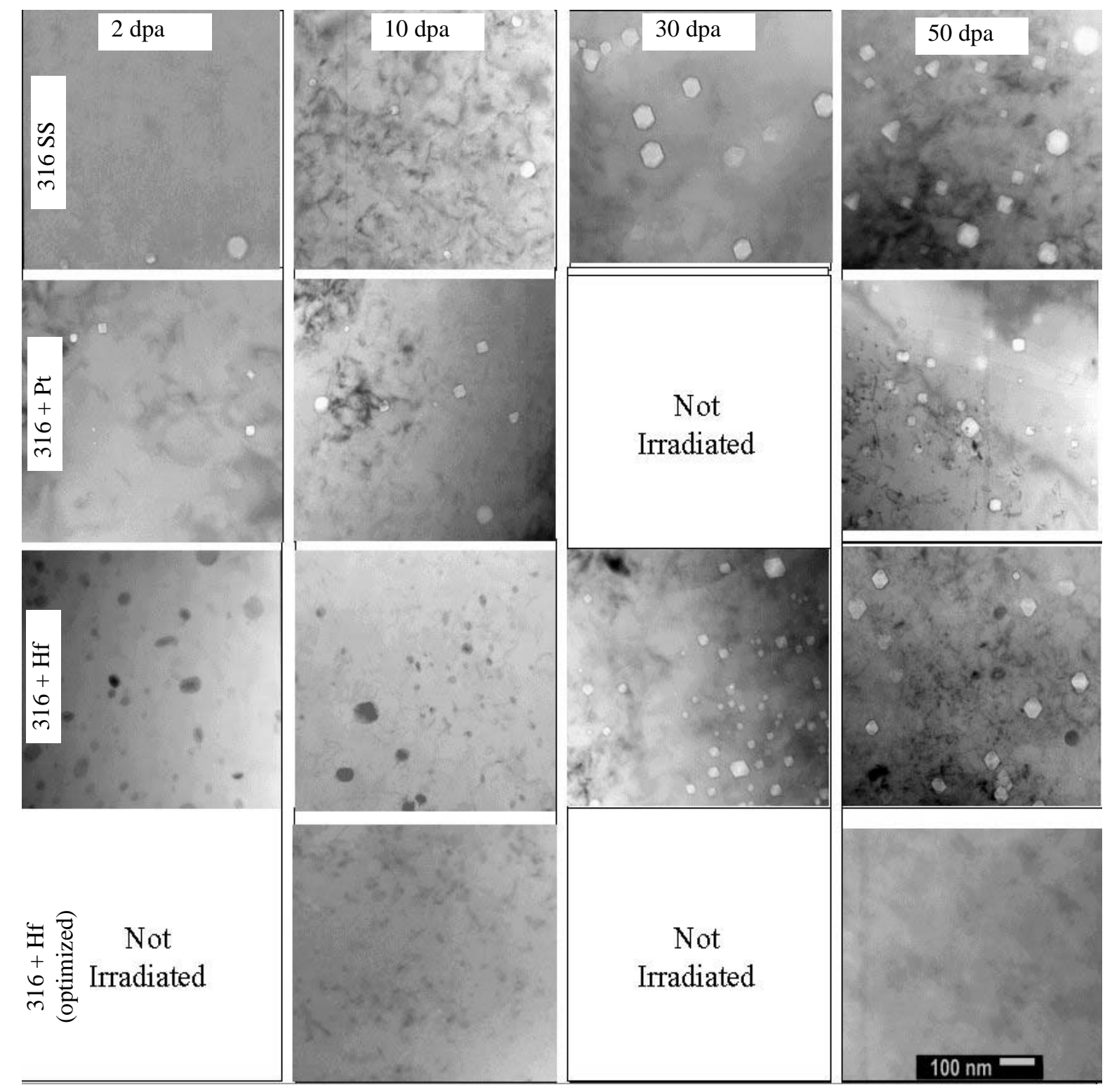

Figure 15. Micrographs of voids for the three alloys irradiated to the indicated doses. Voids show up as white images. Voids are only predominant at $50 \mathrm{dpa}$. The density was marginally high enough to obtain an estimate at $10 \mathrm{dpa}$ for the Pt containing alloy. For the Pt- and Hfcontaining alloys, the images shown represent local areas of higher void density. Typically, a significant fraction of the areas examined showed no voids. The base alloy exhibited a uniform void distribution represented in the figure. The marker in the inset indicates $100 \mathrm{~nm}$. 
Table 2. Loop number density and size as a function of dose.

\begin{tabular}{|c|c|c|c|c|c|c|c|c|}
\hline \multirow[t]{2}{*}{$\begin{array}{l}\text { Dose } \\
\text { (dpa) }\end{array}$} & 316L SS & $316 \mathrm{~L}+\mathrm{Pt}$ & $316 \mathrm{~L}+\mathrm{Hf}$ & $\begin{array}{l}316 \mathrm{~L}+\mathrm{Hf} \\
\text { (optimized) }\end{array}$ & \multicolumn{4}{|c|}{ 316L SS 316L+Pt 316L+Hf $\begin{array}{c}316 \mathrm{~L}+\mathrm{Hf} \\
\text { (optimized) }\end{array}$} \\
\hline & loops $/ \mathrm{cm}^{3}$ & loops $/ \mathrm{cm}^{3}$ & loops $/ \mathrm{cm}^{3}$ & loops $/ \mathrm{cm}^{3}$ & dia., $\mathrm{nm}$ & dia., $\mathrm{nm}$ & dia., $\mathrm{nm}$ & dia., $\mathrm{nm}$ \\
\hline 0.0 & 0 & 0 & 0 & 0 & 0 & 0 & 0 & 0 \\
\hline 0.5 & $4.3 \mathrm{E}+14$ & $2.9 \mathrm{E}+14$ & $6.0 \mathrm{E}+14$ & -- & 22 & 22 & 17 & -- \\
\hline 2.0 & $9.4 \mathrm{E}+14$ & $1.5 \mathrm{E}+15$ & $9.6 \mathrm{E}+14$ & -- & 24 & 32 & 15 & -- \\
\hline 10 & $6.9 \mathrm{E}+14$ & $1.1 \mathrm{E}+15$ & $2.3 \mathrm{E}+15$ & $8.2 \mathrm{E}+14$ & 25 & 28 & 14 & 14 \\
\hline 30 & $7.4 \mathrm{E}+14$ & -- & $4.5 \mathrm{E}+15$ & -- & 25 & -- & 13 & -- \\
\hline 50 & $8.8 \mathrm{E}+14$ & $1.1 \mathrm{E}+15$ & $5.8 \mathrm{E}+15$ & $1.9 \mathrm{E}+15$ & 25 & 20 & 13 & 16 \\
\hline
\end{tabular}

Table 3. Void number density and size as a function of dose.

\begin{tabular}{|c|c|c|c|c|c|c|c|c|}
\hline $\begin{array}{l}\text { dose } \\
\text { (dpa) }\end{array}$ & $\begin{array}{l}316 \mathrm{~L} \mathrm{SS} \\
\text { voids } / \mathrm{cm}^{3}\end{array}$ & $\begin{array}{l}316 \mathrm{~L}+\mathrm{Pt} \\
\text { voids } / \mathrm{cm}^{3}\end{array}$ & $\begin{array}{l}316 \mathrm{~L}+\mathrm{Hf} \\
\text { voids } / \mathrm{cm}^{3}\end{array}$ & $\begin{array}{c}\text { 316L+ Hf } \\
\text { (optimized) } \\
\text { voids } / \mathrm{cm}^{3}\end{array}$ & $\begin{array}{l}316 \mathrm{~L} \mathrm{SS} \\
\text { dia., } \mathrm{nm}\end{array}$ & $\begin{array}{l}316 \mathrm{~L}+\mathrm{P} \\
\text { dia., } \mathrm{nm}\end{array}$ & $\begin{array}{l}\text { 316L+Hf } \\
\text { dia., } \mathrm{nm}\end{array}$ & $\begin{array}{c}\text { 316L+Hf } \\
\text { (optimized) } \\
\text { dia., } \mathrm{nm}\end{array}$ \\
\hline 0.0 & 0 & 0 & 0 & 0 & 0 & 0 & 0 & 0 \\
\hline 0.5 & 0 & 0 & 0 & -- & 0 & 0 & 0 & -- \\
\hline 2.0 & $\begin{array}{c}\text { low, non- } \\
\text { uniform < } \\
2.1 \mathrm{E}+13 \\
\text { low, non- } \\
\text { uniform < }\end{array}$ & $2.8 \mathrm{E}+13$ & 0 & -- & 18 & 26 & 0 & -- \\
\hline & $4.3 \mathrm{E}+13$ & $6.2 \mathrm{E}+13$ & 0 & 0 & 21 & 27 & 0 & 0 \\
\hline 30 & $8.0 \mathrm{E}+13$ & -- & $1.2 \mathrm{E}+14$ & -- & 33 & -- & 25 & -- \\
\hline 50 & $2.1 \mathrm{E}+14$ & $2.7 \mathrm{E}+14$ & $2.5 \mathrm{E}+14$ & 0 & 27 & 30 & 29 & 0 \\
\hline
\end{tabular}

Table 4. Void swelling as a function of dose.

\begin{tabular}{|ccccc|}
\hline $\begin{array}{c}\text { Dose } \\
\text { (dpa) }\end{array}$ & $316 \mathrm{~L}+\mathrm{Hf}$ & $316 \mathrm{~L}+\mathrm{Pt}$ & $316 \mathrm{~L}+\mathrm{Hf}$ & $\begin{array}{c}316 \mathrm{~L}+\mathrm{Hf} \\
\text { (optimized) }\end{array}$ \\
& $(\%)$ & $(\%)$ & $(\%)$ & $(\%)$ \\
0.0 & 0 & 0 & 0 & 0 \\
0.5 & 0 & 0 & 0 & -- \\
2.0 & 0.01 & 0.03 & 0 & -- \\
10 & 0.03 & 0.09 & 0 & - \\
30 & 0.26 & -- & 0.15 & 0 \\
50 & 0.46 & 0.65 & 0.45 & \\
\hline
\end{tabular}

Table 5. Hf precipitate number density, size and volume as a function of dose.

\begin{tabular}{|c|c|c|c|c|c|c|}
\hline \multirow[t]{2}{*}{$\begin{array}{l}\text { Dose } \\
\text { (dpa) }\end{array}$} & $316 \mathrm{~L}+\mathrm{Hf}$ & $\begin{array}{c}316 \mathrm{~L}+\mathrm{Hf} \\
\text { (optimized) }\end{array}$ & $316 \mathrm{~L}+\mathrm{Hf}$ & $\begin{array}{c}316 \mathrm{~L}+\mathrm{Hf} \\
\text { (optimized) }\end{array}$ & $316+\mathrm{Hf}$ & $\begin{array}{c}316 \mathrm{~L}+\mathrm{Hf} \\
\text { (optimized) }\end{array}$ \\
\hline & $\mathrm{ppt} / \mathrm{cm}^{3}$ & $\mathrm{ppt} / \mathrm{cm}^{3}$ & dia., $\mathrm{nm}$ & dia., nm & vol., $\mathrm{nm}^{3}$ & vol., $\mathrm{nm}^{3}$ \\
\hline 0.0 & $6.5 \mathrm{E}+14$ & $9.6 \mathrm{E}+12$ & 20 & 129 & $1.06 \mathrm{E}+4$ & $2.6 \mathrm{E}+6$ \\
\hline 0.5 & $8.6 E+14$ & -- & 22 & -- & $0.95 \mathrm{E}+4$ & -- \\
\hline 2.0 & $8.6 \mathrm{E}+14$ & -- & 20 & -- & $0.93 \mathrm{E}+4$ & -- \\
\hline 10 & $6.0 \mathrm{E}+14$ & $4.0 \mathrm{E}+12$ & 22 & 111 & $1.05 \mathrm{E}+4$ & $1.4 \mathrm{E}+6$ \\
\hline 30 & $2.0 \mathrm{E}+14$ & -- & 26 & -- & $1.76 \mathrm{E}+4$ & -- \\
\hline 50 & $1.9 \mathrm{E}+14$ & $8.5 E+12$ & 26 & 105 & $2.46 \mathrm{E}+4$ & $1.6 \mathrm{E}+6$ \\
\hline
\end{tabular}




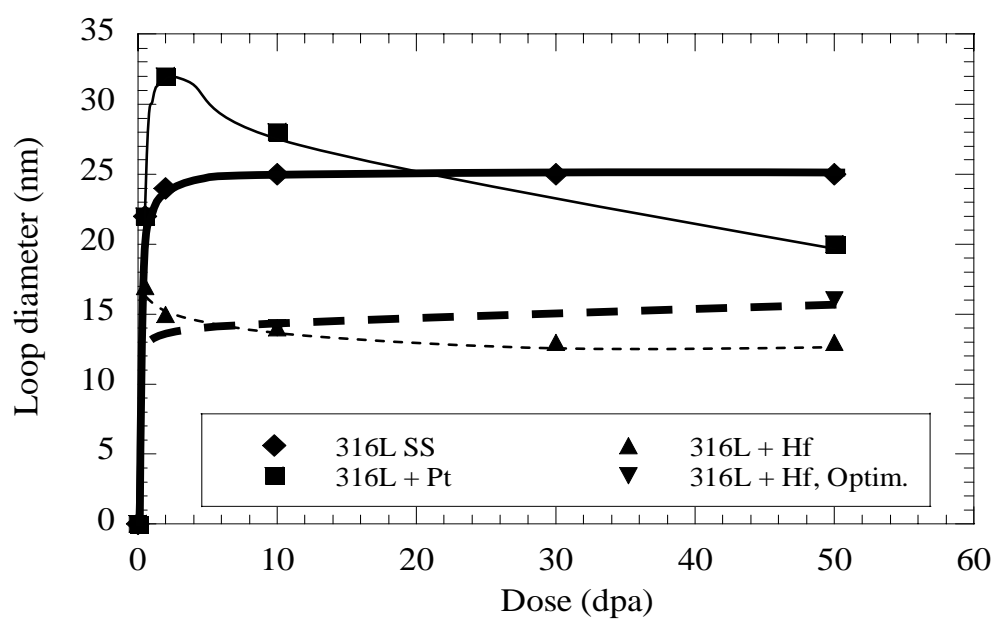

Figure 16. Loop size as a function of dose for the three alloys. The loop size decreases from 2 to $10 \mathrm{dpa}$ because of unfaulting of large loops. After unfaulting these loops glide and become integrated in the network dislocation line length.

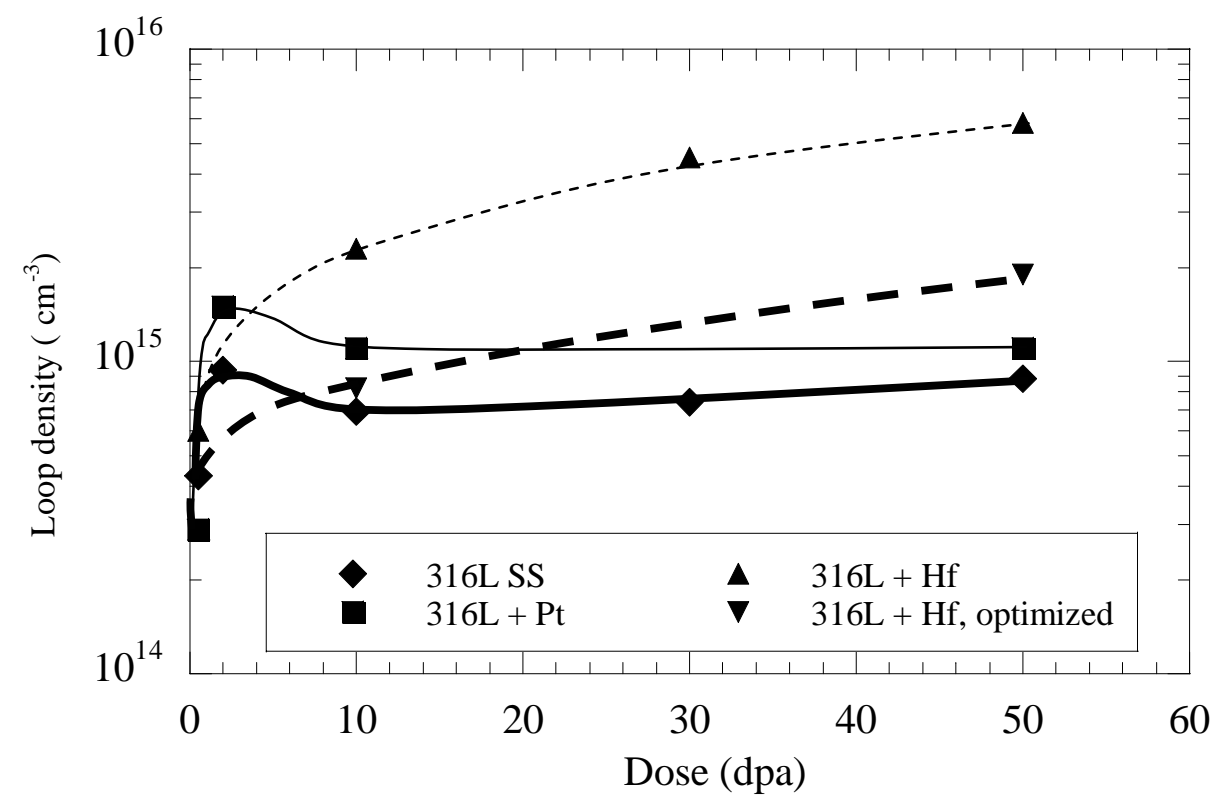

Figure 17. Loop number density as a function of dose for the three alloys. The loop number density decreases from 2 to 10 dpa because of unfaulting of large loops. After unfaulting these loops glide and become integrated in the network dislocation line length.

The size distributions of the loops are shown in Figure 19. The loop sizes ranged up to $70 \mathrm{~nm}$. The interesting feature in the dose dependence for the Hf-containing alloy is the presence of large loops at low dose but an absence of large loops at high dose. This indicates that Hf inhibits unfaulting at low doses but not at high doses. 


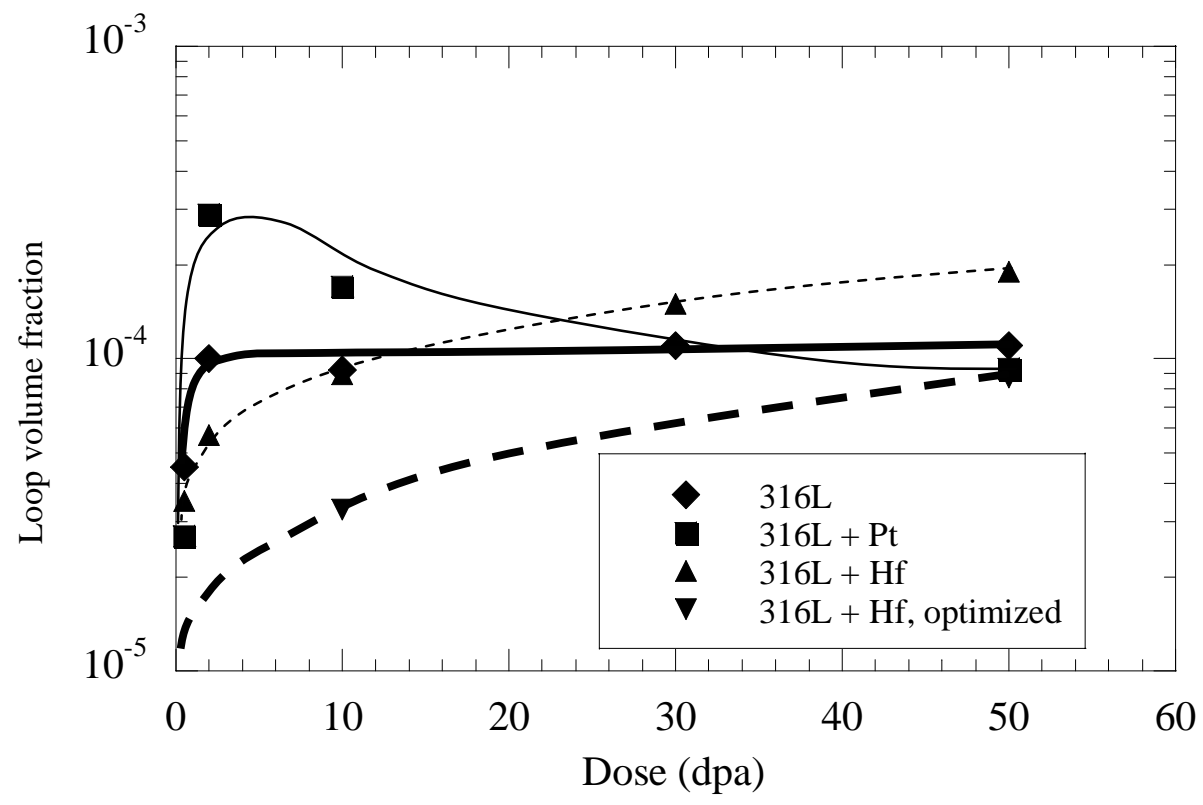

Figure 18. The loop volume fraction is shown as a function of dose for the three alloys. The capacity to store interstitials in loops passes through a strong transient between 0 and $10 \mathrm{dpa}$ for the alloys with Pt or Hf additions but not for the base alloy.

The measured void number densities were very low, often non-uniform and difficult to characterize statistically. The base alloy revealed a low number density of non-uniform voids up to 10 dpa but revealed a large size and high number density at 50 dpa as seen in Figures 20 and 21 , respectively. The Pt-containing alloy revealed a void density higher than the base alloy.

The alloy $316+\mathrm{Hf}$ delayed void swelling up to dose $10 \mathrm{dpa}$ while the optimized alloy $316+\mathrm{Hf}$ completely suppressed the void swelling up to a dose of $50 \mathrm{dpa}$. Measured void sizes in these alloys ranged from $18 \mathrm{~nm}$ to $33 \mathrm{~nm}$ as indicated in the Table 3 . The dependence of void volume fraction (swelling) on dose is shown in Figure 22. At high dose, three of the alloys swell at similar swelling rates of about $0.13 \% / \mathrm{dpa}$ while there is no swelling in the optimized $316+\mathrm{Hf}$ alloy.

The size distributions of the voids at 50 dpa are shown in Figure 23. Void size distributions could only be characterized at 50 dpa because of the lack of voids at the other conditions. The void sizes ranged up to $70 \mathrm{~nm}$. Interestingly, the Hf-containing alloy heat-treated at $900^{\circ} \mathrm{C}$ reveals a narrower size distribution compared to the other alloys.

The Hf-containing alloys exhibited a distribution of Hf-rich precipitates in the non-irradiated condition. These precipitates have a composition suggesting a $\mathrm{HfNi}_{5}$ intermetallic phase. The effect of increasing dose on the precipitate number density and size is shown in Figure 24 for the $316+\mathrm{Hf}$ alloy. The number density decreases as the size increases up to a dose of $30 \mathrm{dpa}$ and then stabilized between $30 \mathrm{dpa}$ and $50 \mathrm{dpa}$. However, as shown in Table 4 the mean volume of the precipitates remains nearly constant up to $10 \mathrm{dpa}$, then increases by a factor of $\sim 2.5$ from 10 

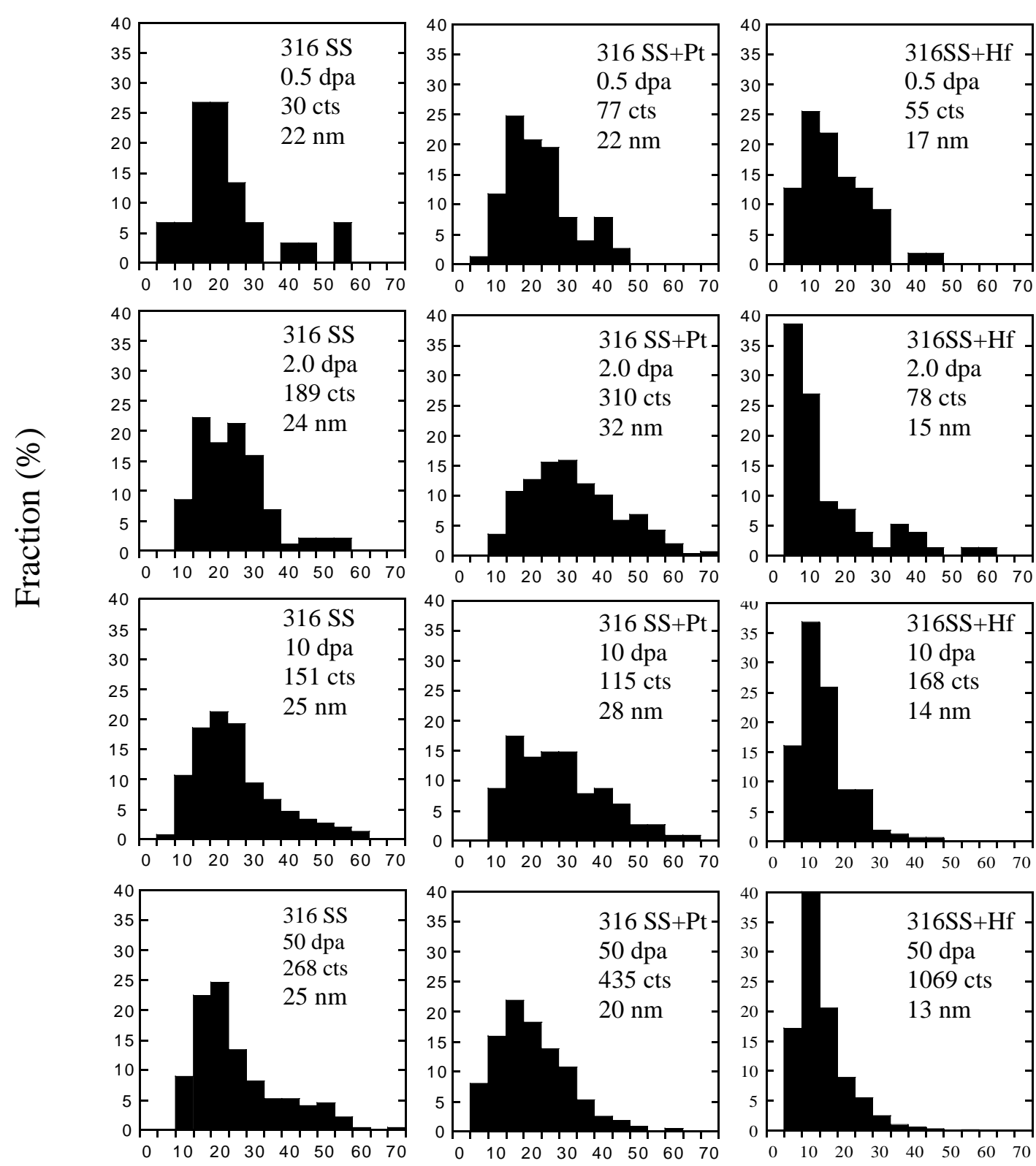

\section{Diameter (nm)}

Figure 19. The faulted loop size distributions are shown as a function of alloy and dpa from 0.5 to $50 \mathrm{dpa}$. The average loop size is insensitive to dose $(15-30 \mathrm{~nm})$. The tail in the distribution is likely caused by unfaulting near $60 \mathrm{~nm}$ followed by adsorption in the network dislocations.

dpa to $50 \mathrm{dpa}$ at which dose the voids were observed in alloy $316+\mathrm{Hf}$ (heat treated at $900^{\circ} \mathrm{C}$ for $20 \mathrm{~min}$ ). The change of precipitate microstructure may play an important role in the development of the irradiated microstructure. This indicates that the irradiation behavior is influenced by both $\mathrm{Hf}$ in solution and by Hf precipitates. The evolution of precipitate structure between doses of $10 \mathrm{dpa}$ to $50 \mathrm{dpa}$ likely contributes to the continuous increase of loop density in the Hf containing alloy to $50 \mathrm{dpa}$. 
The $316+\mathrm{Hf}$ alloy modified using a higher temperature heat treatment $\left(1100^{\circ} \mathrm{C}\right.$ for $30 \mathrm{~min}$.) demonstrated that by reducing the precipitate density down to below $10^{13} / \mathrm{cm}^{3}$, the Hf-doped alloy postponed the void microstructure beyond $50 \mathrm{dpa}$. In addition, loop volume fraction is delayed in the 316+Hf optimized alloy, shown in Figure 18. It is clear that reduction of the Hfenriched precipitate microstructure in the $316+\mathrm{Hf}$ alloy significantly improves the alloy performance by suppressing the irradiated microstructure. The comparison of precipitate

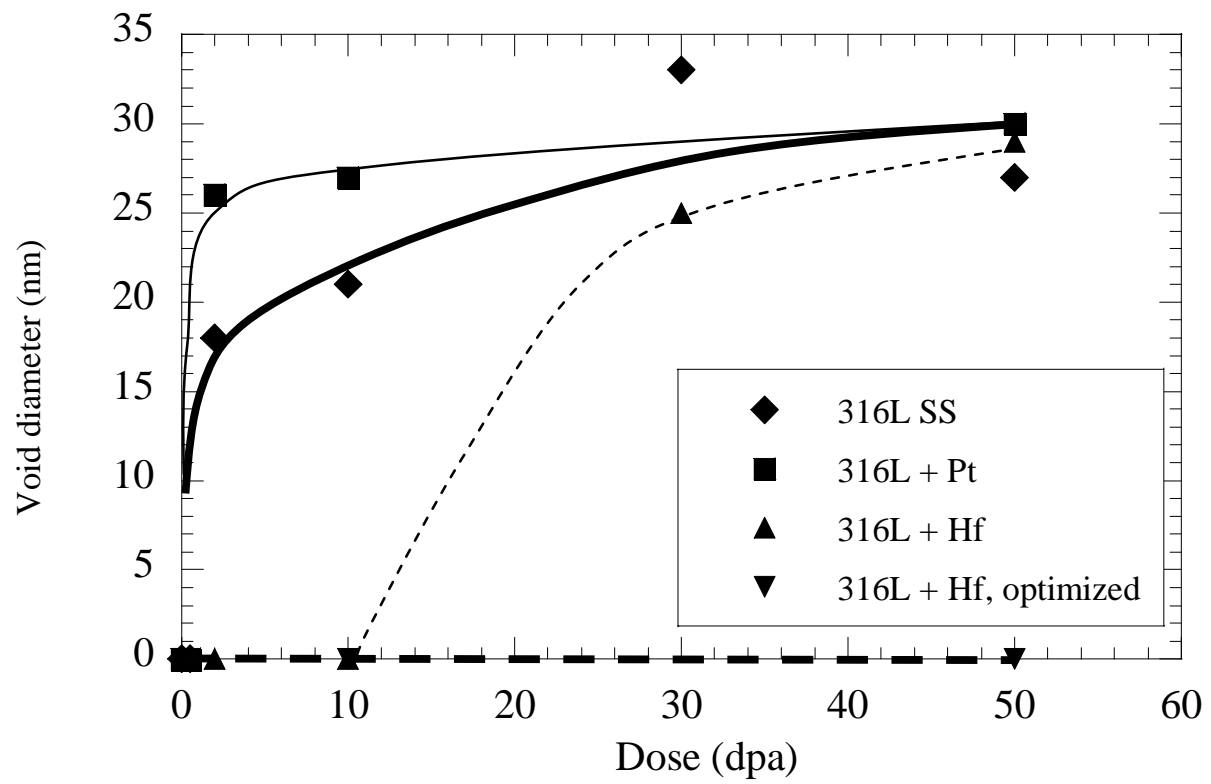

Figure 20. Measured void size as a function of dose.

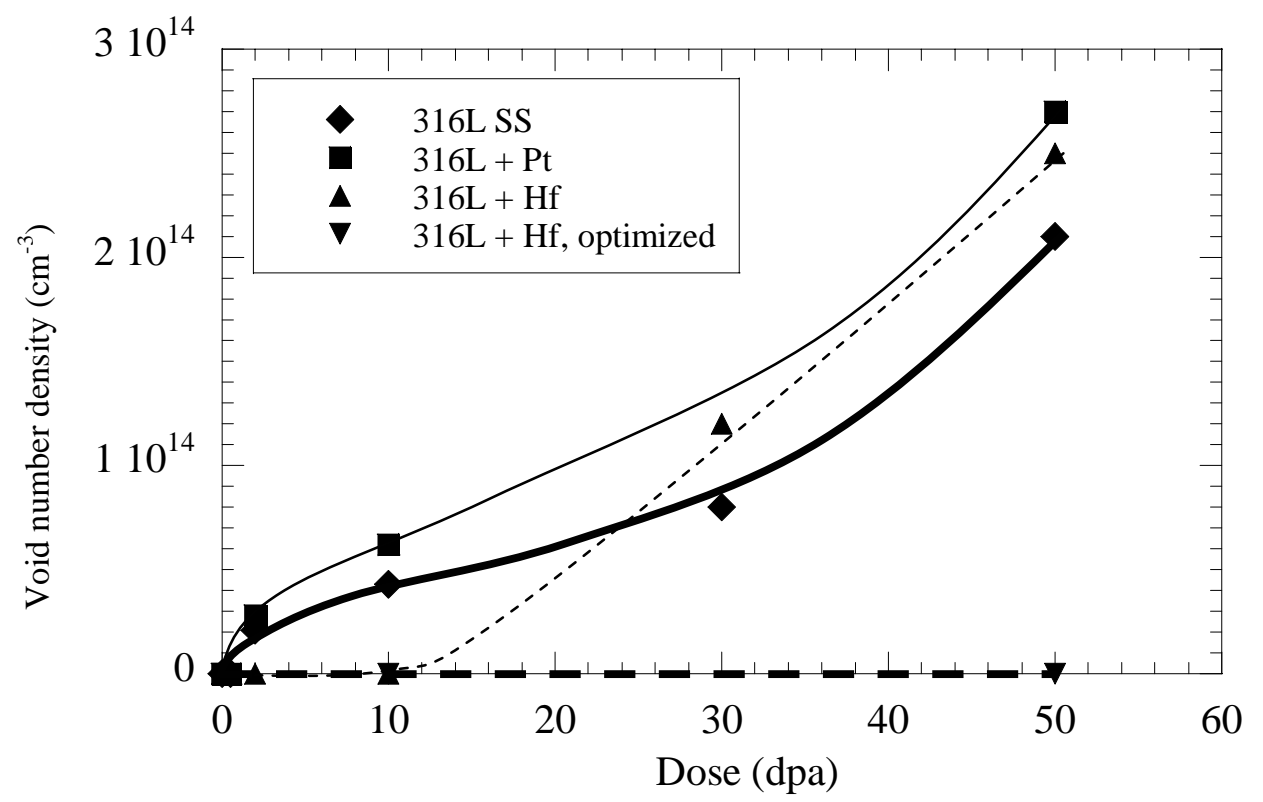

Figure 21. Measured void number density as a function of dose. The base alloy exhibits a much greater void number density at 50 dpa compared to the alloys with Pt or $\mathrm{Hf}$ additions. 
microstructure as a function of dose between the $316+\mathrm{Hf}$ alloy and the modified $316+\mathrm{Hf}$ alloy is shown in Figure 25. Note that the precipiate density in the optimized alloy is below $10^{13} / \mathrm{cm}^{3}$, which is at the lower limit for the number density measurement using TEM analysis.

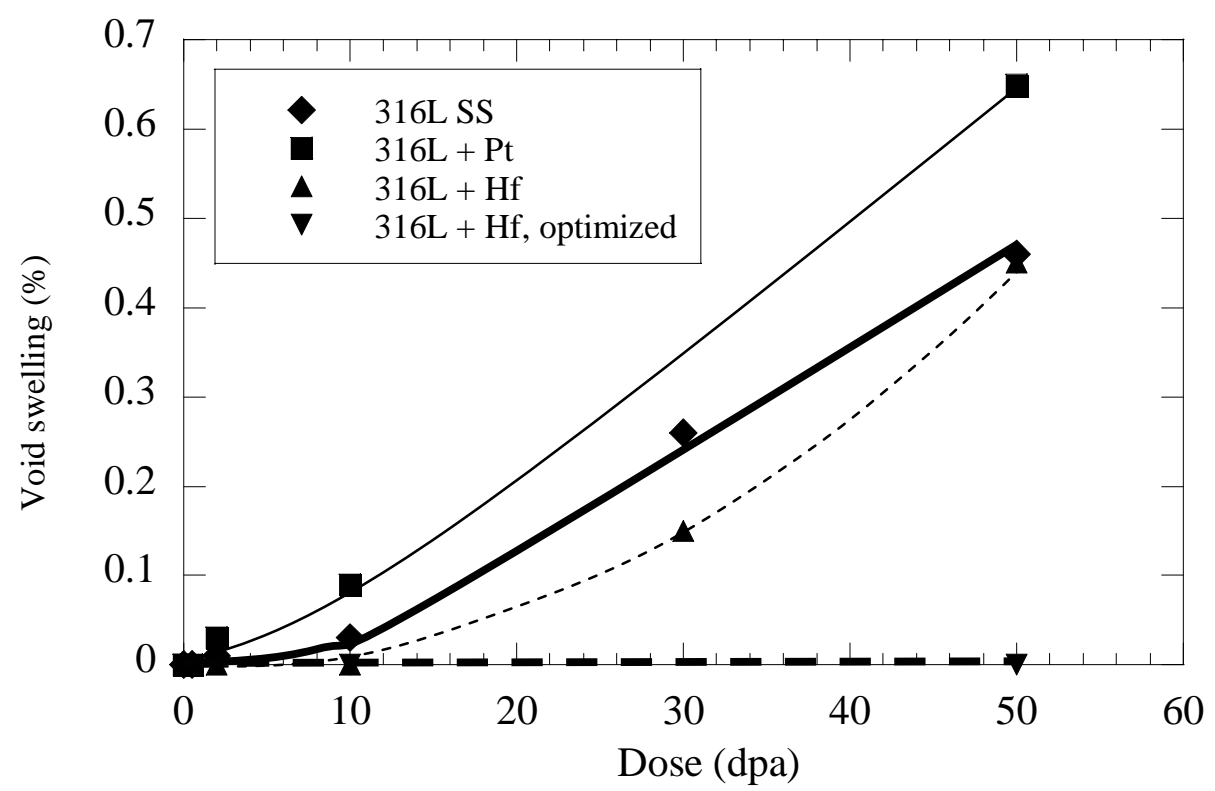

Figure 22. Measured void volume fraction (swelling) is shown as a function of dose. The measured swelling in the alloys having Pt or Hf additions is reduced compared to the base alloy. The alloys with Pt or Hf additions had very non-uniform distributions and therefore the actual swelling is less than indicated. 

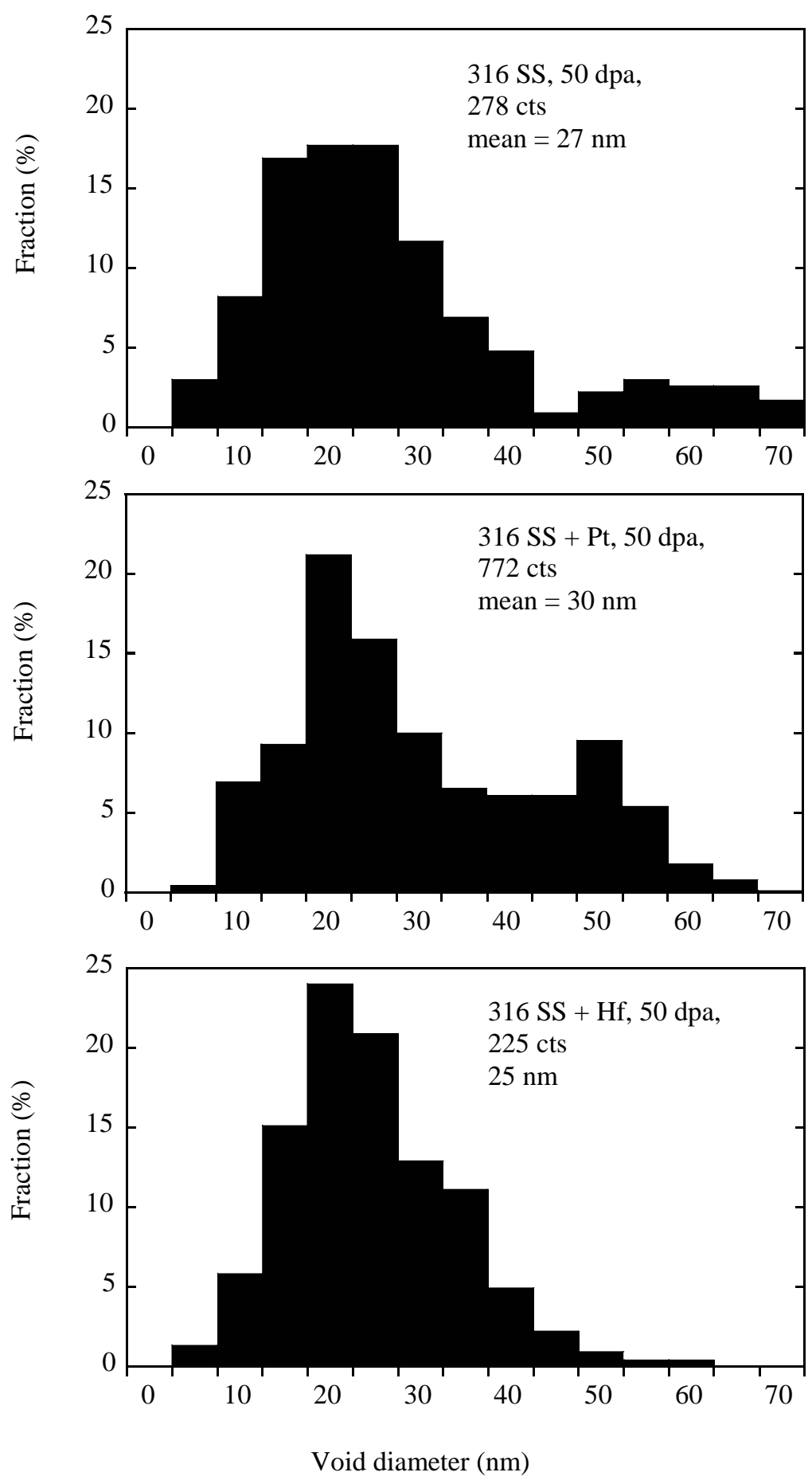

Figure 23. Void size distribution at $50 \mathrm{dpa}$ for the three alloys initially heat treated at $900^{\circ} \mathrm{C}$. The $316 \mathrm{~L}+\mathrm{Hf}$ heat treated at $1100^{\circ} \mathrm{C}$ showed no evidence of void formation at $50 \mathrm{dpa}$. The apparent bimodal distribution in the Pt-containing alloy may be an artifact of the low counts used for the distribution. 


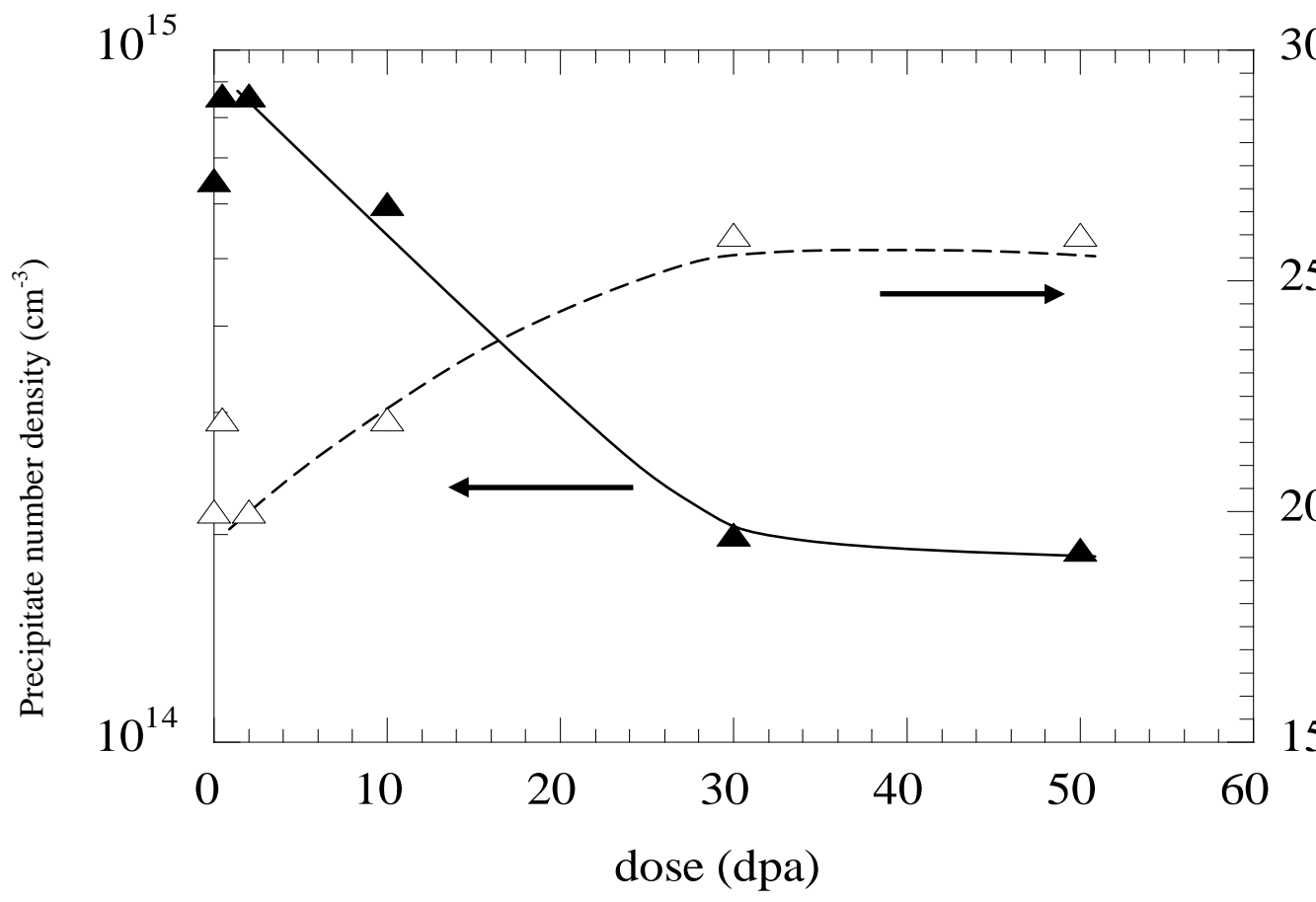

30

25 葛.

20

15

Figure 24. Measured size and number density of Hf-containing precipitates in the 316L+Hf alloy heat-treated at $900^{\circ} \mathrm{C}$. A significant coarsening of the precipitate distribution is observed.

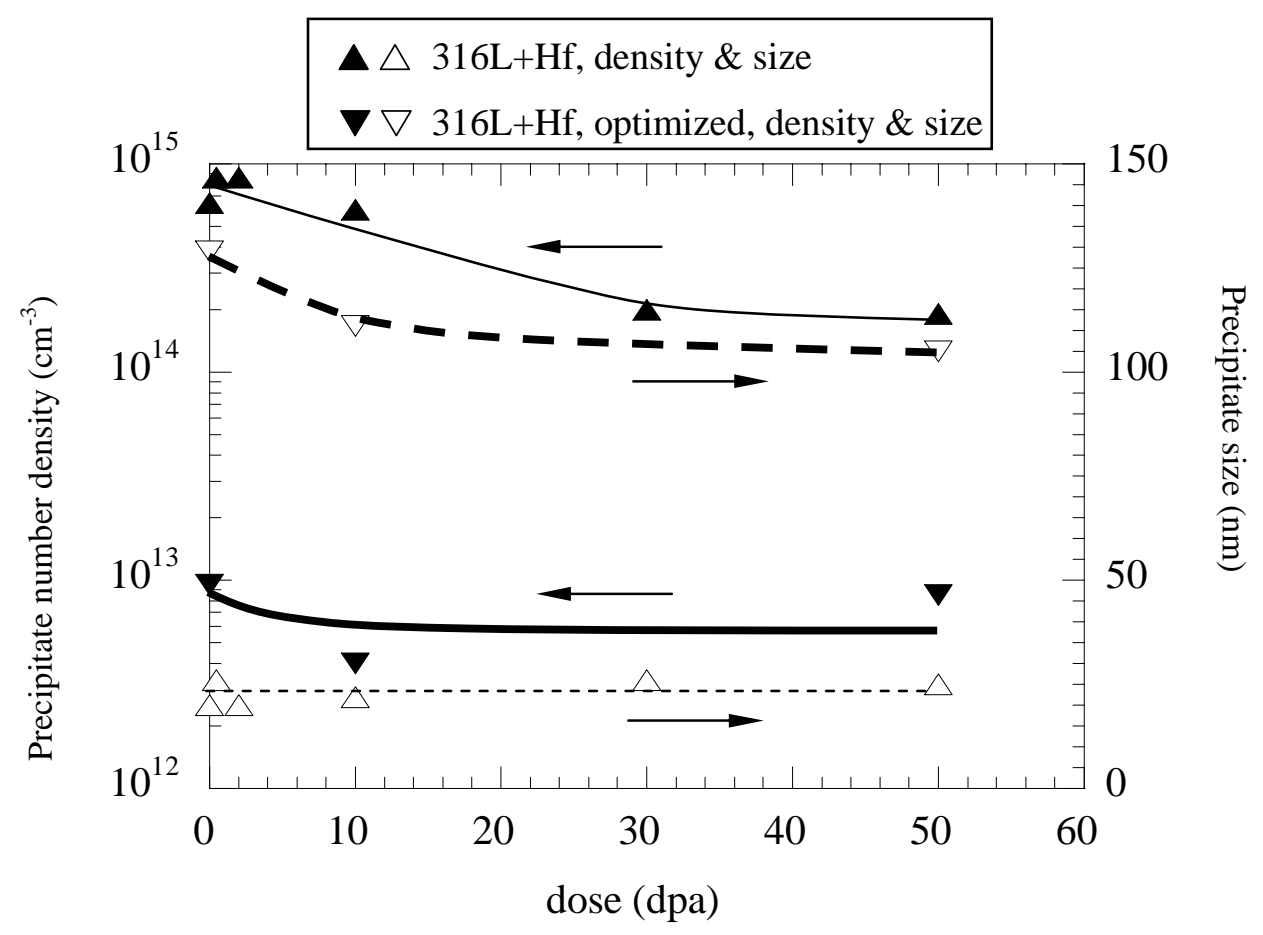

Figure 25. Comparison size and number density of Hf-containing precipitates for the $316 \mathrm{~L}+\mathrm{Hf}$ alloy heat-treated at $900^{\circ} \mathrm{C}$ and $1100^{\circ} \mathrm{C}$ conditions. 


\section{Proton Irradiations at UM}

Proton irradiation produced a significant change in the general microstructure of $316 \mathrm{~L} \mathrm{SS}$, $316 \mathrm{SS}+\mathrm{Pt}$ and $316 \mathrm{SS}+\mathrm{Hf}$ modified stainless steels similar to those observed after $\mathrm{Ni}^{++}$ irradiation. The proton irradiations focused on the $316 \mathrm{SS}+\mathrm{Hf}$ modified alloy heat treated at $1100^{\circ} \mathrm{C}$ and with only cursory exams of the $316 \mathrm{SS}+\mathrm{Hf}$ alloy heat treated at $900^{\circ} \mathrm{C}$.

Microstructures in $316 \mathrm{~L} \mathrm{SS}, 316 \mathrm{SS}+\mathrm{Pt}$ and $316 \mathrm{SS}+\mathrm{Hf}$ irradiated with $3.2 \mathrm{MeV}$ protons at $400^{\circ} \mathrm{C}$ to a nominal dose of $2.5 \mathrm{dpa}$ are shown in Figure 26. Figure 26 (a, b, c) shows bright-field images while Figure 26 (d, e, f) shows faulted Frank loops imaged by the rel-rod technique. A summary of the proton-irradiated microstructure is presented in Table 6 and 7 as a function of dose and alloy composition.

As shown in Figure 26 (a) and (b) and in Figure 27, microstructures of both 316L SS and $316 \mathrm{SS}+\mathrm{Pt}$ exhibited void swelling after $2.5 \mathrm{dpa}$. As shown in Table 6, swelling was larger in $316 \mathrm{SS}+\mathrm{Pt}$ than in 316L SS. Meanwhile the microstructure of 316SS+Hf modified showed only a non-uniform distribution of small voids at $2.5 \mathrm{dpa}$, Figure 28. With increasing dose from 2.5 dpa to 5 dpa, Figures 29-31, more uniform void formation was observed in the microstructure of 316SS+Hf. Void size in 316SS+Hf remained smaller than that in 316L SS, however the void density was greater.
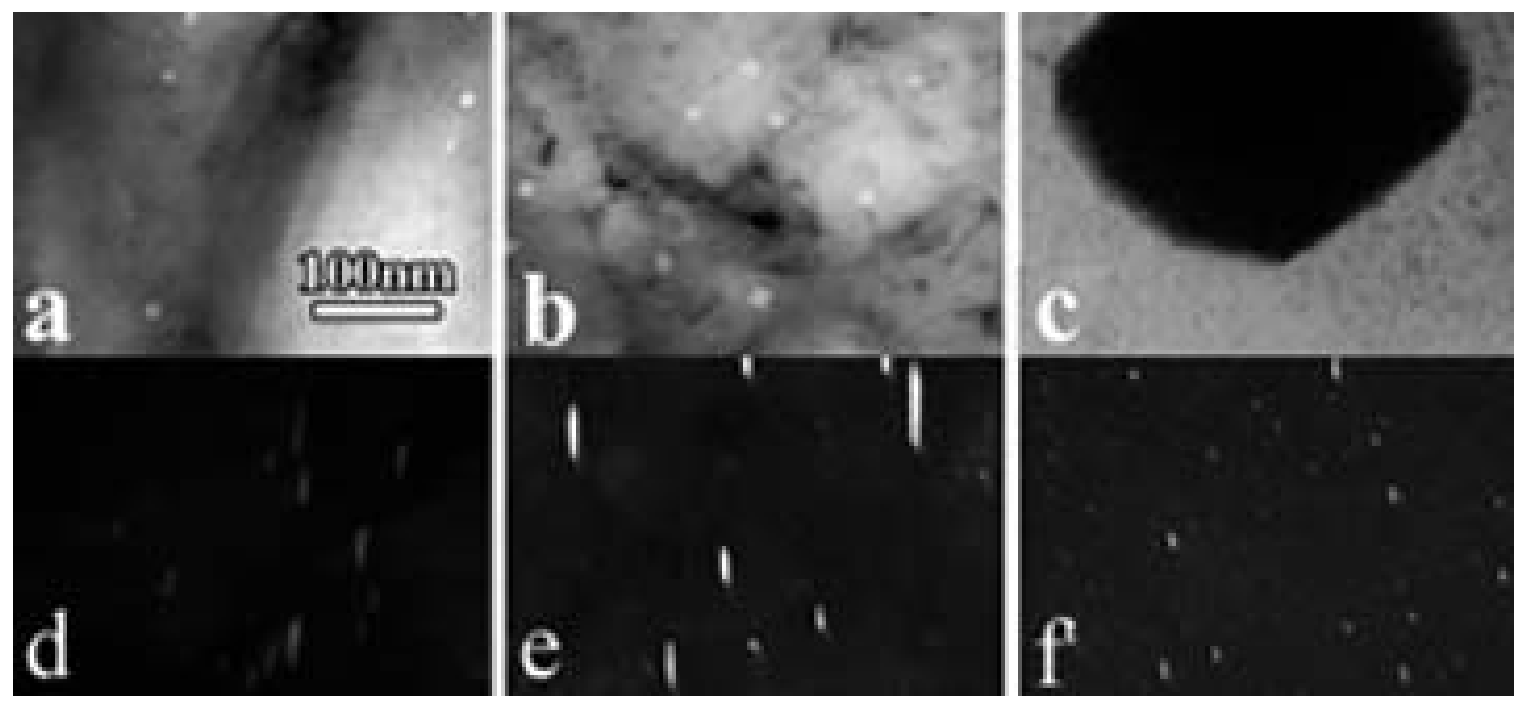

Figure 26. Bright field and dark field (rel-rod) images of the microstructure following irradiation with $3.2 \mathrm{MeV}$ protons to $2.5 \mathrm{dpa}$ at $400^{\circ} \mathrm{C}$ in a and d) $316 \mathrm{~L} \mathrm{SS}, \mathrm{b}$ and e) $316 \mathrm{SS}+\mathrm{Pt}$, c and f) 316SS+Hf modified.

Table 6. Loop microstructure of 316L SS, 316SS+Pt and 316SS+Hf modified irradiated to 2.5 and 5.0 dpa with $3.2 \mathrm{MeV}$ Protons at $400^{\circ} \mathrm{C}$.

\begin{tabular}{|c|c|c|c|c|c|c|}
\hline $\begin{array}{l}\text { Dose } \\
(\mathrm{dpa})\end{array}$ & $\begin{array}{l}316 \mathrm{~L} \mathrm{SS} \\
\text { loops } / \mathrm{m}^{3}\end{array}$ & $\begin{array}{c}316 \mathrm{SS}+\mathrm{Pt} \\
\text { loops } / \mathrm{m}^{3}\end{array}$ & $\begin{array}{c}316 \mathrm{SS}+\mathrm{Hf} \\
1100^{\circ} \mathrm{C} \\
\text { loops } / \mathrm{m}^{3} \\
\end{array}$ & $\begin{array}{l}\text { 316L SS } \\
\text { dia., } \mathrm{nm} \\
\end{array}$ & $\begin{array}{r}316 \mathrm{SS}+\mathrm{Pt} \\
\text { dia., } \mathrm{nm} \\
\end{array}$ & $\begin{array}{c}316 \mathrm{SS}+\mathrm{Hf} \\
1100^{\circ} \mathrm{C} \\
\text { dia., } \mathrm{nm} \\
\end{array}$ \\
\hline 2.5 & & & & 30 & 38 & 13 \\
\hline 5.0 & 1.5E 21 & No data & 3.9E 21 & 19 & No data & 13 \\
\hline
\end{tabular}


Table 7. Void microstructure of 316L SS, 316SS+Pt and 316SS+Hf modified irradiated to 2.5 and 5.0 dpa with 3.2 MeV Protons at $400^{\circ} \mathrm{C}$.

\begin{tabular}{|c|c|c|c|c|c|c|c|c|c|}
\hline $\begin{array}{l}\text { Dose } \\
\text { (dpa) }\end{array}$ & $\begin{array}{l}316 \mathrm{~L} \text { SS } \\
\text { voids } / \mathrm{m}^{3}\end{array}$ & $\begin{array}{c}316 \mathrm{SS}+\mathrm{Pt} \\
\text { voids } / \mathrm{m}^{3}\end{array}$ & $\begin{array}{c}316 \mathrm{SS}+\mathrm{Hf} \\
\text { voids } / \mathrm{m}^{3}\end{array}$ & $\begin{array}{l}\text { 316L SS } \\
\text { dia., } \mathrm{nm}\end{array}$ & $\begin{array}{l}316 \mathrm{SS}+\mathrm{Pt} \\
\text { dia., } \mathrm{nm}\end{array}$ & $\begin{array}{c}316 \mathrm{SS}+\mathrm{Hf} \\
\text { dia., } \mathrm{nm}\end{array}$ & $\begin{array}{c}\text { 316L SS } \\
\text { Swelling } \\
\%\end{array}$ & $\begin{array}{c}316 \mathrm{SS}+\mathrm{Pt} \\
\text { Swelling } \\
\%\end{array}$ & $\begin{array}{c}316 \mathrm{SS}+\mathrm{Hf} \\
\text { Swelling } \\
\%\end{array}$ \\
\hline $\begin{array}{l}2.5 \\
5.0\end{array}$ & $\begin{array}{l}1.0 \mathrm{E} 20 \\
3.5 \mathrm{E} 20\end{array}$ & $\begin{array}{l}4.0 \mathrm{E} 20 \\
\text { No data }\end{array}$ & $\begin{array}{c}0 \\
2.4 \mathrm{E} 21\end{array}$ & $\begin{array}{l}13 \\
13\end{array}$ & $\begin{array}{c}18 \\
\text { No data }\end{array}$ & $\begin{array}{l}0 \\
4\end{array}$ & $\begin{array}{l}0.01 \\
0.04\end{array}$ & $\begin{array}{c}0.13 \\
\text { No data }\end{array}$ & $\begin{array}{c}0 \\
0.01\end{array}$ \\
\hline
\end{tabular}

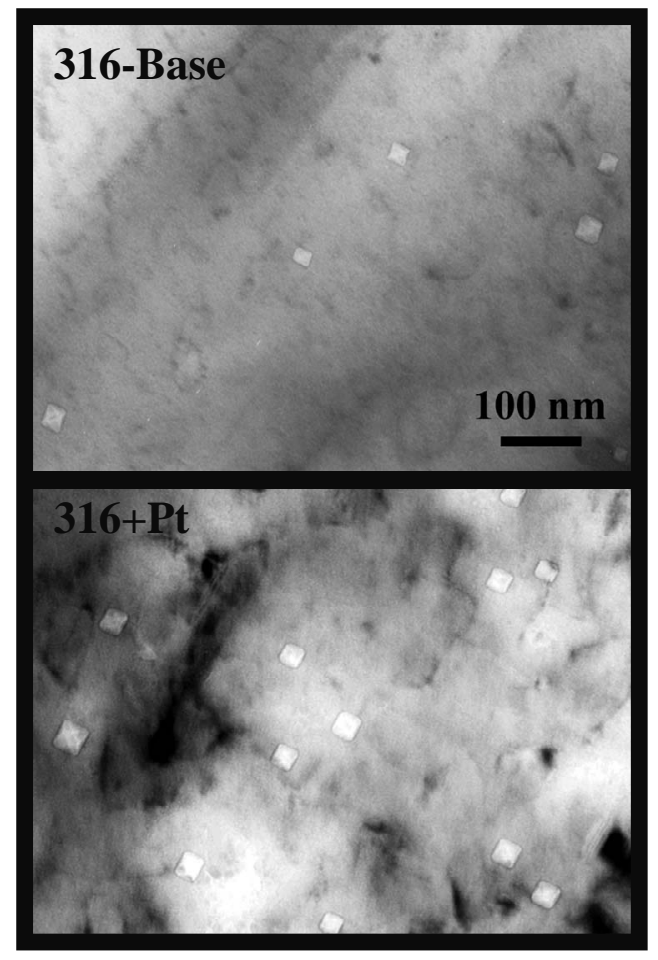

(a)

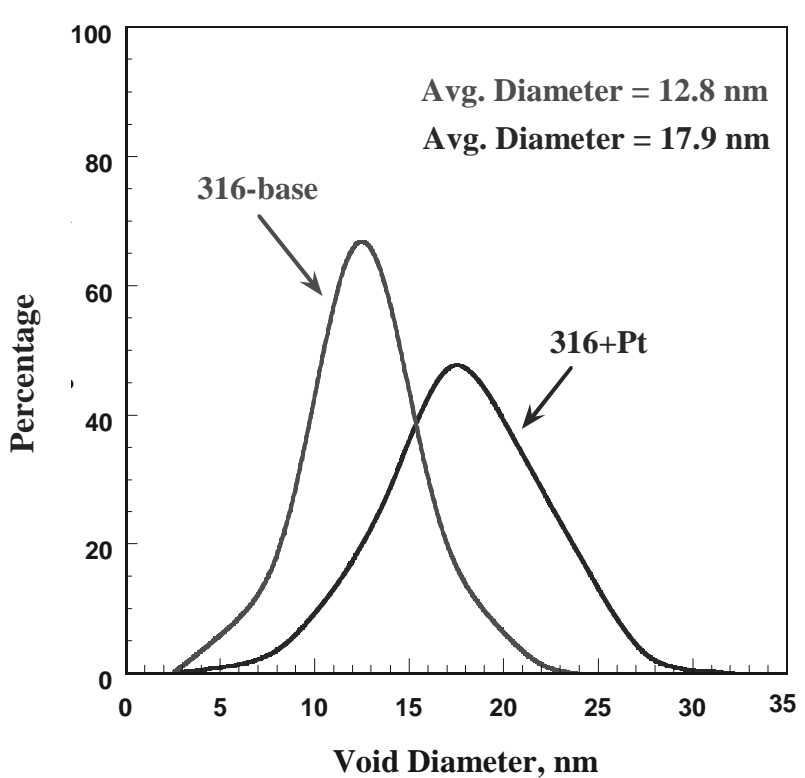

(b)

Figure 27. Void images (a) and void size distributions (b) in 316L SS and 316SS+Pt irradiated with $3.2 \mathrm{MeV}$ protons to $2.5 \mathrm{dpa}$ at $400^{\circ} \mathrm{C}$.

Faulted Frank loops can be seen in Figure $26(\mathrm{~d}, \mathrm{e}, \mathrm{f})$ imaged by the relrod technique. At 2.5 $\mathrm{dpa}$, the $316 \mathrm{SS}+\mathrm{Pt}$ alloy has larger loops and a higher density than does the base alloy. The $316 \mathrm{SS}+\mathrm{Hf}$ alloy has a significantly higher density of smaller loops as compared to the base. After irradiation to $5 \mathrm{dpa}$, the loop density of both the base 316L SS and the 316SS+Hf increased. The loop size in the $316 \mathrm{SS}+\mathrm{Hf}$ remained the same but that in the base alloy decreased from $30 \mathrm{~nm}$ at $2.5 \mathrm{dpa}$ to $19 \mathrm{~nm}$ at $5.0 \mathrm{dpa}$. 


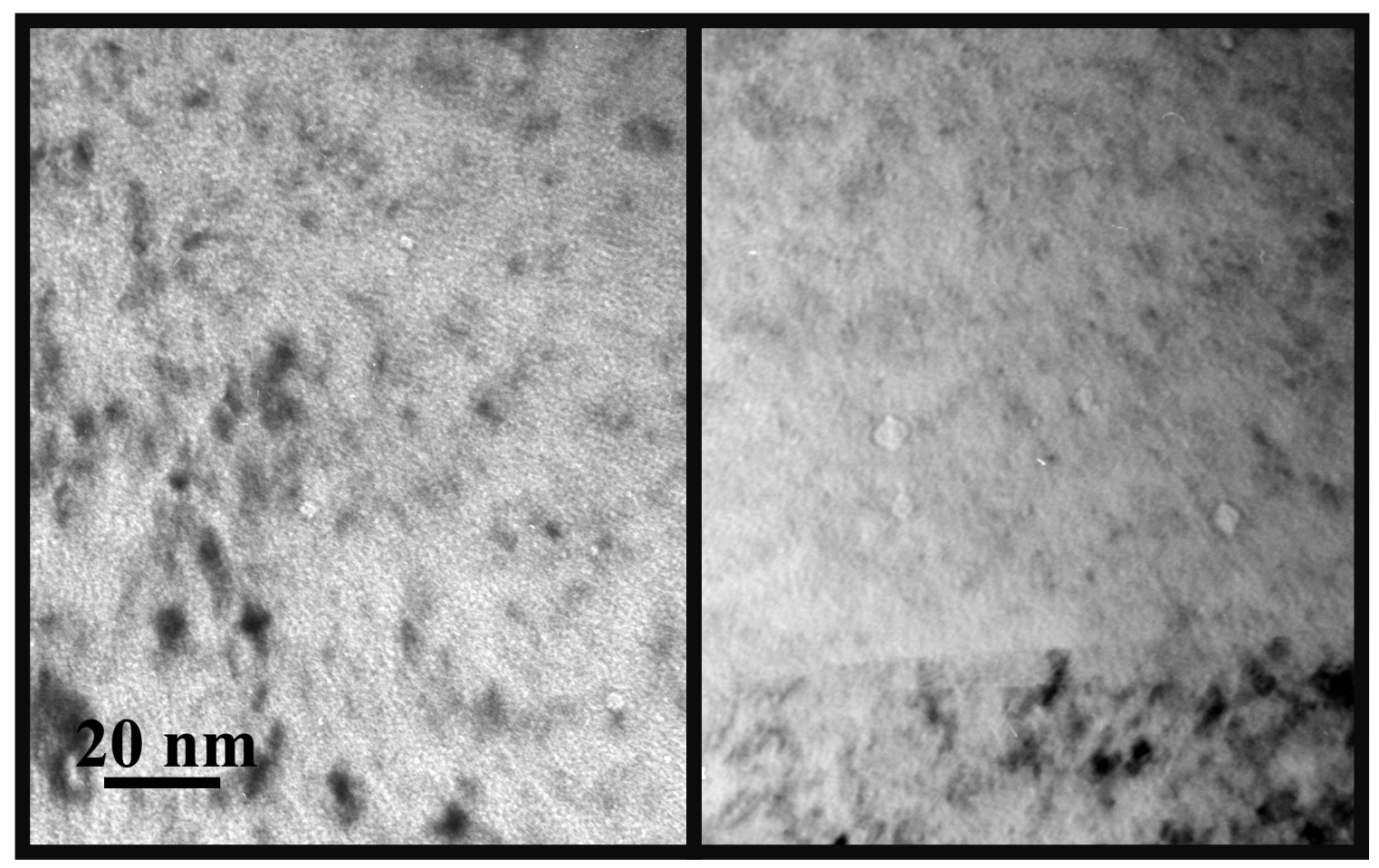

Figure 28. Voids in $316 \mathrm{~L}+\mathrm{Hf}$ alloy after irradiation with $3.2 \mathrm{MeV}$ protons to $2.5 \mathrm{dpa}$ at $400^{\circ} \mathrm{C}$.

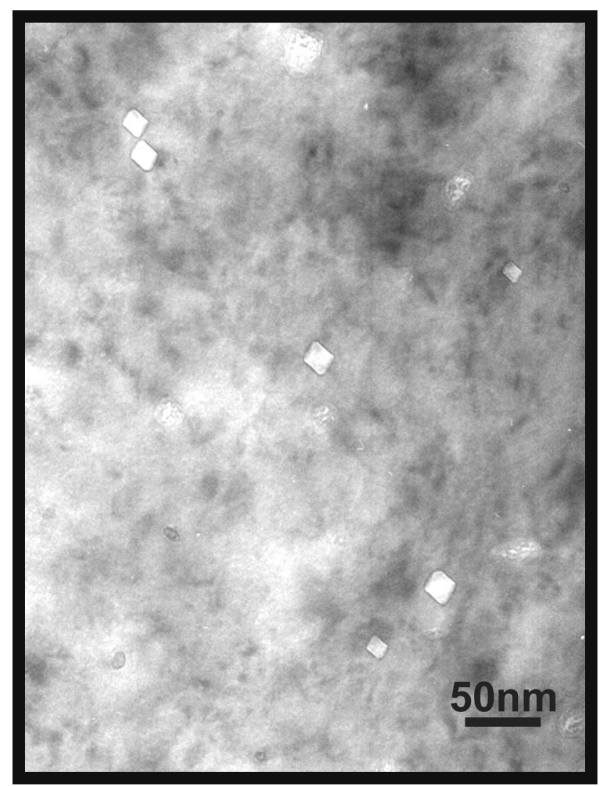

Void density $=3.5 \times 10^{20} \mathrm{~m}^{-3}$ Avg. dia. $=13.1 \mathrm{~nm}$ $\%$ swelling $=0.04$

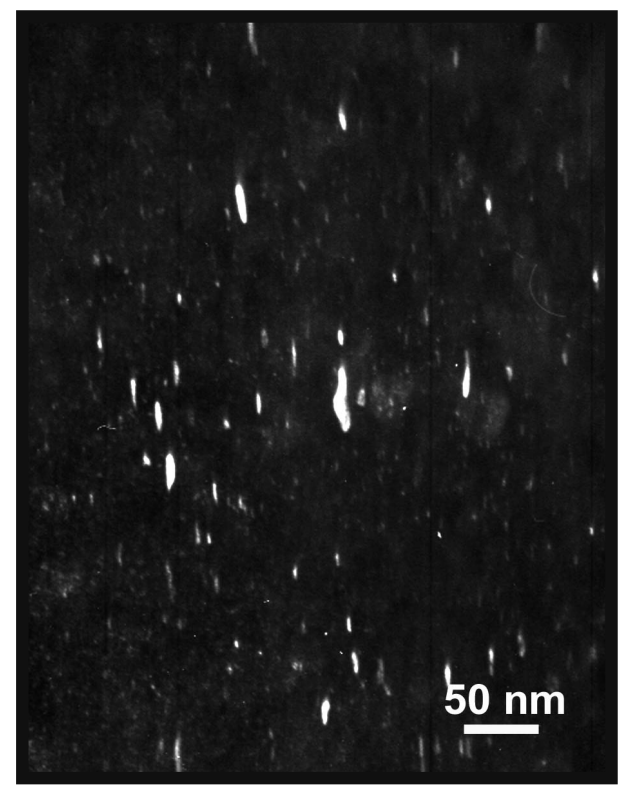

Loop density $=1.5 \times 10^{21} \mathrm{~m}^{-3}$

Avg. dia. $=19.1 \mathrm{~nm}$

Figure 29. Voids and loops in 316L SS after irradiation with $3.2 \mathrm{MeV}$ protons to $5.0 \mathrm{dpa}$ at $400^{\circ} \mathrm{C}$. 


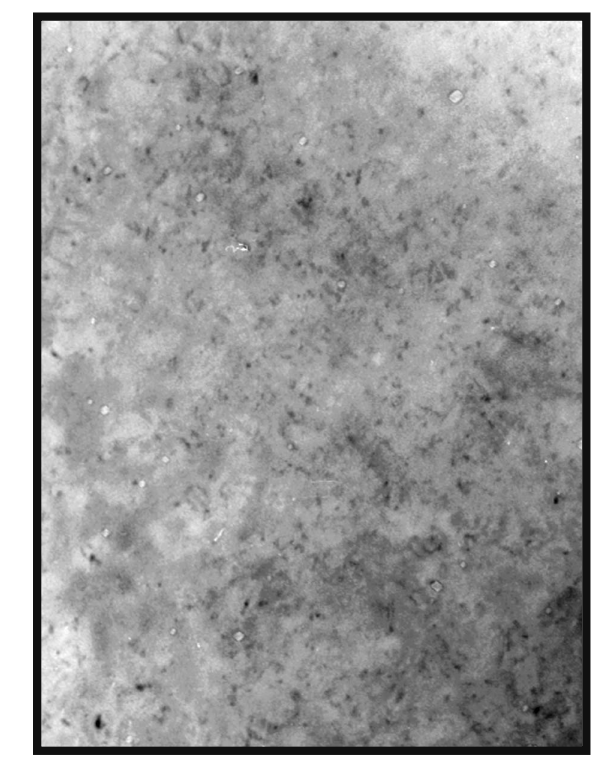

Void density $=2.4 \times 10^{21} \mathrm{~m}^{-3}$

Avg. dia. $=4.2 \mathrm{~nm}$

$\%$ swelling $=0.01$

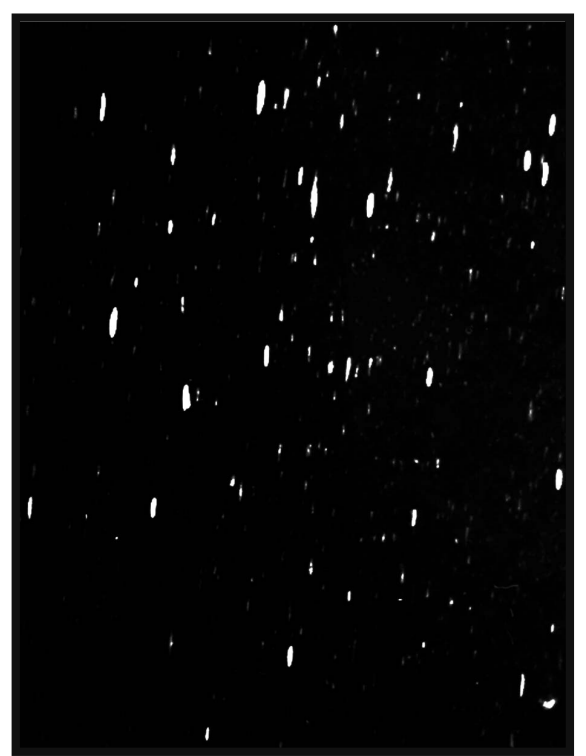

Loop density $=3.9 \times 10{ }^{21} \mathrm{~m}^{-3}$

Avg. dia. $=13 \mathrm{~nm}$

Figure 30. Voids and loops in 316SS+Hf modified alloy after irradiation with $3.2 \mathrm{MeV}$ protons to $5.0 \mathrm{dpa}$ at $400^{\circ} \mathrm{C}$.

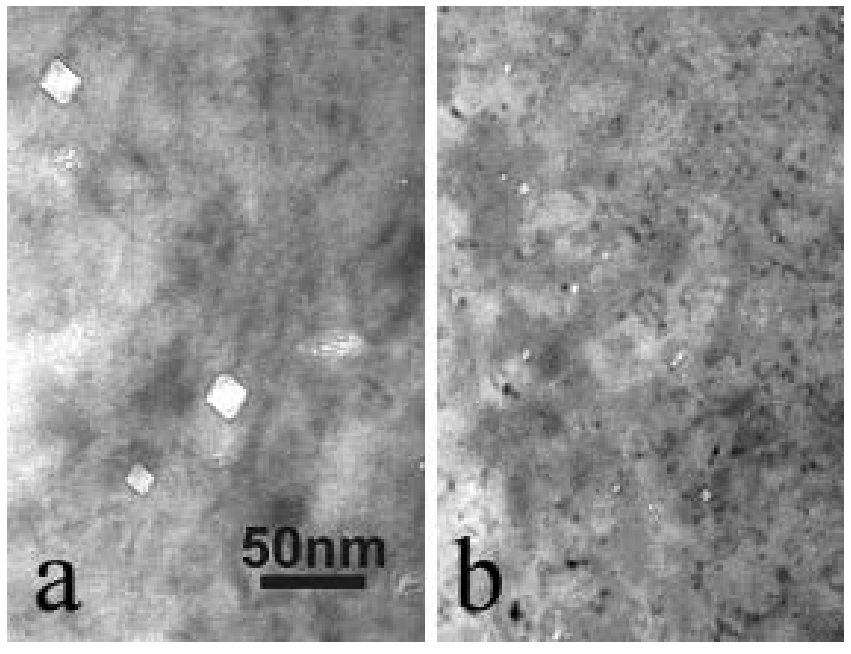

Figure 31. Bright-field images showing voids in 316L SS in (a) and 316SS+Hf modified in (b) irradiated with $3.2 \mathrm{MeV}$ protons at $400^{\circ} \mathrm{C}$ to a nominal dose of $5.0 \mathrm{dpa}$.

The loop size distribution, Figure 32, shows that after $2.5 \mathrm{dpa}$, the loop size is smallest in the $316 \mathrm{SS}+\mathrm{Hf}$ modified alloy and largest in the $316 \mathrm{SS}+\mathrm{Pt}$ alloy. Loop density is the highest in 316SS+Hf modified and the lowest in 316L SS. Loop size was the largest in 316SS+Pt where some of the Frank loops had already unfaulted as shown in Figure 33. Loop unfaulting may occur due to enhanced matrix diffusion leading to voids and larger loops. 


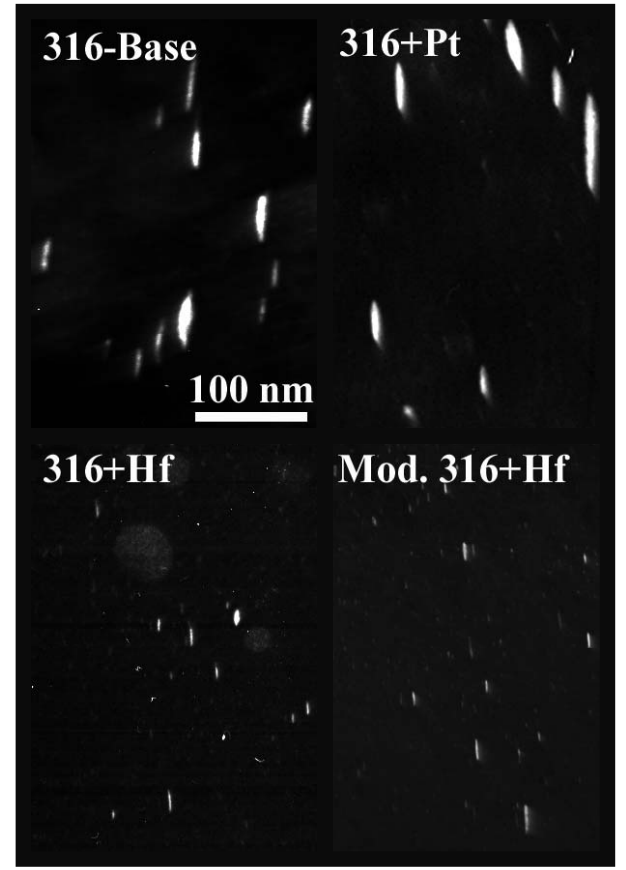

(a)

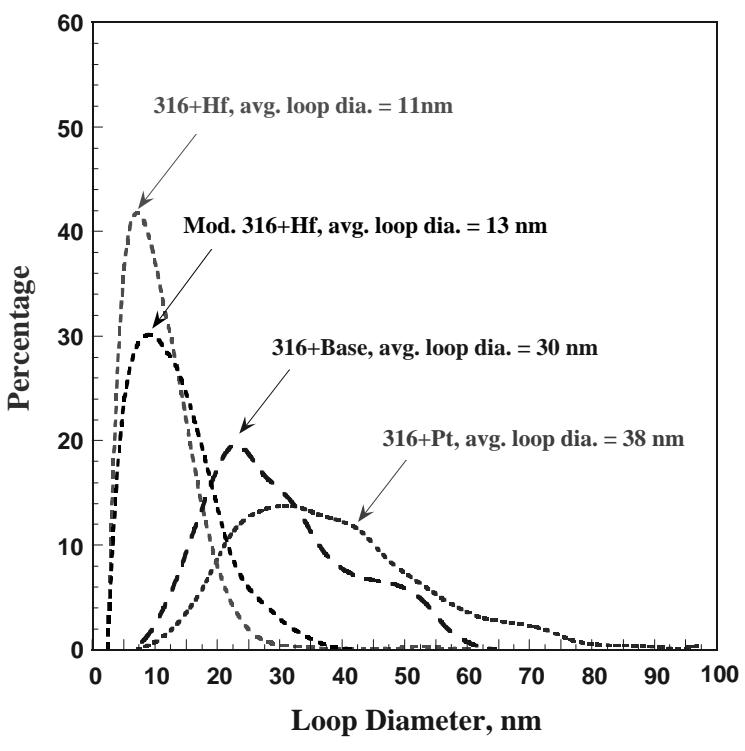

(b)

Figure 32. Dark-field images of faulted loops (a) and loop size distributions (b) in 316L SS alloys after irradiation with $3.2 \mathrm{MeV}$ protons to $2.5 \mathrm{dpa}$ at $400^{\circ} \mathrm{C}$.

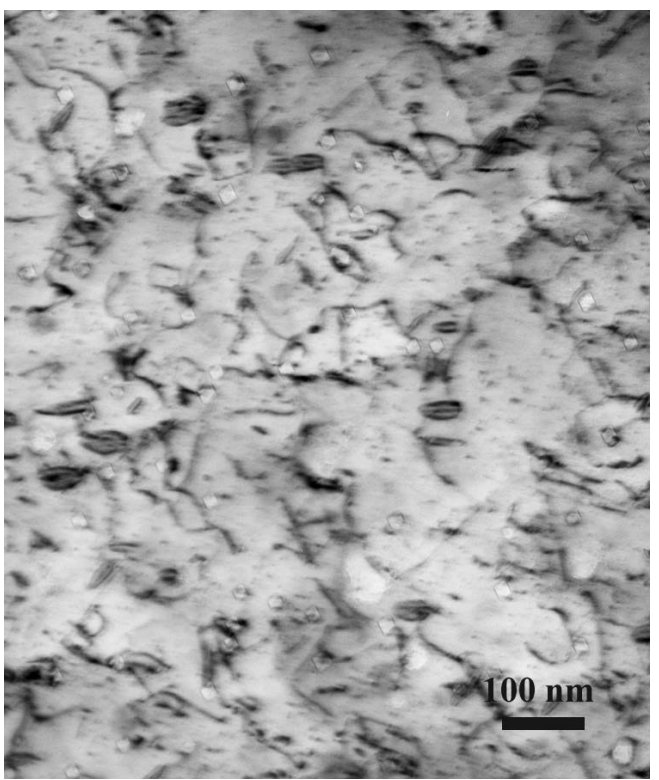

Figure 33. Bright-field image shows dislocations, loops and voids in $316 \mathrm{SS}+\mathrm{Hf}$ modified alloy irradiated to $5 \mathrm{dpa}$ at $400^{\circ} \mathrm{C}$. Image is slightly off-focus condition to observe voids. Unfaulted loops are also discernible. 
The dose dependencies of faulted loop density, faulted loop size, void density, void size and swelling for 316L SS, 316SS+Pt and 316SS+Hf modified irradiated with protons are plotted in Figures 34-36, respectively.

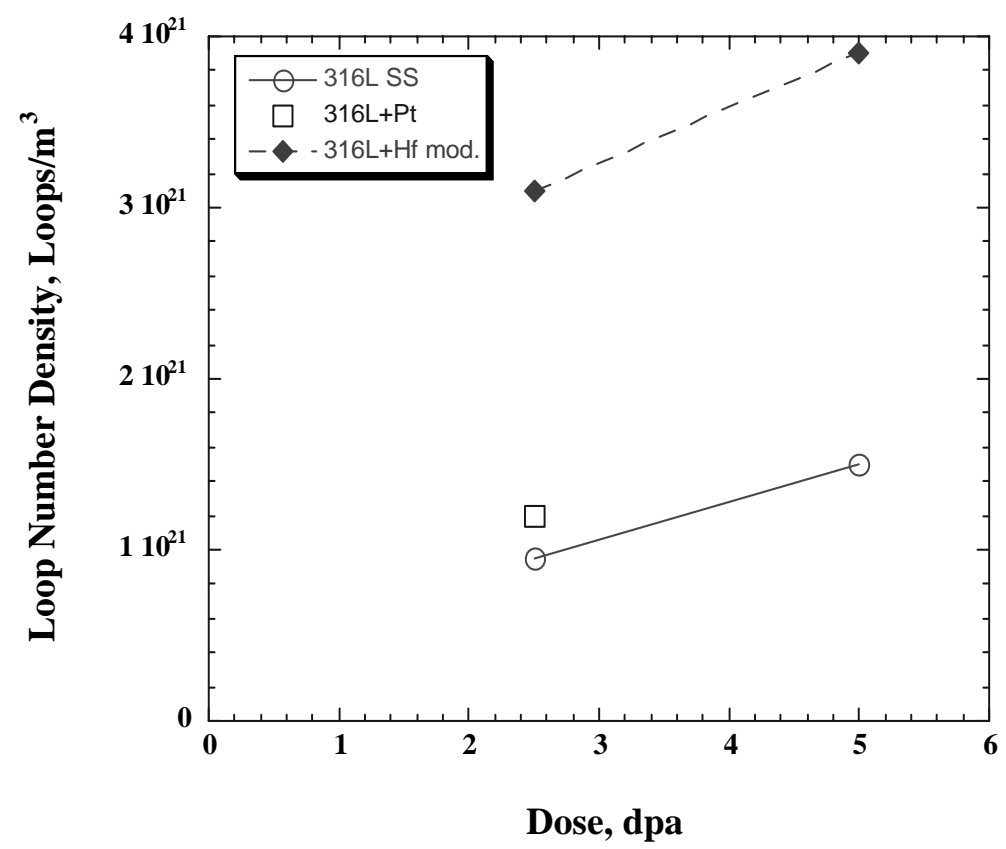

(a)

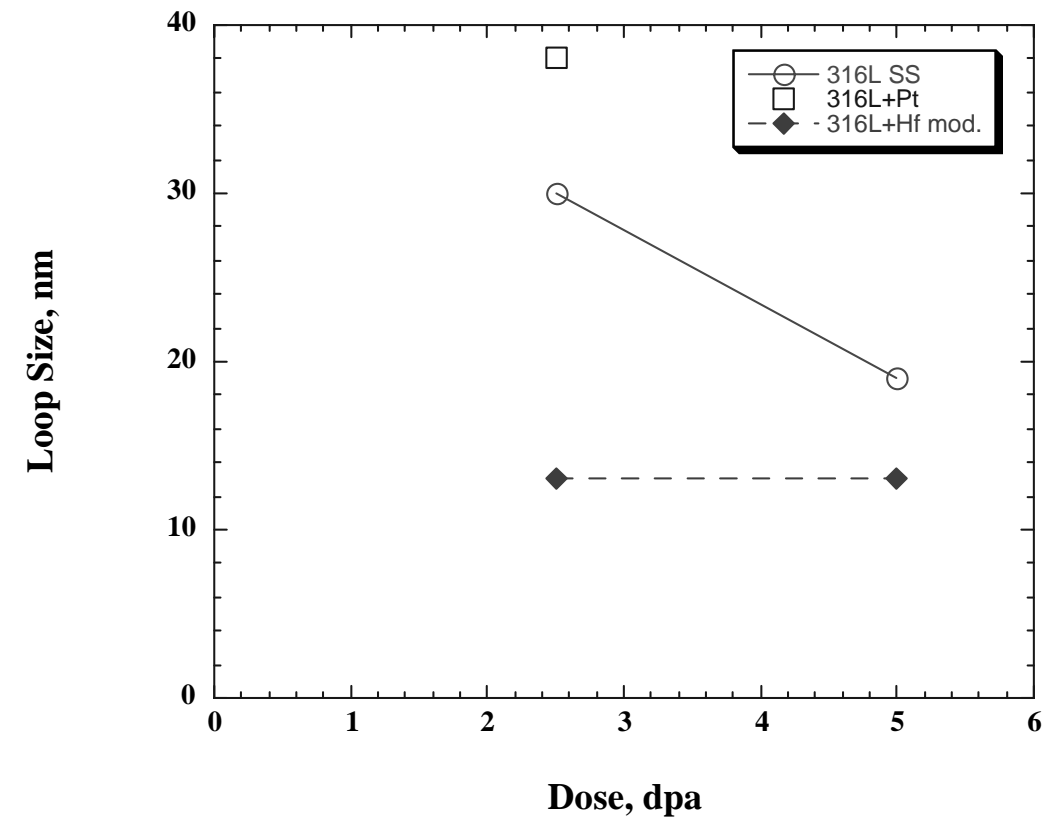

(b)

Figure 34. Dose dependence of faulted loop density (a) mean loop diameter (b) in 316L SS, $316 \mathrm{SS}+\mathrm{Pt}$ and $316 \mathrm{SS}+\mathrm{Hf}$ modified alloys proton irradiated at $400^{\circ} \mathrm{C}$. 


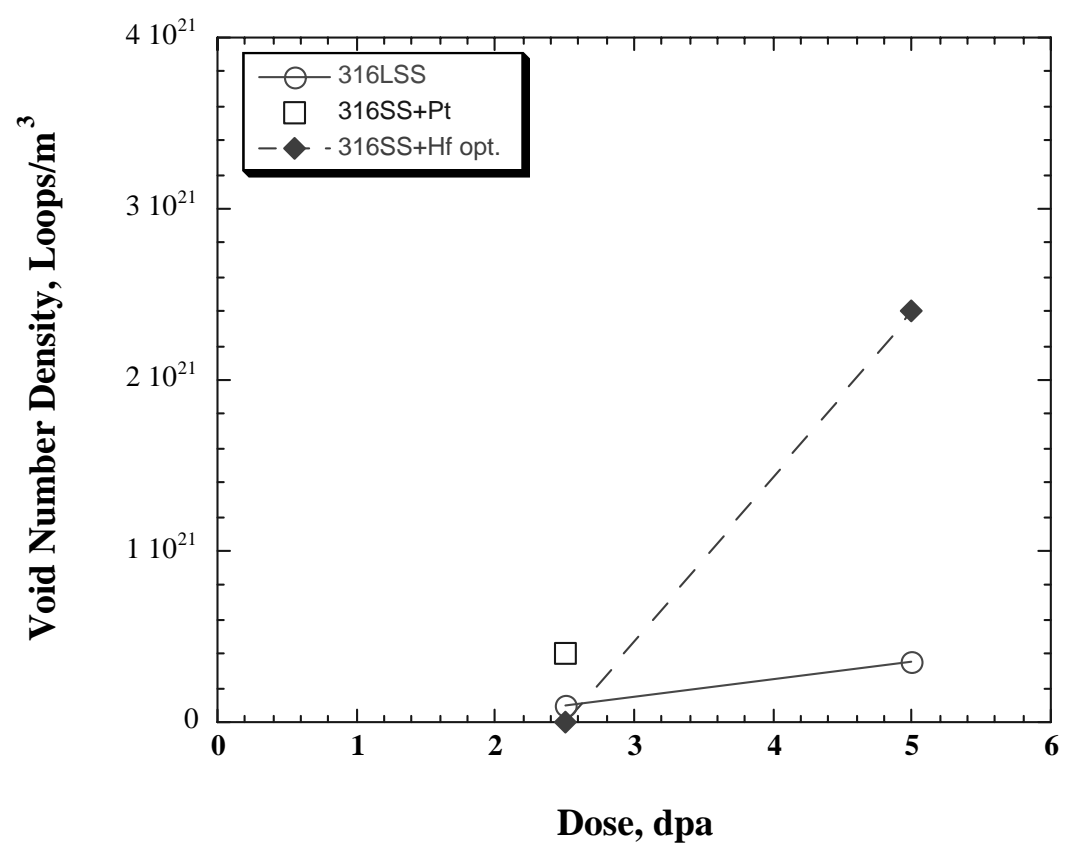

(a)

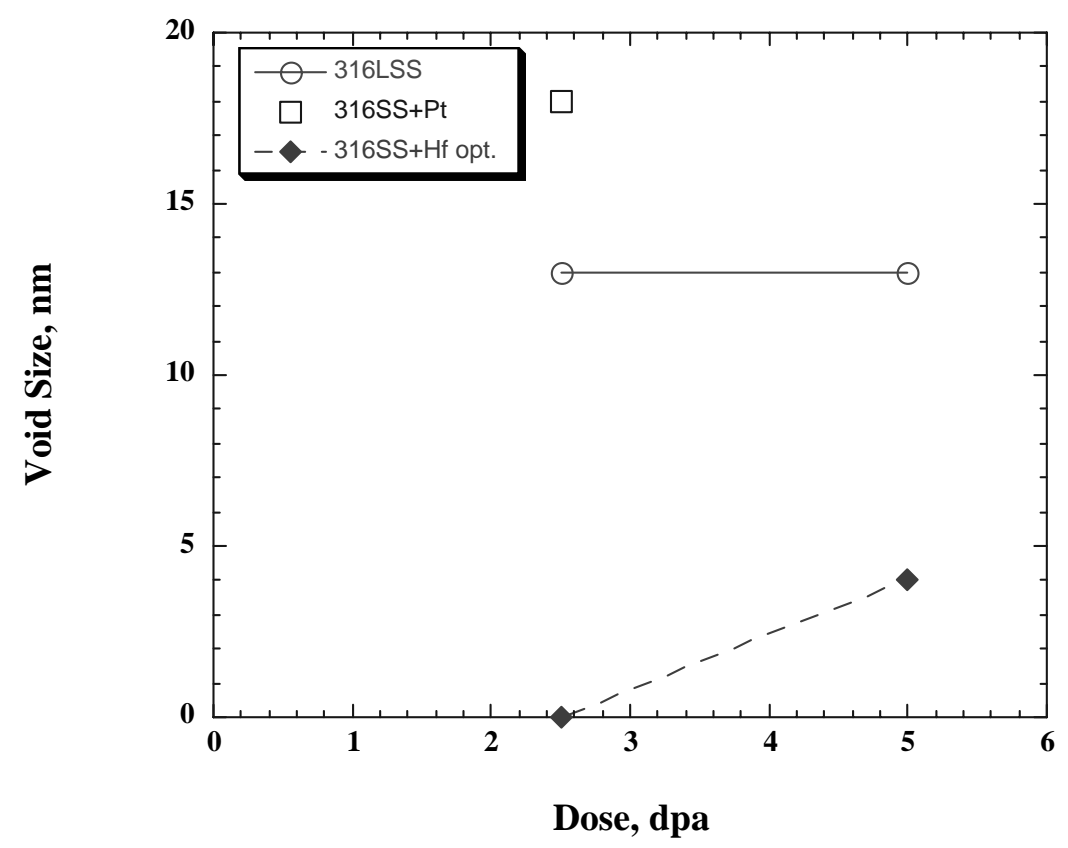

(b)

Figure 35. Dose dependence of void density (a) mean void diameter (b) in 316L SS, 316SS+Pt and $316 \mathrm{SS}+\mathrm{Hf}$ alloys proton irradiated at $400^{\circ} \mathrm{C}$. 


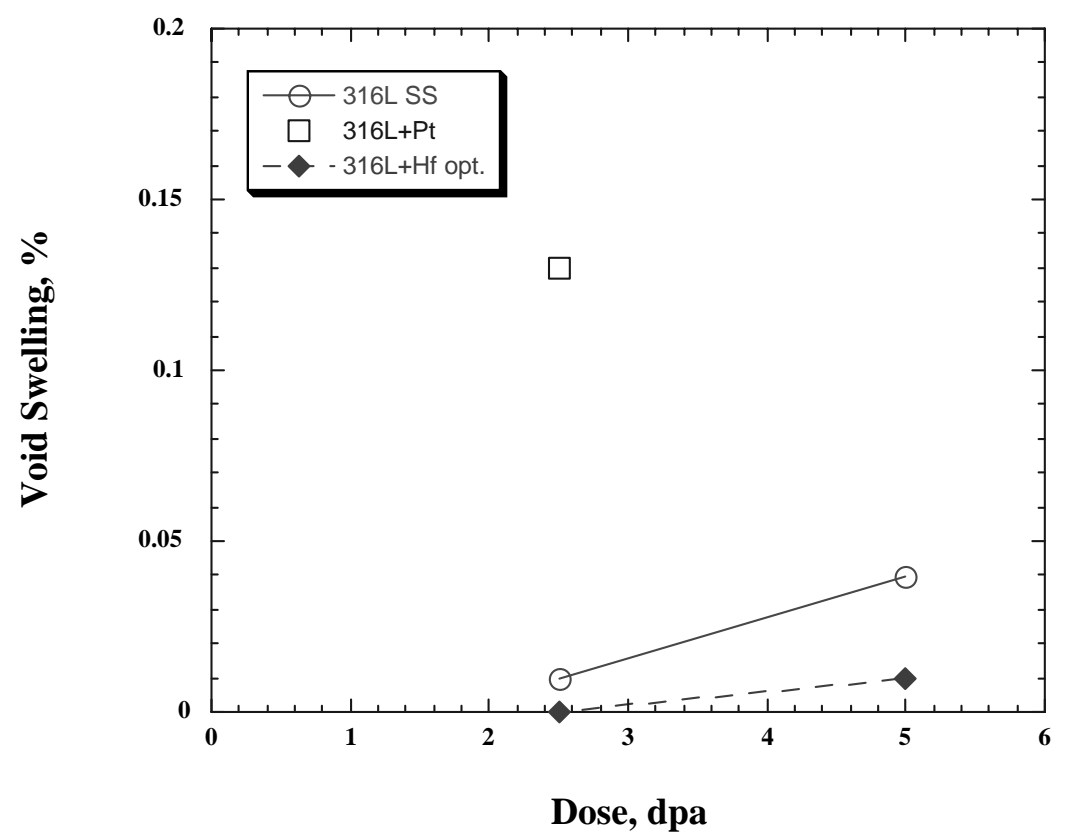

Figure 36. Dose dependence of swelling in 316L SS, 316L+Pt and 316L+Hf alloys protonirradiated at $400^{\circ} \mathrm{C}$.

Characterization of Radiation-Induced Grain Boundary Segregation

\section{Measured Grain Boundary Compositions $-\mathrm{Ni}^{++}$Irradiations}

Microchemical analyses at internal interfaces, primarily high-angle grain boundaries, have been carried out using either the JEOL 2010F FEG (field emission gun) TEM. The primary technique for microchemical analysis is energy dispersive x-ray spectroscopy (EDS) with an incident beam probe size of $0.7 \mathrm{~nm}$. High-efficiency Link analyzers and detectors are present on both instruments. A minimum of 3 grain boundaries has been analyzed for each material with multiple measurements on each boundary and at a distance of about ${ }^{ \pm} 2 \mathrm{~nm}$ and ${ }^{ \pm} 10 \mathrm{~nm}$ adjacent to each interface (i.e. on both sides). Complete composition profiles across the boundary into the matrix has been recorded out to a distance of ${ }^{ \pm} 5 \mathrm{~nm}$ in $0.5-1 \mathrm{~nm}$ steps, and to ${ }^{ \pm} 20 \mathrm{~nm}$ in $5 \mathrm{~nm}$ steps, for two of the boundaries. Specific profile steps are selected to map individual boundaries based on observed composition changes. Matrix compositions in each grain (corresponding to boundaries analyzed) have been obtained in both point mode and area mode at a distance of $\sim 50$ $\mathrm{nm}$ from the boundary. Profiles were taken from regions less than a $100 \mathrm{~nm}$ in thickness. Radiation-induced grain boundary chemistries were evaluated as a function of dose for the base alloy, the alloy with Pt addition and the alloy with Hf addition.

Detailed analyses were performed on the 316L SS base alloy at 0.5, 2.5, 10 and 50 dpa and on the 316SS+Hf modified alloy at 2.5, 10 and $50 \mathrm{dpa}$. Only a single dose of $2.5 \mathrm{dpa}$ was examined for the $316 \mathrm{SS}+\mathrm{Pt}$ alloy.

316L SS Base Alloy

Consistent with prior work on neutron- and ion-irradiated stainless steels, significant enrichment of $\mathrm{Ni}$ was observed balanced by the depletion of $\mathrm{Cr}$ and $\mathrm{Fe}$ at grain boundaries. Representative 
composition profiles are presented in Figure 37 demonstrating this behavior for each dose level. The sharp change in composition for each major alloying element is seen over a few $\mathrm{nm}$ surrounding the grain boundary plane. In general, $\mathrm{Ni}$ enrichment and $\mathrm{Fe}$ depletion tend to be narrower than for $\mathrm{Cr}$ depletion.

The increased width for Cr corresponds to "shoulders" for the Fe profiles where the concentration peaks above the matrix levels. This local Fe enrichment adjacent to the boundary depletion is seen for all three of the lower doses. A completely different profile is seen for the 50 dpa sample due to apparent grain boundary migration during irradiation exposure. Movement of the boundary increases the width of the resultant profile and results in the maximum segregation shifted to one side of the final boundary position.

Several minor alloying elements in these high-purity alloys also segregated during irradiation. The most significant was Mo as shown in Figure 38 for doses of 0.5, 2.5 and 10 dpa. A "W-

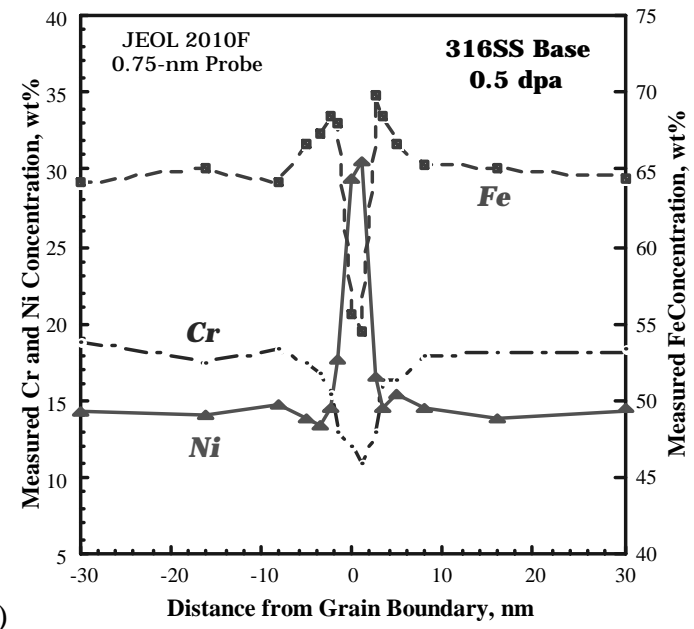

(a)

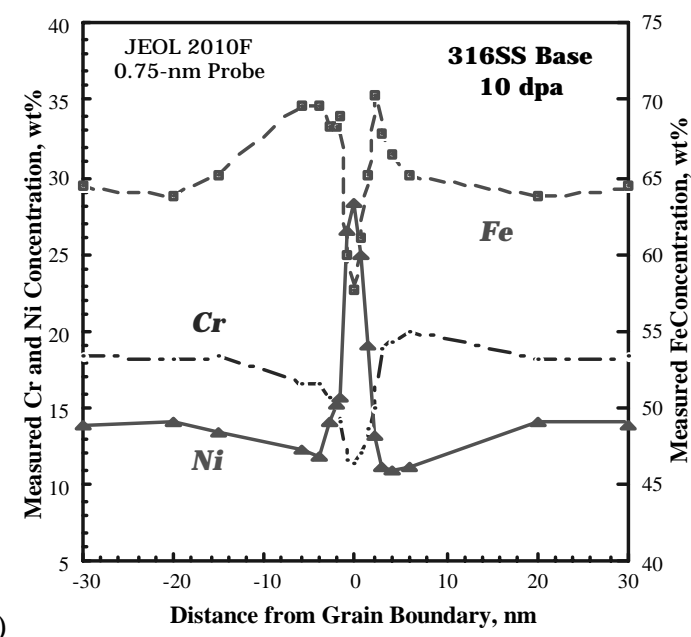

(c)

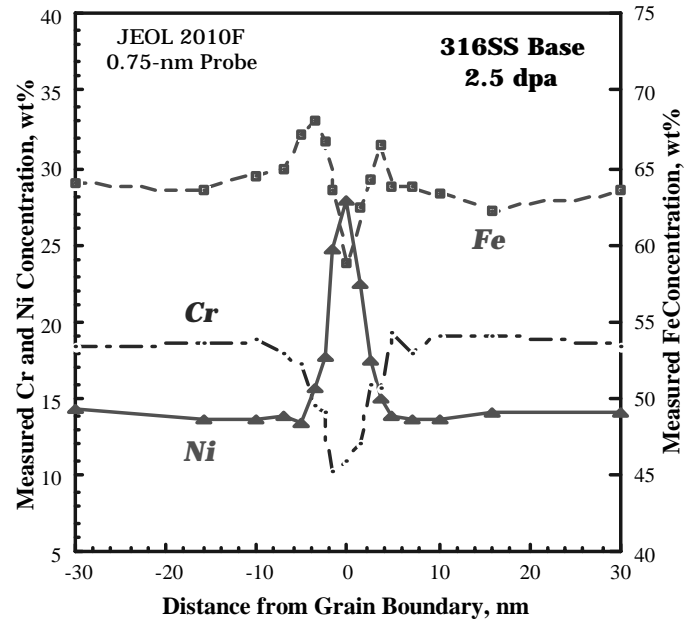

(b)

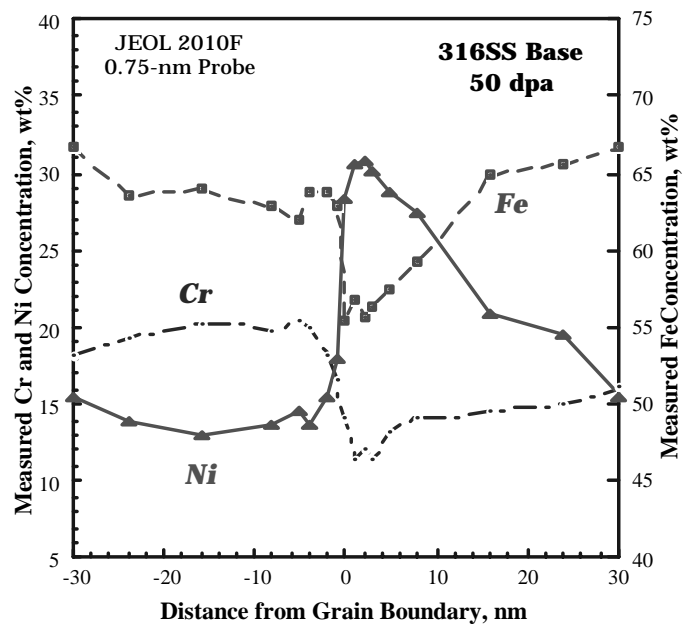

Figure 37. FEG-TEM grain boundary composition profiles for the $316 \mathrm{SS}$ base alloy after Ni-ion irradiations to (a) $0.5 \mathrm{dpa}$, (b) $2.5 \mathrm{dpa}$, (c) $10 \mathrm{dpa}$ and (d) $50 \mathrm{dpa}$. 
shaped" profile is present at the lowest dose due to pre-segregation of Mo during the initial processing heat treatments. After $0.5 \mathrm{dpa}$, Mo grain boundary levels exceed $4 \mathrm{wt} \%$ with depletion immediately adjacent to the boundary down to $\sim 1 \mathrm{wt} \%$. A slight indication of this presegregation remains at $2.5 \mathrm{dpa}$, but is eliminated by $10 \mathrm{dpa}$. As a result of this pre-segregation, Mo reveals the most significant changes as a function of dose. It is interesting that the Mo depletion widths tend to be quite broad in comparison to the major alloying elements shown above. The final minor elements that redistribute under irradiation are Mn (depletes from $\sim 1$ $\mathrm{wt} \%$ in the bulk to less that $0.4 \mathrm{wt} \%$ at the grain boundary) and $\mathrm{Si}$ (enriches from $\sim 0.1 \%$ in the bulk to $\sim 0.5 \%$ at the boundary).

\section{$\underline{316 S S+H f}$ Modified Alloy}

The influence of Hf on RIS response was mapped in detail since Hf addition had a large effect on the radiation microstructure evolution. Examples of grain boundary composition profiles for the major alloying elements are presented in Figures 39(a), (b) and (c). A very narrow profile was seen at $2.5 \mathrm{dpa}$ for both grain boundaries examined. The width of these profiles was approximately half of that measured for the 316L SS base alloy at the same dose. However although the width was reduced, the degree of segregation was enhanced at the boundaries. For example, grain boundary $\mathrm{Cr}$ minimums dropped to less than $8 \mathrm{wt} \%$ at 2.5 dpa while $\mathrm{Cr}$ minimum levels for the base alloy were $\sim 10 \mathrm{wt} \%$. The trend for $\mathrm{Cr}$ radiation-induced depletion continued at high dose with grain boundary minimums approaching $5 \mathrm{wt} \%$.

Profiles shown in Figure 39(c) did not show of grain boundary migration in the 50 dpa sample as noted for the base alloy in Figure 37(d). The increased resistant to boundary migration may result from Hf segregation that was clearly seen at $50 \mathrm{dpa}$. Grain boundary compositions reached $\sim 2.5 \mathrm{wt} \%$ as shown in Figure 39(d) and was localized with a $\mathrm{nm}$ or so of the boundary plane. This strong enrichment in combination with Ni could lead to the formation of intergranular precipitation of an intermetallic phase at higher doses. It is also important to note that measured $\mathrm{Hf}$ concentrations in the matrix were typically very low $(\sim 0.1$ to $0.3 \mathrm{wt} \%)$ at all doses in comparison to the bulk concentration for the alloy $(\sim 1 \mathrm{wt} \%)$. Measured Mo depletion at $50 \mathrm{dpa}$ is also presented in Figure 39(d) again illustrating the wider profile for this element than for others.

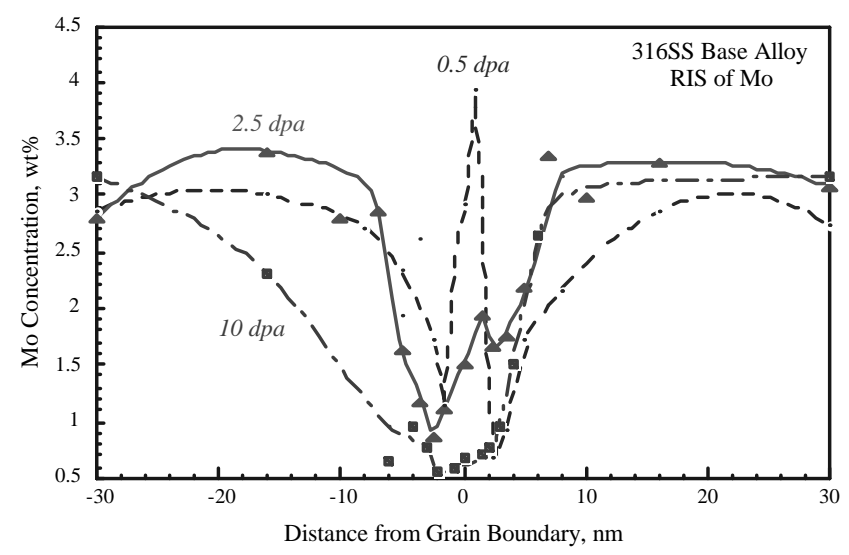

Figure 38. Grain boundary composition profiles for Mo as a function of radiation dose. 

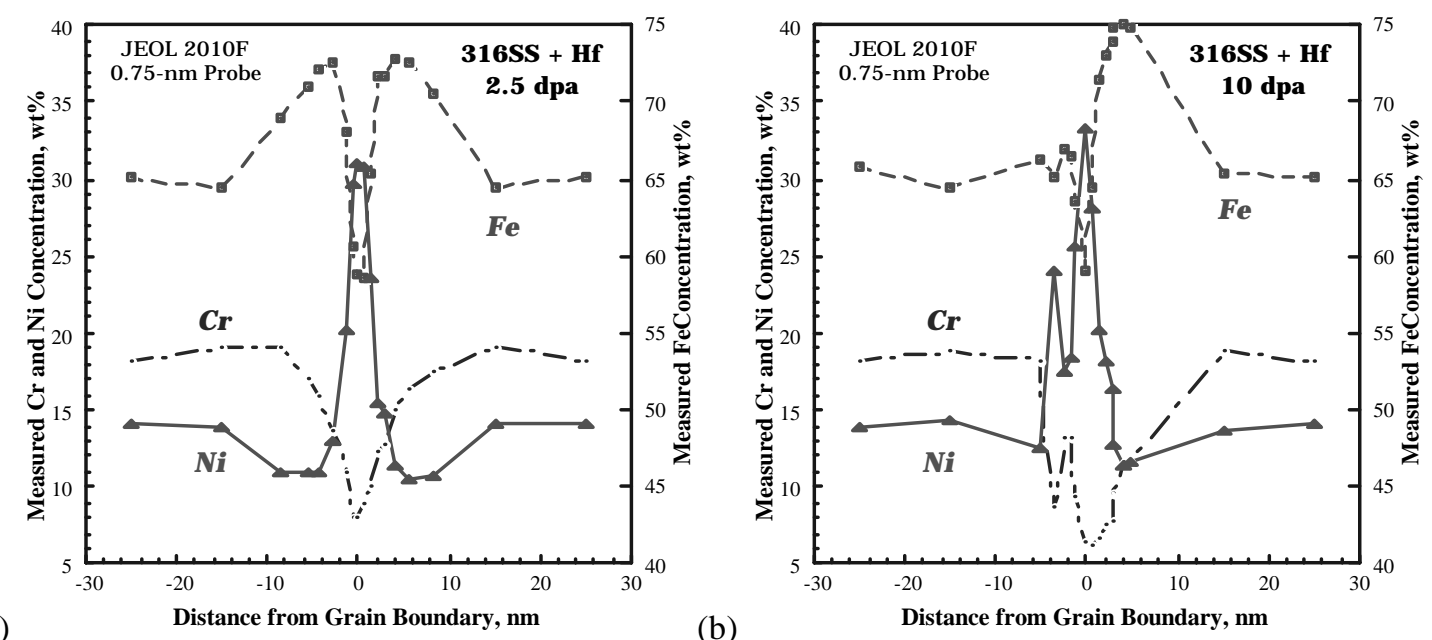

(a)

(b)

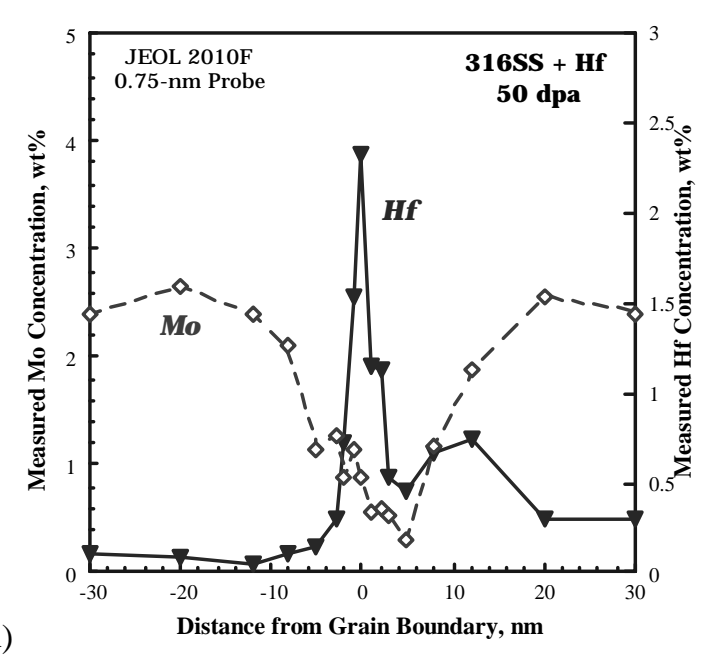

(c)

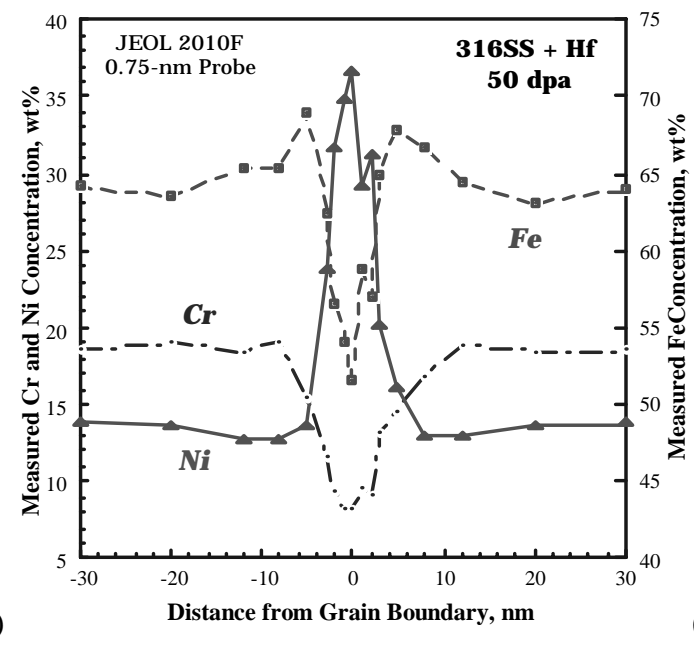

(d)

Figure 39. Radiation-induced grain boundary segregation profiles for $\mathrm{Fe}, \mathrm{Cr}$ and $\mathrm{Ni}$ in the $316 \mathrm{SS}+\mathrm{Hf}$ modified alloy as a function of dose in (a-c) and for Mo and Hf at $5 \mathrm{dpa}$ in (d).

Although its difficult to make quantitative assessments of RIS behavior without additional grain boundary analyses, trends can be established from the current data where $~ 10$ composition measurements are available for at least two grain boundaries in each irradiated condition. Averages were taken from this data set and normalized to bulk concentrations enabling dosedependent comparisons shown in Figures 40 and 41. Stronger RIS of $\mathrm{Cr}$ and $\mathrm{Ni}$ is indicated for the 316SS+Hf modified alloy in all dose conditions. Grain boundary $\mathrm{Cr}$ levels decrease by $\sim 12$ wt $\%$ in $316 \mathrm{SS}+\mathrm{Hf}$ modified versus only $\sim 7 \mathrm{wt} \%$ in 316L SS, while Ni levels increase by $17-24 \%$ $\mathrm{wt} \%$ in $316 \mathrm{SS}+\mathrm{Hf}$ modified versus only $14-17 \mathrm{wt} \%$ in $316 \mathrm{~L} \mathrm{SS}$. Interestingly, the Fe grain boundary concentration is similar over this dose range for the two alloys and Mo shows slightly less depletion in the Hf-doped alloy.

\section{$\underline{316 \mathrm{~L}+\mathrm{Pt} \text { Alloy }}$}

Detailed RIS examinations were conducted on only one irradiated condition ( $2.5 \mathrm{dpa})$ for the 316SS+Pt alloy. This was thought to be sufficient based on the dose-dependent RIS response for 


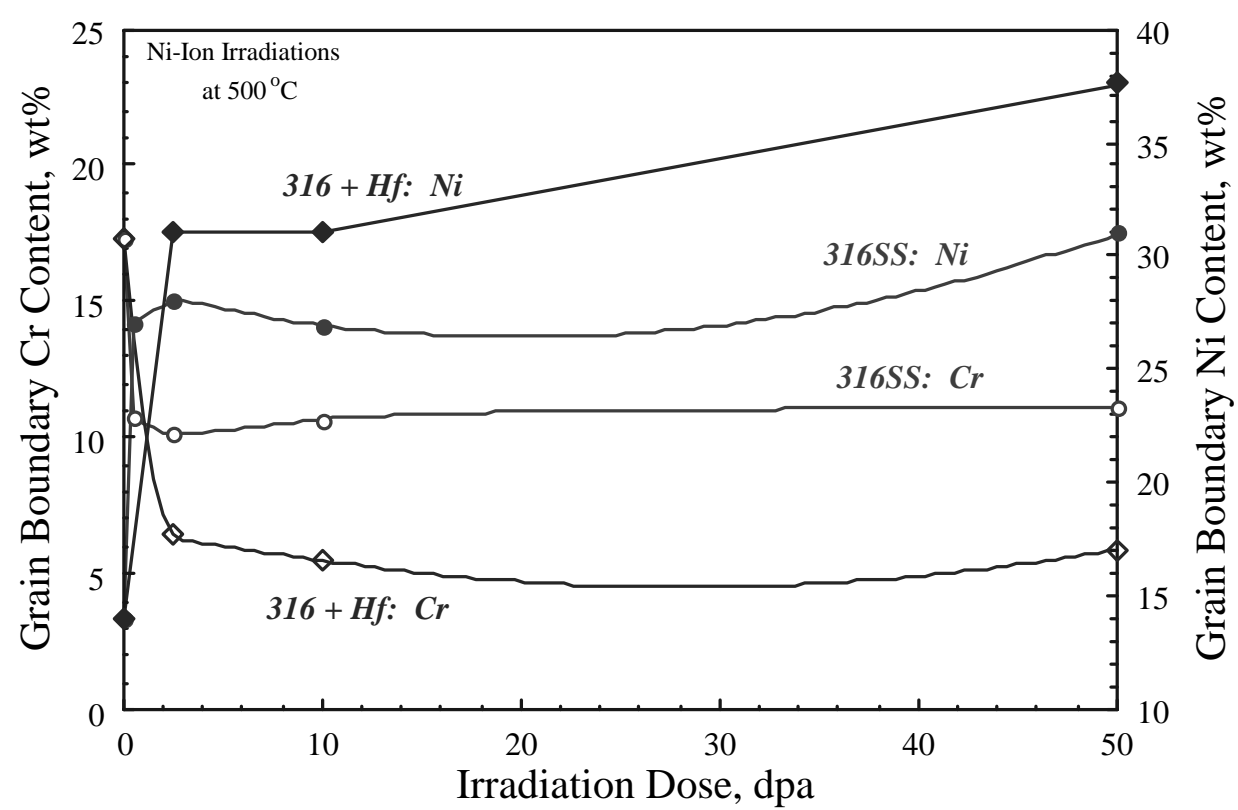

(a)

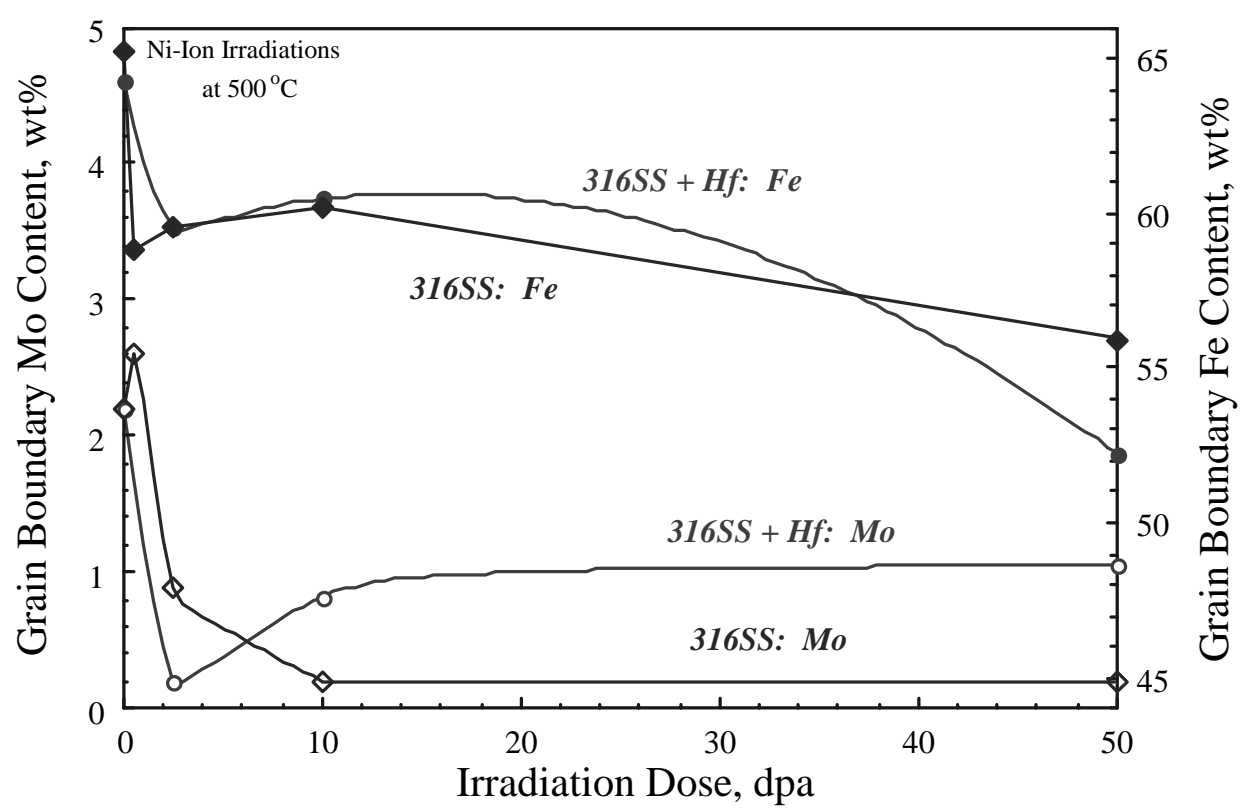

(b)

Figure 40. Average grain boundary composition measured in the 316L SS base and the $316 \mathrm{SS}+\mathrm{Hf}$ modified alloys as a function of irradiation dose.

the 316L SS base and 316SS+Hf modified alloys. Measured composition profiles for the 2.5dpa sample are presented in Figure 42. Based on this data at 2.5 dpa and limited exams on a 10dpa sample, the addition of Pt to the 316L SS base alloy does not appear to have a strong effect on RIS. Cr, Fe and Mo depletion and Ni enrichment are all comparable to that measured on the base alloy. Platinum was not seen to segregate to grain boundaries as illustrated in Figure 42(b). 


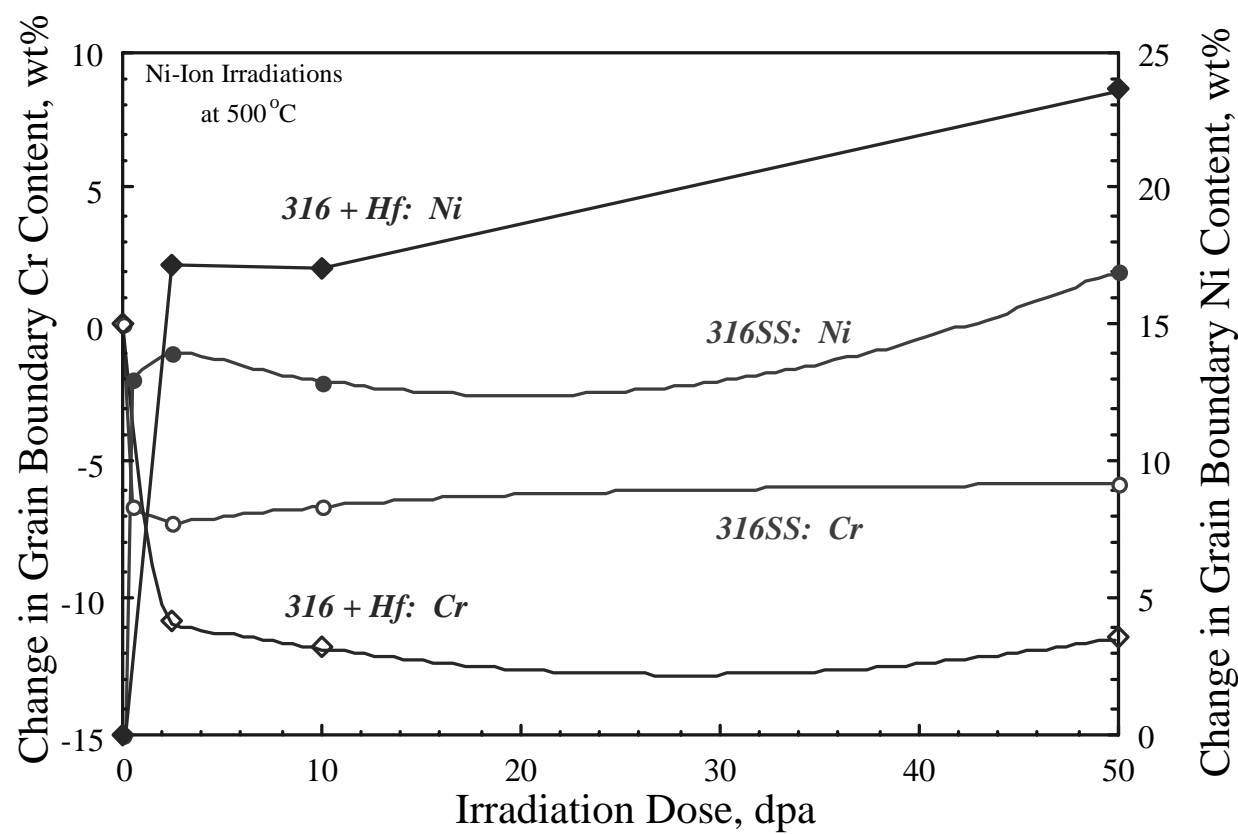

(a)

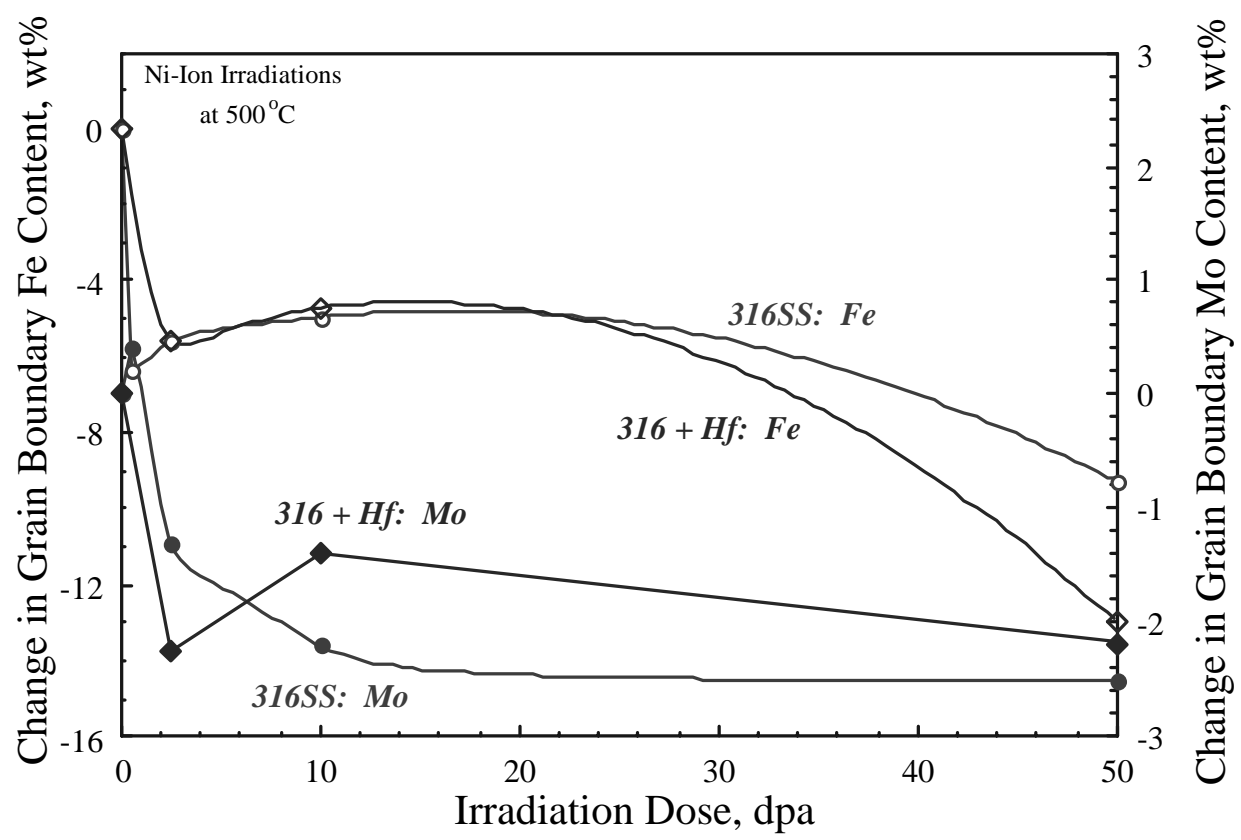

(b)

Figure 41. Measured change in grain boundary composition versus the matrix in the 316L SS base and the $316 \mathrm{SS}+\mathrm{Hf}$ modified alloys as a function of irradiation dose.

The measured variability in the grain boundary compositions is shown in Figures 43(a) and (b) for irradiation of the base alloy to $2.5 \mathrm{dpa}$ and the Hf doped alloy to $10 \mathrm{dpa}$. The graphs demonstrate the consistent measurement of RIS at multiple grain boundaries. 


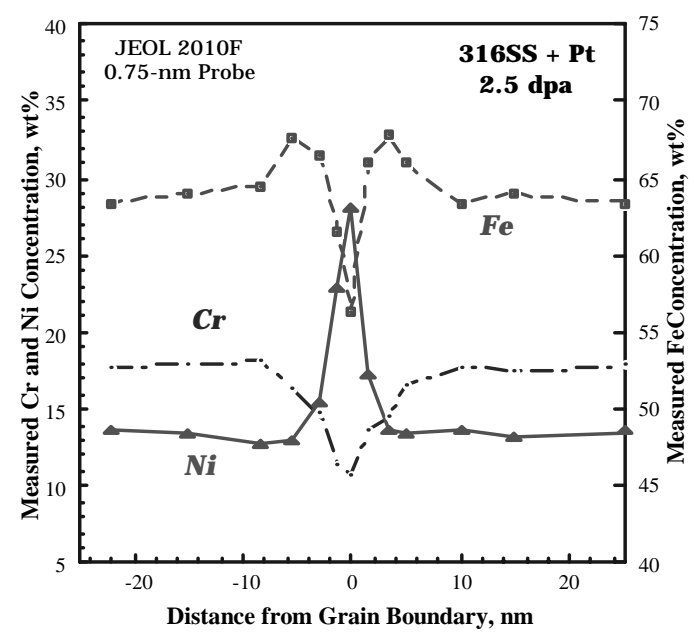

(a)

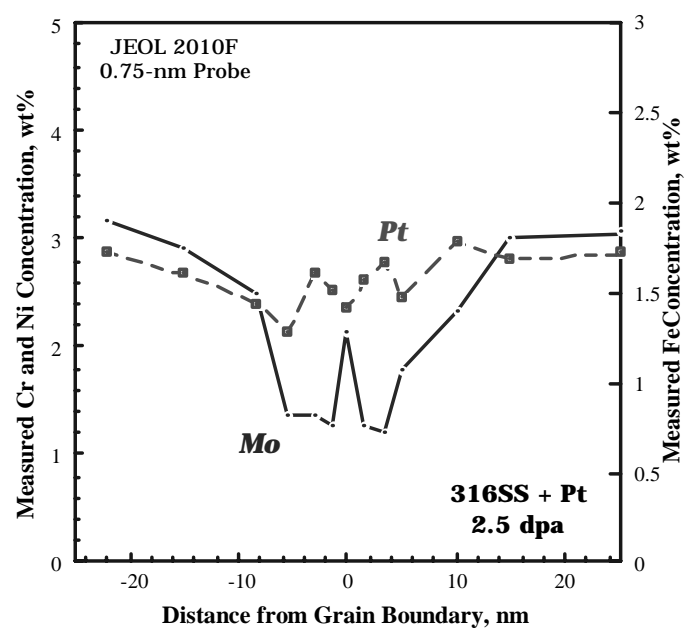

(b)

Figure 42. Measured grain boundary composition profiles for $\mathrm{Cr}, \mathrm{Fe}, \mathrm{Ni}, \mathrm{Mo}$ and $\mathrm{Pt}$ in the $316 \mathrm{SS}+\mathrm{Pt}$ alloy irradiated to $2.5 \mathrm{dpa}$.
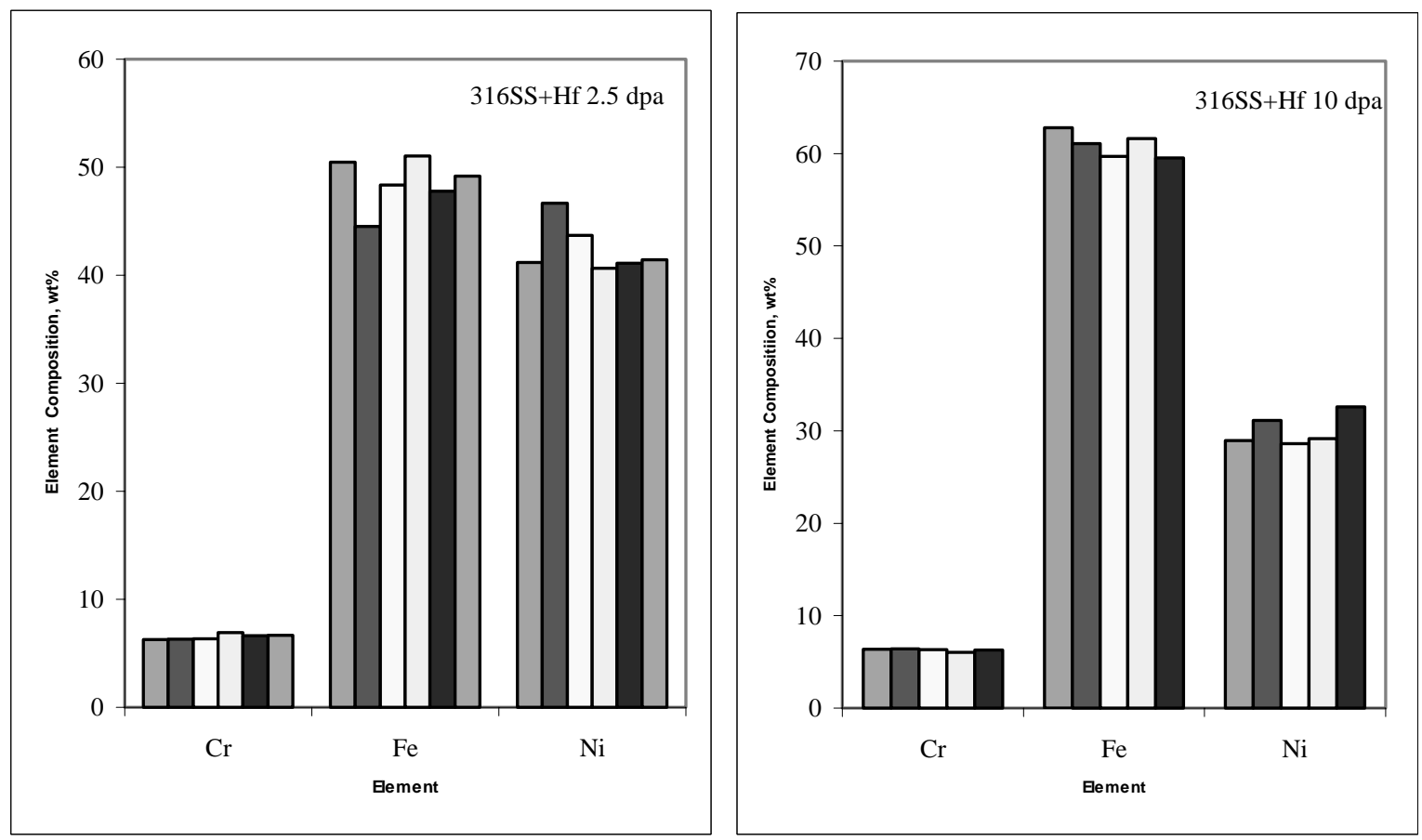

Figure 43. (a) Variability in measured grain boundary composition after 2.5 dpa irradiation of the Hf doped alloy; (b) Variability in measured grain boundary composition after (a) 2.5 dpa and (b) 10 dpa irradiation of the Hf-doped alloy.

\section{Measured Grain Boundary Compositions - Proton Irradiations}

The results of grain boundary composition measurements on 316L SS, 316SS+Pt and 316SS+Hf modified alloys proton-irradiated to $2.5 \mathrm{dpa}$ as well as 316L SS and 316SS+Hf modified alloys irradiated to $5 \mathrm{dpa}$ are summarized in Table 8. In addition, typical comparative grain boundary composition profiles of $316 \mathrm{~L} \mathrm{SS}, 316 \mathrm{SS}+\mathrm{Pt}$ and $316 \mathrm{SS}+\mathrm{Hf}$ alloys after proton irradiations are 
Table 8. Measured grain boundary compositions (wt \%) after proton irradiation.

\begin{tabular}{|c|c|c|c|c|c|c|c|c|c|c|c|}
\hline Alloy & $\begin{array}{l}\text { Dose } \\
(\text { dpa })\end{array}$ & $\# G B$ & \# GB meas. & $F e$ & $\mathrm{Cr}$ & $\mathrm{Ni}$ & Mo & $M n$ & $\overline{S i}$ & $P t$ & $H f$ \\
\hline$\underline{316 S S}$ & 2.5 & & & & & & & & & & \\
\hline Avg. $\overline{G B \text { comp. }}$ & & 2 & 6 & 63.3 & 12.4 & 22 & 1.4 & 0.8 & 0.1 & & \\
\hline Bulk comp & & & & 65.5 & 17.5 & 13.3 & 2.4 & 1.3 & $<0.1$ & & \\
\hline$\underline{316 S S+P t}$ & 2.5 & & & & & & & & & & \\
\hline Avg. GB comp. & & 2 & 6 & 64.8 & 15.4 & 15.7 & 2 & 1 & $<0.1$ & 1 & \\
\hline Bulk comp & & & & 65.2 & 16.9 & 13.6 & 2 & 1.2 & $<0.1$ & 1 & \\
\hline$\underline{316 S S+H f \mathrm{mod} .}$ & 2.5 & & & & & & & & & & \\
\hline Avg. GB comp. & & 2 & 6 & 64 & 15.8 & 15.5 & 2.5 & 1.1 & $<0.1$ & & 1 \\
\hline Bulk comp & & & & 64.8 & 17 & 13.5 & 2.4 & 1.3 & $<0.1$ & & 0.9 \\
\hline$\underline{316 S S}$ & 5 & & & & & & & & & & \\
\hline Avg. GB comp. & & 2 & 6 & 58.9 & 10.8 & 27.9 & 1.7 & 0.6 & $<0.1$ & & \\
\hline Bulk comp & & & & 65.7 & 16.8 & 14.4 & 1.8 & 1.19 & $<0.1$ & & \\
\hline$\underline{316 S S+H f \mathrm{mod} .}$ & 5 & 2 & 6 & & & & & & & & \\
\hline Avg. GB comp. & & & & 63.2 & 12.3 & 22.2 & 1.54 & 0.7 & $<0.1$ & & - \\
\hline Bulk comp & & & & 64.4 & 17.3 & 14 & 2.2 & 1.2 & $<0.1$ & & 0.9 \\
\hline
\end{tabular}

shown in Figures $44-46$. Comparisons among the three heats for individual element are illustrated in Figure 49.

Strong RIS of alloying elements and impurities was observed for the 316L SS alloy proton irradiated to $2.5 \mathrm{dpa}$. Cr, Fe, Mo and Mn concentrations at the grain boundary are depleted while $\mathrm{Ni}$ and $\mathrm{Si}$ are enriched after irradiation. In good agreement with the evolution of RIS as a function of the dose reported in the literature, ${ }^{11}$ even stronger RIS of alloying elements and impurities was observed for the 316L SS alloy proton irradiated to $5 \mathrm{dpa}$. The composition profiles of the alloying elements are nearly symmetrical, decaying to near-matrix levels within a few $\mathrm{nm}(<10 \mathrm{~nm})$ from the grain boundary as illustrated in Figure 44.

The addition of oversized solutes was found to significantly modify RIS at grain boundaries. RIS was significantly reduced for $316 \mathrm{SS}+\mathrm{Pt}$ and was almost completely suppressed for the $316 \mathrm{SS}+\mathrm{Hf}$ modified alloy irradiated to $2.5 \mathrm{dpa}$. A small amount of segregation is still observed (Figure 45) on the grain boundary composition profile of the 316L+Pt alloy proton-irradiated to $2.5 \mathrm{dpa}$. However, it should be emphasized here that grain boundary composition profiles plotted in Figures 44 - 47 are typical grain boundary composition profiles and not the average of the five grain boundary composition profiles taken while the grain boundary compositions given in Table 8 represent average values.

Irradiation to 5.0 dpa resulted in increased RIS in both the 316 SS and 316SS+Hf modified alloys. In the base alloy, the $\mathrm{Cr}$ concentration dropped to about $8 \mathrm{wt} \%$, and the $\mathrm{Ni}$ concentration increased to $34 \mathrm{wt} \%$, indicative of severe segregation. Significant RIS occurred in the 316SS+Hf modified alloy, but the degree of segregation was considerably less than in the base 316L SS alloy. After $5.0 \mathrm{dpa}$, the grain boundary $\mathrm{Cr}$ concentration was $12 \mathrm{wt} \%$ and the grain boundary $\mathrm{Ni}$ concentration was $22 \mathrm{wt} \%$. In fact, the amount of grain boundary segregation in $316 \mathrm{SS}+\mathrm{Hf}$ 
modified alloy at $5.0 \mathrm{dpa}$ was similar to that for the $316 \mathrm{~L} \mathrm{SS}$ alloy at $2.5 \mathrm{dpa}$, indicating that the $\mathrm{Hf}$ addition still influenced the radiation-induced grain boundary composition at $5.0 \mathrm{dpa}$, but not as dramatically as at $2.5 \mathrm{dpa}$.

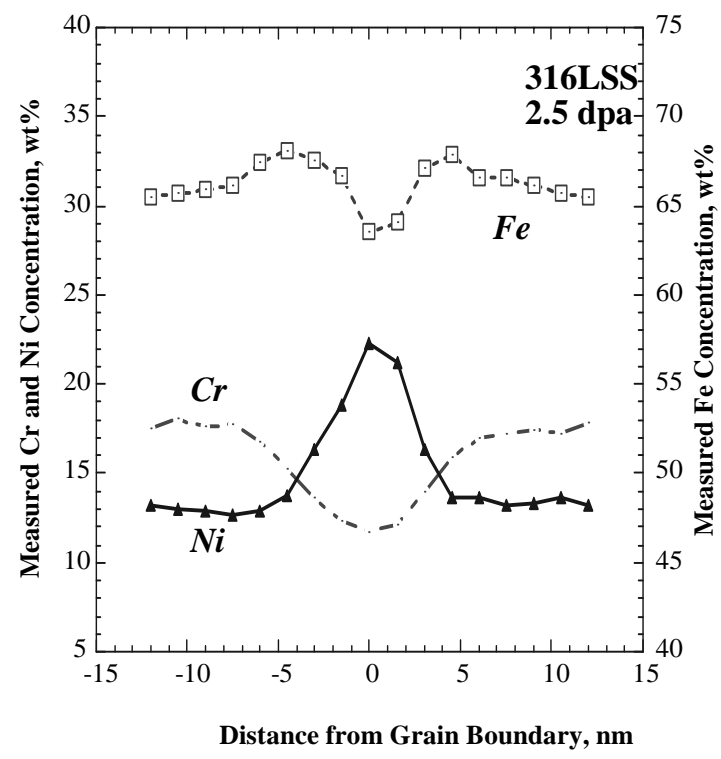

(a)

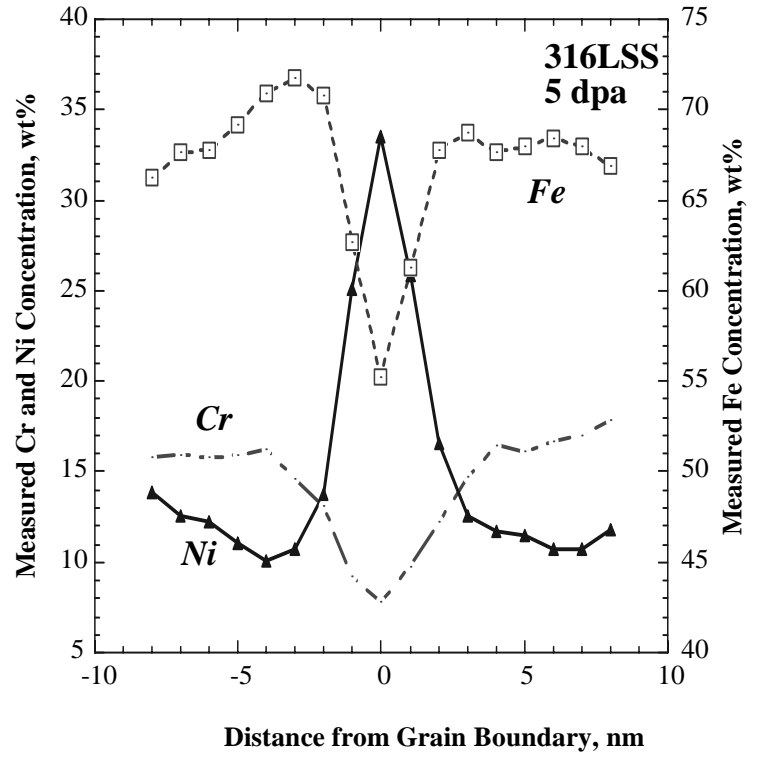

(b)

Figure 44. Radiation-induced grain boundary segregation for the 316L SS base alloy after proton irradiation to (a) $2.5 \mathrm{dpa}$ and (b) $5.0 \mathrm{dpa}$ at $400^{\circ} \mathrm{C}$.

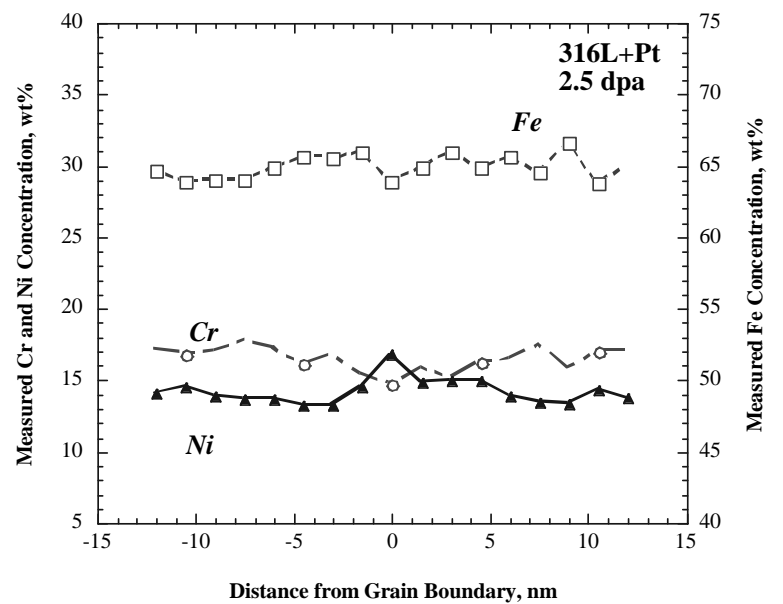

Figure 45. Typical grain boundary composition profiles for the $316 \mathrm{SS}+\mathrm{Pt}$ alloy after proton irradiation to $2.5 \mathrm{dpa}$ at $400^{\circ} \mathrm{C}$. 


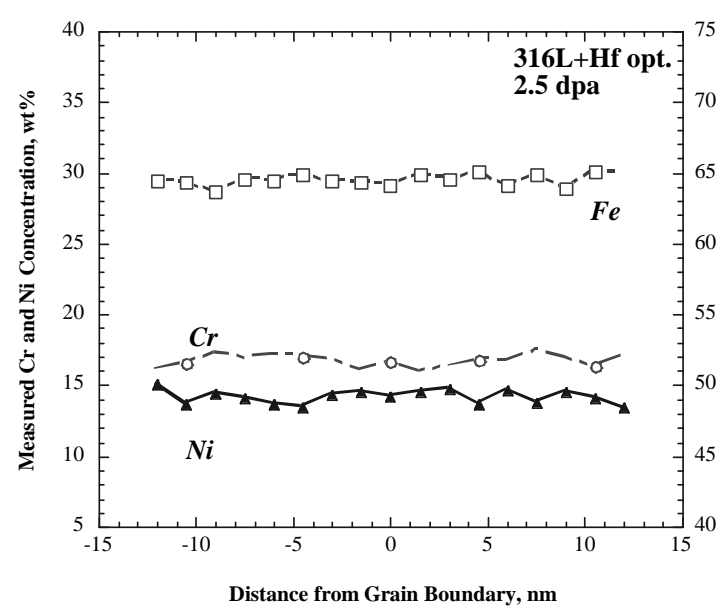

(a)

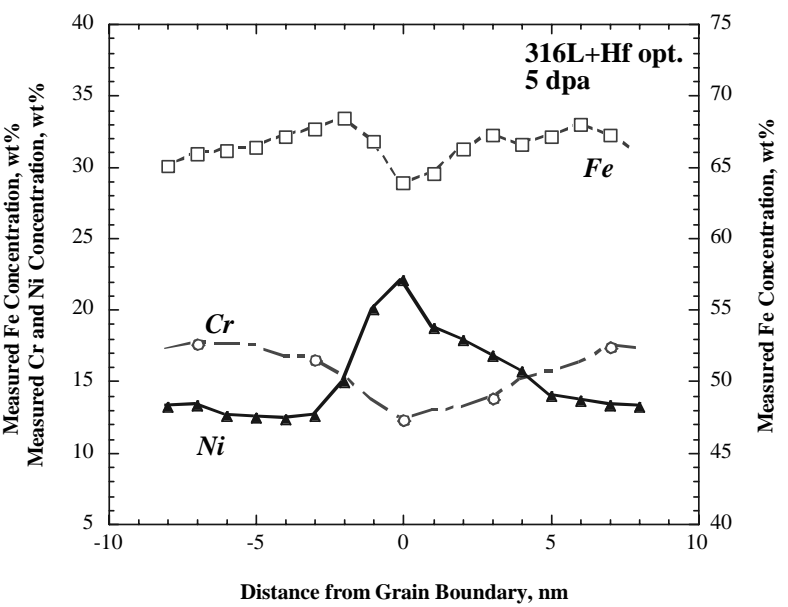

(b)

Figure 46. Composition profiles across grain boundaries fro the $316 \mathrm{SS}+\mathrm{Hf}$ alloy after proton irradiation to (a) $2.5 \mathrm{dpa}$ and (b) $5.0 \mathrm{dpa}$ at $400^{\circ} \mathrm{C}$.

\section{Discussion of Oversized Element Concept for Radiation-Damage Resistance}

The thesis of the oversized solute concept was to inhibit control of detrimental radiation damage by the addition of a very large misfit solute. The hypothesis was that Hf addition should affect the evolution of radiation damage because of its very larger size, very higher mass and known influences on modifying the distribution of solutes in the host matrix. Comparison experiments delineated effects from the study of variation in material composition and processing as well as variation in radiation environment. Nickel-ion irradiation demonstrated resistance to swelling to $50 \mathrm{dpa}$ and proton irradiation demonstrated resistance to IASCC initiation to $5 \mathrm{dpa}$. Oversized solutes affect the production, migration and aggregation of point defects during irradiation. The findings of this NERI project have elucidated proposed mechanisms for improved damage resistance.

The addition of oversized solute to 316SS affects the accumulation of radiation damage through specific influences on point defects and their aggregates. The controlling mechanisms for the development of damage-resistant alloys were explored by the addition of Hf or Pt to 316SS and documenting the radiation response after either $\mathrm{Ni}^{++}$irradiation or proton irradiation. Key findings that elucidate mechanistic understanding include (1) the significant improvement in void swelling for the $316 \mathrm{SS}+\mathrm{Hf}\left(1100^{\circ} \mathrm{C}\right)$ alloy and (2) no beneficial effect for Pt addition on radiation-induced microstructure and (3) generally consistent results between $\mathrm{Ni}$-ion and proton irradiation except for RIS response.

Fundamentally, radiation effects are dominated by the production, migration and annihilation of vacancies and interstitials. The mechanistic influence of oversized solute can be understood in terms of either mutual recombination or partitioning of defects into accumulating microstructure and microchemistry. Mutual recombination mechanisms affect radiation-induced processes in general because it reduces the number of defects that are long-lived enough to participate in persistent changes. More complex mechanisms affect radiation-induced processes by altering defect aggregation processes that leave an imprint on the alloy microstructure and 

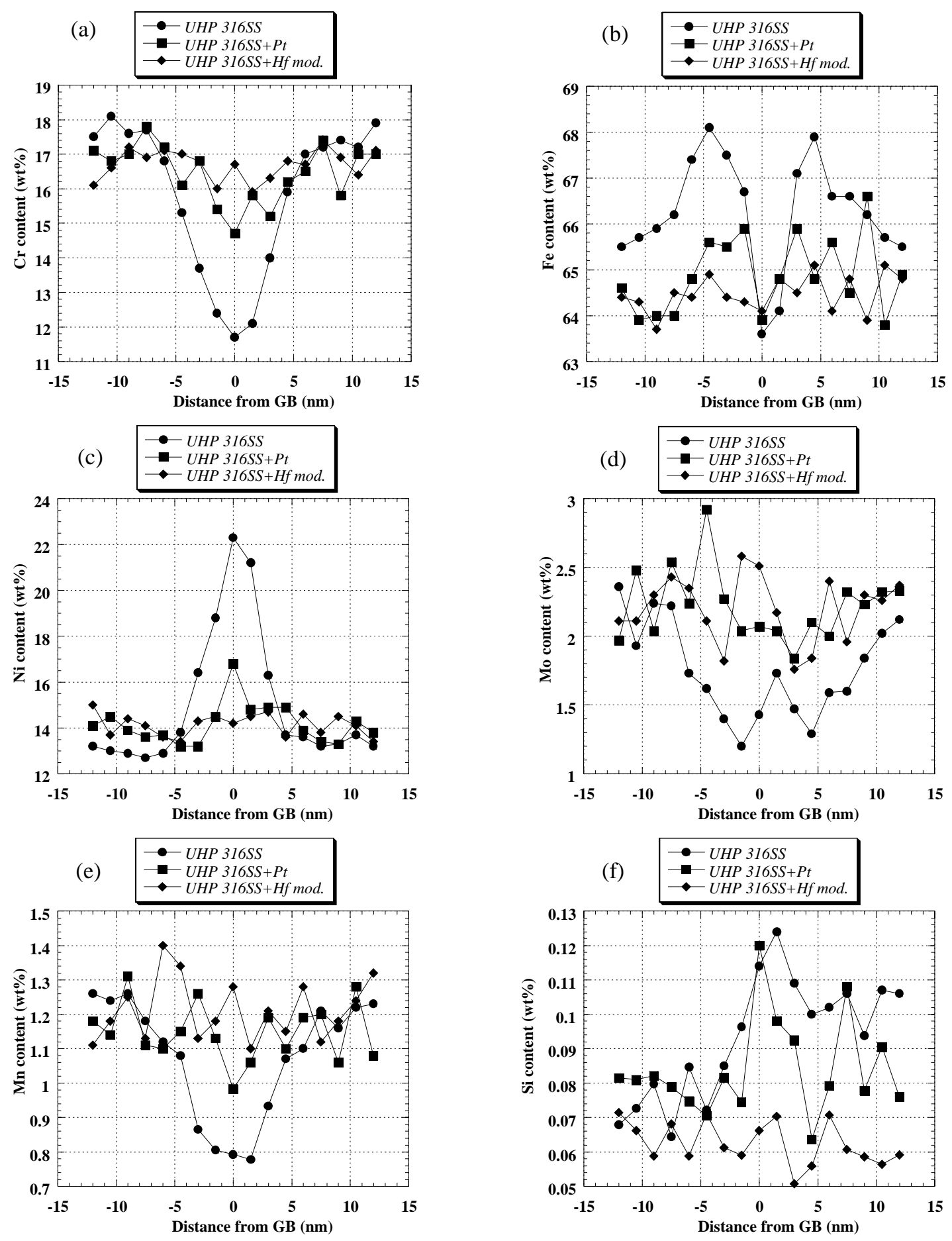

Figure 47. Typical grain boundary composition profiles for (a) $\mathrm{Cr}$, (b) $\mathrm{Fe}$, (c) $\mathrm{Ni}$, (d) $\mathrm{Mo}$, (e) $\mathrm{Mn}$ and (f) Si comparing RIS for the 316L SS base alloy, 316SS+Pt and 316SS+Hf after proton irradiation to $2.5 \mathrm{dpa}$ at $400^{\circ} \mathrm{C}$. 
microchemistry. Microstructural and microchemical characterization document this imprint and provide evidence of damage resistance. This research has systematically examined radiation responses in comparative alloys based on 316SS. In addition, radiation responses from $\mathrm{Ni}^{++}$ irradiation (high damage rates and large cascades) were compared with responses from proton irradiation (low damage rate and small cascades).

The effect of Hf addition on the irradiated microstructure is complex both physically and chemically. The large size promotes binding with single vacancies and perhaps single interstitials. This slows the migration of vacancies and promotes recombination with interstitials. Elastic interactions between oversized Hf and multiple vacancies may promote void embryo formation by stabilizing small clusters of vacancies. Large oversized solute elastically alters sink efficiencies and the partitioning of defects to the evolving microstructure. In contrast to longterm defect aggregation, the short-term production efficiency of point defects and clusters is affected by a more rapid cascade quench for the high $\mathrm{Hf}$ electron concentration and is affected by disrupted collision sequences caused by the heavy Hf mass. Although the above physical characteristics mostly likely dominate damage processes, the chemical reactivity of Hf is known to affect the non-irradiated metallurgical behavior of alloys. Similar processes will influence radiation behavior. Hf-rich precipitates remove solute (i.e., $\mathrm{C}$ and $\mathrm{Ni}$ ) from the matrix and hence may alter damage evaluation.

Mutual Recombination Influence

The simplest Hf physical influence on radiation behavior is by promotion of mutual recombination. This mechanism is expected from rate theory for defect migration and aggregation. Oversized elements interact elastically with vacancies because of elastic accommodation between the empty lattice site and the oversized element. Mutual recombination affects both microstructural evolution as well as microchemical evolution. Sakaguchi et al. ${ }^{\text {, }}$ assuming that the oversized solute atoms interact with vacancies and form additive-vacancy complexes, modeled the influence of the linear size factor of the oversized solute atoms on RIS. The linear size factor, $\mathrm{L}_{\mathrm{sf}}$, is defined as:

$$
L_{\text {sf }}=\left\{\left(\Omega_{\text {add }} / \Omega_{\text {sol }}\right)^{1 / 3}-1\right\} \times 100(\%)
$$

where $\Omega_{\text {sol }}$ is the atomic volume of the solvent, and $\Omega_{\text {add }}$ is the atomic volume of the additive. The amount of segregation was observed to decrease linearly with increases in the linear size factor of the oversized solute atoms. Model predictions were in good agreement with RIS measurements performed by Kato et al. ${ }^{8}$ on model 316L stainless steel alloys doped with different oversized solute atoms and electron irradiated to $10.8 \mathrm{dpa}$ at $500^{\circ} \mathrm{C}$. On the basis of this comparison, Sakaguchi et al. ${ }^{7}$ concluded that the additive-vacancy binding energy is related to the linear size factor and controls the influence of oversized solute atoms on RIS. Point defects are destroyed in the matrix prior to their ability to migrate and influence properties. In the present study, the addition of Hf generally did retard void formation while its influence on RIS was unclear.

After $\mathrm{Ni}^{++}$irradiation, the degree of major alloying element $(\mathrm{Cr}, \mathrm{Ni}$ and $\mathrm{Fe}$ ) RIS in the alloy with Hf was similar or greater than that measured in the irradiated base alloy without Hf. This indicates a lack of effect on mutual recombination. However, a much-reduced Ni enrichment 
and $\mathrm{Cr}$ depletion was observed after proton irradiation as illustrated in Figure 47. At 2.5 dpa, only minimal RIS was measured in $316 \mathrm{SS}+\mathrm{Hf}$ compared to significant segregation in the base 316SS. At a higher dose of $5 \mathrm{dpa}$, the Hf influence on proton RIS can be seen by comparing Figures 44 (b) and 46 (b). Based on these results, Hf additions appear to delay the evolution of RIS during proton irradiation at $400^{\circ} \mathrm{C}$. This is quite different than observed for the Ni-ion irradiated samples at $500^{\circ} \mathrm{C}$.

After electron irradiation, Kato et al. ${ }^{8}$ reported that addition of $\mathrm{Hf}$ had a profound influence on reducing RIS. The electron-irradiation experiment required use of very thin specimens that exhibited extensive grain boundary migration during irradiation. The concentration profiles were measured using a 10-nm, through-thickness probe, five-to-ten times larger then the current measurements. Comparison of the electron-induced profiles with the ion-induced profiles is complicated by these differences in experimental technique. The electron-induced profile widths were of the order of $100 \mathrm{~nm}$ whereas the ion-induced profiles widths were less than $10 \mathrm{~nm}$. The larger analysis probe size for the electron irradiation analysis makes detection of $\mathrm{nm}$ variations in concentration difficult. None-the-less, the Cr-depletion magnitudes measured after ion irradiation would have appeared as a depletion using the $10-\mathrm{nm}$ resolution but the depletion would have been much less. The observation of a beneficial effect for electron and proton irradiations at low dose may suggest that the Hf influence is dependent on irradiation particle type and hence cascade characteristics.

The influence of binding on mutual recombination can be explored by examining the effect of a larger vacancy migration energy on the expected change in RIS. The calculated effect of an assumed binding energy on $\mathrm{Cr}$ depletion and Ni enrichment is shown in Figure 48. The vacancy migration energy is assumed to be the sum of the migration energy without oversized solute (1.0 $\mathrm{eV})$ plus a factor attributed to binding. The calculation indicates that to eliminate RIS a vacancy migration energy in excess of $2 \mathrm{eV}$ is required. This magnitude of energy increase is not expected for elastic binding between vacancies and oversized solutes. The expected binding energy, and the effect on the migration energy, is only a few tenths of an eV. Therefore, Hf's influence on mutual recombination is not consistent with measured RIS behavior for proton and electron experiments. However, it should be noted that this calculation refers to the steady-state case and as shown in proton irradiations, the effect of $\mathrm{Hf}$ is transient, and its affect may be better captured by modeling it as a saturable sink.

\section{Oversized Solute Influence on Defect Aggregation}

In addition to solute-point defect interactions, solute can influence aggregation of either vacancytype or interstitial-type clusters. The large Hf solute stress is relieved by associating with multiple vacancies. However, Hf additions were observed to retarded void formation in contrast to the vacancy cluster stabilization concept. Pt addition did accelerate the evolution of voids to a small degree which is consistent with vacancy cluster stabilization. In general, there is no consistent observation that oversized solutes promote void nucleation.

The aggregation of interstitials in loops showed a systematic difference when comparing irradiation behavior in alloys with and without Hf addition. The loop sizes were smaller in the alloys with $\mathrm{Hf}$ addition and the loop number density exhibited a persistent increase in density with increasing dose in the $316 \mathrm{SS}+\mathrm{Hf}$ alloy. In contrast, the alloy with Pt addition was most 
swelling prone and the loop density exhibited the most rapid increase in loop number density to 2 dpa and exhibited a maximum in loop volume fraction at this dose. These empirical observations demonstrate that $\mathrm{Hf}$ and $\mathrm{Pt}$ additions have distinctly different impacts on the development of loop microstructure and void formation. The oversized solute addition mechanism is more likely related to interstitial aggregation in loops and is less likely related to vacancy trapping and vacancy aggregation processes.

\section{Hf Precipitate Influence on Damage}

Hf-rich precipitates formed during processing of the Hf-doped alloys. These precipitates influence the accumulation of damage by promoting mutual recombination of point defects at precipitate/matrix interfaces. This recombination mechanism is not expected to be significant unless the precipitate sink strength is large compared to the sink strength of the radiation damage. The calculated sink strengths (controlled by the product of the loop or precipitate size and density) shown in Figure 49 indicate that the loop development was not retarded by the high Hf-rich precipitate sink strength when compared to the base alloy with no precipitates. Therefore, it cannot be concluded that Hf precipitates retard the development of loop damage. Furthermore, the loop sink strength increase with dose was not significantly altered by the large reduction in precipitate sink strength at high dose. The Hf-modified alloy had a low precipitate sink strength, but the loop volume fraction and sink strength increased at a lower rate compared to the Hf-reference alloy.

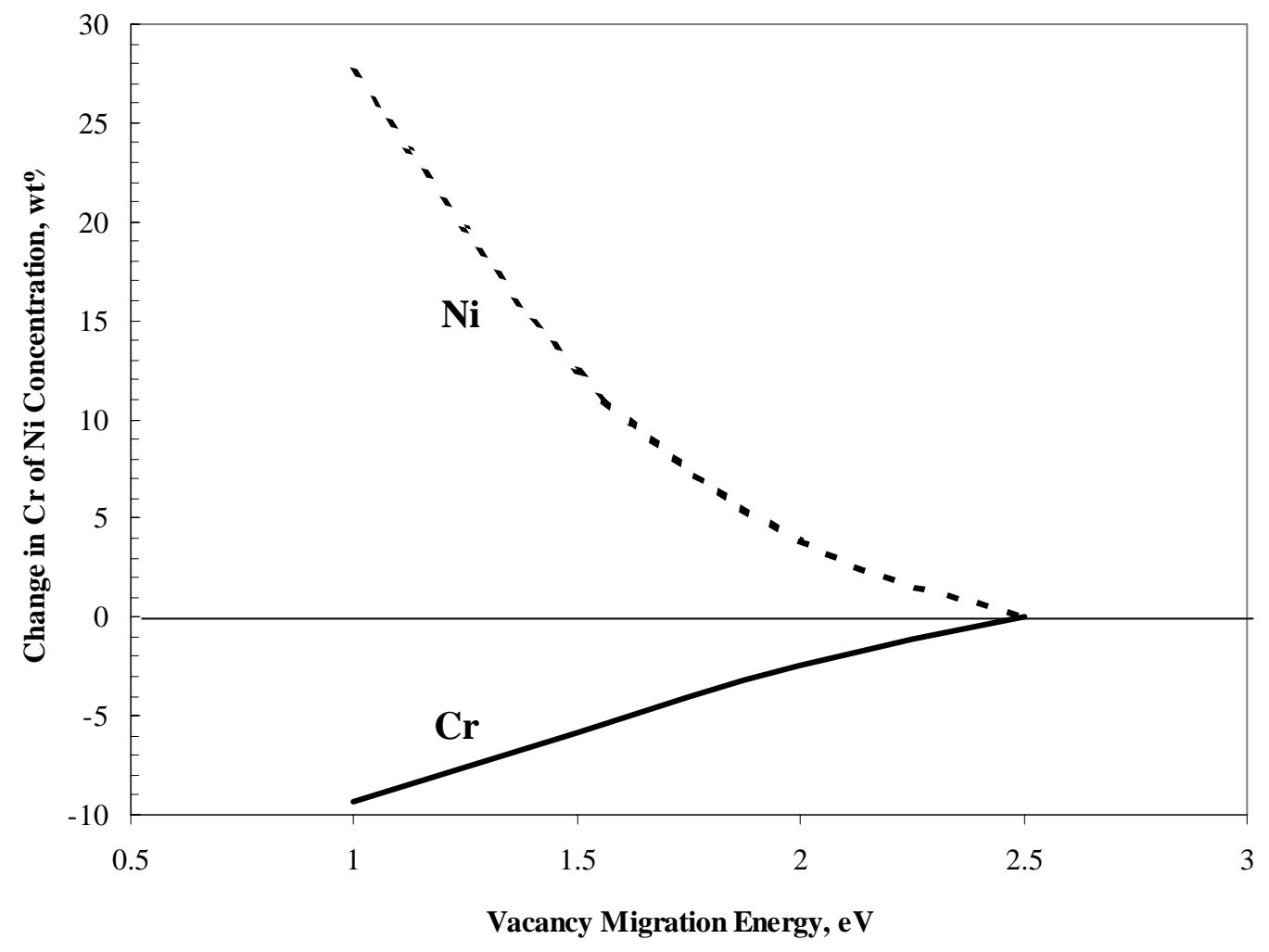

Figure 48. The predicted effect of an elevated migration energy for vacancies caused by vacancy-solute trapping. 
Indirect effects of Hf precipitation could influence damage development by removing solutes in addition to $\mathrm{Hf}$ from the matrix. In particular, $\mathrm{Ni}$ and $\mathrm{C}$ are solutes observed in the Hf-containing precipitates. Removing Ni from the matrix should promote swelling, whereas it is observed that the $316 \mathrm{SS}+\mathrm{Hf}$ alloy with precipitates containing Hf and Ni exhibited less swelling then the base alloy. Similarly, it is expected that removing $C$ from the matrix should promote swelling. Again, only the $316 \mathrm{SS}+\mathrm{Hf}$ alloy contained Hf carbides and it exhibited the least swelling. However, $1100^{\circ} \mathrm{C}$ heat treatment that decreased the number of $\mathrm{Hf} \mathrm{Ni}_{5}$ and $\mathrm{HfC}$ precipitates did show improved swelling resistance over the $900^{\circ} \mathrm{C}$ heat-treated alloy.

These results indicate that influences of Hf precipitation on removing solute from the matrix is likely not responsible for the observed benefit of $\mathrm{Hf}$ additions to $316 \mathrm{~L} \mathrm{SS}$.

Influence of Irradiation Particle Type

Nickel-ion irradiation and proton irradiation have significant differences in cascade morphology. ${ }^{9}$ Nickel ions produce point defects mainly in large cascades whereas protons produce damage mainly through wildly separated small cascades, which is a consequence of the bombarding particle mass. If the effect of oversized solute addition on microstructure is mainly

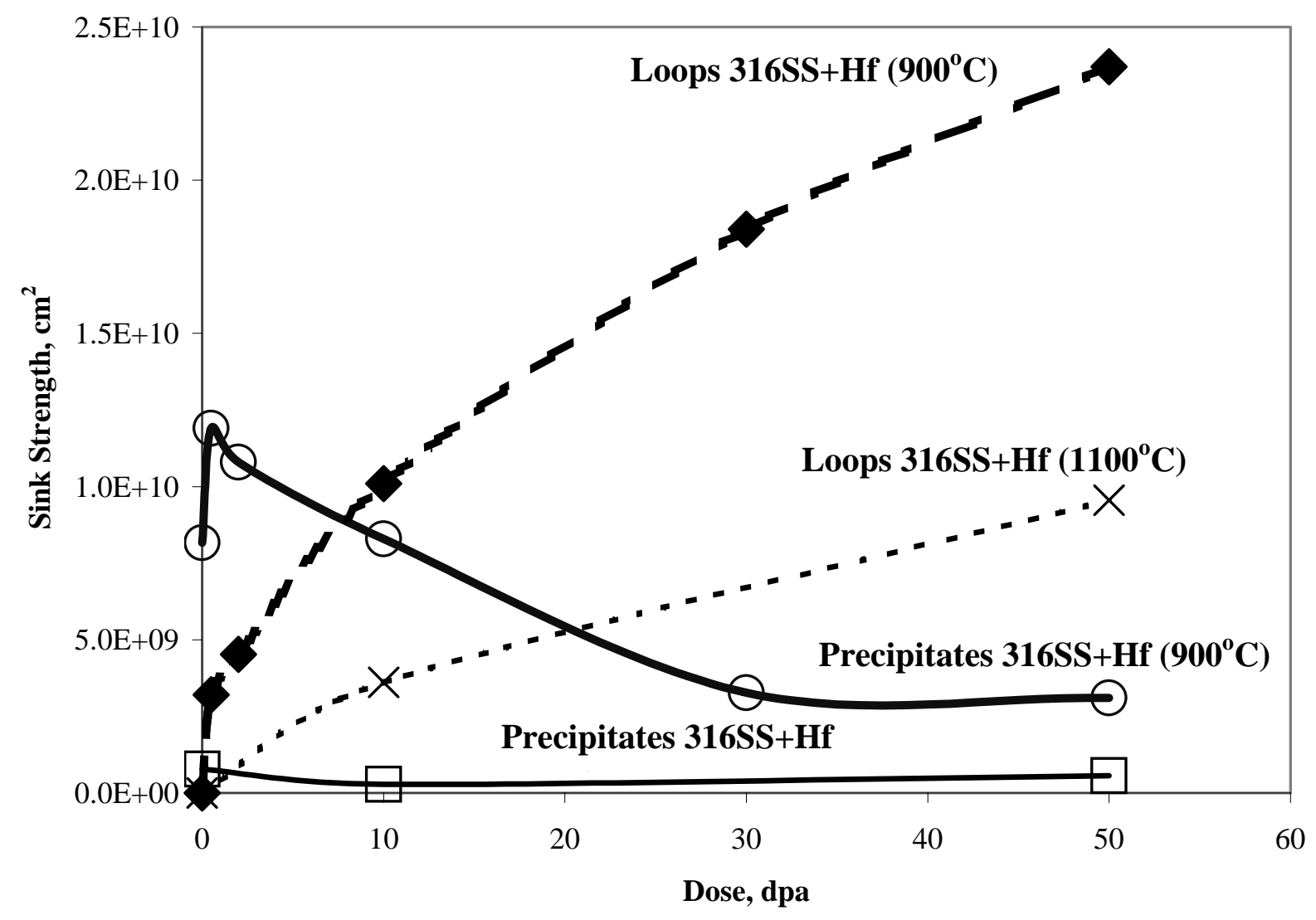

Figure 49. The measured sink strengths for the two Hf-doped alloys are shown as a function of dose. The reference Hf doped alloy exhibited a high precipitate sink strength early in the irradiation. The modified Hf-doped alloy has an insignificant precipitate sink strength. 
within the cascade process, Ni-ion irradiation should show stronger effects than proton irradiation. The generally good agreement in radiation-induced microstructures evolution between the two different types of irradiation indicates that the major effect of the oversized solute addition is not in the cascade process but in the post-cascade defect survival and partitioning to the matrix microstructure and grain boundary microchemistry.

For comparison, radiation-induced microstructure characterization results of the same alloys irradiated with $\mathrm{Ni}$-ions up to $10 \mathrm{dpa}$ are included in Table 8 . As observed for proton irradiation at $2.5 \mathrm{dpa}$, Pt addition is shown to increase faulted loop density and faulted loop size relative to the base alloy in Ni-ion irradiated specimens in the dose range 2 to $10 \mathrm{dpa}$. Also in good agreement with proton irradiation data, the Ni-ion-irradiated 316SS+Pt alloy exhibited a higher void density and void size, resulting in increased swelling, in comparison to the 316L SS alloy in the dose range 2 to $10 \mathrm{dpa}$.

The results for $316 \mathrm{SS}+\mathrm{Hf}$ modified alloy Ni-ion irradiated at $500^{\circ} \mathrm{C}$ to $10 \mathrm{dpa}$ were reasonably consistent with $400^{\circ} \mathrm{C}$ proton data, but revealed several differences. A smaller faulted loop size $(14 \mathrm{~nm})$ is measured after Ni-ion irradiation to $10 \mathrm{dpa}$, than proton-irradiated to $5 \mathrm{dpa}(25 \mathrm{~nm})$. The absence of swelling for the 316SS+Hf modified alloy Ni-ion irradiated to 50 dpa was quite different than the slight swelling detected after proton irradiation to $5 \mathrm{dpa}$. However, it should be noted here that in $316 \mathrm{SS}+\mathrm{Hf}$ alloys that the void distribution was non-uniform after proton irradiation, rendering statistical characterization difficult.

None-the-less, the greater tendency for swelling from proton irradiation may relate to the dose rate effect on the swelling. The dose rate for proton irradiation is about two orders of magnitude higher than that for neutron irradiation and the Ni-ion dose rate is two orders of magnitude higher than that for protons. For the same dose and temperature, swelling is more pronounced at lower neutron dose rates. ${ }^{10}$ The effect of Pt addition on promoting void nucleation is more obvious in proton irradiation than in Ni-ion irradiation. Because protons are more effective in producing freely migrating point defects, point-defect interactions with solute addition may be more pronounced in proton irradiation.

Ohnuki, et al. ${ }^{12}$ have also observed that $\mathrm{Hf}$ addition to $316 \mathrm{~L}$ SS improved swelling resistance after neutron irradiation at $425^{\circ} \mathrm{C}$ to a dose of $35 \mathrm{dpa}$. The comparison of Ni-ion results at $500^{\circ} \mathrm{C}$ to $50 \mathrm{dpa}$ in the alloy $316 \mathrm{SS}+\mathrm{Hf}\left(1100^{\circ} \mathrm{C}\right)$ with neutron irradiation at $425^{\circ} \mathrm{C}$ to $35 \mathrm{dpa}$ is in agreement in that $\mathrm{Hf}$ has a beneficial effect on suppressing void swelling. However, it is also in contrast in that no voids were observed for Ni-ion irradiation at $50 \mathrm{dpa}$ but minor void microstructure was observed after neutron irradiation to $35 \mathrm{dpa}$. This is consistent with a doserate effect between heavy-ion and neutron experiments. Complete suppression of swelling depends on details of irradiation and material condition.

Oversized Solute Influences on the RIS Mechanism

The relative diffusivities of major elements in 316SS may be influenced by the addition of oversized solutes. This influence can be explored by comparing the relative changes in $\mathrm{Ni}$ and $\mathrm{Cr}_{\text {RIS }}{ }^{11}$ for the alloys. Comparison of the present response for the base alloy with previous studies of neutron-irradiated stainless steel is shown in Figure 50a. The Ni-ion RIS is similar to 


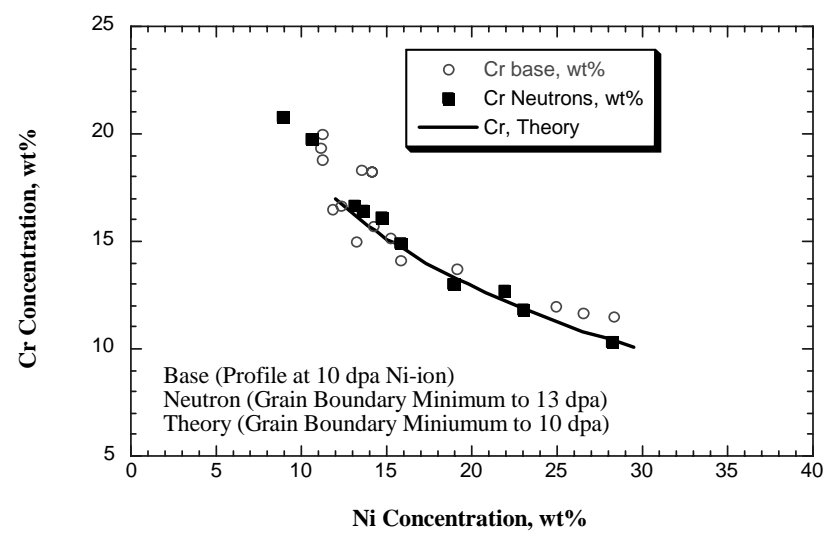

(a)

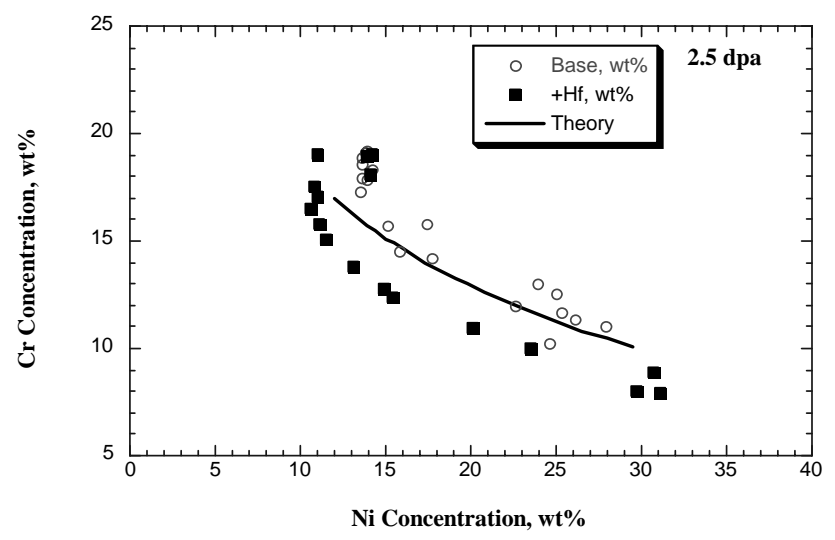

(b)

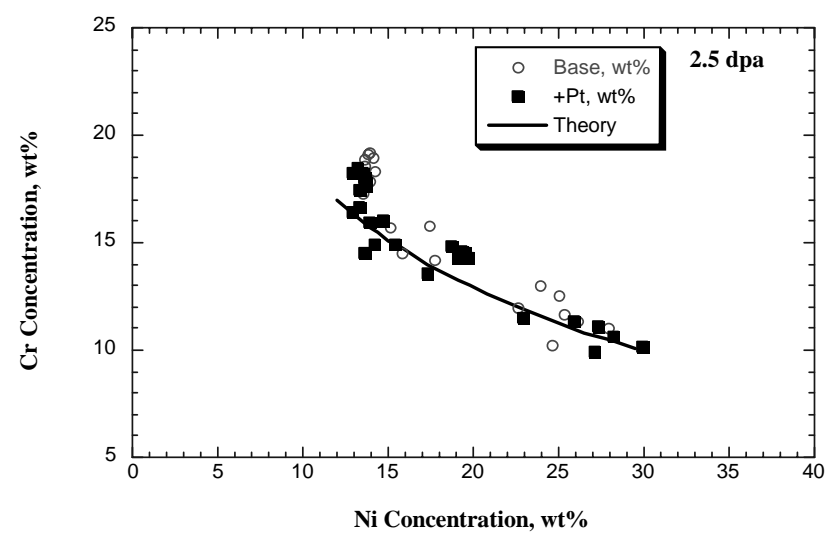

(c)

Figure 50. Cr concentration as a function of Ni concentration is in agreement when comparing RIS induced by Ni ions and neutrons; (b) shown to be somewhat different when comparing $\mathrm{Ni}$-ion RIS in the Hf-doped alloy to the base alloy and (c) similar when comparing RIS in the Pt-doped alloy to the 316L SS base alloy. 
measured neutron RIS and the general model prediction in stainless steel. The favorable comparison indicates that RIS observed in the base alloy is occurring as expected. The RIS response for the Hf-doped alloy differs from the base alloy in that $\mathrm{Cr}$ depletion initially is greater than expected at low Ni concentrations as shown in Figure 50(b). This indicates that the ratio of $\mathrm{Cr} / \mathrm{Ni}$ diffusion rates is greater for the Hf-doped alloys compared to the base alloy. The addition of Pt does not alter the mechanism as shown in Figure 50(c) even though Pt is oversized and has a mass similar to Hf.

Insights on Current Understanding for Oversized Solute

The current research represents the first quantitative measurements mapping both microstructural and microchemical evolution to help isolate the effect of oversize solutes on radiation damage. Results clearly demonstrated that Hf solute addition to 316L SS inhibits swelling and improved IASCC resistance described in a later section. Conventional concepts for the influences of oversized solute as discussed in the previous sections do not effectively rationalize the significant improvement in behavior. Hafnium precipitate effects are not responsible based on their initial distribution and alterations with radiation dose. This leaves $\mathrm{Hf}$ as a solute as the cause in delaying detrimental material changes. However as noted in the prior sections, many aspects of this explanation do not agree with rate theory concepts for defect production and annihilation. The fate of point defects is shown schematically in Figure 51.

Annihilation of defects by recombination inside and outside of cascades was expected to be the primarily mechanism for resistance to void formation and radiation-induced segregation. Rough calculations on the effect of Hf on the steady state RIS profile cannot account for the observed changes in RIS following proton irradiation. Furthermore as shown in Figure 51, recombination should affect both microstructural evolution and RIS because recombination annihilates freely migrating defects required for both processes. The significant delay in the onset of swelling for $\mathrm{Ni}$-ion-irradiated $316 \mathrm{SS}+\mathrm{Hf}$ is difficult to rationalize by effects on recombination since RIS is unaffected or perhaps even enhanced in the Hf-doped alloy.

Unexpectedly, measured RIS for the oversized element alloys was found to depend on the irradiation particle type. Prior joint research between UM and PNNL has documented similar RIS behavior among protons, Ni ions and neutrons. The current study is our first comparative examinations of oversized elements effects and significantly less RIS is seen after low-dose proton irradiations than for heavy-ion irradiations. This effect implies an influence of oversized solute on point-defect loss in displacement cascades for proton irradiation. After heavy-ion irradiation, the magnitude of RIS was not reduced by the addition of Hf even at a low dose of 0.5 dpa suggesting limited in-cascade recombination. The electron-irradiation experiment of Kato et al. also revealed a significant effect of Hf addition of reducing RIS in $316 \mathrm{SS}$. Low-energy displacement irradiation (protons and electrons) may be more strongly influenced by oversized solute addition compared to high-energy displacements characteristic of heavy ions and neutrons. This identifies the critical need to evaluate microstructural and microchemical evolution in neutron-irradiated materials. As noted earlier in this report, such irradiations are being performed in the Bor-60 fast reactor and samples will be available for characterization at doses of $5,10,20,40$ and 70 dpa. 


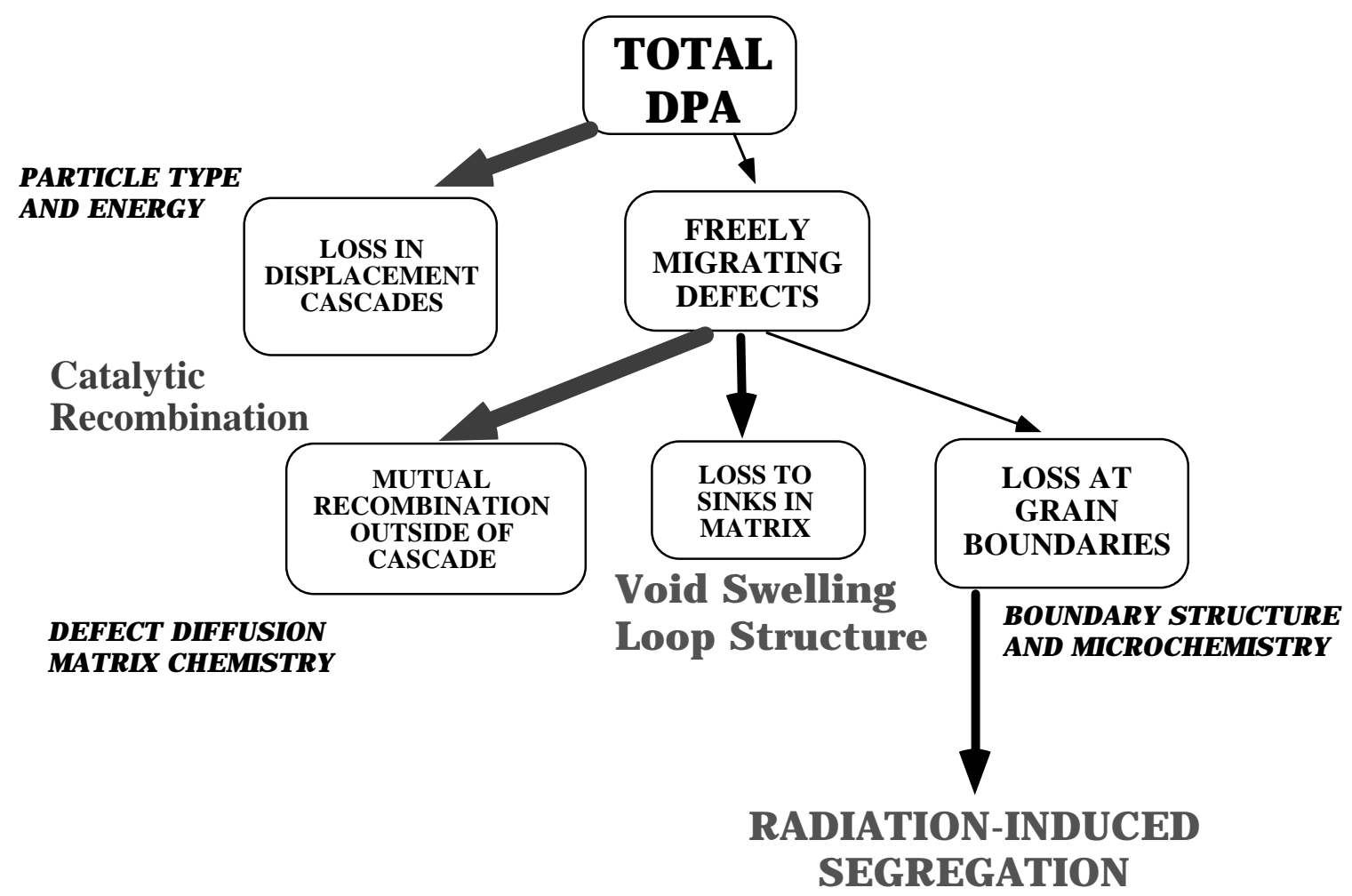

Figure 51. Schematic for the fate of point defects produced by displacement damage.

Another possible reason for the difference in RIS between $\mathrm{Ni}$ and proton results could be the displacement rate. As lower displacement rates are more effective in void formation, they could also affect RIS. If the displacement rate is too high, then the vacancy and interstitial concentrations and fluxes are so high that perhaps, small changes in sink density can't make an impact on the net defect fluxes and hence, on RIS.

Unlike recombination effects, theories for partitioning of point defects into vacancy (void) and interstitial (loop) aggregates are not easily applied. The primary factors affecting partitioning are the bias factors that defect sinks have for either vacancies or interstitials. Bias factors are typically scaling factors for rationalizing rates of microstructural evolution and are difficult to determine theoretically. A consistent observation in this study is that Hf addition promoted loop nucleation, inhibited loop growth and inhibited void formation for both proton and heavy-ion irradiations. This suggests that oversized $\mathrm{Hf}$ is affecting bias parameters for microstructural evolution. The bias factor is related to the stress field surrounding lattice defects such as line dislocations, dislocation loops and voids. The large size misfit, 27\%, for Hf, clearly must induce strong influences on these stress fields. The consequence is that loop growth is inhibited because interstitials are weakly attracted to sinks in the presence of Hf compared to sinks in the absence of Hf. The lack of swelling is related to the inhibited loop development and integration into the dislocation network. Typically, void formation does not occur until the irradiation-induced dislocation network evolves. Oversized solute effects on defect bias factors are likely the primary mechanism for the beneficial influence of $\mathrm{Hf}$ addition to $316 \mathrm{~L} \mathrm{SS}$. 


\section{Multiphase Microstructures}

Radiation exposure of Fe- and Ni-base stainless alloys can accelerate or retard the precipitation of various second phases, modify existing precipitates, and produce new phases that do not form during thermal treatments. A primary influence of radiation on second phase formation and stability will depend on solute segregation that will be described in the following section. For example, radiation-induced segregation (RIS) of $\mathrm{Ni}$ and $\mathrm{Si}$ to sinks in stainless alloys can lead to $\gamma^{\prime}$ or $\mathrm{G}$ phase precipitation and depletion of $\mathrm{Cr}$ can retard carbide precipitation at grain boundaries. These studies ${ }^{13,14}$ suggest that radiation-induced precipitation in 300-series stainless steels is different below $\sim 400^{\circ} \mathrm{C}$. Although characterization of materials after high-dose irradiations at pertinent temperatures is lacking, radiation-induced precipitation does not appear to be an issue for LWRs unless significant off-normal compositions are present in the stainless steel. The only second phase that is commonplace in 300-series stainless steels is metal carbides that appear to be stable under LWR irradiation. However, high-strength Ni-base alloys such as alloy 718 have several second phases that dissolve $\left(\gamma^{\prime \prime}\right)$, redistribute $\left(\gamma^{\prime}\right)$ or become amorphous (Laves) during LWR irradiation. ${ }^{14}$ These changes can significantly influence the alloy properties.

Many alloys of engineering interest are inherently metastable in nature, continuing to evolve slowly in solute distribution and often in precipitate identity and configuration. For most low-tomoderate temperature applications, the rate of return toward some other more stable state is so slow that most alloys can be considered to be effectively stable during service lifetimes. Radiation can strongly enhance diffusion compared to conventional thermal diffusion, however, and provides new modes of solute distribution at rates far above those characteristic of thermally driven processes.

Radiation can cause phase instability because displacement processes persistently disrupt the fine details of precipitate structure and interfaces with the surrounding matrix. Ballistic dissolution of the precipitate phases continually places the solute elements into solution. Small second-phase particles can potentially be completely dissolved and another phase precipitate in return. In some cases, the original phase may be so stable that it simply reforms, but in a different morphology and spatial distribution. Due to the complexity of the processes involved, radiationstable states (dynamic stability) cannot be easily or reliably predicted from thermally stable states based on minimum free energy (thermodynamic stability) considerations. Some phases can be stable during irradiation or be destroyed with the balance of stability often dependent on temperature and displacement rate. Precipitates also serve as potential repositories for elements that may be either detrimental or beneficial if released to, or removed, from the matrix. Therefore, intuition arising from conventional metallurgy experience may be at odds with new strategies proposed for designing damage-resistant alloys.

Radiation can either drive precipitates toward or away from equilibrium. In the first case (toward equilibrium), irradiation can enhance precipitation and erase the benefits of thermomechanical processing used to optimize non-radiation properties. In the second case (away from equilibrium), irradiation can induce precipitate formation of entirely new phases that strongly alter the matrix composition. The rate-determining step for void swelling (and several forms of embrittlement) is the radiation-induced microchemical changes that occur in the matrix. 
This process often removes important elements known to retard the onset of accelerated swelling, e.g., Ni, Si and P. These elements exert their influence primarily on vacancy diffusivity, a property that influences all stages of microstructural evolution.

Moreover, precipitation can promote both hardening and susceptibility to environmental cracking. The balance of precipitation hardening versus the combined effect of solute hardening and dislocation strengthening is always shifted by irradiation, sometimes with very undesirable consequences. In Inconel alloy 706 irradiated at temperatures near $600^{\circ} \mathrm{C}$, the matrix becomes very hard as a result of the enhanced precipitation and changes in dislocation microstructure, and the grain boundaries become very brittle as a result of radiation-induced $\gamma$ ' formation. This is a very unfortunate combination that has disqualified the use of this strong and very swellingresistant alloy at its most desired temperature range. The phase balance consists of both metastable and stable precipitates, but the sum effect of the two is undesirable.

Control of stable and metastable precipitates is required for the development of effective damage-resistant alloys. The balance between metastable and stable precipitation must be understood as a function of accumulating damage to accommodate changes in matrix solute composition induced by both segregation and transmutation. In addition, the radiation-induced introduction of dislocation loops and cavities must be accounted for. The presence of transmutation species becomes much more important for new reactor designs that will reach higher radiation dose levels. This balance of stability approach has the potential to maintain desired initial properties at high damage levels. Sacrificial unstable phases might be tailored to disappear during irradiation and compensate for the introduction of new strengthening components. The balanced stability approach also requires that the shift in strengthening balance not be accompanied by net depletion of solutes from the matrix that are beneficial with respect to cracking resistance or void swelling.

The concept of metastable sacrificial precipitates has been observed in alloy 718 irradiated with neutrons in a PWR and also irradiated with $800 \mathrm{MeV}$ protons. ${ }^{15}$ Depending on the irradiation temperature, there is either total or partial dissolution of $\gamma^{\prime}$ and $\gamma^{\prime \prime}$ precipitates. This loss compensates for most of the radiation-induced hardening arising from clusters, dislocation loops and cavities. Another feature of this approach is a refinement of size in precipitate distribution. This process has been observed and successfully modeled at PNNL, i.e., $\gamma^{\prime}$ and $\gamma^{\prime \prime}$ precipitate behavior in Inconel 718 during neutron irradiation at $300^{\circ} \mathrm{C} .{ }^{14}$ An initial distribution of $20-\mathrm{nm}$ precipitates was replaced by a fine distribution of 1-nm precipitates after $20 \mathrm{dpa}$. Model calculations based on a recoil jump distance of $20 \mathrm{~nm}$ and a recoil event rate of $10^{-7} \mathrm{~s}^{-1}$ is in accord with the observed steady-state precipitate distribution. This study indicates that the goal of achieving a durable steady-state precipitate distribution in balance with an evolving new microstructure is plausible. At lower temperatures $\left(<100^{\circ} \mathrm{C}\right)$ characteristic of the $800 \mathrm{MeV}$ proton irradiation, the diffusion-driven repair was slow enough not to allow precipitates of any size to survive. This demonstrates the need to incorporate the dependence of stability on both temperature and displacement rate. 
Ni-Base Alloy Concept Evaluation

\section{Material}

The Ni-base alloy with metastable precipitates (alloy 718) was obtained from a prior neutron irradiation study. Prior to irradiation the alloy was processed according to the following treatment: $954^{\circ} \mathrm{C} / 1 \mathrm{~h}+718^{\circ} \mathrm{C} / 8 \mathrm{~h}+$ furnace cool to $621^{\circ} \mathrm{C} / 8 \mathrm{~h}$. Tubing samples were wet-polished with a SiC paper to 600 grit, diamond abrasive file to 3 凶m and a final polish of $0.3-凶 \mathrm{~m}$ alumina. The alloy composition is given in Table 9 .

Table 9. Alloy 718 Composition in wt\%.

\begin{tabular}{|l|l|l|l|l|l|l|l|l|l|l|l|l|l|l|l|}
\hline $\mathrm{C}$ & $\mathrm{Fe}$ & $\mathrm{Ni}$ & $\mathrm{Cr}$ & $\mathrm{Mn}$ & $\mathrm{Mo}$ & $\mathrm{Nb}$ & $\mathrm{Ti}$ & $\mathrm{Al}$ & $\mathrm{Si}$ & $\mathrm{N}$ & $\mathrm{S}$ & $\mathrm{P}$ & $\mathrm{Cu}$ & $\mathrm{B}$ & $\mathrm{Co}$ \\
\hline .06 & 19.2 & 52.0 & 18.1 & 0.2 & 3.1 & 5.3 & 1.0 & 0.6 & 0.23 & 0.007 & 0.001 & 0.015 & 0.10 & 0.006 & 0.24 \\
\hline
\end{tabular}

TEM characterization of non-irradiated Alloy 718

SEM and TEM examinations of the non-irradiated samples showed extensive precipitation along grain boundaries as well as in the grain interiors. Grain boundaries contained coarse lath-shaped precipitates identified as $\delta$ phase, the normal overaging product of $\mathrm{Ni}_{3} \mathrm{Nb}-\gamma^{\prime \prime}$. Average grain sizes were about $20 \mu \mathrm{m}$. Lenticular particles of Mo- and Si-rich Laves phase were also found on the grain boundaries. Intergranular $\delta$ phase also appeared in the material used for the proton and spallation neutron irradiations, but was not specifically analyzed. Phase compositions determined by TEM-EDS analyses are given in Table 10.

Table 10. Precipitate compositions in alloy 718 measured by TEM-EDS, wt\%.

\begin{tabular}{|c|c|c|c|c|c|c|c|c|}
\hline Phase & $\underline{\text { Al }}$ & $\underline{\mathbf{S i}}$ & $\underline{\mathbf{T i}}$ & $\mathrm{Cr}$ & $\mathrm{Fe}$ & $\underline{\mathrm{Ni}}$ & $\mathbf{N b}$ & Mo \\
\hline$\delta$ & $\overline{0.2}$ & 0.2 & 2.2 & $\overline{2.1}$ & $\overline{3.8}$ & $\overline{61.4}$ & 29.6 & 0.5 \\
\hline Laves & 0.2 & 2.6 & 0.3 & 12.1 & 12.4 & 30.1 & 28.6 & 13.8 \\
\hline$\gamma^{\prime \prime}$ & 0.1 & 0.4 & 1.9 & 2.9 & 3.6 & 64.9 & 24.2 & 2.0 \\
\hline $\mathrm{NbC}$ & 0.0 & 0.2 & 7.9 & 1.7 & 1.5 & 3.4 & 91.9 & 0.0 \\
\hline $\mathrm{TiC}$ & 0.1 & 0.1 & 64.6 & 0.9 & 0.9 & 1.6 & 31.4 & 0.4 \\
\hline $\mathrm{G}$ & 1.0 & 15.9 & 1.6 & 3.7 & 6.3 & 50.1 & 21.4 & 0.0 \\
\hline
\end{tabular}

TEM examinations of unirradiated samples further showed fine spheroidal $\gamma^{\prime}$ and disk-shaped $\gamma^{\prime \prime}$ throughout the grain interiors. Enlarged $\gamma^{\prime \prime}$ (but no $\gamma^{\prime}$ ) also appeared on the grain boundaries between coarse $\delta$ particles. The intragranular $\gamma^{\prime}$ and $\gamma^{\prime \prime}$ particles in respectively averaged $\sim \mathrm{nm}$ and $10 \mathrm{~nm}$ in size. The $\gamma^{\prime}-\gamma^{\prime \prime}$ darkfield image in Figure 52 shows enlarged $\gamma^{\prime \prime}$ along a grain boundary, a narrow precipitate-free zone along the boundary, and coprecipitated $\gamma^{\prime}$ and $\gamma^{\prime \prime}$ particles in the matrix. This darkfield imaging condition shows all the $\gamma^{\prime}$ and one of three $\gamma^{\prime \prime}$ orientation variants in one grain). Lattice images, as in Figure 52, revealed that the intragranular $\gamma^{\prime}$ and $\gamma^{\prime \prime}$ particles generally occurred in pairs, each $\gamma^{\prime}$ particle sharing an interface with a $\gamma^{\prime \prime}$ particle.

Phase Instabilities during Neutron Irradiation at $288^{\circ} \mathrm{C}$

Both the $\gamma^{\prime}$ and $\gamma^{\prime \prime}$ age-hardening phases in Alloy 718 exhibited instabilities under neutron irradiation at $288^{\circ} \mathrm{C}$. After $3.5 \mathrm{dpa}$, the original $\gamma^{\prime \prime}$ particles throughout the matrix and at grain 

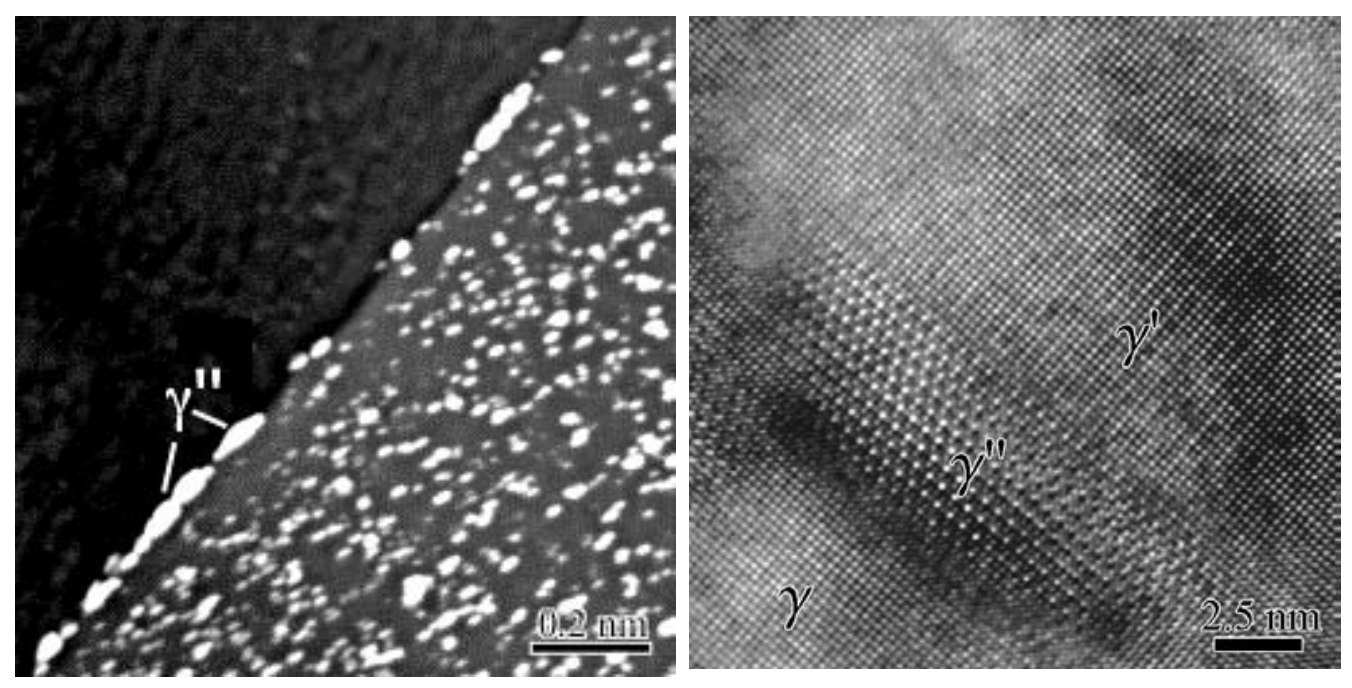

Figure 52. The left micrograph is a $\gamma^{\prime}-\gamma^{\prime \prime}$ darkfield image showing enlarged $\gamma^{\prime}$ and denuded zone at grain boundary in alloy 718. Both $\gamma^{\prime}$ and $\gamma^{\prime \prime}$ are present in matrix. Lattice image of coprecipitated $\gamma^{\prime}-\gamma^{\prime \prime}$ particle is shown on right.

boundaries disappeared and the $\gamma^{\prime}$ particles began disintegrating. This striking loss of $\gamma^{\prime \prime}$ was evident from changes in the diffracted spot intensities. As shown in Figure 53, only $\gamma^{\prime}$ reflections remained after $3.5 \mathrm{dpa}$. The $\gamma^{\prime \prime}$ reflections were still faintly visible after $2.5 \mathrm{dpa}$. No other spots from irradiation-induced phases appeared in diffraction patterns from the irradiated samples. The only extra reflections not accounted for by $\gamma^{\prime}$ and $\gamma^{\prime \prime}$ phases were relrod satellites from radiationinduced Frank loops. These satellite spots appear prominently around the off-Bragg matrix spots in diffraction patterns taken a few degrees off a [001] orientation, as shown in the diffraction pattern at the upper right in Figure 53. The Frank loops should not be mistaken for radiationinduced precipitates as done in a recent paper on alloy $718 .^{15}$

The $\gamma^{\prime}-\gamma^{\prime \prime}$ darkfield images in Figure 53 further illustrate the redistribution of $\gamma^{\prime}$. After 3.5 dpa, the original $\gamma^{\prime}$ particles were still clearly visible but appeared in the process of breaking up into smaller discrete particles. Newly formed $\gamma^{\prime}$ particles with much finer sizes also appeared the matrix between the original, disintegrating particles. After $20 \mathrm{dpa}$, the original 10-nm particles were entirely replaced by dispersed $1-\mathrm{nm} \gamma^{\prime}$ particles.

Solute redistribution at grain boundaries, dislocations, and $\gamma^{\prime}$ particles was measured by nanoprobe EDS. The irradiation leveled initially high levels of Mo at the grain boundaries and decreased the $\mathrm{Nb}$ concentrations from intergranular $\gamma^{\prime \prime}$ particles. $\mathrm{Ni}$ at the grain boundaries increased greatly with dose at the expense of $\mathrm{Cr}$ and Fe. Surprisingly, no segregation was detected on dislocation loops. Fine-spot analyses of radiation-induced Frank loops, illustrated by Figure 54, showed no compositional difference between the loop plane and the adjacent alloy matrix away from $\gamma^{\prime}$ particles. This result also showed that the dislocation loops occurred in the matrix rather than inside $\gamma^{\prime}$, and therefore cannot be considered a distinct phase (such as $\eta$ ). High $\mathrm{Nb}$ concentrations in redistributed $\gamma^{\prime}$ particles (Figure 54) indicated that the new $\gamma^{\prime}$ formed at the expense of the original $\gamma^{\prime \prime}$ phase, reflecting altered stability of the $\gamma^{\prime}$ phase under irradiation. 


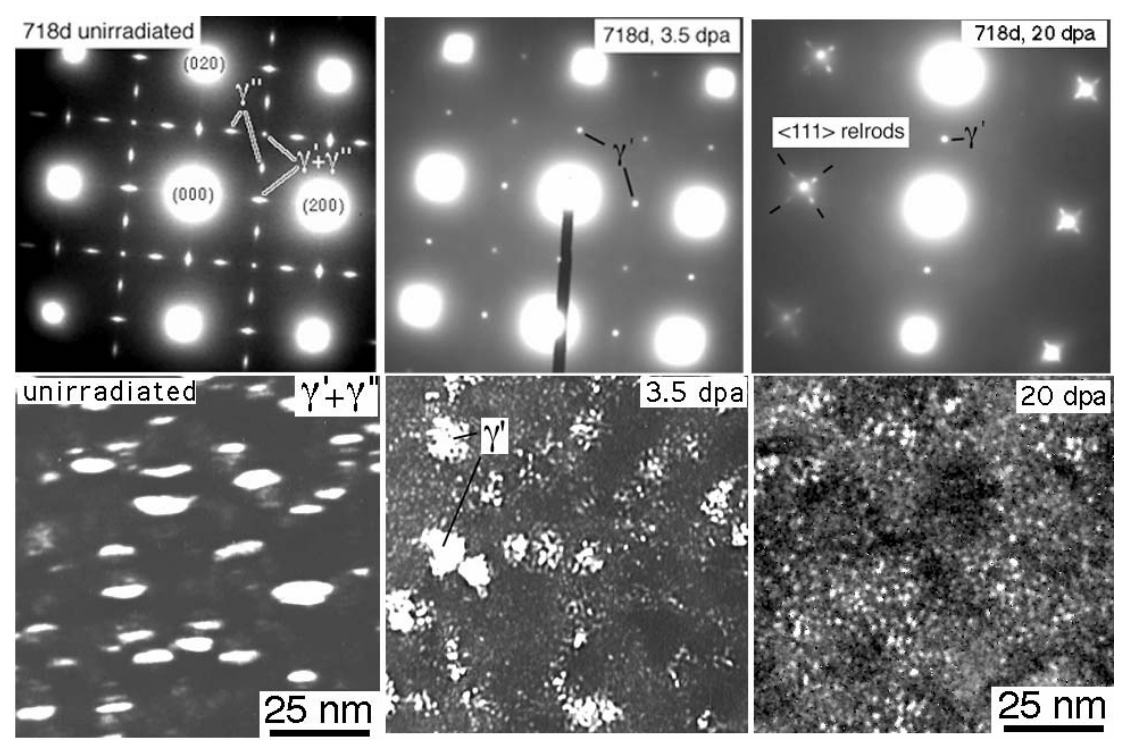

Figure 53. Diffraction patterns and precipitate darkfield micrographs showing irradiationinduced dissolution of $\gamma^{\prime \prime}$ and reprecipitation of $\gamma^{\prime}$ phase at $288^{\circ} \mathrm{C}$. Only $\gamma^{\prime}$ remained after 3.5 dpa. Relrod diffraction spots indicated in off-zone pattern at top right were caused by radiationinduced Frank dislocation loops in the austenite matrix.
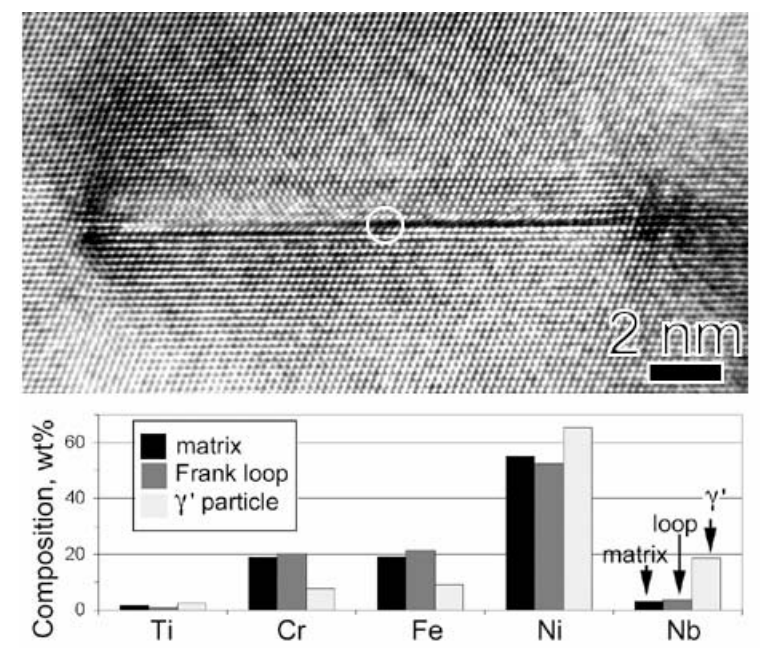

(a)
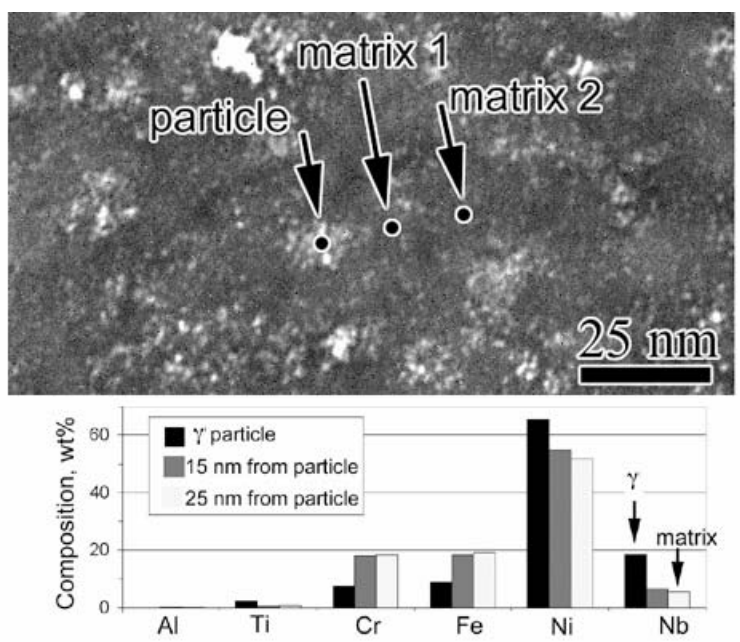

(b)

Figure 54. Solute redistribution after neutron irradiation to $3.5 \mathrm{dpa}$ at $288^{\circ} \mathrm{C}$ : (a) EDS compositional analysis of Frank loops showing absence of solute segregation to loop plane and (b) EDS analysis of re-precipitated $\gamma^{\prime}$ showing $\mathrm{Nb}$-rich particle composition of particles.

Phase Instabilities during $\mathrm{Ni}$-Ion Irradiation at $500^{\circ} \mathrm{C}$

In order to establish the ability to once again use heavy ions to assess radiation-induced microstructure evolution, the same alloy 718 heat was irradiated to 2, 10 and 50 dpa with $5 \mathrm{MeV}$ $\mathrm{Ni}$ ions. The stability of the $\gamma^{\prime}$ and $\gamma^{\prime \prime}$ precipitates is shown in Figures 55 and 56 as a function of dose. As was the case for neutron irradiation, $\gamma^{\prime \prime}$ precipitates become unstable and begin 
disappearing at intermediate doses. The density of $\gamma^{\prime \prime}$ precipitates is dramatically reduced by 2 dpa and no evidence of this phase can be seen in the 10 or 50 dpa samples. Phase instability was also identified for $\gamma^{\prime}$ precipitates with a reduction in diffraction spot intensity at higher doses corresponding to a change in precipitate size. The $\gamma^{\prime}$ precipitates are only a few nm in diameter in comparison to $\sim 10 \mathrm{~nm}$ before irradiation. The dose dependence of phase instability for $\gamma^{\prime}$ and $\gamma^{\prime \prime}$ is quite similar to that seen for LWR neutron irradiation at $288^{\circ} \mathrm{C}$.

The dramatic changes to the matrix during irradiation impacts the development of the dislocation structure and suppresses void formation even at 50 dpa. Faulted loops are shown in Figures 57 and 58. Loop size and density increases with dose from 2 to $10 \mathrm{dpa}$ as documented in Table 11. No further increase is seen at the highest dose of $50 \mathrm{dpa}$. Loop sizes are smaller than for the base

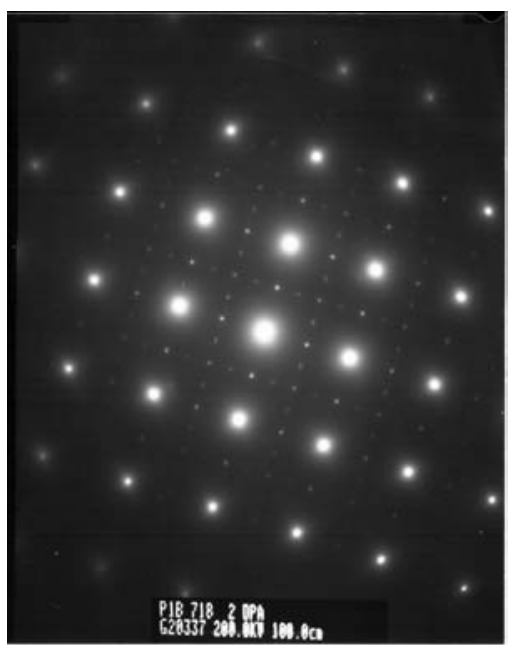

(a)

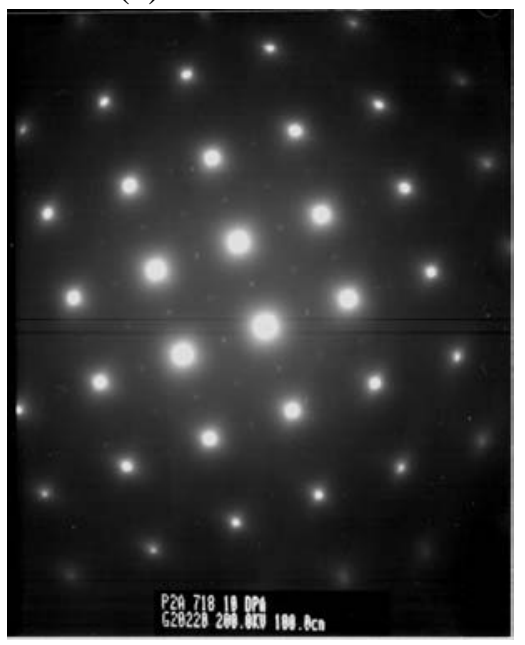

(c)

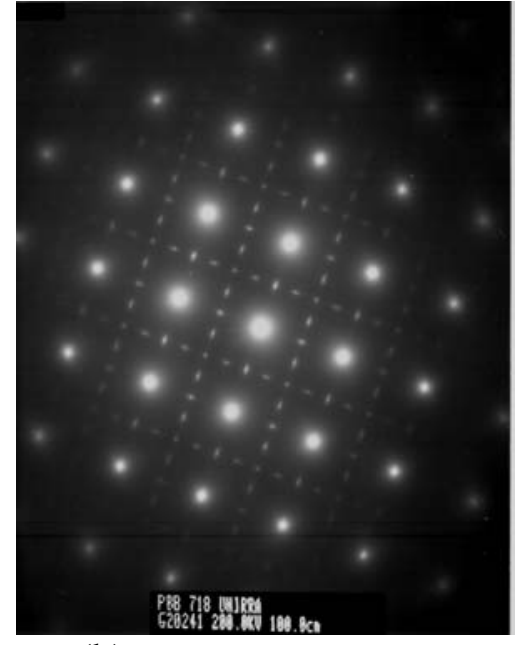

(b)

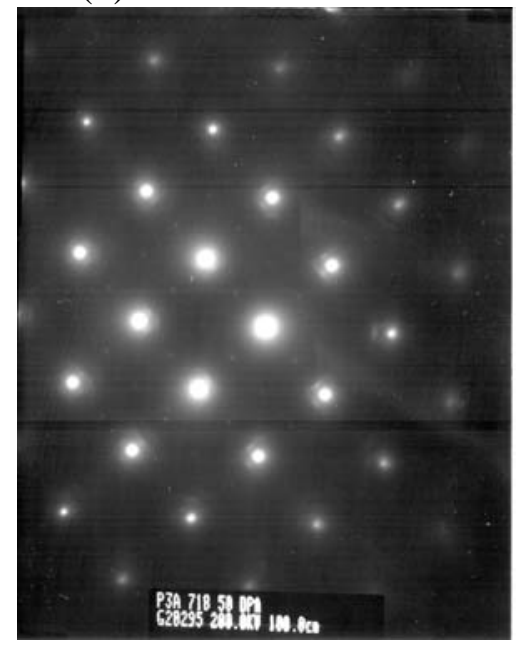

(d)

Figure 55. Electron diffractions patterns illustrating Ni-ion irradiation effects on $\boldsymbol{\gamma}^{\prime}-\boldsymbol{\gamma}^{\prime}$ phase stability: (a) non-irradiated, $\gamma^{\prime}$ and $\gamma^{\prime \prime}$ spots bright; (b) 2 dpa, $\gamma^{\prime}$ spots bright and $\gamma^{\prime \prime}$ spots weak; (c) $10 \mathrm{dpa}, \gamma^{\prime}$ spots weak and $\gamma^{\prime}$ ' spots have disappeared; and (d) $50 \mathrm{dpa}, \gamma^{\prime}$ spots very weak and $\gamma$ ' spots missing. 
Table 11. Faulted Loop Density and Size as a Function of Dose for Alloy 718.

\begin{tabular}{|c|c|c|c|}
\hline Dose (dpa) & Density $(\mathrm{cm}-3)$ & $\underline{\text { Size }(\mathrm{nm})}$ & \# loops measured \\
\hline 2 & $2.7 \mathrm{E}+15$ & 9.2 & 138 \\
\hline 10 & $7.1 \mathrm{E}+15$ & 14.5 & 307 \\
\hline 50 & $5.6 \mathrm{E}+15$ & 14.0 & 479 \\
\hline
\end{tabular}

316L SS, comparable to the $316 \mathrm{~L}+\mathrm{Hf}$ at higher doses. The density of faulted loops in the Niion-irradiated, alloy 718 is slightly higher than for the 316L SS heats.

Fe-Base Alloy Concept Evaluation

\section{Materials}

The multiphase alloys chosen for study were $17-4 \mathrm{PH}$ and $17-7 \mathrm{PH}$, the designations indicating that the alloys contained approximately $17 \% \mathrm{Cr}$ with levels of $\mathrm{Ni}$ as indicated in an Fe-base alloy that can be precipitation hardened (PH). These alloys were chosen based on their reliance on fine-scale precipitates for achieving desirable properties. 17-4PH includes additions of $\mathrm{Cu}$ and $\mathrm{Nb}$, and 17-7PH includes additions of $\mathrm{Al}$ for precipitation hardening, and the alloy class is described as maraging because precipitation is promoted after the alloy is allowed to transform from austenite to martensite.

Alloy compositions, heat treatment, irradiation and preparation details for $17-4 \mathrm{PH}$ and $17-7 \mathrm{PH}$ are summarized below. The alloys were received as plate in a solution-annealed condition. Table 12 gives alloy compositions. The heat treatment given 17-4PH specimens was $550^{\circ} \mathrm{C} / 1 \mathrm{~h} / \mathrm{AC}$ and that given $17-7 \mathrm{PH}$ was $760 \mathrm{C} / 1.5 \mathrm{~h} / \mathrm{AC}(20 \mathrm{~min})+550^{\circ} \mathrm{C} / 1 \mathrm{~h} / \mathrm{AC}$ where $\mathrm{AC}$ defines air-cooling. The resultant hardnesses (Vickers with 500g load,) were 419 and 438 $\mathrm{kg} / \mathrm{mm}^{2}$, respectively. $\mathrm{Ni}^{++}$irradiations were performed at $500^{\circ} \mathrm{C}$ to doses of 2 and $10 \mathrm{dpa}$ at a dose rate of $1 \mathrm{dpa} / 12 \mathrm{~min}$, so that samples remained at temperature for at least $24 \mathrm{~min}$ for $2 \mathrm{dpa}$ and $2 \mathrm{~h}$ for $10 \mathrm{dpa}$. Specimen TEM disks were electropolished in a solution of $2 \%$ perchloric acid, $15 \%$ ethylene glycol and $83 \%$ methanol held at $-60^{\circ} \mathrm{C}$. Specimens were polished from one side at $25 \mathrm{~V}$ for $5 \mathrm{~s}$ in order to remove a $0.5 \mu \mathrm{m}$ layer and then polished from the other side at $45 \mathrm{~V}$ to perforation. Examinations were performed on a JEOL JEM 1200EX.

\section{TEM characterization of non-irradiated Fe-base alloys}

Both alloys contained precipitation-strengthened tempered martensite with well-defined regions of $\delta$-ferrite strongly decorated with carbide. The $\delta$-ferrite grain size in $17-4 \mathrm{PH}$ was on the order of $1 \mu \mathrm{m}$ at a volume fraction below $10 \%$, but the $\delta$-ferrite grain size and volume fraction in 17 $7 \mathrm{PH}$ was much larger. Both alloys contained fairly high dislocation densities in martensite regions but delta ferrite regions were almost dislocation free. Examples of these microstructures are provided in Figure 59. Figure 59 allows comparison of the microstructures of unirradiated $17-4 \mathrm{PH}$ and $17-7 \mathrm{PH}$ at low magnification in a) and c), and at higher magnification in the remaining micrographs. A bright-field image and two weak-beam, dark-field images are shown for each condition, one using $g=011$ in e) and $g$ ) and the other using $g=200$ in $f$ ) and $h$ ), respectively, for different areas. 
(a)

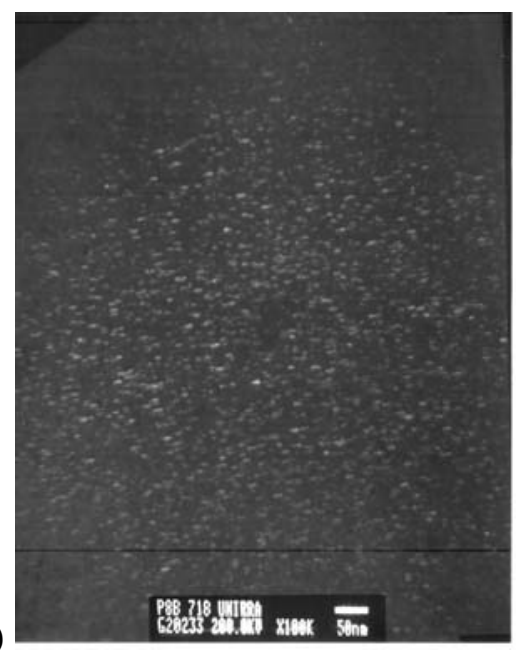

(c)

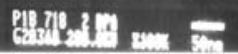

(e)

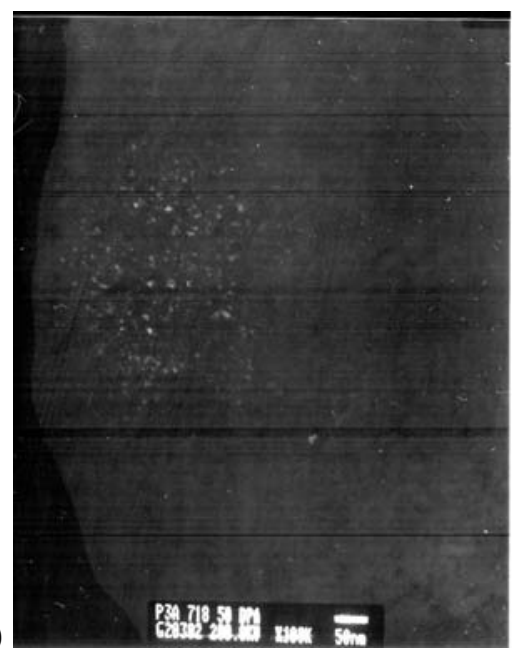

(b)

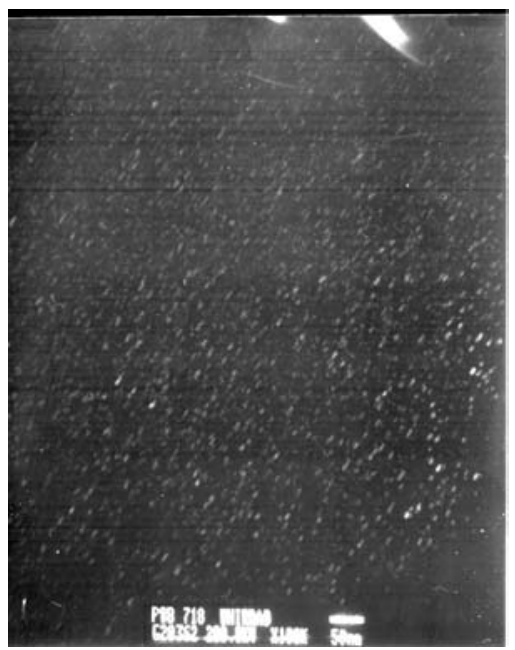

(d)

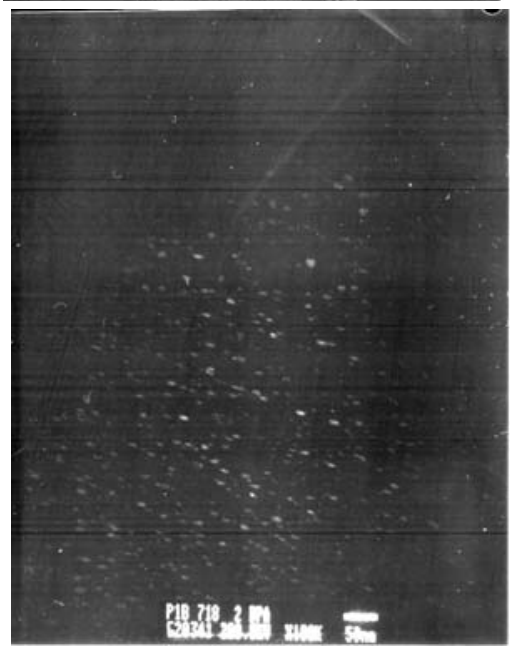

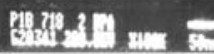

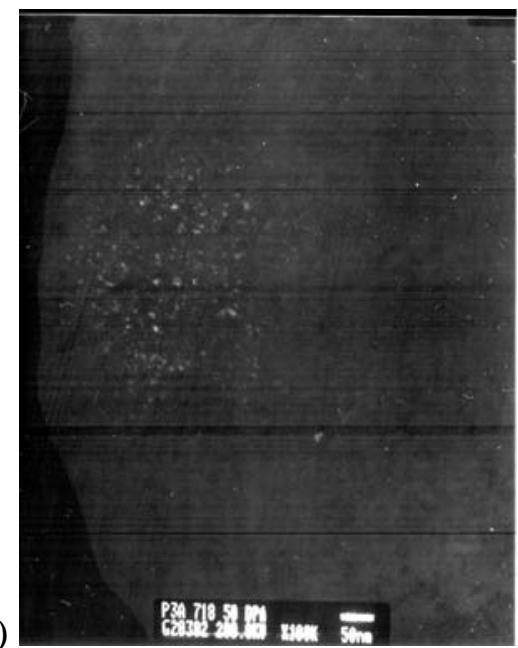

Figure 56. TEM dark field images illustrating Ni-ion irradiation effects on $\gamma^{\prime}-\gamma^{\prime \prime}$ stability in alloy 718: (a) non-irradiated, $\gamma^{\prime} / \gamma^{\prime \prime}$ image; (b) non-irradiated, $\gamma^{\prime \prime}$ image; (c) $2 \mathrm{dpa}, \gamma^{\prime} / \gamma^{\prime \prime}$ image ; (d) 2 dpa, $\gamma^{\prime \prime}$ image; (e) $10 \mathrm{dpa}$, only $\gamma^{\prime}$ present; and (d) $50 \mathrm{dpa}$, only $\gamma^{\prime}$ present. 

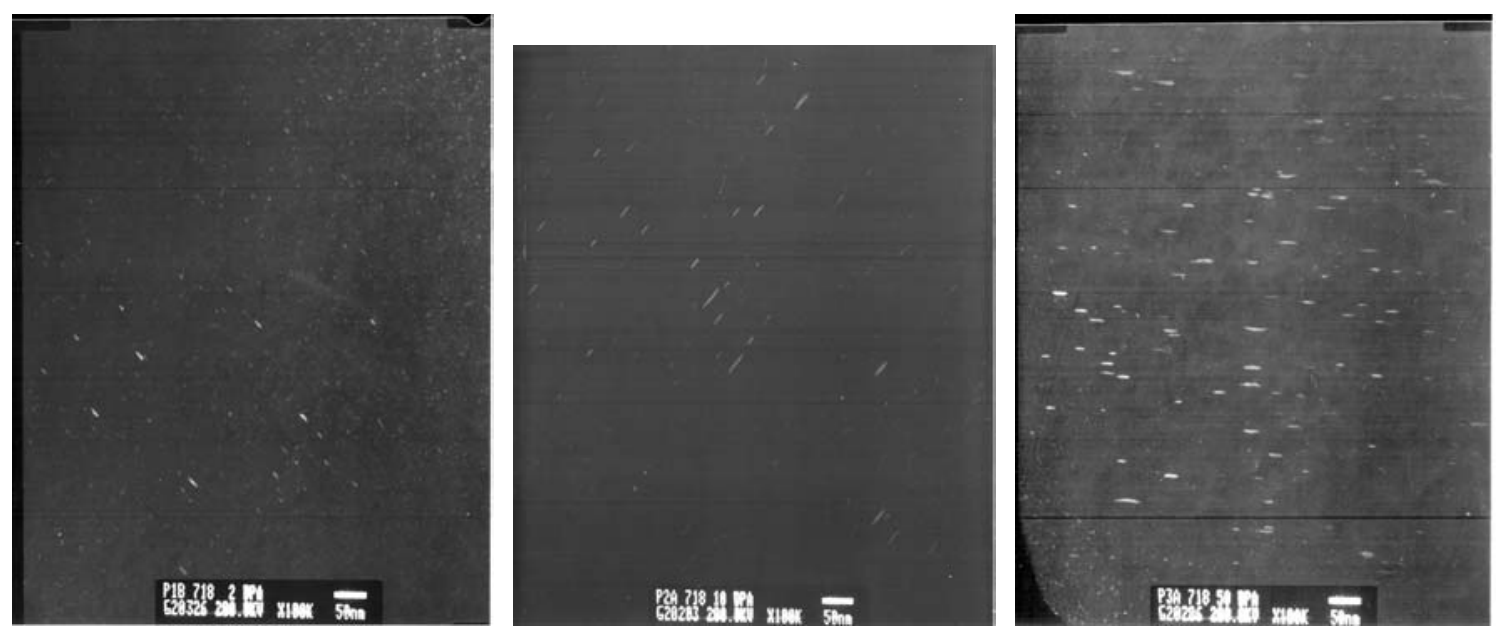

Figure 57. TEM relrod images showing faulted Frank loops in the alloy 718 matrix.

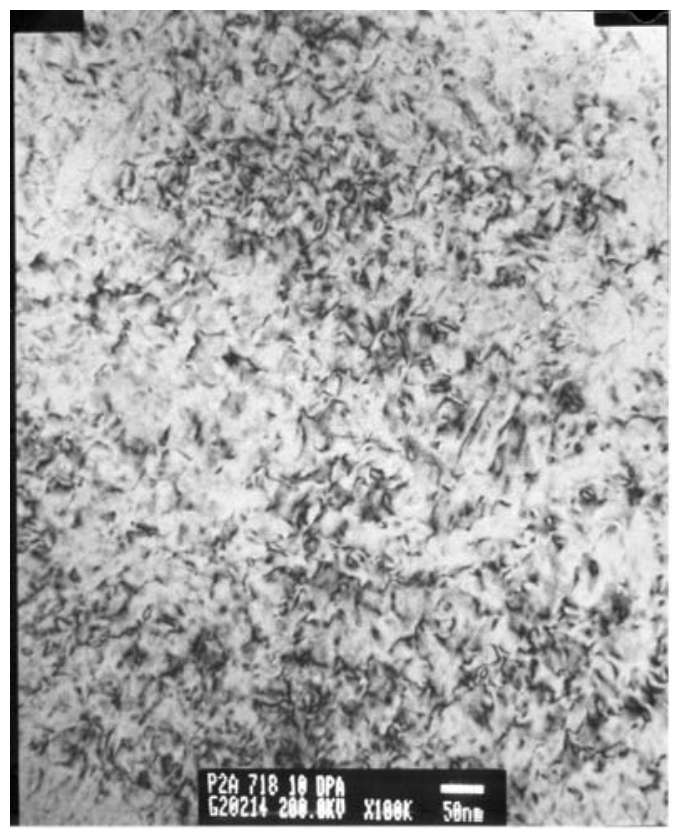

(a)

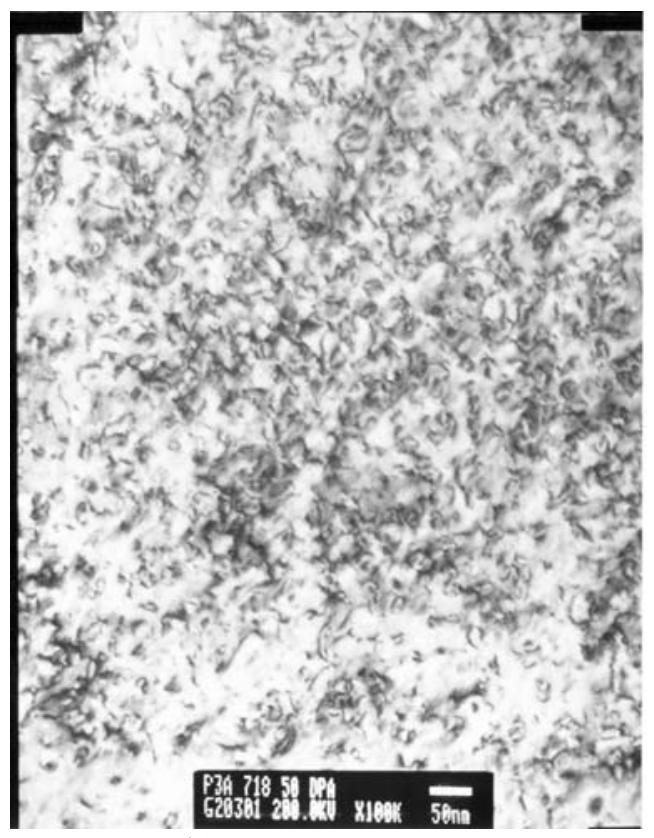

(b)

Figure 58. TEM brightfield images showing strain contrast from radiation-induced dislocation loops in alloy 718 after Ni-ion irradiations to (a) $10 \mathrm{dpa}$ and (b) 50 dpa.

Table 12. Heat Compositions for 17-7PH and 17-4PH Alloys in wt $\%$.

\begin{tabular}{|l|l|l|l|l|l|l|l|l|l|l|c|}
\hline Alloy & $\mathrm{C}$ & $\mathrm{Mn}$ & $\mathrm{P}$ & $\mathrm{S}$ & $\mathrm{Si}$ & $\mathrm{Ni}$ & $\mathrm{Cr}$ & $\mathrm{Mo}$ & $\mathrm{Cu}$ & $\mathrm{Al}$ & $\mathrm{Nb}$ \\
\hline $17-7 \mathrm{PH}$ & .073 & .84 & .023 & .0003 & .40 & 6.97 & 16.9 & .20 & .20 & 1.08 & - \\
$17-4 \mathrm{PH}$ & .040 & .63 & .028 & .014 & .48 & 4.31 & 15.3 & .19 & 3.21 & - & .28 \\
\hline
\end{tabular}




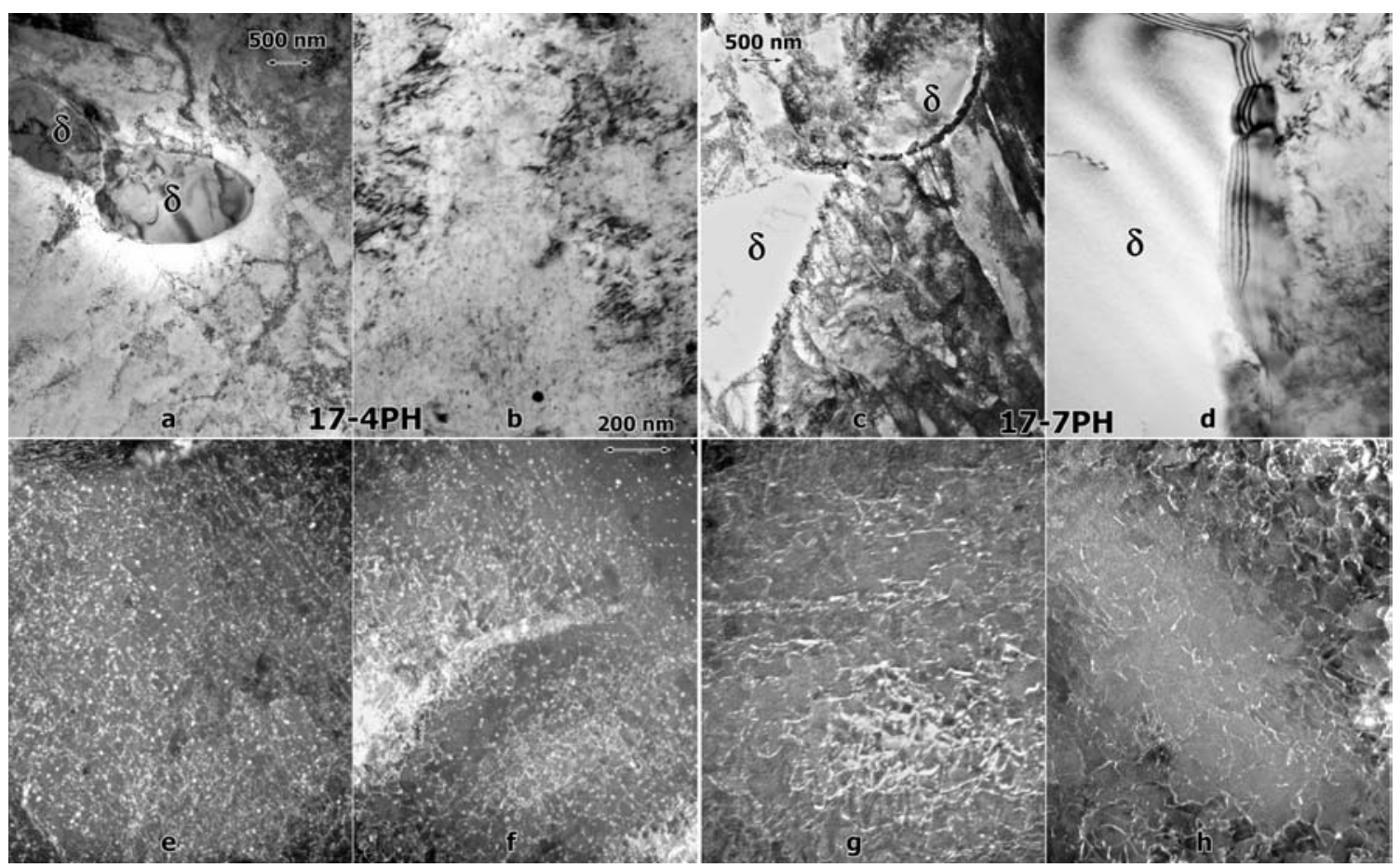

Figure 59. The microstructures of control specimens of $17-4 \mathrm{PH}$ and $17-7 \mathrm{PH}$.

Figures 59(a) and (c) show examples of the microstructures of 17-4PH and 17-7PH at low magnification with regions labeled that contain $\delta$-ferrite grains. In comparison, the regions containing tempered lath martensite plates have a much finer structure. These structures are shown at higher magnification in Figures 59(b) and (d). The example for 17-4PH only contains a martensite region and reveals both fine and somewhat coarser precipitate particles, but the example for $17-7 \mathrm{PH}$ shows that the $\delta$-ferrite contains a low dislocation density and is bounded by coarse precipitates and that the martensite contains finer structure without precipitates easily visible. The dark-field images are intended to provide an understanding of the dislocation structure prior to irradiation, and show that the dislocation structures are generally a loose tangle. However, it is noteworthy that precipitation can be easily identified as equiaxed features in the dark-field images of 17-4PH sometimes in linear arrays probably decorating dislocations, but similar features are not visible for 17-7PH.

\section{TEM characterization of irradiated Fe-base alloys}

Following $\mathrm{Ni}^{++}$ion irradiation at $500^{\circ} \mathrm{C}$, changes in the microstructure of $17-4 \mathrm{PH}$ are difficult to identify. Dislocation structures remain as loose tangles and precipitate sizes change little.

Examples are provided in Figure 60 showing examples of damage at 2 dpa on the left and $10 \mathrm{dpa}$ on the right. Figures 60(a), (b) (e) and (f) all show the same area of a martensite region with different imaging conditions after irradiation to $2 \mathrm{dpa}$. Figures 60(a) and (e) provide $\mathrm{g}=011$ bright-and dark-field images (with $\mathrm{g}$ vertical) and Figure 60(b) provides comparison with $\mathrm{g}=$ 200. However, these images do not show the precipitates clearly, although the dark-field image again reveals their presence. Figure 60(f) gives a precipitate dark-field image so that it can be shown that little change in precipitate size is apparent after a dose of $2 \mathrm{dpa}$ at $500^{\circ} \mathrm{C}$. 


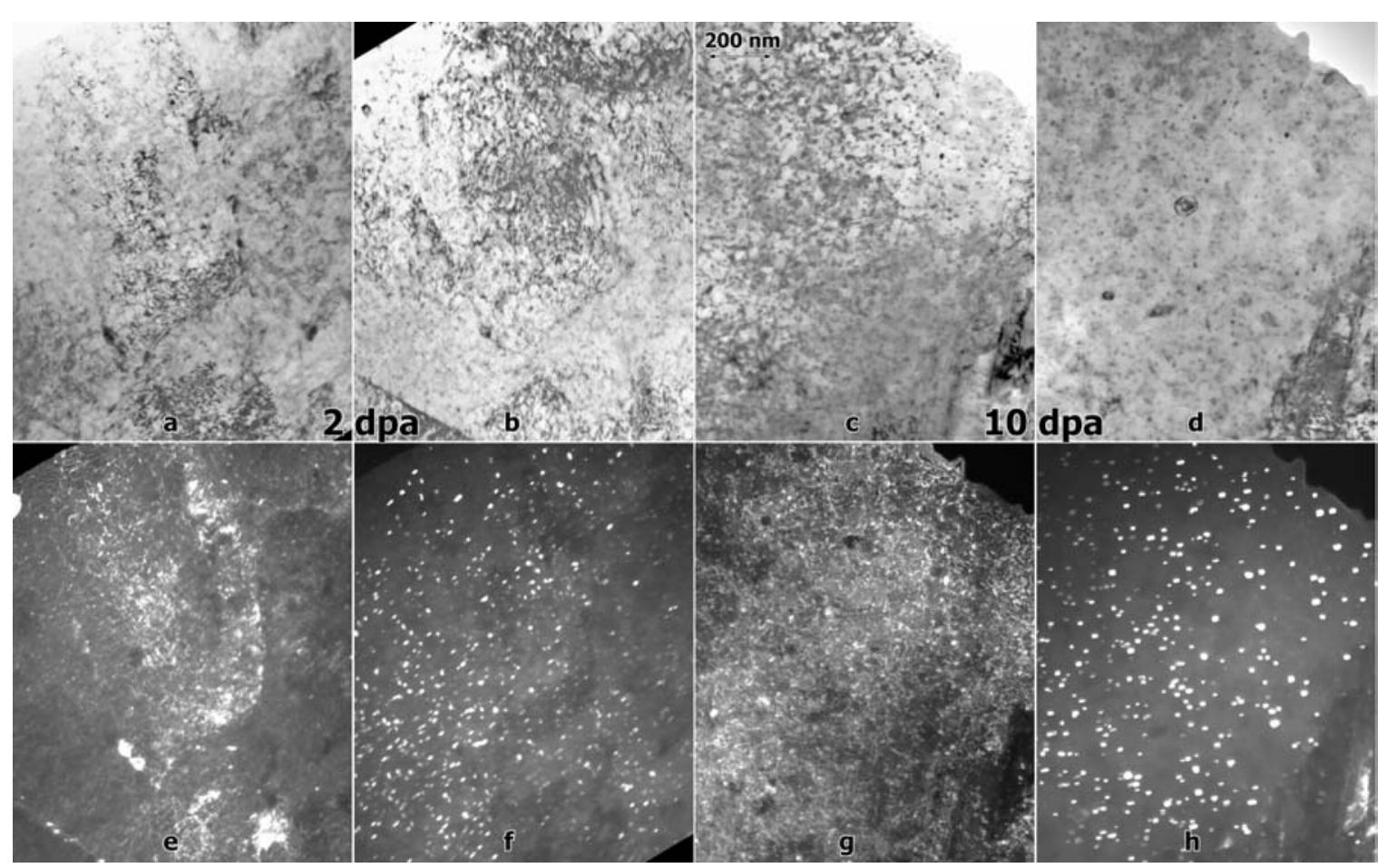

Figure 60. The microstructures of $17-4 \mathrm{PH}$ after $\mathrm{Ni}^{++}$ion irradiation at $500^{\circ} \mathrm{C}$.

Figures $60(\mathrm{c})$ and $(\mathrm{g})$ provide examples of the dislocation structure after irradiation to $10 \mathrm{dpa}$ using $\mathrm{g}=011$ (with $\mathrm{g}$ vertical) in bright-field and dark-field contrast. Again, the dislocation structure is found to be a loose tangle, but it is likely that the dislocations are often connected to precipitate particles as is more evident in the bright-field image. Figures 60(d) and (h) show the same area in void and precipitate dark-field contrast, respectively. Features that may be interpreted as voids can be identified, but the features are highly elongated which is atypical of voids in cubic materials, and uniformly distributed indicating that they are probably associated with the surface and therefore are artifacts. Precipitates that can be seen after 10 dpa may be slightly coarser than those appearing after $2 \mathrm{dpa}$.

Changes in the microstructure of $17-7 \mathrm{PH}$ following $\mathrm{Ni}^{++}$ion irradiation at $500^{\circ} \mathrm{C}$ could be identified in $\delta$-ferrite regions, but not in martensite regions. The $\delta$-ferrite was found to contain precipitation following irradiation, whereas precipitates could not be identified in martensite regions. Examples of microstructures in irradiated 17-7PH are given in Figure 61. Again, on the left is material irradiated to $2 \mathrm{dpa}$ and on the right the result of irradiation to $10 \mathrm{dpa}$.

Figures 61(a) and (c) give examples of dislocation structures in martensite regions and the remaining images show behavior in $\delta$-ferrite. The dislocation structures in Figures 61 (a) and (c) are similar indicating again that dose does not significantly alter dislocation configurations in these martensitic steels. Figures 61 (b), (e) and (f) show a $\delta$-ferrite region at 2 dpa in dislocation contrast (b) using $\overline{\mathbf{g}}=200$ (horizontal), and (e) and (f) with $\overline{\mathbf{g}}=011$ (vertical) with f) in dark-field contrast. The dislocation density is non-uniform with a higher density towards the upper left eminating from a grain boundary that is likely induced during specimen preparation. The remaining dislocations are more typical of structure elsewhere exhibiting radiation-induced climb. In the background of Figure 61(b), both a fine precipitation and coarser particles are clearly 
visible, only the coarser precipitate can be seen in Figure 61(f) most notable towards the center right. Figure $61(\mathrm{~d}),(\mathrm{g})$ and $(\mathrm{h})$ show the precipitation in $\delta$-ferrite irradiated to $10 \mathrm{dpa}$ at higher magnification, d) in bright field, and g) and $h$ ) in precipitate dark-field contrast. No voids are apparent, but two different sets of precipitates can be identified. Figure $61(\mathrm{~g})$ includes the corresponding (011) diffraction pattern inset at the upper right. The spot at $(1 / 2)<200>$ was used for Figure $(\mathrm{g})$ and probably corresponds to $\gamma^{\prime}$ precipitates of type $\mathrm{Ni}_{3} \mathrm{Al} . \gamma^{\prime}$ precipitates are often difficult to see in bright field imaging. Three different size distributions are apparent, but the largest particles are apparently not associated with voids. The spot at approximately $(3 / 8)<200>$ was used for Figure 61 (h) and shows a second set of particles of intermediate size. Many of these features correspond to the intermediated sized $\gamma^{\prime}$ precipitates such that $\gamma^{\prime}$ appears to be coating them. Therefore, Ni-ion irradiation produces a moderate density of dislocations and at least two different precipitate types in $\delta$-ferrite, but the structure is sufficiently complex in martensite so that similar differences are not apparent.

\section{Discussion of Fe-based Alloy Concept}

Effects of $5 \mathrm{MeV} \mathrm{Ni}^{++}$irradiation at $500^{\circ} \mathrm{C}$ to $17-4 \mathrm{PH}$ and $17-17 \mathrm{PH}$ in the fully heat-treated conditions were most evident in retained $\delta$-ferrite. The microstructure of martensitic regions was sufficiently complex so that details may have been masked, but more likely, the structure was sufficiently fine to provide point defect sink distributions that prevented significant effects of radiation damage. The damage found in $\delta$-ferrite included both dislocation evolution and precipitation, but no evidence for void swelling was observed. The dislocation evolution produced loose tangles and loops, but the imaging in Figure 61b) is typical of $(\mathrm{a} / 2)<111>$ Burgers vectors indicating that $\mathrm{a}<100>$ Burgers vectors are not common. [The $\mathrm{a}<100>$ dislocations

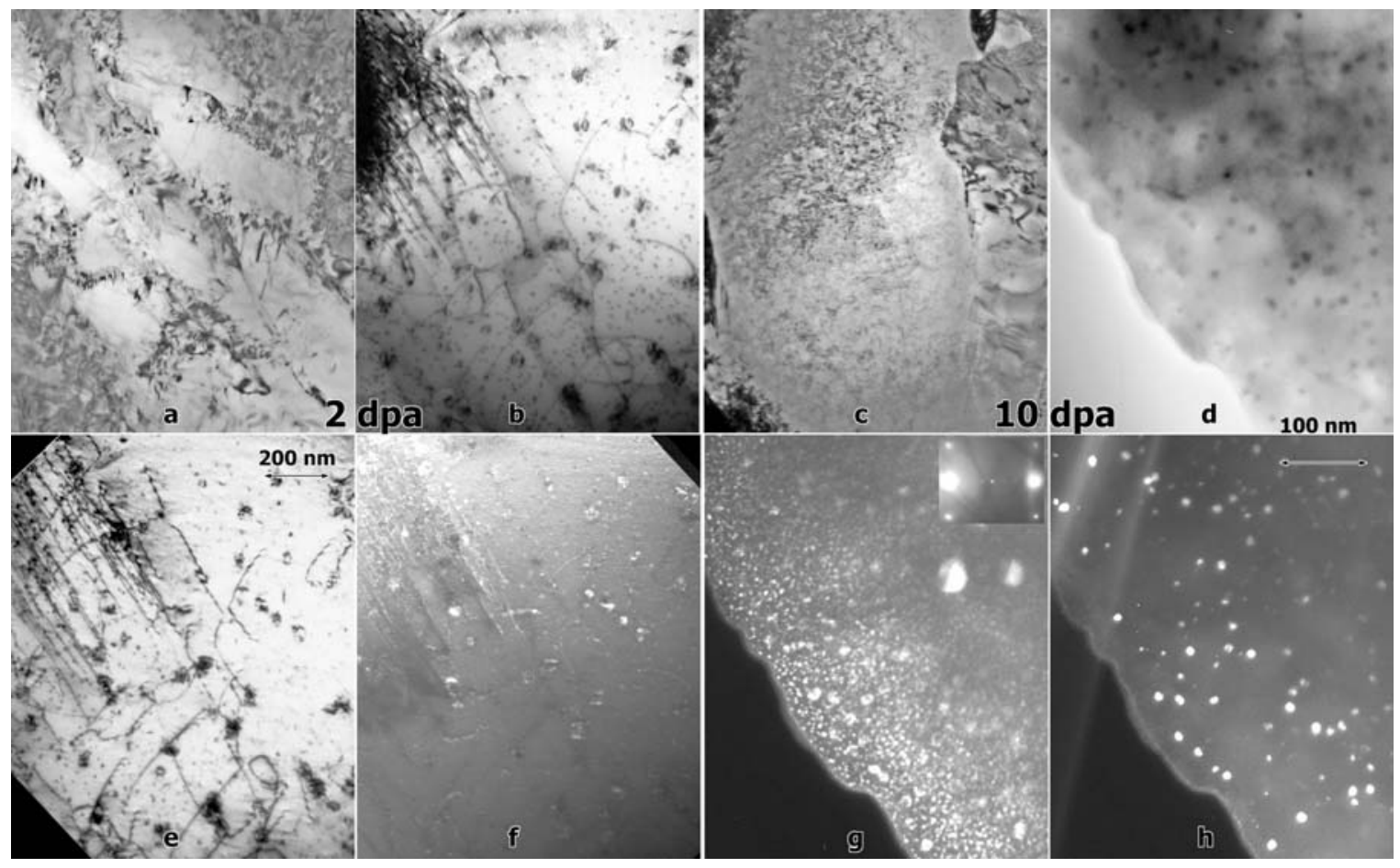

Figure 61 . The microstructures of $17-7 \mathrm{PH}$ after $\mathrm{Ni}++$ ion irradiation at $500^{\circ} \mathrm{C}$. 
would appear in stronger contrast as elongated images normal to the operating $\left.\overline{\mathbf{g}} .{ }^{16}\right]$ Two different precipitate types developed during irradiation in each alloy. In 17-4PH, each was of uniform size, that imaged only in Figure 61(b) at higher number density than the one in found also in Figure 61(f). In 17-7PH, one of the precipitates was comparable to that found in 17-4PH, but the other, tentatively identified as $\mathrm{Ni}_{3} \mathrm{Al} \gamma^{\prime}$ was found in three distinctly different size classes, one large, one medium and associated with the other precipitate type as a coating and the third, uniformly distributed on a finer scale. Such behavior is typical of $\gamma^{\prime}$ precipitation when solute segregation and coarsening occur simultaneously during irradiation. ${ }^{17}$ However, the origin of the largest size class is not yet understood. The effective diffusion needed to create a particle of this size is typical of pre-irradiation heat treatment or extensive solute segregation. For example, it may have formed during $760^{\circ} \mathrm{C}$ heat treatment, but no evidence of such precipitation was found in unirradiated microstructures. Alternatively, it may have formed as a coating on large voids, but no evidence of void swelling was found. Finally, it can be noted that the fine distribution of $\gamma^{\prime}$ precipitates in Figure 61(g) is probably characteristic of coarsening behavior under the irradiation enhanced diffusion conditions imparted by $5 \mathrm{MeV} \mathrm{Ni}^{++}$irradiation at $500^{\circ} \mathrm{C}$. Further understanding of $\gamma^{\prime}$ precipitation in this alloy would suggest an inferred diffusion coefficient in a manner similar to that done by Gelles ${ }^{17}$ doing work on alloy PE16.

The precipitation strengthened Fe-base alloys exhibited precipitate stability to high dose similar to that observed in the Ni-base alloy 718. In both classes of alloys, the precipitate distribution was altered by irradiation but their presence was not eliminated. The ferritic/martensitic alloy class is an example of potential alloys exhibiting stable mechanical properties and acceptable irradiation microstructures at high dose and high temperature.

\section{Ferritic/Martensitic Concept Evaluation}

The promise for using multiphase $\mathrm{Ni}$-and Fe-based alloys for nuclear structural materials was followed by an effort to assess ferritic/martensitic ODS steels. A review of ODS steels development for irradiation environments is provided to summarize international research for liquid-metal, fast-breederreactor and fusion-reactor technology.

\section{Introduction}

Ferritic/martensitic steels have demonstrated excellent promise for use as structural materials in neutron irradiation environments. The $12 \% \mathrm{Cr}$ steel HT-9 was chosen to replace all internal components in the liquid-metal fast-breeder reactor, Fast Flux Test Facility (FFTF), made initially of SS316, ${ }^{18}$ and the low-activation alloy class of 7-9\% Cr steels containing $1-2 \% \mathrm{~W}$ are considered the front-runners for Tokamak fusion reactor DEMO designs. ${ }^{19}$ These choices are a result of good physical and high temperature properties, and excellent dimensional stability to neutron doses as high as $200 \mathrm{dpa}$. Taken into account are poor toughness properties for HT-9 following irradiation at low temperatures, an issue that is much less pertinent for low activation steels, as well as an upper-temperature operating limit on the order of $550-600^{\circ} \mathrm{C}$. HT-9 was chosen after extensive testing of many classes of materials including superalloys and refractory metals.

The opportunity exists for significant improvements in high-temperature operation limits for ferritic/martensitic steels by use of technology known as oxide-dispersion strengthening (ODS). The basis for the alloy class is to provide very stable precipitates, usually $\mathrm{Y}_{2} \mathrm{O}_{3}$, distributed on a 
very fine scale. This alloy class was first considered for fast-breeder technology in Europe by Huet, ${ }^{20,21}$ but it must be noted that his DT series of alloys were in fact chi phase strengthen due to high levels of Mo and Ti that created thick coatings of chi on grain boundaries. Concurrently, the International Nickel Company developed a series of patents for mechanical alloying which employed powder processing techniques for distributing a dispersoid such as $\mathrm{Y}_{2} \mathrm{O}_{3}$ on a fine scale. $^{22}$ This led to a series of mechanically alloyed, (MA) materials described in a series of conferences ${ }^{23,24}$ entitled "Frontiers of High Temperature Materials." An alloy was developed specifically designed for fast-breeder technology applications and designated MA957. ${ }^{25,26}$ However, the testing programs using MA957 were not successfully completed, in part because of a technical flaw in the MA957 bar stock, as will be discussed. Work is continuing for development of ODS materials in other alloy systems, particularly for aerospace applications. ${ }^{27}$

The advantages of the ODS technology can best be demonstrated with stress rupture behavior using Larson-Miller representation. An example is given in Figure 62, comparing a standard martensitic steel with data for a wide range of ODS ferritic steels. The significant difference between these two classes of steel is the slope of the data, because values to the upper right represent long times at high temperatures. This behavior is a direct consequence of the thermal stability of the oxide dispersoid. Differences between the various ODS materials can be attributed to composition, microstructure and dispersion differences.

\section{MA957 Fabrication Studies}

The patent for MA957 specifies an alloy $\sim 13-25 \% \mathrm{Cr}, \sim 0.2-2.0 \% \mathrm{Ti},<2.0 \% \mathrm{Mo}$ and $<2.0 \% \mathrm{Y}_{2} \mathrm{O}_{3}$, with a recommended composition of $14 \mathrm{Cr}-1 \mathrm{Ti}-0.3 \mathrm{Mo}-0.25 \mathrm{Y}_{2} \mathrm{O}_{3}$. The $\mathrm{Y}_{2} \mathrm{O}_{3}$ is added as the dispersoid, the Mo and Ti to improve high-temperature strength and retain ductility, the Ti to reduce vaporization of $\mathrm{Cr}$ and to control excess oxygen absorption during mechanical alloying, and the $\mathrm{Cr}$ to provide corrosion resistance and maintain a fully -ferritic microstructure (that does not transform to austenite on heating). Mechanical alloying is required to distribute the dispersoid uniformly.

Early efforts on ODS materials were intended for application as fuel cladding in fast-breeder reactors. Therefore, it was necessary to produce tubing to the required size. This was possible in Europe, the U.S. and Japan, but major difficulties were encountered in the U.S. ${ }^{29,30}$ MA957 is extremely strong, with yield strength over $1000 \mathrm{Mpa}$ at room temperature. Annealing to soften the material requires temperatures approaching $1300^{\circ} \mathrm{C}$ and results in secondary recrystallization in MA957 with drastically different properties. An example of annealing behavior is given in Figure 63 and a processing window for tubing fabrication needed to retain properties is shown in Figure 64. Unfortunately, after complete secondary recrystallization, strength drops dramatically and is never recovered. This is because the $\mathrm{Y}_{2} \mathrm{O}_{3}$ disappears following recrystallization and is replaced by a much coarser phase related to ruby. The problem was traced to $\mathrm{Al}_{2} \mathrm{O}_{3}$ contamination that was introduced as a contaminant in the $\mathrm{Cr}$ during production of MA957. Given the requirement that annealing treatments were restricted to about $1000^{\circ} \mathrm{C}$ and cold work levels to $\sim 12 \%$, a large number of draws were needed to produce tubing of the required size with many opportunities for creation of defects. 


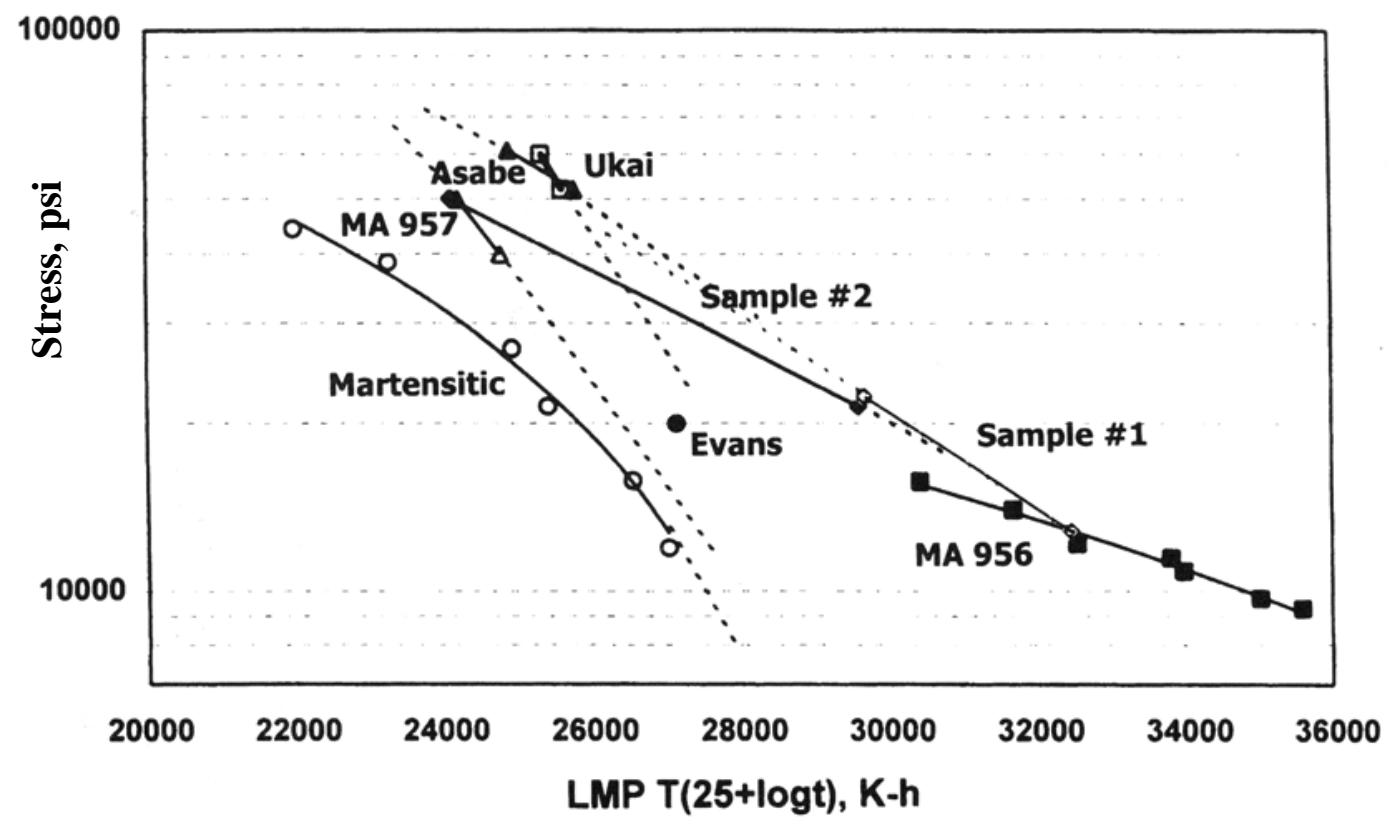

Figure 62. Larson-Miller plot comparing creep rupture strength of ODS ferritic steels with those of martensitic steels. ${ }^{28}$
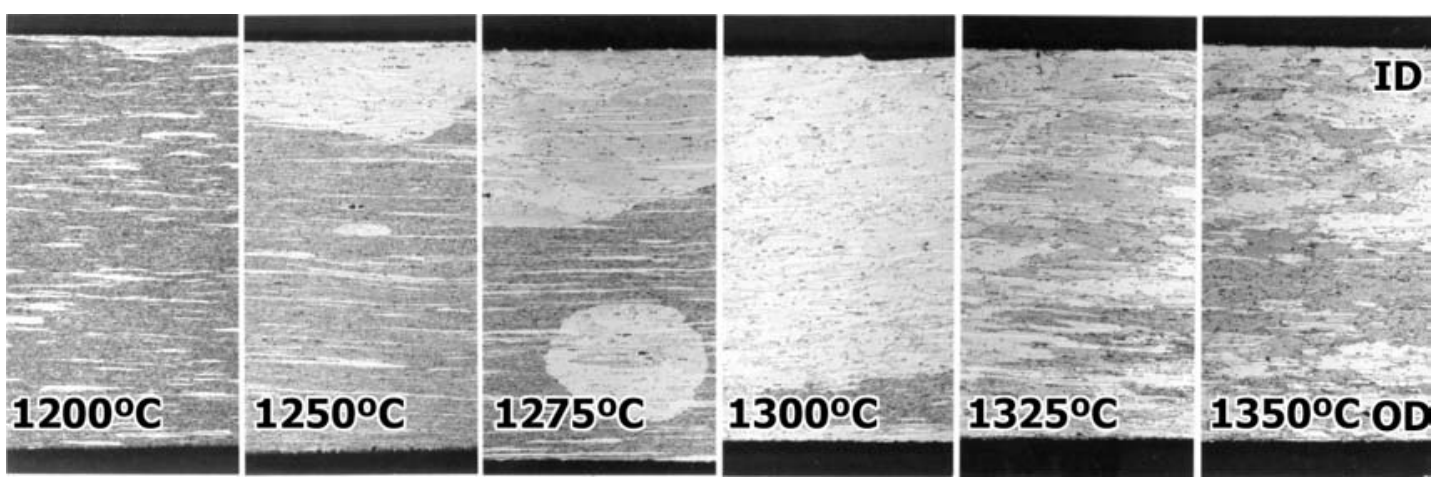

Figure 63. MA957 annealing study for tubing at $24 \% \mathrm{CW}$ annealed for $30 \mathrm{~min}$ at the temperature indicated.

Further alloy development efforts

ODS ferritic steel efforts have been continued on two fronts. In Japan, PNC arranged with Kobe Steel Ltd. and Sumitomo Metal Industries to redesign ODS ferritic steels in order to eliminate anisotropy in the microstructure, shifted from Mo to $\mathrm{W}$ for solute strengthening, Ti was reduced but similar levels of $\mathrm{Y}_{2} \mathrm{O}_{3}$ were considered. The study showed that $\mathrm{Y}_{2} \mathrm{O}_{3}$ levels increased strength and reduced ductility with increasing concentration, that excess oxygen controlled formation of ultra-fine oxide complexes of $\mathrm{Ti}, \mathrm{Y}$ and $\mathrm{O}$ and that anisotropy could be avoid by the use of an austenite/ferrite transformation, i.e. by reduction of the $\mathrm{Cr}$ content. ${ }^{31}$ These new compositions could be successfully fabricated into tubing with excellent properties but maximum high-temperature strength was obtained for maximum levels of Ti and $\mathrm{Y}_{2} \mathrm{O}_{3}{ }^{32}$ 


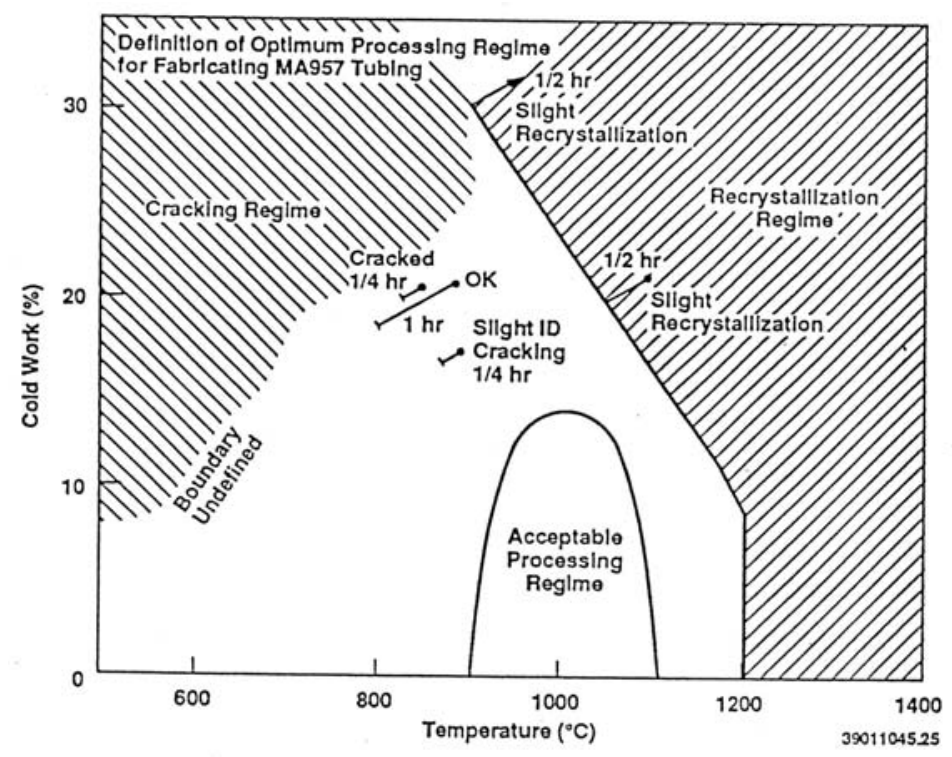

Figure 64. Definition of the optimum processing regime for fabrication of MA957 tubing to avoid recrystallization.

ODS ferritic steel development by mechanical alloying was also considered for fusion-reactor applications. Early efforts to shift to compositions that avoided elements with long-lived radioactive isotopes were not very successful. The approach used was based on MA957 and maintained $\mathrm{Ti}$ and $\mathrm{Y}_{2} \mathrm{O}_{3}$ levels but substituted $\mathrm{W}$ for Mo and considered two levels of $\mathrm{Cr}, 14$ and $9 \%$, the latter in order to consider use of an austenite/ferrite transformation (and using $-2 \mathrm{~W}$ and $0.3 \mathrm{~V}$ as is done for reduced activation martensitic steels). First attempts at extrusion failed and it was learned that a $\mathrm{C}-\mathrm{Y}_{2} \mathrm{O}_{3}$ reaction could occur that destroyed the original dispersoid distribution. ${ }^{33}$ A second attempt after $\mathrm{C}$ additions were avoided was successfully extruded into bar stock, ${ }^{34}$ but tubing could not be fabricated.

A later effort was intended to reduce $\mathrm{Cr}$ levels as low as possible and still maintain a fully ferritic structure and to demonstrate the applicability of this class of materials by performing creep tests at $900^{\circ} \mathrm{C}$. The limit on $\mathrm{Cr}$ was determined to be $13.5 \mathrm{Cr}$ and creep rupture results were shown in Figure 62 marked as sample \#1 and sample \#2. ${ }^{28}$

Most recently, a European effort is intended to produce large experimental batches of material based on the basic steel EUROFER 97 ( Fe-8.9Cr-1.1W-0.2V-0.14Ta-0.42Mn-0.11C) gas atomized, with $\mathrm{Y}_{2} \mathrm{O}_{3}$ added (at levels of 0.3 and $0.5 \mathrm{wt} \%$ ), mechanically alloyed and then HIPed, ${ }^{35}$ and various laboratories around the world are recommending other novel compositions for fusion ODS alloys. ${ }^{36,37}$

\section{Effects of Irradiation}

A limited number of irradiation experiments have been performed on ODS ferritic steels. These include microstructure and swelling, tensile deformation, charpy impact, biaxial creep, and stress rupture to neutron doses as high as $200 \mathrm{dpa} .{ }^{30,37,38-41}$ Electron and Fe+ with He-ion irradiation studies on microstructural change have also been performed. ${ }^{42-44}$ However, it must be emphasized that this data base was sufficient so that MA957 was being seriously considered for 
use in fuel cladding tests in FFTF. Data was available to show that at least to moderate doses, no significant problems could be identified.

Two early experimental batches of MA967 have been irradiated to $206 \mathrm{dpa}$ at $\sim 420^{\circ} \mathrm{C}$ in FFTF for density change and microstructural characterization in comparison with HT-9, T91 and MA956. ${ }^{38}$ It was found that density change was less than $2 \%$, attributed at least in part to isolated regions showing voidage at levels as high as $5 \%$ in recrystallized grains. Other unrecrystalized regions contained only a few cavities perhaps remaining from fabrication and retained fine dispersoids and with $\alpha^{\prime}$ precipitation during irradiation. It was concluded that such microstructures can be expected to retain stable mechanical properties to high dose.

In order to qualify MA957 for fuel cladding, experimental batches of tubing were tested in the FFTF over the temperature range $410-730^{\circ} \mathrm{C}$ covering fluences as high as $\sim 80 \mathrm{dpa}$ for creep response, but generally in the range $22-36 \mathrm{dpa}$ in order to provide tensile, impact, stress rupture and microstructural behavior due to irradiation. ${ }^{30}$ It was found that for tensile tests or irradiation temperatures above about $500^{\circ} \mathrm{C}$ there was very little change in yield strength due to irradiation. On the other hand, specimens irradiated at $410^{\circ} \mathrm{C}$, showed yield strength increases by $\sim 20 \%$ with elongation degraded somewhat. The behavior is plotted in Figure 65.

Irradiation of MA957 at $410^{\circ} \mathrm{C}$ to $\sim 36$ dpa produced a large increase in DBTT to $\sim 325^{\circ} \mathrm{C}$ from $\sim 100^{\circ} \mathrm{C}$ for unirradiated specimens. Irradiation at $540^{\circ} \mathrm{C}$ caused a smaller increase in DBTT of about $100^{\circ} \mathrm{C}$. The data is plotted in Figure 66.

Irradiation creep measurements on both pressurized tubes made from gun-drilled rod stock and drawn tubing of MA957 showed excellent behavior, particularly at the highest temperature of $750^{\circ} \mathrm{C}$ even in comparison with other ferritic/martensitic steels. Creep strains generally did not exceed $\sim 1 \%$, and diameter change measurements of unstressed tubes at low temperatures showed negligible change, indicating negligible void swelling had occurred. Furthermore, in cases where gun-drilled specimens were re-irradiated to higher dose, further increases in strain were reduced indicating that some of the strain measured was likely due to primary creep.

Five tests produced failures in-reactor, allowing comparison with stress rupture behavior under thermal conditions. The failures covered temperature conditions from 565 to $660^{\circ} \mathrm{C}$. All tests provided response similar to that out of reactor, indicating that irradiation in this temperature range did not lead to a change in failure mechanism. Results are shown in Figure 67. The parameter indicated is the Larson Miller parameter and results of regression analysis of thermal test data based on the Dorn Parameter equation gave:

$$
\log _{10} t_{r}=-7.0156+23049 / T-7.67081 \log _{10}(\sigma)
$$

Microstructural changes due to irradiation in MA957 could be divided into two regimes. Below $550^{\circ} \mathrm{C}$, dislocation development, $\alpha^{\prime}$ formation, and void evolution in the matrix were found, providing explanation for the observed increase in strength. Above $550^{\circ} \mathrm{C}$, damage appeared to be restricted to cavity formation within $\mathrm{TiO}_{2}$ particles. The initial subgrain structure and the fine dispersion of $\mathrm{Y}_{2} \mathrm{O}_{3}$ particles were retained following irradiation at all temperature over the range 365 to $750^{\circ} \mathrm{C}$. An example showing cavities formed in $\mathrm{TiO}_{2}$ particles following irradiation at 


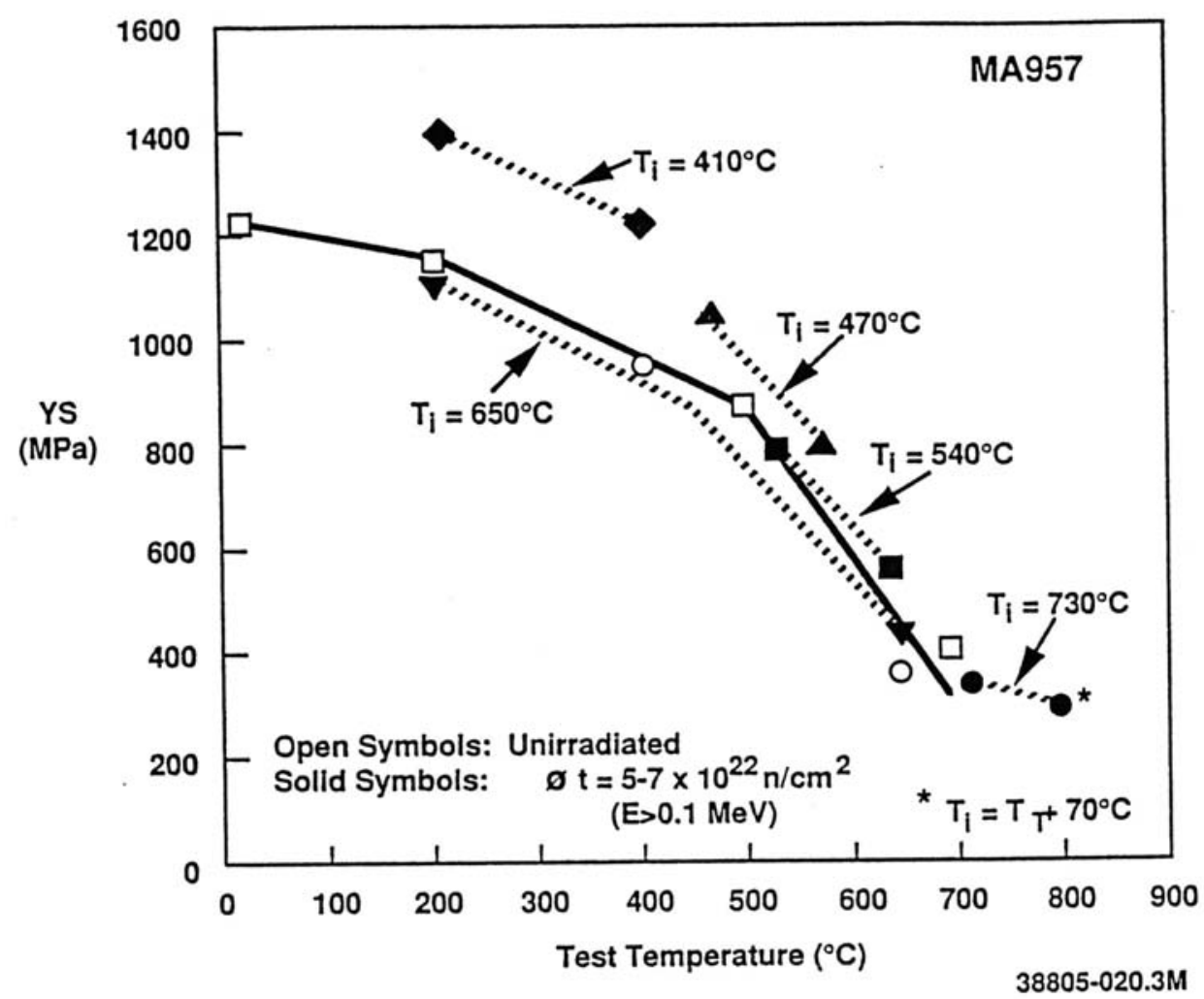

Figure 65. Yield strength of MA957 for tests performed at various temperatures relative to the irradiation temperature.

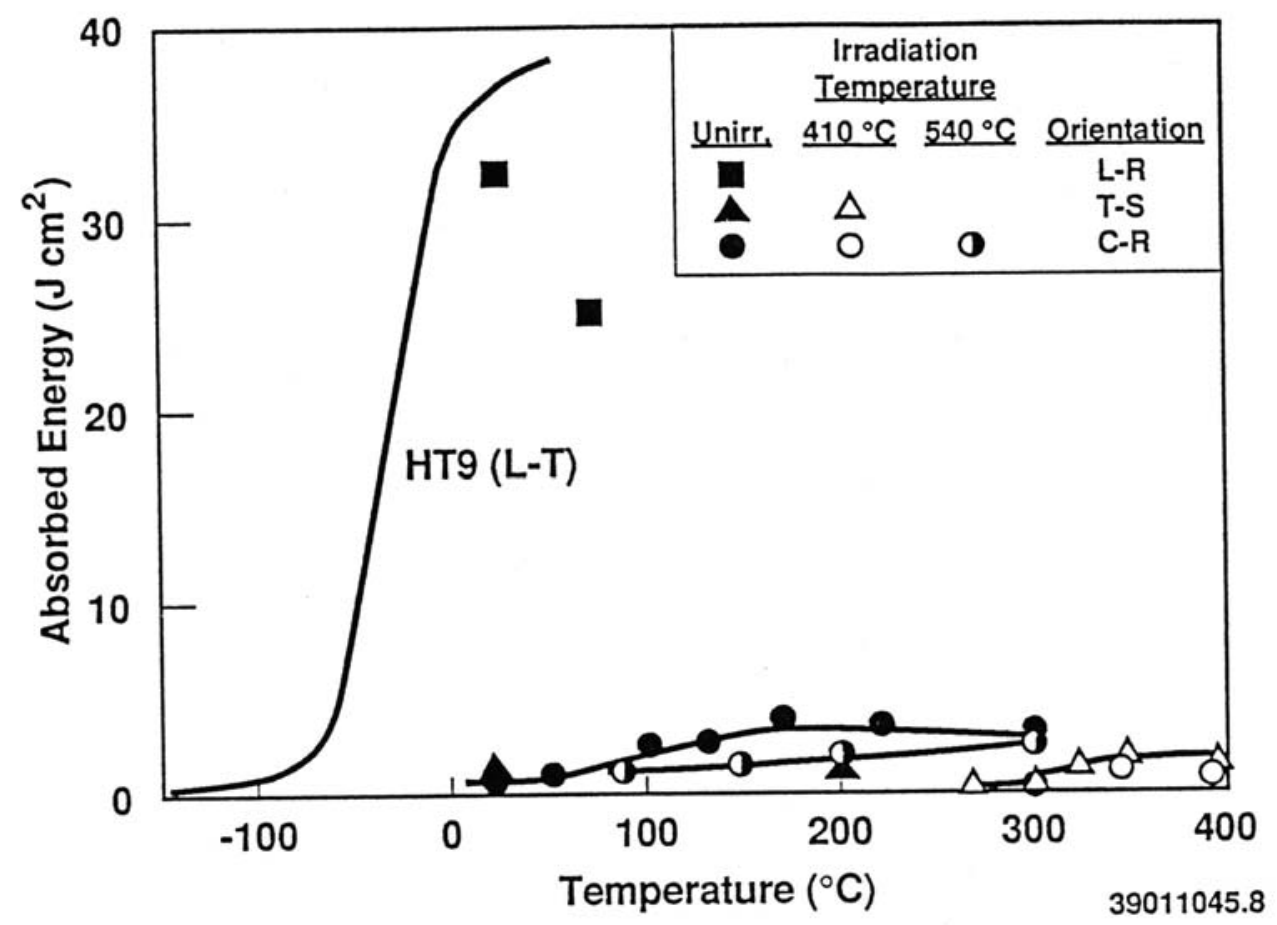

Figure 66. Fracture energy of 1/3 size Charpy impact specimens of MA957 irradiated at 410 and $540^{\circ} \mathrm{C}$. 
$670^{\circ} \mathrm{C}$ to $\sim 28 \mathrm{dpa}$ is provided in Figure 68 . Based on irradiation creep and in-reactor stress rupture response, this cavitation causes negligible problems.

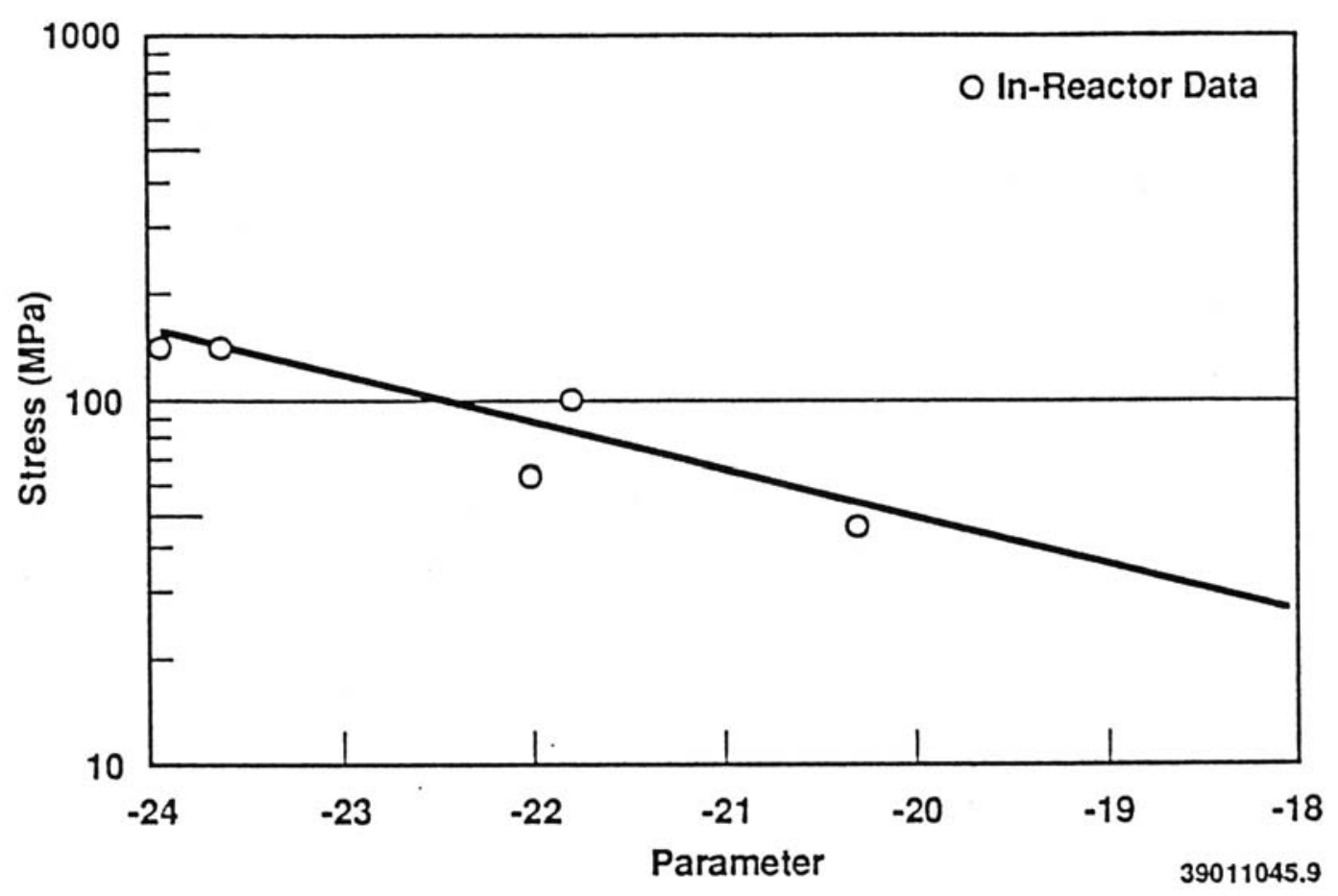

Figure 67. Rupture behavior of pressurized tubes of MA957 tested in-reactor.

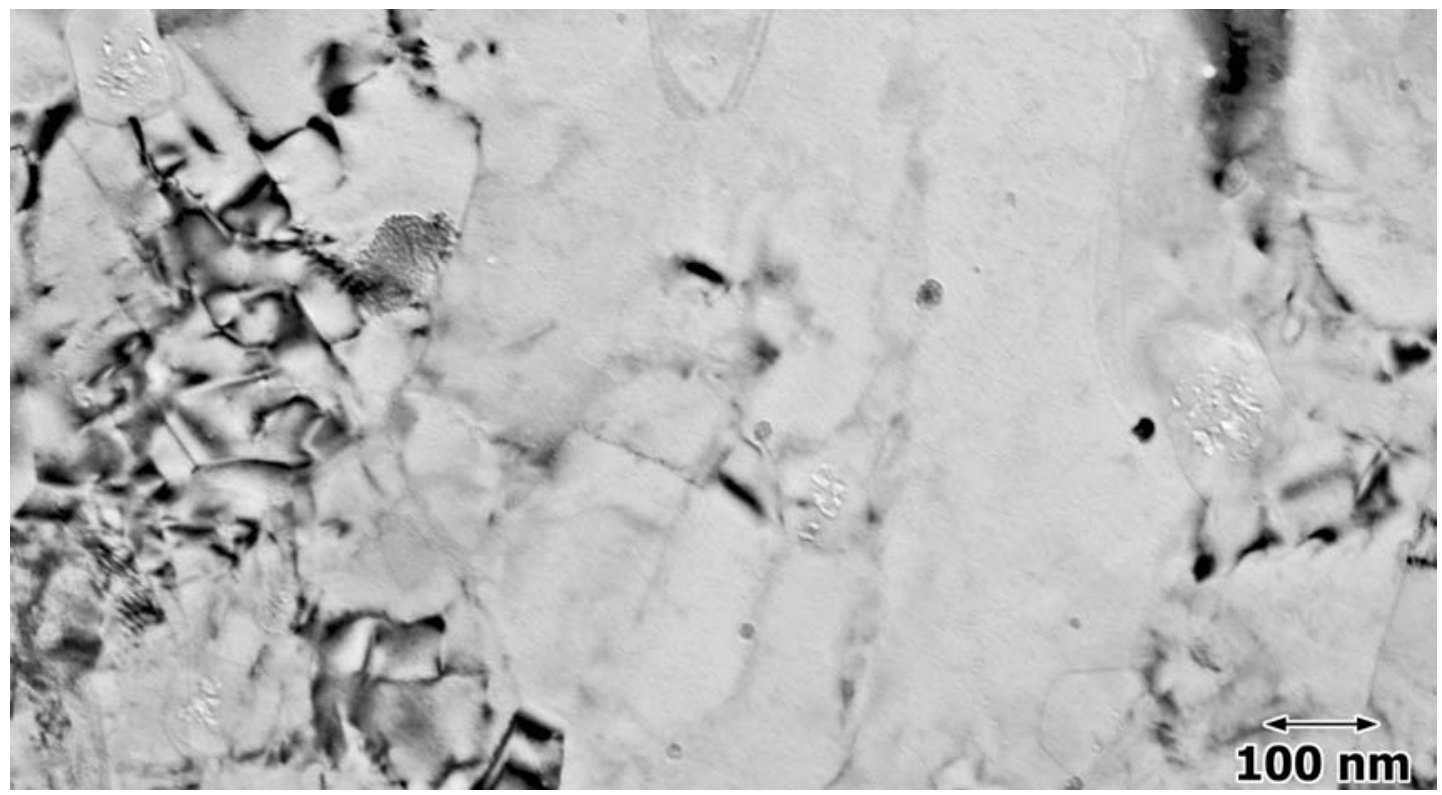

Figure 68. Cavities in MA957 following irradiation at $670^{\circ} \mathrm{C}$ to $\sim 28 \mathrm{dpa}$ are found to be restricted to large $\mathrm{TiO}_{2}$ particles. 
Two reports on effects of irradiation on the DT type alloys from Belgium are noteworthy. Asbroeck and co-workers reported that following neutron irradiation to moderate doses only as high as $7 \mathrm{dpa}$ at $700^{\circ} \mathrm{C}$, flat tensile specimens showed significant reductions in total elongation, but in the worst case, $9.1 \%$ elongation was measured. ${ }^{40}$ Also, a CEA effort by Dubuisson and coworkers have reported microstructural changes based on irradiation of fueled cladding of a similar alloy with lower $\mathrm{Ti}$ and Mo levels following irradiation in the Phenix reactor over the temperature range 400 to $580^{\circ} \mathrm{C}$ to doses as high as $\sim 80 \mathrm{dpa}$. During mechanical cutting, they did encounter brittle longitudinal failure of two tubes indicating embrittlement, with minimum elongation found at maximum dose, corresponding to an irradiation temperature of $\sim 500^{\circ} \mathrm{C}$. However, the general microstructure did not appear to be modified by in-pile service. For example, negligible void swelling could be identified, and those voids found were associated with oxide particles that could be titania. X-ray microanalysis showed a increase in $\mathrm{Cr}$ - and Mo levels and a decrease in the Ti levels in the grain boundary chi phase that was best correlated with irradiation temperature. A uniform distribution of tiny particles that formed at low temperatures during irradiation could be identified, anticipated to be $\mathrm{Cr}$-rich $\alpha^{\prime}$, chi-phase precipitation developed at $532^{\circ} \mathrm{C}$ and Laves was identified after irradiation at $580^{\circ} \mathrm{C}$. Finally, evidence for precipitate redistribution of larger particles was found following irradiation at $480^{\circ} \mathrm{C}$, also observable following irradiation at 508 and $532^{\circ} \mathrm{C}$. Redistribution resulted in replacement of the outer regions of large precipitate particles with a fine distribution of tiny particles around the original oxide particles. These results must be interpreted with care, they do not necessarily describe embrittlement behavior in all ODS alloys. DT type alloys contain very high levels of Ti and Mo.

Russian ODS alloys with 1.1-2.2Ti and 0.2-1.4Mo have been irradiated up to $20 \mathrm{dpa}$ at $410^{\circ} \mathrm{C}$ to provide post-irradiation mechanical properties. ${ }^{37}$ Insignificant increases in strength were observed following irradiation, but ductility was reduced to $1.1 \%$ for the alloy containing highest $\mathrm{Ti}$ and Mo levels. In comparison, irradiation at low temperature irradiation $\left(-200^{\circ} \mathrm{C}, 77 \mathrm{~K}\right)$ to less than 10 dpa produced failure prior to yield, similar to that found in other bcc alloys. Finally, irradiation testing has been performed on Japanese ODS materials but limited results have not yet been reported. ${ }^{45,46}$

\section{Discussion}

This review of ferritic/martensitic ODS alloy development demonstrates a great deal of interest in this alloy class around the world. A summary of the composition space being considered has been compiled in Table 13. In effect, these efforts can be divided into two types and four classes of alloys based on the $\mathrm{Cr}$ and $\mathrm{Ti}$ levels being considered. Choice of $\mathrm{Cr}$ determines if a martensite structural transformation is being employed. Levels on the order of $9 \%$ are chosen to encourage an austenite to ferrite transformation on cooling producing martensite if cooling rates are rapid enough, and should produce alloys less sensitive to $\mathrm{Cr}$-rich $\alpha^{\prime}$ formation during irradiation at low temperatures. Levels on the order of $14 \% \mathrm{Cr}$ avoid transformation on cooling. The martensite transformation can be used to refine the microstructure and eliminate anisotropy. However, it can be noted that Lindau and co-workers found that a martensite transformation only occurred as a result of rapid quenching in their ODS-EUROFER. ${ }^{35}$ Also, Ukai and co-workers have noted that a fine grain size leads to higher creep rates and a lower stress exponent. ${ }^{45}$ As a function of $\mathrm{Ti}$, four levels can be defined; high, intermediate, low and none. It can be claimed that each level has 
Table 13. Compositions of ODS ferritic/martensitic alloys.

\begin{tabular}{|l|l|l|l|l|l|l|l|l|}
\hline ID & $\mathrm{Cr}$ & $\mathrm{Ti}$ & $\mathrm{Mo}$ & $\mathrm{W}$ & $\mathrm{Y}_{2} \mathrm{O}_{3}$ & $\mathrm{C}$ & Other & Ref \\
\hline DT2203Y01 & 13 & 1.5 & 2.2 & - & 1.0 & - & - & {$[4]$} \\
\hline DT3503Y005 & 13 & 1.5 & 3.5 & - & 0.5 & - & - & {$[4]$} \\
\hline K2-7 & 13 & $1.1-2.2$ & $.2-1.4$ &,- 2 & 0.4 & $.1-.2$ & - & {$[20]$} \\
\hline MA957 & 14 & 1 & 0.3 & - & 0.25 & - & - & {$[8]$} \\
\hline Fe-14Cr & 14 & 1 & - & 0.5 & 0.25 & - & - & {$[17]$} \\
\hline Fe-9Cr & 9 & 1 & - & 0.5 & 0.25 & 0.036 & - & {$[17]$} \\
\hline Fe-13.5CrW & 13.5 & 0.5 & - & 2 & 0.25 & - & - & {$[11]$} \\
\hline M1-3 & 9 & 0.2 & - & 2 & 0.3 & .012 & - & {$[29]$} \\
\hline F1-4 & 11.8 & 0.2 & - & 2 & 0.2 & - & - & {$[30]$} \\
\hline LAFY & 8.8 & & - & 2 & 0.4 & .011 & $0.3 \mathrm{~V}, 0.01 \mathrm{Ta}$ & {$[19]$} \\
\hline $\begin{array}{l}\text { ODS- } \\
\text { EUROFER }\end{array}$ & 8.9 & - & - & 1.1 & $0.3-.5$ & .12 & $0.2 \mathrm{~V}, 0.1 \mathrm{Ta}$ & {$[18]$} \\
& & & & & & & & \\
\hline
\end{tabular}

distinct differences. At high levels, and probably requiring high levels of Mo as well, intermetallic phase formation is encouraged producing both chi and Laves, in the DT alloys for example. ${ }^{21,41}$ Intermediate levels, on the order of $1.0 \%$ appear to produce stable $\mathrm{Y}_{2} \mathrm{O}_{3}$ dispersoids, provided $\mathrm{Al}_{2} \mathrm{O}_{3}$ or $\mathrm{C}$ is not present. Low levels, on the order of 0.2 apparently allow excess oxygen to be present enabling the formation of $\mathrm{Y}_{2} \mathrm{O}_{3}-\mathrm{TiO}_{2}$ dispersoid on a very fine scale during heat treatment. Finally, no Ti additions leave excess oxygen levels introduced by mechanical alloying in solution, to react with other oxide forming element that are present. It is not yet clear which levels are superior.

ODS alloys provide materials that are intended to operate at high temperatures. As a result, it can be anticipated that several issues regarding irradiation effects can be avoided. For example, ODS alloys, like their ferritic/martensitic steel counterparts, show irradiation embrittlement. The embrittlement is apparent following irradiation at temperatures of $\sim 500^{\circ} \mathrm{C}$ and below. Reducing $\mathrm{Cr}$ contents below $9 \%$ and controlling alloying to match low activation fusion alloy development can be expected to mitigate embrittlement. However, it must be emphasized that for irradiation temperatures of $\sim 550^{\circ} \mathrm{C}$ and above, effects of irradiation can be expected to be small because point defect populations will be controlled by thermal vacancy concentrations. Therefore, the need for extensive irradiation testing can be significantly reduced, and many properties can be successfully predicted based on behavior out-of-reactor.

It is possible that other alloys can be considered within the definition of ODS ferritic steels. An alternate dispersoid may be practical. Inevitably, key criteria involve thermal stability, both with regard to coarsening and transformation to other less-stable phases. Coarsening is generally controlled by diffusion of precipitate species left in solid solution. In ODS alloys, this is mitigated by removing oxygen from solid solution, for which $\mathrm{Y}$ and Ti are very effective additives. A good measure of effectiveness is the heat of formation required to form the oxide. Therefore, a search is required for oxides with heats of formation higher that that for $\mathrm{Y}_{2} \mathrm{O}_{3}$ if a better dispersoid can be found. 
It is important to note that little commercial capability now exists for ODS alloy production, both with regard to making the alloy and producing components in useful configurations. By their very nature ODS alloys are very strong with limited ductility, so fabricability is of concern. Tubing has been made but special vendor education was required. ${ }^{29}$ Any program intending to develop ODS technology must include efforts regarding production and fabrication.

In conclusion, oxide-dispersion-strengthened ferritic/martensitic steels appear to be excellent candidates for advanced reactor systems. Operating limits on the order of 750 to $800^{\circ} \mathrm{C}$ may be possible. Development of irradiation effects databases is not so important because they will operate above the temperature regime where radiation-induced point defects control behavior. However, a very limited commercial capability exists for these materials and any alloy development program must include production and fabrication as key elements.

\section{Mechanical Behavior and Irradiation-Assisted Stress Corrosion Cracking}

\section{Radiation-Induced Hardening}

Hardening in proton-irradiated samples was measured by Vickers indentation (MICROMET II) with a load of $25 \mathrm{~g}$. This low load was used to confine the plastic zone ahead of the indenter tip to a depth within the proton range $(\sim 40 \mu \mathrm{m})$ to ensure that non-irradiated material is not being sampled. Samples for hardness testing were mechanically wet-polished using $\mathrm{SiC}$ paper (grit $300-4000$ ) and then electropolished for $3 \mathrm{~min}$ in a $60 \%$ phosphoric acid - 40\% sulfuric acid solution. A total of 20 hardness indents were made for each irradiation condition.

The measured hardness of 316LSS, 316SS+Pt and 316SS+Hf alloys before and after proton irradiation to 2.5 and $5.0 \mathrm{dpa}$ at $400^{\circ} \mathrm{C}$ are listed in Table 3 . The hardness value of the irradiated condition was subtracted from that of the non-irradiated condition to arrive at a hardness increase due to irradiation. The yield strength increase due to irradiation was also estimated using the following empirical relation ${ }^{47}$ :

$$
\Delta \sigma=3.55 \Delta \mathrm{H}_{\mathrm{V}}
$$

where $\Delta \sigma$ is the increment in yield strength (MPa), and $\Delta \mathrm{H}_{\mathrm{V}}$ is the increment in hardness $\left(\mathrm{kg} / \mathrm{mm}^{2}\right)$. For the 316LSS alloy, the hardness increase at $2.5 \mathrm{dpa}$ was determined to be 136 $\mathrm{kg} / \mathrm{mm}^{2}$, corresponding to an estimated increase in the yield strength of $483 \mathrm{MPa}$. Interestingly, $316 \mathrm{SS}+\mathrm{Hf}$ alloy exhibits a significantly smaller increase in hardness than the 316SS alloy at 2.5 dpa. Further hardening is observed in both the 316SS and the 316SS+Pt alloy with an increase in the dose. For the 316LSS alloy, the hardness increase at 5 dpa was determined to be 179 $\mathrm{kg} / \mathrm{mm}^{2}$, corresponding to an estimated increase in the yield strength of $635 \mathrm{MPa}$. Conversely, the $316 \mathrm{SS}+\mathrm{Hf}$ alloy exhibits a decrease in hardness with an increase of the dose.

The yield strength increase derived from hardness measurements was also compared with the yield strength increase determined from microstructural characterization using the dispersedbarrier hardening model $^{48}$ :

$$
\Delta \sigma=3.06 \alpha \mu b(\mathrm{Nd})^{0.5}
$$


where $\Delta \sigma$ is the increment in yield strength (MPa), $\alpha$ is the strength of the barrier, $\mu$ is the shear modulus (76 GPa), $b$ is the Burgers vector $(0.255 \mathrm{~nm}), \mathrm{N}$ is the barrier density and $\mathrm{d}$ is the size of the barrier. When both voids and faulted loops are present in the microstructure, the yield strength change is calculated using the following relation:

$$
\Delta \sigma=\left[\left(\Delta \sigma_{\text {Loops }}\right)^{2}+\left(\Delta \sigma_{\text {Voids }}\right)^{2}\right]^{0.5}
$$

where $\alpha$ is 1.0 for voids and 0.4 for faulted loops.

As shown in Table 14, the calculated yield strength changes from measured microstructure using the dispersed-barrier hardening model are in poor agreement with the calculated yield strength changes from measured hardness. As a matter of fact, using the dispersed-barrier hardening model leads to a significant underestimation of the radiation-induced yield change. This result is surprising since a good agreement between calculated yield strength change from measured microstructure using this model and calculated yield strength change from measured hardness was previously observed by Was et al. ${ }^{3}$ on commercial grade $316 \mathrm{SS}$ proton irradiated to similar doses and characterized following the same procedure.

Several authors ${ }^{6,49-51}$ have considered the source-hardening model as an alternative model to the dispersed hardening one to explain radiation-induced hardening. In the source-hardening model, clusters of small defects (self-interstitial atoms) that cannot be resolved by TEM are believed to decorate the grown-in dislocations so that they cannot act as dislocation sources. Trinkaus et al. ${ }^{50}$ suggested that this effect may arise from either (i) migration and enhanced agglomeration of single self-interstitial atoms (SIA) in the form of loops in the strain field of the dislocation or (ii) glide and trapping of SIA loops (directly produced in the cascades) in the strain field of the dislocation. This is an important consideration for alloys doped with oversize solutes, where the formation of vacancy-solute clusters is the intended result.

In light of the source-hardening model, molecular dynamics simulation of the effect of oversized solute addition on displacement cascades may be useful in order to explain the beneficial effect of Hf addition on decreasing the degree of radiation-induced hardening.

Table 14. Results of microhardness measurements of 316SS, 316SS+Pt and 316SS+Hf alloys irradiated with $3.2 \mathrm{MeV}$ protons to $2.5 \mathrm{dpa}$ and 5.0 at $400^{\circ} \mathrm{C}$.

\begin{tabular}{lcccccc}
\hline \multicolumn{1}{c}{ Alloy } & $\begin{array}{c}\text { Dose } \\
(\mathrm{dpa})\end{array}$ & $\begin{array}{c}\mathrm{H}_{\mathrm{v}} \text { unirrad. } \\
\left(\mathrm{kg} / \mathrm{mm}^{2}\right)\end{array}$ & $\begin{array}{c}\mathrm{H}_{\mathrm{v}} \text { irrad. } \\
\left(\mathrm{kg} / \mathrm{mm}^{2}\right)\end{array}$ & $\begin{array}{c}\Delta \mathrm{H}_{\mathrm{v}} \\
\left(\mathrm{kg} / \mathrm{mm}^{2}\right)\end{array}$ & $\begin{array}{c}\Delta \sigma \text { from } \\
\text { hardness } \\
(\mathrm{MPa})\end{array}$ & $\begin{array}{c}\Delta \sigma \text { from } \\
\text { microstructure } \\
(\mathrm{MPa})\end{array}$ \\
\hline 316SS & 2.5 & 167 & 303 & 136 & 483 & 144 \\
316SS+Pt & 2.5 & 173 & 258 & 85 & 302 & 226 \\
316SS+Hf & 2.5 & 189 & 288 & 99 & 351 & 150 \\
& & & & & & \\
316SS & 5.0 & 167 & 346 & 179 & 635 & 179 \\
316SS+Pt & 5.0 & 173 & 285 & 113 & 401 & No data \\
316SS+Hf & 5.0 & 189 & 252 & 63 & 224 & 250 \\
\hline
\end{tabular}


Irradiation-Assisted Stress Corrosion Cracking

The results of the CERT tests performed in normal BWR water chemistry at $288^{\circ} \mathrm{C}$ on the 316LSS alloy, the 316SS+Pt alloy and the 316SS+Hf alloy, proton irradiated to 2.5 and 5.0 dpa, as well as on a non-irradiated 316SS alloy are summarized in Table 15. The strain-to-failure, failure mode, number of intergranular surface cracks, total crack length in the irradiated region and \% IG on the fracture surface were tabulated for each sample. Figure 69 shows the stressstrain curves for the samples irradiated to $2.5 \mathrm{dpa}$. The non-irradiated 316L base alloy attained the highest value of strain, followed by the irradiated $316+\mathrm{Hf}$ alloy, the irradiated base 316 alloy and finally, the irradiated $316+\mathrm{Pt}$ alloy. Figure 70 contains low-magnification images of the irradiated surfaces of the samples after removal from the SCC test and Figures 71 and 72 show the fracture surfaces of the samples after the test.

As expected, no environment-assisted cracking was observed for the non-irradiated 316LSS. This alloy failed at the largest strain in a fully ductile manner by microvoid coalescence after a total strain of $36 \%$.

In contrast, the $316 \mathrm{LSS}$ alloy irradiated to $2.5 \mathrm{dpa}$ was found to be extremely sensitive to postirradiation IGSCC. This alloy exhibited a loss of ductility of $12 \%$ in comparison to the 316LSS unpredicted alloy. Figures 71 and 72 show the fracture surfaces at low and high magnification, respectively. Note that there was significant IG cracking (43\%) in the irradiated region, i.e. in the first $40 \mu \mathrm{m}$, and then transgranular ductile failure below the irradiated depth for the irradiated 316LSS.

Table 15. Summary of CERT tests performed in simulated BWR normal water chemistry (2 ppm $\mathrm{O}_{2}$ and 0.2 凶S/cm) at $288^{\circ} \mathrm{C}$ on non-irradiated 316 alloy and on $316 \mathrm{SS}, 316 \mathrm{SS}+\mathrm{Pt}$ and $316 \mathrm{SS}+\mathrm{Hf}$ alloys irradiated with $3.2 \mathrm{MeV}$ protons to 2.5 and $5 \mathrm{dpa}$ at $400^{\circ} \mathrm{C}$.

\begin{tabular}{|l|c|c|c|c|c|c|c|c|c|}
\hline Alloy & $\begin{array}{c}\text { Dose } \\
(\mathrm{dpa})\end{array}$ & $\begin{array}{c}\text { Strain } \\
\text { at } \\
\text { failure } \\
(\%)\end{array}$ & $\begin{array}{c}\text { Failure } \\
\text { location }\end{array}$ & $\begin{array}{c}\text { Failure } \\
\text { mode }\end{array}$ & $\begin{array}{c}\# \\
\text { Cracks }\end{array}$ & $\begin{array}{c}\text { Total } \\
\text { Crack } \\
\text { length } \\
(\mathrm{mm})\end{array}$ & $\begin{array}{c}\text { Crack } \\
\text { length/strain } \\
(? \mathrm{~m} / \% \text { strain })\end{array}$ & $\begin{array}{c}\text { \%IG on } \\
\text { fracture } \\
\text { surface }\end{array}$ & $\begin{array}{c}\% \text { IG/ } \\
\% \text { strain }\end{array}$ \\
\hline $316 \mathrm{SS}$ & - & 36 & - & ductile & 0 & 0 & 0 & 0 & 0 \\
\hline $316 \mathrm{SS}$ & 2.5 & 24 & $\begin{array}{c}\text { irrad. } \\
\text { region }\end{array}$ & ductile & 11 & 10.1 & 421 & 43 & 1.8 \\
\hline $316 \mathrm{SS}+\mathrm{Pt}$ & 2.5 & 19 & $\begin{array}{c}\text { irrad. } \\
\text { region }\end{array}$ & IG+TG & 4 & 6.1 & 321 & 34 & 1.8 \\
\hline $316 \mathrm{SS}+\mathrm{Hf}$ & 2.5 & 32 & $\begin{array}{c}\text { irrad. } \\
\text { region }\end{array}$ & IG+TG & 2 & 1.3 & 41 & 0 & 0 \\
\hline $316 \mathrm{SS}$ & 5.0 & 17 & $\begin{array}{c}\text { irrad. } \\
\text { region }\end{array}$ & IG+TG & 24 & 22.6 & 1329 & 48 & 2.8 \\
\hline $316 \mathrm{SS}+\mathrm{Pt}$ & 5.0 & 27 & $\begin{array}{c}\text { irrad. } \\
\text { region }\end{array}$ & IG+TG & 19 & 11.9 & 441 & 40 & 1.5 \\
\hline $316 \mathrm{SS}+\mathrm{Hf}$ & 5.0 & 32 & $\begin{array}{c}\text { irrad. } \\
\text { region }\end{array}$ & ductile & 3 & 1.6 & 50 & 0 & 0 \\
\hline
\end{tabular}




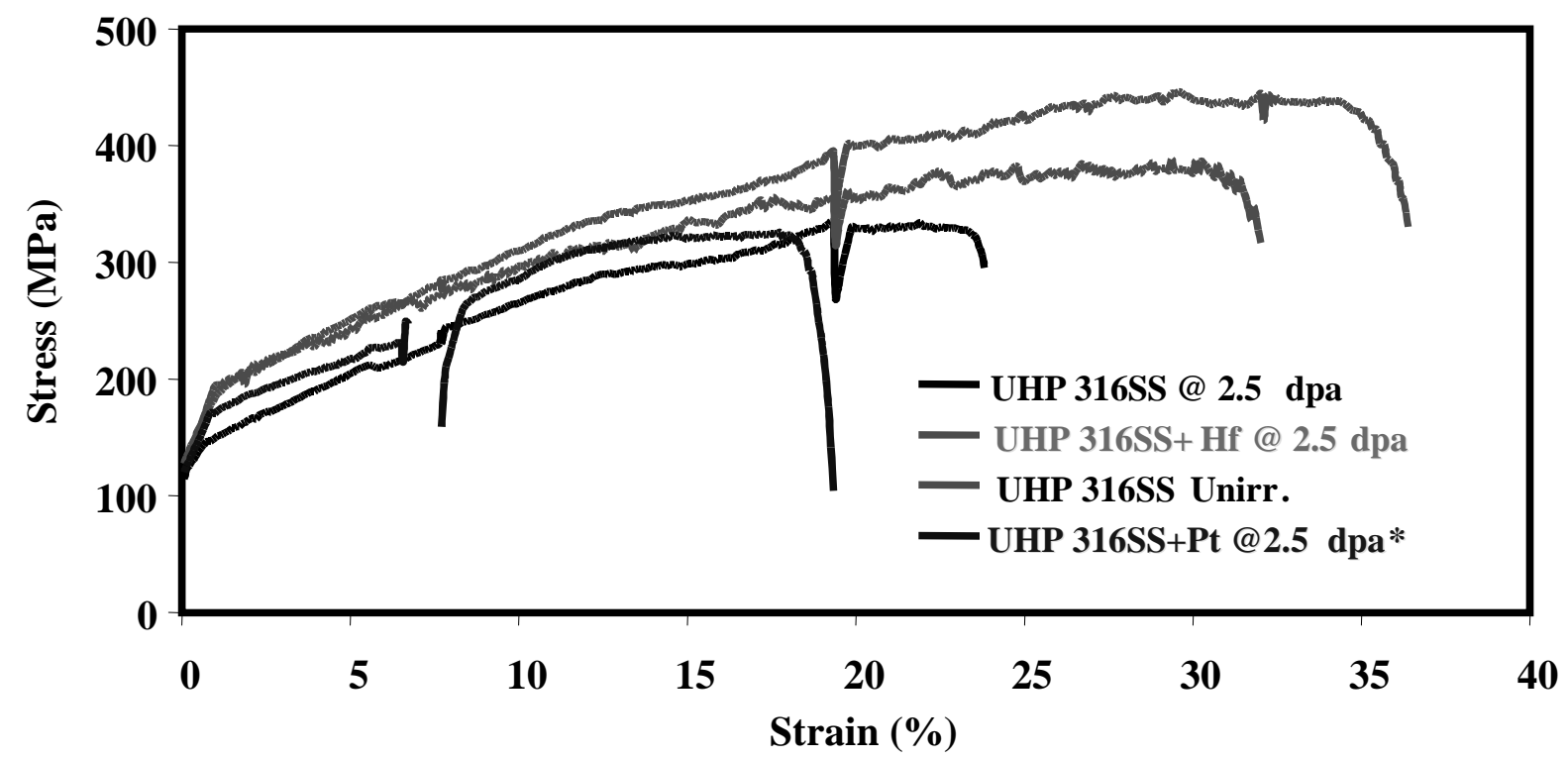

Figure 69. Stress-strain curves from constant-extension-rate tests of non-irradiated 316SS and of $316 \mathrm{SS}, 316 \mathrm{SS}+\mathrm{Pt}$ and $316 \mathrm{SS}+\mathrm{Hf}$ irradiated with $3.2 \mathrm{MeV}$ protons to $2.5 \mathrm{dpa}$ at $400^{\circ} \mathrm{C}$. Samples were strained at $3 \times 10^{-7} \mathrm{~s}^{-1}$ in $288^{\circ} \mathrm{C}$ normal water chemistry containing $2 \mathrm{ppm} \mathrm{O}_{2}$ and conductivity of $0.2 \otimes \mathrm{S} / \mathrm{cm}$.

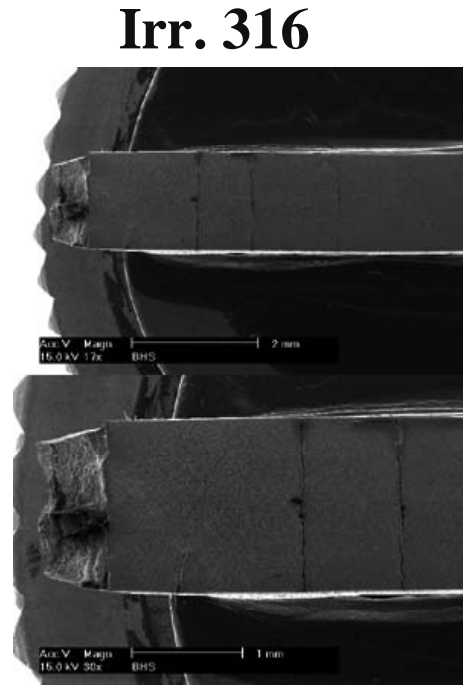

Irr. 316

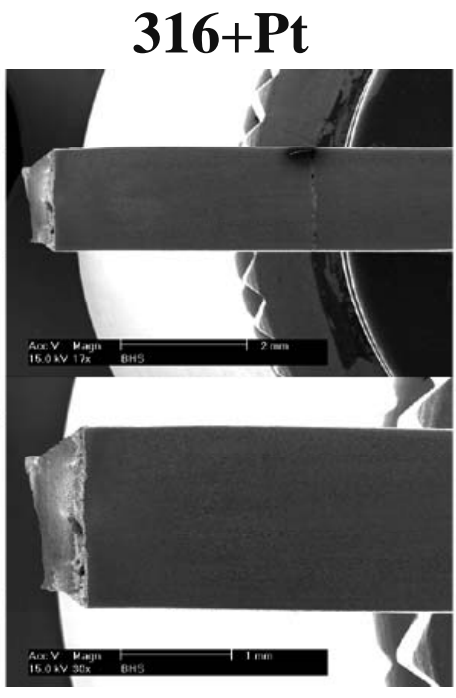

$316+\mathbf{P t}$

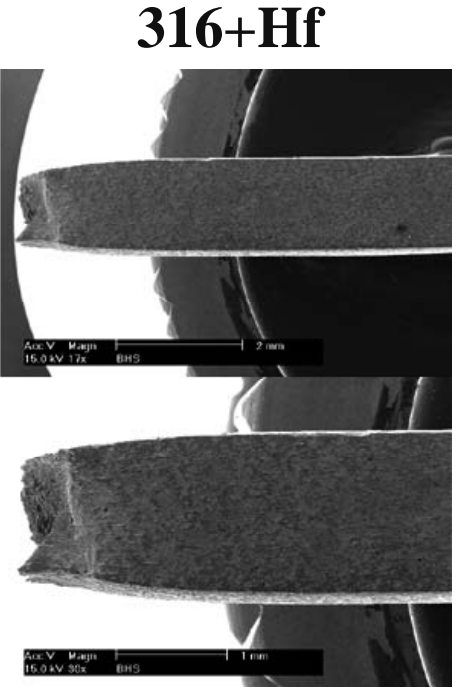

316+Hf

Figure 70. Low magnification photos of the irradiated surfaces of 316SS, 316SS+Pt and $316 \mathrm{SS}+\mathrm{Hf}$ irradiated with $3.2 \mathrm{MeV}$ protons to $2.5 \mathrm{dpa}$ at $400^{\circ} \mathrm{C}$ following CERT tests in $288^{\circ} \mathrm{C}$ normal water containing $2 \mathrm{ppm} \mathrm{O}_{2}$ and conductivity of $0.2 \mathrm{mS} / \mathrm{cm}$ and strained at $3 \times 10^{-7} \mathrm{~s}^{-1}$. 
non-irradiated 316
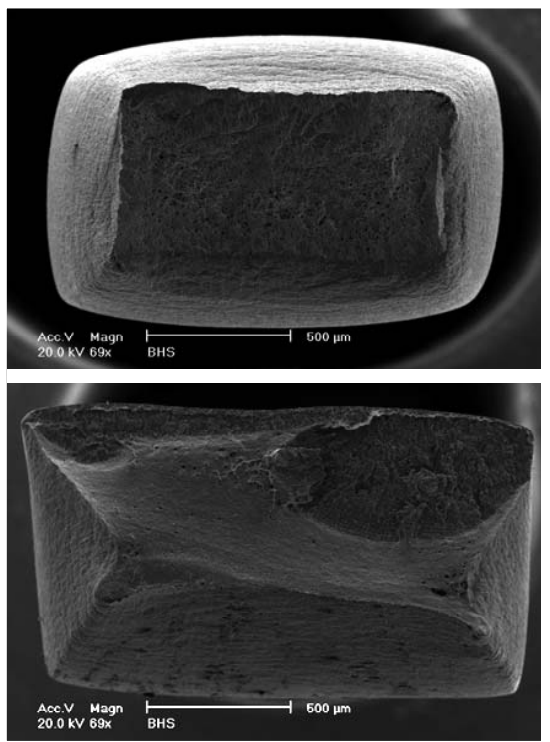

irradiated 316+Pt irradiated 316
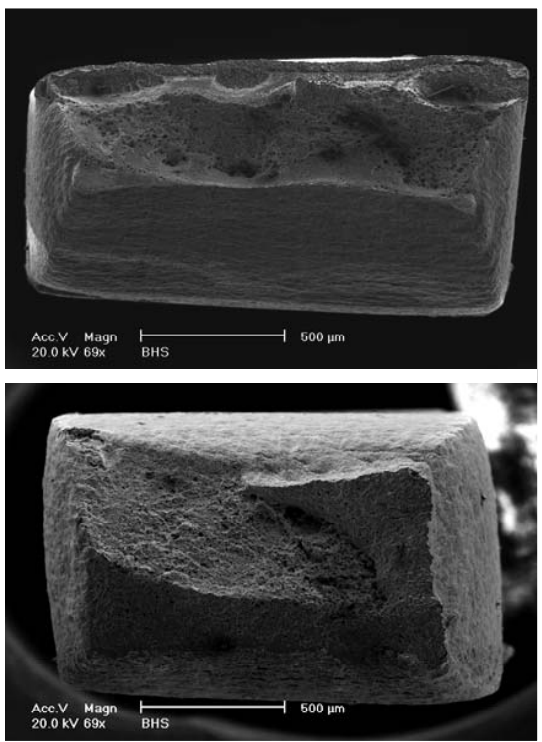

irradiated $316+\mathrm{Hf}$

Figure 71. Fracture surfaces of non-irradiated 316SS, and 316SS, 316SS+Pt and 316SS+Hf irradiated with $3.2 \mathrm{MeV}$ protons to $2.5 \mathrm{dpa}$ at $400^{\circ} \mathrm{C}$ following CERT tests in $288^{\circ} \mathrm{C}$ normal water containing $2 \mathrm{ppm} \mathrm{O}_{2}$ and conductivity of $0.2 \mathrm{mS} / \mathrm{cm}$ and strained at $3 \times 10^{-7} \mathrm{~s}^{-1}$.

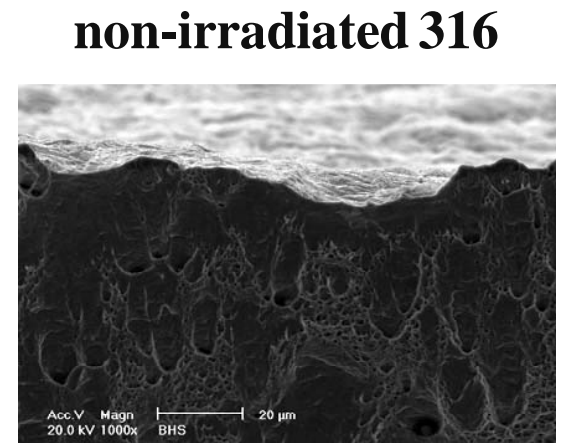

irradiated 316

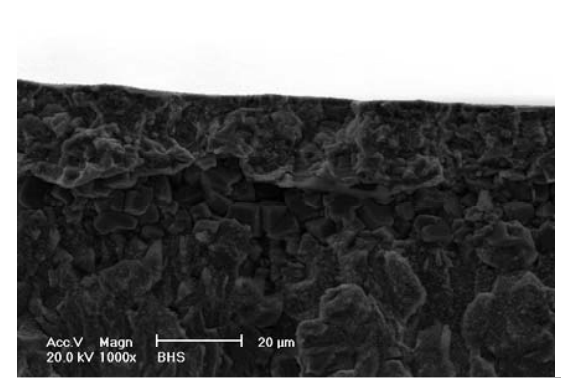

irradiated $316+\mathbf{P t}$
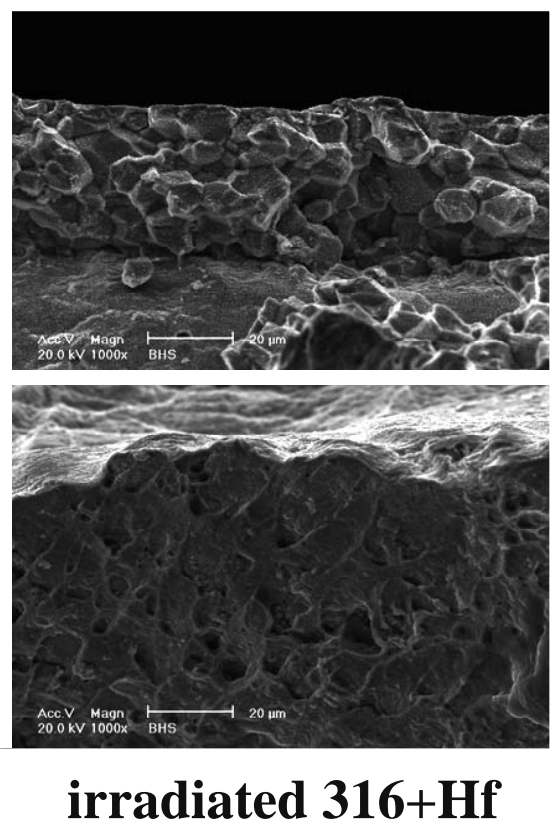

Figure 72. High-magnification fracture surfaces of non-irradiated 316SS, and 316SS, 316SS+Pt and $316 \mathrm{SS}+\mathrm{Hf}$ irradiated with $3.2 \mathrm{MeV}$ protons to $2.5 \mathrm{dpa}$ at $400^{\circ} \mathrm{C}$ following CERT tests in $288^{\circ} \mathrm{C}$ water containing $2 \mathrm{ppm} \mathrm{O}_{2}$, a conductivity of $0.2 \mathrm{mS} / \mathrm{cm}$ and strained at $3 \times 10^{-7} \mathrm{~s}^{-1}$. 
Figures 73-75 show the irradiated surfaces and the fracture surfaces of the three alloys CERT tested in the 5.0 dpa condition. IGSCC in 316LSS after 5.0 dpa was significantly worse as indicated by the strain to failure (decreased from $24 \%$ to $17 \%$ ), the number of IG cracks in the irradiated region (increased from 11 to 24 ) and the $\%$ IG on the fracture surface (increased from $43 \%$ to $48 \%$ ).

The 316SS+Pt alloy also exhibited significant IG cracking after both $2.5 \mathrm{dpa}$ and $5.0 \mathrm{dpa}$, but not quite as severe as the base 316 alloy. Between 2.5 and $5.0 \mathrm{dpa}$, the total number of IG cracks in the irradiated region increased from 4 to 19 and the $\%$ IG increased from $34 \%$ to $40 \%$. However, the strain to failure actually increased from $19 \%$ to $27 \%$, which was unexpected, but not unusual. (This point will be discussed later).

The 316SS+Hf alloy was remarkably resistant to IGSCC at both doses. At $2.5 \mathrm{dpa}$, there only two very small surface cracks and the fracture surface of the sample was completely ductile with no evidence of IG cracking, as shown in Figure 76. After 5.0 dpa, only 3 short cracks were observed on the irradiated surface and neither of them led to failure as the fracture occurred by ductile rupture due to microvoid coalescence. The strains to failure were also the largest at $32 \%$ for both doses, and close to the value of $36 \%$ for the non-irradiated 316 alloy.

The proton-irradiated samples are unique in their geometry since only 40 冈m of the 1500 冈m sample thickness is irradiated. Cracks will form on the irradiated surface to the depth of the irradiation (40 凶m) and then progress across the surface of the sample. Consequently, cracks can run completely across the sample and not result in failure of the entire sample. Sample failure occurs after an IG crack nucleates a TG crack that propagates either through the thickness of the sample or to a depth where ductile overload results in complete failure. This process can also

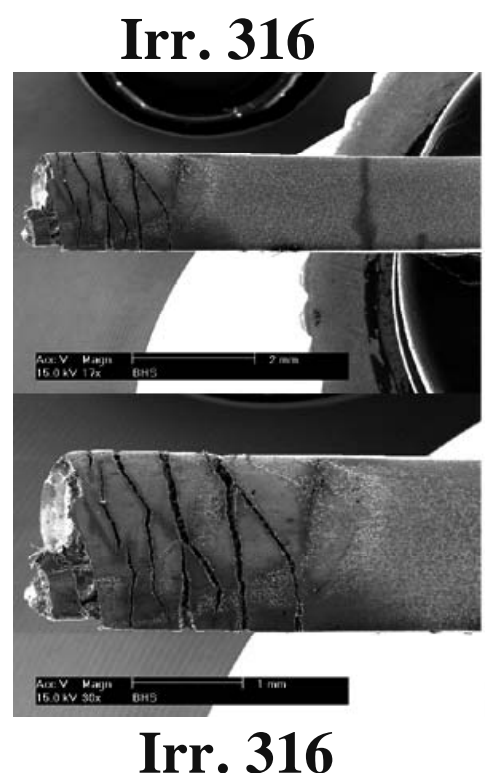

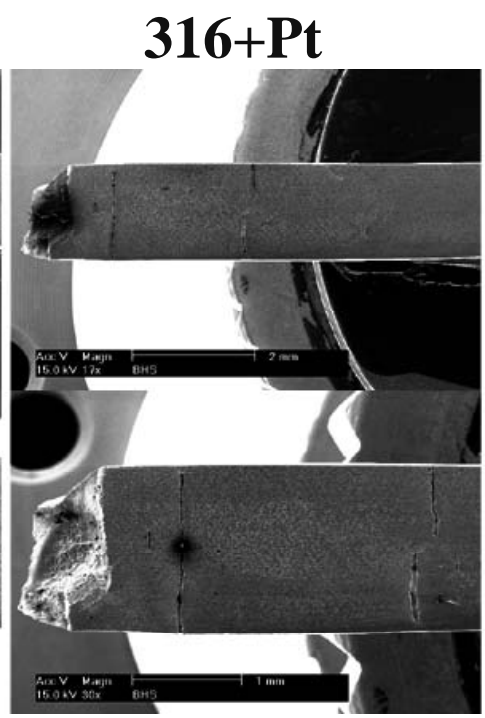

$316+P t$

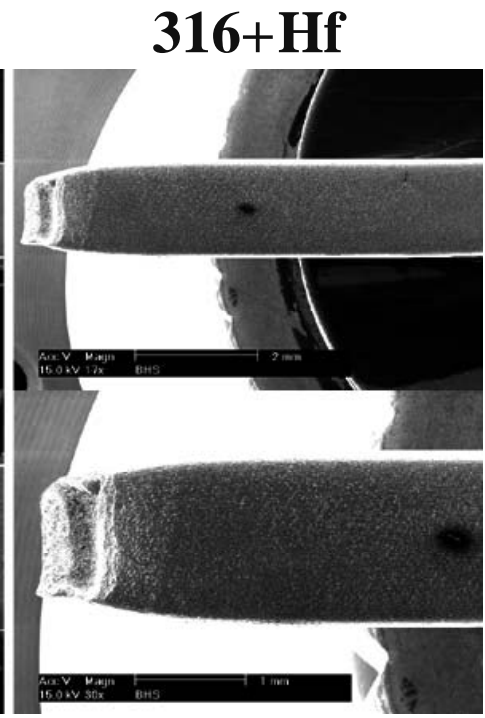

316+Hf

Figure 73. Low-magnification photos of the irradiated surfaces of $316 \mathrm{SS}, 316 \mathrm{SS}+\mathrm{Pt}$ and $316 \mathrm{SS}+\mathrm{Hf}$ irradiated with $3.2 \mathrm{MeV}$ protons to $5.0 \mathrm{dpa}$ at $400^{\circ} \mathrm{C}$ following CERT tests in $288^{\circ} \mathrm{C}$ 
water containing $2 \mathrm{ppm}_{2}$, a conductivity of 0.2 区S/cm and strained at $3 \times 10^{-7} \mathrm{~s}^{-1}$.

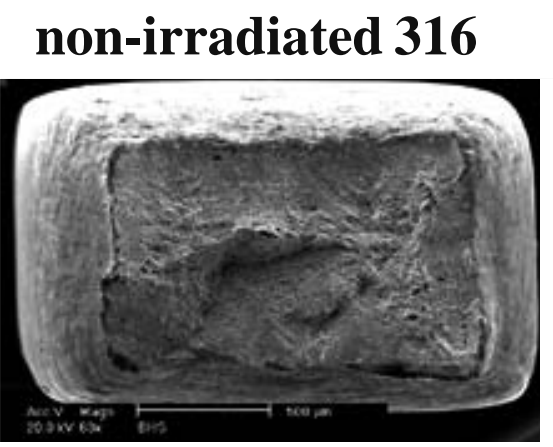
irradiated 316

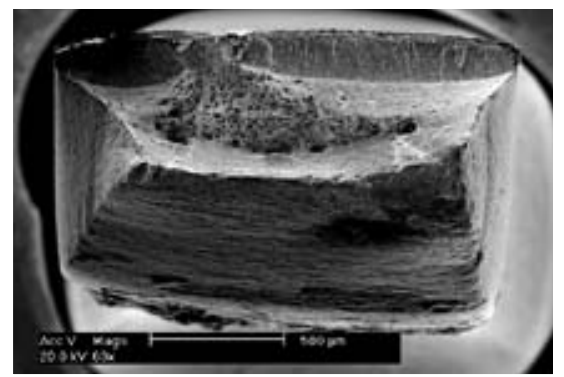

$316+\mathbf{P t}$
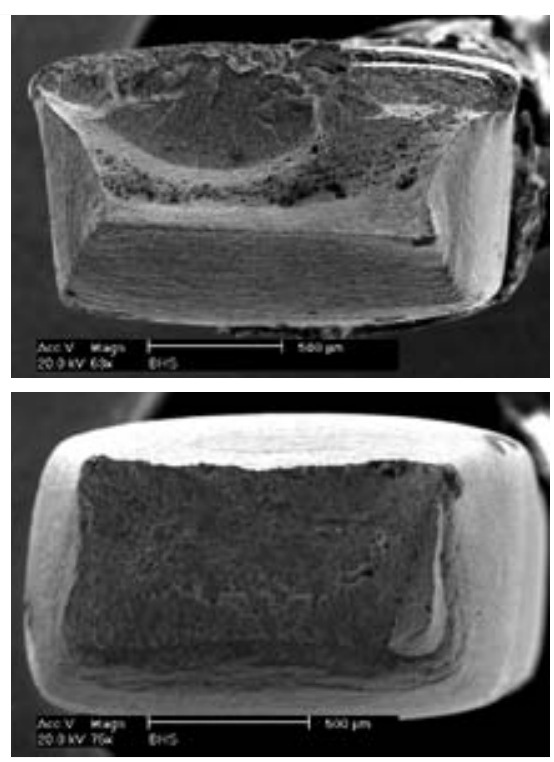

$316+$ Hf

Figure 74. Fracture surfaces of non-irradiated 316SS, and 316SS, 316SS+Pt and 316SS+Hf irradiated with $3.2 \mathrm{MeV}$ protons to $5.0 \mathrm{dpa}$ at $400^{\circ} \mathrm{C}$ following CERT tests in $288^{\circ} \mathrm{C}$ water containing $2 \mathrm{ppm} \mathrm{O}_{2}$, a conductivity of $0.2 \mathrm{mS} / \mathrm{cm}$ and strained at $3 \times 10^{-7} \mathrm{~s}^{-1}$.
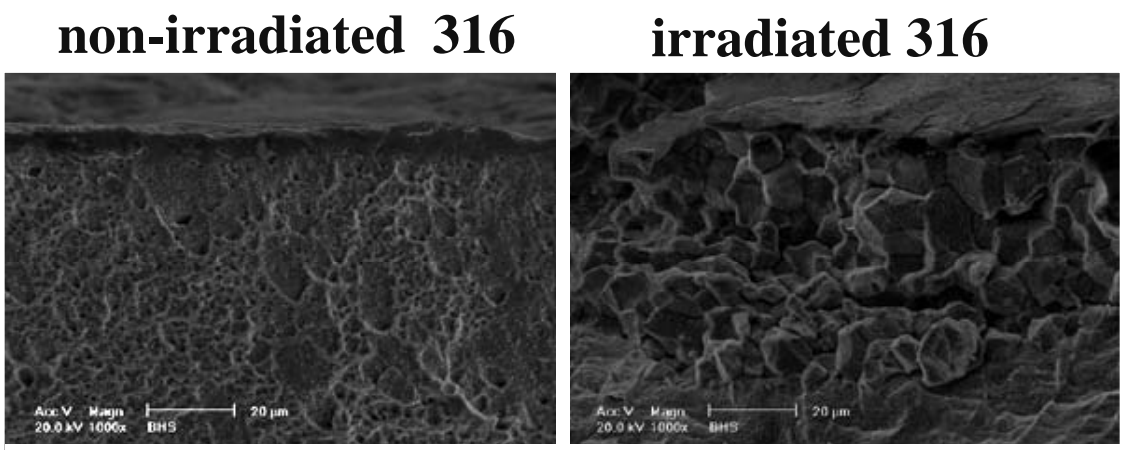

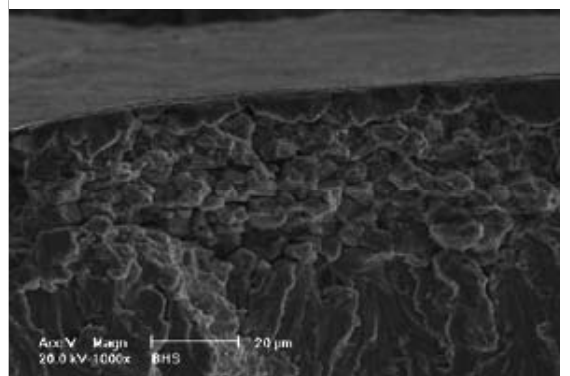

$316+\mathbf{P t}$

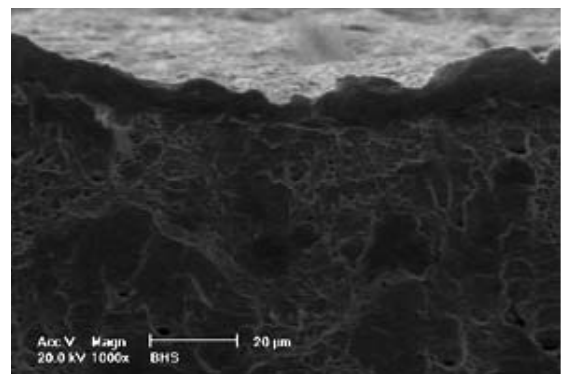

$316+$ Hf

Figure 75. Higher-magnification images of fracture surfaces for non-irradiated 316SS, and 316SS, 316SS+Pt and 316SS+Hf proton irradiated to $5.0 \mathrm{dpa}$ following CERT tests in $288^{\circ} \mathrm{C}$ 
water containing $2 \mathrm{ppm} \mathrm{O}_{2}$, a conductivity of $0.2 \mathrm{mS} / \mathrm{cm}$ and strained at $3 \times 10^{-7} \mathrm{~s}^{-1}$.

$316 S S @ 2.5 d p a$
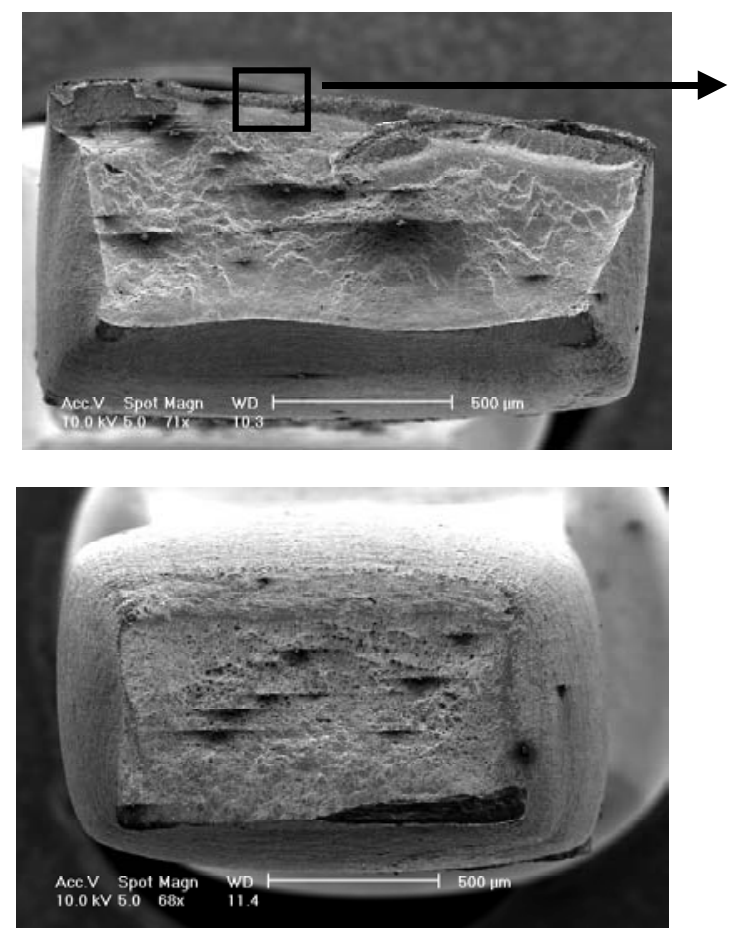

UHP 316SSUnirr.
$316 S S @ 2.5 d p a$
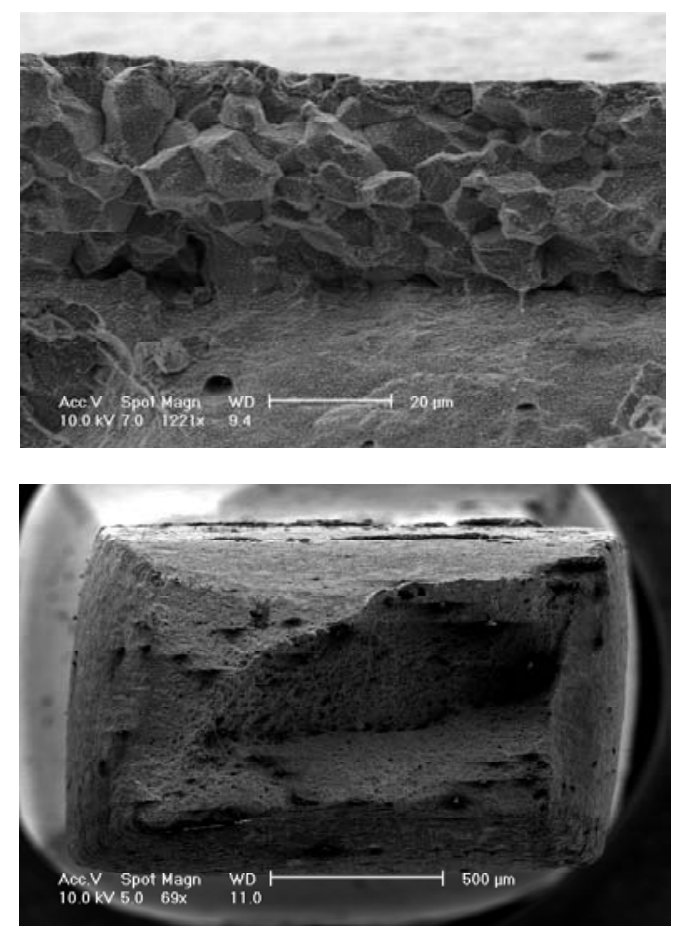

UHP 316SS+Hf mod. @ 2.5dpa

Figure 76. Comparison of fracture surfaces for non-irradiated 316SS and irradiated 316SS alloys with $3.2 \mathrm{MeV}$ protons to $2.5 \mathrm{dpa}$ at $400^{\circ} \mathrm{C}$ following CERT tests in $288^{\circ} \mathrm{C}$ normal water containing $2 \mathrm{ppm} \mathrm{O}_{2}$ and conductivity of $0.2 \mathrm{mS} / \mathrm{cm}$ and strained at $3 \times 10^{-7} \mathrm{~s}^{-1}$. Ductile transgranular failure is observed for the non-irradiated 316LSS and for the irradiated 316SS+Hf alloy in comparison to extensive IG cracking in the irradiated 316SS.

produce a "scatter" in the strain to failure since the total strain is dependent on nucleation and propagation of TG cracks below the irradiated region. Because the irradiated surface can support multiple cracks, the number and total length of these cracks (Figure 77) becomes a measure of the IASCC susceptibility, in addition to the \%IG on the fracture surface. Since samples can be strained to varying levels and the number of cracks that can be supported by the sample will increase with the amount of strain, a normalized cracking propensity was constructed by dividing the number of cracks by the $\%$ strain, and the $\%$ IG by the $\%$ strain. As shown in Table 10 , the total number of cracks, the normalized number of cracks, the \%IG and the normalized \%IG all consistently showed that the propensity for IASCC increases with dose and that $316 \mathrm{SS}+\mathrm{Hf}$ is much more resistant to cracking then 316SS+Pt with the base 316SS showing the highest susceptibility. 


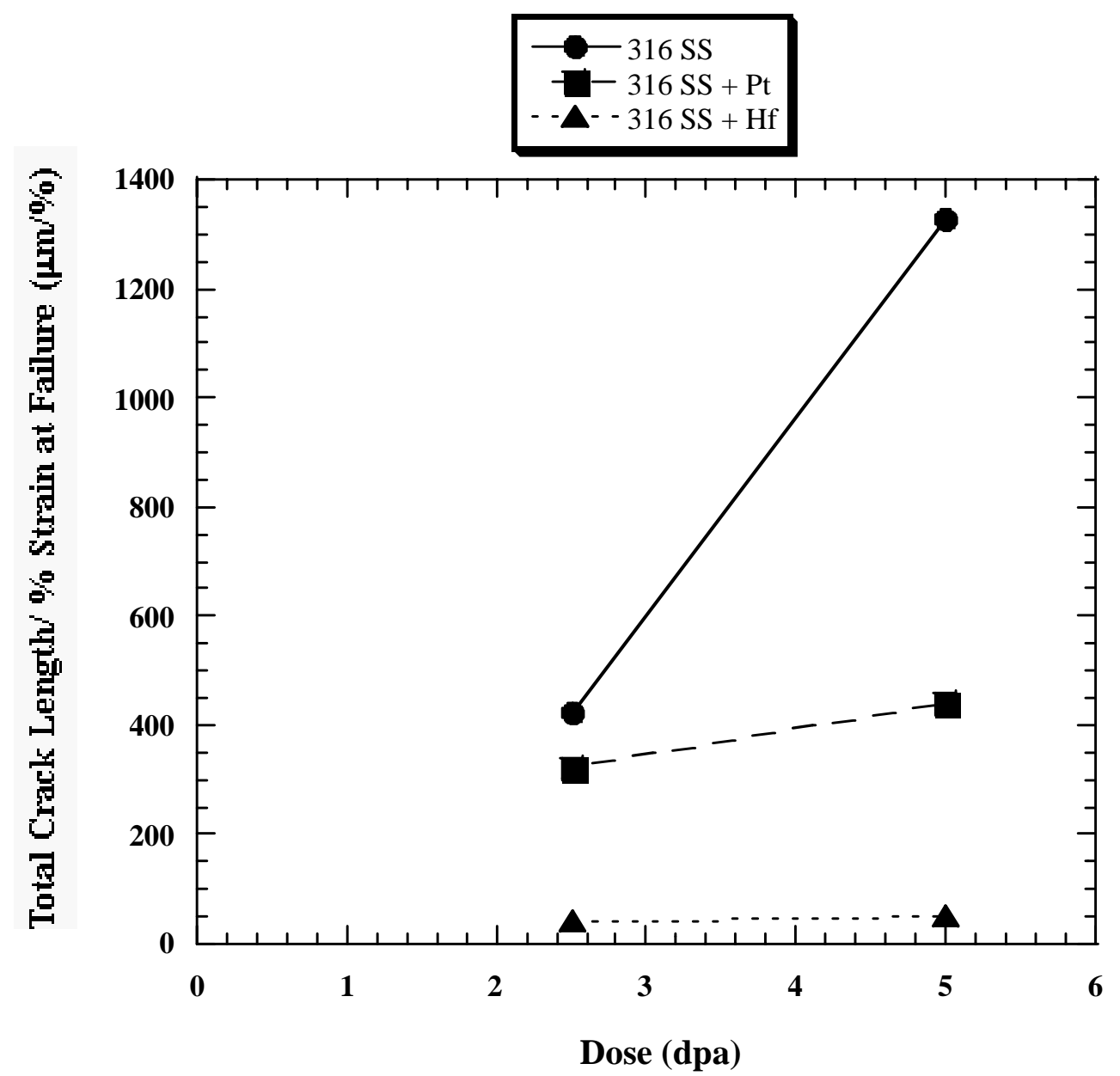

Figure 77. Normalized crack length as a function of dose for 316SS, 316SS+Pt and 316SS+Hf following CERT test in $288^{\circ} \mathrm{C} \mathrm{BWR}$ water chemistry and strained at $3 \times 10^{-7} \mathrm{~s}^{-1}$. 


\section{Crack-Growth Rate Evaluation of Stress Corrosion Cracking}

\section{Background}

This report summarizes the SCC crack-growth data on various grades of stainless steel in 288, 320 , and $340{ }^{\circ} \mathrm{C}$ pure water. The objective was to elucidate aspects of environmental cracking in hot water related to the roles of:

- yield strength, using cold work to simulate other hardening mechanisms, especially radiation hardening,

- corrosion potential,

- temperature, covering the range of temperatures relevant to boiling water reactors and pressurized water reactors,

- hydrogen fugacity and permeation,

- compositional and phase stability effects associated with different grades of stainless steel, and

- grain boundary carbides in the absence of $\mathrm{Cr}$ depletion.

Stress corrosion cracking tests are complex and require exacting control and measurement of many critical chemistry, temperature, metallurgical, electrochemical, and mechanical parameters. Continuous, high resolution monitoring of crack length is also essential, because it cannot be assumed that the cracks will grow in a stable, well-behaved fashion using simple "exposure tests." Other factors, like the need to transition from a transgranular fatigue precrack to an IGSCC crack, are widely overlooked but are very important.

The studies on the effects of yield strength, corrosion potential, hydrogen, composition and temperature were evaluated using Types 304, 304L and 316L stainless steels that were rolled by $10 \%, 20 \%$ or $50 \%$. Hydrogen permeation data were also obtained in companion measurements during some SCC tests. The effect of grain-boundary carbides was evaluated using type 304 stainless steel that was sensitized, "healed," then cold worked, which decorates the grain boundaries with $\mathrm{Cr}$ carbides but eliminates $\mathrm{Cr}$ depletion, so that the effect of carbides alone on deformation processes and SCC susceptibility can be quantified.

Understanding the effect on SCC of these factors - increased yield strength, corrosion potential, hydrogen fugacity, $\mathrm{Cr}$ depletion, grain boundary carbides, composition and phase stability variations among the $18 \mathrm{Cr}-8 \mathrm{Ni}$ family of stainless steels, and temperature - is highly relevant to both irradiated and non-irradiated stainless steels in both boiling and pressurized water reactors. The resulting perspective - that the behavior of these alloys follows a well-behaved continuum across many material, water chemistry, stress and radiation conditions - provides powerful insights, mechanistic synergy, and improved qualitative and quantitative understanding of cracking kinetics in light water reactors.

A focal point in these studies has been the role of yield strength - alone, or in combination with other factors (sensitization, grain boundary carbides without sensitization, compositional variations, etc.). With neutron irradiation, the yield strength rises rapidly as shown in Figure 4, particularly in the range of about 0.2 to $3 \mathrm{dpa}^{52,53}$ While the microstructural origins of this 
strengthening are different than that produced by cold work, SCC data show a pronounced and similar effect of both mechanisms of yield strength elevation, although clearly more data and more careful separation of the contribution of the effect of irradiation hardening are needed. Yield strength is important under unirradiated conditions, as most failures in light-water-reactor structural components show the presence of a cold worked surface layer from fabrication, machining, grinding, etc. Additionally, weld shrinkage produces deformation in the weld heataffected zone, which is equivalent to $5-15 \%$ tensile strain at room temperature. This residual deformation increases the local yield strength and is now recognized as an important contributor to SCC susceptibility in non-sensitized stainless steels. ${ }^{54-59}$

Materials and Experimental Procedures

The compositions of the Types 304, 304L and 316L stainless steels used in this program are listed in Table 16 . The materials were rolled at $+140{ }^{\circ} \mathrm{C}$ by $10 \%, 20 \%$ or $50 \%$. Equal reduction, cross-rolling passes were used for the $20 \%$ and $50 \%$ reductions in thickness (forging was used with specimens c184, c185, and c201). Since rolling at $<10 \%$ per pass produces very inhomogeneous deformation, the $10 \%$ reduction was produced in a single pass. Standard solution annealing $\left(1050{ }^{\circ} \mathrm{C}\right.$ for 30 minutes + water quench) and sensitization $\left(621{ }^{\circ} \mathrm{C}\right.$ for 24 hours) were used preceding the cold working. The "healed" specimens were solution annealed at $1050{ }^{\circ} \mathrm{C}$ for 30 minutes + water quench, sensitized at $621^{\circ} \mathrm{C}$ for 24 hours, then given a healing treatment at $950{ }^{\circ} \mathrm{C}$ for 5 hours + water quench that retained the grain boundary carbides but equilibrated the grain boundary $\mathrm{Cr}$ profile to eliminate $\mathrm{Cr}$ depletion $\left(950{ }^{\circ} \mathrm{C}\right.$ is the equilibrium temperature, where the $\mathrm{Cr}$ activity in the metal is identical to that in the $\mathrm{Cr}_{23} \mathrm{C}_{6}$ carbides).

Table 16. Compositions of Stainless Steels for Crack-Growth Tests.

\begin{tabular}{|c|c|c|c|c|c|c|c|c|c|c|}
\hline $\begin{array}{c}\text { alloy } \\
\text { (heat) }\end{array}$ & Fe & $\mathbf{C}$ & $\mathbf{N}$ & $\mathbf{P}$ & $\mathbf{S}$ & $\mathbf{C r}$ & $\mathbf{N i}$ & $\mathbf{M o}$ & $\mathbf{M n}$ & $\mathbf{S i}$ \\
\hline $\mathbf{3 0 4}$ & & & & & & & & & & \\
\hline AJ9139 & Bal. & 0.066 & 0.25 & 0.026 & 0.028 & 18.37 & 8.13 & 0.25 & 0.93 & 0.47 \\
\hline 304L & & & & & & & & & & \\
\hline Grand Gulf & bal. & 0.015 & 0.069 & 0.015 & 0.010 & 18.60 & 8.89 & 0.33 & 1.78 & 0.45 \\
\hline 316L & & & & & & & & & & \\
\hline A14128 & bal. & 0.014 & 0.085 & 0.015 & 0.001 & 17.64 & 12.40 & 2.60 & 1.78 & 0.47 \\
\hline
\end{tabular}

Crack-growth specimens were machined into $0.5 \mathrm{~T}$ compact-tension type (CT) with 5\% side grooves on each side, Figure 78. The CT specimens were instrumented with platinum current and potential probe leads for dc potential drop measurements of crack length. In this technique, current flow through the sample is reversed about once per second primarily to reduce measurement errors associated with thermocouple effects and amplifier offsets. The computer control of current reversal, data acquisition, data averaging techniques, and the relationship between measured potential and crack length have been presented previously. ${ }^{60-63}$ Depending on the test conditions, data were stored in a permanent disk file approximately once every 0.3 to $6 \mathrm{~h}$. In addition to the data record number, total elapsed and incremental time, and crack length, the system measured and stored the temperature, current corrosion potential, effluent dissolved 


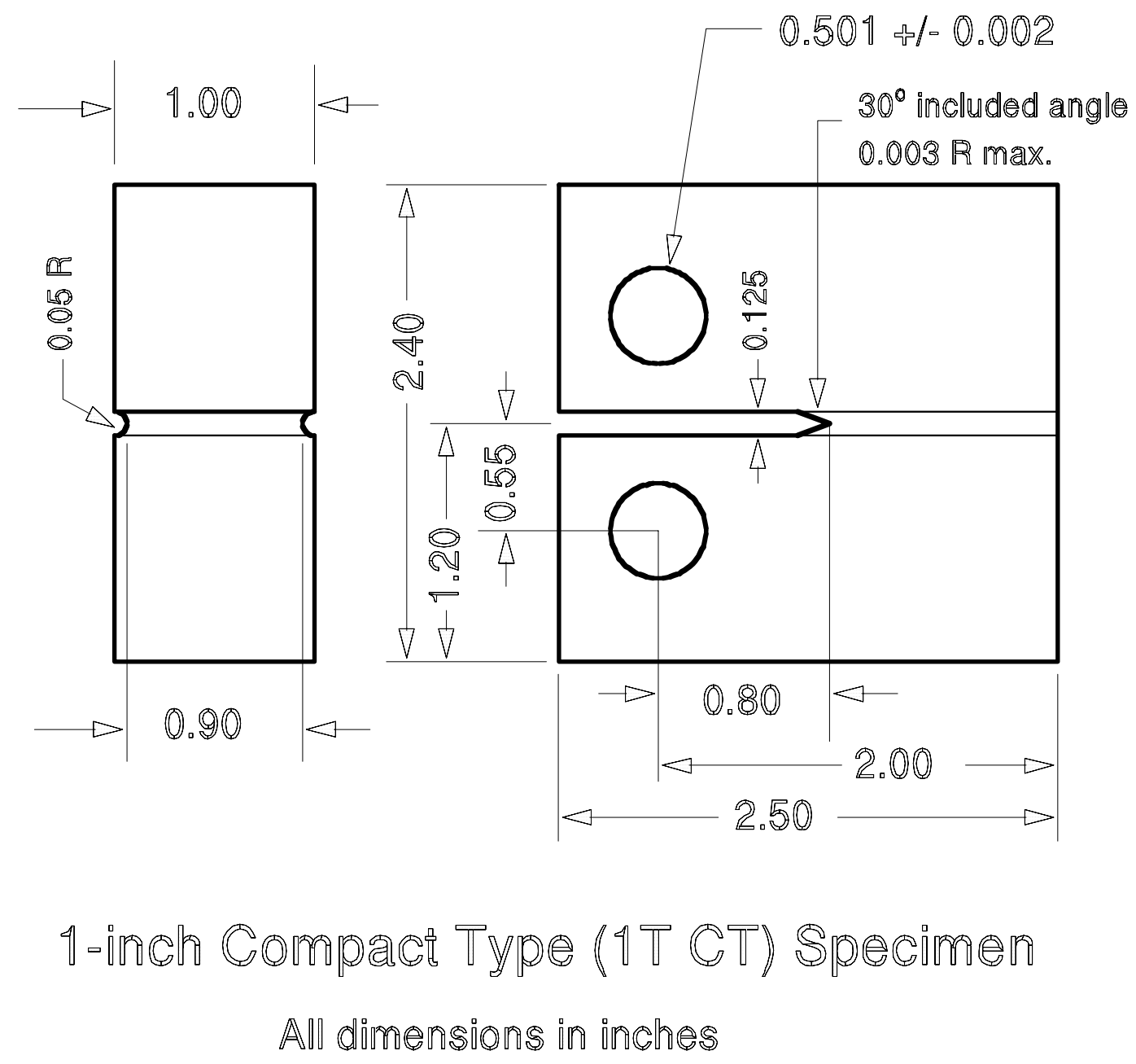

Figure 78. Schematic drawing of a 1TCT specimen in inches. 0.5TCT specimens are scaled in all dimensions by $0.5 \mathrm{X}$.

oxygen, effluent conductivity, load and time/date. Statistical information on temperature and current fluctuations are also recorded. Additionally, messages describing changes in test conditions were a permanent part of the data record.

Most CT specimens were electrically insulated from the pins using zirconia sleeves inserted into the specimen, whose loading holes were machined slightly oversized to accommodate the sleeves. The loading clevises and pull rods were electrically insulated within the autoclave using a zirconia washer to isolate the upper pull rod from the internal load frame. The lower pull rod was electrically isolated from the autoclave using an Omiseal pressure seal and from the loading actuator using an insulating washer. Since the CT specimens and loading linkage were made of very similar material, there was no consequential difference in their corrosion potential. Even if there was a difference, the corrosion potentials in pure water are localized on the specimen, so that at the crack plane of a CT specimen, very little polarization from galvanic effects would occur. However as "seen" by a reference electrode several $\mathrm{cm}$ from the specimen, any difference in corrosion potential between the specimen and electrically connected loading linkage would be 
"averaged." Therefore, the primary effect would be on measurement of potential, not on the actual potential of the specimen. Ground isolated instrumentation was used for the platinum current and potential probe attachments to the specimen.

Initial crack extension from the machined notch by 0.5 to $1.0 \mathrm{~mm}$ was typically performed in air or in the environment at $\geq 1 \mathrm{~Hz}$ at a load ratio $\left(\mathrm{K}_{\min } / \mathrm{K}_{\max }\right) \mathrm{R}=0.3$ to 0.5 and a $\mathrm{K}_{\max }$ somewhat below the test value of about $27.5 \mathrm{MPa} \sqrt{ } \mathrm{m}$. When specimens were tested in tandem, they were fatigue pre-cracked individually in air, then loaded together into the autoclave for subsequent transitioning from transgranular fatigue conditions to IGSCC.

Testing was performed using Instron Model 1362 servo-electric testing machines, Instron Model 1350 servo-hydraulic machines (equipped with a single-stage, slow-strain servovalve to ensure optimal, non-noisy response), or various models of Interactive Instruments load controllers. All systems are equipped with digital controls that provide improved machine control and full computer interface/control capabilities. The potential-drop software has been modified to interface with the digital control electronics and to automate several facets of the load and waveform setup procedure. Automatic K control was employed in most tests, with load corrections applied after very small increases in stress intensity, typically $<0.1 \%$.

Crack-growth rates can be treated as statistically significant when the crack-length increment is at least 10 times the limiting resolution of the technique, which is typically $<0.002$ to $0.005 \mathrm{~mm}$. Thus, crack-length increments are typically $\geq 0.05 \mathrm{~mm}$, although for very low-growth-rate conditions, smaller increments are occasionally used to reduce testing time from several months per datum to several weeks. The correlation coefficients from linear-regression analyses of the crack lengths vs. time data from which growth rates are calculated are typically $>0.98$.

Optimization of the reversed-DC, potential-drop technique has provided incremental cracklength resolution on a micron scale. For example, even without compensation for minor fluctuations in temperature and applied current, the typical resolution on a 1TCT specimen in $288^{\circ} \mathrm{C}$ water is about 2 to $5 \mu \mathrm{m}$-h, corresponding to $\approx 1 \mu \mathrm{m}$ resolution if data are averaged over a few hour period. Subtle changes in apparent crack length during cyclic loading can also be observed.

An analysis of the accuracy technique/was performed based on eleven Type 304L, 316L and $316 \mathrm{NG}$ SS $1 \mathrm{TCT}$ specimens tested in $288^{\circ} \mathrm{C}$ water and revealed that the crack depth determined by potential drop was, on average, $2.2 \%$ below the actual depth (with a standard deviation, $\sigma_{\mathrm{n}}$, of $5.2 \%$ ) on growth increments of 4.2 to $13.6 \mathrm{~mm}$ from an initial (as-machined) a/ $\mathrm{W}$ of 0.384 to 0.463 . The worst case error was $11 \%$ on $20 \mathrm{~mm}$ of growth; the errors for 6 of 11 specimens were $<2 \%$. Similar results have also been observed for sensitized Type 304 stainless steel and turbine steel specimens. Larger errors inherently occur when the crack fronts are less uniform, because dc potential drop is very sensitive to regions of uncracked metal in the wake of the crack.

Water chemistry control is vital given the high sensitivity of cracking in stainless steel to corrosion potential and anionic activity. Deaereated, demineralized water (Figure 79) was drawn through another demineralizer and submicron filter to ensure ultra-high purity $(\approx 0.055 \mu \mathrm{S} / \mathrm{cm})$ and then into a glass column (6.4-cm diameter by $183 \mathrm{~cm}$ long). A low-pressure pump provided 
positive pressure to the high-pressure pump, and drew water from and recirculated excess water (water that did not go into the high pressure pump) back into the glass column. The autoclave effluent was back-pressure regulated, then measured for conductivity using a Sybron Barnstead Model PM-512 and dissolved oxygen using an Orbisphere Model 2606. The oxygen concentration was controlled by bubbling gas mixtures blended by a Tylan Model RO-20-A mass flow controller. Impurities were added to the glass column using a metering pump (Fluid Metering Model RP-G50/H1 GKC) which was controlled by the Sybron Barnstead conductivity meter. Typical impurity additions were, for instance, $0.3 \mu \mathrm{S} / \mathrm{cm} \mathrm{H}_{2} \mathrm{SO}_{4}$, which corresponds to $6.79 \times 10^{-7} \mathrm{~N}$, or $32.6 \mathrm{ppb}$ sulfate, as shown in Figure 80.

Tests were performed in 4-liter stainless steel autoclaves at $288^{\circ} \mathrm{C}$ and $10.3 \mathrm{MPa}(1500 \mathrm{psi})$. The autoclave effluent water was continuously monitored for solution conductivity using a second Sybron Barnstead Model PM-512 meter and some tests, as needed, were periodically monitored for dissolved oxygen using an Orbisphere Model 2606. A zirconia-membrane reference electrode $^{64}$ was employed for continuous measurement of the corrosion potential of the CT specimen.

To help ensure state-of-the-art water chemistry, both influent and effluent water was continuously monitored. Influent water was $\approx 0.055 \mu \mathrm{S} / \mathrm{cm}$. In the absence of impurity additions, the effluent will be generally $<0.07 \mu \mathrm{S} / \mathrm{cm}$, which requires (among other things) that the autoclave flow rate be moderately high, typically $>125 \mathrm{cc} / \mathrm{min}$. Two major contributions to degraded effluent water purity may be identified as chromic acid release from the passive film on the stainless steel autoclave components and carbonic acid from thermal decomposition of organics. The effect of chromic acid on effluent conductivity was small in de-aerated environments (using nitrogen or argon - 6\% hydrogen), although in oxygenated environments its effect could be appreciable. Details of these issues have been discussed previously. ${ }^{65-66}$

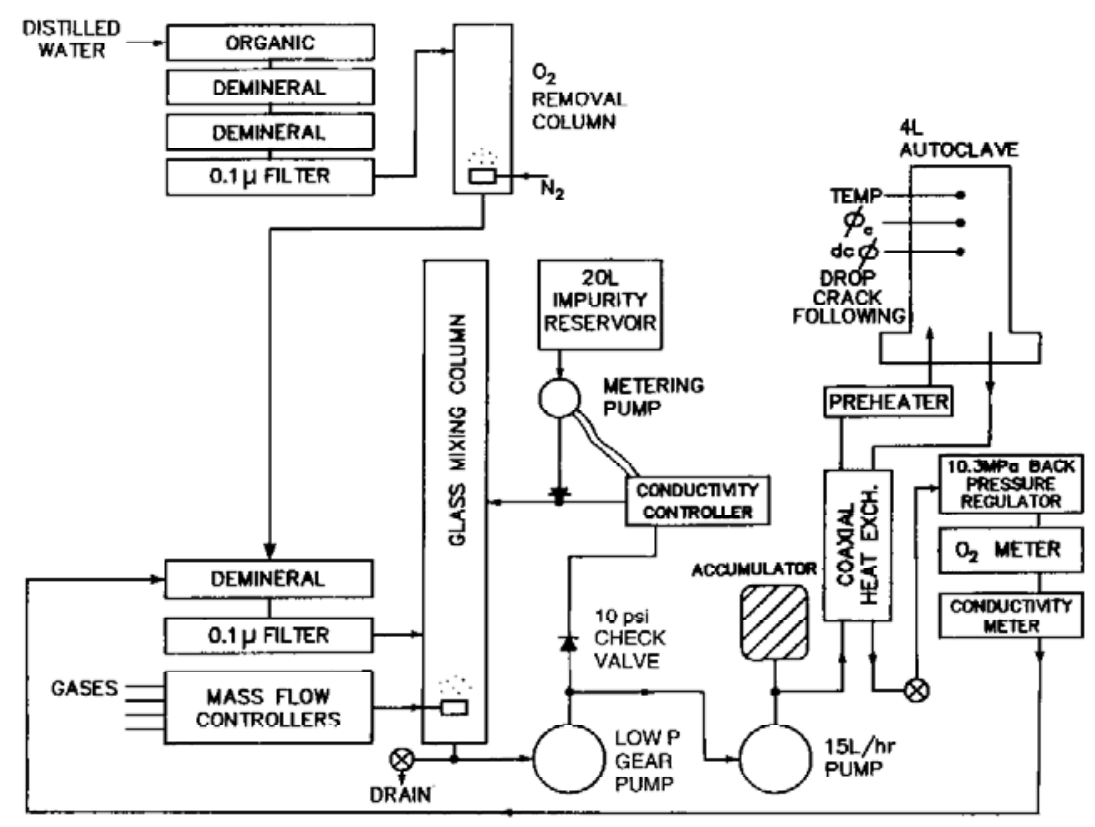

Figure 79. Water chemistry control loop. 


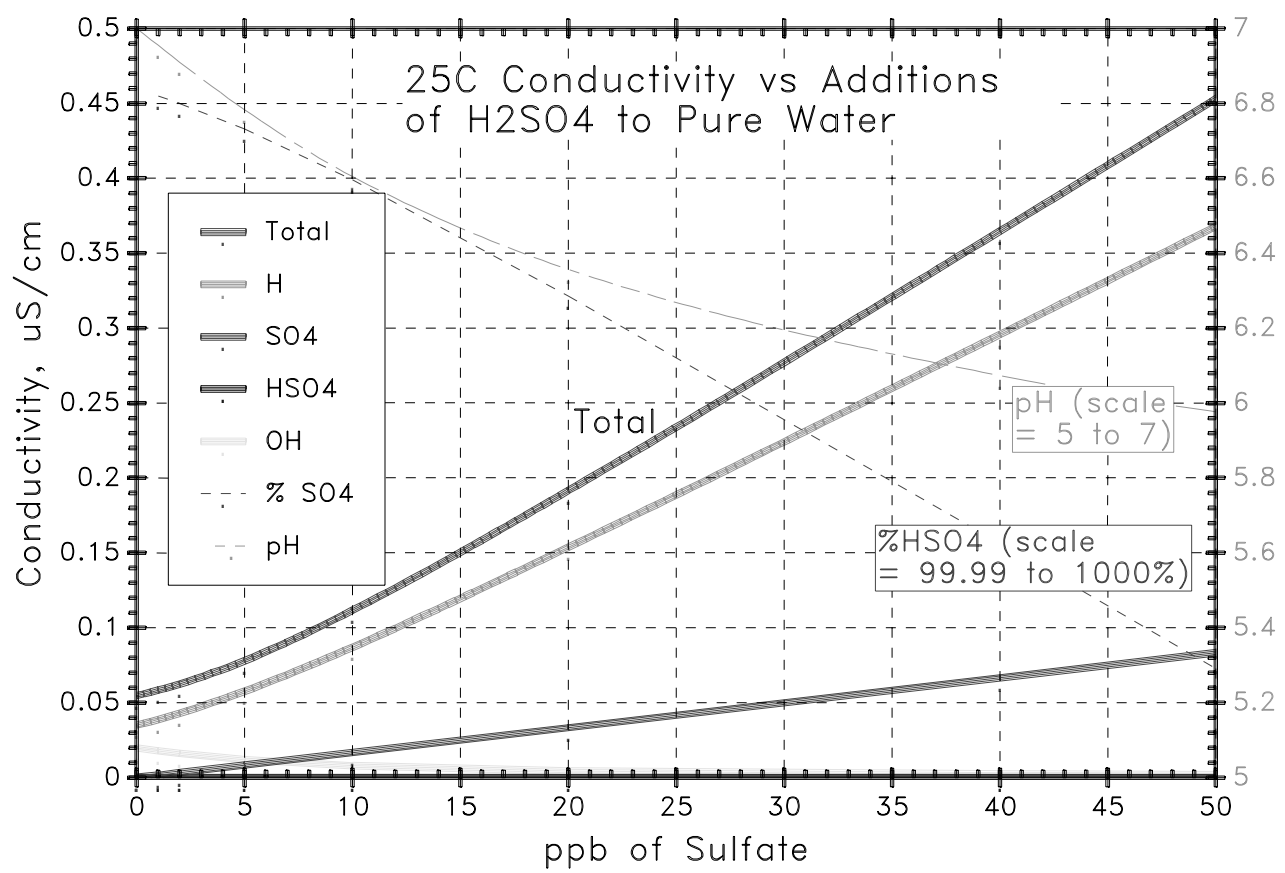

Figure 80. Concentration vs. conductivity for $\mathrm{H}_{2} \mathrm{SO}_{4}$ showing the contributions of individual ions, total conductivity, $\mathrm{pH}$ and percentage of $\mathrm{HSO}_{4}$.

Crack Growth Test Results and Discussion

\section{Stress Corrosion Cracking of Cold-Worked Stainless Steel}

Prior studies performed at the GE Global Research Center ${ }^{54-59}$ on the effect of cold work on stainless steel helped defined the most critical areas to evaluate in this study. It is widely understood that sensitization (grain boundary $\mathrm{Cr}$ depletion) in stainless steels produces an increased susceptibility to SCC in general, and faster crack-growth kinetics in particular (Figure 81). ${ }^{54-59}$ Sensitization was responsible for pipe cracking in boiling water reactors from the late 1960 s to the early 1980s, at which point most sensitized stainless steel piping had been replaced. Figure 81 also shows that annealed (i.e., non-sensitized and non-cold-worked) stainless steel is not immune to SCC, although the growth rates are lower than for sensitized stainless steel.

What was not well recognized is that cold work (and other sources of elevated yield strength, like radiation hardening) is in many ways more damaging than sensitization. It not only elevates the SCC growth rates at high corrosion potential to roughly the same extent as sensitization (Figure 81), it also elevates the growth rates at low corrosion potential (where Cr depletion plays a lesser role, and annealed and sensitized stainless steels respond similarly). Thus, the benefit of the widely adopted techniques to mitigation SCC by lowering corrosion potential (hydrogen water chemistry and NobleChem ${ }^{\mathrm{TM}}$ ) are less effective with cold-worked stainless steel than sensitized (or annealed) stainless steel (Figure 81).

The importance of yield strength in interpreting field observations of SCC during the last decade cannot be over-emphasized, as most of these incidents in BWRs have occurred in non-sensitized 

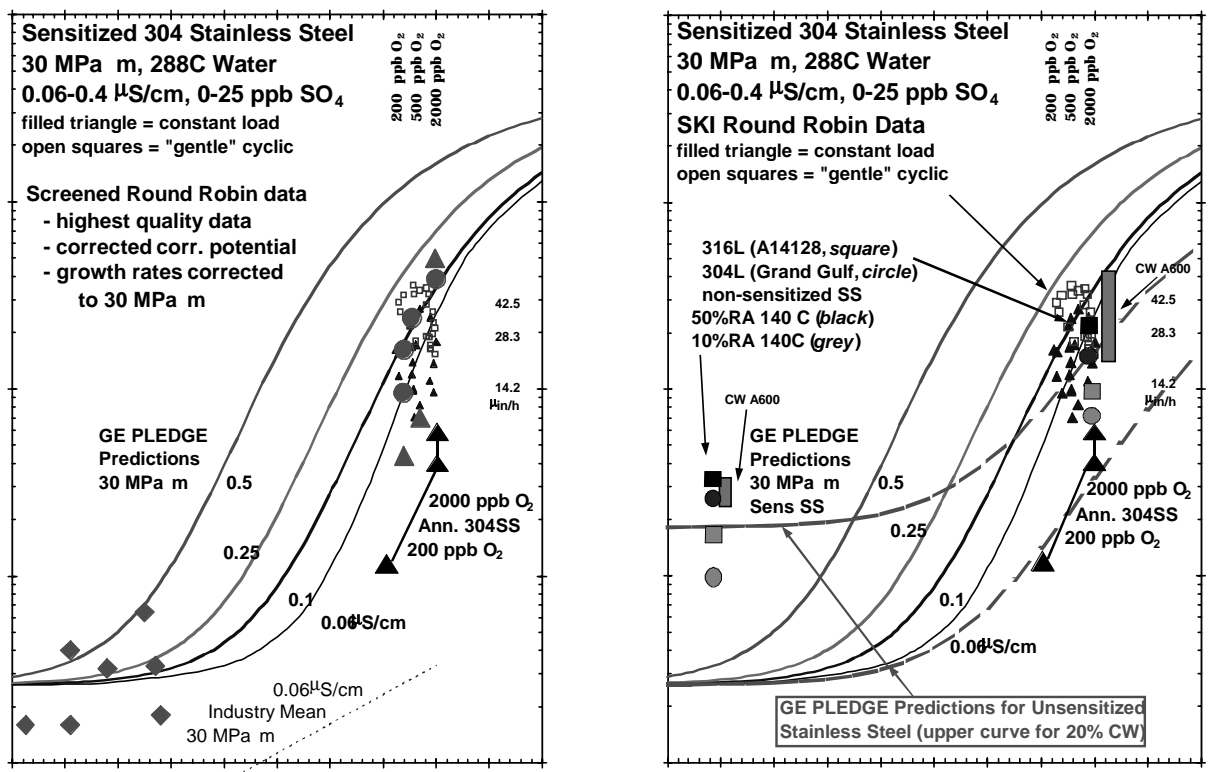

Figure 81. SCC growth rate vs. corrosion potential for stainless steels tested in $288^{\circ} \mathrm{C}$ high purity water containing $2000 \mathrm{ppb}_{2}$ and $95-3000 \mathrm{ppb} \mathrm{H}_{2}$.

stainless steel, particularly the core shroud and core spray piping. While there is a role of irradiation in some instances, in many others the neutron fluence is far below $10^{20} \mathrm{n} / \mathrm{cm}^{2}$, the point at which some measurable radiation-induced changes first appear. The origin of these cracking observations is primarily associated with weld shrinkage strains (Figure 82, which produces an increased yield strength near the heat-affected zone), weld residual stresses, and the high corrosion potential near the core. Similar non-sensitized stainless steels have not exhibited consequential SCC when used in external piping, where the corrosion potential is enough lower to account for the difference in the emergence of the SCC indications.

The pragmatic and fundamental value of understanding yield strength effects relates to the many mechanisms of yield-strength enhancement: solid-solution hardening, cold work, weld shrinkage strains, radiation hardening, oxide-dispersion hardening and precipitation hardening. While the microstructural origins of hardening varies dramatically, their effect on SCC shows strong commonality. This has been attributed to the importance of redistribution of the strain field in front of a growing crack, which is responsible for the dynamic strain at the crack tip that sustains SCC. In high yield strength materials, the plastic zone size is smaller and the strain gradients near the crack tip are accordingly higher. This leads to greater crack-tip strain for a given increment in crack advance.

The synergy between crack advance and dynamic crack-tip strain is an inherent characteristic of SCC. Unlike corrosion fatigue or slow-strain-rate testing (both of which have much in common with SCC under constant load), there is (in theory) no external source of dynamic strain (vs. reversed slip from cycling, or monotonically increasing strain - however, pragmatically there may be a number of contributions to dynamic strain, including vibrations, water hammers, start up, rising stress intensity from growing cracks and weld residual stress profiles). Any initial change in loading would produce transient creep that would decay with time. However, crack 


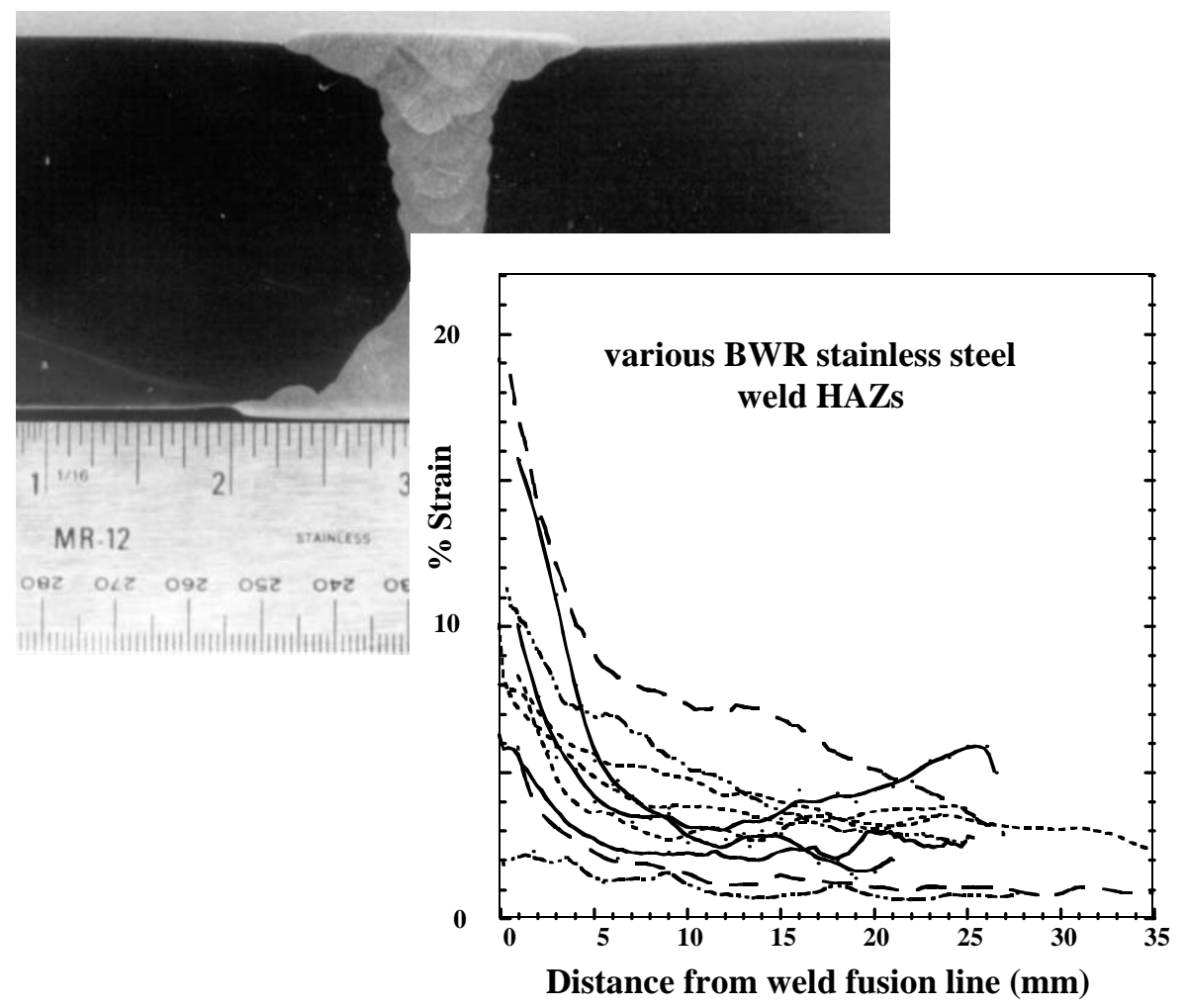

Figure 82. Profile of the equivalent room temperature tensile strain that develops in a weld heat affected zone from shrinkage strain.
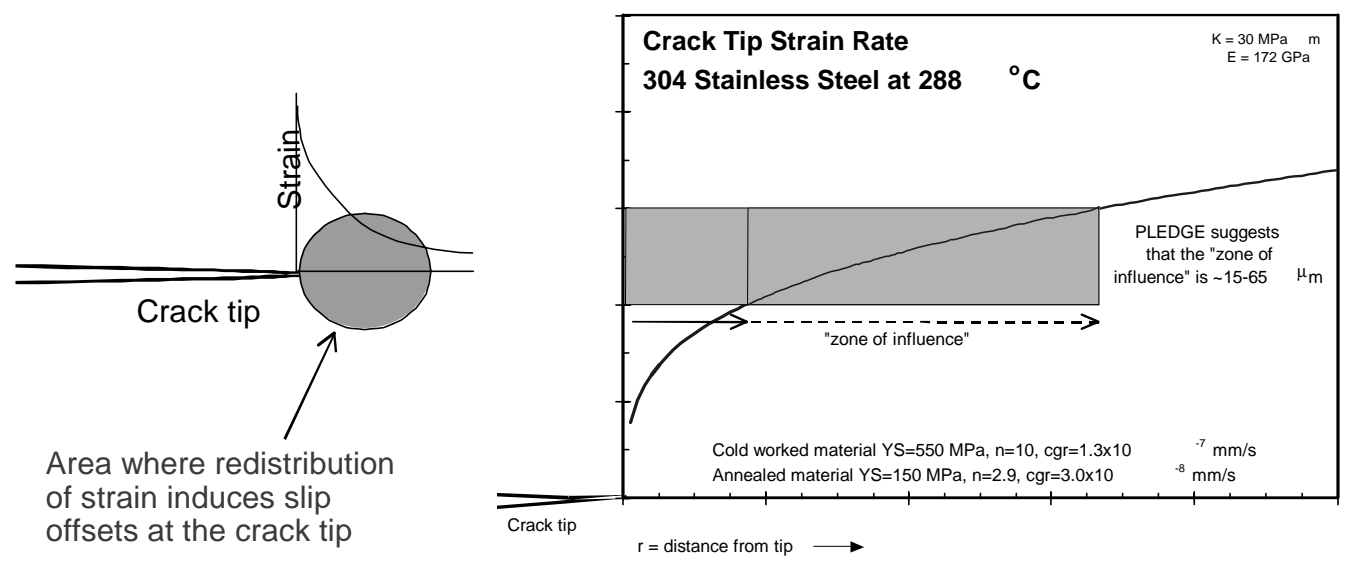

Figure 83. Schematic of the strain distribution at a crack tip. Under static-load SCC conditions, sustained dynamic strain results from the redistribution of the stress and strain fields ahead of the crack as the crack advances. This creates an inter-dependency in which the crack-tip strain rate is responsible for crack advance, and crack advance then produces stress/strain field redistribution, influencing the crack-tip strain rate. This synergy can occur in a complex fashion, since crack advance does not occur instantaneously in time or space. Conceptually, there is a zone of influence within which strain redistribution directly affects the crack tip by creating slip offsets. 
advance causes redistribution of the stress/strain field in front of the crack tip, which sustains dynamic strain and the stress corrosion process (Figure 83).

The initial observations of SCC on cold-worked stainless steels included data in aerated and deaerated $288^{\circ} \mathrm{C}$ water. The observation that IGSCC was readily sustained in hydrogendeaerated water was a surprise to many in the PWR community, where significant efforts to obtain IG cracking in cold-worked stainless steels using simpler techniques were unsuccessful. One concern raised in various discussions was that the prior exposure at high corrosion potential had somehow permanently contaminated the crack - i.e., such results might not be obtained if the specimens were exposed only to hydrogen-deaerated water. This concern was accounted for in the initial testing in this program, which examined the role of temperature and hydrogen fugacity/permeation on SCC.

Specimen c147 of 20\% CW 316L Stainless Steel Specimen c147 was fabricated from Type 316L stainless steel (heat A14128) that was solution annealed, then cold worked by $20 \%$ reduction in thickness at $+140^{\circ} \mathrm{C}$. The objective was to perform test in $\mathrm{H}_{2}$ deaerated water at $320^{\circ} \mathrm{C}$ and $340^{\circ} \mathrm{C}$. In these tests, all in-situ fatigue presoaking and "SCC transitioning" was performed in $1.58 \mathrm{ppm} \mathrm{H}_{2}\left(17.7 \mathrm{cc} / \mathrm{kg}\right.$, or $100 \% \mathrm{H}_{2}$ bubbled in pure water at STP $)$ - i.e., there was no exposure to aerated / high corrosion potential conditions. As expected, these data also show strong SCC susceptibility and moderately high growth rates despite exposure to $\mathrm{H}_{2}$ deaerated water during all phases of fatigue/SCC testing.

The specific crack length vs. time response for this specimen (c147) is shown in Figures $84-92$. An overview of the entire test is shown in Figure 84. Pre-cracking was performed in-situ in ultra high-purity water containing $1.58 \mathrm{ppm}(17.6 \mathrm{cc} / \mathrm{kg}) \mathrm{H}_{2}$. Because transitioning from a transgranular fatigue precrack to an IGSCC crack is often crucial to obtaining good data, the latter part of the fatigue precrack (Figure 85) and initial phase of SCC testing (Figure 86) is designed to help ensure that a completely IG crack front exists along the entire crack. In addition, the plastic zone should be as similar as possible to a monotonically formed (vs. cyclically hardened) plastic zone. In this specimen, this transition was made with great success, as the shift from continuous cycling at $\mathrm{R}=0.7,0.001 \mathrm{~Hz}$ to a $9,000 \mathrm{~s}$ hold time at $\mathrm{K}_{\max }$ at 185 hours (Figure 87 ) occurs very smoothly, and the crack growth response is very well behaved.

At 521 hours (Figure 88) the loading is changed to "static load" (actually constant stress intensity, using very small incremental changes in load as the crack grows), and only a very small perturbation in the crack-growth response is observed (as expected). A very linear, wellbehaved growth rate of $4.5 \times 10^{-8} \mathrm{~mm} / \mathrm{s}$ is sustained for over 300 hours (Figures 88 and 89) before the temperature was increased to $340^{\circ} \mathrm{C}$ at 832 hours. The growth rate increased to $\approx 9 \mathrm{x}$ $10^{-8} \mathrm{~mm} / \mathrm{s}$ for nearly 200 hours before slowing somewhat to $7.6 \times 10^{-8} \mathrm{~mm} / \mathrm{s}$, which was sustained for about 800 hours.

At this point the specimen had to be unloaded (the test stayed at $340^{\circ} \mathrm{C}$ ), and an error was made on reloading the specimen that resulted in an overload of about $35 \%$. While not extreme in terms of causing obvious physical damage to the specimen, it did seriously disturb the SCC response of the specimen. Indeed, over the course of the next $\approx 3000$ hours many different attempts were made to produce well-behaved SCC response (Figures 89 - 92), and they were all basically 


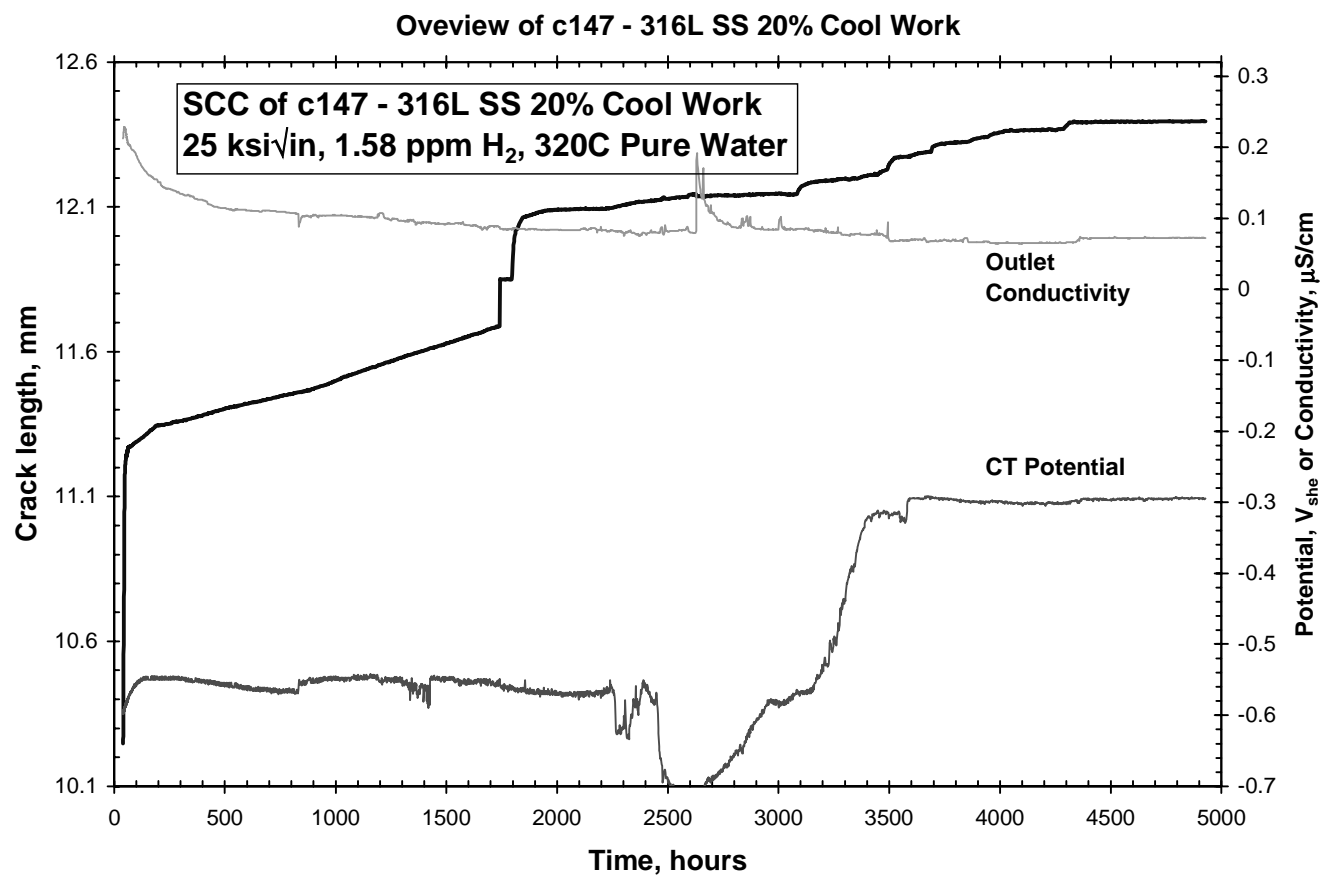

Figure 84. Overview of CT specimen c147, Type 316L SS (heat A14128) cool rolled 50\% at $+140^{\circ} \mathrm{C}$.

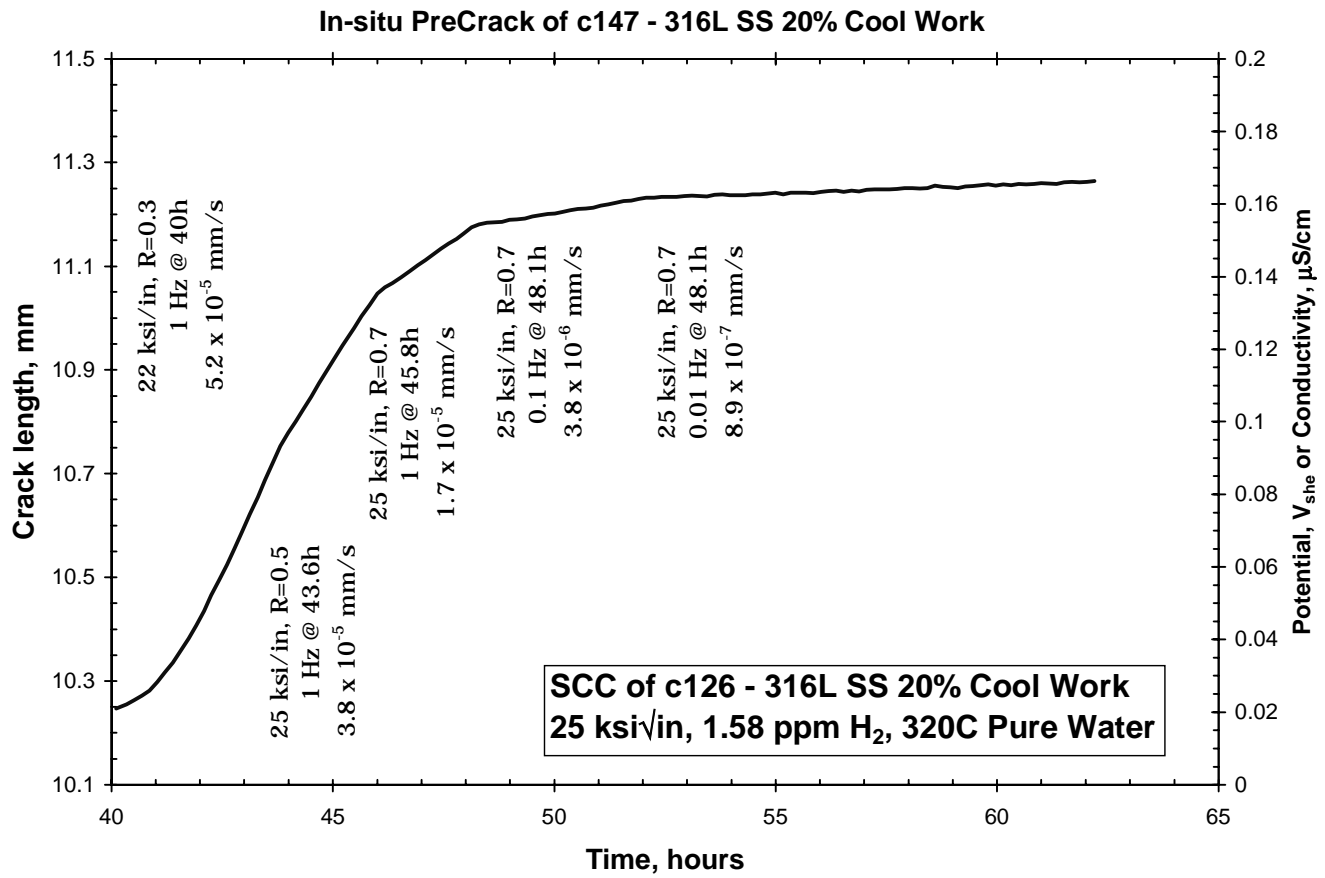

Figure 85. In-situ fatigue presoaking response of CT specimen c147, Type 316L SS (heat A14128) cool rolled $50 \%$ at $+140^{\circ} \mathrm{C}$.

unsuccessful. This is another among many examples of the sensitivity of SCC measurements to laboratory practice, particularly the methods of pre-cracking, transitioning from a transgranular 


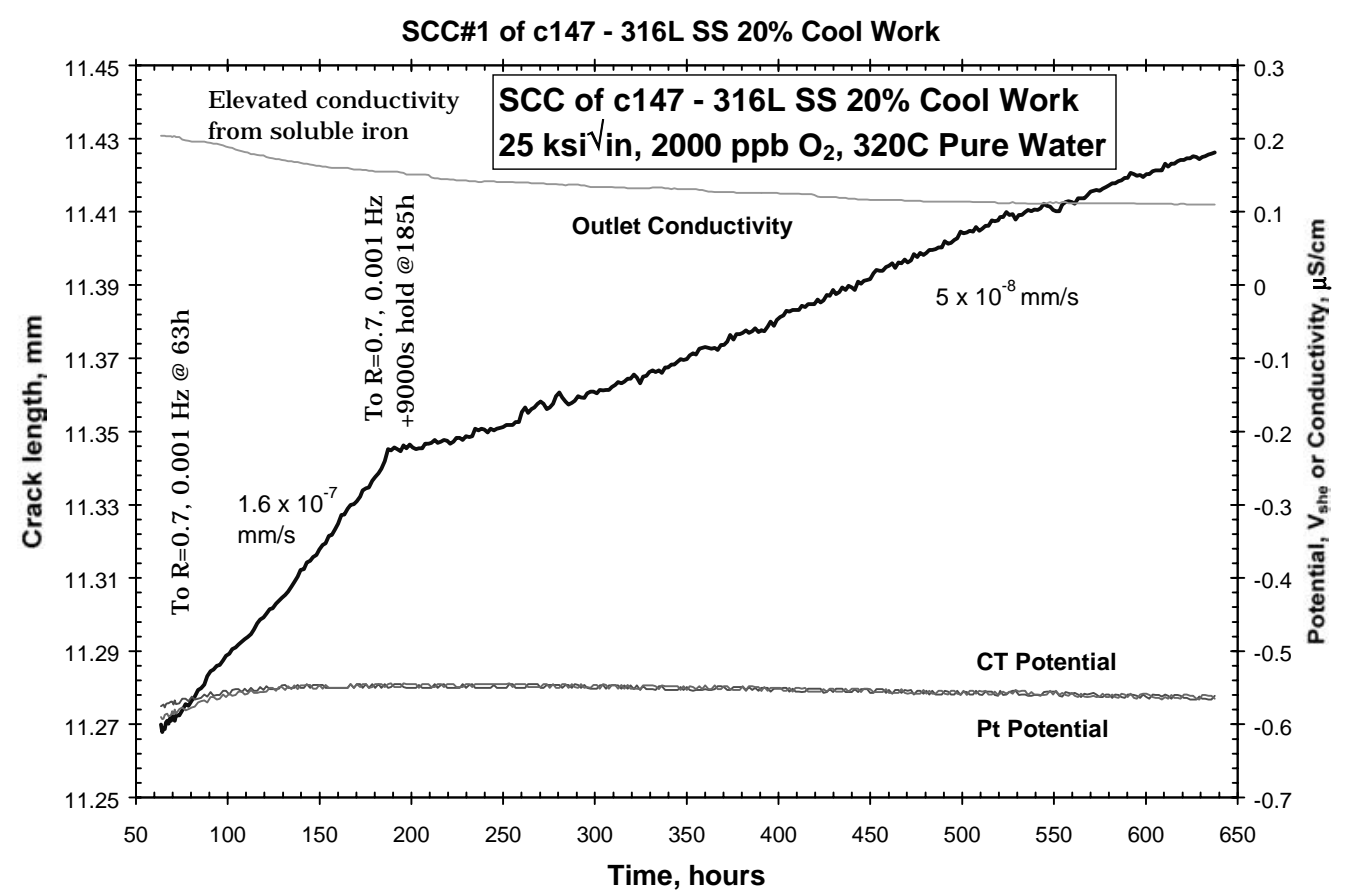

Figure 86. SCC response immediately following presoaking of CT specimen c147, Type 316L SS (heat A14128) cool rolled $50 \%$ at $+140^{\circ} \mathrm{C}$.

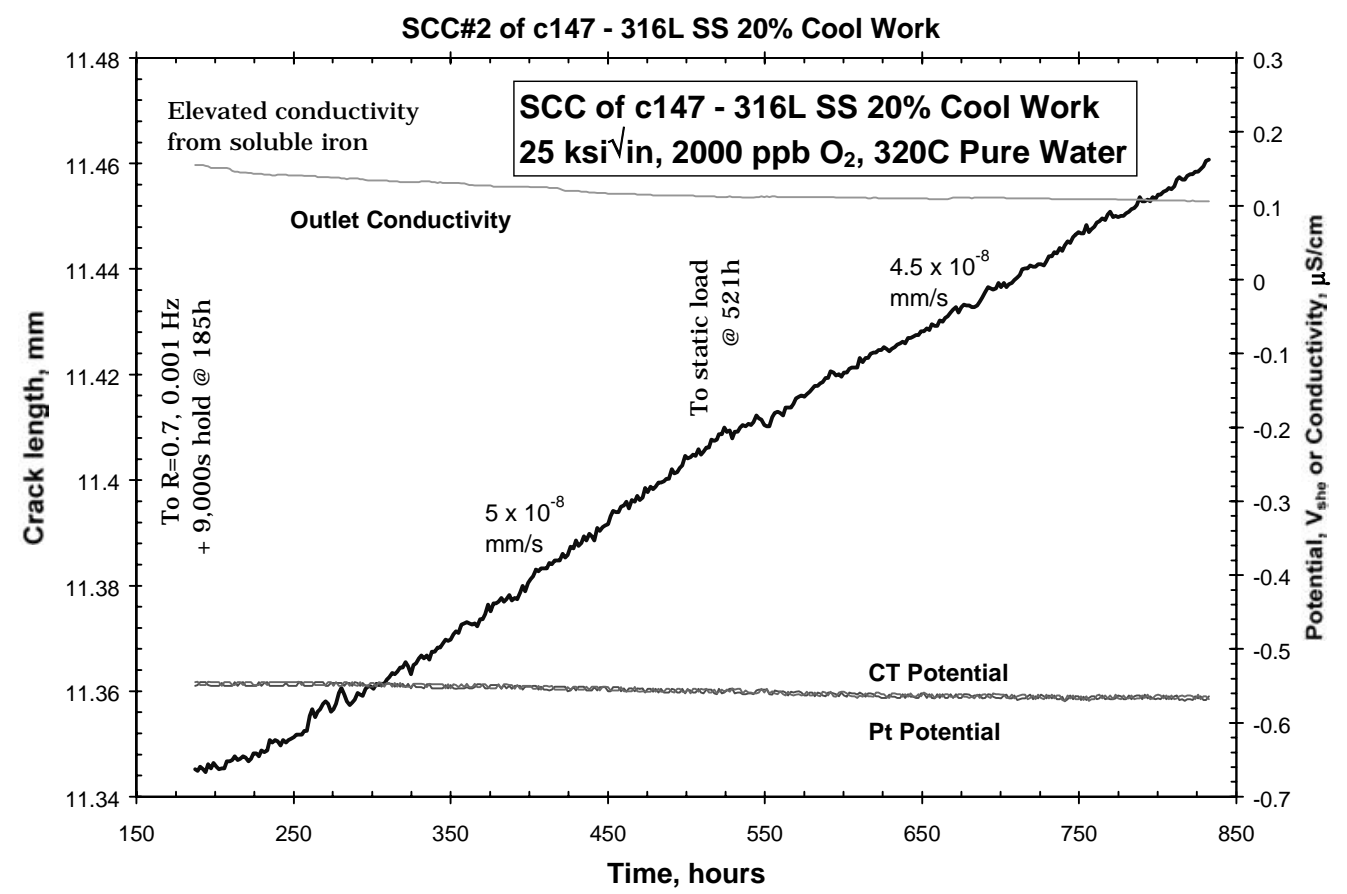

Figure 87. SCC response of CT specimen c147, Type 316L SS (heat A14128) cool rolled 50\% at $+140^{\circ} \mathrm{C}$. 


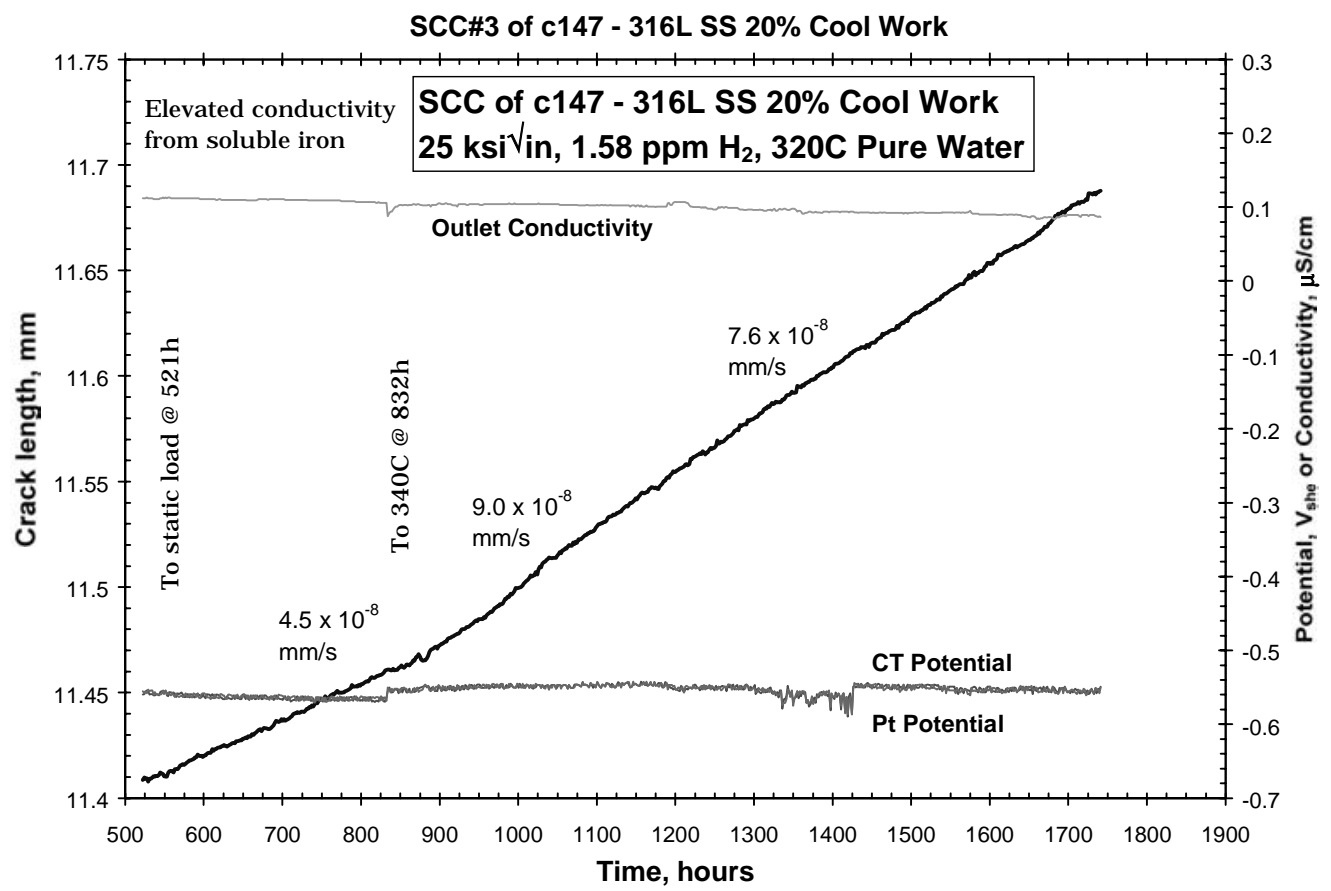

Figure 88. SCC response of CT specimen c147, Type 316L SS (heat A14128) cool rolled 50\% at $+140^{\circ} \mathrm{C}$ showing the transition in fast temperature from 320 to $340^{\circ} \mathrm{C}$.

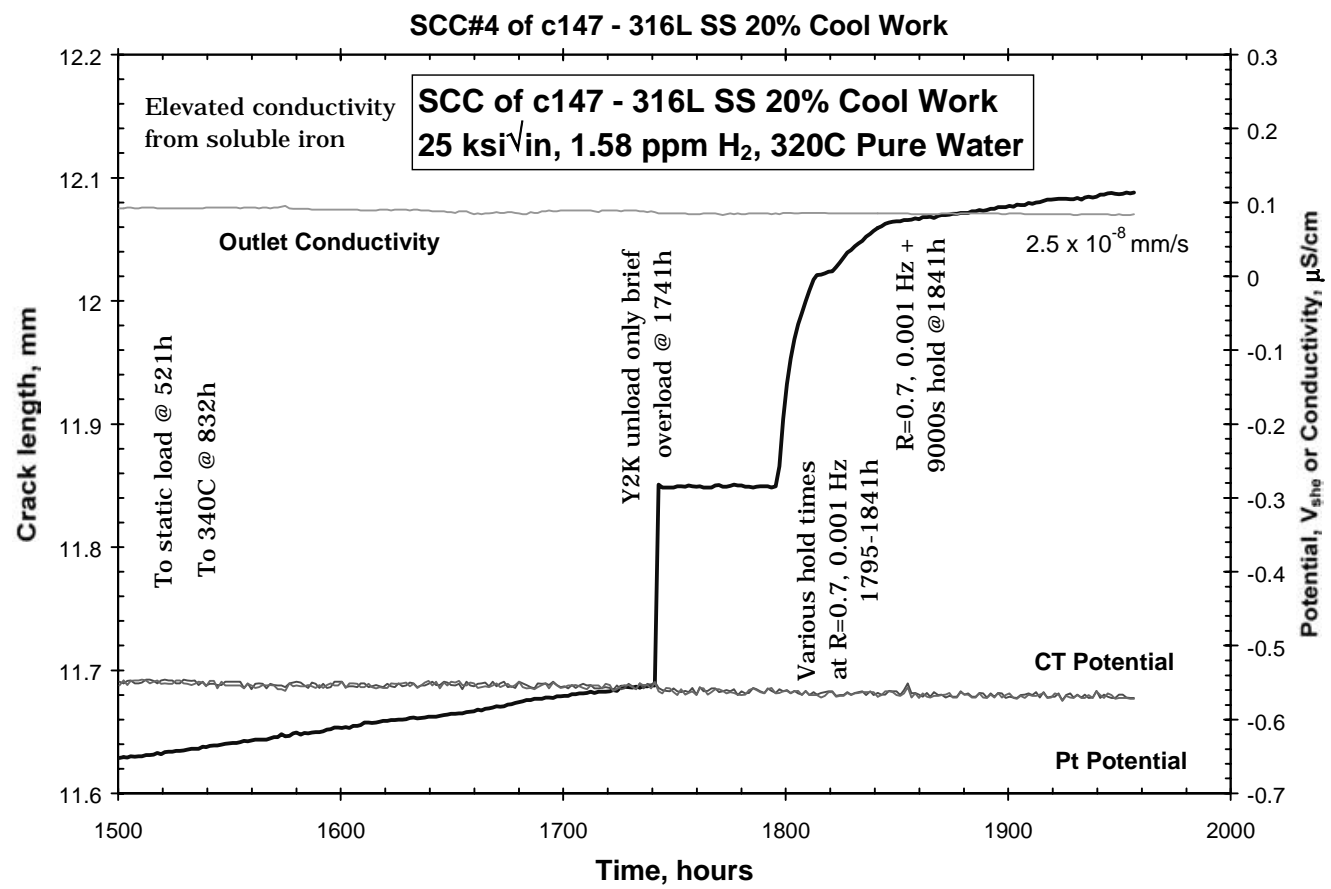

Figure 89. SCC response of CT specimen c147, Type 164L SS (heat A14128) cool rolled 50\% at $+140^{\circ} \mathrm{C}$ after an overload event. 


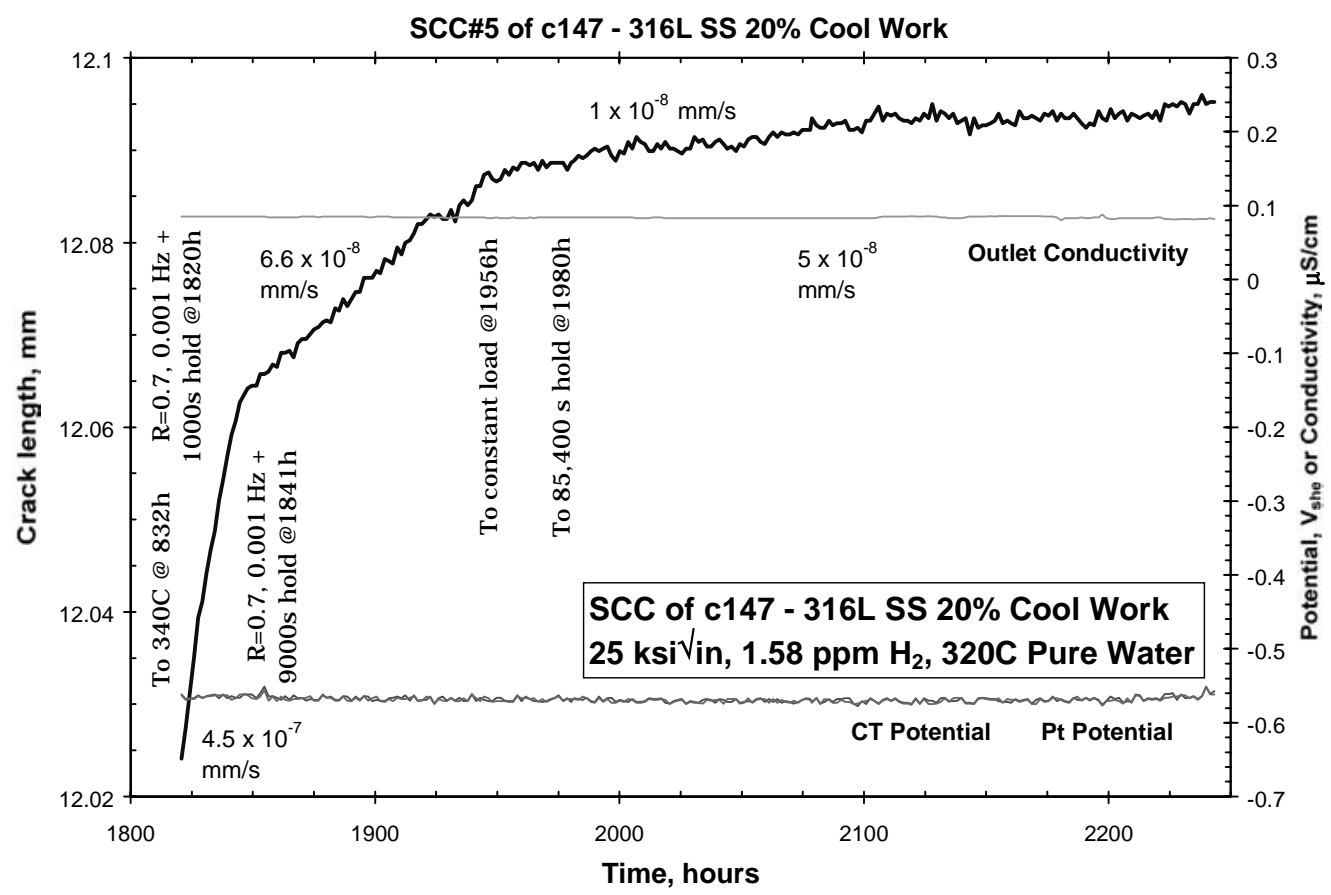

Figure 90. SCC response of CT specimen c147, Type 1316L SS (heat A14128) cool rolled 50\% at $+140^{\circ} \mathrm{C}$ showing cyclic loading in an attempt to recover well-balanced crack growth.

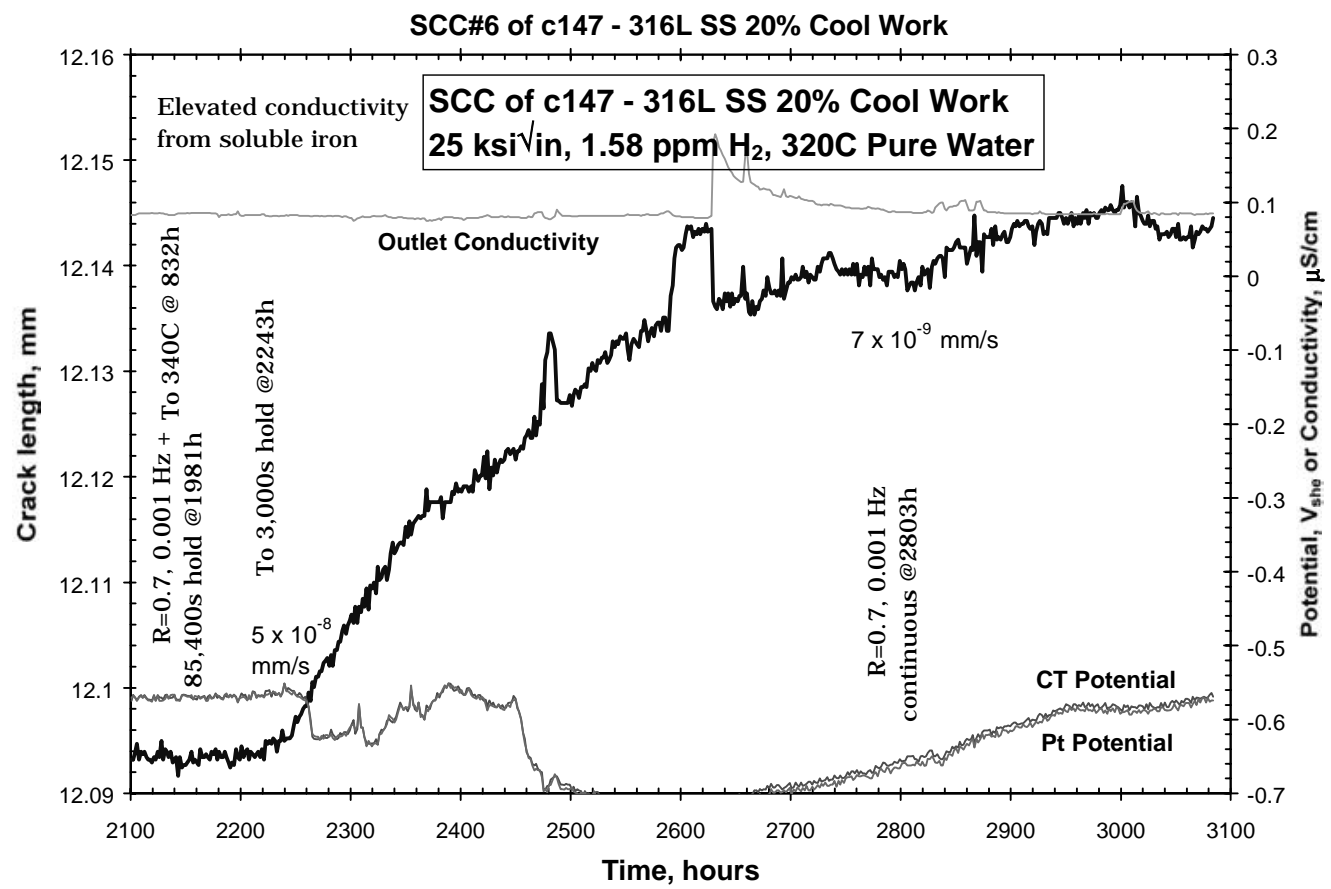

Figure 91. SCC response of CT specimen c147, Type 316L SS (heat A14128) cool rolled 50\% at $+140^{\circ} \mathrm{C}$ continuing attempts to re-establish well-behaved crack growth. 
to an IG crack front, ensuring that the loads and plastic zone characteristics are as close as possible to the conditions that exist an SCC crack forms and grows in a structure.

The test was ended at 4933 hours. Figure 93 shows a low magnification macrograph of the fracture appearance, and Figure 94 shows fractography by scanning electron microscopy. The dc potential drop indicated growth is $2.14 \mathrm{~mm}$, while the average, maximum and minimum of the actual crack depth are $2.44 \mathrm{~mm}, 2.74 \mathrm{~mm}$ and $1.78 \mathrm{~mm}$. No corrections to the crack length or stress intensity data of specimen c147 were performed.

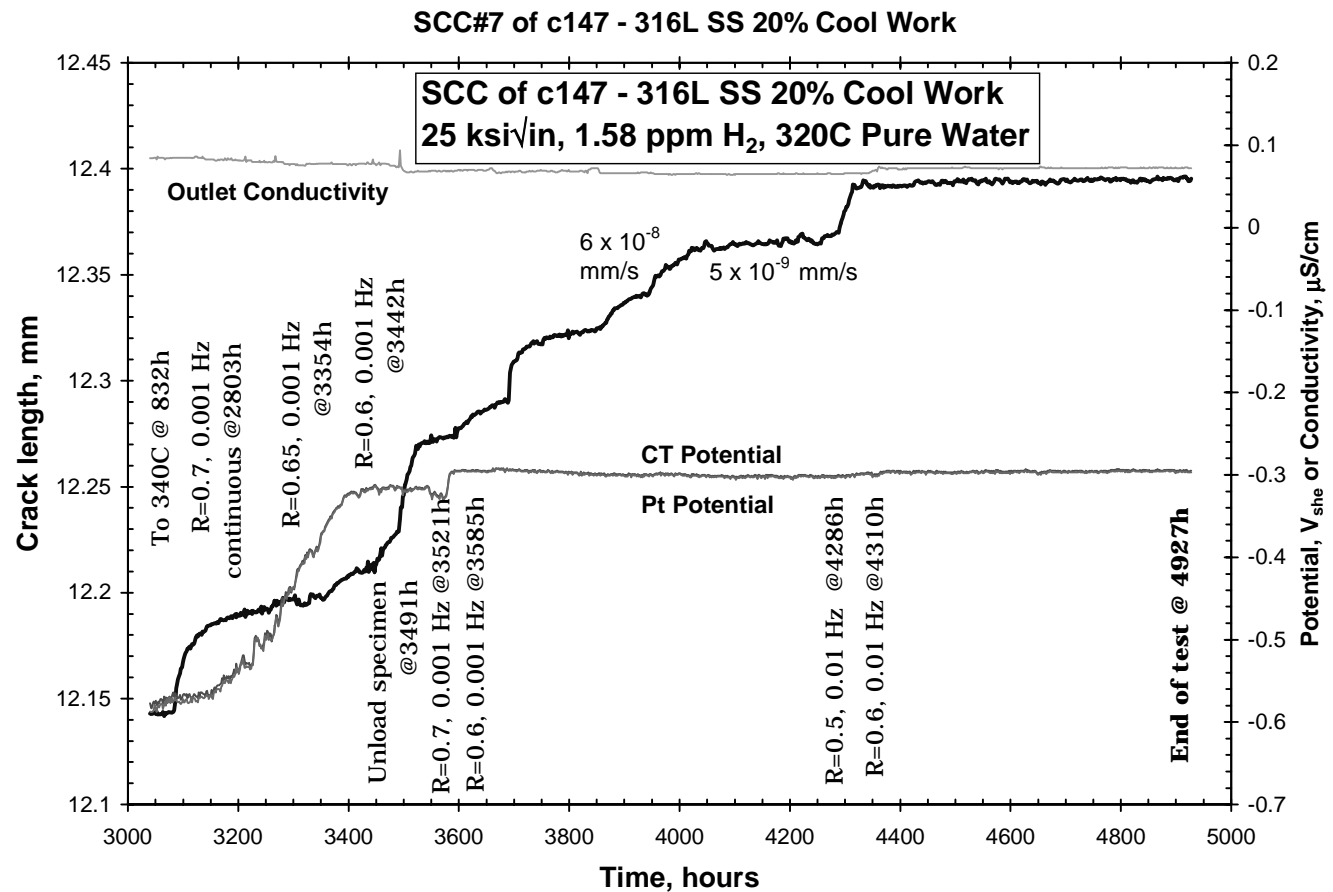

Figure 92. Final attempts to re-gain consistent SCC response of CT specimen c147, Type 316L SS (heat A14128) cool rolled $50 \%$ at $+140^{\circ} \mathrm{C}$.

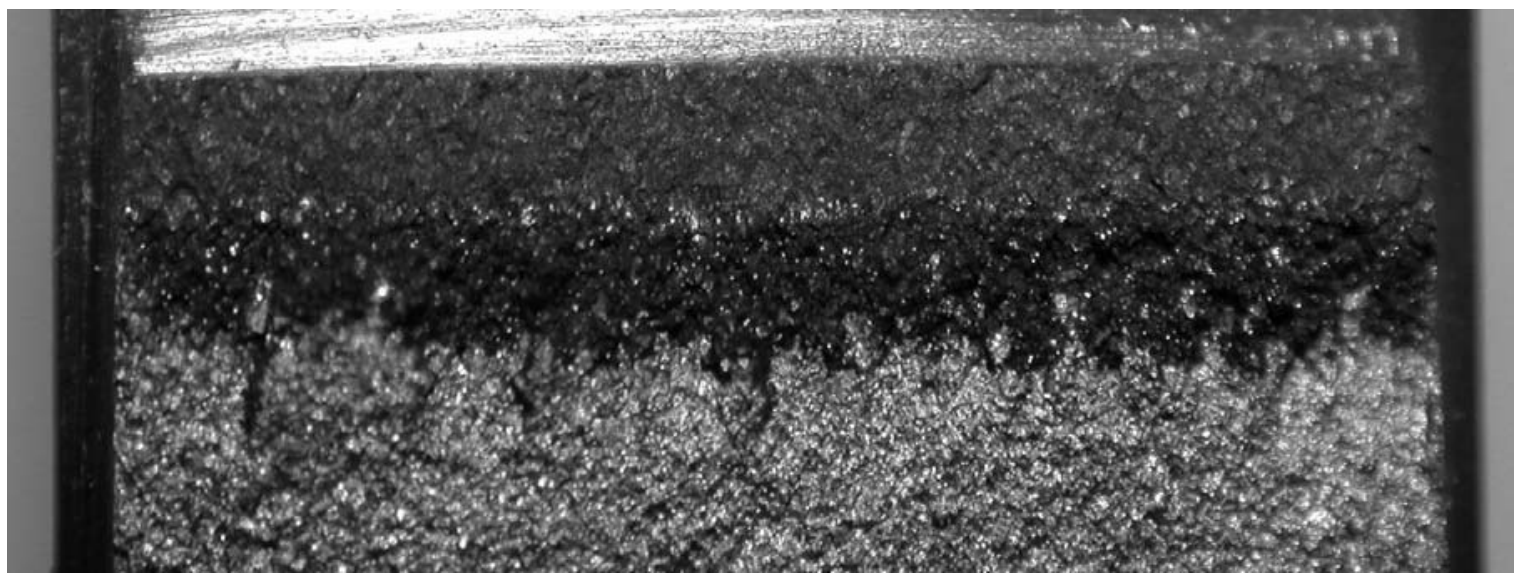

Figure 93. Light macrograph of specimen c147, Type 316L SS (heat A14128) cool rolled 50\% at $+140^{\circ} \mathrm{C}$. 

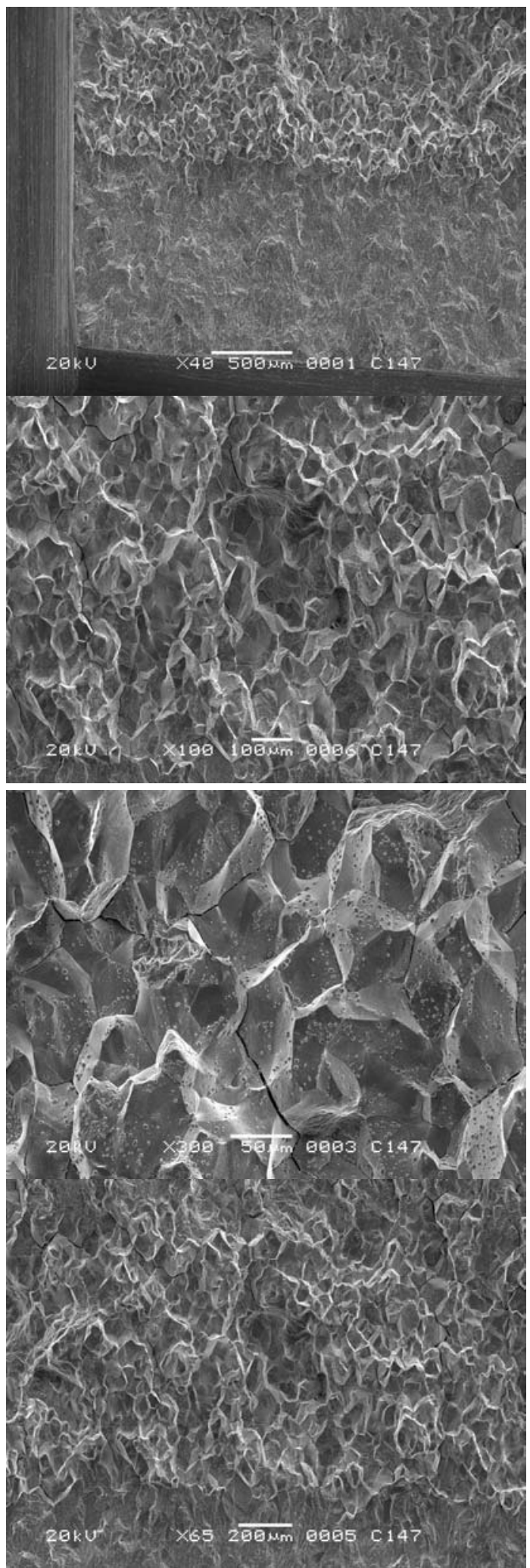

Figure 94. SEM micrograph of fracture surface for specimen c147, Type 316L SS (heat A 14128) cool rolled $50 \%$ at $+140^{\circ} \mathrm{C}$ showing IG crack path. 
Specimen c156 of 50\% CW 304L Stainless Steel Specimens c156 and c157 were tested in the autoclave in tandem (loaded in series). While this permits data on two specimens to be obtained simultaneously, there is always the risk that the specimens will behave enough differently to make it difficult to get good data on both specimens. Even under the best of circumstances, one specimen will inevitably exhibit more rapid crack propagation that the other, so the stress intensities will diverge. If this occurs to a limited extent $(10-20 \%$ difference in stress intensity), the result is not that important. In general, the desired stress intensity is controlled constant on the specimen that is growing faster, so it drops on the other specimen. The decreasing stress intensity can put the slower specimen in danger of crack arrest, so the decision on how best to control the test depends on the specific responses.

An overview of this test is shown in Figure 95, which includes crack length vs. time, the corrosion potential of the CT specimen and a Pt electrode, and outlet solution conductivity. Conditions during the test were very well controlled, and 6043 hours of continuous crack growth testing were completed before the test was ended. While crack lengths and growth rates were not corrected for post-test fractography, the effect on stress intensity of corrected crack length is noted in the figures.

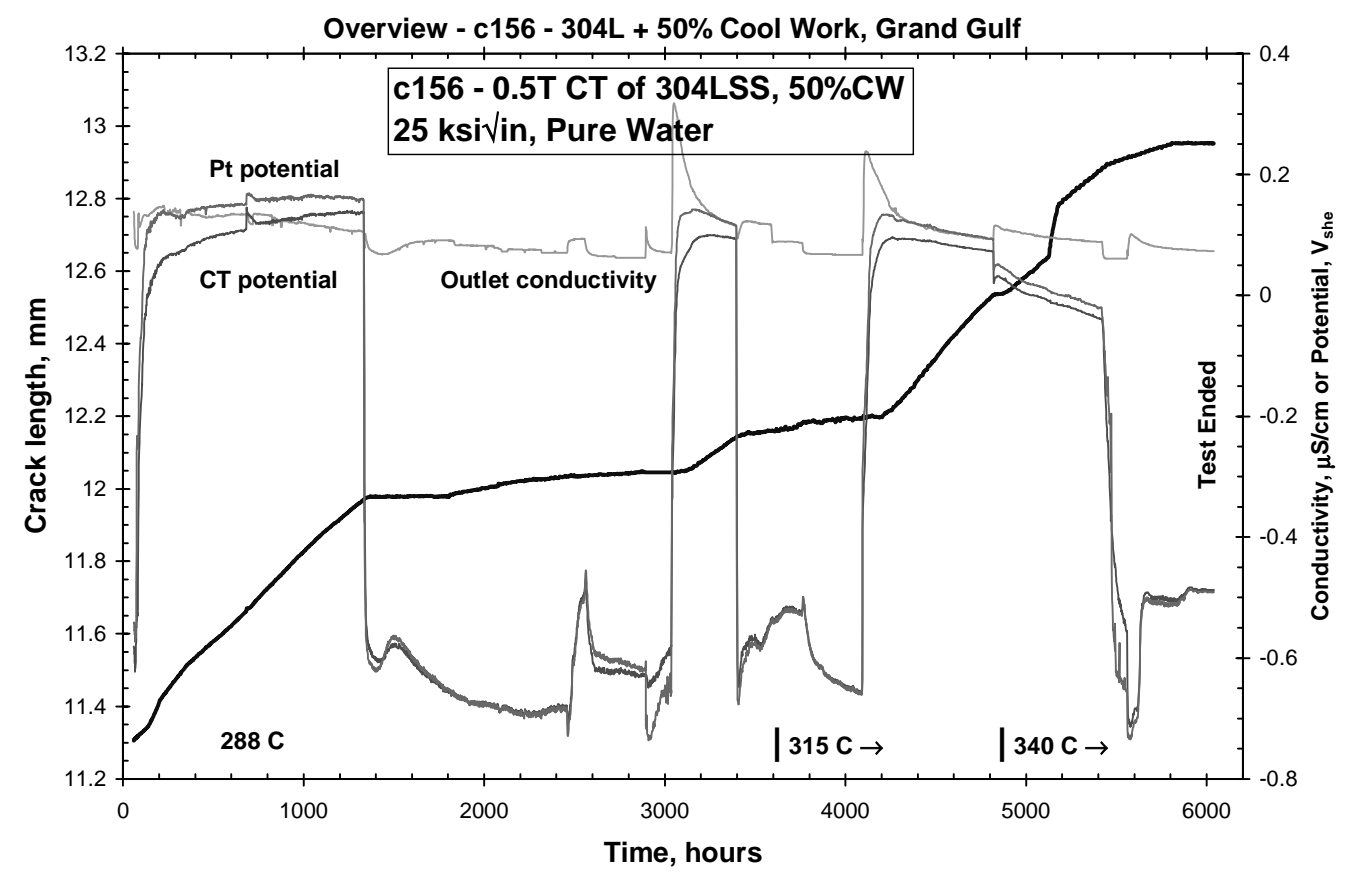

Figure 95. Overview of CT specimen c156, Type 304L SS (Grand Gulf heat) cool rolled 50\% at $+140^{\circ} \mathrm{C}$.

The air fatigue precracking response is shown in Figure 96. The specimen was then assembled into the autoclave, heated and equilibrated, than loaded to transition from fatigue pre-cracking conditions (where cracking is fully transgranular) to SCC (where cracking is predominately IG) - one of the most critical ingredients in achieving good SCC response. Figure 97 shows the response in $288^{\circ} \mathrm{C}$ pure water containing $2000 \mathrm{ppb} \mathrm{O}_{2}$ as the loading is changed toward constant stress intensity conditions. Crack-growth rates are quite high compared to prior experience with 
non-sensitized, low-strength, annealed stainless steel, and there was no difficulty in establishing a well-behaved crack-growth rate under fully static loading conditions, initiated at 491 hours. Note that there is minimal change in crack-growth rate following the change from a 3000 -second hold time, to 9000 -second hold time, and finally to constant stress intensity.

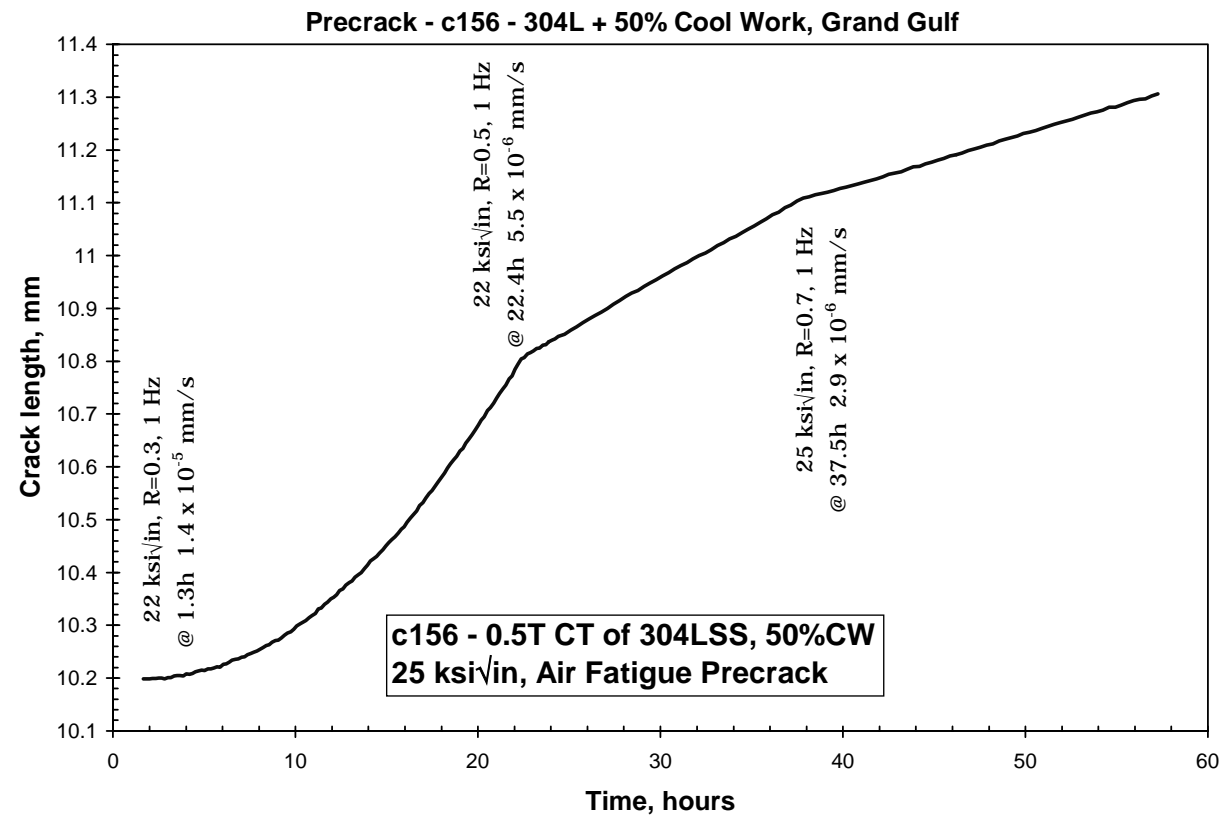

Figure 96. Air fatigue pre-cracking of CT specimen c156, Type 304L SS (Grand Gulf heat) cool rolled $50 \%$ at $+140^{\circ} \mathrm{C}$.

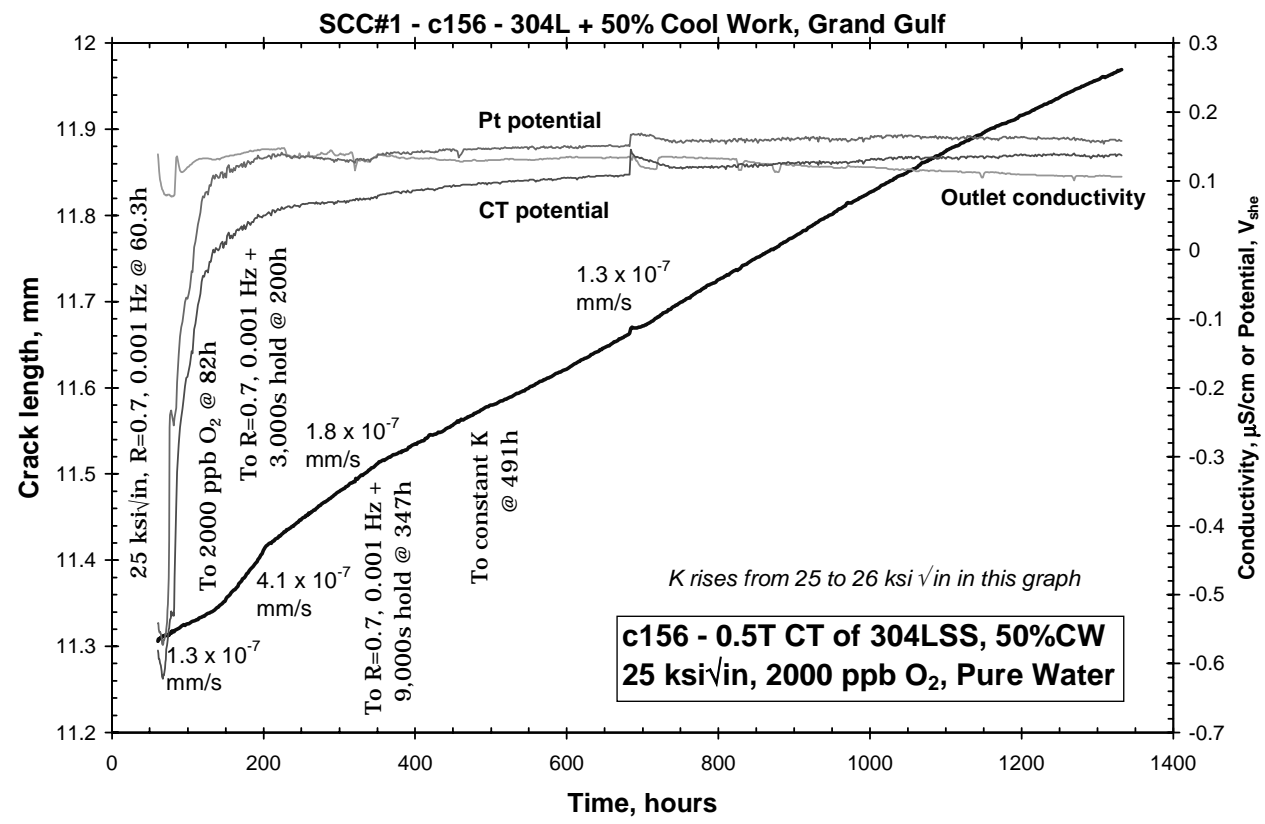

Figure 97. Transition from transgranular in-situ fatigue pre-cracking to IGSCC of specimen c156, Type 304L SS (Grand Gulf heat) cool rolled $50 \%$ at $+140^{\circ} \mathrm{C}$. 
Figure 98 shows the longer-term response under static loading conditions, which is quite linear and well-behaved at a crack-growth rate of $1.3 \times 10^{-7} \mathrm{~mm} / \mathrm{s}$ until the test conditions were changed at $1332 \mathrm{~h}$. Figures 98 and 99 show the change to $95 \mathrm{ppb} \mathrm{H}_{2}$ at $1332 \mathrm{~h}$, which produced a rapid decrease in crack-growth rate to $1.5 \times 10^{-9} \mathrm{~mm} / \mathrm{s}$. Because this rate was so low, at $1808 \mathrm{~h}$ a change to one unloading cycle $(\mathrm{R}=0.7,0.001 \mathrm{~Hz})$ per day was initiated. This increased the crack-growth rate to $3 \times 10^{-8} \mathrm{~mm} / \mathrm{s}$. The growth rate slowly decayed with time to $\approx 1.5 \times 10^{-8}$ $\mathrm{mm} / \mathrm{s}$, and was essentially unchanged to shifts to $1580 \mathrm{ppb} \mathrm{H}_{2}$ at 2459 , or to $100 \% \mathrm{~N}_{2}$ at $2555 \mathrm{~h}$ (Figure 99).

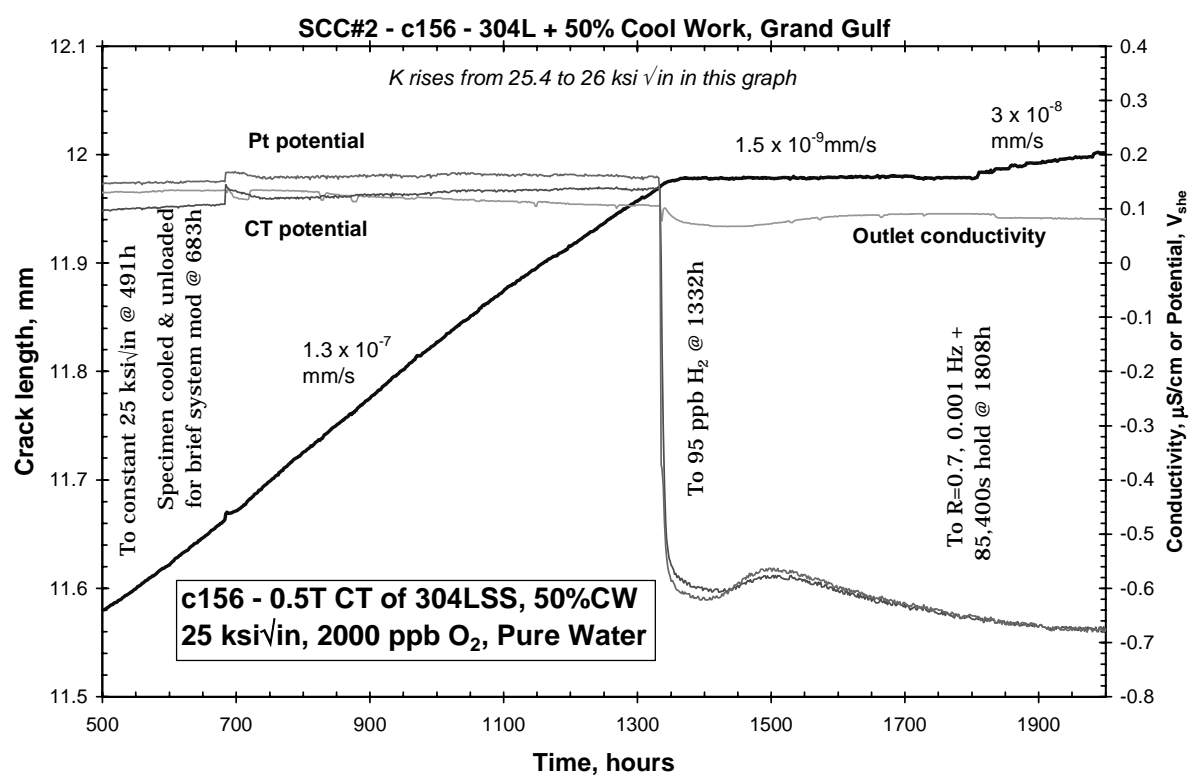

Figure 98. SCC behavior at static load of specimen c156, Type 304L SS (Grand Gulf heat) cool rolled $50 \%$ at $+140^{\circ} \mathrm{C}$.

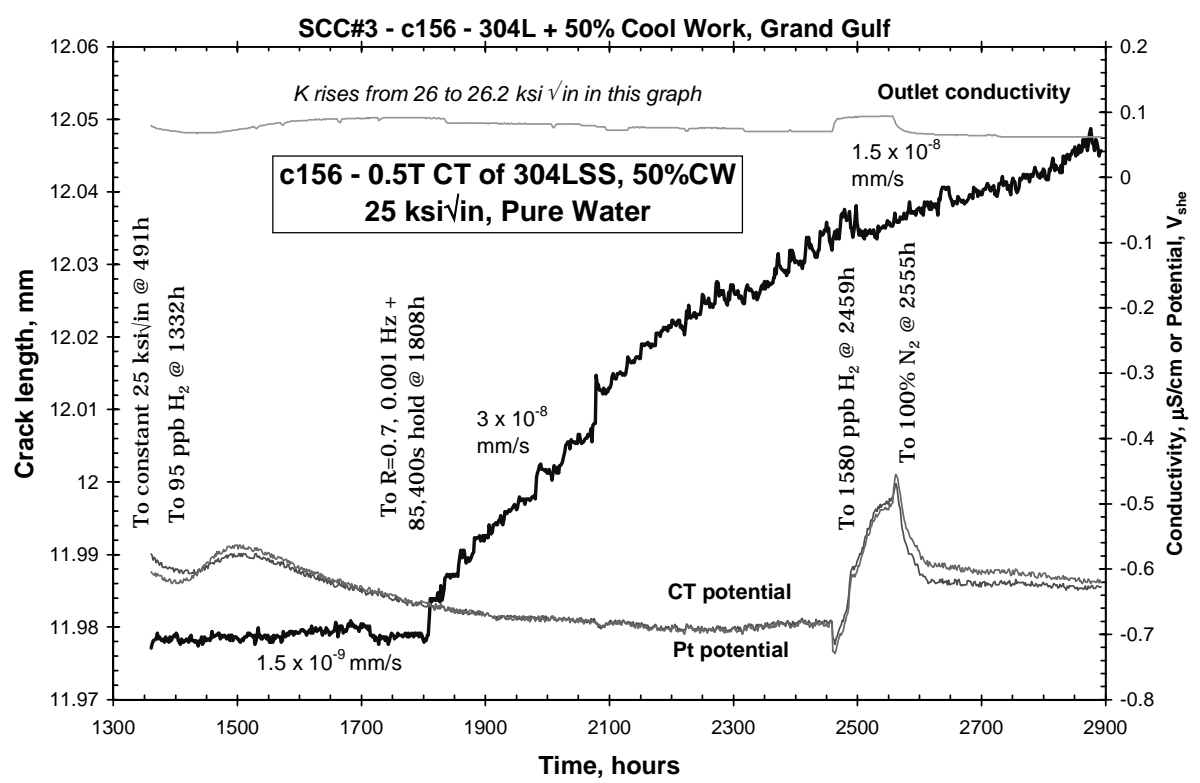

Figure 99. SCC behavior of specimen c156 showing graduate decrease in crack-growth rate and minimal effects of changes to the environment. 
From 2893 to $3037 \mathrm{~h}$, the temperature was decreased to $150^{\circ} \mathrm{C}$ to establish that no leakage was occurring in a hydrogen permeation tube that was a passive part of this experiment. At $3037 \mathrm{~h}$, the temperature was increased back to $288^{\circ} \mathrm{C}$ and the dissolved gas was changed from $\mathrm{H}_{2}$ to 2000 $\mathrm{ppb} \mathrm{O}_{2}$ (Figure 100). A growth rate of $1 \times 10^{-7} \mathrm{~mm} / \mathrm{s}$ was observed, only slightly lower than the previous observation of $1.3 \times 10^{-7} \mathrm{~mm} / \mathrm{s}$. At $3396 \mathrm{~h}$, the dissolved gas was changed to $1.58 \mathrm{ppm}$ $\mathrm{H}_{2}$ and about $200 \mathrm{~h}$ later, the test temperature was increased to $315^{\circ} \mathrm{C}$. While the higher temperatures are not strictly relevant to BWR operation (they are to PWR operation), it is very valuable to be able to understand the SCC response in BWRs in the context of the broader temperature conditions that occur in PWRs. Under these conditions the crack growth rate increased from $1.5 \times 10^{-8} \mathrm{~mm} / \mathrm{s}$ observed earlier in Figure 100 to about $2.7 \times 10^{-8} \mathrm{~mm} / \mathrm{s}$.

At $4092 \mathrm{~h}$ (Figure 101), the dissolved gas conditions were changed to $2000 \mathrm{ppb} \mathrm{O}_{2}$ and the growth rate increased to $1.8 \times 10^{-7} \mathrm{~mm} / \mathrm{s}$. This rate was sustained as the change was made from a gentle unloading cycle $(\mathrm{R}=0.7,0.001 \mathrm{~Hz})$ once per day to constant $\mathrm{K}$ conditions at $4483 \mathrm{~h}$.

The test temperature was increased to $340^{\circ} \mathrm{C}$ at $4818 \mathrm{~h}$ (Figure 102) and there was a short-lived slowing of the crack-growth rate, returning to a rate of $1.2 \times 10^{-7} \mathrm{~mm} / \mathrm{s}$. This rate was expected to be higher than at $315^{\circ} \mathrm{C}$, so gentle cycling was initiated at $5126 \mathrm{~h}$, which produced a significant increase in growth rate to $6.5 \times 10^{-7} \mathrm{~mm} / \mathrm{s}$ (Figure 103). At $5176 \mathrm{~h}$ the hold time at $\mathrm{K}_{\max }$ was increased from $9,000 \mathrm{~s}$ to $85,400 \mathrm{~s}$ and the growth rate slowed back to $1.2 \times 10^{-7} \mathrm{~mm} / \mathrm{s}$. The dissolved gas was changed to $100 \% \mathrm{~N}_{2}$ at $5419 \mathrm{~h}$ and then to $1.58 \mathrm{ppmH}_{2}$ at $5559 \mathrm{~h}$. The growth rate slowed to $5 \times 10^{-8} \mathrm{~mm} / \mathrm{s}$ (Figure 104). At $5802 \mathrm{~h}$, the loading was changed to constant stress intensity and the growth rate slowed to $\approx 0 \mathrm{~mm} / \mathrm{s}$.

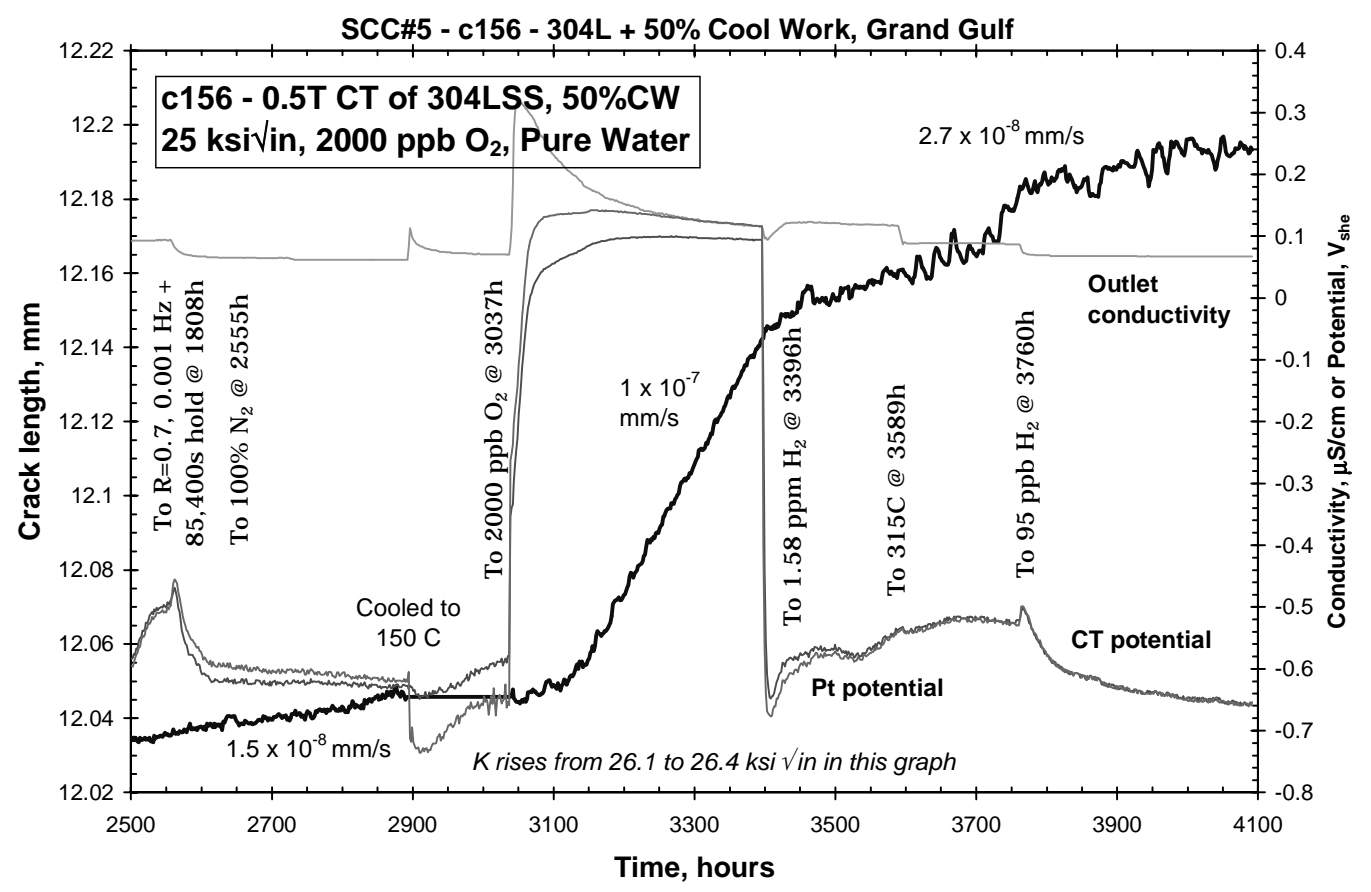

Figure 100. SCC behavior showing the effect of test temperature for specimen c156, Type 304L SS (Grand Gulf heat) cool rolled $50 \%$ at $+140^{\circ} \mathrm{C}$. 


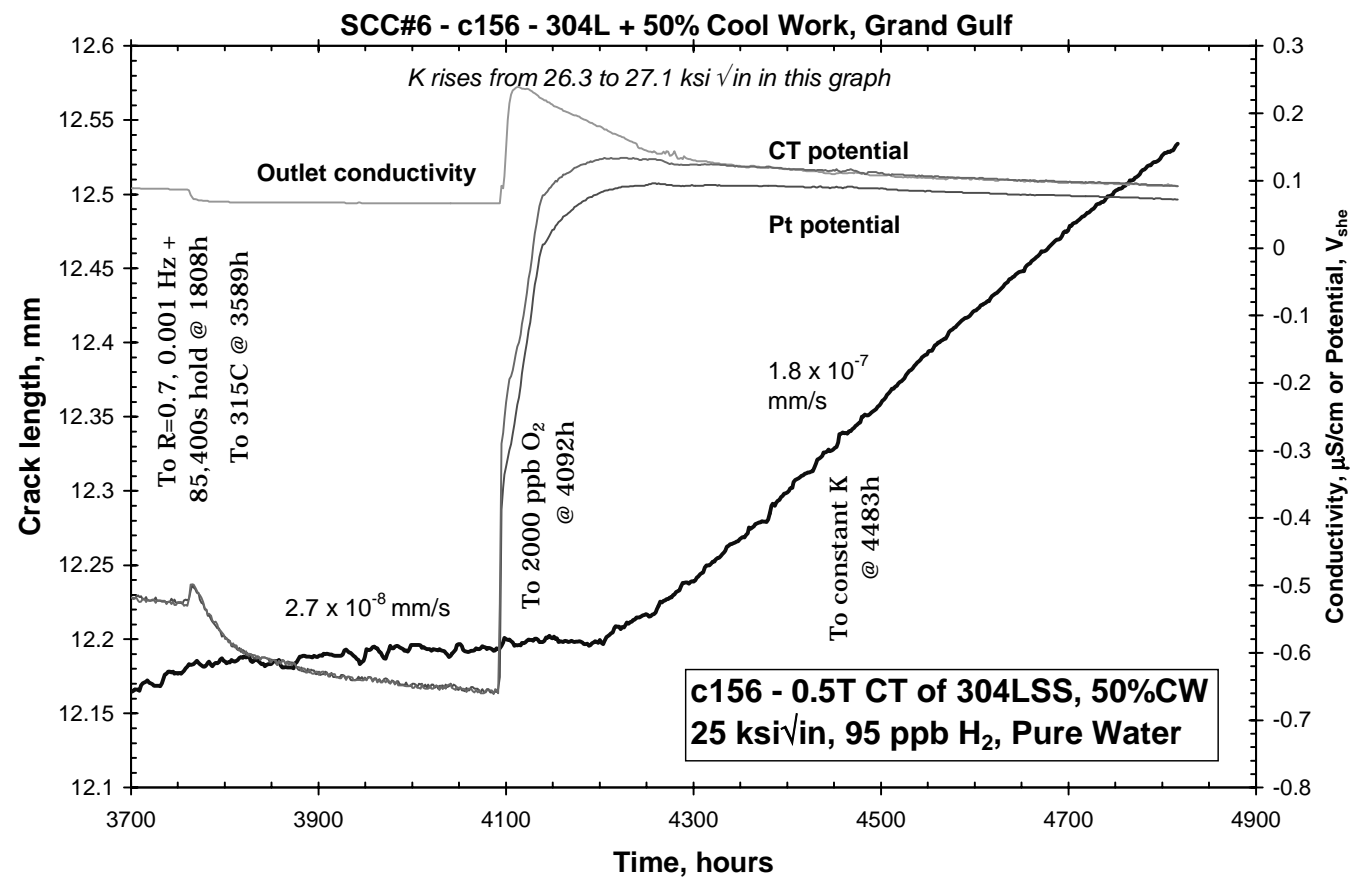

Figure 101. SCC behavior of specimen c156 at a test temperature of $315^{\circ} \mathrm{C}$.

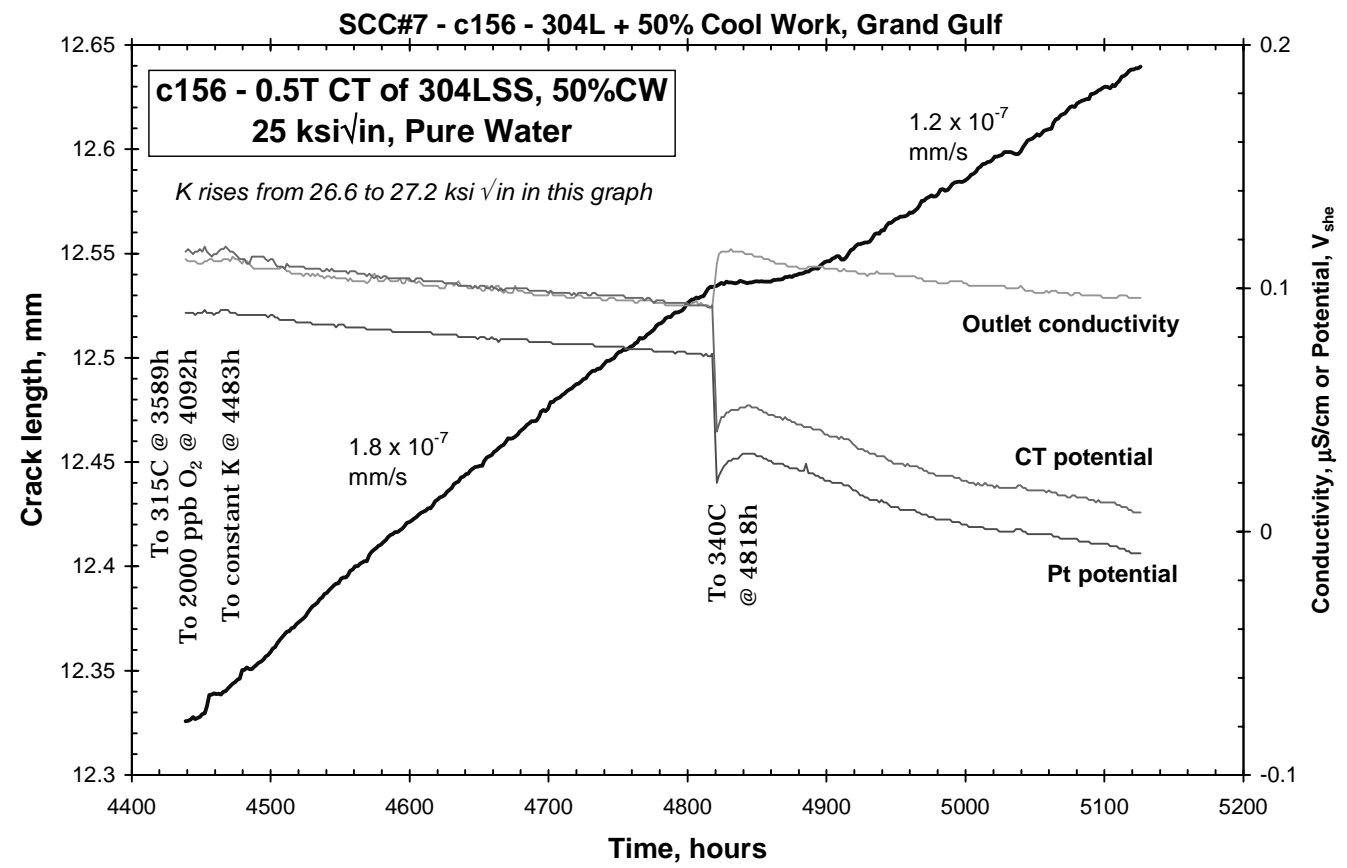

Figure 102. SCC behavior of specimen c156, transitioning to a test temperature of $340^{\circ} \mathrm{C}$. 


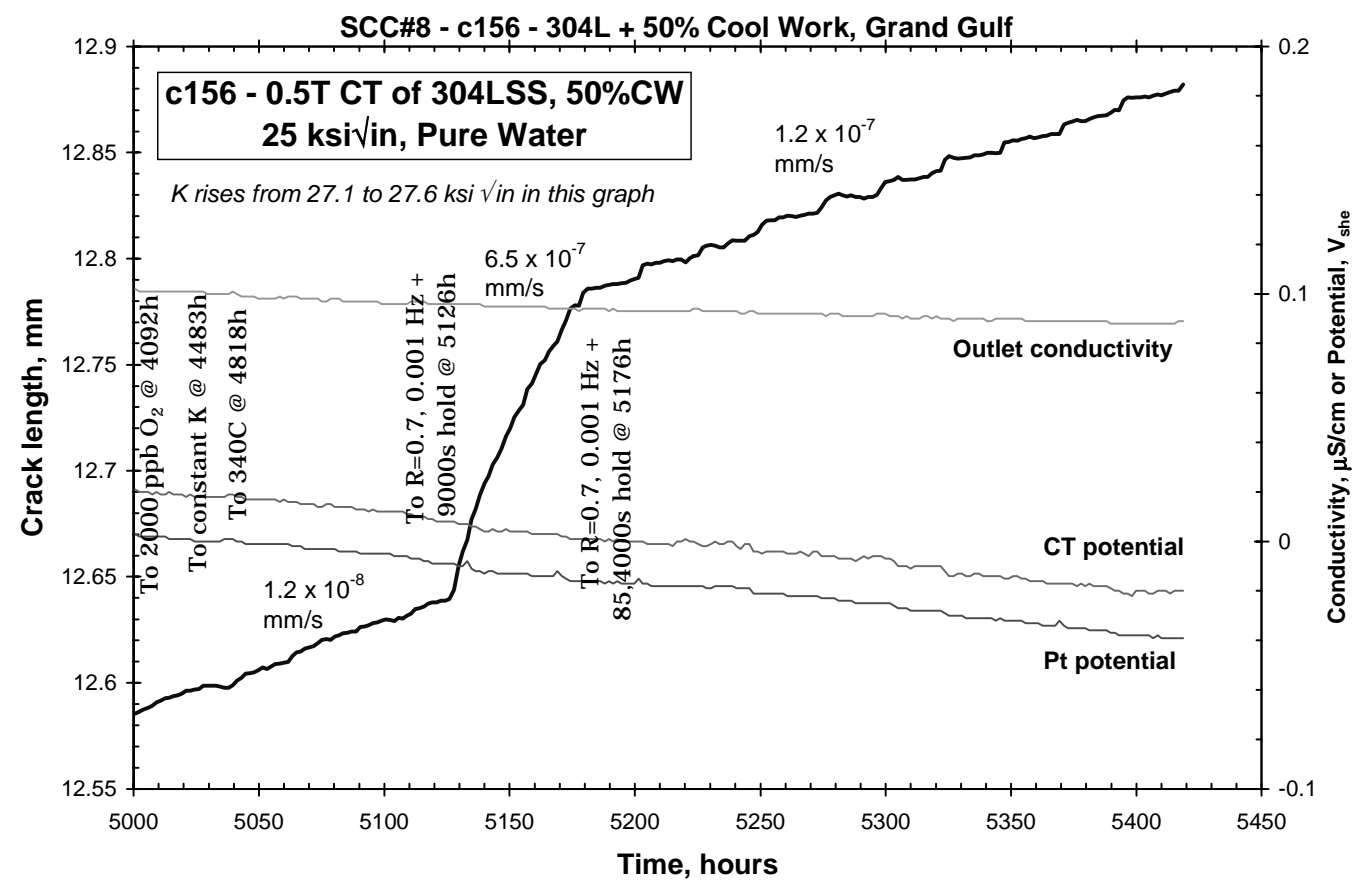

Figure 103. SCC behavior of specimen c156, showing the effect of gentle cycling to increase the crack-growth rate.

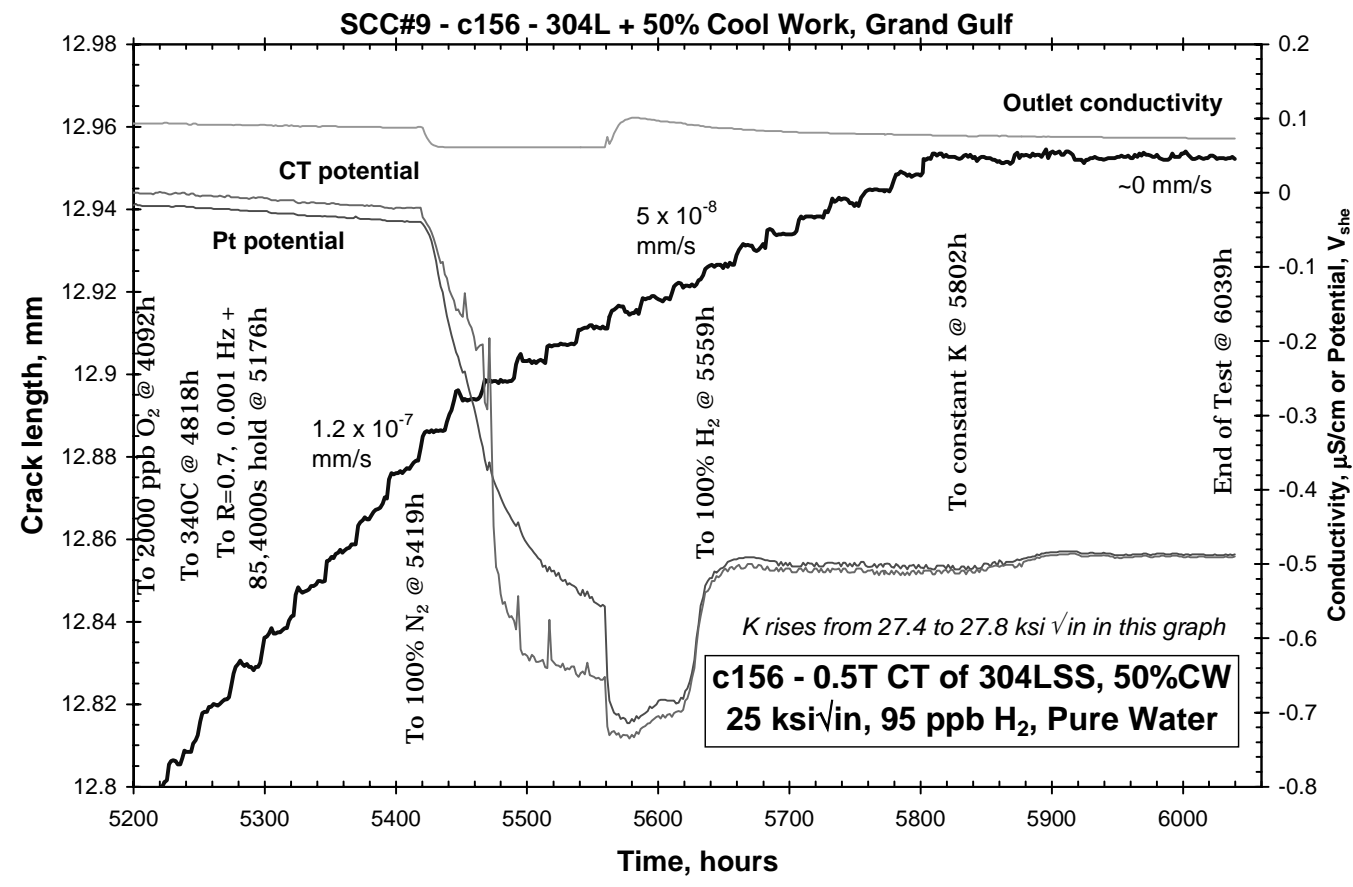

Figure 104. SCC behavior of specimen c156, showing the effect of $\mathrm{N}_{2}$ and $\mathrm{H}_{2}$ addition on the crack-growth rate. 
The test was ended at $6040 \mathrm{~h}$. Figure 105 shows a low-magnification macrograph of the fracture appearance, and fractography is documented by SEM in Figure 106.

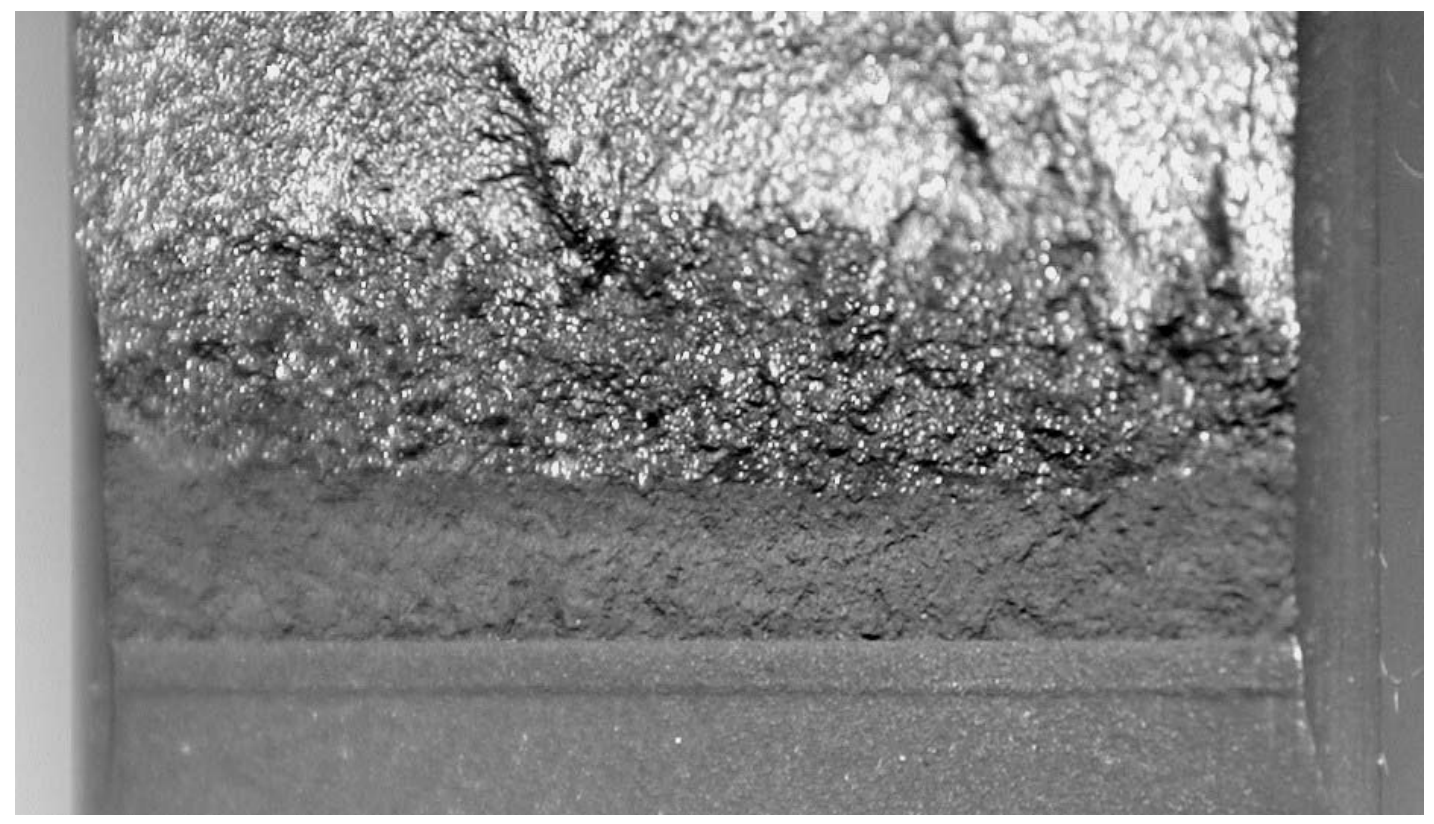

Figure 105. Light macrograph of specimen c156, Type 304L SS (Grand Gulf heat) cool rolled $50 \%$ at $+140^{\circ} \mathrm{C}$.

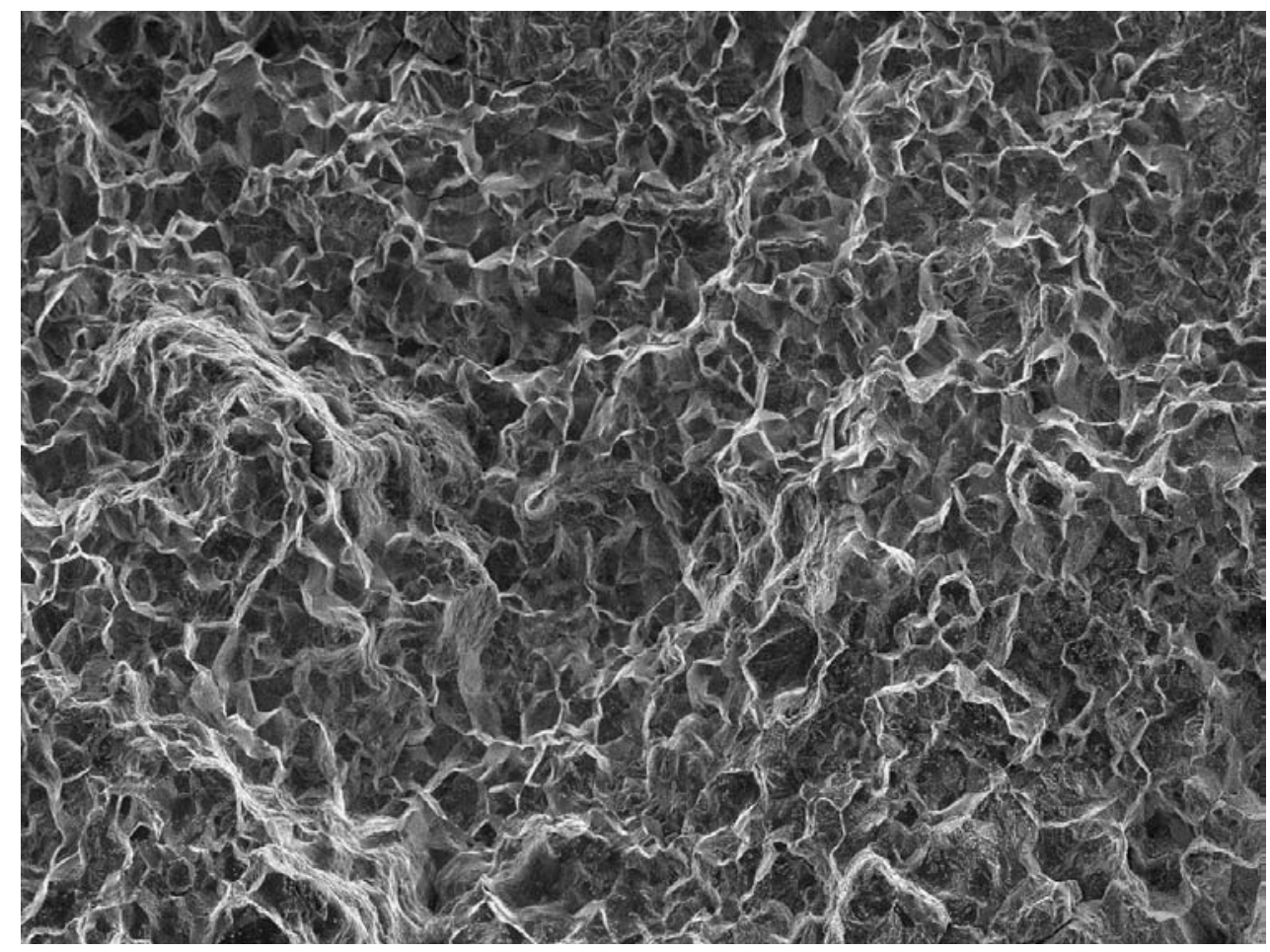

Figure 106. SEM micrograph of IG fracture surface for specimen c156. 
The dc potential drop indicated growth is $2.59 \mathrm{~mm}$, while the average, maximum and minimum of the actual crack depth are $3.89 \mathrm{~mm}, 5.44 \mathrm{~mm}$ and $2.5 \mathrm{~mm}$. This is reasonable agreement, given that potential drop is heavily influenced by the minimum crack depth. Although the error is about $50 \%$, no corrections to the crack length or stress intensity data of specimen c156 were performed because there is no accurate way to back correct the data. Given that the correction to the average actual crack depth is $\approx 1.5 \mathrm{X}$, there is some justification for correction. The effect of corrected crack length on stress intensity is noted in the figures.

Specimen c157 of 50\% CW 316L Stainless Steel The overview of this test is given in Figure 107 , which shows crack length vs. time, the corrosion potential of the CT specimen and a Pt electrode, and outlet solution conductivity. Conditions during the test were very well controlled, and $2000 \mathrm{~h}$ of continuous crack-growth testing has been performed to date.

Figure 108 shows the air-fatigue pre-cracking response of specimen c157. As is typical of fatigue and corrosion fatigue response, once the crack has nucleated from the machined notch, the changes in crack-growth rate are reflected quite crisply following changes in $\Delta \mathrm{K}$ or frequency. The growth rates in this $316 \mathrm{~L}$ stainless steel specimen are consistently higher during this transition period and subsequent test phases than in specimen c156 of 304L stainless steel.

Figure 109 shows the transition from fatigue pre-cracking conditions (where cracking is fully transgranular) to $\mathrm{SCC}$ (where cracking is IG). This was performed in $288^{\circ} \mathrm{C}$ pure water containing $2000 \mathrm{ppb} \mathrm{O}_{2}$, and the test showed well-behaved crack growth rate. The small but

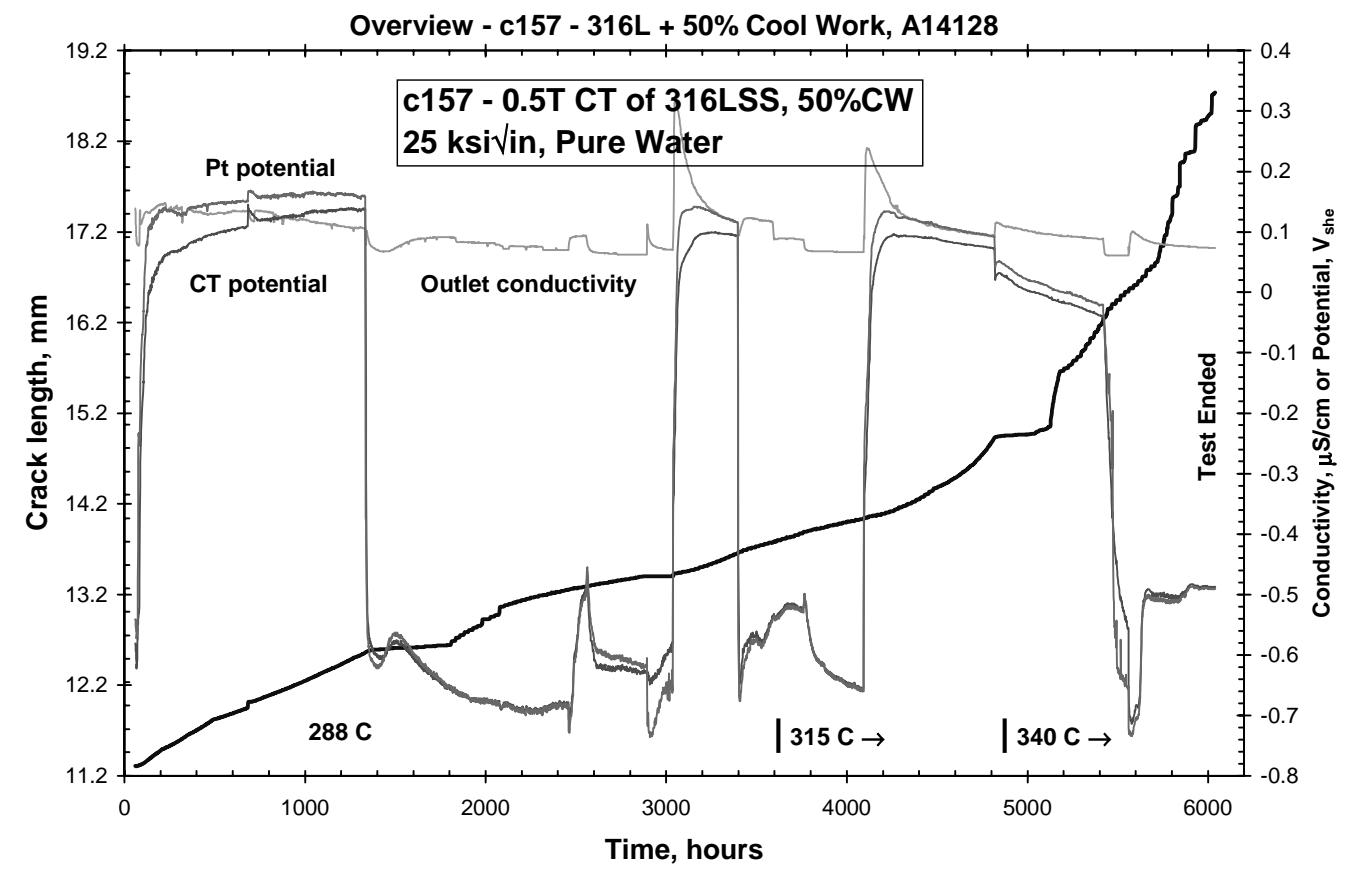

Figure 107. Overview of CT specimen c157, Type 316L SS (heat A14128) cool rolled 50\% at $+140^{\circ} \mathrm{C}$. 


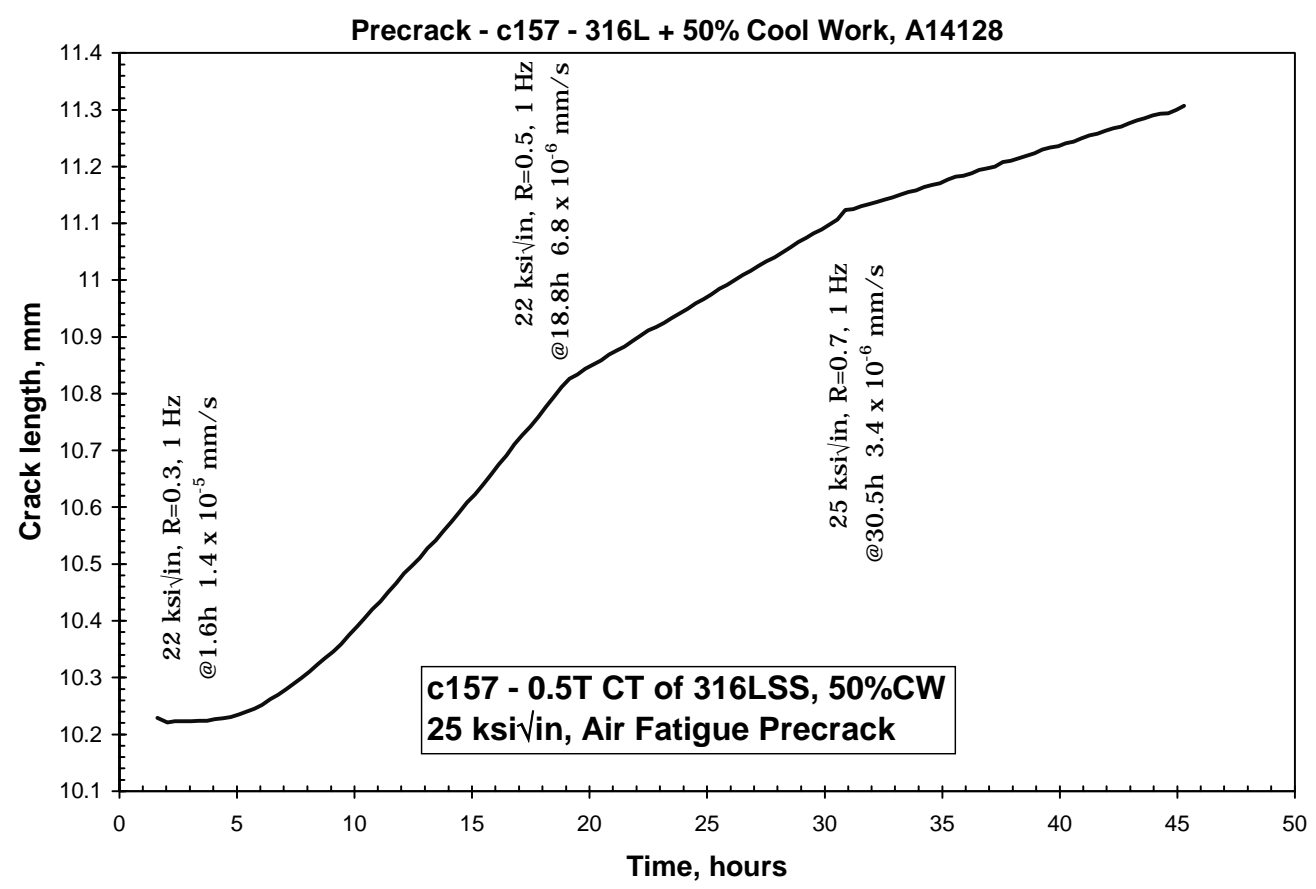

Figure 108. Air fatigue pre-cracking of CT specimen c157, Type 316L SS (heat A14128) cool rolled $50 \%$ at $+140^{\circ} \mathrm{C}$.

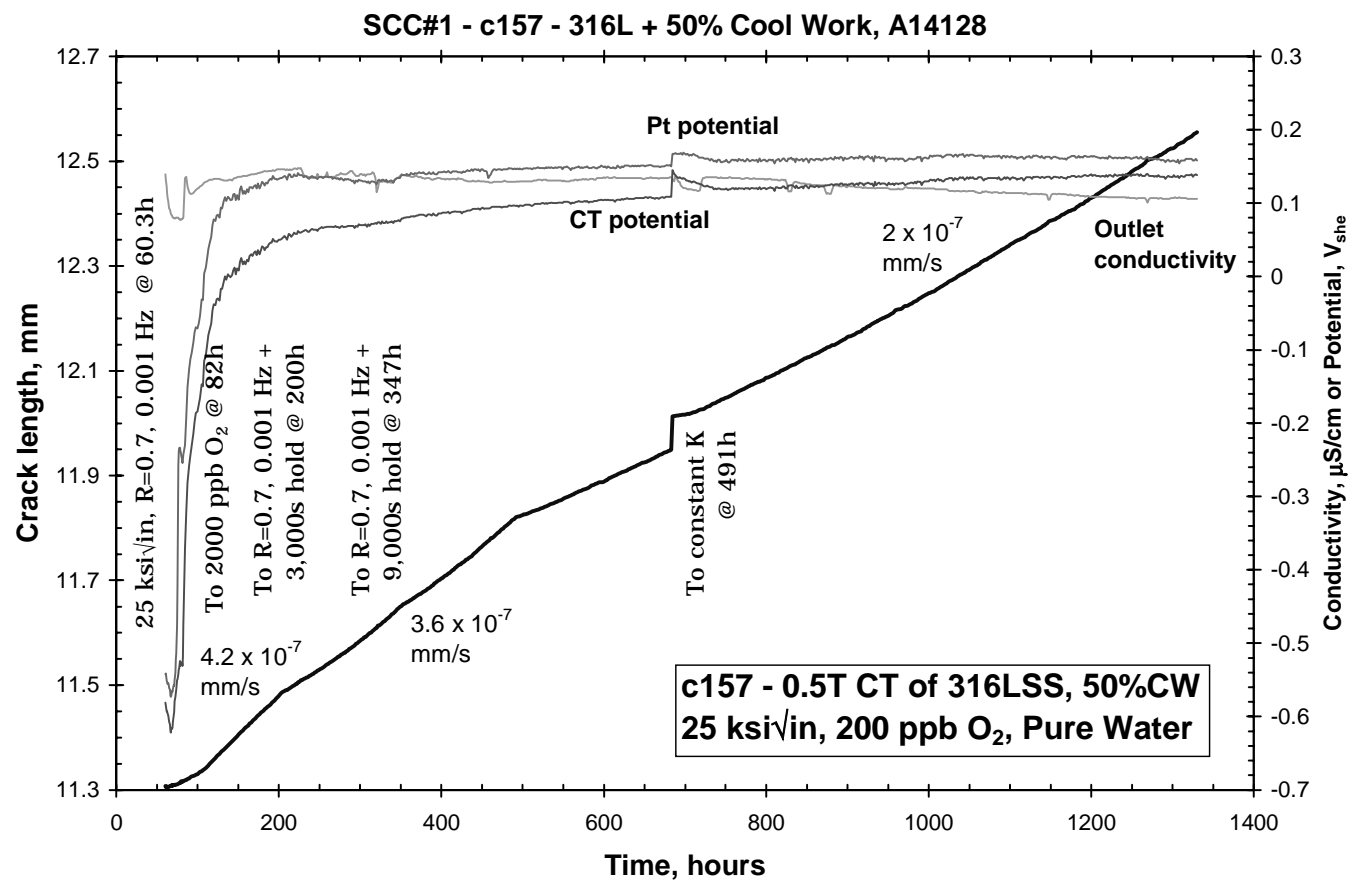

Figure 109. Transition from transgranular in-situ fatigue pre-cracking to IGSCC of 316L SS specimen c157. 
abrupt change in growth rate at $\approx 500 \mathrm{~h}$ does not correspond to any change in experimental conditions, and similar behavior was not observed in the tandem specimen, c156. Static loading (actually, constant stress intensity) was initiated at $491 \mathrm{~h}$. Figure 110 shows the well-behaved longer-term response under static loading conditions, where a crack growth rate of $\approx 2.0 \times 10^{-7}$ $\mathrm{mm} / \mathrm{s}$ was observed. Because $\mathrm{c} 157$ is growing faster than $\mathrm{c} 156, \mathrm{~K}$ is slowly rising from 28 to 30 $\mathrm{MPa} \sqrt{ } \mathrm{m}$ in this graph.

Figure 111 shows the change from $2000 \mathrm{ppb} \mathrm{O}_{2}$ to $95 \mathrm{ppb} \mathrm{H}_{2}$ at $1332 \mathrm{~h}$, which produced a rapid decrease in crack-growth rate to $3.5 \times 10^{-8} \mathrm{~mm} / \mathrm{s}$. At $1808 \mathrm{~h}$, a change to one unloading cycle $(\mathrm{R}=0.7,0.001 \mathrm{~Hz})$ per day was initiated. This increased the crack-growth rate very significantly to $3.5 \times 10^{-7} \mathrm{~mm} / \mathrm{s}$, an unexpectedly high value, although there is clearly some effect of the two complete unloading cycles at 1981 and $2077 \mathrm{~h}$ (which are neglected in calculating the growth rate). Over the next thousand hours, the growth rate slowed to $\approx 1 \times 10^{-7} \mathrm{~mm} / \mathrm{s}$. Because c157 is growing faster than c156, $\mathrm{K}$ is slow rising from 29 to $32 \mathrm{MPa} \sqrt{\mathrm{m}}$ in this graph.

The growth rates in between each "step" increase in crack length is $\approx 4 \times 10^{-8} \mathrm{~mm} / \mathrm{s}$ (Figures 112 and 113), a much more reasonable value for static loading conditions. It is unclear what is producing the step changes in crack length, although they correspond to the daily unloading cycles. This is an unusual observation in our laboratory, and it is certainly not paralleled in specimen c156 with which this specimen was tested in series. Most likely crack front unevenness that develops during constant stress intensity exposure is responsible for this behavior, although this could also be an indication of accelerated crack growth during reloading in the presence of $\mathrm{H}_{2}$.

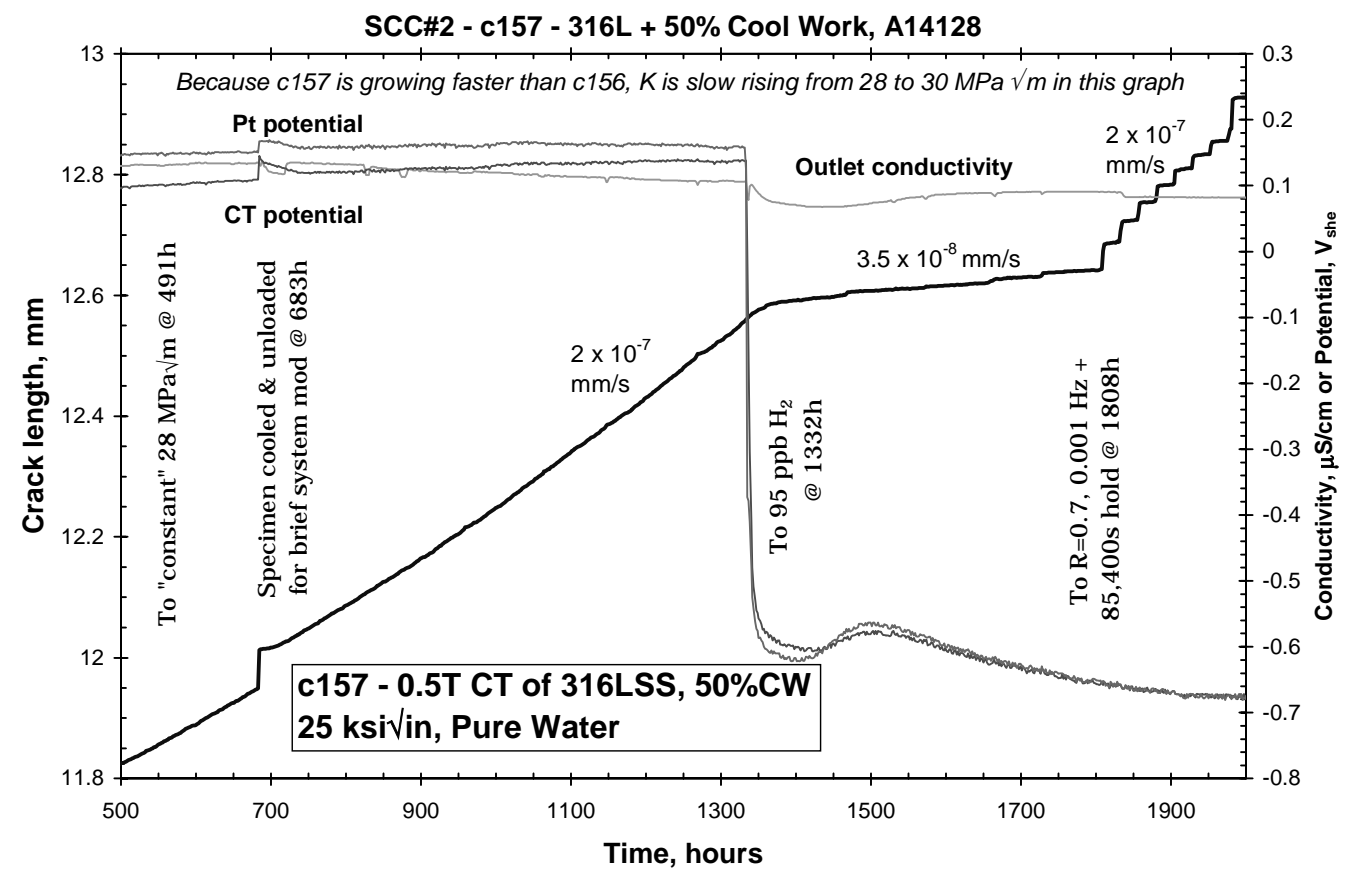

Figure 110. SCC behavior at static load of specimen c157, Type 316L SS (heat A14128) cool rolled $50 \%$ at $+140^{\circ} \mathrm{C}$. 


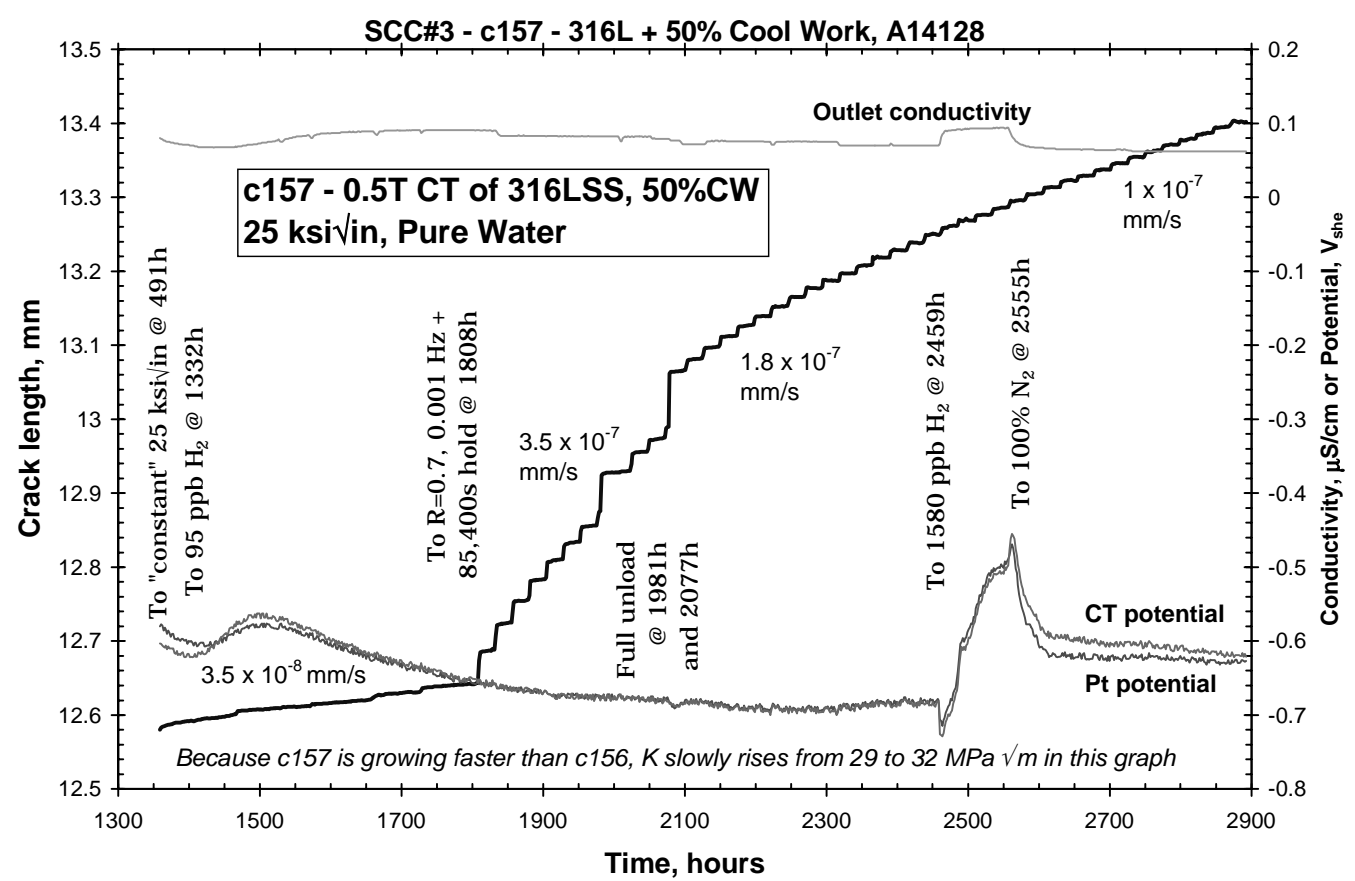

Figure 111. SCC behavior of specimen c157, Type 316L SS (heat A14128) cool rolled 50\% at $+140^{\circ} \mathrm{C}$ showing the effect of environment change.

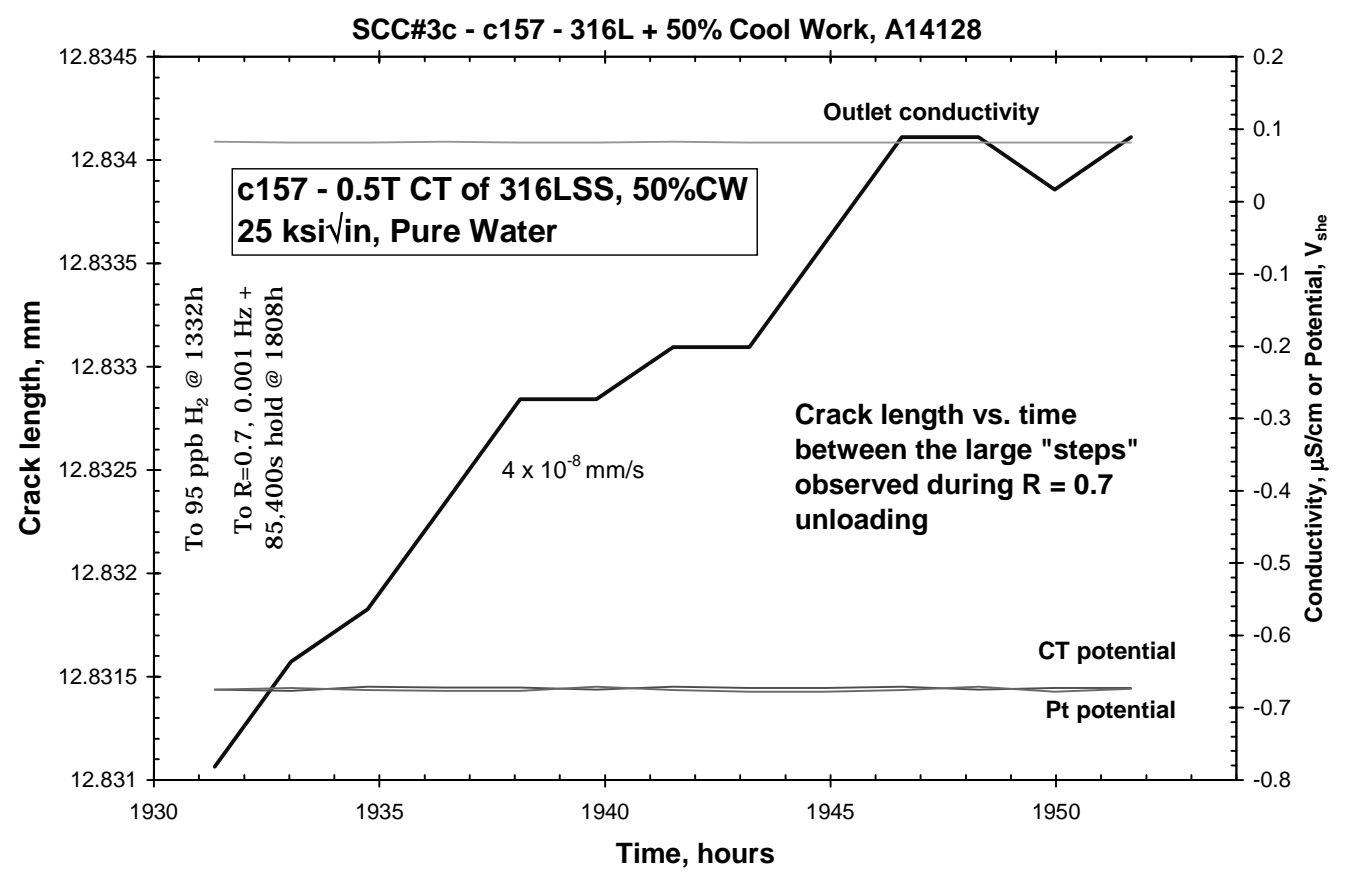

Figure 112. SCC behavior of specimen $\mathrm{c} 157$, unloaded at $\mathrm{R}=0.7,0.001 \mathrm{~Hz}$ per day. A rate of $2.0 \times 10^{-7} \mathrm{~mm} / \mathrm{s}$ is observed, but the rate in between each "step" is $\approx 4 \times 10^{-8} \mathrm{~mm} / \mathrm{s}$. 
From 2893 to $3037 \mathrm{~h}$, the temperature was decreased to $150^{\circ} \mathrm{C}$ to establish that no leakage was occurring in a hydrogen permeation tube that was a passive part of this experiment. At $3037 \mathrm{~h}$, the temperature was increased back to $288^{\circ} \mathrm{C}$ and the dissolved gas was changed from $\mathrm{H}_{2}$ to 2000 $\mathrm{ppb} \mathrm{O}_{2}$ (Figure 114). A growth rate of $2.2 \times 10^{-7} \mathrm{~mm} / \mathrm{s}$ was observed, very similar to the

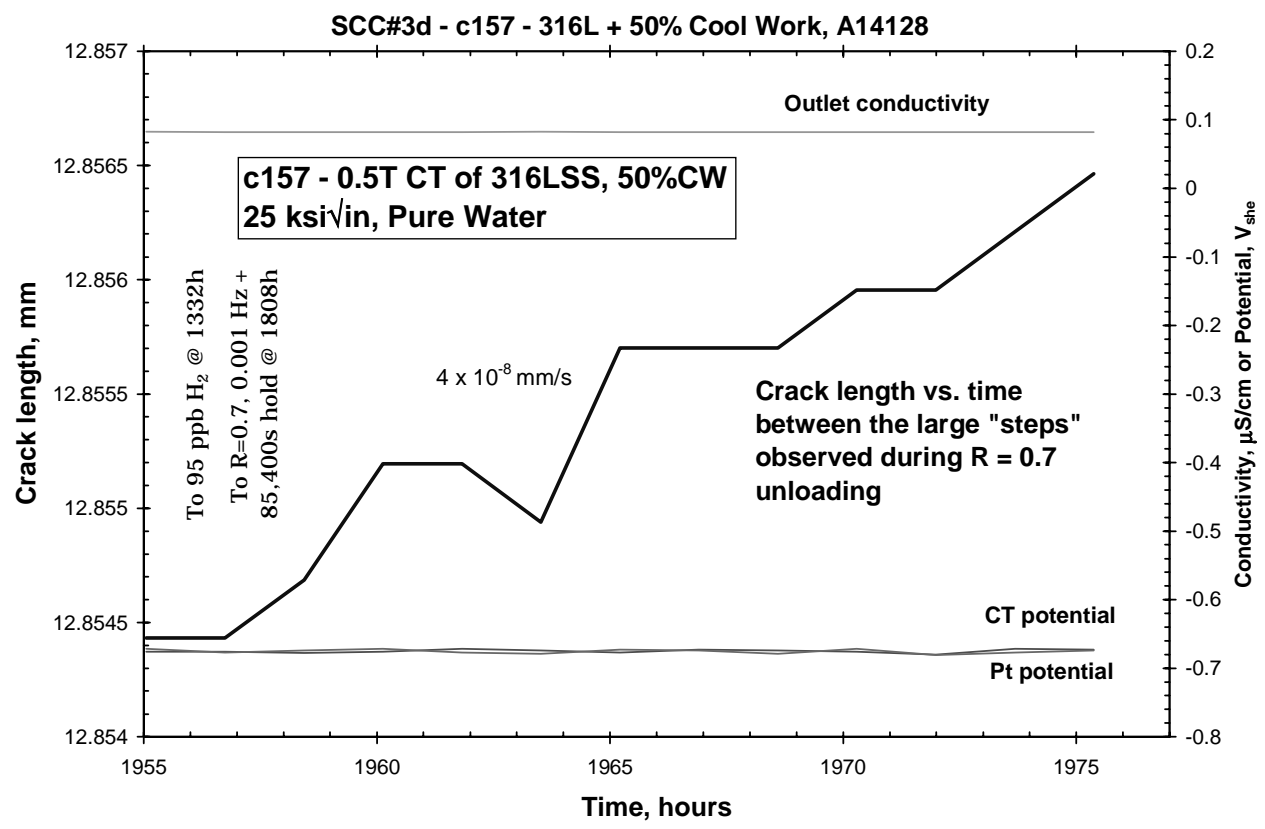

Figure 113. SCC behavior of $\mathrm{c} 157$, unloaded at $\mathrm{R}=0.7,0.001 \mathrm{~Hz}$ per day. A rate of $2.0 \times 10^{-7}$ $\mathrm{mm} / \mathrm{s}$ is observed, but the rate in between each "step" is $\approx 4 \times 10^{-8} \mathrm{~mm} / \mathrm{s}$.

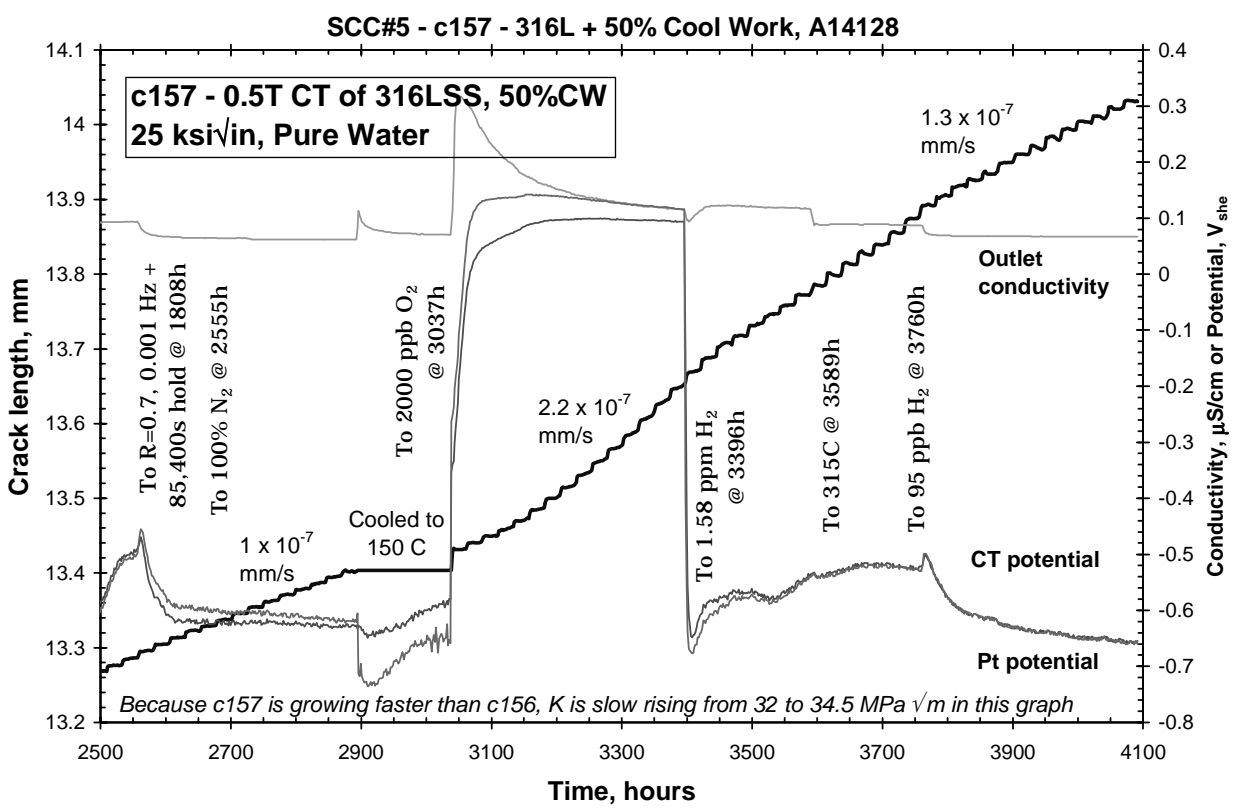

Figure 114. SCC behavior of specimen c157, Type 316L SS (heat A14128) cool rolled 50\% at $+140^{\circ} \mathrm{C}$, showing effects of temperature and environmental changes on crack growth. 
previous observation of $2.0 \times 10^{-7} \mathrm{~mm} / \mathrm{s}$ (Figure 110). At $3396 \mathrm{~h}$, the dissolved gas was changed to $1.58 \mathrm{ppm} \mathrm{H}_{2}$ and about $200 \mathrm{~h}$ later; the test temperature was increased to $315^{\circ} \mathrm{C}$. Under these conditions the crack growth rate increased from $1 \times 10^{-7} \mathrm{~mm} / \mathrm{s}$ observed earlier in Figure 114 to about $1.3 \times 10^{-7} \mathrm{~mm} / \mathrm{s}$. These rates are notably higher than were observed on Type 304L stainless steel.

At $4092 \mathrm{~h}$ (Figure 115), the dissolved gas conditions were changed to $2000 \mathrm{ppb} \mathrm{O}_{2}$ and the growth rate increased to $3.6 \times 10^{-7} \mathrm{~mm} / \mathrm{s}$, about twice that of $304 \mathrm{~L}$ stainless steel (Figure 113). This rate was initially sustained as the change was made from a gentle unloading cycle $(\mathrm{R}=0.7,0.001 \mathrm{~Hz})$ once per day to constant $\mathrm{K}$ conditions at $4483 \mathrm{~h}$. The change from a stepped or scalloped curve to a smooth curve shows the stepping appearance is caused by the occasional partial unloading cycles. With time, the growth rate increased to $8 \times 10^{-7} \mathrm{~mm} / \mathrm{s}$, a very high rate. At this point in the test, the stress intensity has increased from its original value of 27.5 $\mathrm{MPa} \sqrt{\mathrm{m}}$ to $\approx 36 \mathrm{MPa} \sqrt{\mathrm{m}}$.

The change from $315^{\circ} \mathrm{C}$ to $340^{\circ} \mathrm{C}$ at $4818 \mathrm{~h}$ (Figure 116) produced a dramatic reduction in crackgrowth rate, although the rate slowly increased from $4 \times 10^{-8}$ to $2 \times 10^{-7} \mathrm{~mm} / \mathrm{s}$ at this temperature. Note that the reference electrode requires different conversion factors at different temperatures, and the $\mathrm{pH}$ of pure water increases above about $260^{\circ} \mathrm{C}$, so direct comparison of reported corrosion potentials across multiple temperatures is misleading.

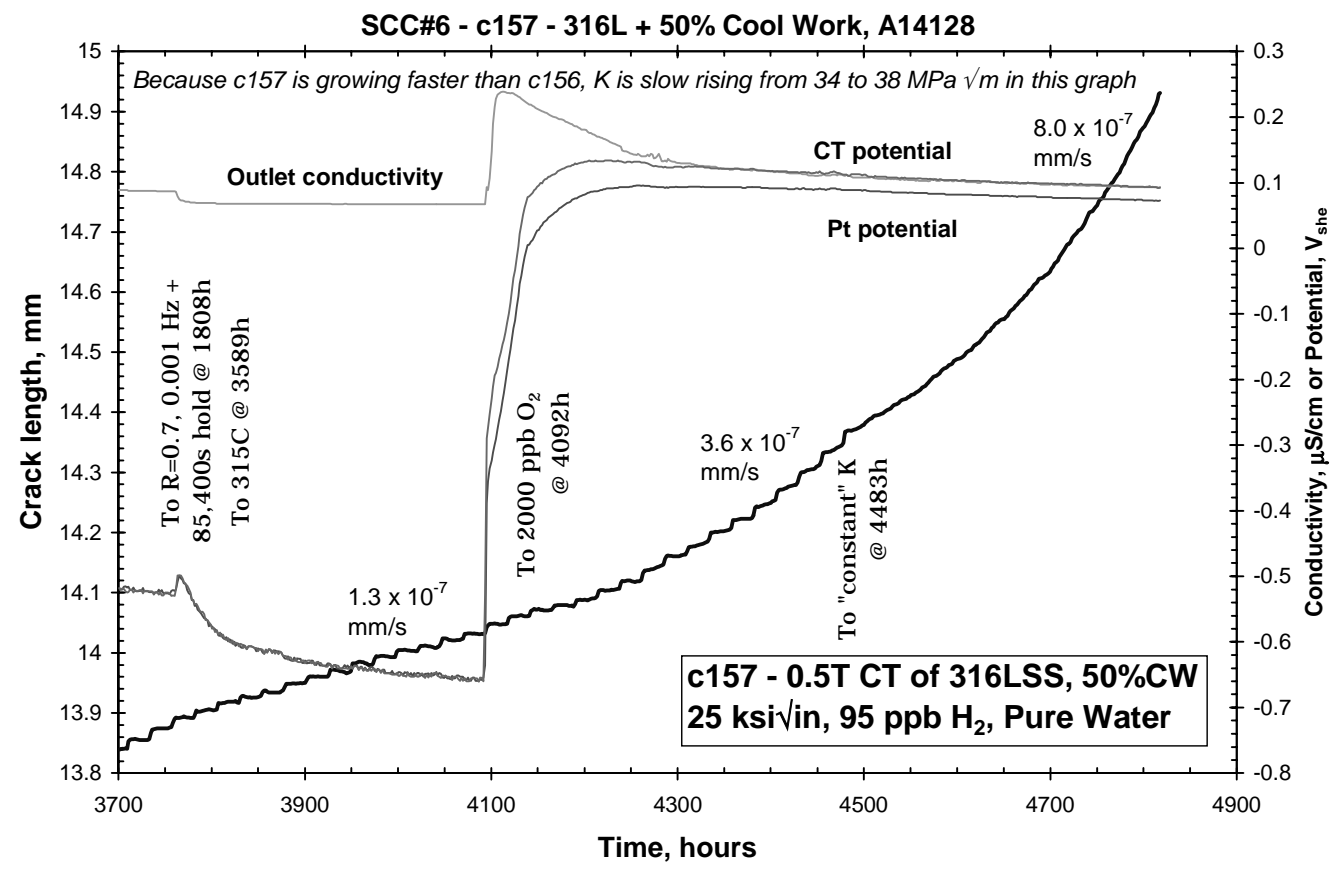

Figure 115. SCC behavior of Type 316L SS, 50\% cool-rolled specimen c157 showing the influence of $\mathrm{O}_{2}$ content and ECP in crack growth. 


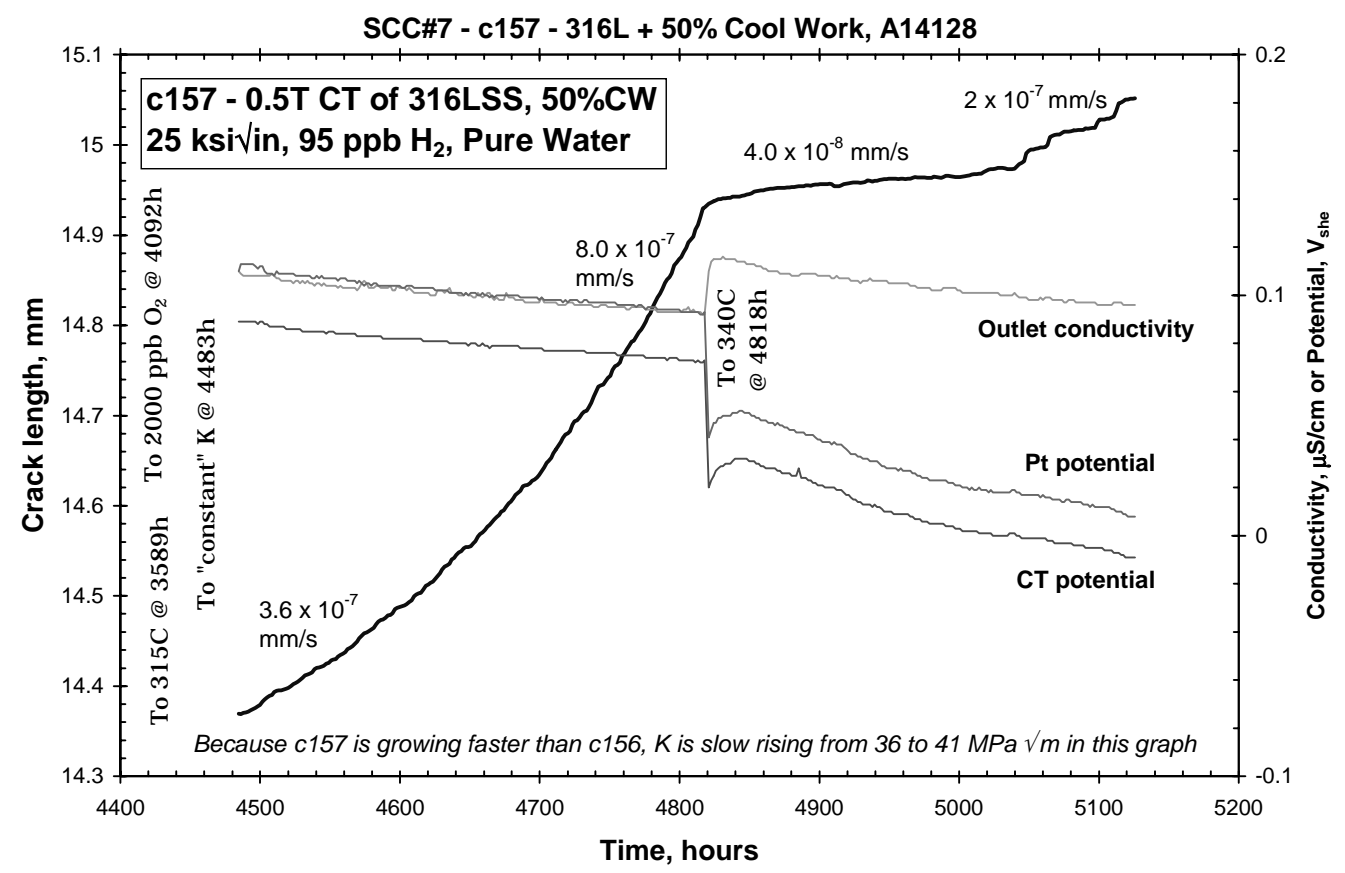

Figure 116. SCC behavior of specimen c157, showing decrease in crack growth rate with the increase in temperature from $315^{\circ} \mathrm{C}$ to $340^{\circ} \mathrm{C}$.

This growth rate was expected to increase with increasing water temperature, so gentle cycling was initiated at $5126 \mathrm{~h}$, which produced a large increase in growth rate to $2.5 \times 10^{-6} \mathrm{~mm} / \mathrm{s}$ (Figure 117). At $5176 \mathrm{~h}$ the hold time at $\mathrm{K}_{\max }$ was increased from $9,000 \mathrm{~s}$ to $85,400 \mathrm{~s}$ and the growth rate slowed back to $8.8 \times 10^{-7} \mathrm{~mm} / \mathrm{s}-$ still a very high rate. While this $316 \mathrm{~L}$ stainless steel specimen has consistently exhibited higher growth rate than the companion 304L stainless steel specimen (c156), the stress intensity in $\mathrm{c} 157$ has increased markedly from its original value of $27.5 \mathrm{MPa} \sqrt{\mathrm{m}}$ to $\approx 38 \mathrm{MPa} \sqrt{\mathrm{m}}$ at the start of Figure 117 to $\approx 45 \mathrm{MPa} \sqrt{\mathrm{m}}$ at the end of this graph.

At $5419 \mathrm{~h}$, the dissolved gas was changed to $100 \% \mathrm{~N}_{2}$ and then to $1.58 \mathrm{ppm}_{2}$ at $5559 \mathrm{~h}$. The growth rate slowed to $5 \times 10^{-7} \mathrm{~mm} / \mathrm{s}$ but then began to increase to $1.3 \times 10^{-6} \mathrm{~mm} / \mathrm{s}$ (Figures 118 and 119), despite changing to constant load conditions at $5802 \mathrm{~h}$. This high rate under static loading conditions in $\mathrm{H}_{2}$-deaerated water can be explained by the very high stress intensity factors that developed as the crack grew. Because c157 is growing faster than $\mathrm{c} 156, \mathrm{~K}$ is slow rising from 41 to $\approx 74 \mathrm{MPa} \sqrt{ } \mathrm{m}$ in Figure 118 . The test was stopped as the $\mathrm{a} / \mathrm{W} \approx 0.738$, so the remaining ligament is $\approx 6.6 \mathrm{~mm}$. Using the $2.5\left(\mathrm{~K} / \sigma_{\mathrm{YS}}\right)^{2}$ from E399 (and also employing the "flow stress" calculation for yield stress of $\left.\sigma_{\mathrm{FS}}=\sigma_{\mathrm{YS}}+\sigma_{\mathrm{UTS}}\right) / 2$ ), the maximum allowable $\mathrm{K}$ is about $43 \mathrm{MPa} \sqrt{\mathrm{m}}$. Using the E647 criteria of $(4 / \pi)\left(\mathrm{K} / \sigma_{\mathrm{YS}}\right)^{2}$, the maximum allowable $\mathrm{K}$ is about $60 \mathrm{MPa} \vee \mathrm{m}$. Thus, much of the data obtained with within LEFM validity. This may be surprising for a $0.5 \mathrm{TCT}$ specimen, but there is a huge effect of a $\sigma_{\mathrm{FS}}=780 \mathrm{MPa}$.

The test was ended at $6040 \mathrm{~h}$. Figure 120 shows a low magnification macrograph of the fracture appearance, and more detailed fractography is documented by SEM in Figure 21. The 


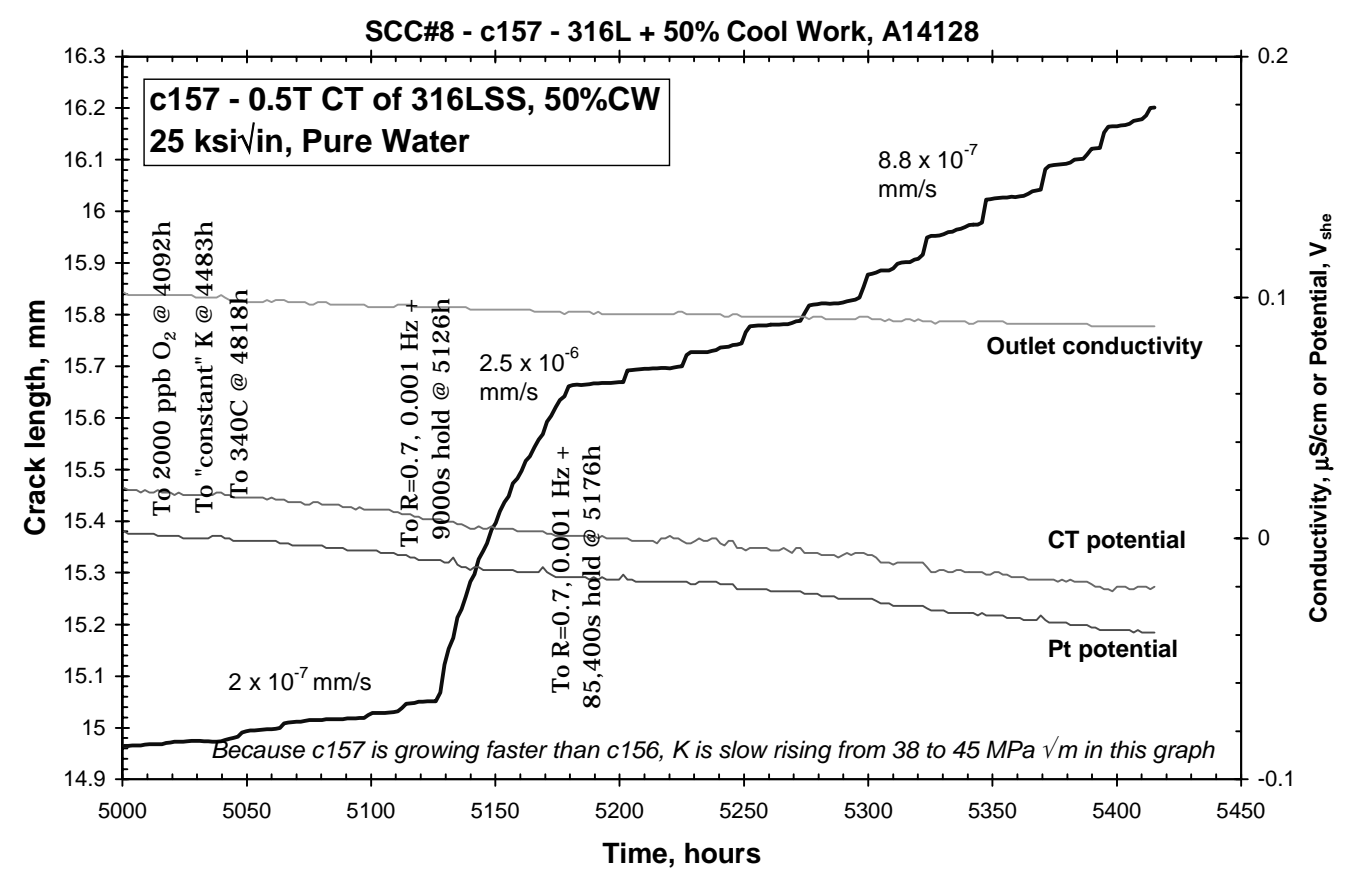

Figure 117. SCC behavior of 50\% cold-rolled, 316L SS specimen c157, showing a large increase in crack growth rate with cyclic loading.

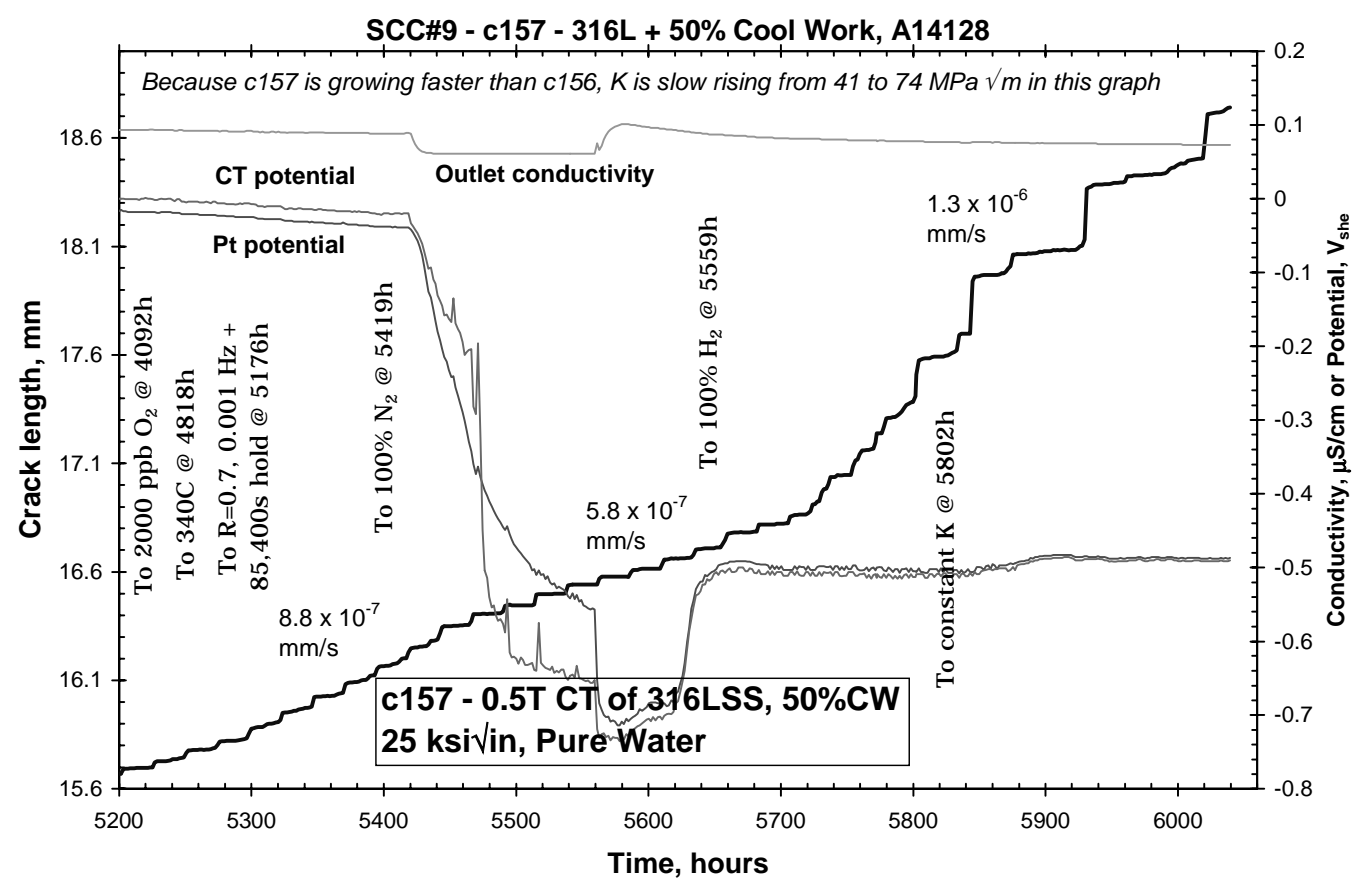

Figure 118. Complex effects of environment change and increasing K on SCC behavior of specimen $\mathrm{c} 157$. 
dc potential drop indicated growth is $8.51 \mathrm{~mm}$, while the average, maximum and minimum of the actual crack depth are $10.4 \mathrm{~mm}, 12.6 \mathrm{~mm}$ and $\approx 7.4 \mathrm{~mm}$. Because there was only an $18 \%$ error, no corrections to the crack length data of specimen c157 were performed. However, because c156 was used to control the stress intensity and the crack grew faster in c157, the effect on stress intensity factor is noted on the figures.

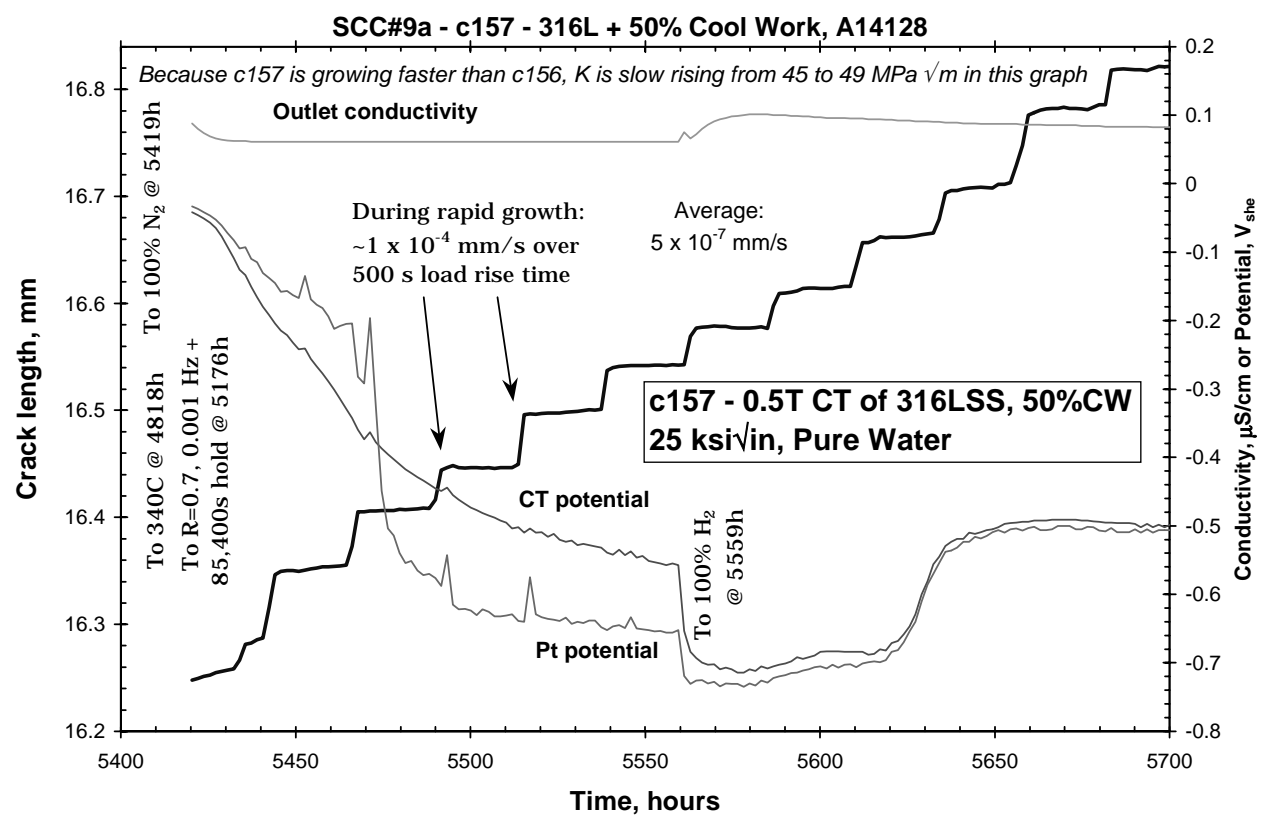

Figure 119. SCC behavior at the end of this experiment on specimen c157, Type 316L SS (heat A14128) cool rolled $50 \%$ at $+140^{\circ} \mathrm{C}$.

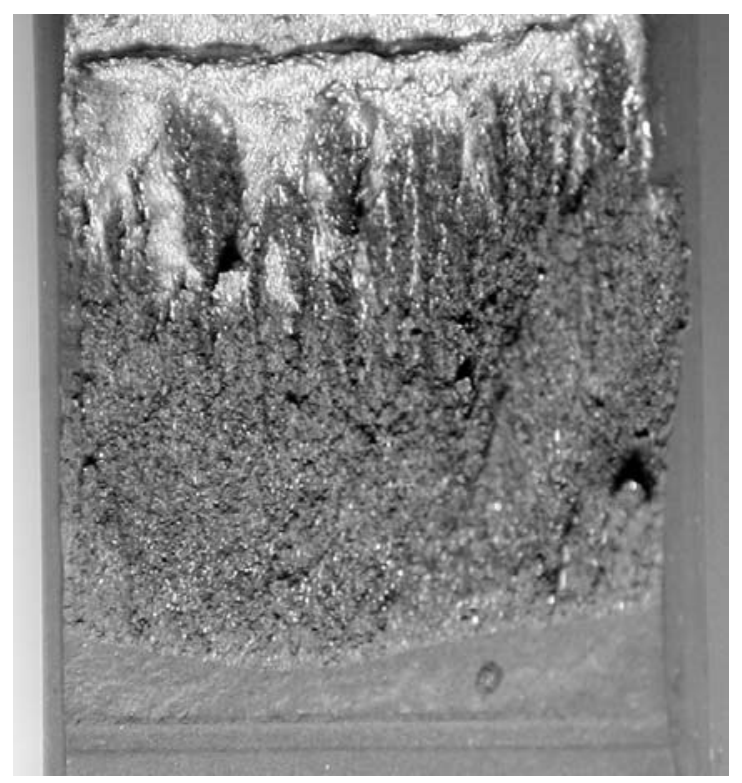

Figure 120. Light macrograph of 50\% cold-rolled, 316L SS specimen c157. 


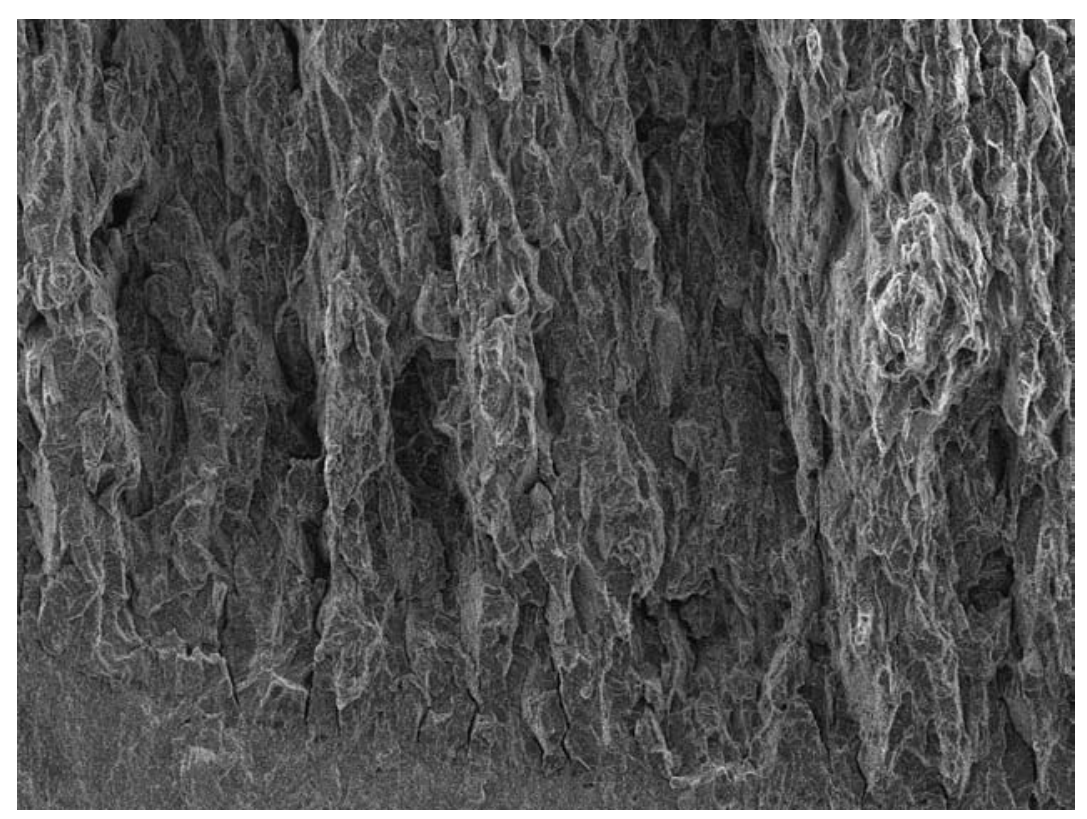

Figure 121. SEM micrograph of fracture surface for specimen c157, Type 316L SS (heat A 14128) cool rolled $50 \%$ at $+140^{\circ} \mathrm{C}$.

Specimen c158 of 10\% CW 304L Stainless Steel Figure 122 provides an overview of this test, which includes crack length vs. time, the corrosion potential of the CT specimen and a $\mathrm{Pt}$ electrode, and outlet solution conductivity. Conditions during the test were very well controlled throughout the $7885 \mathrm{~h}$ of crack-growth testing.

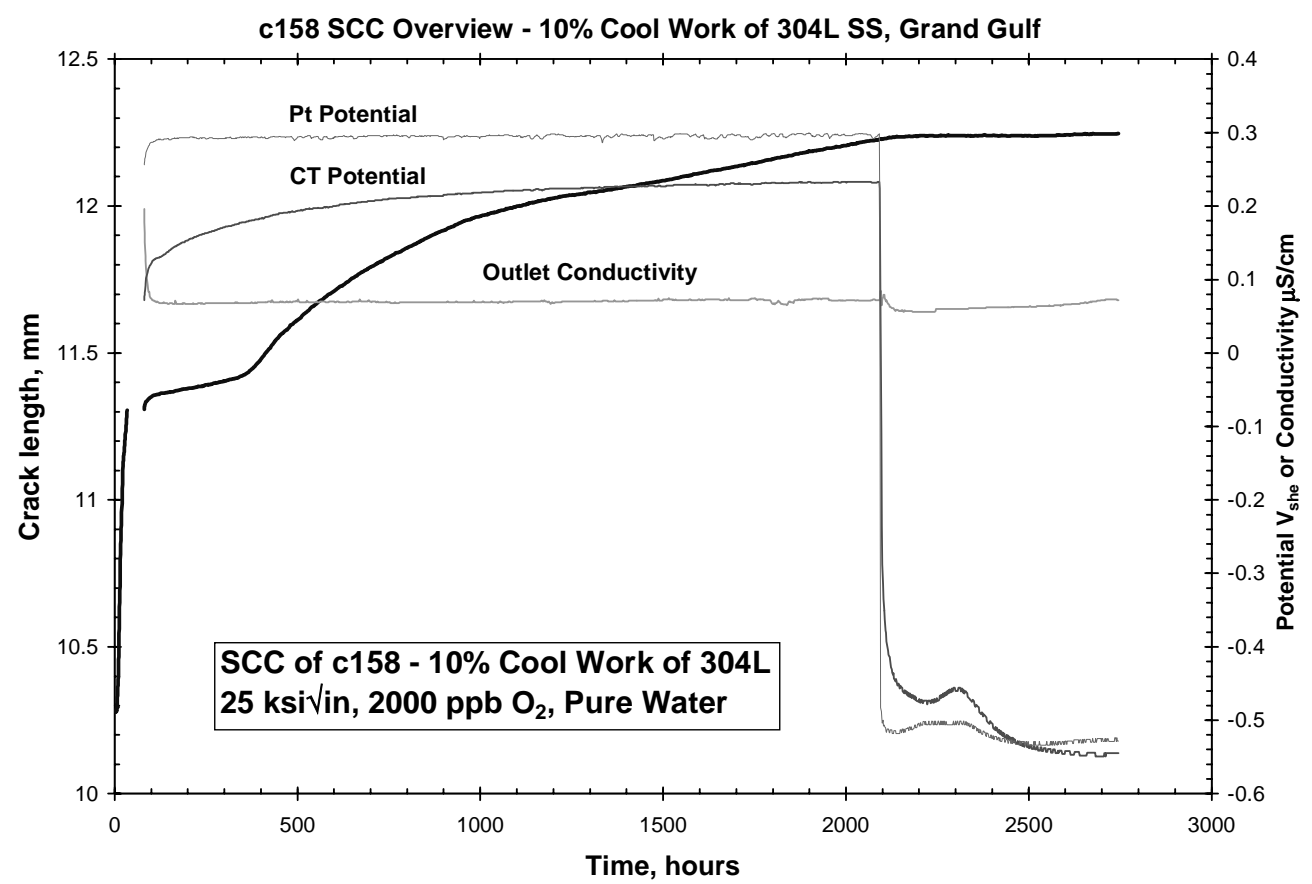

Figure 122. Overview of CT specimen c158, type 304L SS (Grand Gulf heat) cool rolled 10\% at $+140^{\circ} \mathrm{C}$. 
0.5TCT specimens c158 and c159 were tested in tandem, so their fatigue pre-cracking was performed in air. The air fatigue pre-cracking response of specimen c158 is shown in Figure 123. As in prior tests, once the crack has nucleated from the machined notch, the fatigue and corrosion fatigue crack growth rate response reflects the changes in $\Delta \mathrm{K}$ or frequency.

Figure 124 shows the IG transition response in $288^{\circ} \mathrm{C}$ pure water containing $2000 \mathrm{ppb} \mathrm{O}_{2}$ following fatigue precracking in air. A fairly long transition period is observed after

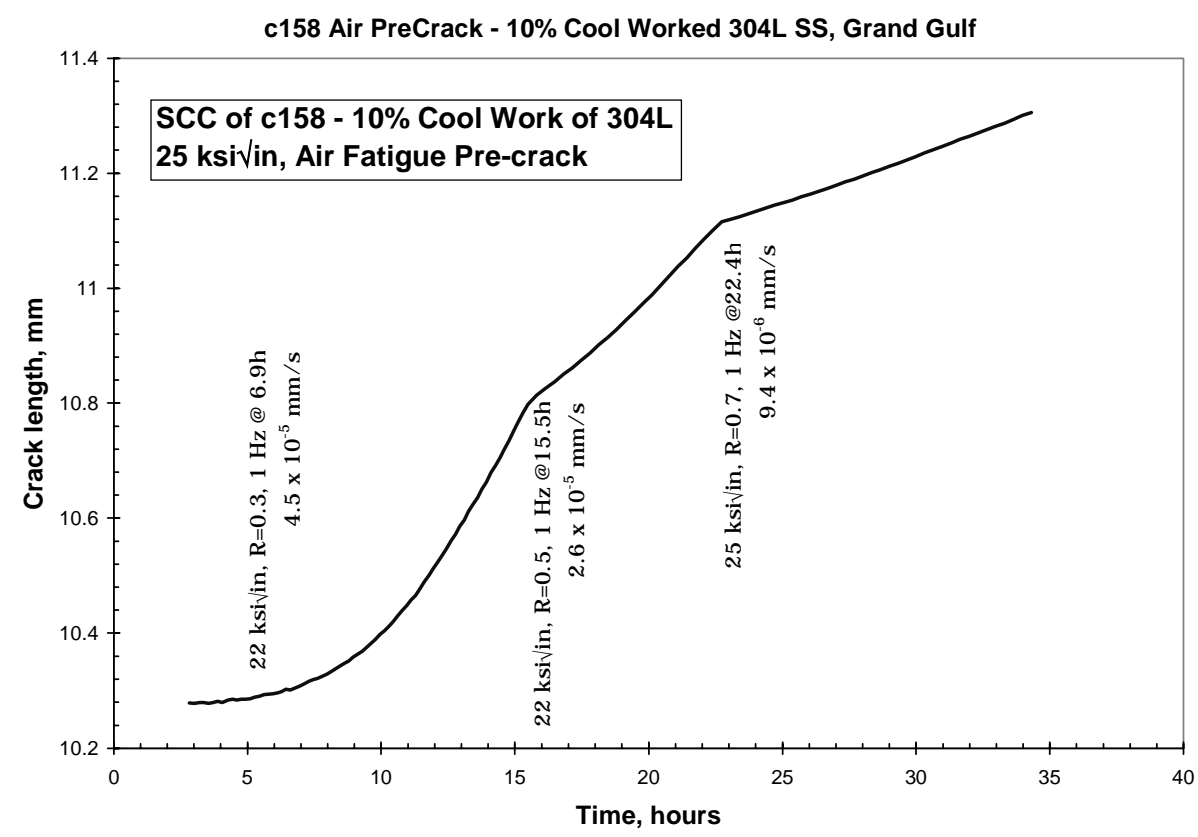

Figure 123. Air fatigue pre-cracking of CT specimen c158.

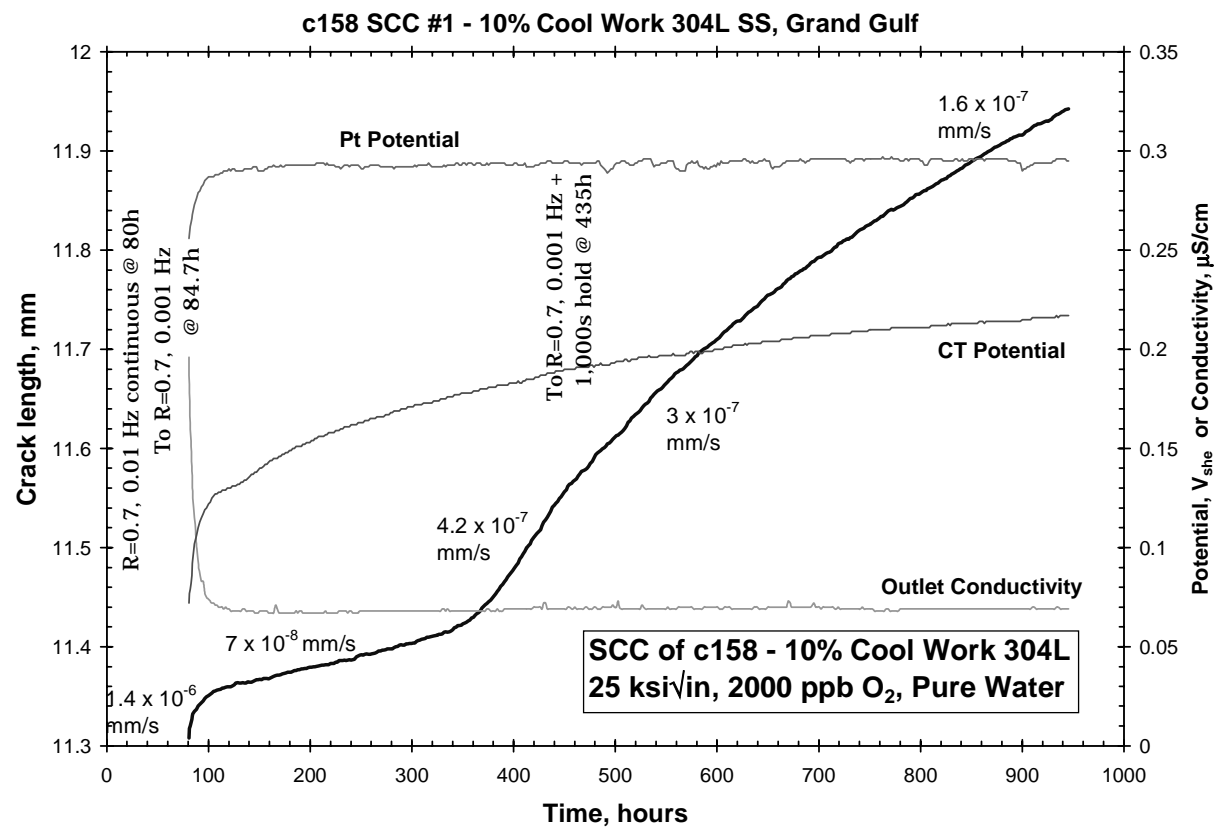

Figure 124. Transition from transgranular in-situ fatigue to IGSCC. 
changing to $\mathrm{R}=0.7,0.001 \mathrm{~Hz}$ loading at $84.7 \mathrm{~h}$. It's not until about $350 \mathrm{~h}$ that the growth rate increases to the expected value. On changing to $\mathrm{R}=0.7,0.001 \mathrm{~Hz}$ loading with a 1,000 -s hold time at $435 \mathrm{~h}$, the growth rate slowly decreased from $3 \times 10^{-7} \mathrm{~mm} / \mathrm{s}$ to $1.6 \times 10^{-7} \mathrm{~mm} / \mathrm{s}$. The crack growth rate then decreased to $8.0 \times 10^{-8} \mathrm{~mm} / \mathrm{s}$ after increasing the hold time to $9,000 \mathrm{~s}$ (Figure 125). After changing to constant load at $1235 \mathrm{~h}$, the growth rate changed to $6.5 \times 10^{-8}$ $\mathrm{mm} / \mathrm{s}$, and was sustained in a highly linear fashion for over $900 \mathrm{~h}$.

Figure 126 shows the effect of the change to $95 \mathrm{ppb} \mathrm{H}_{2}$ at $2091 \mathrm{~h}$, which immediately reduced the crack growth rate to $\approx 1 \times 10^{-9} \mathrm{~mm} / \mathrm{s}$. At $2575 \mathrm{~h}$, a once-a-day unloading cycle at $\mathrm{R}=0.7$, $0.001 \mathrm{~Hz}$ was initiated, and the growth rate increased to $\approx 6 \times 10^{-9} \mathrm{~mm} / \mathrm{s}$. The dissolved gas was changed to $2000 \mathrm{ppb} \mathrm{O}_{2}$ at $3437 \mathrm{~h}$, and the growth rate rapidly increased to $1 \times 10^{-7} \mathrm{~mm} / \mathrm{s}$ (Figure 127). At $4281 \mathrm{~h}$, the dissolved gas was changed back to $95 \mathrm{ppb} \mathrm{H}_{2}$ and the growth rate rapidly dropped to $\approx 3 \times 10^{-9} \mathrm{~mm} / \mathrm{s}$.

The dissolved gas was changed back to $2000 \mathrm{ppb}_{2}$ at $5796 \mathrm{~h}$, and the growth rate seemed to rapidly increase, then slow somewhat to $4 \times 10^{-8} \mathrm{~mm} / \mathrm{s}$ (Figure 128). At $6486 \mathrm{~h}$, a $\mathrm{R}=0.7,0.001$ $\mathrm{Hz}$ with a 9,000-s hold was initiated, and the growth rate increased to $1.2 \times 10^{-7} \mathrm{~mm} / \mathrm{s}$, where it remained steady for several hundred hours.

Repeating the previous sequence, the dissolved gas was again changed to $95 \mathrm{ppb}_{2}$, which produced a rapid decrease in crack growth rate to $4 \times 10^{-9} \mathrm{~mm} / \mathrm{s}$ (Figures 129 and 130). At 7401 $\mathrm{h}$, the hold time was increased to $85,400 \mathrm{~s}$, which produced no notable change in growth rate.

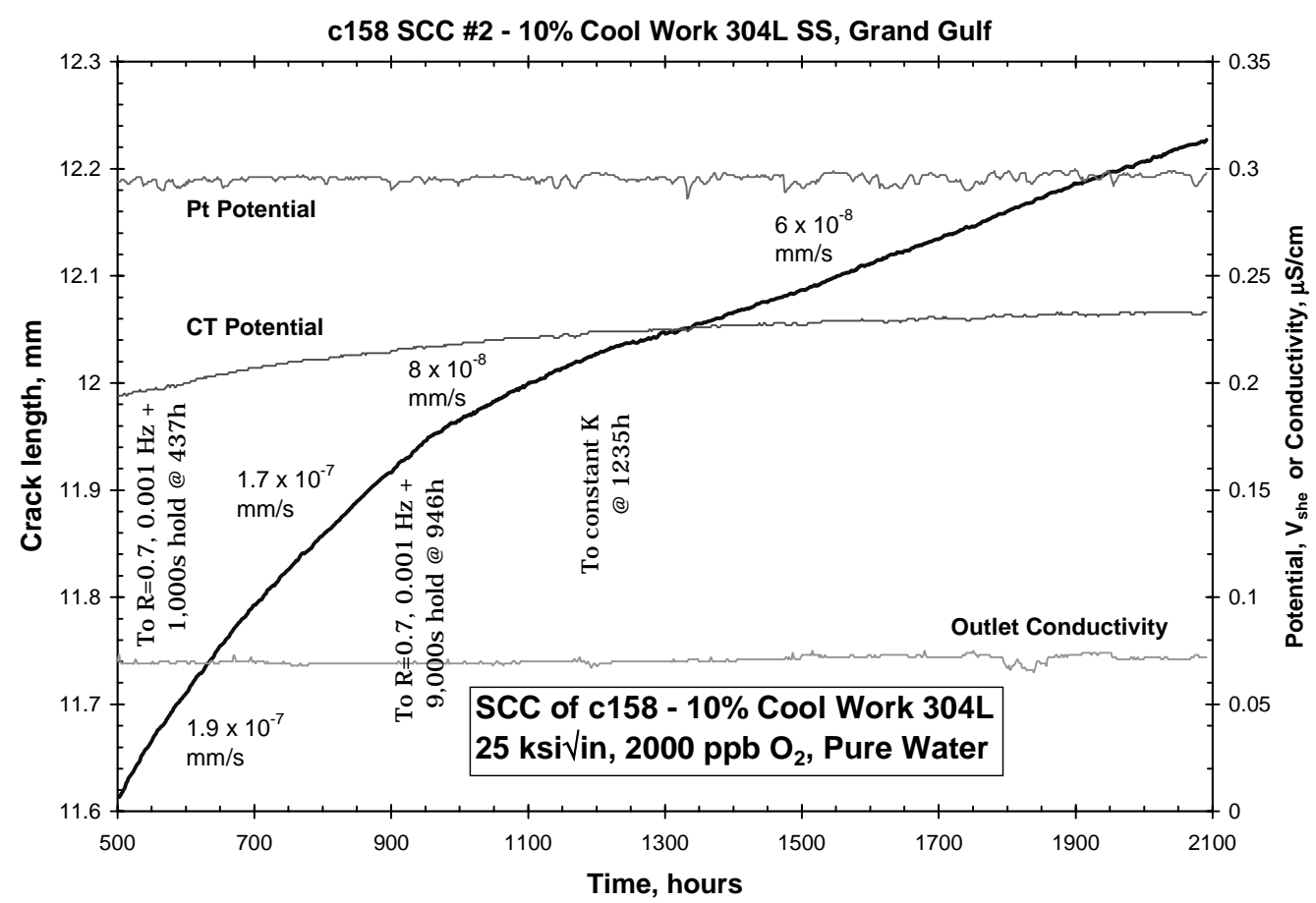

Figure 125. SCC behavior at static load of specimen c158, Type 304L SS (Grand Gulf heat) cool rolled $10 \%$ at $+140^{\circ} \mathrm{C}$. 


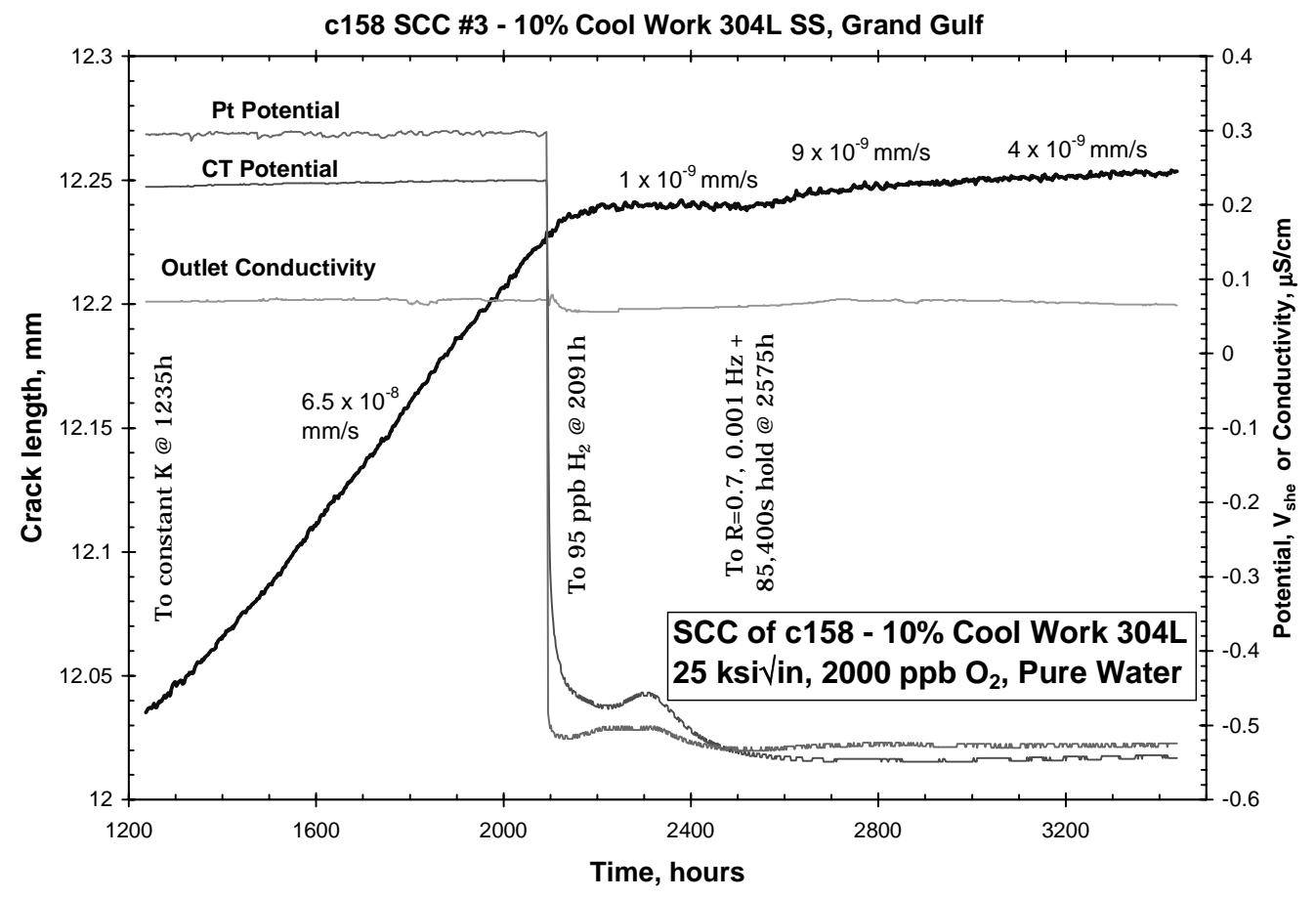

Figure 126. SCC behavior at static load of specimen c158 showing effect of $\mathrm{H}_{2}$ addition.

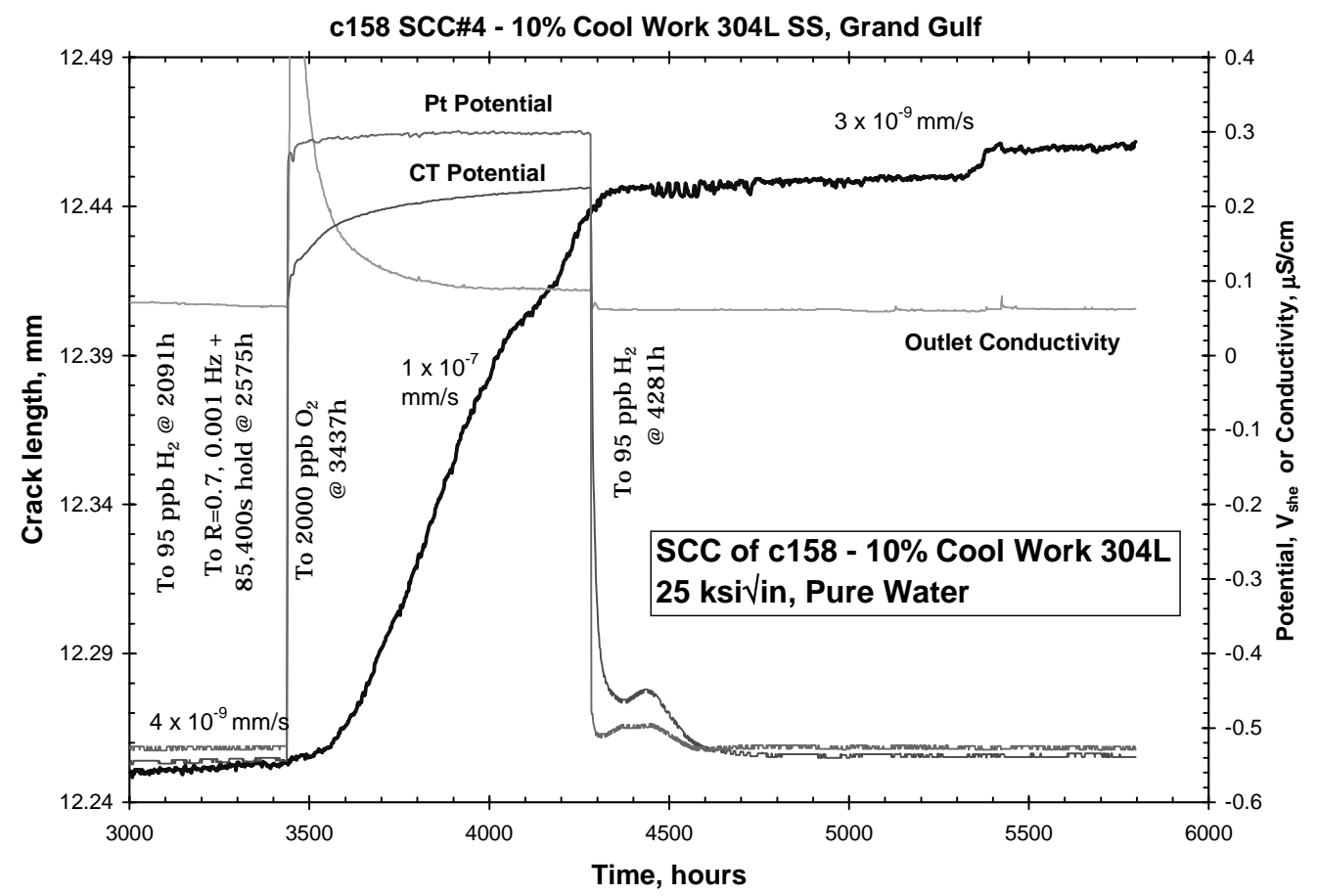

Figure 127. SCC behavior at static load of Type 304LSS specimen c158 repeating effect of $\mathrm{O}_{2}$ and $\mathrm{H}_{2}$ on crack growth rate. 


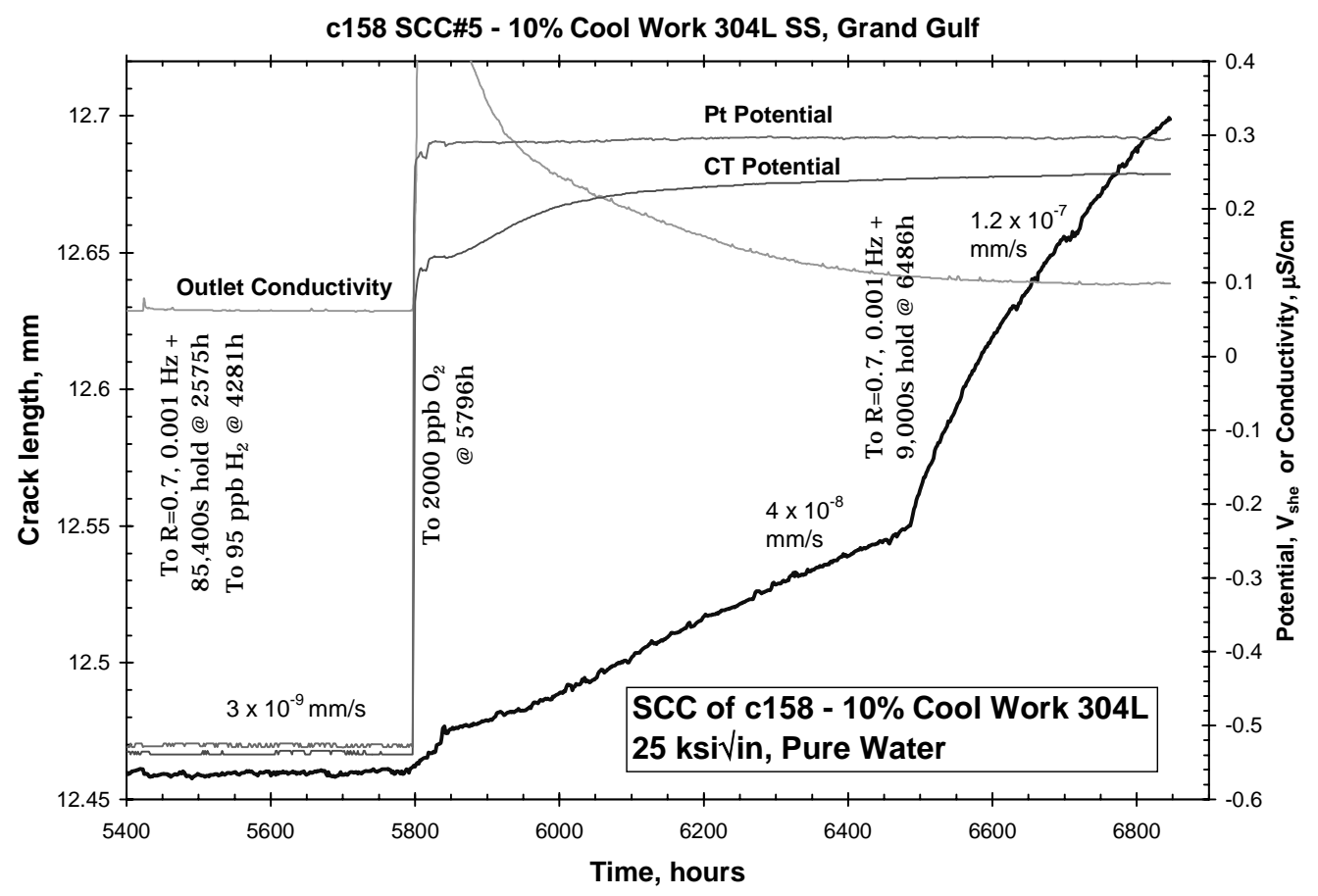

Figure 128. SCC behavior at static load of specimen c158, showing effect of environment change and cyclic loading.

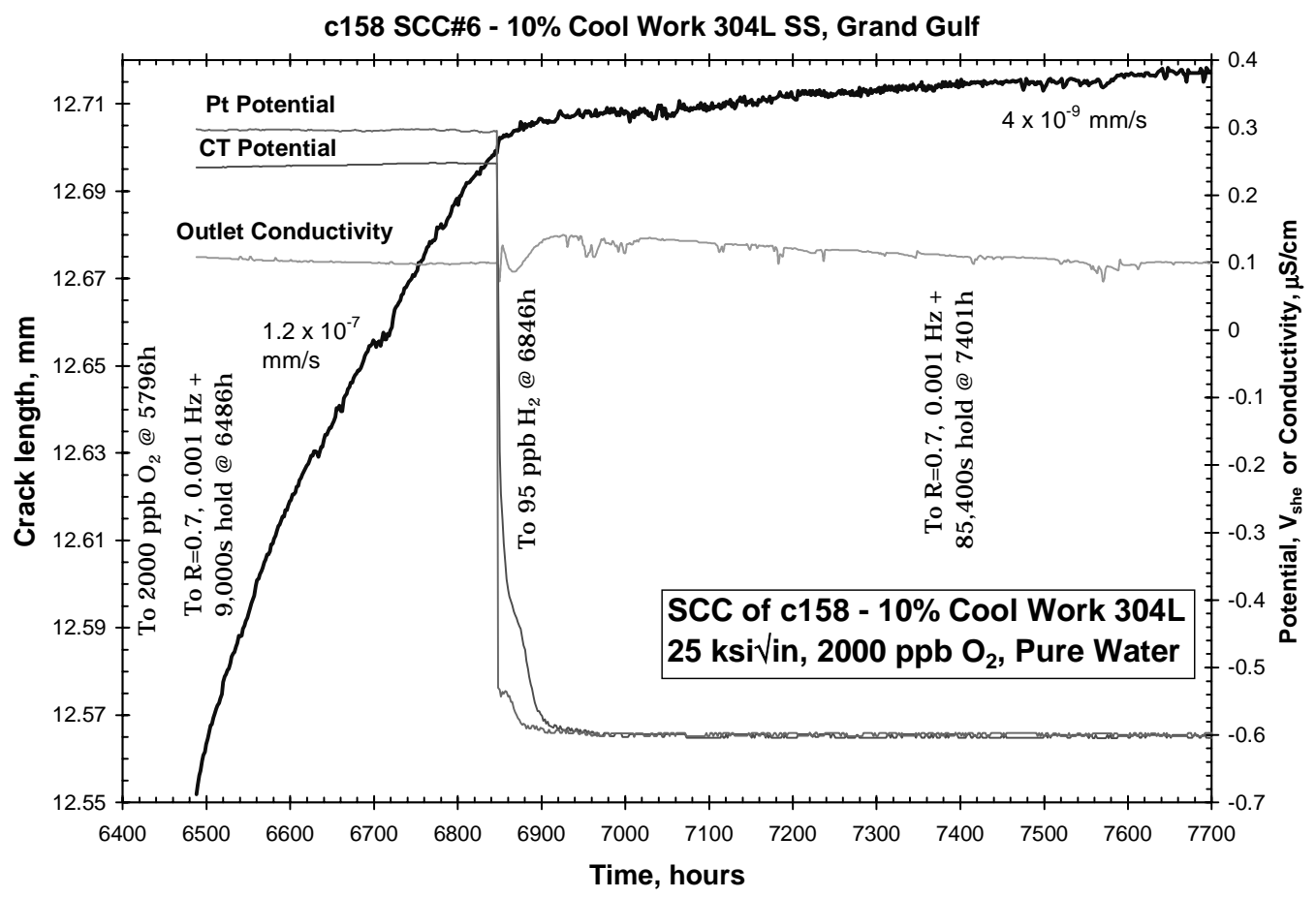

Figure 129. Influence of $\mathrm{H}_{2}$ addition on the SCC behavior at static load of specimen c158, Type 304L SS (Grand Gulf heat) cool rolled $10 \%$ at $+140^{\circ} \mathrm{C}$. 
The test was ended at $7885 \mathrm{~h}$. Figure 131 shows a low magnification macrograph of the fracture appearance, and Figure 132 documents the predominantly IG fracture path by SEM. The dc potential drop indicated growth is $2.44 \mathrm{~mm}$, while the average, maximum and minimum of the actual crack depth are $2.78 \mathrm{~mm}, 2.60 \mathrm{~mm}$ and $2.44 \mathrm{~mm}$. No corrections to the crack length or stress intensity data of specimen c158 were performed. While fairly high crack growth rates have been observed on this $10 \%$ cold worked specimen, they are not as uniformly observed (nor as high) as on the specimens having higher levels of cold work.

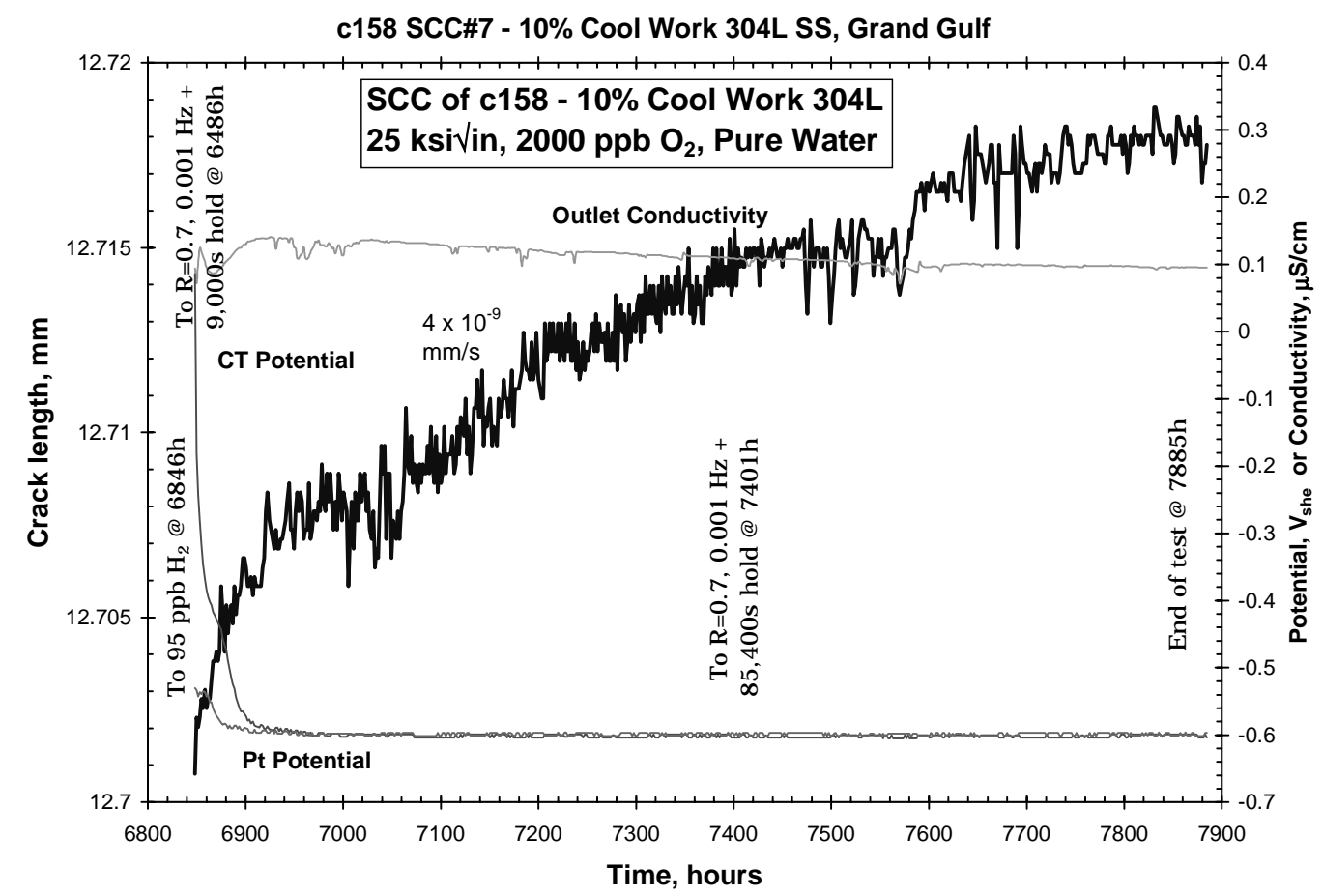

Figure 130. Crack growth response under static load at the end of the experiment on specimen c158.

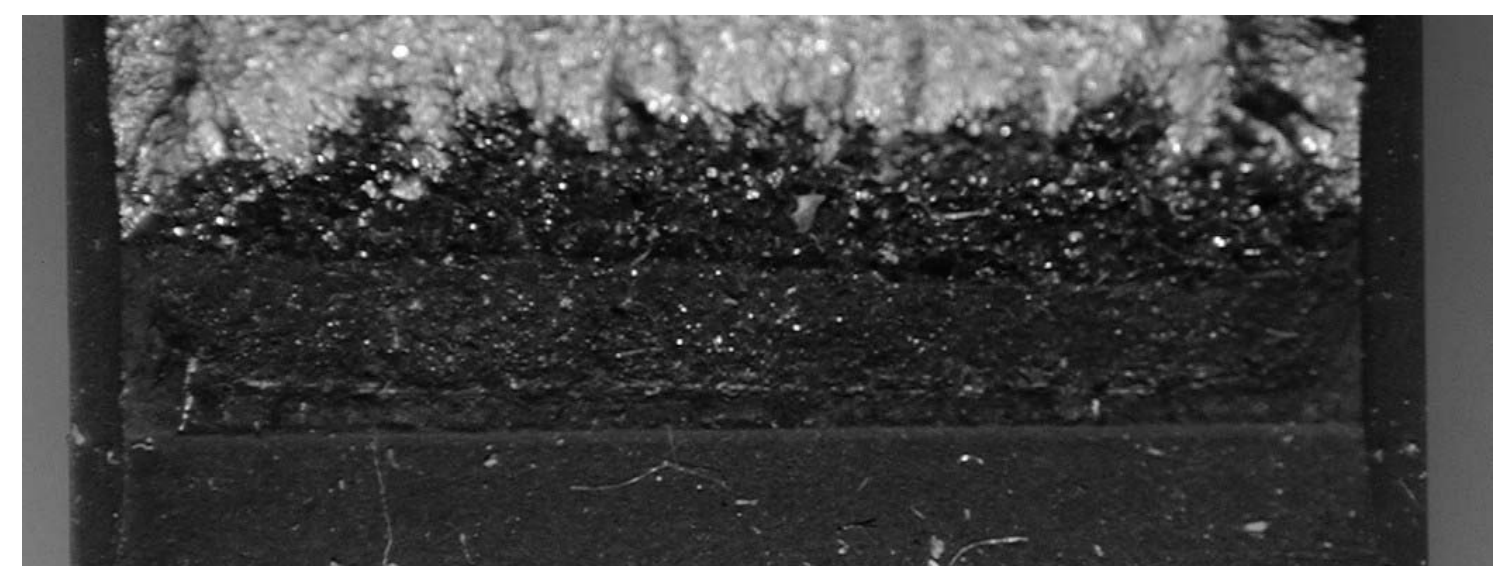

Figure 131. Light macrograph of fracture surface for 0.5T CT specimen c158, Type 304L SS (Grand Gulf heat) cool rolled $10 \%$ at $+140^{\circ} \mathrm{C}$. 

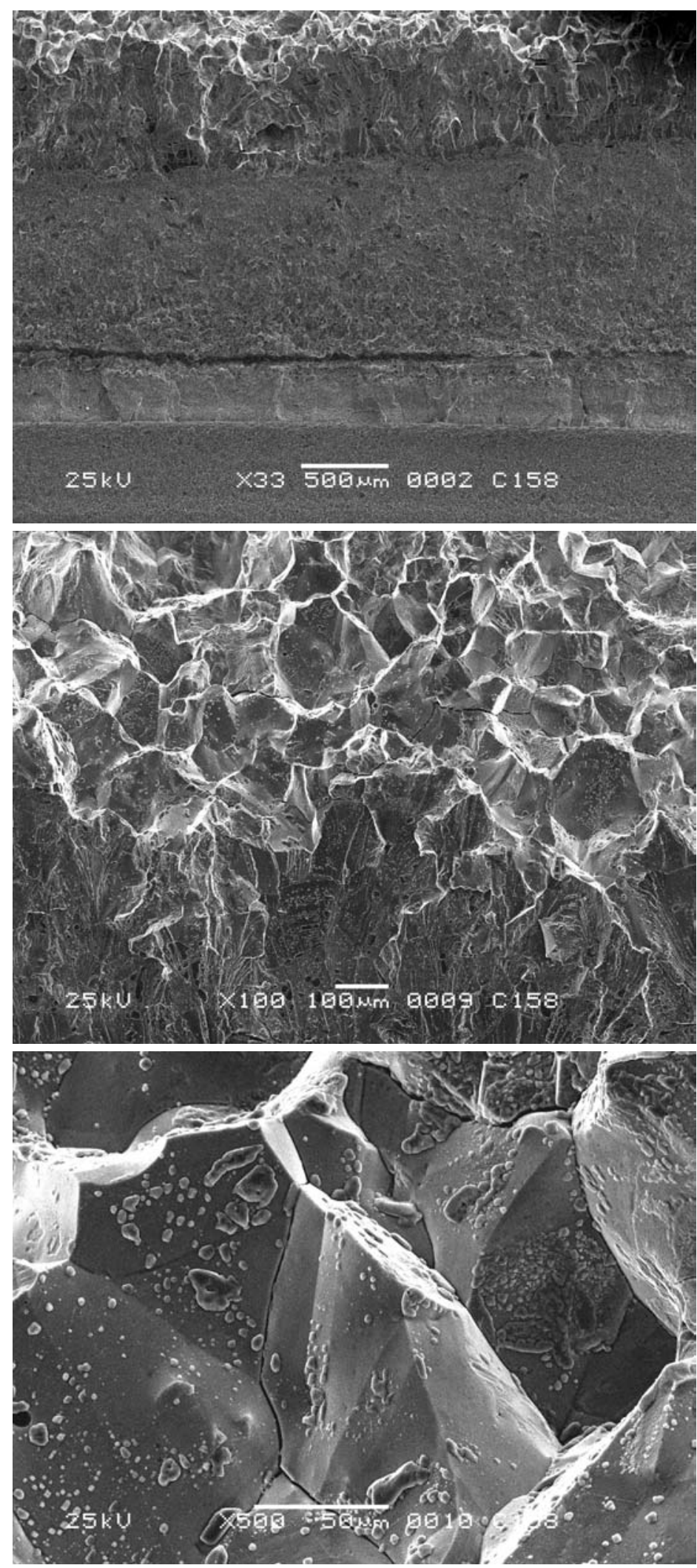

Figure 132. SEM micrographs documenting IG fracture on specimen c158, Type 304L SS (Grand Gulf heat) cool rolled $10 \%$ at $+140^{\circ} \mathrm{C}$. 
Specimen c159 of $10 \%$ CW 316L Stainless Steel An overview of this test is shown in Figure 133, which includes crack length vs. time, the corrosion potential of the CT specimen and a $\mathrm{Pt}$ electrode, and outlet solution conductivity. Conditions during the test were very well controlled throughout the $7885 \mathrm{~h}$ of crack-growth testing.

Since specimens c158 and c159 were tested in tandem, the fatigue pre-cracking of specimen c159 in air is recorded in Figure 134. As with the other specimens, there is an incubation and delayed growth period associated with nucleating a crack from the machined notch, then the fatigue crack-growth rates reflect the changes in loading.

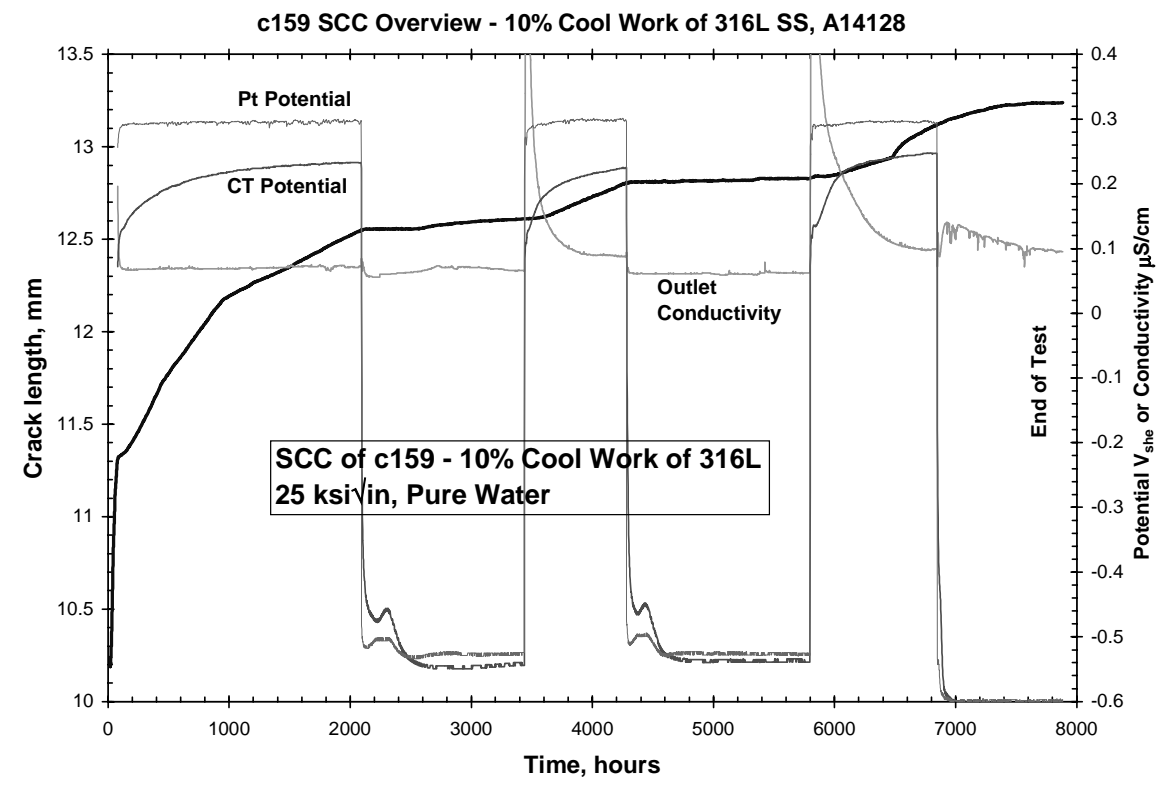

Figure 133. Overview of CT specimen c159, Type 316L SS (heat A14128) cool rolled 10\% at $+140^{\circ} \mathrm{C}$.

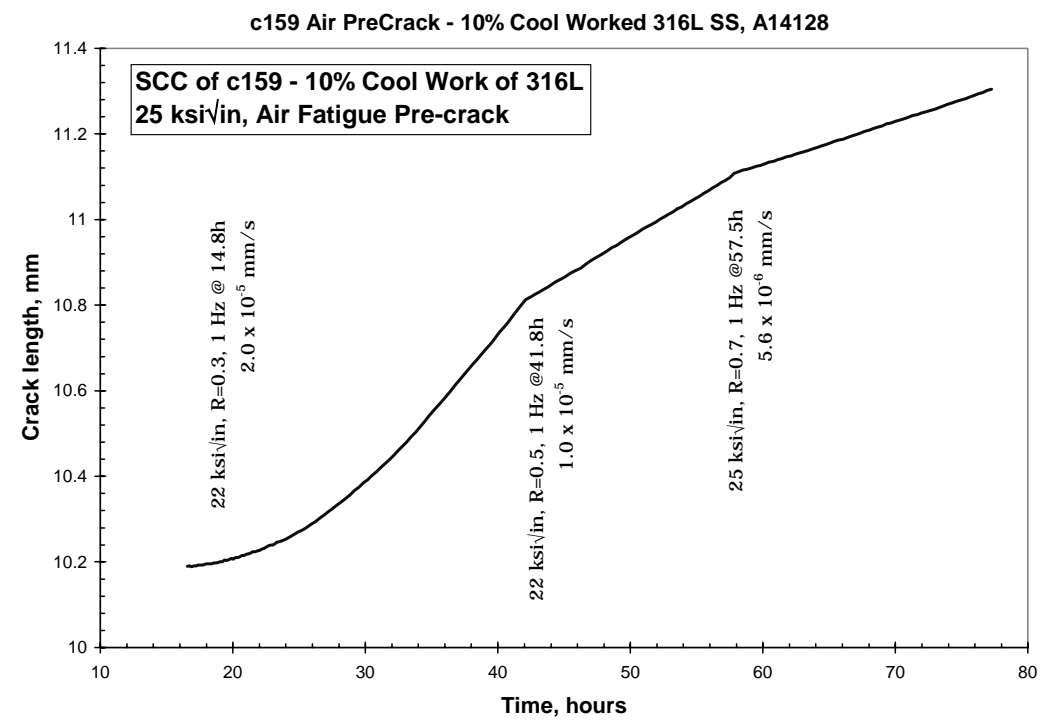

Figure 134. Air fatigue pre-cracking of CT specimen c159. 
Figure 135 shows the response after starting SCC testing in $288^{\circ} \mathrm{C}$ pure water containing 2000 $\mathrm{ppb}_{2}$. A fairly short transition period is observed after changing to $\mathrm{R}=0.7,0.001 \mathrm{~Hz}$ loading at $84.7 \mathrm{~h}$, with an observed growth rate of $3.7 \times 10^{-7} \mathrm{~mm} / \mathrm{s}$. On changing to $\mathrm{R}=0.7$, $0.001 \mathrm{~Hz}$ loading with a 1,000 -s hold time at 435 , the growth rate slow decreased to $2.5 \times 10^{-7}$ $\mathrm{mm} / \mathrm{s}$. The crack growth rate dropped by $\approx 2 X$ after increasing the hold time to $9,000 \mathrm{~s}$, Figure 136. After changing to constant load at $1235 \mathrm{~h}$, the growth rate changed to $1 \times 10^{-7} \mathrm{~mm} / \mathrm{s}$, which

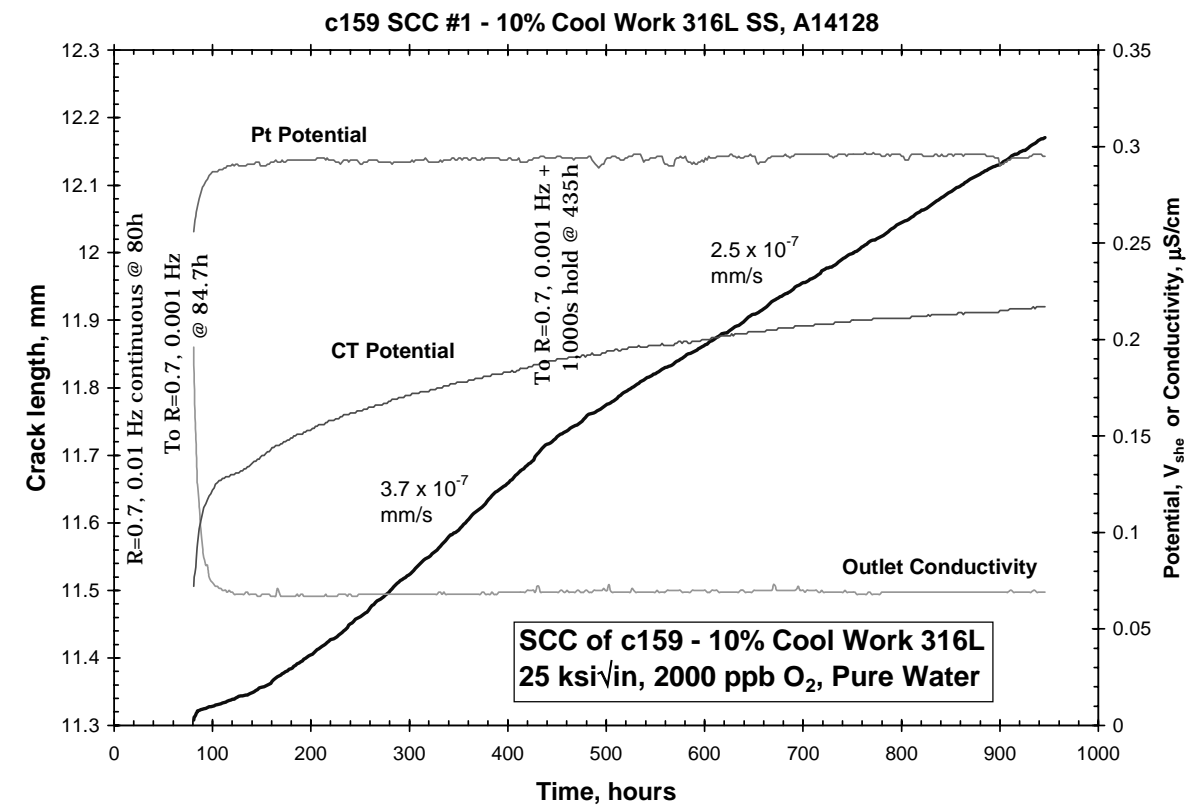

Figure 135. Transition from transgranular in-situ fatigue pre-cracking to IGSCC of $10 \%$ coolrolled, 316L SS specimen c159.

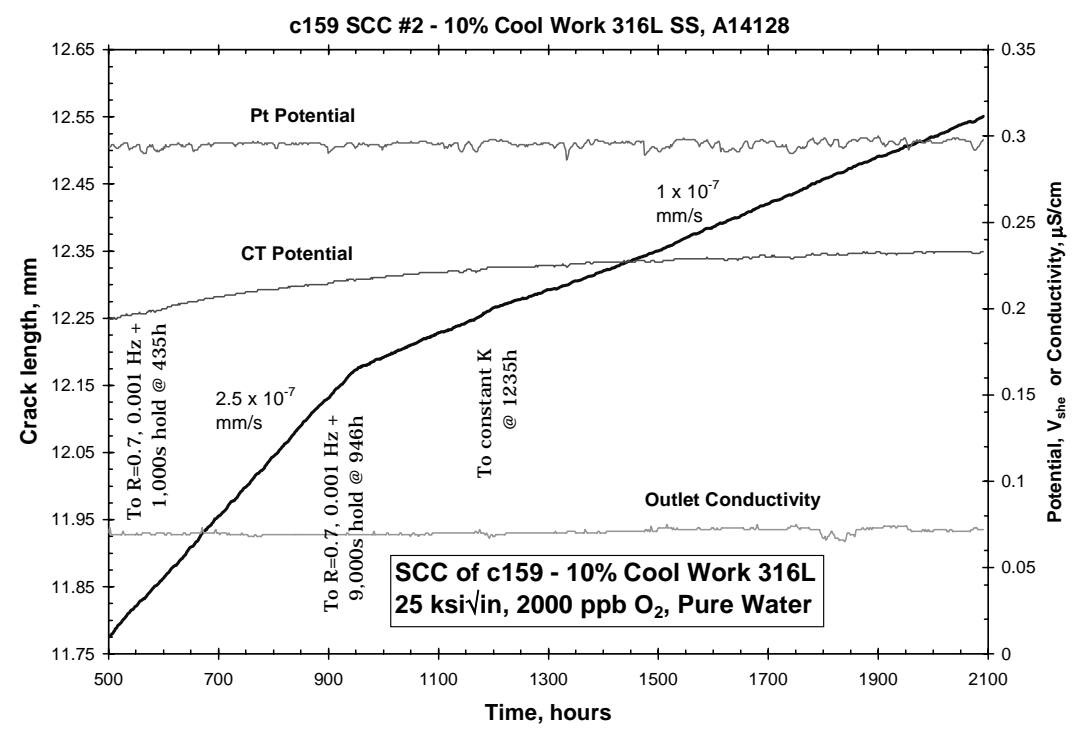

Figure 136. SCC behavior at static load of specimen c159, Type 316L SS (heat A14128), cool rolled $10 \%$ at $+140^{\circ} \mathrm{C}$. 
was sustained for over $900 \mathrm{~h}$. This is a somewhat higher growth rate than was observed on the companion 304L stainless steel specimen (c158) during this period $\left(6.5 \times 10^{-8} \mathrm{~mm} / \mathrm{s}\right)$.

Figure 137 shows the effect of changing to $95 \mathrm{ppb} \mathrm{H}_{2}$ at $2091 \mathrm{~h}$, which immediately reduced the crack growth rate to $\approx 1 \times 10^{-9} \mathrm{~mm} / \mathrm{s}$. At $2575 \mathrm{~h}$, a once-a-day unloading cycle at $\mathrm{R}=0.7,0.001$ $\mathrm{Hz}$ was initiated, and the growth rate increased to $\approx 3 \times 10^{-8} \mathrm{~mm} / \mathrm{s}$. The dissolved gas was then changed to $2000 \mathrm{ppb} \mathrm{O}_{2}$ and the growth rate rapidly increased to $8 \times 10^{-8} \mathrm{~mm} / \mathrm{s}$, Figure 138 . At $4281 \mathrm{~h}$, the dissolved gas was changed back to $95 \mathrm{ppb} \mathrm{H}_{2}$ and the growth rate rapidly dropped to $\approx 4 \times 10^{-9} \mathrm{~mm} / \mathrm{s}$.

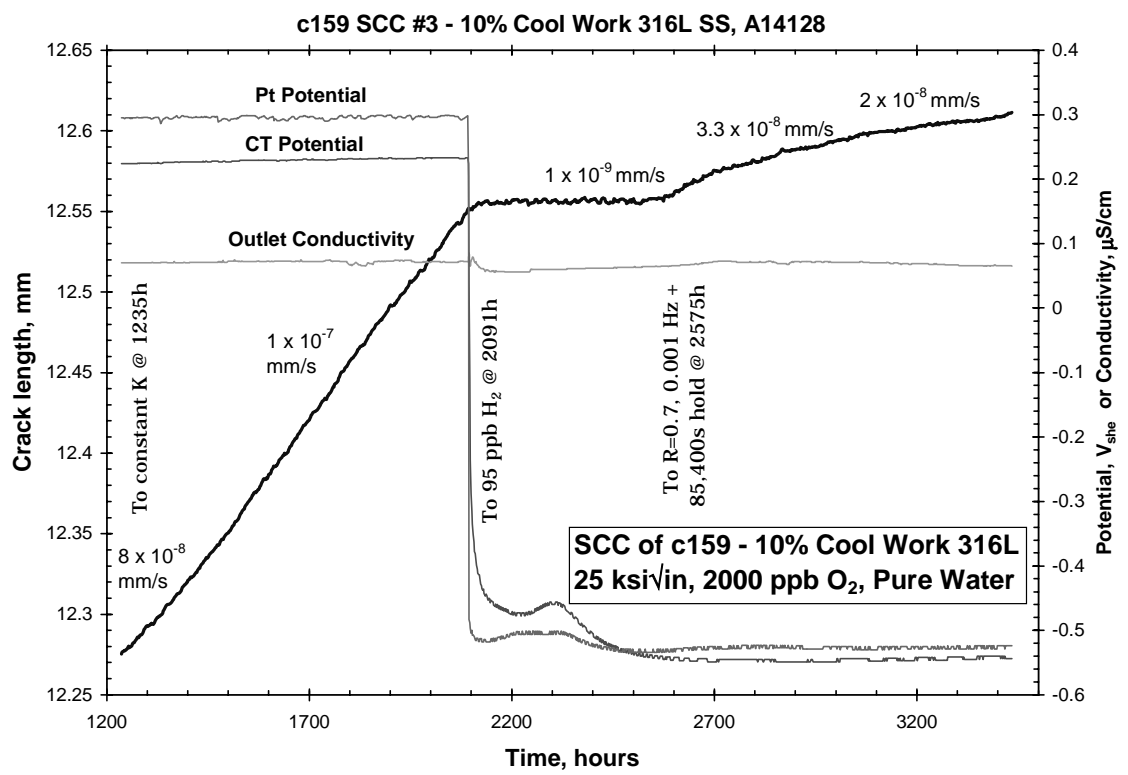

Figure 137. SCC behavior at static load of specimen c159, showing sharp decrease in growth rate with $\mathrm{H}_{2}$ addition.

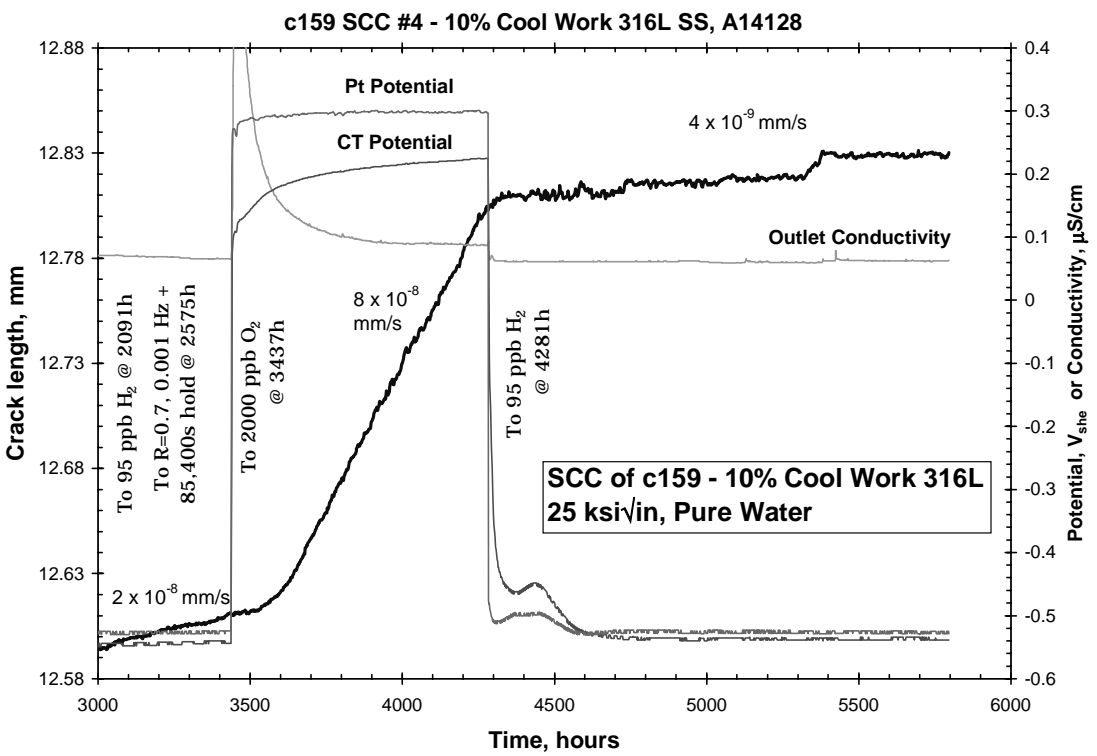

Figure 138. Influence of environment changes on the SCC behavior at static load of specimen c159. 
The dissolved gas was again changed to $2000 \mathrm{ppb} \mathrm{O}_{2}$ at $5796 \mathrm{~h}$ and the growth rate seemed to rapidly increase, then slow somewhat to $6 \times 10^{-8} \mathrm{~mm} / \mathrm{s}$ Figure 139 . At 6486 , a $\mathrm{R}=0.7,0.001 \mathrm{~Hz}$ with a 9,000-s hold was initiated, and the growth rate increased to $1.6 \times 10^{-7} \mathrm{~mm} / \mathrm{s}$, where it remained steady for several hundred hours. The dissolved gas was then changed back to $95 \mathrm{ppb}$ $\mathrm{H}_{2}$, which surprisingly did not produce an immediate decrease in crack-growth rate, Figures 140 and 141 . At $7401 \mathrm{~h}$, the hold time was increased to $85,400 \mathrm{~s}$, and this did produce a dramatic

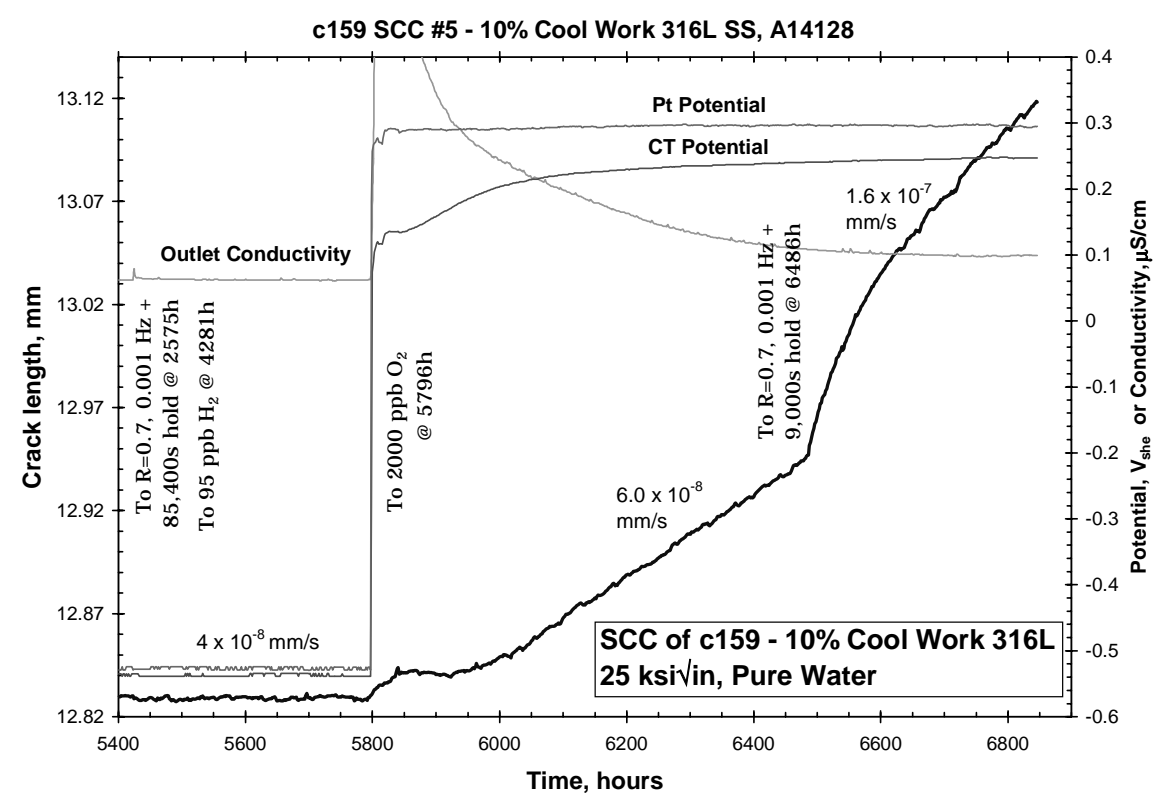

Figure 139. SCC behavior at static load of Type 316L SS specimen c159 with changes in environment and cyclic loading.

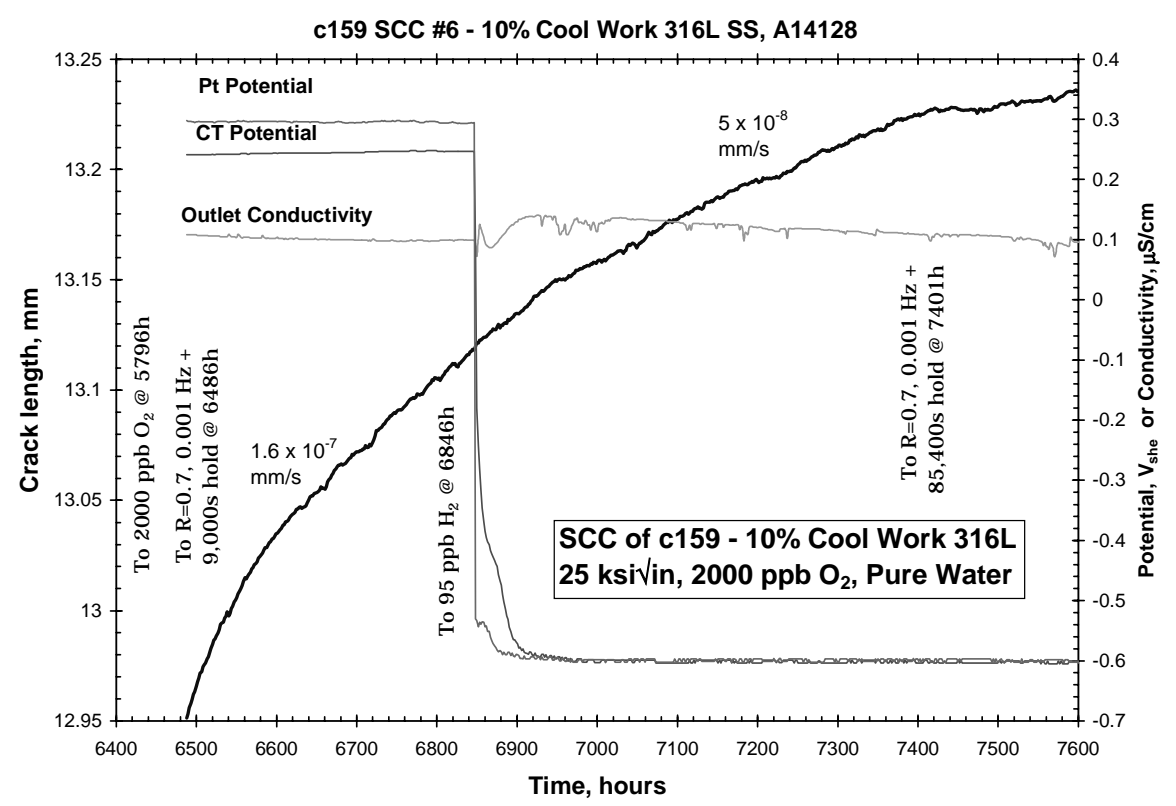

Figure 140. Crack-growth response of specimen c159 after the addition of $\mathrm{H}_{2}$. 


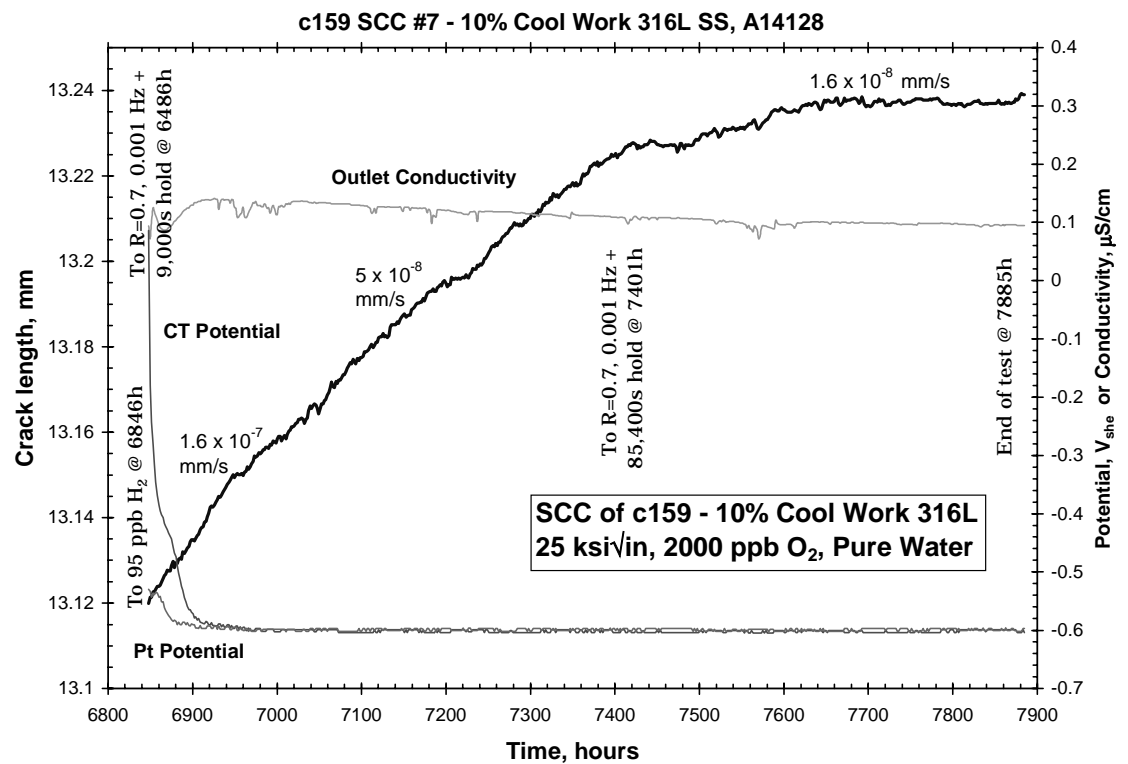

Figure 141. SCC behavior at static load of 10\% cool-rolled, Type 316SS specimen c159, showing crack-growth decrease after a decrease in hold time.

decrease in growth rate to $1.6 \times 10^{-9} \mathrm{~mm} / \mathrm{s}$. The failure of the decrease in corrosion potential to produce an immediate change in crack growth rate is unusual and distressing, although there have been a number of similar observations in our laboratory on high yield strength materials under periodic cyclic unloading.

At 7885 h the test was ended. Figure 142 shows a low magnification light macrograph of the fracture appearance, and high-magnification details of the fracture surface are documented in Figure 143. The dc potential drop indicated growth is $3.05 \mathrm{~mm}$, while the average, maximum and minimum of the actual crack depth are $3.51 \mathrm{~mm}, 4.27 \mathrm{~mm}$ and $\approx 2.77 \mathrm{~mm}$. No corrections to the crack length data of specimen c159 were performed. While fairly high crack-growth rates

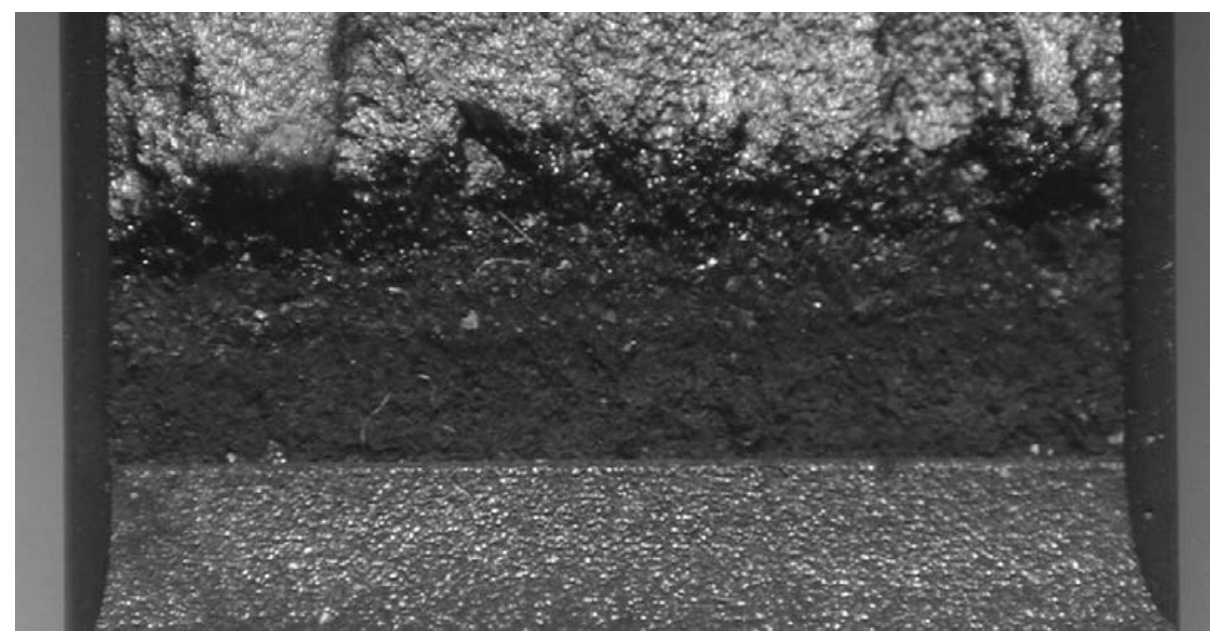

Figure 142. Light macrograph of fracture surface for specimen c159, Type 316L SS (heat a14128) cool rolled $10 \%$ at $+140^{\circ} \mathrm{C}$. 
have been observed on this $10 \%$ cold-worked specimen, they are not as uniformly observed (nor as high) as on the specimens having higher levels of cold work. In general, the 316L SS specimen has exhibited slightly higher crack growth rates than the companion 304LSS specimen, which was also true of the companion tests c156 and c157.

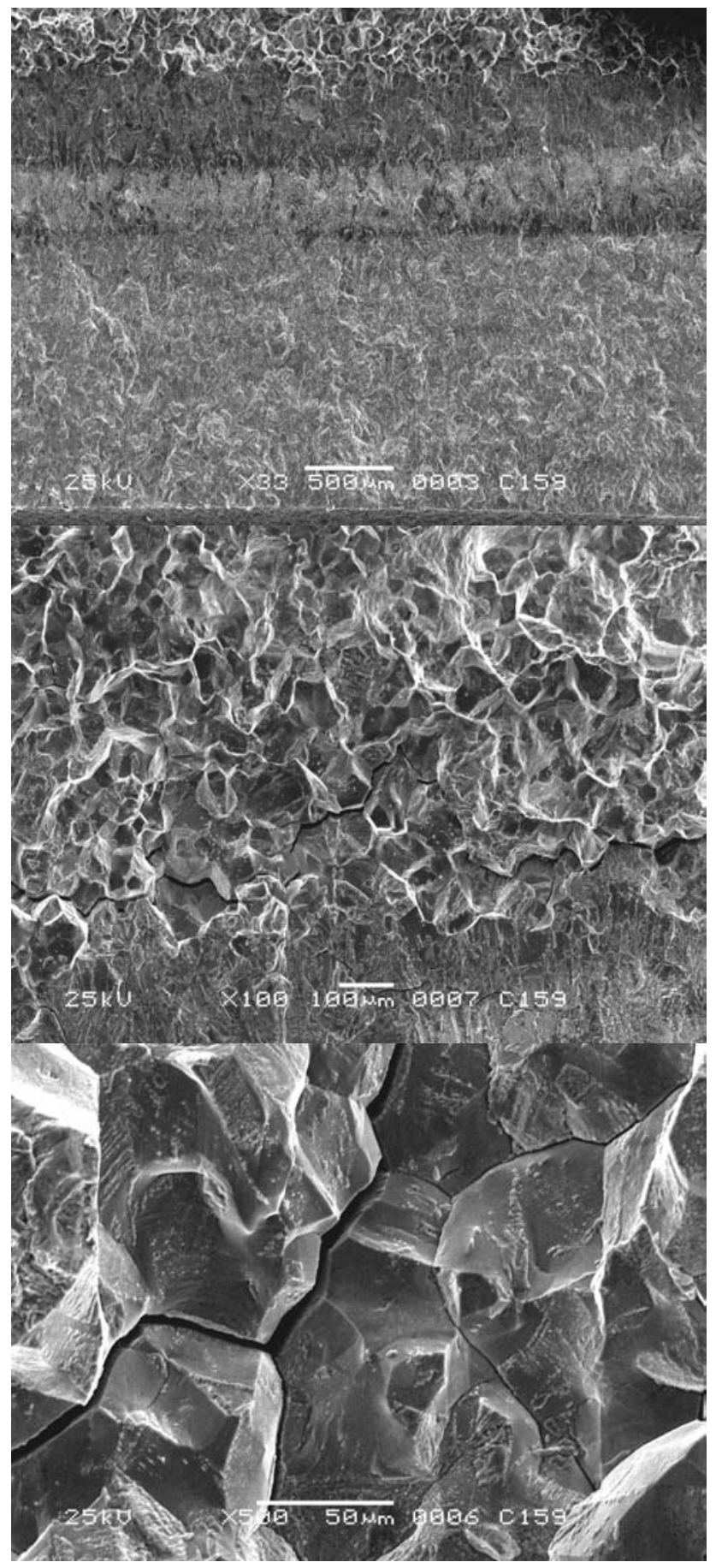

Figure 143. SEM micrographs illustrating IG crack path in specimen c159, Type 316L SS (heat a14128) cool rolled $10 \%$ at $+140^{\circ} \mathrm{C}$. 
Stress Corrosion Cracking of Sensitized and Desensitized Stainless Steels

Specimen c184, sensitized 304 SS with $50 \%$ reduction by forging at $+140^{\circ} \mathrm{C}$, heat AJ3193 This test was designed to evaluate SCC crack-growth rates in sensitized plus highly cold worked Type 304 stainless steel (not an L-grade). The specimen was solution annealed at $1050{ }^{\circ} \mathrm{C}$ for 30 minutes and water quenched, then sensitized at $621^{\circ} \mathrm{C}$ for 24 hours, forged to $50 \%$ reduction in thickness at $140^{\circ} \mathrm{C}$ and machined into a $0.5 \mathrm{TCT}$ specimen. An overview of this test is shown in Figure 144, which includes crack length vs. time, the corrosion potential of the CT specimen and a Pt electrode, and outlet solution conductivity. The air-fatigue precracking response is shown in Figure 145 , and the subsequent exposure to $288^{\circ} \mathrm{C}$ pure water and transitioning to IGSCC is shown in Figure 146.

This specimen did not exhibit high growth rates consistent with the presence of both sensitization and cold work. Even at R=0.7, $0.003 \mathrm{~Hz}$, the growth rate was only $8.3 \times 10^{-8} \mathrm{~mm} / \mathrm{s}$ (Figure 146). When the frequency was slowed to $0.001 \mathrm{~Hz}$ at $738 \mathrm{~h}$ (Figure 147), the growth rate slowed further to $4 \times 10^{-8} \mathrm{~mm} / \mathrm{s}$. On introducing a 9,000-s hold time at $2081 \mathrm{~h}$, the growth rate increased to $8 \times 10^{-8} \mathrm{~mm} / \mathrm{s}$, then slowly decayed. The growth rate was already quite slow when $95 \mathrm{ppb}_{2}$ was introduced at 2,919 $\mathrm{h}$.

To accelerate the crack-growth rate and to evaluate the effect of changes in corrosion potential in mixed $\mathrm{O}_{2}-\mathrm{H}_{2}$ mixtures, $\mathrm{Na}_{2} \mathrm{SO}_{4}$ was added at $0.326 \mu \mathrm{S} / \mathrm{cm}(100 \mathrm{ppb}$ sulfate $)$ with $2000 \mathrm{ppb} \mathrm{O}_{2}$ and continuous cycling at $\mathrm{R}=0.7,0.001 \mathrm{~Hz}$. The growth rate increased, but only to $7 \times 10^{-8}$ $\mathrm{mm} / \mathrm{s}$ (Figure 148). At 3,377 h, a 9,000-s hold time was introduced, and the growth rate slowed markedly, down to $2 \times 10^{-8} \mathrm{~mm} / \mathrm{s}$ as shown in Figure 148 .

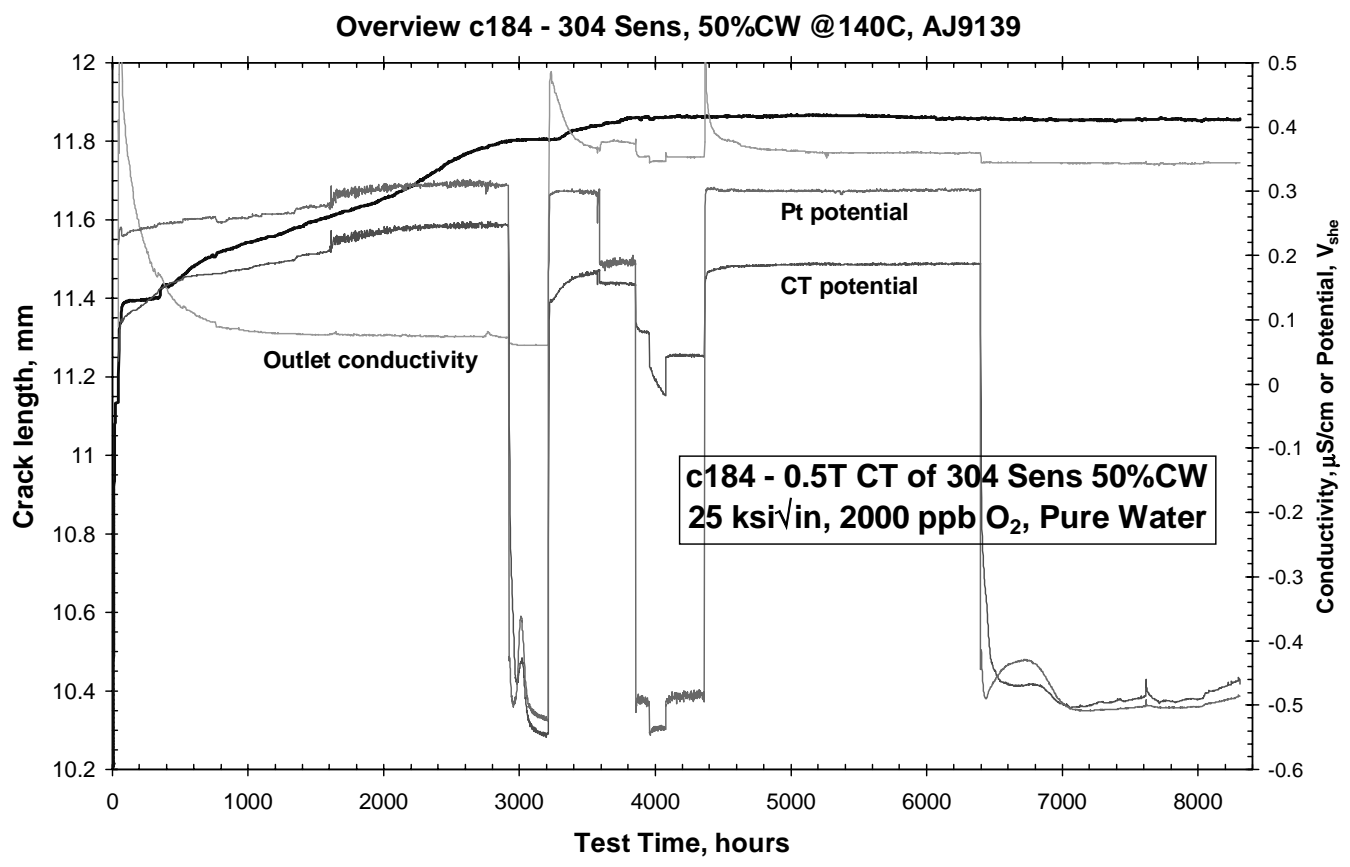

Figure 144. Overview of sensitized specimen c184, Type 304 SS (heat AJ9139) with 50\% reduction in thickness by forging at $+140^{\circ} \mathrm{C}$. 


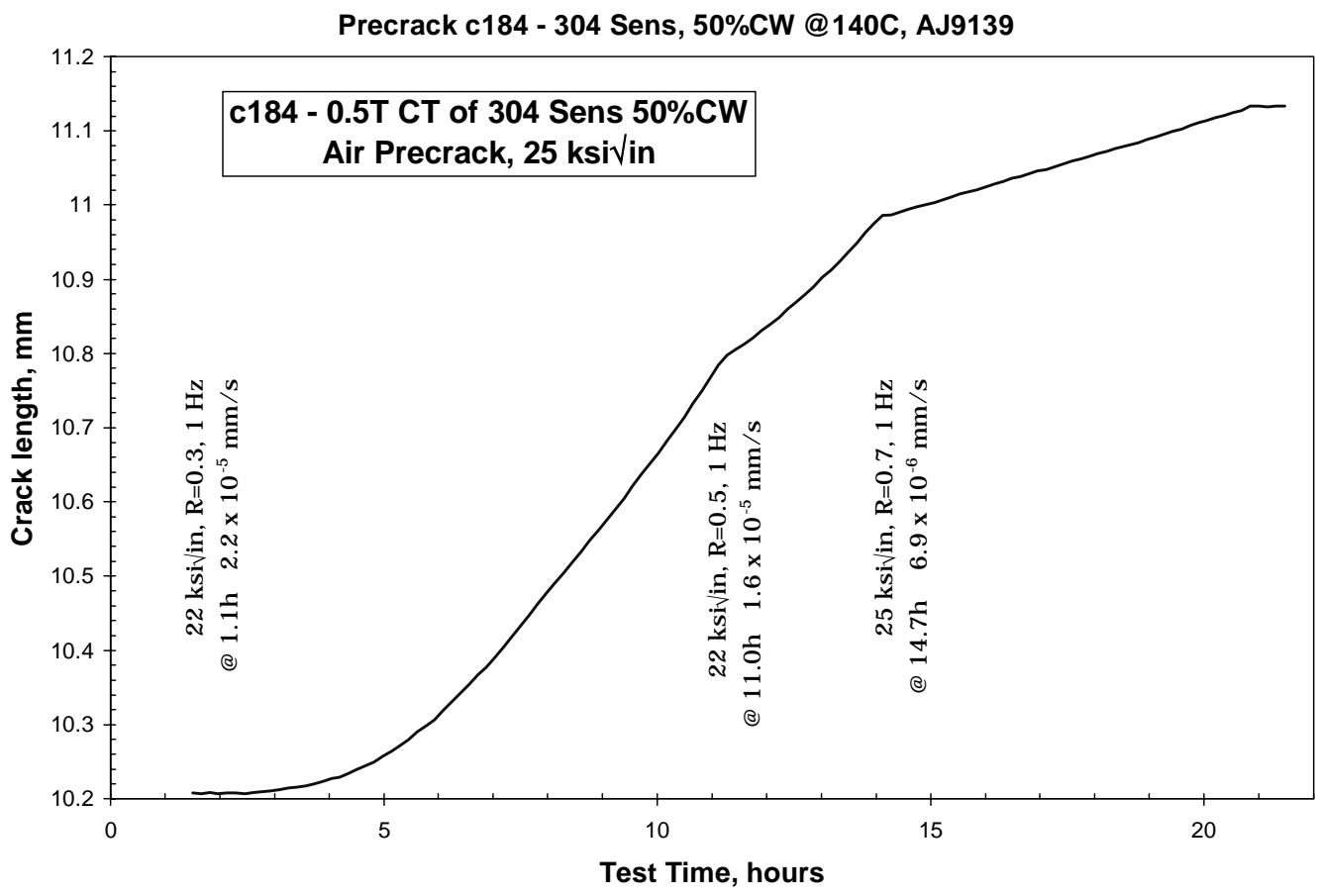

Figure 145. Air fatigue pre-cracking of sensitized Type 304 SS specimen c184.

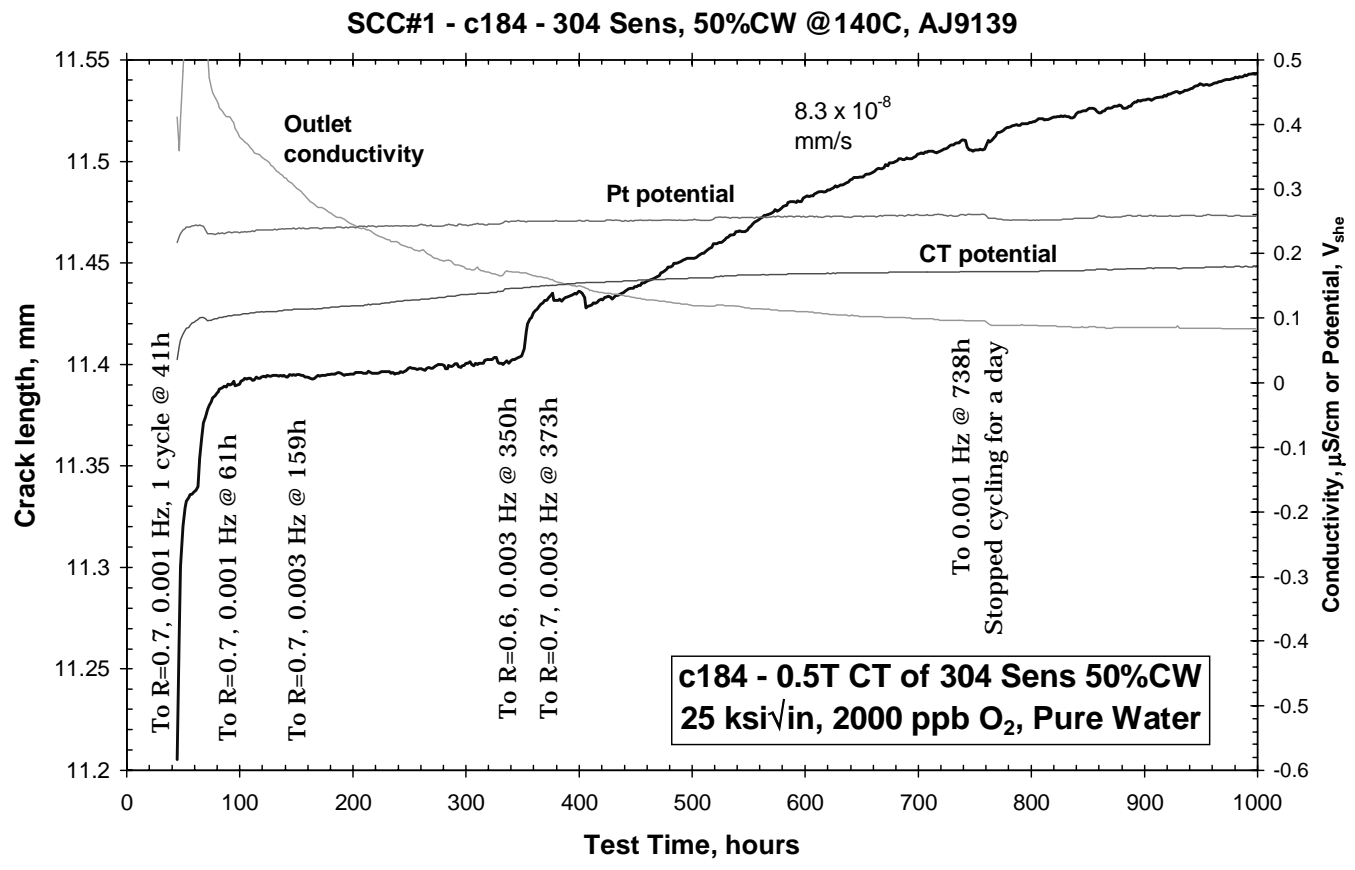

Figure 146. Early crack-growth response with varying cycling conditions for sensitized specimen c184. 


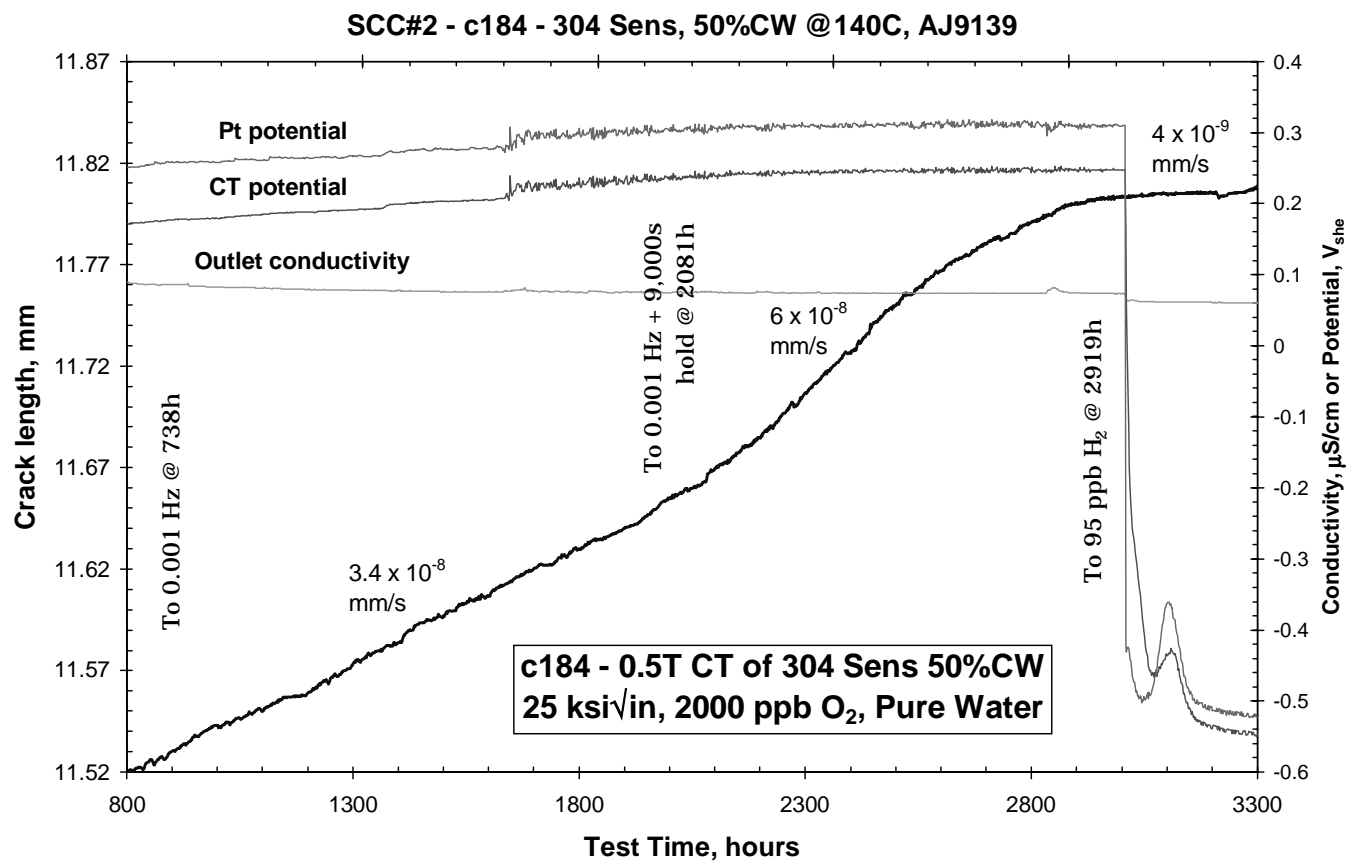

Figure 147. Initial SCC behavior of sensitized 304 SS specimen c184.

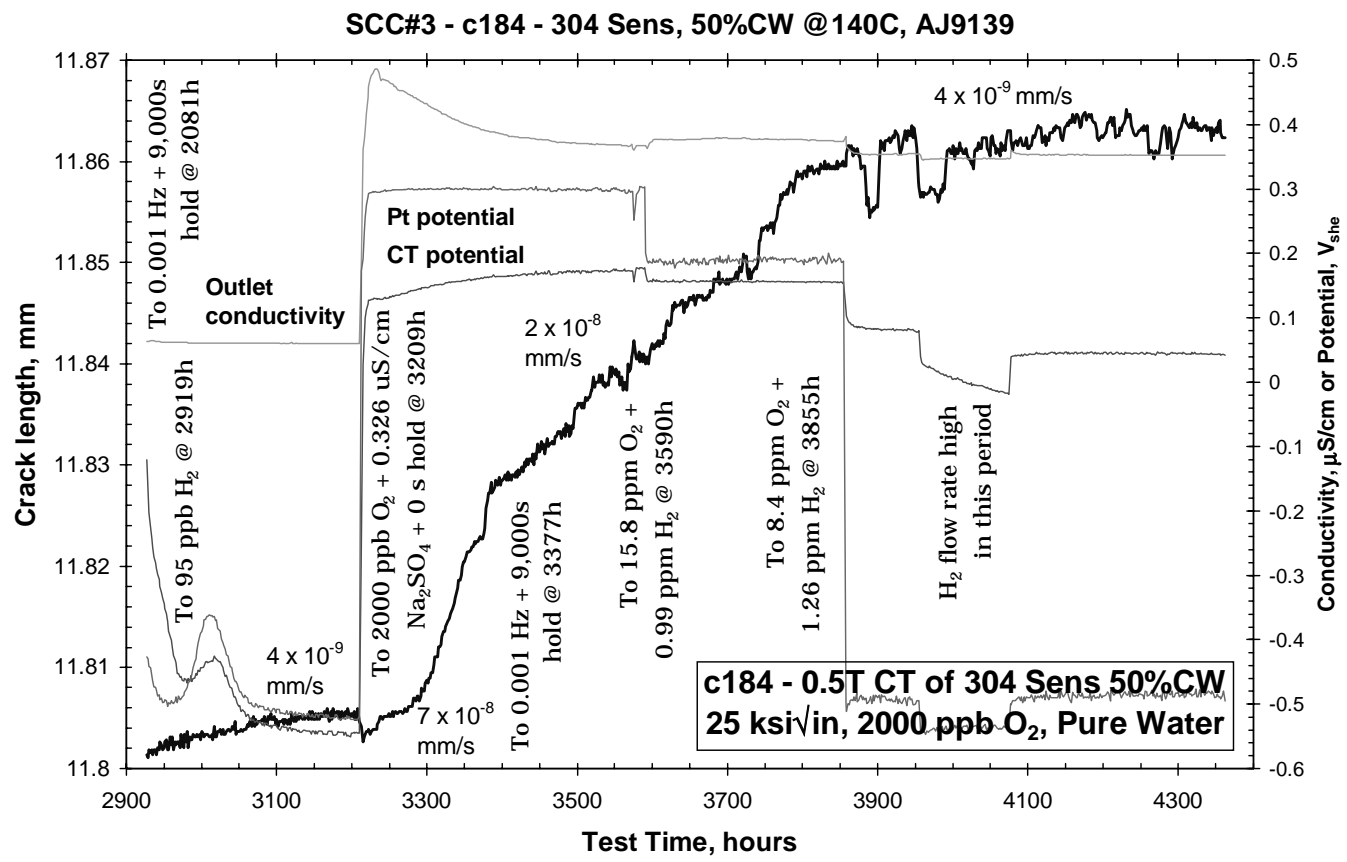

Figure 148. Complex SCC response of sensitized specimen c184 after addition of $\mathrm{Na}_{2} \mathrm{So}_{4}$ and changes in $\mathrm{O}_{2}$ and $\mathrm{H}_{2}$ concentration.

At 3,590 h, a period began when various $\mathrm{O}_{2}$ and $\mathrm{H}_{2}$ mixtures were evaluated. The growth rate decreased, and as the $\mathrm{H}_{2}$ level increased and corrosion potential decreased the growth rate 
slowed dramatically to $\leq 4 \times 10^{-9} \mathrm{~mm} / \mathrm{s}$ as shown in Figure 148. The dissolved gas mixture was returned to $2000 \mathrm{ppb} \mathrm{O}_{2}$ at $4,326 \mathrm{~h}$ and the growth rate remained very low, Figure 149. It is apparent that this specimen exhibits slow growth rates because the post-sensitization cold work disrupts the grain boundary $\mathrm{Cr}$ depletion path, leaving grain boundary carbides to retard the deformation processes in the grain boundary.

The test was ended at $9030 \mathrm{~h}$ and the specimen was fatigued apart in air. Figure 150 shows a low-magnification macrograph of the fracture appearance, and Figure 151 documents the fractography by SEM. For reasons that are quite unclear, the SCC crack growth was not very

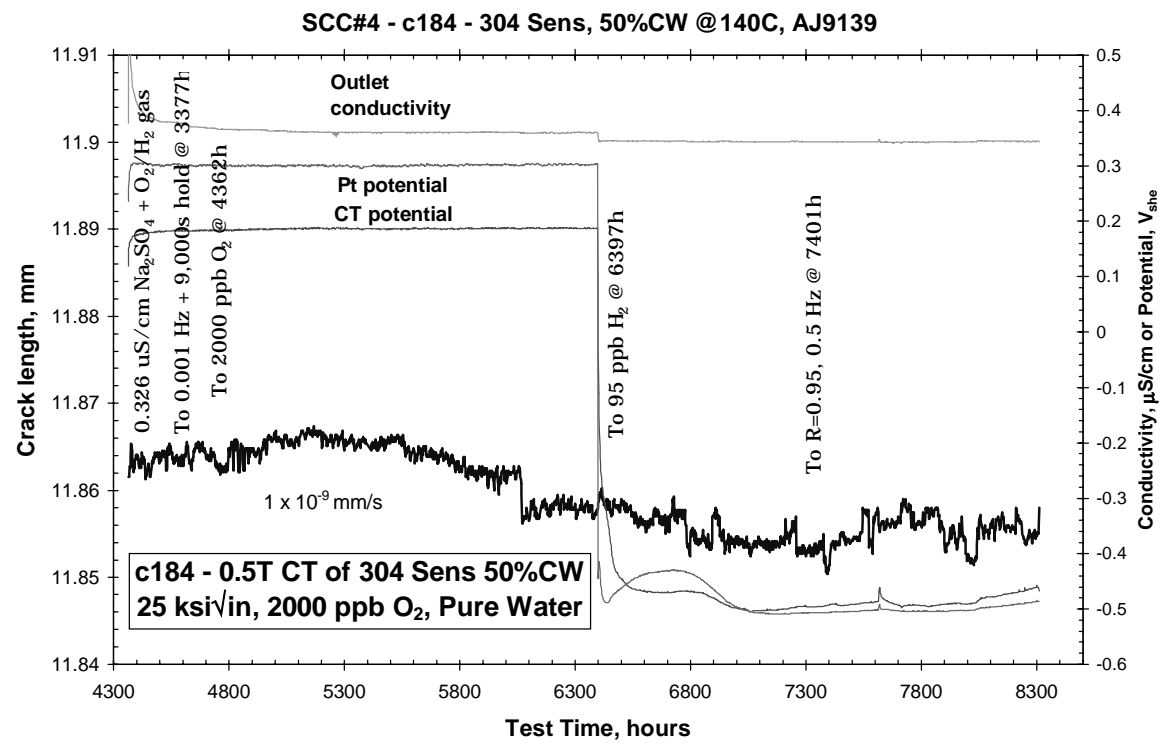

Figure 149. Final attempt to accelerate crack-growth by increasing $\mathrm{O}_{2}$ content for the sensitized specimen c 184 .

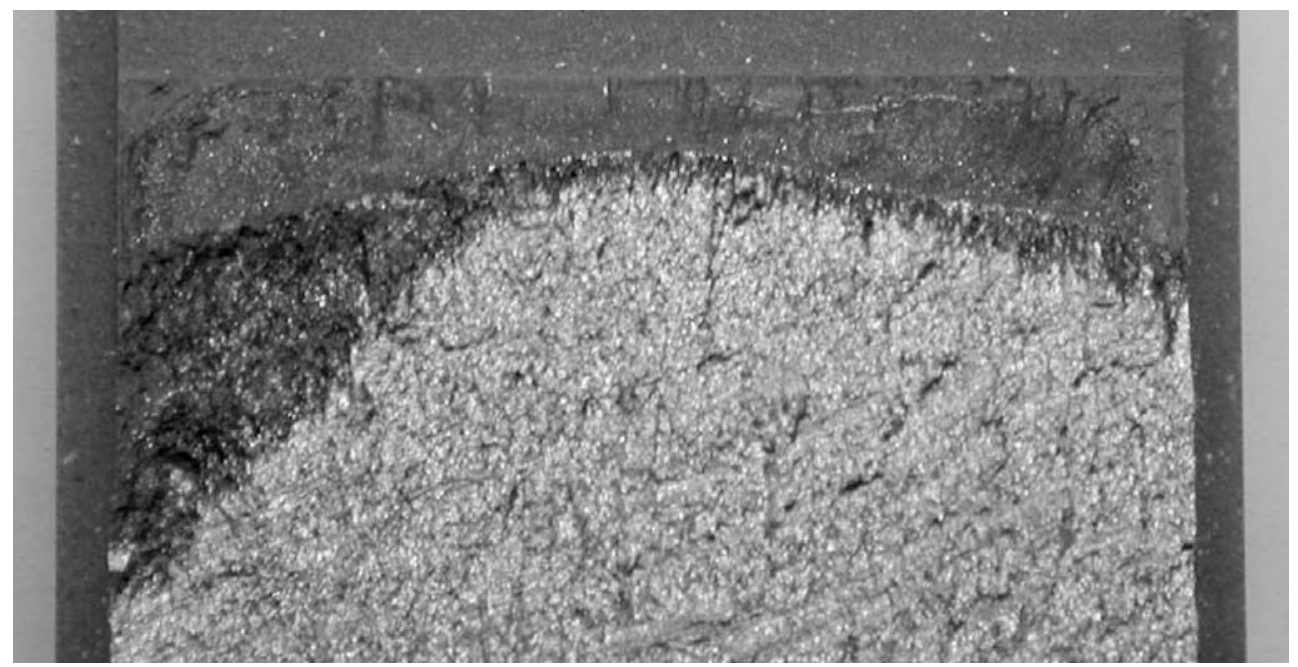

Figure 150. Light macrograph of the fracture surface for specimen c184, Type 304 SS (heat AJ9139) with $50 \%$ reduction in thickness by forging at $+140^{\circ} \mathrm{C}$. 
even along the crack front, despite having a reasonably straight fatigue precrack and having nucleated IG cracking along the entire crack front. Based on extensive prior experience, the processing should not have caused a consequential gradient in material properties. The dc potential drop indicated growth is $1.62 \mathrm{~mm}$, while the average, maximum and minimum of the actual crack depth are $2.27 \mathrm{~mm}, 5.46 \mathrm{~mm}$ and $1.25 \mathrm{~mm}$. No corrections to the crack length data of specimen c184 were performed.

Specimen c185, non-sensitized 304 SS $50 \%$ reduction by forging at $+140^{\circ} \mathrm{C}$, heat AJ3193 This test was designed to evaluate SCC crack-growth rates in non-sensitized plus highly cold-worked Type $304 \mathrm{SS}$. The specimen was solution annealed at $1050^{\circ} \mathrm{C}$ for 30 minutes and water quenched, forged to $50 \%$ reduction in thickness at $140^{\circ} \mathrm{C}$ and machined into a 0.5 TCT specimen. An overview of this test is shown in Figure 152, which includes crack length vs. time, the corrosion potential of the CT specimen and a Pt electrode, and outlet solution conductivity. The air-fatigue pre-cracking response is shown in Figure 153, and the subsequent exposure to $288^{\circ} \mathrm{C}$ pure water and transitioning to IGSCC is shown in Figure 154.
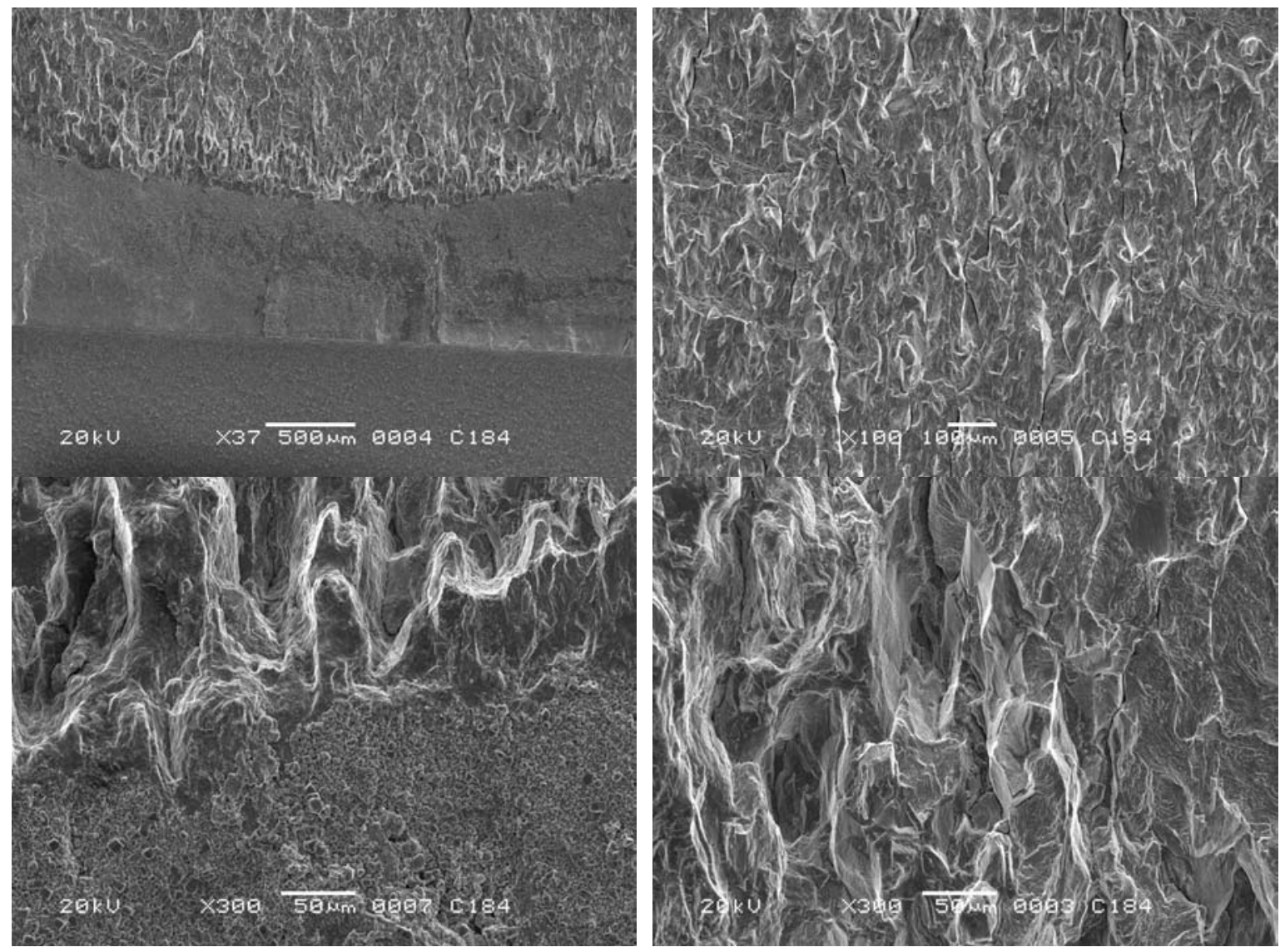

Figure 151. SEM micrograph of fracture surface for specimen c184, Type 304 SS (heat AJ9139) with $50 \%$ reduction in thickness by forging at $+140^{\circ} \mathrm{C}$. 


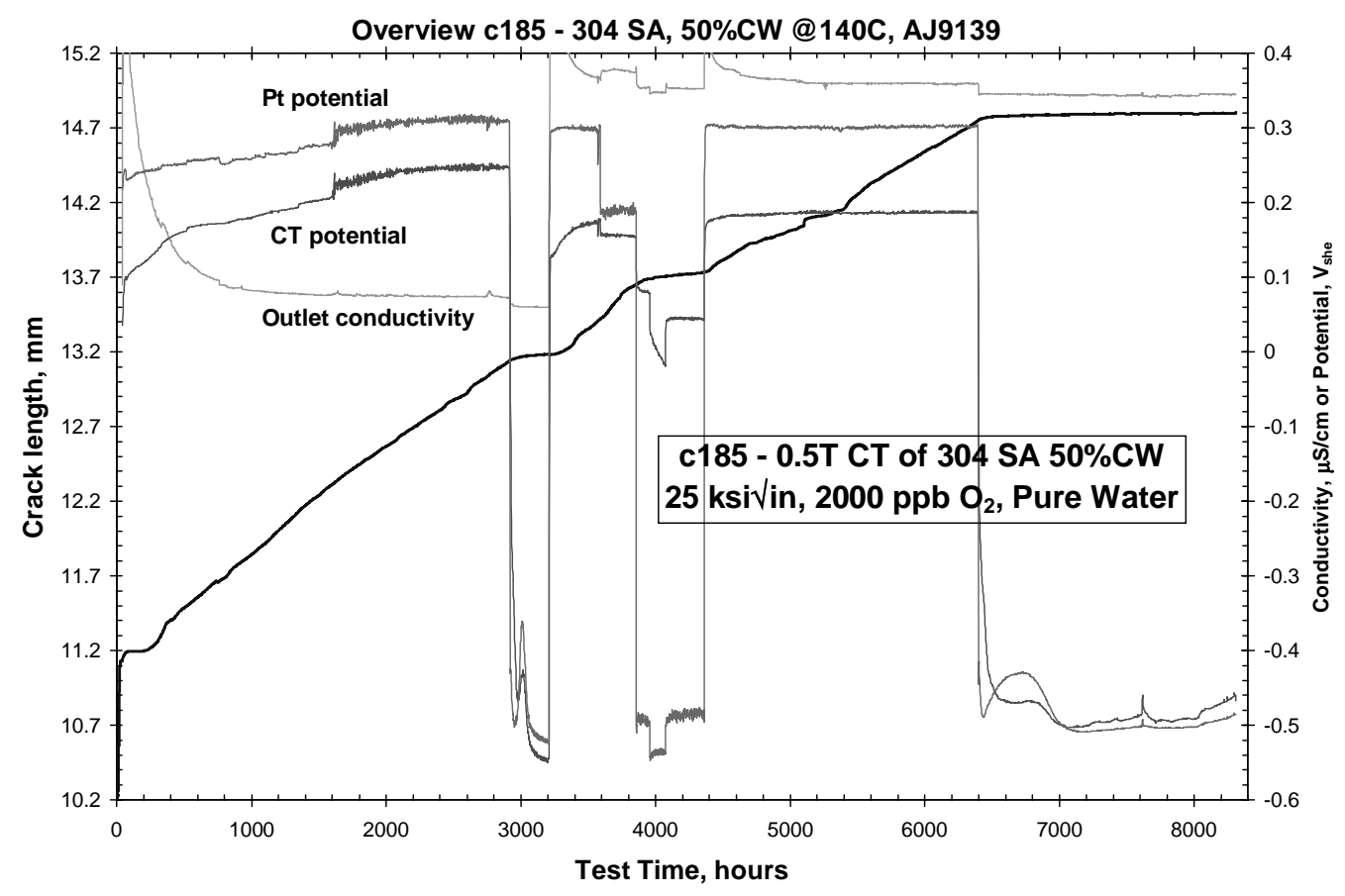

Figure 152. Overview of non-sensitized specimen c185, Type 304 SS (heat AJ9139) with 50\% reduction in thickness by forging at $+140^{\circ} \mathrm{C}$.

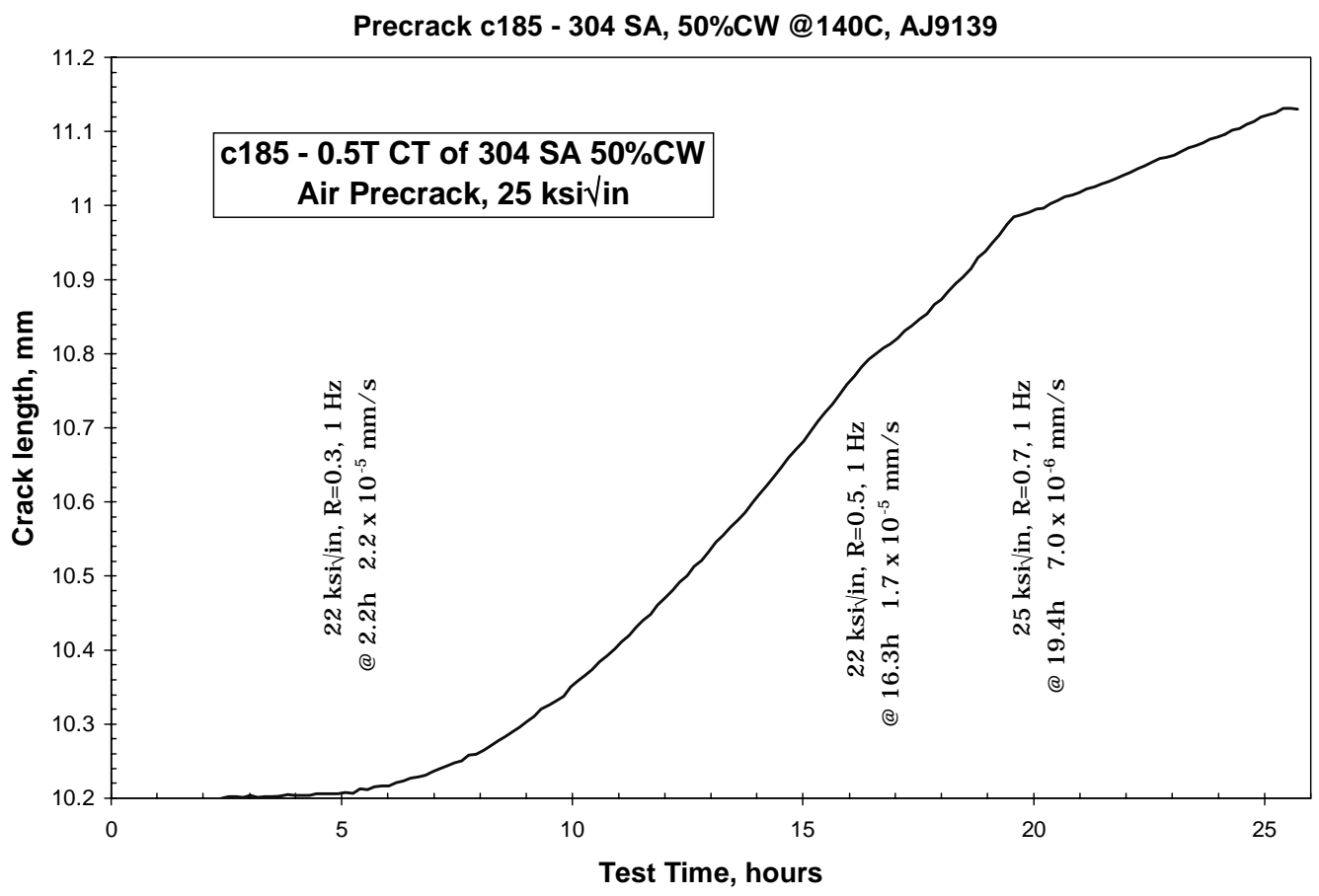

Figure 153. Air fatigue pre-cracking of non-sensitized specimen c185. 
This specimen has responded better than c184. At $\mathrm{R}=0.7,0.003 \mathrm{~Hz}$ the growth rate was $5 \times 10^{-7}$ $\mathrm{mm} / \mathrm{s}$, much faster than c184 at $8.3 \times 10^{-8} \mathrm{~mm} / \mathrm{s}$. When the frequency was slowed to $0.001 \mathrm{~Hz}$ at $738 \mathrm{~h}$ (Figure 155), the growth rate slowed somewhat to $2.4 \times 10^{-7} \mathrm{~mm} / \mathrm{s}$. On introducing a

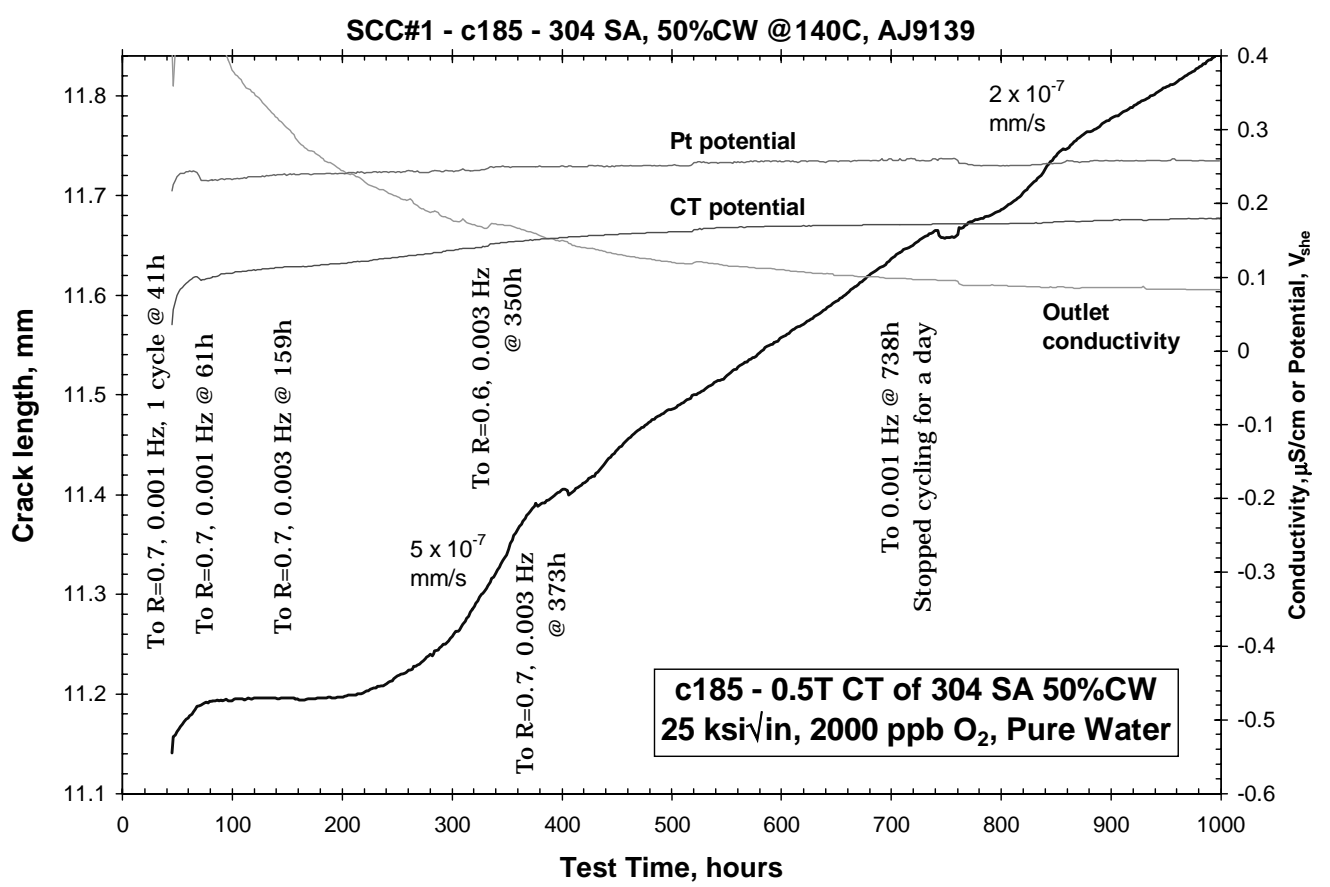

Figure 154. SCC behavior of non-sensitized specimen c185 showing transition to IGSCC.

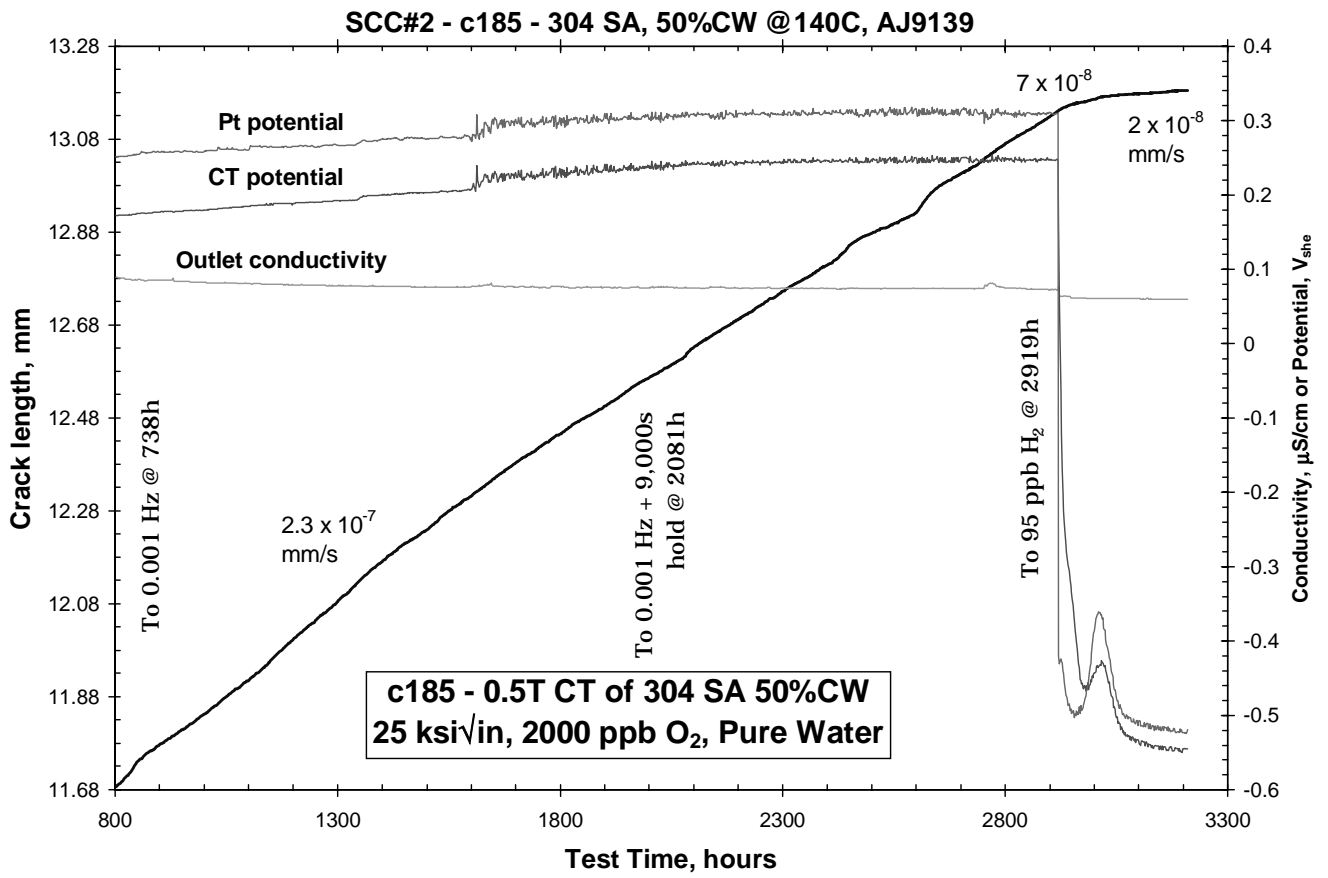

Figure 155. SCC behavior of non-sensitized specimen c185 during initial cycling. 
9,000-s hold time at $2081 \mathrm{~h}$, the growth rate was only reduced slightly to $2 \times 10^{-7} \mathrm{~mm} / \mathrm{s}$, and was quite stable. Ninety-five ppb $\mathrm{H}_{2}$ was introduced at $2,919 \mathrm{~h}$, and the growth rate slowed to $7 \times 10^{-8} \mathrm{~mm} / \mathrm{s}$ and then eventually to $2 \times 10^{-8} \mathrm{~mm} / \mathrm{s}$.

To accelerate the crack-growth rate and to evaluate the effect of changes in corrosion potential in mixed $\mathrm{O}_{2}-\mathrm{H}_{2}$ mixtures, $\mathrm{Na}_{2} \mathrm{SO}_{4}$ was added at $0.326 \mu \mathrm{S} / \mathrm{cm}\left(100 \mathrm{ppb}\right.$ sulfate) with $2000 \mathrm{ppb} \mathrm{O}_{2}$ and continuous cycling at $\mathrm{R}=0.7,0.001 \mathrm{~Hz}$ (Figure 156). The growth rate increased, but increased further when a 9,000-s hold time was introduced at 3,377 h, where a growth rate of $4.3 \times 10^{-7} \mathrm{~mm} / \mathrm{s}$ was observed. The growth rate decayed somewhat with time, then stabilized at $3.4 \times 10^{-7} \mathrm{~mm} / \mathrm{s}$.

At 3,590 h, a period began when various $\mathrm{O}_{2}$ and $\mathrm{H}_{2}$ mixtures were evaluated. The growth rate decreased to $1.5 \times 10^{-7} \mathrm{~mm} / \mathrm{s}$, and as the $\mathrm{H}_{2}$ level increased and corrosion potential decreased, the growth rate dropped to $2.5 \times 10^{-8} \mathrm{~mm} / \mathrm{s}$ (Figure 157). The dissolved gas mixture was returned to $2000 \mathrm{ppb} \mathrm{O}_{2}$ at $4,326 \mathrm{~h}$, and the growth rate increased to $1.6 \times 10^{-7} \mathrm{~mm} / \mathrm{s}$. At 4,692 $\mathrm{h}$, a shift to constant $\mathrm{K}$ was implemented, and the growth rate varied somewhat over the next $1,800 \mathrm{~h}$, stabilizing at about at $2 \times 10^{-7} \mathrm{~mm} / \mathrm{s}$. A shift was made to $95 \mathrm{ppb}_{2}$ at $6,397 \mathrm{~h}$ and the growth rate decreased to $1.2 \times 10^{-8} \mathrm{~mm} / \mathrm{s}$ as shown in Figure 158 .

The test was ended at $9030 \mathrm{~h}$, and the specimen was fatigued apart in air. Figure 159 shows a low magnification light macrograph of the fracture appearance, and detailed fractography is presented in Figure 160. The fatigue pre-crack front is relative straight, and complete IG nucleation along the crack front occurred. However, it's clear that some unusual behavior occurred at the sides, and the macrographs show that there was some vertical cracking from

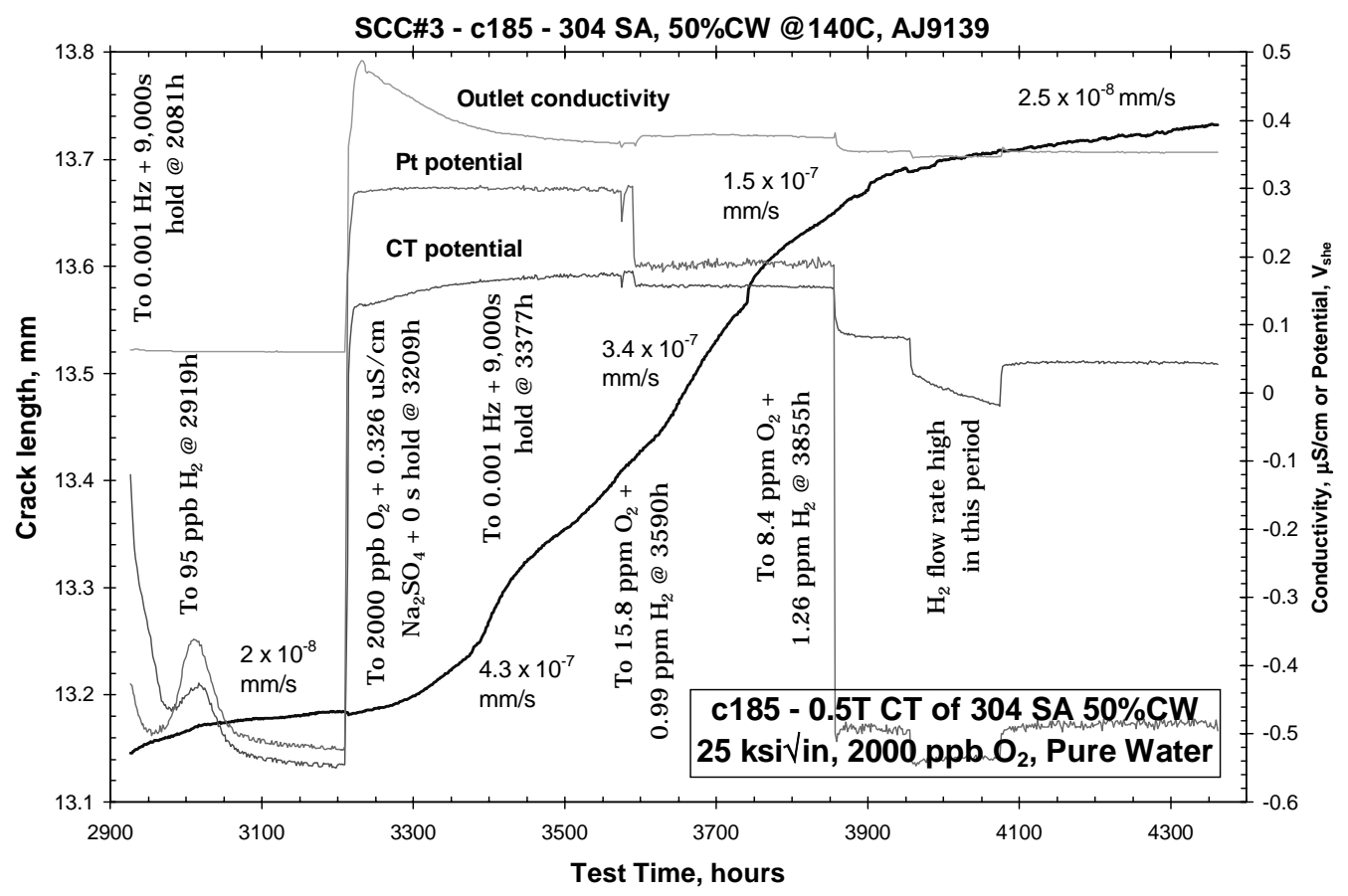

Figure 156. SCC behavior of non-sensitized Type 304 SS specimen c185 after Na2 So4 addition. 


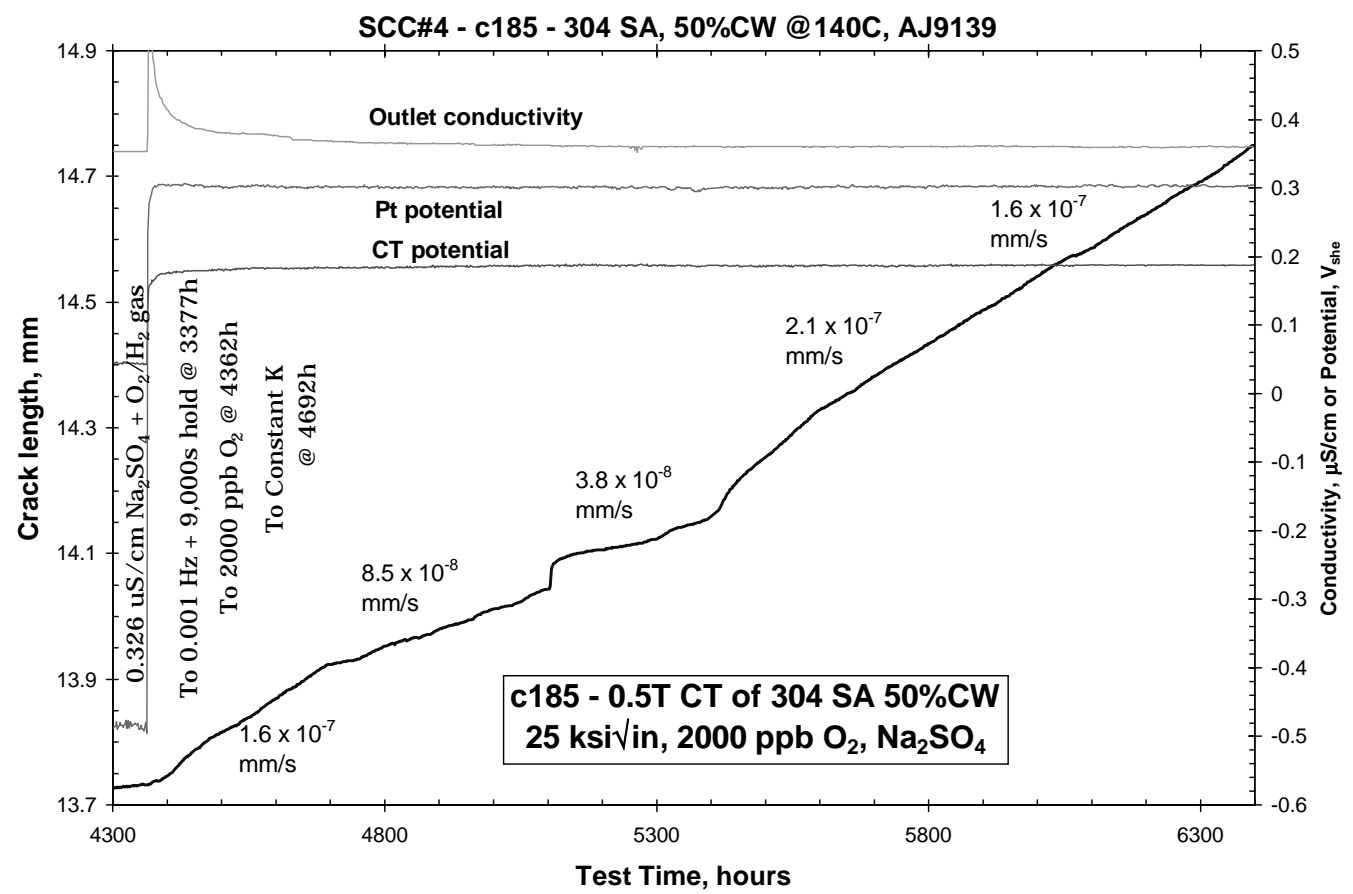

Figure 157. SCC behavior at constant K for the non-sensitized specimen c185, Type 304 SS (heat AJ9139) with $50 \%$ reduction in thickness by forging at $+140^{\circ} \mathrm{C}$.

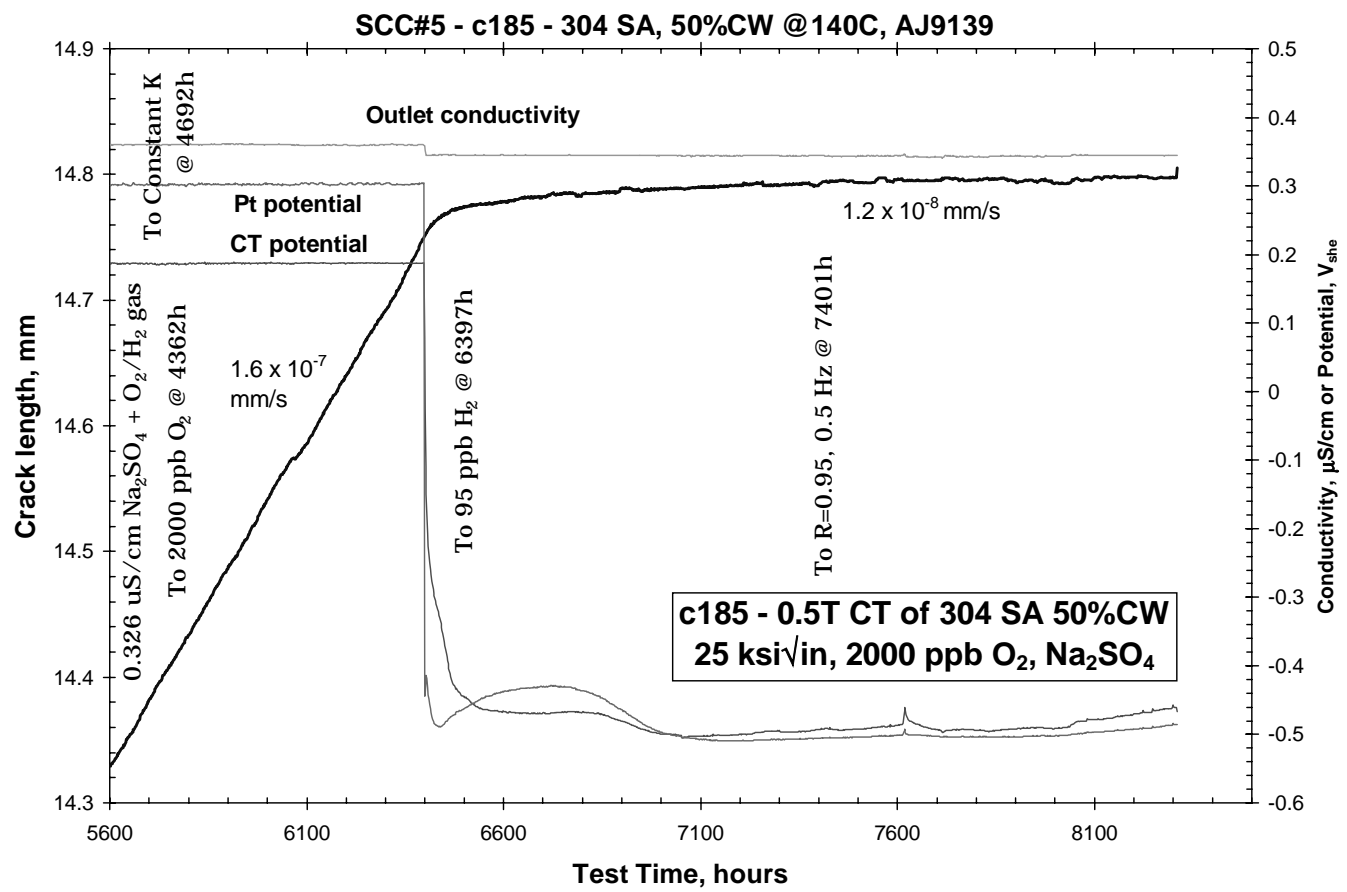

Figure 158. Influence of the $\mathrm{H}_{2}$ addition on the SCC behavior of non-sensitized specimen c185.

fabrication. It is more extensive and deeper on one side, but a small amount is also visible on the other side. The dc potential drop indicated growth is $4.71 \mathrm{~mm}$, while the average, 

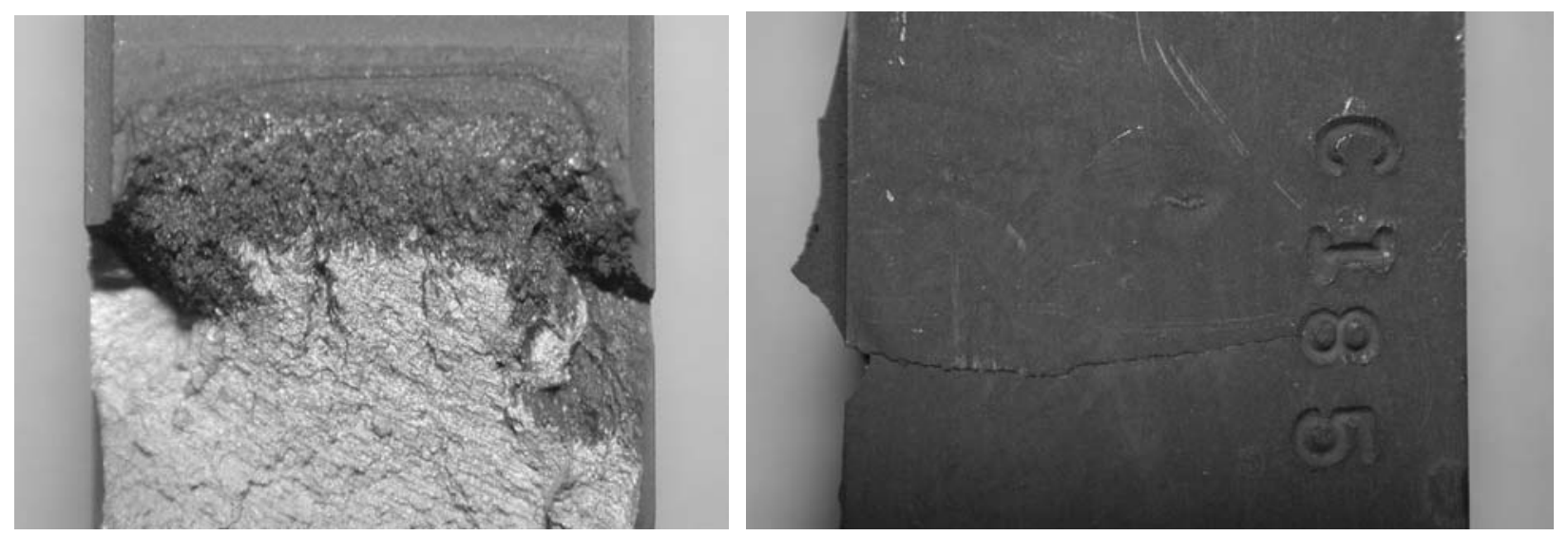

Figure 159. Light macrographs of specimen c185, 304 SS (heat AJ9139) with 50\% reduction by forging at $+140^{\circ} \mathrm{C}$ showing unusual cracking behavior at sample sides.
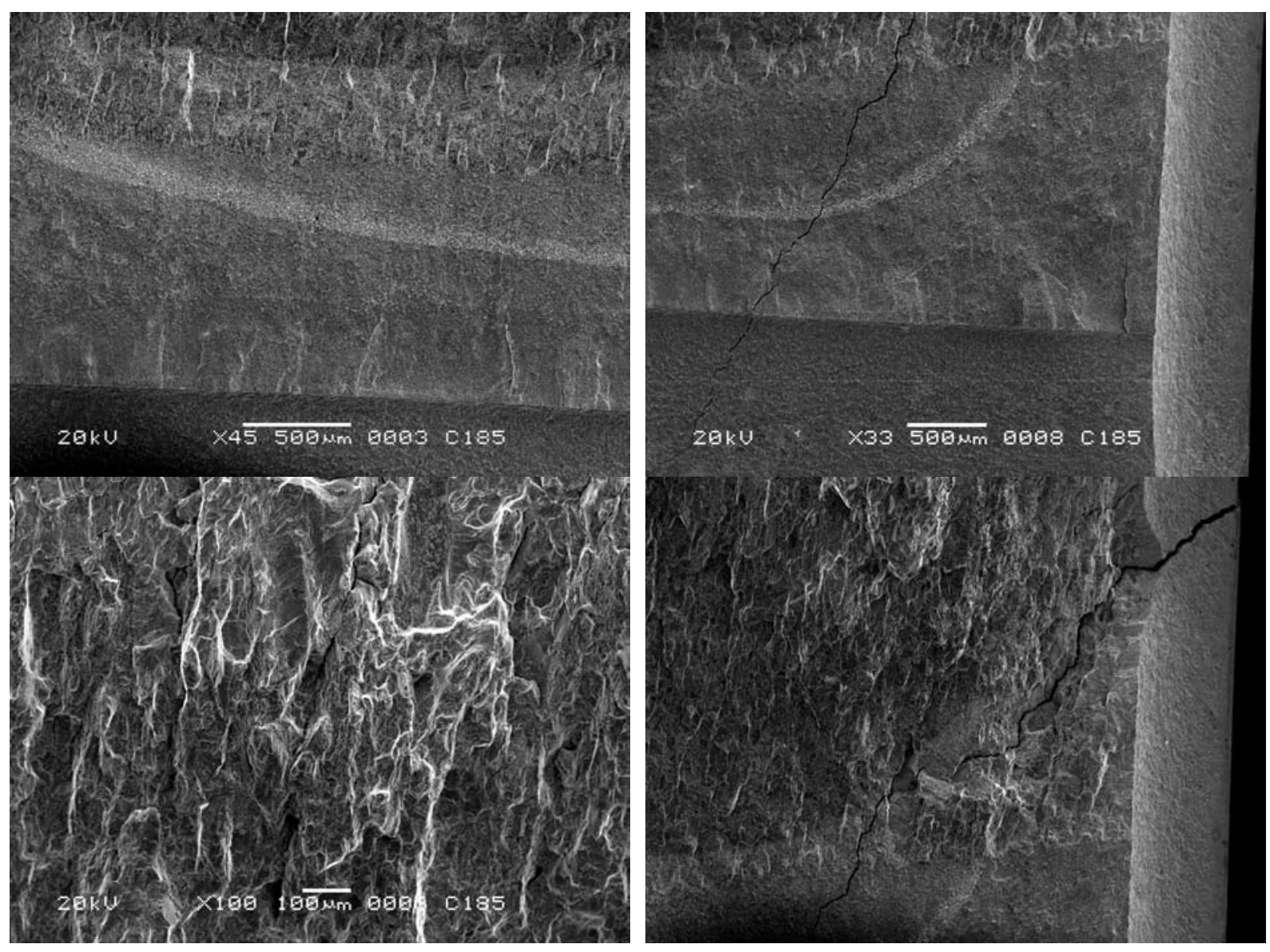

Figure 160. SEM micrograph of fracture surface for specimen c185, Type 304 SS (heat AJ9139) with $50 \%$ reduction in thickness by forging at $+140^{\circ} \mathrm{C}$.

maximum and minimum of the actual crack depth are $5.61 \mathrm{~mm}, 8.92 \mathrm{~mm}$ and $4.45 \mathrm{~mm}$. No corrections to the crack length or stress intensity data of specimen c185 were performed. 
Specimen c201 of Healed + Cold-Worked Type 304 SS (heat AJ9139) In this study, alloy strength and grain boundary characteristics have been individually controlled by material processing to allow crack-growth-rate measurements on non-irradiated stainless steels. This specimen was designed to produce grain boundary carbides without $\mathrm{Cr}$ depletion in alloys of high strength, with irradiation hardening emulated using cold work. Type 304 SS was solution annealed at $1050^{\circ} \mathrm{C}$ for 30 minutes and water quenched, then sensitized at $621^{\circ} \mathrm{C}$ for 24 hours and air cooled. A healing heat treatment was then performed at $950^{\circ} \mathrm{C}$ for 5 hours with water quenching, with the objective of eliminating grain boundary $\mathrm{Cr}$ depletion while retaining the grain boundary carbides. The yield strength of the material was then increased by forging at $+140^{\circ} \mathrm{C}$ to $20 \%$ reduction in thickness.

The initial healing treatment $\left(925^{\circ} \mathrm{C}\right.$ for $5 \mathrm{~h}+$ water quench) was evaluated by analytical electron microscopy to ensure that grain boundary $\mathrm{Cr}$ depletion was eliminated. The $\mathrm{Cr}$ profile showed one point at the boundary that was $1-2 \%$ lower in $\mathrm{Cr}$; this is so narrow that it probably did not come from the $925^{\circ} \mathrm{C}$ phase of the heat treatment, but perhaps from thermal changes before or during water quenching. To determine whether a higher "healing" temperature would resolve this, the sensitized material was heat treated at $950^{\circ} \mathrm{C}$ for $5 \mathrm{~h}$ with water quenching; it also showed a single point along the grain boundary where the $\mathrm{Cr}$ concentration was slightly suppressed, but about $1-1.5 \%$. Since this is a very small value, and (if it had any effect whatsoever in these tests) would tend to lead to conservative conclusions with regard to the effect of grain boundary carbides. That is, the reduction in observed growth rate associated with the presence of grain boundary carbides might be somewhat greater if absolutely no $\mathrm{Cr}$ depletion existed. Note that thermal sensitization often reduces the matrix Cr level by $50-75 \%$ at the grain boundary (e.g., from $18 \% \mathrm{Cr}$ to $\sim 10 \% \mathrm{Cr}$ ).

An overview of the entire test is shown in Figure 161, and Figure 162 shows the response during air-fatigue pre-cracking. Figure 163 shows the response following assembly, set up, and exposure to $288^{\circ} \mathrm{C}$ pure water under loading conditions designed to transition from transgranular fatigue cracking to IGSCC. The response is reasonably well behaved, with the environmentally assisted crack-growth rates being somewhat lower than have been previously observed on coldworked stainless steels without grain boundary carbides.

The subsequent transition to constant stress intensity factor conditions is shown in Figure 164, and the long term SCC growth rate is only about $3.5 \times 10^{-8} \mathrm{~mm} / \mathrm{s}$. This is much lower $(4-10 \mathrm{X}$ lower) than is routinely observed in materials of similar yield strength, as compared later. Figure 165 shows the response as the loading conditions are changed from constant load, to gentle cyclic loading ( $\mathrm{R}=0.7,0.001 \mathrm{~Hz}+9000 \mathrm{~s}$ hold) at $1549 \mathrm{~h}$, then back to constant load at $2362 \mathrm{~h}$. At about $3000 \mathrm{~h}$ (Figure 166), there was a transient increase in crack growth rate that was not associated with any change in the test conditions. Over the longer term, the crack growth rate slowed, and the average crack growth rate over the entire period at constant load is $2 \times 10^{-8}$ $\mathrm{mm} / \mathrm{s}$.

The test was ended at $4491 \mathrm{~h}$, and the specimen was fatigued apart in air. Figure 167 shows a low-magnification macrograph of the fracture appearance, and Figure 168 documents the fractography by SEM. The fatigue pre-crack is relatively straight, although there is an 


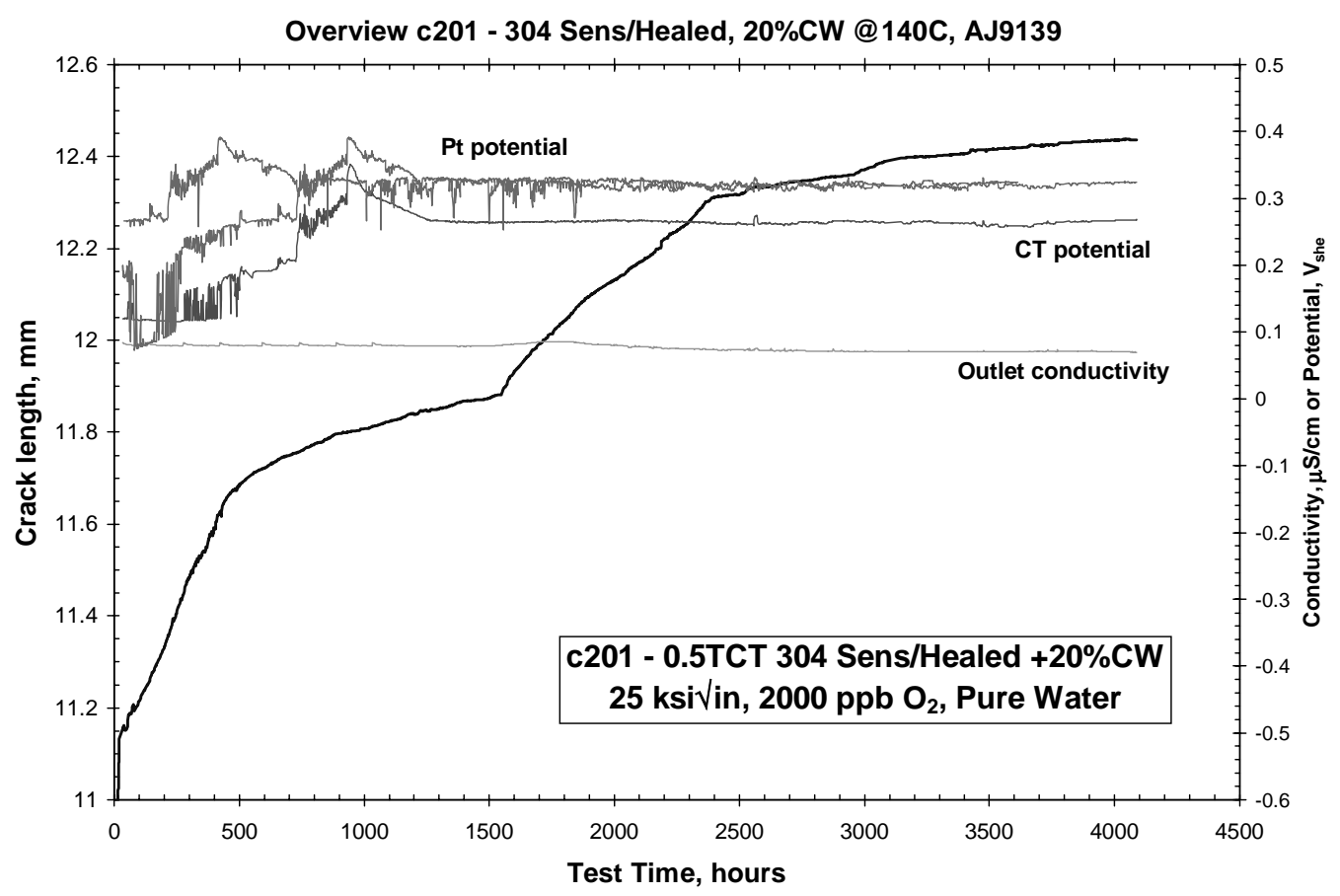

Figure 161. Overview of test c201 fabricated from Type 304 stainless steel that was annealed, sensitized to produce grain boundary carbides, "healed" to eliminate Cr depletion, then cold forged by $20 \%$ reduction in thickness.

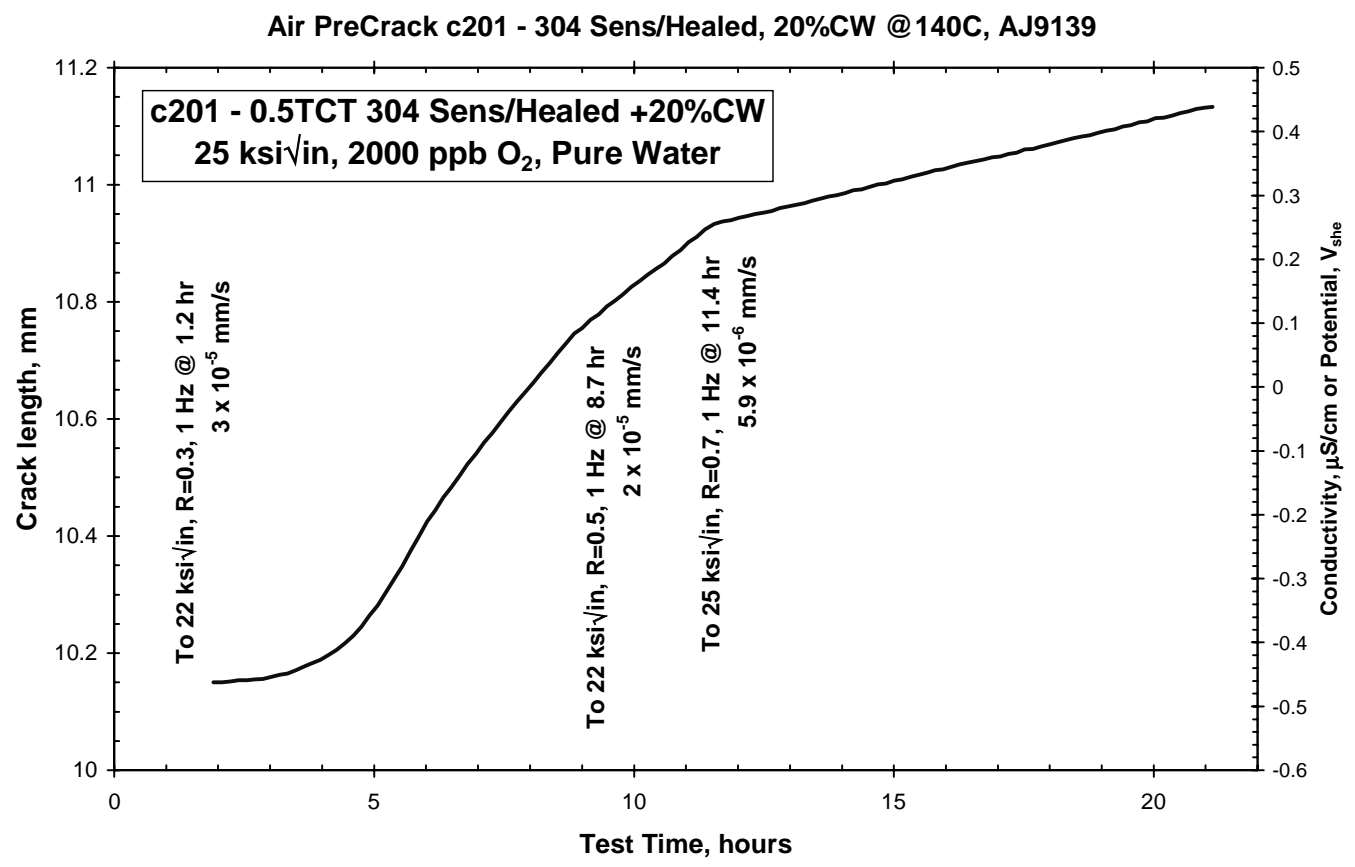

Figure 162. Crack length vs. time during air fatigue pre-cracking for desensitized specimen c201. 


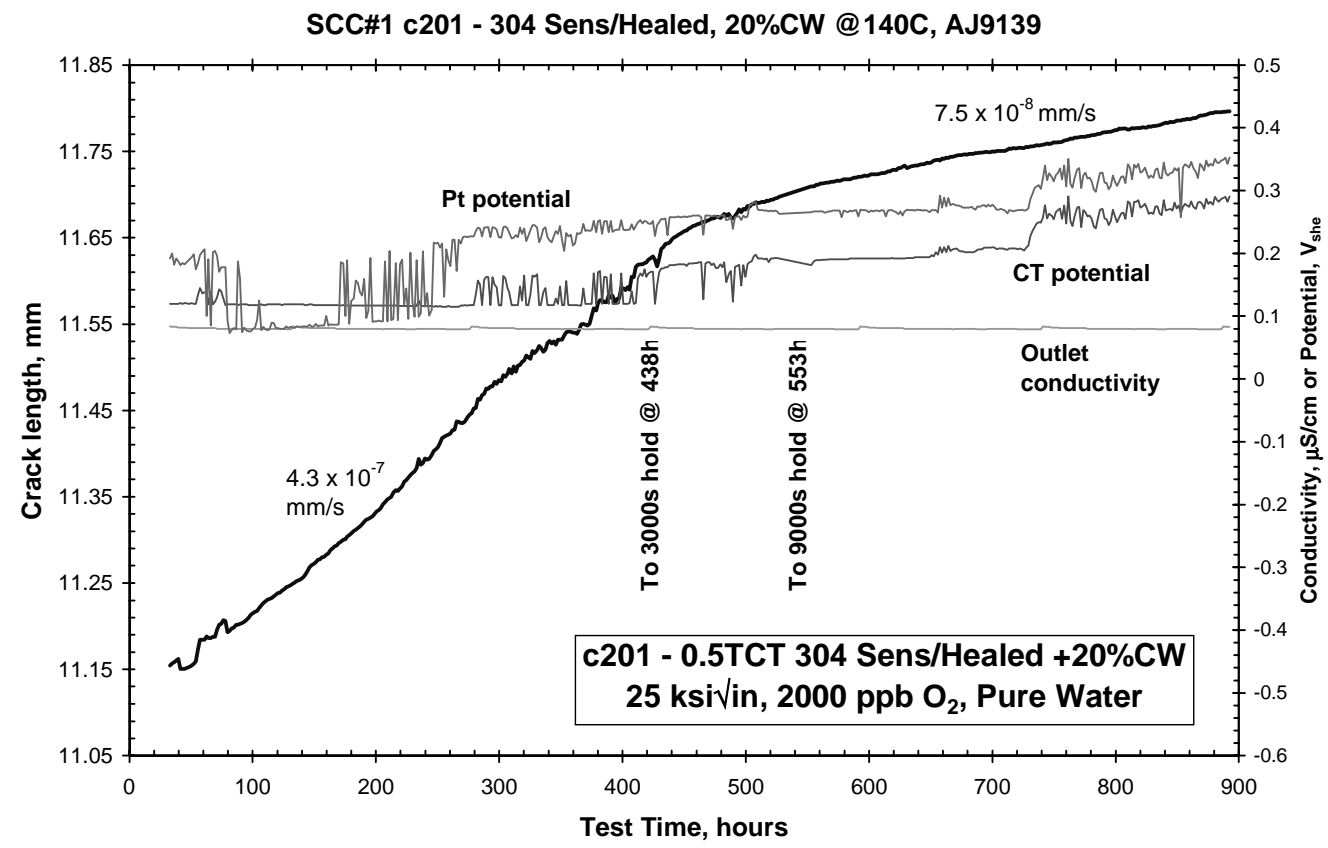

Figure 163. Crack length vs. time during initial exposure to high temperature water and transitioning from transgranular fatigue to IGSCC for specimen c201.

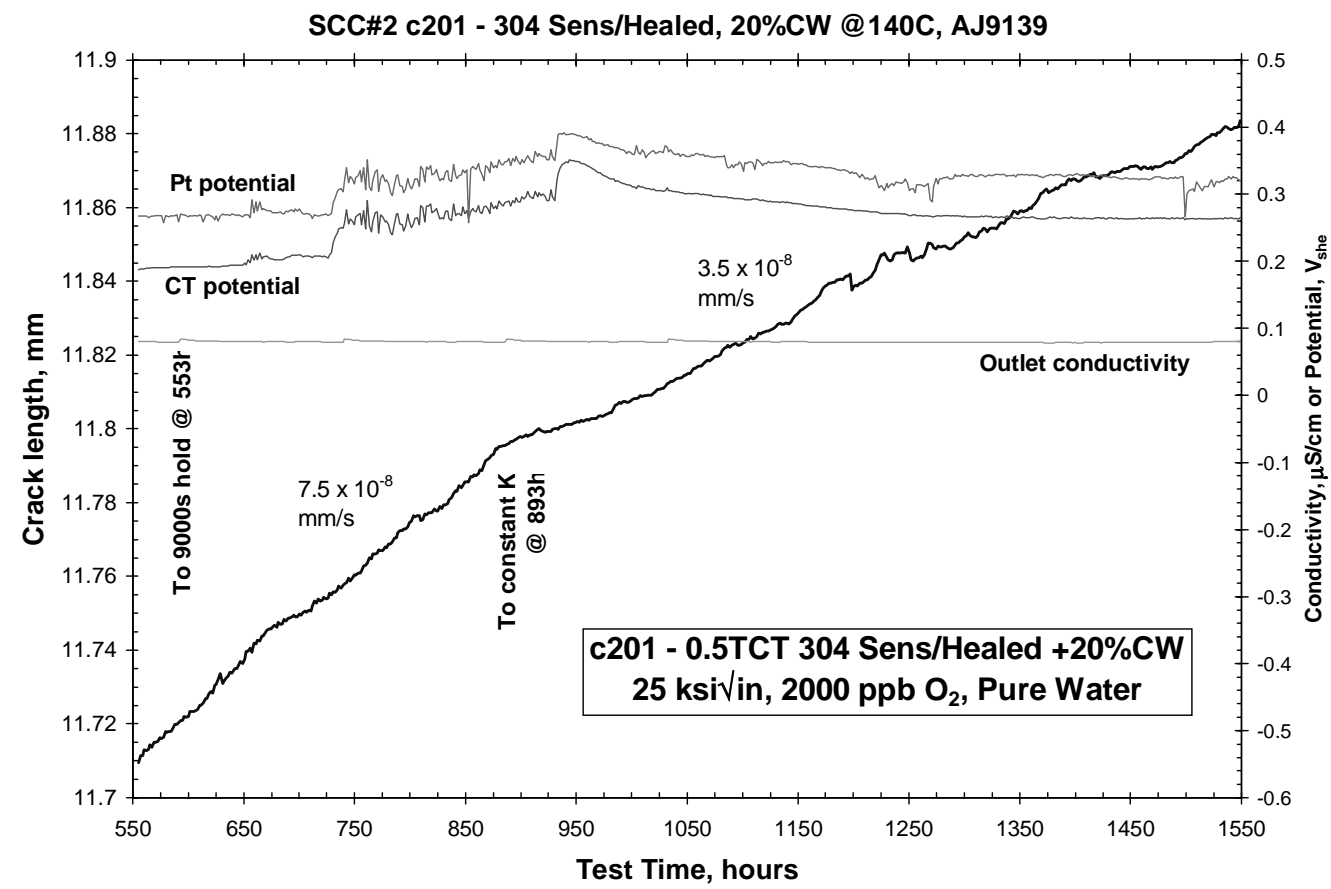

Figure 164. Crack length vs. time in $288^{\circ} \mathrm{C}$ pure water under very gentle cyclic loading and constant $\mathrm{K}$ conditions for desensitized specimen c201. 


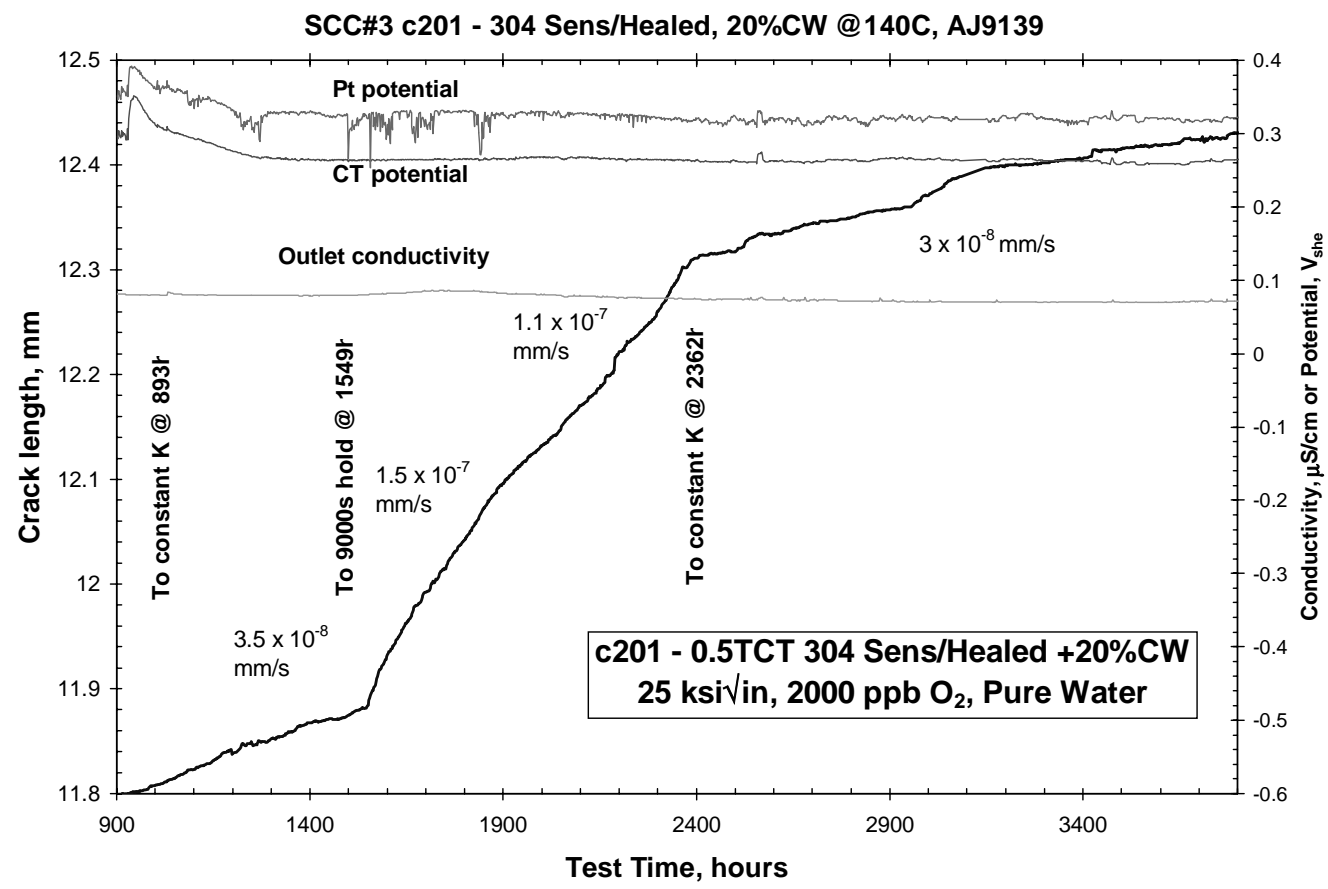

Figure 165. SCC behavior under very gentle cyclic loading and constant K conditions for desensitized specimen c201.

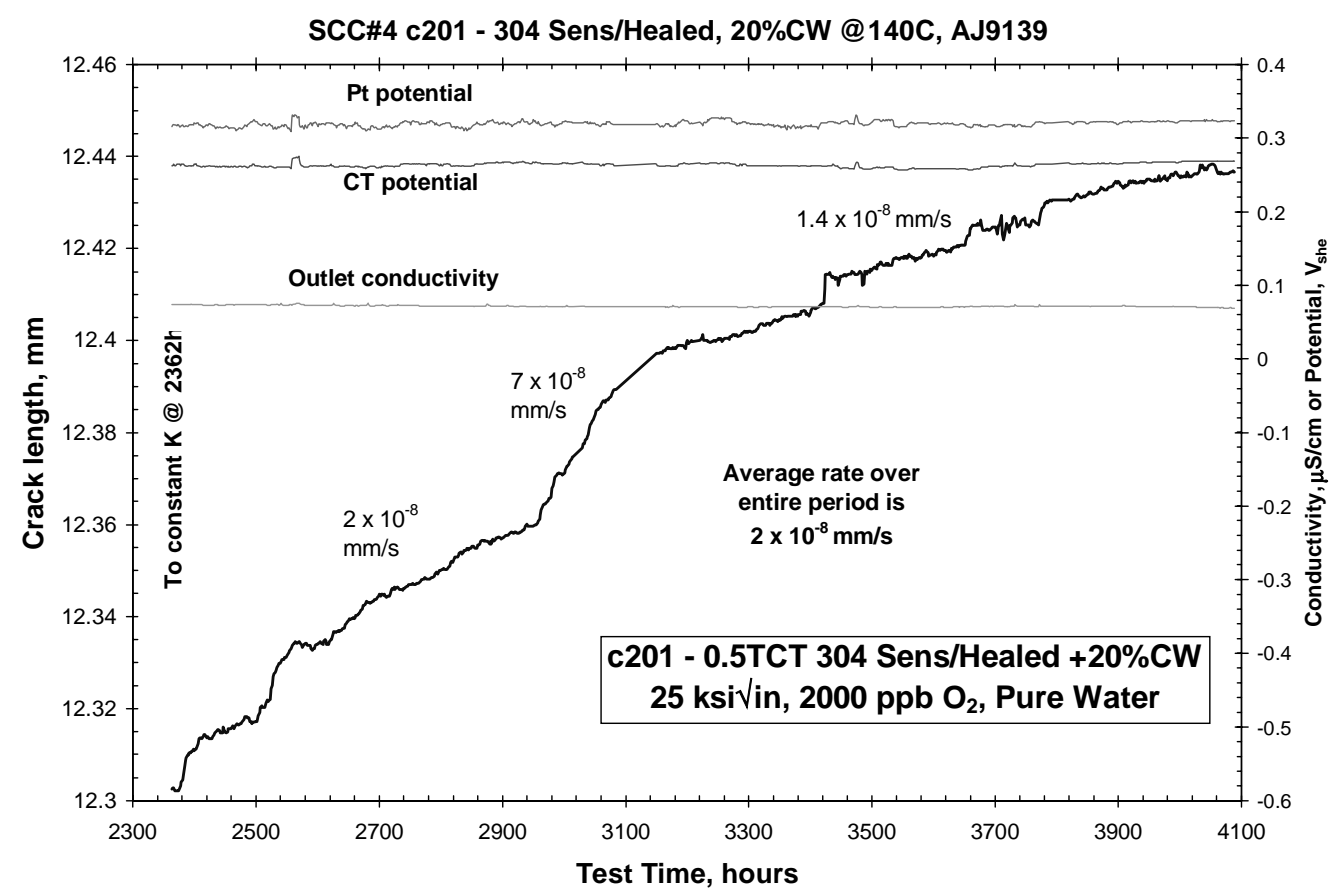

Figure 166. Crack length vs. time in $288^{\circ} \mathrm{C}$ pure water under constant $\mathrm{K}$ conditions for specimen c201. 
unusual U-shaped appearance in the center of the specimen. Intergranular nucleation from the transgranular fatigue crack occurred more successfully in this central area, but even there the uniformity of a crack front that is desired and often achieved did not occur. The dc potential drop indicated growth is $2.29 \mathrm{~mm}$, while the average, maximum and minimum of the actual crack depth are $4.47 \mathrm{~mm}, 5.51 \mathrm{~mm}$ and $2.54 \mathrm{~mm}$. No corrections to the crack length or stress intensity data of specimen c201 were performed.

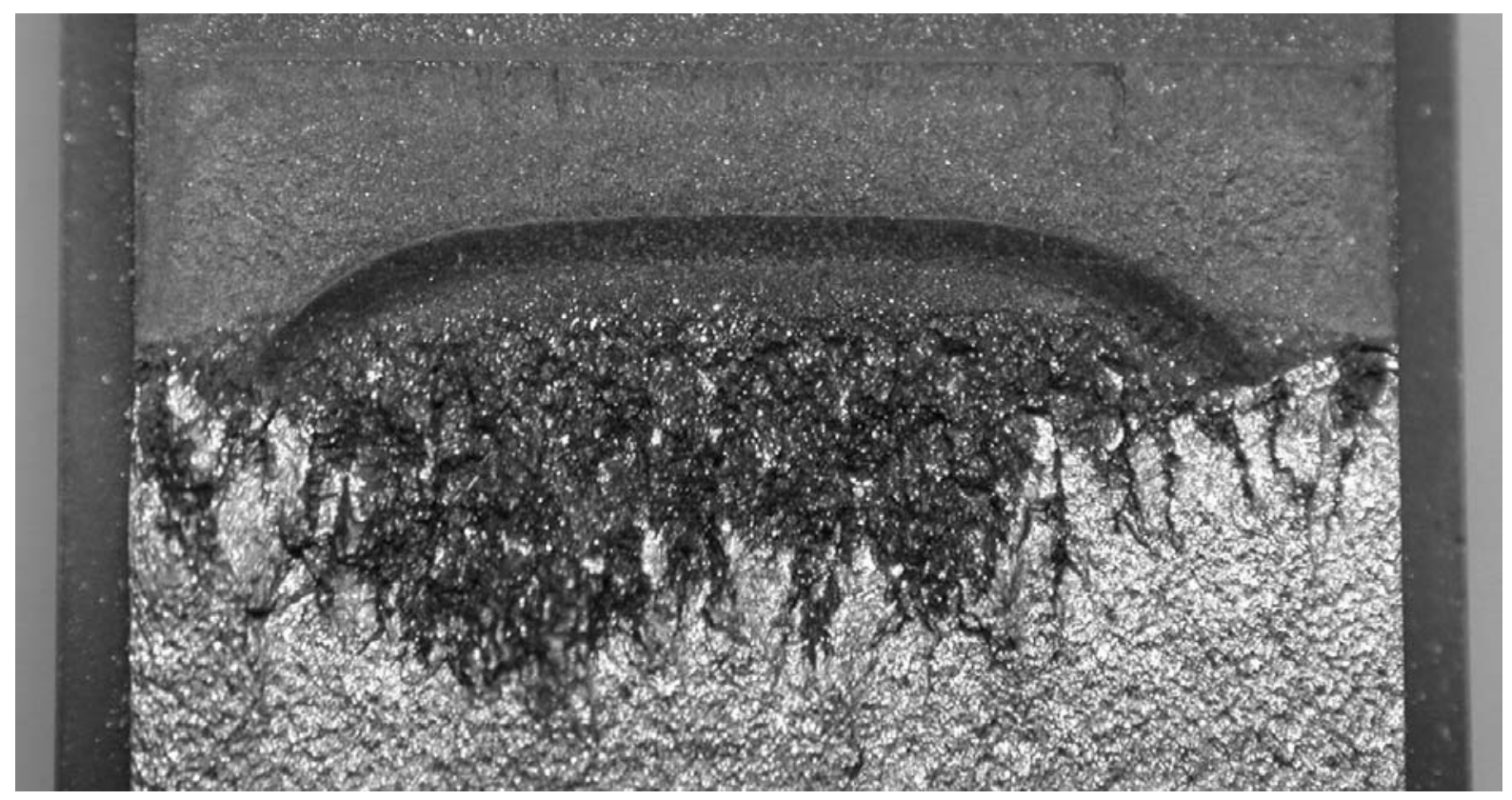

Figure 167. Light macrograph of specimen c201, desensitized Type 304 SS (heat AJ9139) showing unusual appearance of the SCC crack front. 

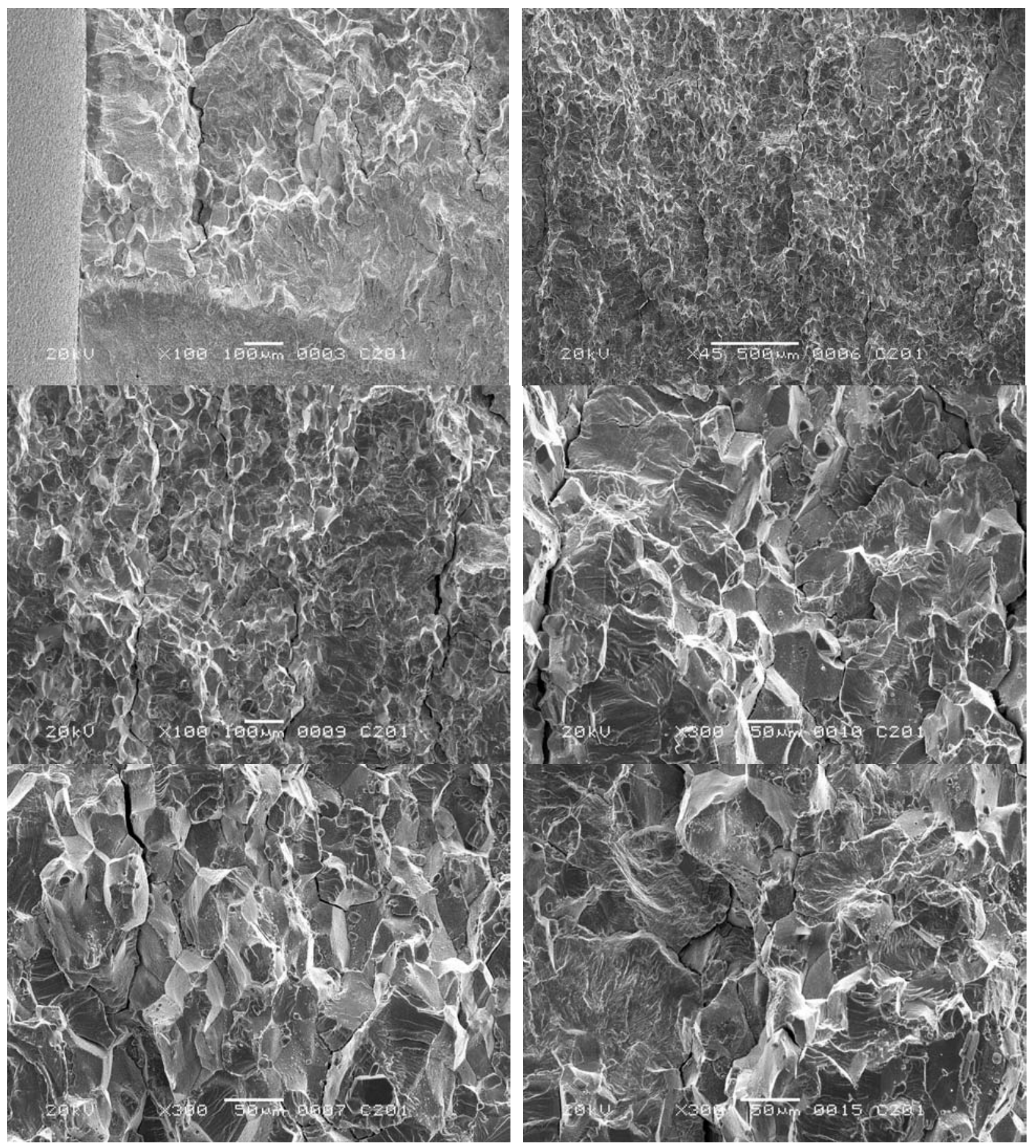

Figure 168. SEM micrograph of the fracture surface for desensitized 304 SS specimen c201. 
Comparisons and Summary of Stress Corrosion Response

A preeminent issue is whether SCC occurs only on some stainless steels and not on others; that is, do variations in composition, processing, temperature, corrosion potential, etc. produce variations in SCC susceptibility or a complete shift from susceptibility to immunity. The concept of SCC immunity clearly does not apply for these non-sensitized Types 304, 304L and 316L SS, and the observations reported here are consistent with prior observations of SCC on annealed and cold-worked stainless steels (Figures 169 - 174), and even alloy 600 (Figure 175). There are a

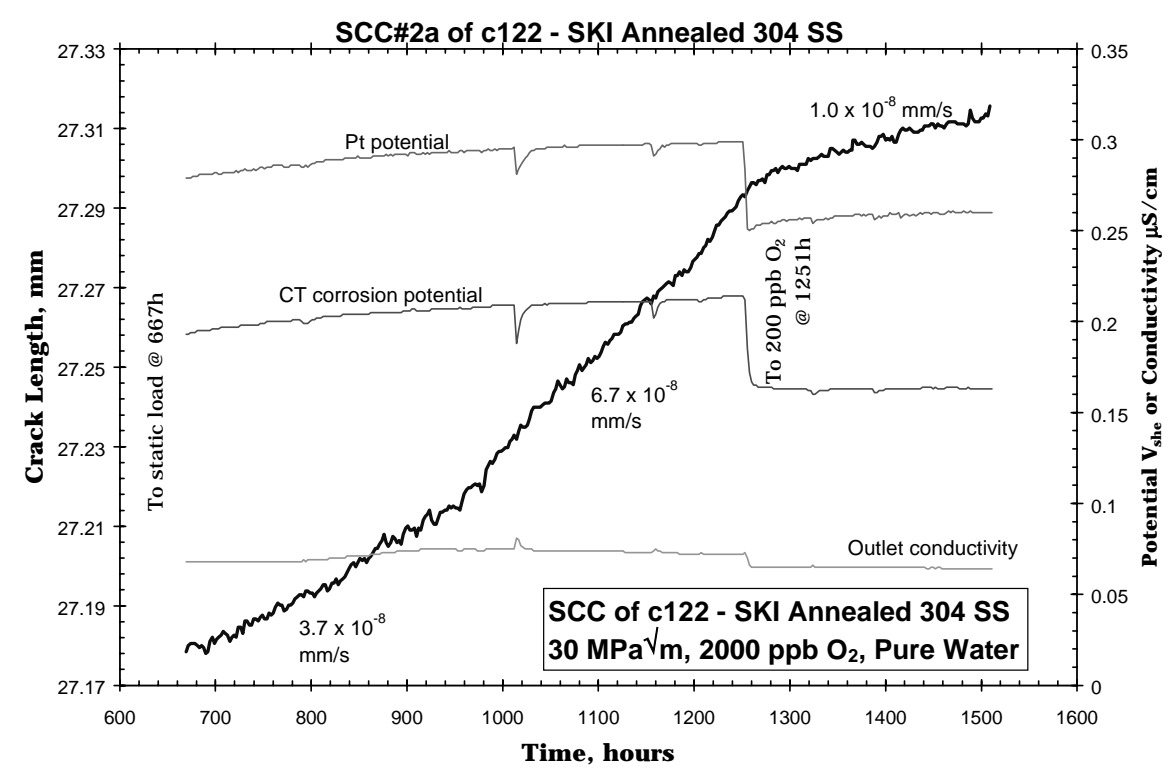

Figure 169. SCC behavior of specimen c122 of solution-annealed Type 304 SS, identical to that used in the SKI/EPRI Round Robin.

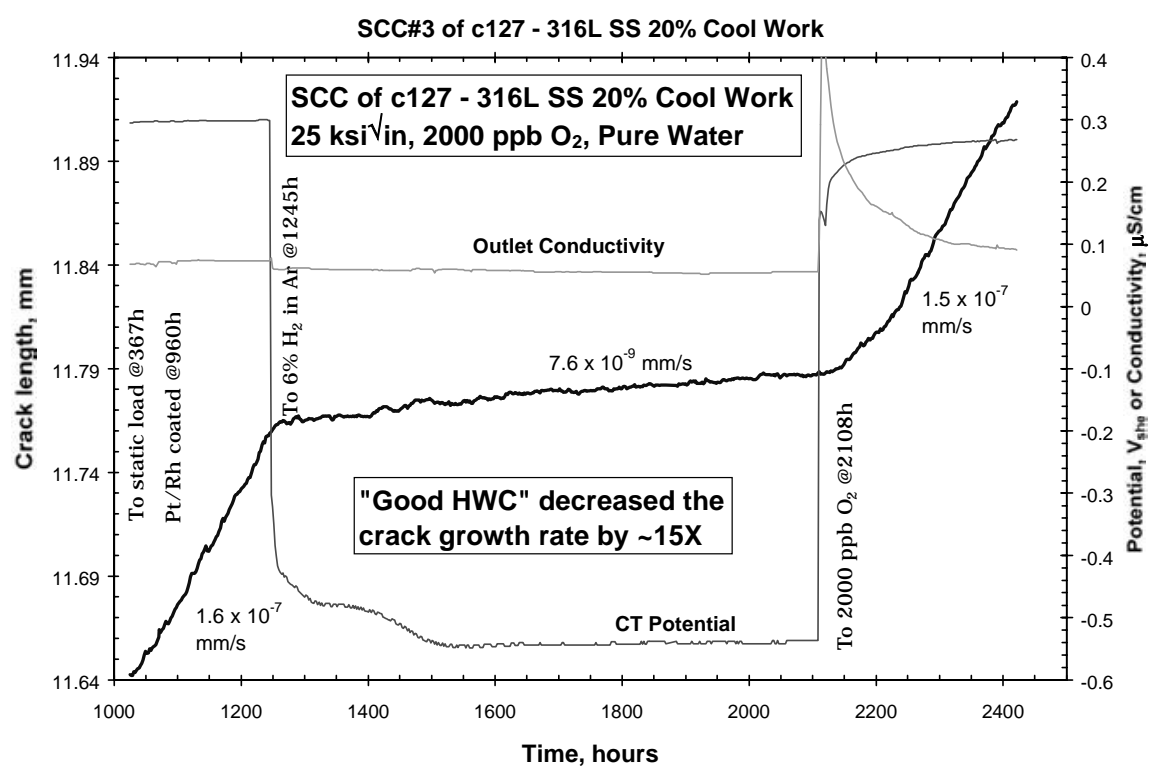

Figure 170. SCC response of CT specimen c127 of Type 316L SS (heat A14128) cool rolled $20 \%$ at $+140^{\circ} \mathrm{C}$ and tested in a separate GE GRC program. 


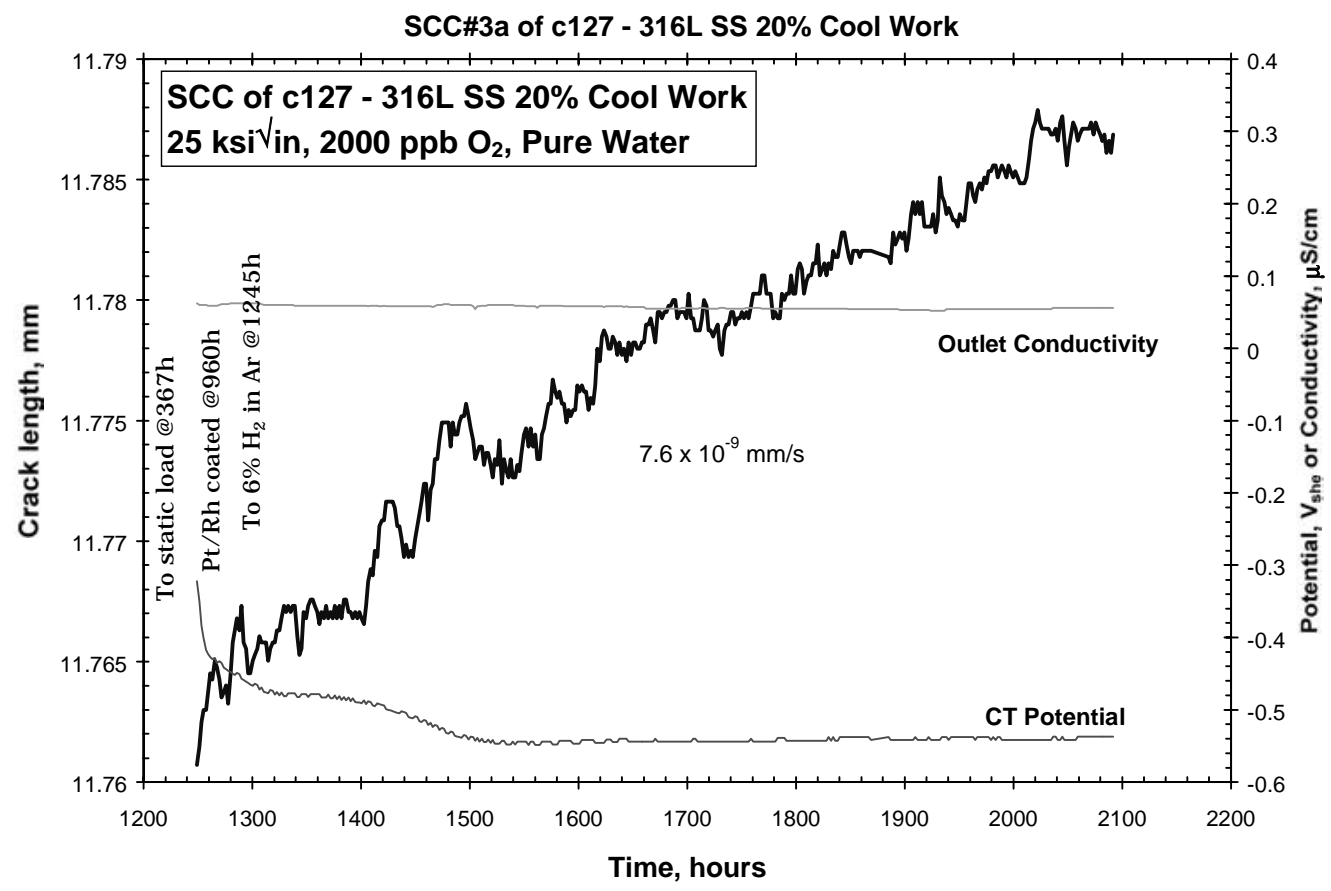

Figure 171. SCC response of CT specimen c127 of Type 316L SS (heat A14128) cool rolled $20 \%$ at $+140^{\circ} \mathrm{C}$ and tested in a separate GE GRC program.

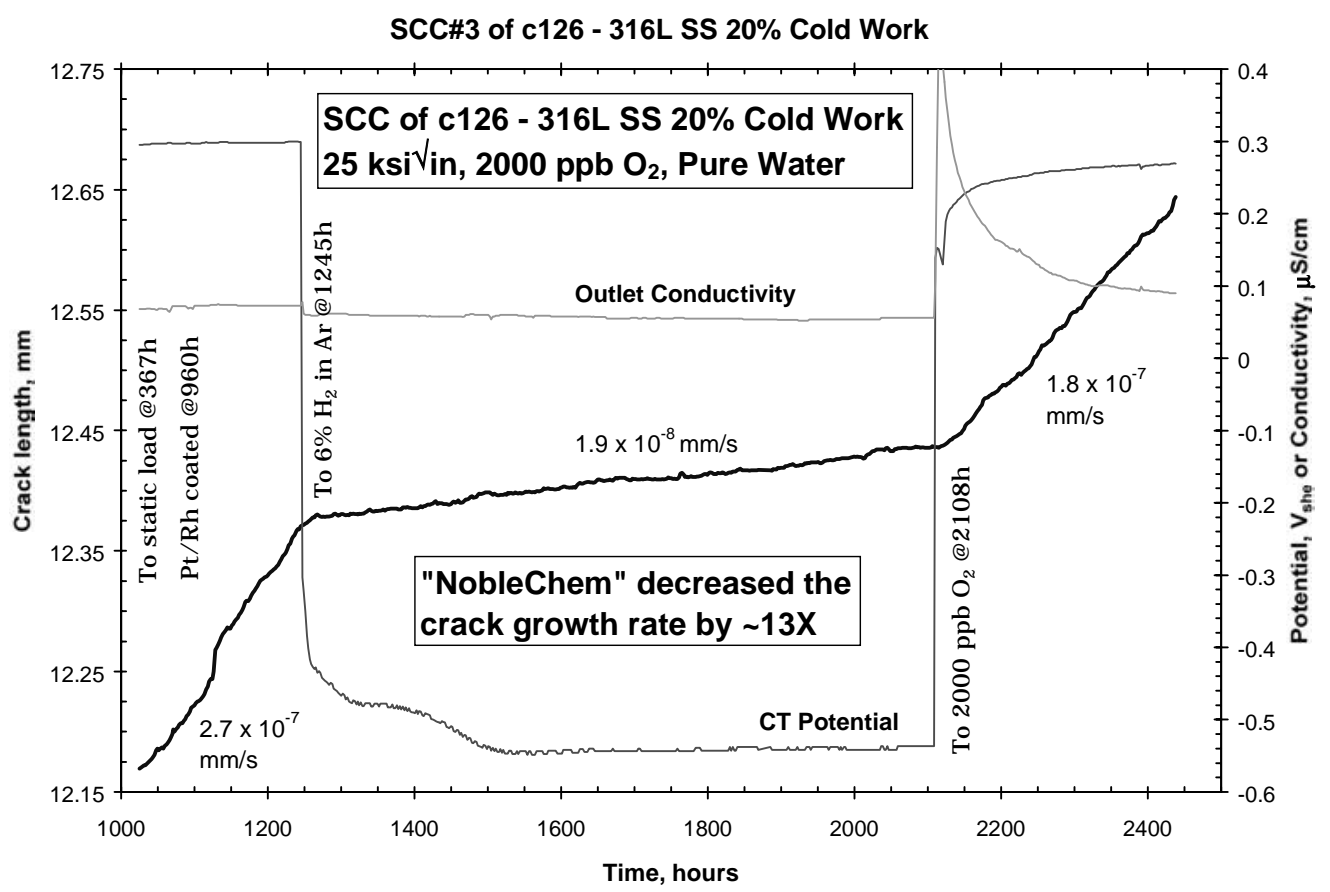

Figure 172. SCC response of CT specimen c126 of Type 316L SS (heat A14128) cold rolled $20 \%$ at $-55^{\circ} \mathrm{C}$ and tested in a separate GE GRC program. 


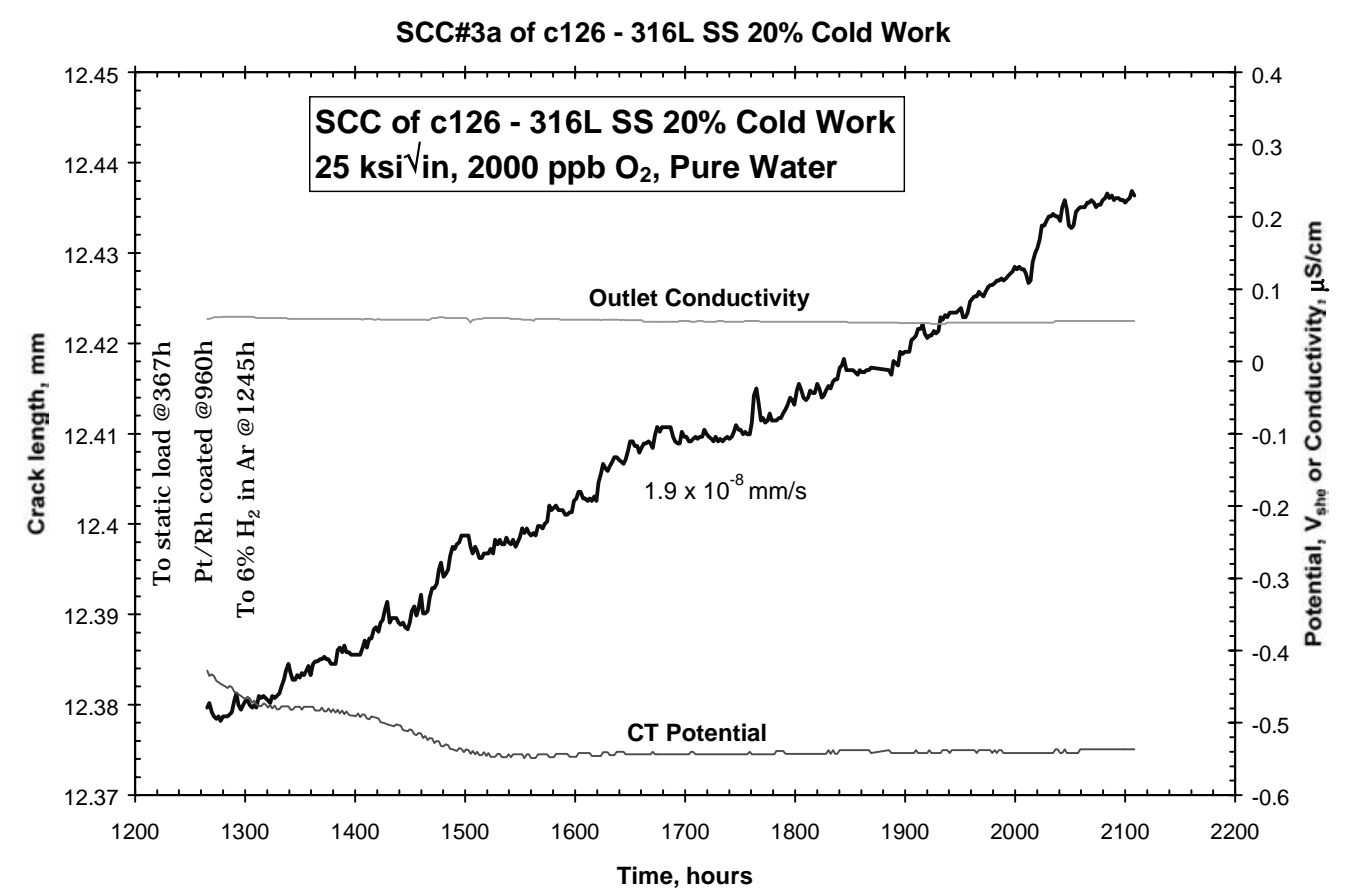

Figure 173. SCC response of CT specimen c126 of Type 316L SS (heat A14128) cold rolled $20 \%$ at $-55^{\circ} \mathrm{C}$ and tested in a separate GE GRC program.

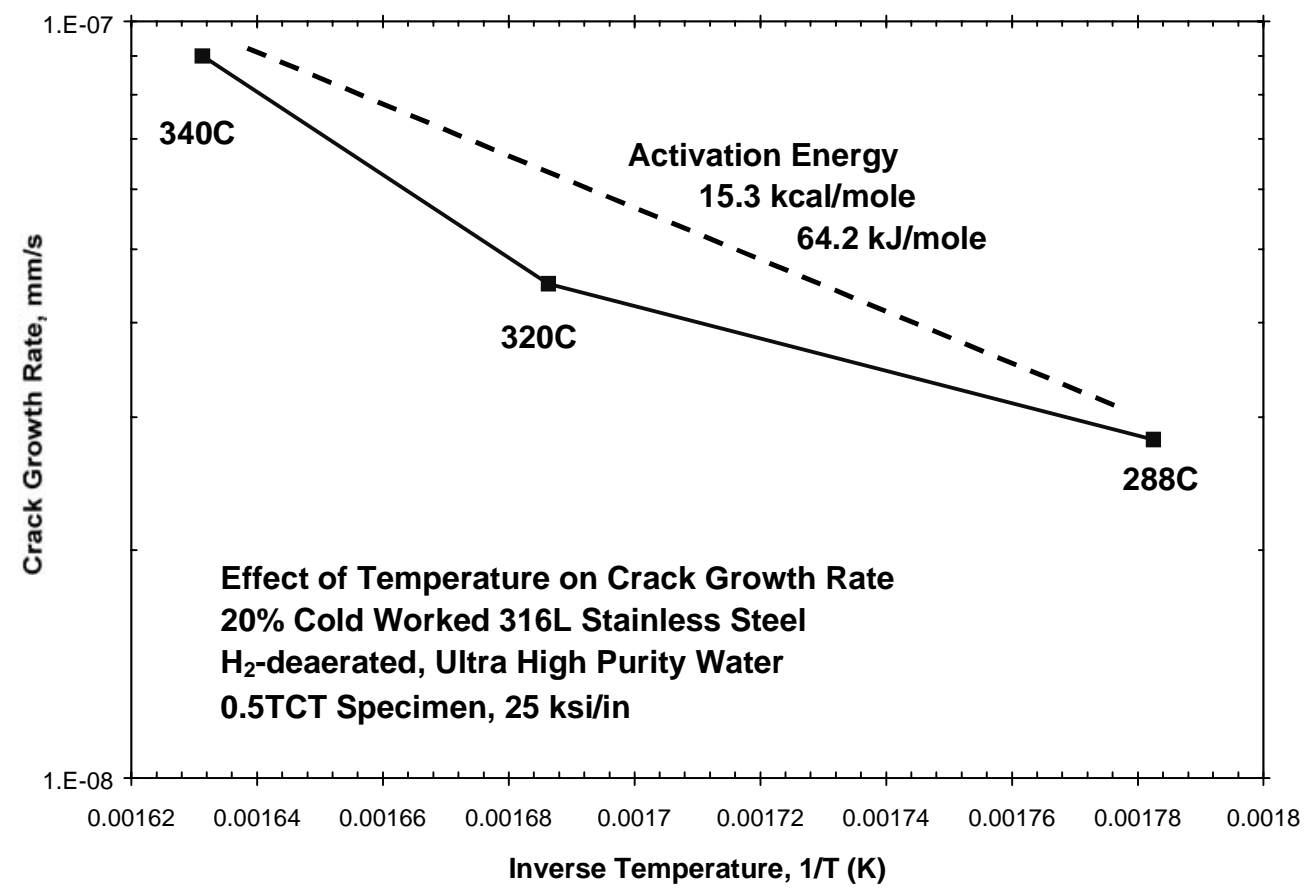

Figure 174. Crack growth rate vs. temperature for Type $316 \mathrm{~L} \mathrm{SS} 20 \%$ cold rolled at $+140^{\circ} \mathrm{C}$ and tested in $\mathrm{H}_{2}$ deaerated, pure water. 
variety of factors that enhance SCC growth rates, including temperature (Figure 174), corrosion potential (Figures 176 and 177), sensitization (Figure 176), cold work (Figure 177), yield strength (Figures 178 and 179), etc. However, even solution-annealed stainless steels are susceptible to SCC in ultra-high-purity water (Figure 169). Similarly, cold-worked stainless steels undergo SCC in ultra-high-purity, $\mathrm{H}_{2}$-deaerated water, as shown in the data summarized in this report as well as from prior work.

The importance of sensitization has long been recognized, although there has been significant disagreement about the magnitude of the crack-growth rates in sensitized stainless steels under plant-relevant conditions. Figure 176 shows the data obtained on sensitized stainless steels in the SKI / EPRI Round Robin by five highly experienced, international laboratories, along with predictions by PLEDGE (GE's SCC prediction methodology). These rates are much higher (about 50X) than the mean of a separate collection of 120 readily available data from three labs, which was carefully evaluated statistically in a major industry initiative. This highlights the need for improved techniques to be used for studying SCC.

However, it is also clear that even solution-annealed stainless steels can have moderate SCC susceptibility and, when cold worked, can have about the same susceptibility as sensitized stainless steel (Figure 177). Fortunately, the SCC crack-growth rates are greatly reduced as the corrosion potential is reduced, and this is the strategy widely adopted in the operation of modern BWRs via the introduction of NobleChem ${ }^{\mathrm{TM}} \cdot{ }^{67,68}$ However, the reduction in growth rate at low corrosion potential for cold-worked stainless steels is not as great as for sensitized stainless steels, although at $288^{\circ} \mathrm{C}$ the rates remain acceptably low in most instances.

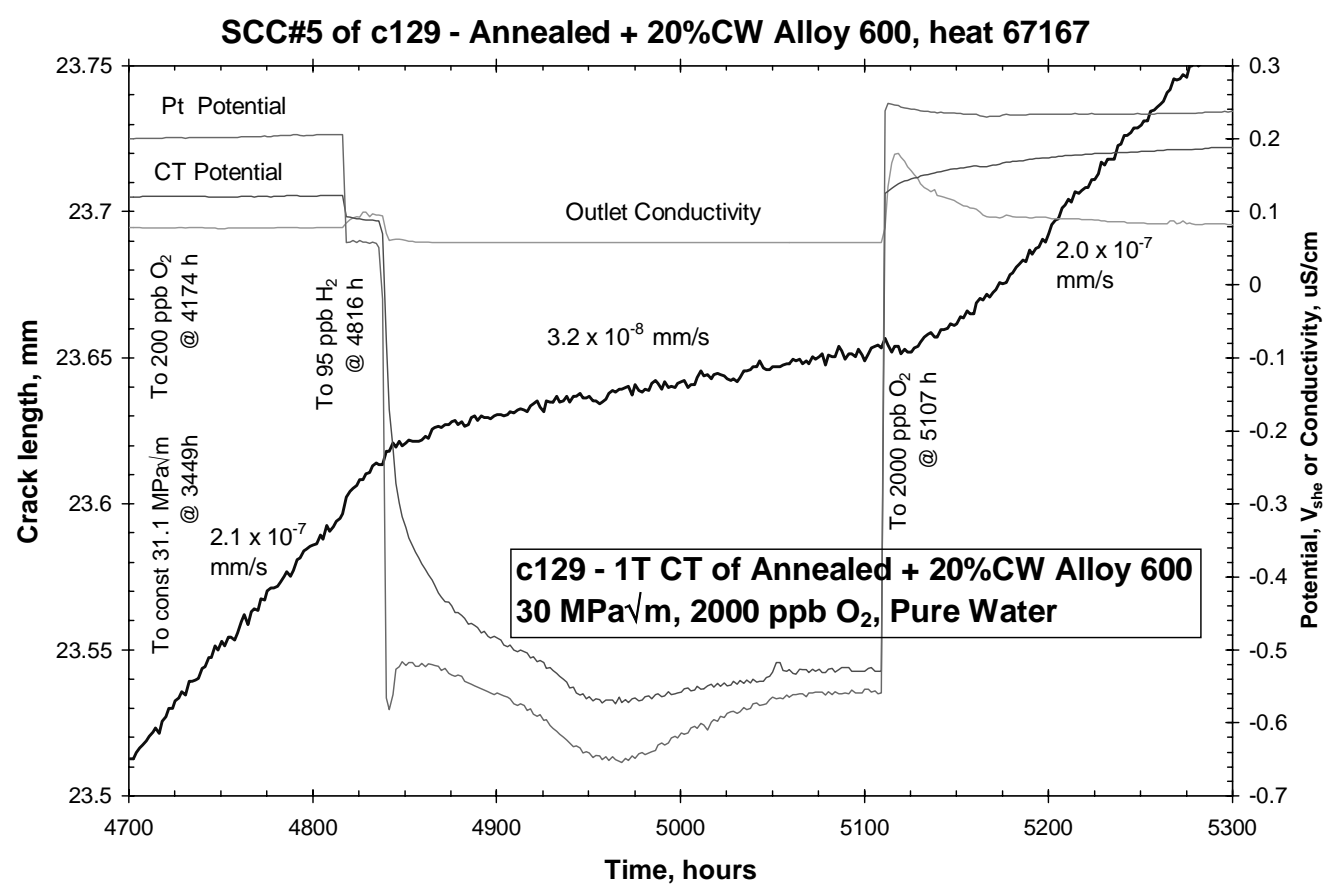

Figure 175. Crack depth vs. time for non-sensitized alloy 600 cold rolled to $20 \%$ reduction in thickness at $25^{\circ} \mathrm{C}$ and tested in $288^{\circ} \mathrm{C}$ pure water. 


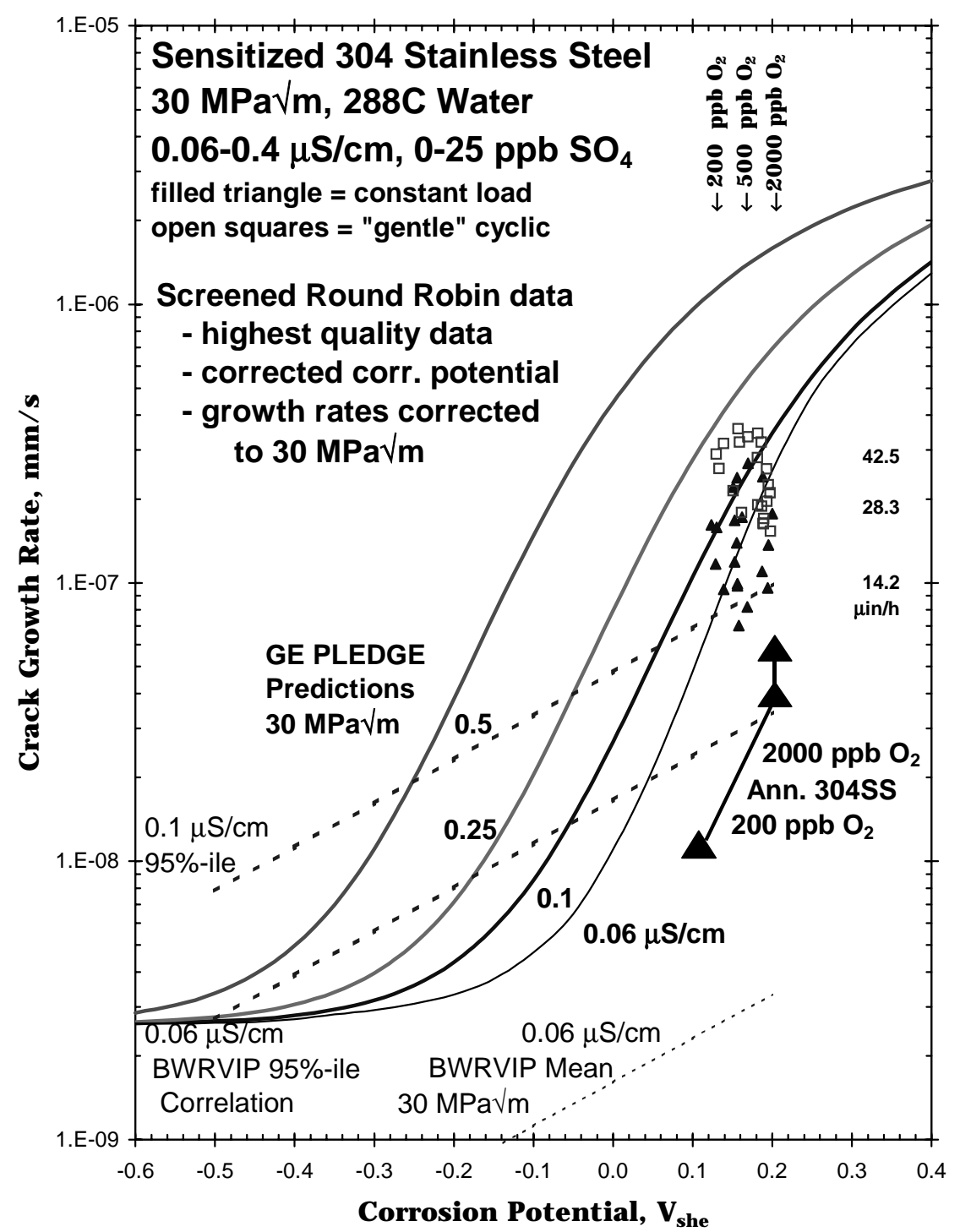

Figure 176. Crack growth rate vs. corrosion potential for annealed and sensitized type 304 SS tested in $288^{\circ} \mathrm{C}$ pure water.

As temperature is increased (for example in PWRs from $290^{\circ} \mathrm{C}$ to $323^{\circ} \mathrm{C}$, typical core inlet and outlet temperatures), the susceptibility to SCC rises as illustrated in Figure 174. Temperatures at or above $340^{\circ} \mathrm{C}$ can exist in the pressurizers and from in-core from gamma heating. Under these conditions, the SCC growth rates can approach those in sensitized or cold worked material at high corrosion potential in $288^{\circ} \mathrm{C}$ water.

While grain boundary chromium carbides accompanied by sensitization clearly accelerate crack advance, the studies on specimen c201 show that in the absence of $\mathrm{Cr}$ depletion the presence of grain boundary carbides is beneficial, reducing growth rates in cold-worked stainless steels by 3 to 10X in Figures 164-166. However, the combination of sensitization and cold work (specimen c184) was not as damaging as expected if their contributions to crack-growth rate were additive Because the growth rates were similar to the companion cold-worked Type 304 stainless steel 


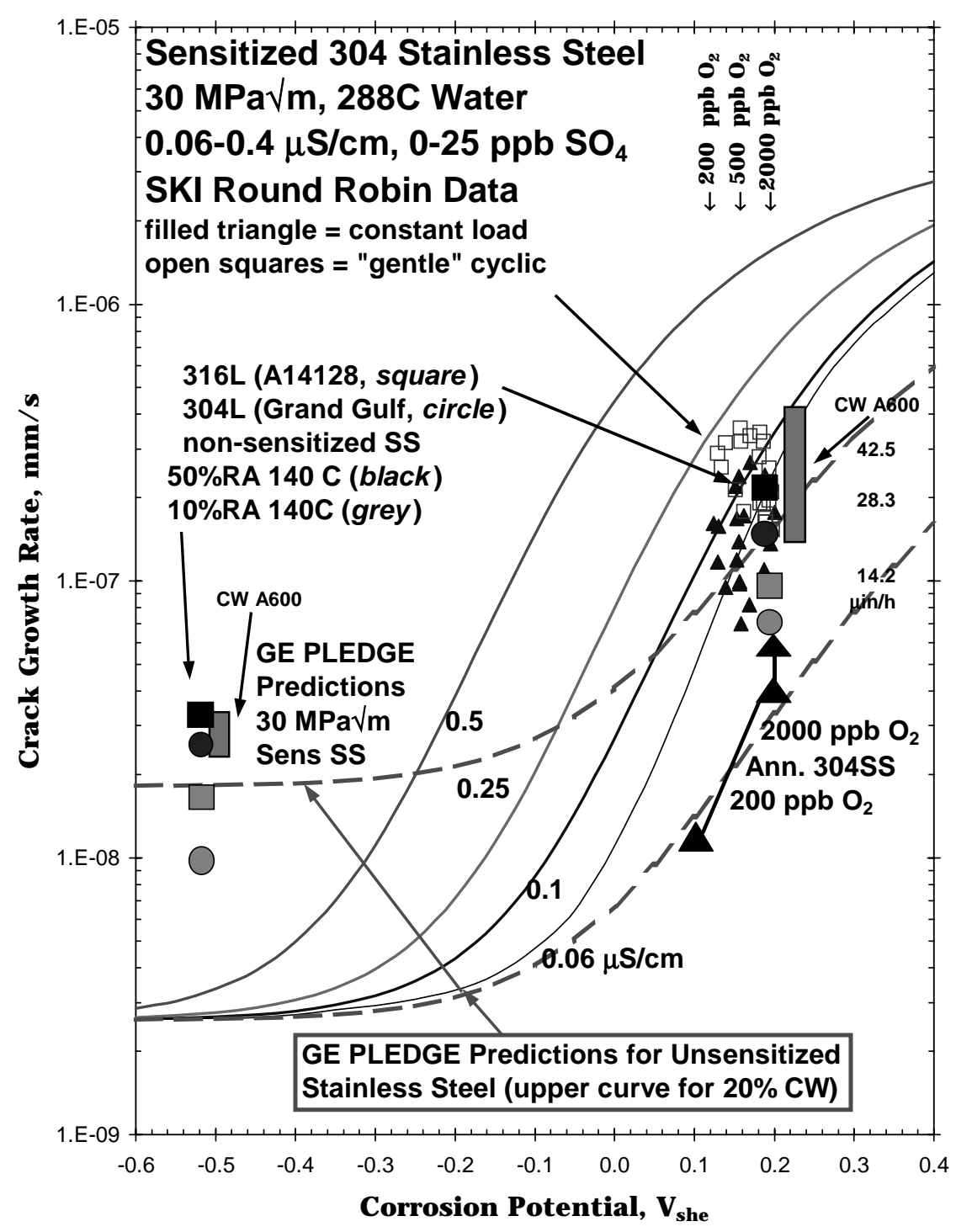

Figure 177. Crack-growth rate vs. corrosion potential for cool-rolled Types 304L and 316L SS tested in $288^{\circ} \mathrm{C}$ pure water. The numerous data at high potential and $\approx 10^{-7} \mathrm{~mm} / \mathrm{s}$ were obtained in the SKI/EPRI Round Robin, and the three black triangles were obtained in a separate GE GRC program on the same (SKI) material after solution annealing. The large circles and squares represent the best estimate of the observed behavior in this program.

(c185), it is possible that cold work alters the Cr-depleted pathway, although the peculiarities noted on the fracture surface also create some ambiguities about the data. Note that radiation produces the opposite effect of that studied in specimen c201 by inducing grain boundary $\mathrm{Cr}$ depletion without any carbides.

Relevance to Radiation Hardening and IASCC Of particular relevance to this program is the similarity in SCC response of materials whose yield strength is increased by cold work and irradiation. While more data is needed on irradiated stainless steels, the enhancement in crackgrowth rate from radiation hardening has long been implicated as an important element in 


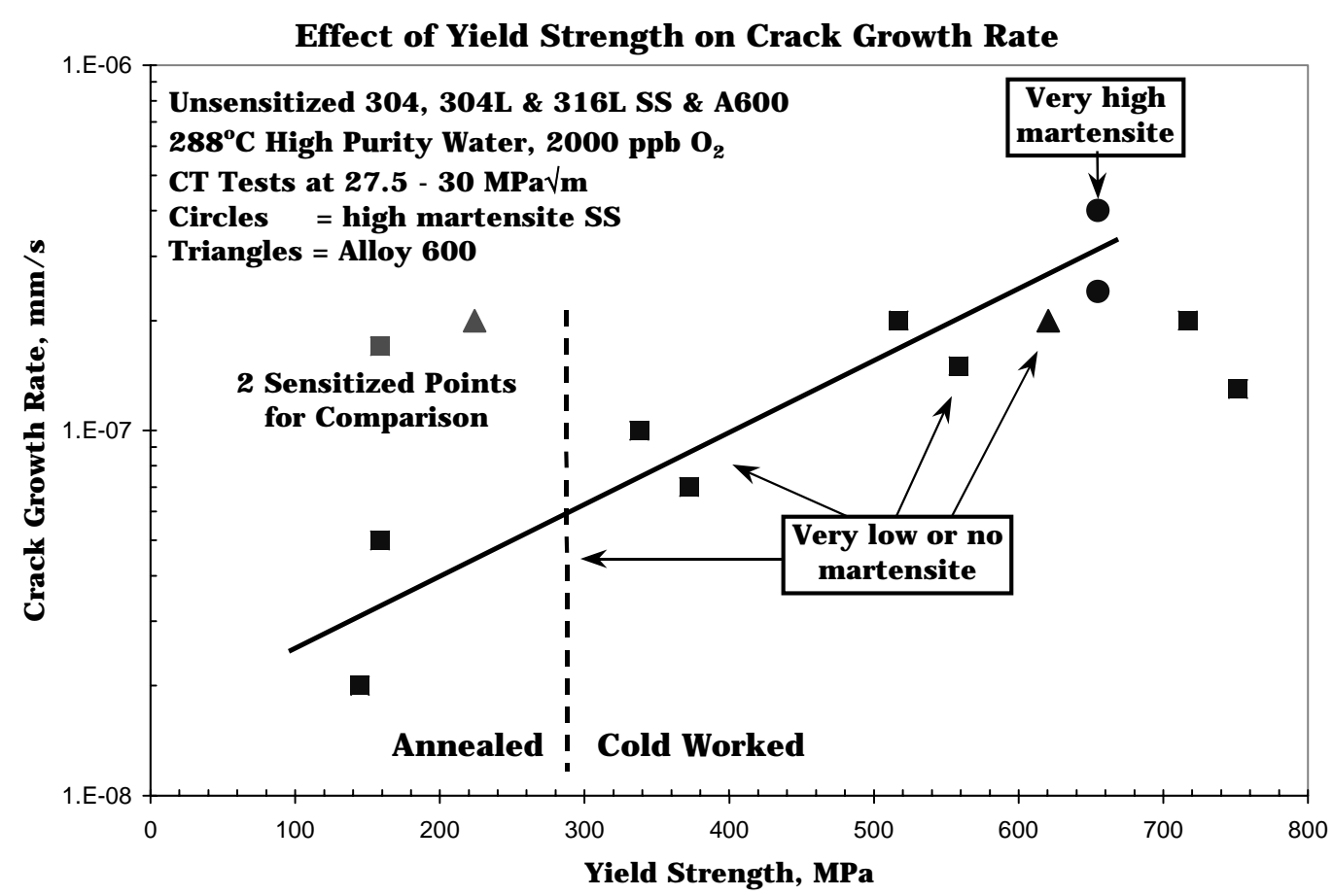

Figure 178. Effect of yield strength and martensite on the stress corrosion crack growth rate on stainless steel and alloy 600 in $288^{\circ} \mathrm{C}$, high purity water $(<0.10 \mu \mathrm{S} / \mathrm{cm}$ outlet $)$ containing 2000 ppb $\mathrm{O}_{2}$.

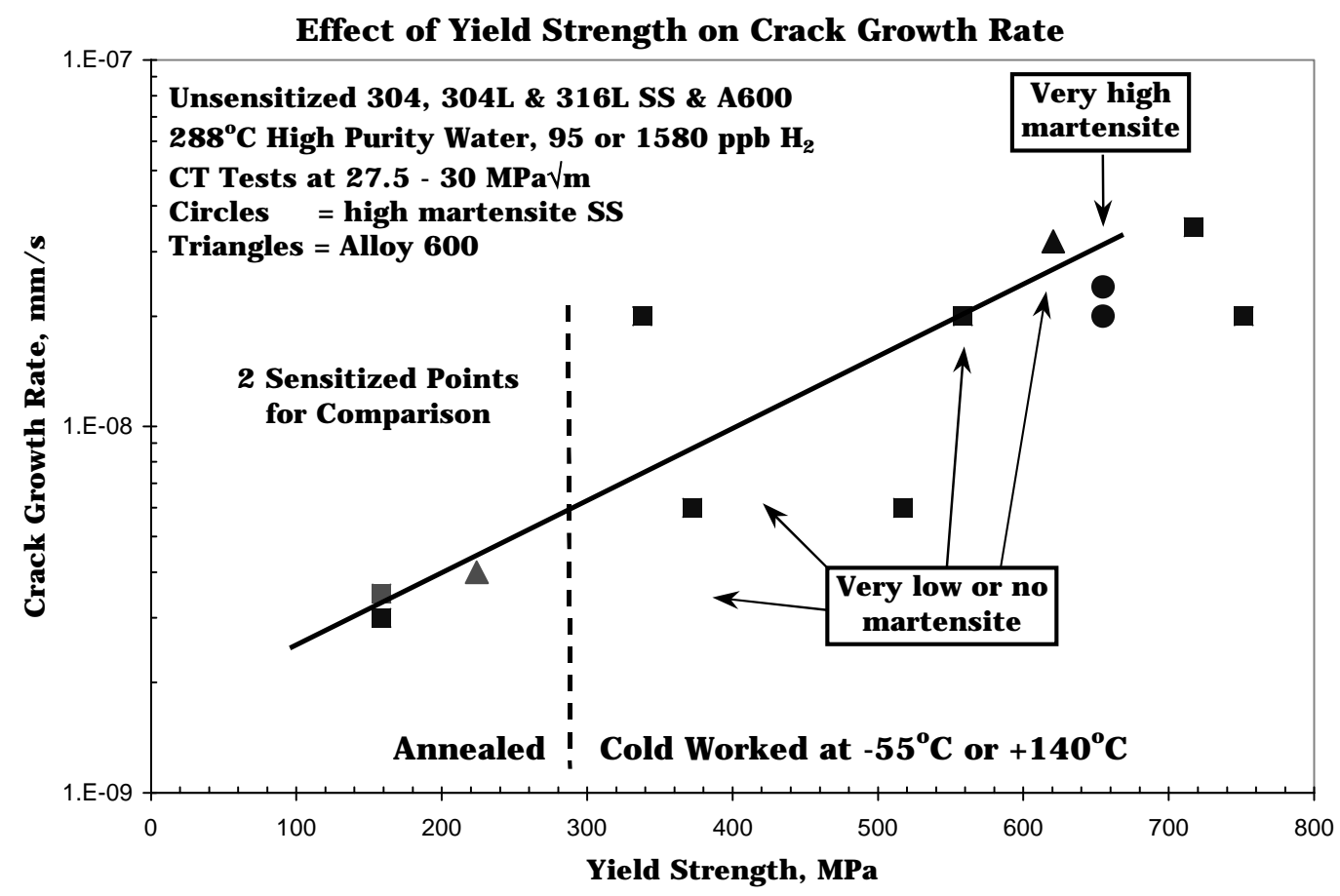

Figure 179. Effect of yield strength and martensite on the stress corrosion crack growth rate on stainless steel and alloy 600 in $288^{\circ} \mathrm{C}$, high purity water $(\approx 0.06 \mu \mathrm{S} / \mathrm{cm}$ outlet $)$ containing 95 or $1580 \mathrm{ppb} \mathrm{H}_{2}$. 
IASCC. These studies demonstrate that an increase in yield stress from cold work also produces a large increase in SCC susceptibility, which helps isolate radiation-hardening effects on cracking.

To examine the concept that similar effects on SCC result from an increase in yield strength from cold work and radiation hardening, high quality crack-growth data on irradiated stainless steel under similar condition are needed for comparison. The quantity of irradiated crack-growth data is very limited, but data were obtained in GE programs ${ }^{69,70}$ under comparable conditions to the non-irradiated data obtained in this program. Because the experiments were focused on the effect of corrosion potential on crack-growth rate, both high and low corrosion potentials were evaluated as shown in Figures 180 and 181. Figure 180 shows the crack length vs. time response for a CT specimen of irradiated $304 \mathrm{SS}$ tested at $25 \mathrm{ksi} V$ in in $288^{\circ} \mathrm{C}$ water to which no intentional impurities were added (the primary constituent to the elevated outlet conductivity is chromate).

It must be recognized that at high corrosion potential there are two primary contributing factors present in the irradiated material - radiation segregation (i.e. Cr depletion) and radiation hardening. The much greater role of $\mathrm{Cr}$ depletion in oxidizing solution compared to reducing solutions is widely recognized. Thus, the crack-growth rate under high corrosion potential conditions is above the rates obtained on non-irradiated stainless steel that is only sensitized or cold worked (Figure 182). Some additional elevation in crack-growth rate is expected in the irradiated data because of the higher outlet conductivity.

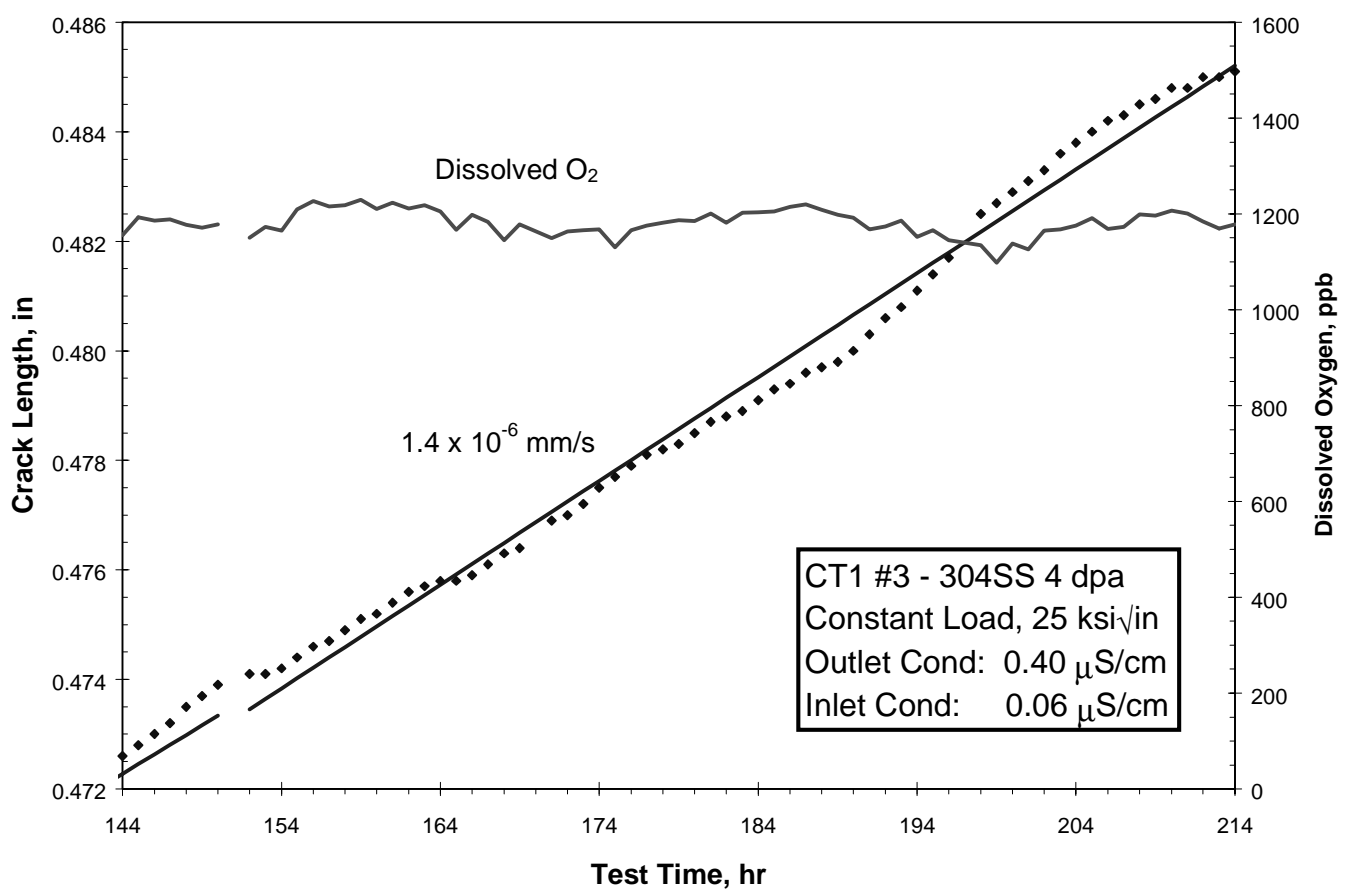

Figure 180. Crack length vs. time for at CT specimen of irradiated Type 304 SS tested at constant load in $288^{\circ} \mathrm{C}$ pure water at high corrosion potential at $25 \mathrm{ksi} \sqrt{ }$ in. ${ }^{69,70}$ 


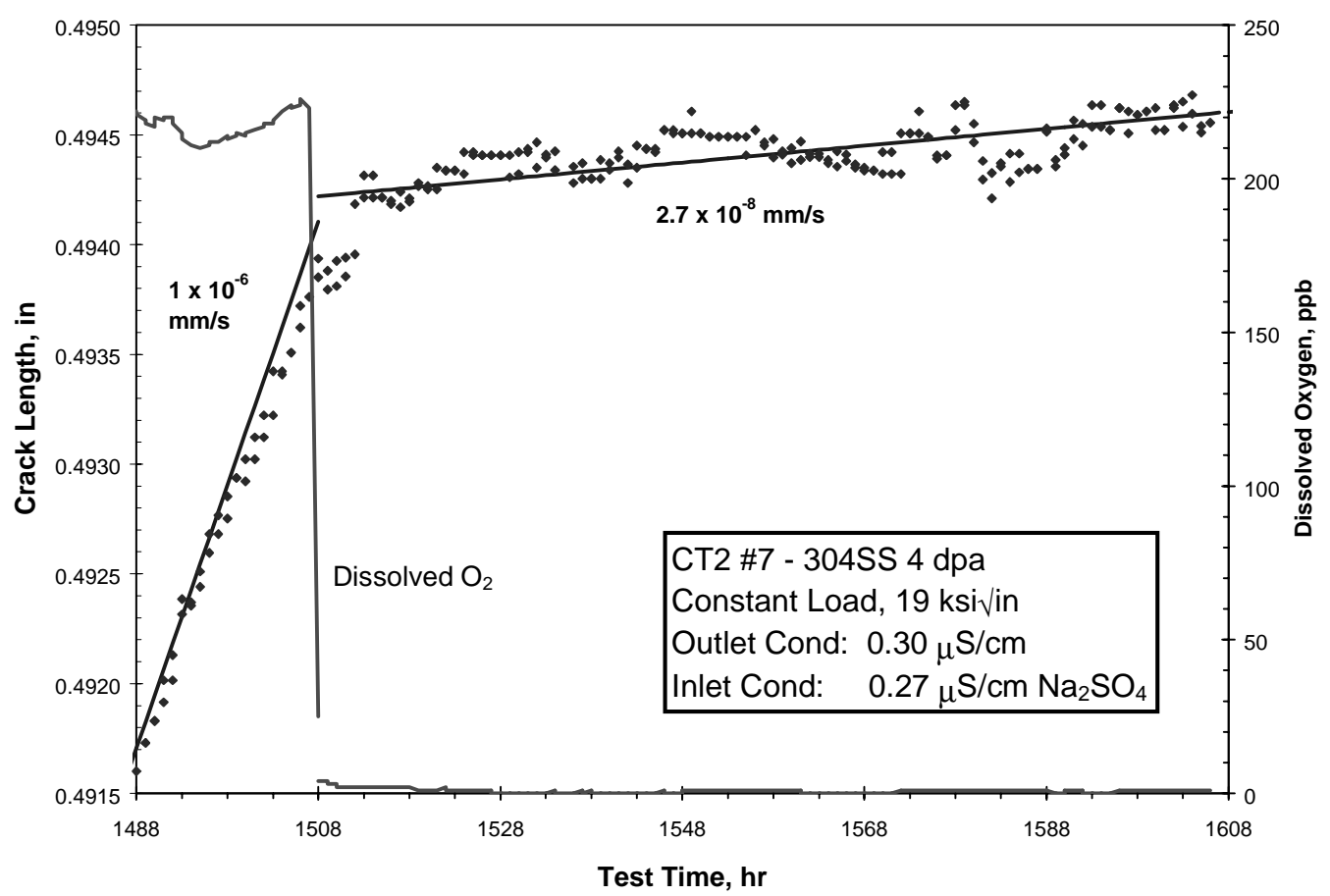

Figure 181. Crack length vs. time for at CT specimen of irradiated type 304 stainless steel tested at constant load in $288^{\circ} \mathrm{C}$ water at both high and low corrosion potential at $19 \mathrm{ksi}{ }_{\mathrm{in}}{ }^{69,70}$

The irradiated crack-growth rate response is corroborated by the data in Figure 181. The identical irradiated material was used, but the testing was performed at slightly lower stress intensity factor (19 ksi $\sqrt{ }$ in) and different water chemistry (220 ppb $\mathrm{O}_{2}$ and $0.27 \mu \mathrm{S} / \mathrm{cm} \mathrm{Na}_{2} \mathrm{SO}_{4}$ ). On switching from $220 \mathrm{ppb}$ to $0 \mathrm{ppb} \mathrm{O}_{2}$ at $1508 \mathrm{~h}$, a rapid transition downward in crack growth rate occurs to $2.7 \times 10^{-8} \mathrm{~mm} / \mathrm{s}$. This rate compares very favorably with that observed on nonirradiated, cold-worked stainless steel of similar (700 - 850 MPa) yield strength (Figure 182), and the comparison is a very strong one because all data were obtained at constant load, in $288^{\circ} \mathrm{C}$ pure water (the presence of $\mathrm{Na}_{2} \mathrm{SO}_{4}$ has very little effect at low corrosion potential), and similar stress intensity factors. Further corroboration in the form of unpublished data from Halden $^{71}$ and a recent GE program conducted for the CIR program ${ }^{72}$ exists at both low (and high) corrosion potential, although the comparisons are (only somewhat) complicated by greater differences in stress intensity factor, test temperature, yield strength, etc. The similarity in response of non-irradiated and irradiated materials is also shown in their similarity of $\mathrm{K}$ dependence on SCC. Figure 183 shows a K dependency of about $\mathrm{K}^{2.3}$ for non-irradiated coldworked stainless steel tested over a very wide range in K. Figure 184 shows comparable data on irradiated stainless steel tested under very similar water chemistry conditions, with a $\mathrm{K}$ dependency of about $\mathrm{K}^{2.5}$. Similar comparisons exist for other non-irradiated and irradiated data.

Figure 182 compares the SCC response predicted by PLEDGE for yield strength, corrosion potential, and sensitization effects. The strong equivalence of cold work and irradiation contributions to yield strength in terms of enhancing SCC seems surprising from a microstructural perspective - the microstructures of cold worked and irradiated stainless steels are vastly different. However, the primary effect of increased yield strength appears to be strongly related to their far-field effect in reducing the size of the plastic zone and thereby 


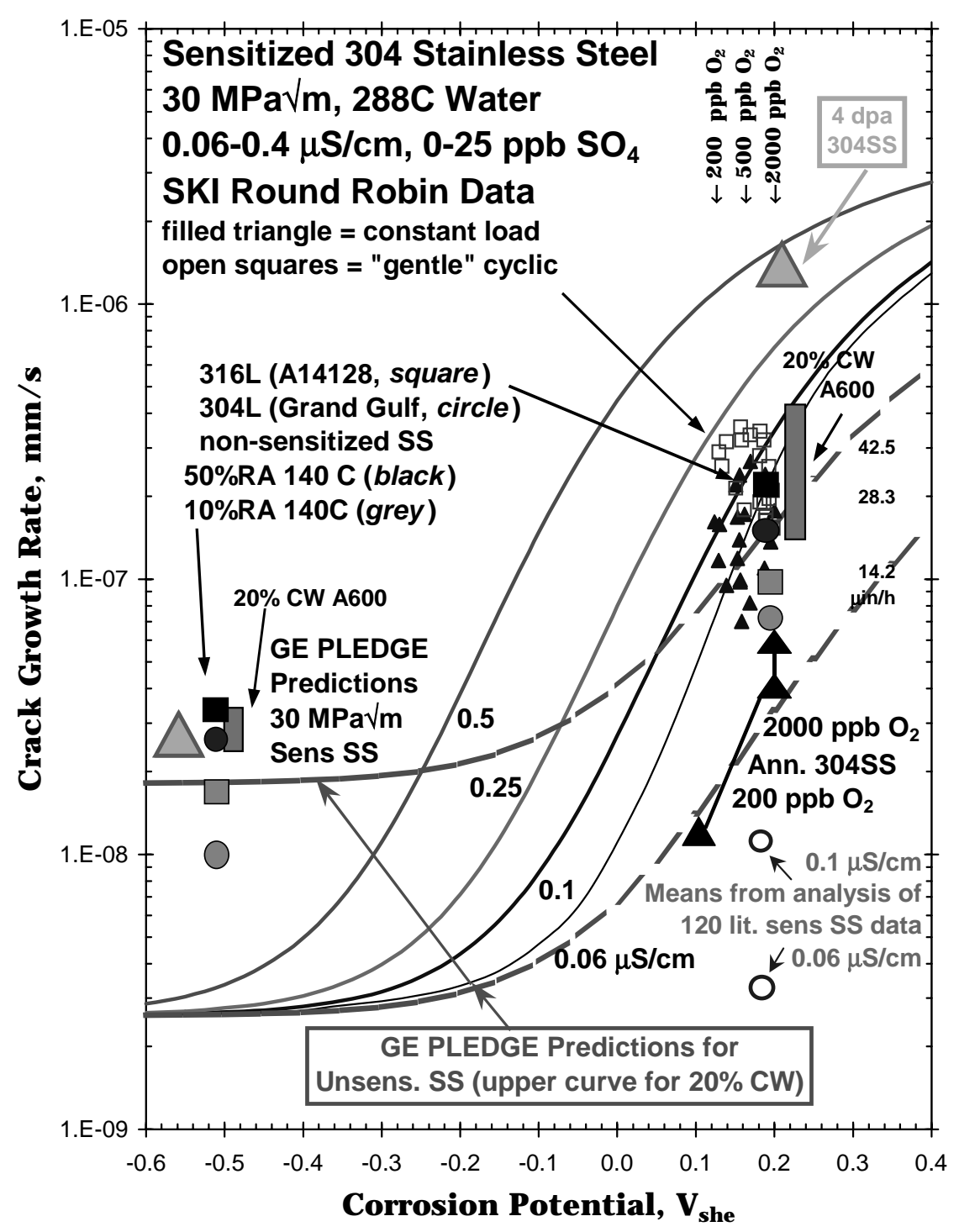

Figure 182. Stress corrosion crack-growth rate vs. corrosion potential cold-worked, sensitized or irradiated stainless steel tested in $288^{\circ} \mathrm{C}$ water. Non-irradiated and irradiated materials of similar yield strength show similar SCC response at low corrosion potential. At high corrosion potential, the combined effect of radiation hardening and radiation segregation produces a higher growth rate than either factor alone (i.e., in the non-irradiated data that is either cold worked or sensitized).

increasing the strain gradient in the smaller plastic zone (and near the crack tip, Figure 183). The distribution of strain throughout the entire plastic zone is not directly important to the deformation kinetics at the crack tip. Slip that impinges near the crack tip is limited to a "zone of influence" near the crack tip, estimated to be within $20-60 \mu \mathrm{m}$ of the tip. Because the strain density and triaxiality of strain is very high near the crack tip, differences between cold-worked and irradiated microstructures are minimized (at very high strain and with extensive cross-slip, the dominant effect of dislocation channeling is reduced). 


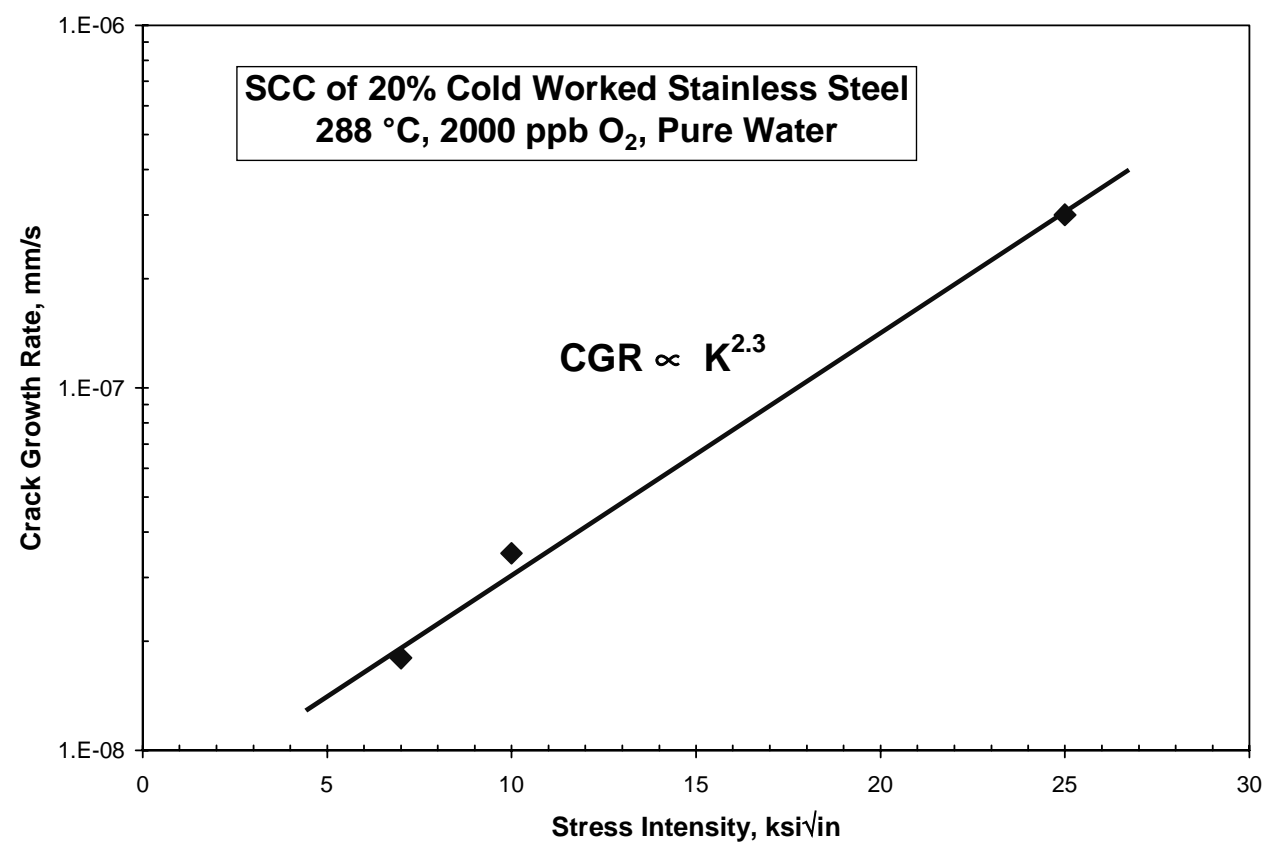

Figure 183. Crack length vs. time and the effect of stress intensity on SCC growth rate of cold worked $316 \mathrm{~L} \mathrm{SS}$ in $288^{\circ} \mathrm{C}$ pure water at high corrosion potential.

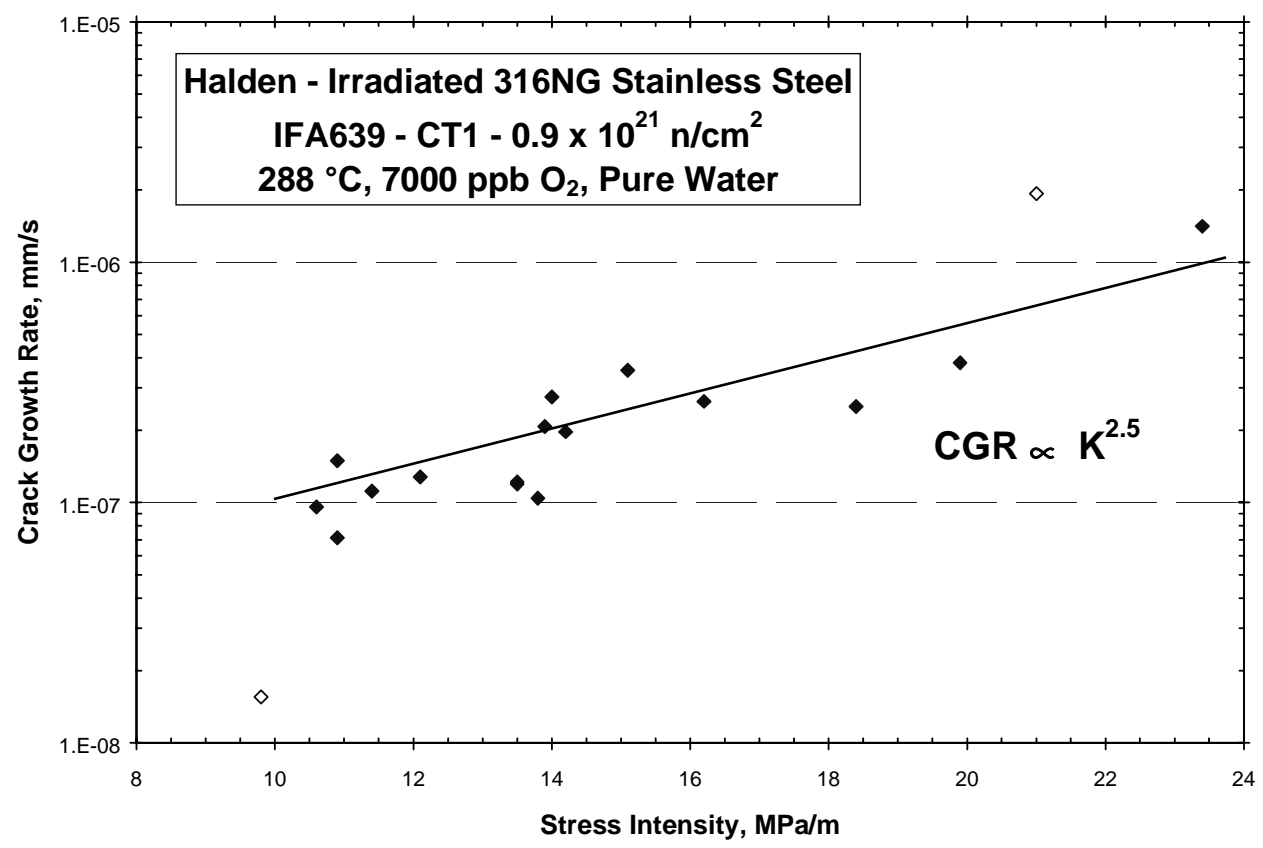

Figure 184. The effect of stress intensity on SCC growth rate of $316 \mathrm{NG}$ stainless steel irradiated to $0.9 \times 10^{21} \mathrm{n} / \mathrm{cm}^{2}$ in $288^{\circ} \mathrm{C}$ pure water at high corrosion potential.

This interpretation recognizes the importance of dynamic strain at the crack tip as a fundamental element in SCC, related primarily to the exposure of bare metal at the slip steps that form and 
impose huge shear strains on the protective oxide film. Under static-load SCC conditions, dynamic (creep) strain would decay to zero if not for the synergistic relationship of dynamic strain and crack advance - dynamic strain causes SCC, and crack advance causes redistribution of the stress and strain fields ahead of the crack, and thereby sustained dynamic strain (Figure $183)$.

H Permeation and Mechanisms of Stress Corrosion Crack Advance There continues to be debate about a primary, causal role (or even a secondary, indirect role) of hydrogen in SCC crack advance. For this reason, experiments were performed in which hydrogen permeation measurements through stainless steel were made under various water chemistry and temperature conditions. These measurements were made using closed-end, $6.4 \mathrm{~mm}$ outside diameter 0.46 stainless steel tubes inserted into the autoclave through a Swagelock fitting. The tubes were abraded on the inside and outside; the inside was exposed only to vacuum and hydrogen after heating. Hydrogen permeation into the tube was recorded by monitoring the increase in pressure to a maximum of 10 torr (10,000 microns) using an MKS Baratron Model 660 with a Model 622A11TCE pressure transducer. He leak checking was used to confirm the absence of leaks, and the system was operated at $150^{\circ} \mathrm{C}$ and $288^{\circ} \mathrm{C}$ under $\mathrm{N}_{2}$-deaerated conditions to confirm its leak-tightness under system operating conditions.

These measurements were performed in a similar fashion to parallel experiments on alloy $600 .^{73}$ The alloy 600 tests were performed using a controlled gage section of $\sim 15 \mathrm{~cm}$ length $(0.6-\mathrm{cm}$ OD, 0.46-mm ID), while the data on stainless steel were obtained on a simpler closed end tube without a gage section, so that the equivalent gage section for the stainless steel specimen was about 1.5 times as long. The total gas volume measured by the pressure transducer was also similar: $\approx 19 \mathrm{cc}$ for these stainless steel tests vs. $24.8 \mathrm{cc}$ for the alloy 600 measurements.

The crack-growth rate response in $288^{\circ} \mathrm{C}$ water of stainless steels and alloy 600 is quite similar when comparisons are separately made for sensitized, non-sensitized or cold-worked materials. There is clearly a strong effect of elevated corrosion potential (e.g., 200 or 2000 ppb dissolved $\mathrm{O}_{2}$ ) under all conditions. However at low potential, there is no evidence of any differences in the crack-growth rate of stainless steel with smaller changes in potential associated with variations in the dissolved $\mathrm{H}_{2}$ concentration. Here the changes in potential are limited to $\approx 56 \mathrm{mV}$ per decade change in $\mathrm{H}_{2}$ at $288^{\circ} \mathrm{C}$; as the $\mathrm{H}_{2}$ level is changed from $95 \mathrm{ppb}$ (about $1.06 \mathrm{cc} / \mathrm{kg}$ ) to $1580 \mathrm{ppb}$ $(17.7 \mathrm{cc} / \mathrm{kg}$ ), the change in potential is only about $68 \mathrm{mV}$ (vs. a $600-700 \mathrm{mV}$ potential change

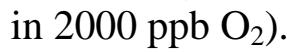

However, such small changes in potential can be important for Ni alloys, because (unlike Fe alloys) the transition from $\mathrm{NiO}$ (or spinel) stability to Ni metal stability occurs in precisely this potential regime. One might anticipate that as the primary alloy constituent becomes phase stable, the SCC susceptibility should drop, as is observed. One explanation for why it does not drop to zero when Ni metal is stable is that there is nearly $30 \%$ of other oxidizable constituents in alloy 600 , including $\mathrm{Cr}, \mathrm{Fe}, \mathrm{Si}$, and $\mathrm{Mn}$. The 3-D percolation threshold (the minimum percentage of a uniformly distributed element needed to maintain a connected path through the structure) is about $18 \%$. Of course, SCC in these materials occurs intergranularly, and the concentration of oxidizable species in the grain boundary is often above the nominal alloy composition. 
Hydrogen permeation data were obtained for stainless steel, and for alloy $600 .^{73}$ All data were very linear and highly reproducible (Figures $185-188$ ), and show that hydrogen permeation is primarily controlled by the coolant fugacity, temperature and material (alloy 600 is about twice as permeable to hydrogen at a given temperature). The important observation for the purpose of this discussion is that the $\mathrm{H}_{2}$ fugacity in the coolant dominates hydrogen permeation through the metal, following (as expected) a square root dependency on $\mathrm{H}_{2}$ fugacity over the temperature range studied $\left(\approx 200\right.$ to $\left.360^{\circ} \mathrm{C}\right)$. Of particular note is that the effective surface fugacity of $\mathrm{H}_{2}$ becomes very low in $\mathrm{O}_{2}$-containing solutions, as reflected in the rapid drop in $\mathrm{H}_{2}$ partial pressure in the tube (Figures 186 and 188). This also shows how readily $\mathrm{H}_{2}$ gas in the tube dissociates to adsorbed $\mathrm{H}^{\mathrm{o}}$ on the tube ID.

The crack-growth rate data obtained on non-sensitized, cold-worked stainless steels and alloy 600 are not consistent with a hydrogen-dominated, crack-advance mechanism in hightemperature water. Considering only the crack-growth-rate data, the similarity in growth rates for Types 304L and 316L stainless steel and alloy 600 under both aerated and deaerated $288^{\circ} \mathrm{C}$ water conditions show that martensite per se does not influence crack growth rates. This is reinforced in Figures 178 and 179 which plot SCC growth rate vs. yield strength for high potential and low potential conditions, respectively (specimens with extremely high or low martensite are identified). No martensite is present in the cold-worked alloy 600, and the difference in martensite in the stainless steels varies by nearly zero in the "cool-worked" $\left(+140^{\circ} \mathrm{C}\right)$ Type $316 \mathrm{~L}$ stainless steel to very high in the "cold-worked" $\left(-55^{\circ} \mathrm{C}\right)$ Type $304 \mathrm{~L}$ stainless steel. Prior studies have shown a large effect of martensite on SCC of stainless steels at temperatures below about $125^{\circ} \mathrm{C}$.

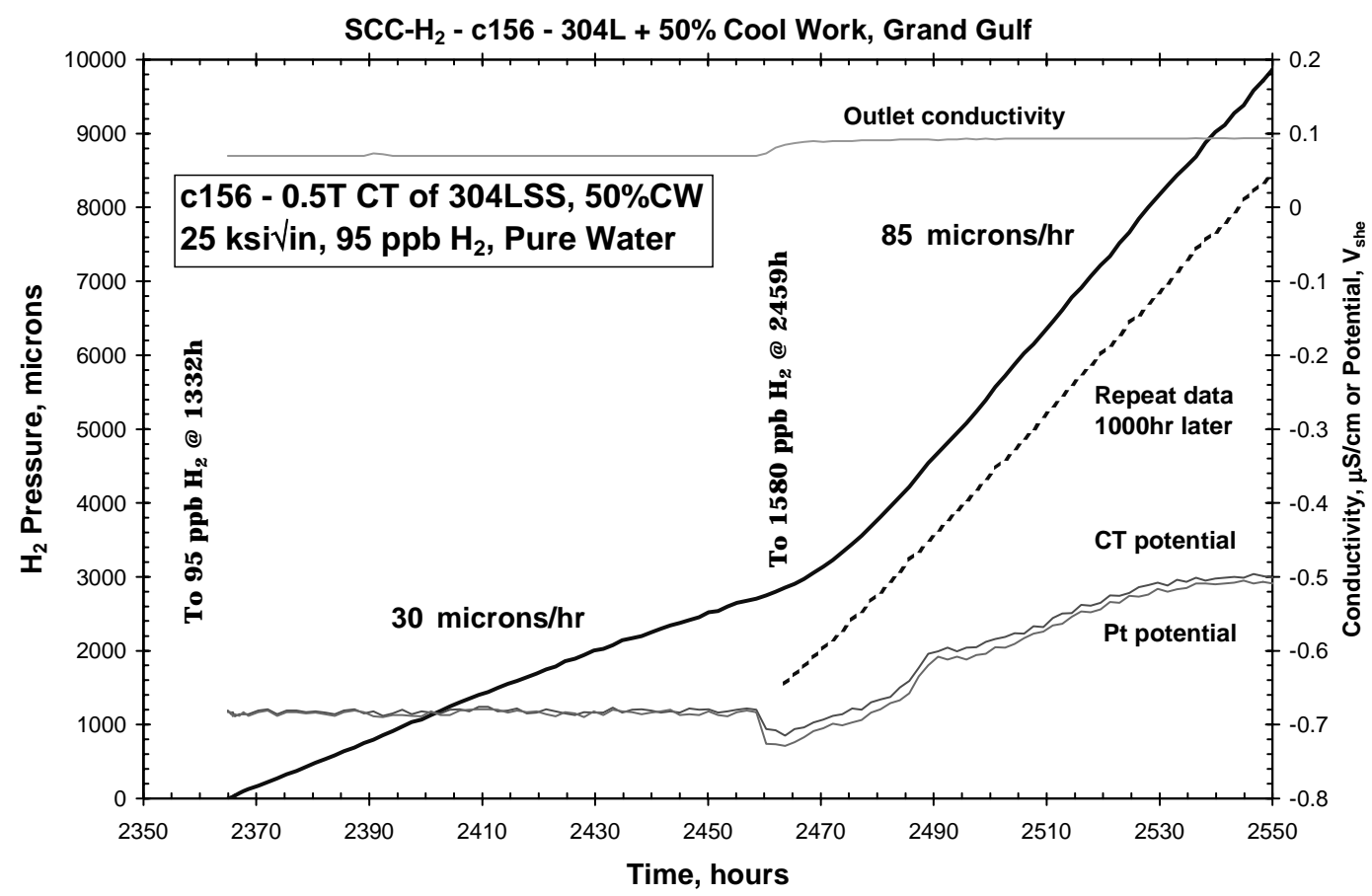

Figure 185. Hydrogen permeation vs. time and coolant $\mathrm{H} 2$ fugacity unsensitized Type 304L stainless steel. Testing was performed in $288^{\circ} \mathrm{C}$ high purity water containing $95 \mathrm{ppb} \mathrm{H}_{2}(1.06$ $\mathrm{cc} / \mathrm{kg})$ and $1580 \mathrm{ppb} \mathrm{H}_{2}(17.7 \mathrm{cc} / \mathrm{kg})$. 


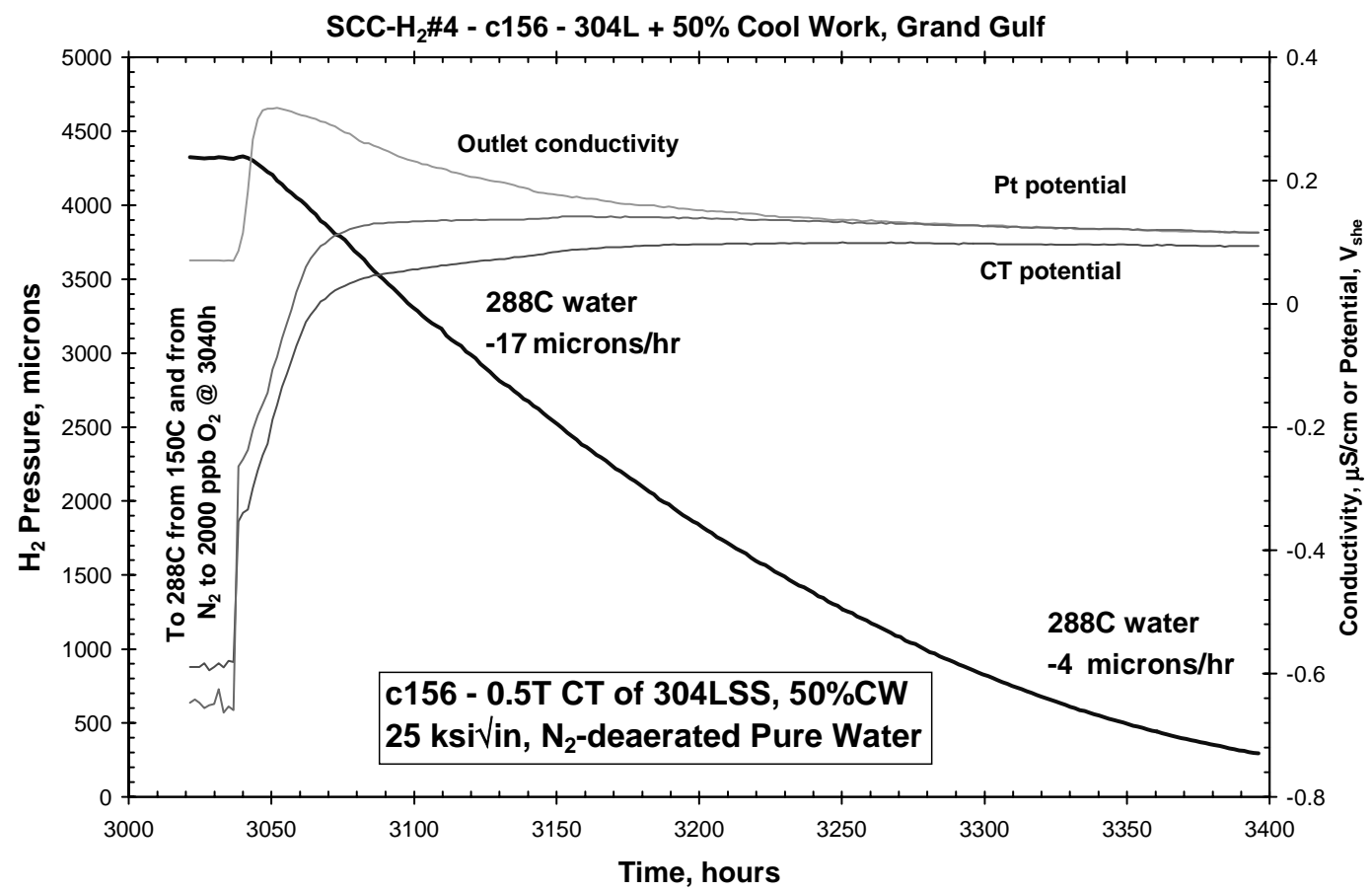

Figure 186. Hydrogen permeation vs. time and coolant $\mathrm{H}_{2}$ fugacity non-sensitized Type 304L SS. Testing was performed in $288^{\circ} \mathrm{C}$ high-purity water containing $\mathrm{N}_{2}$ or $2000 \mathrm{ppb} \mathrm{O}_{2}$.

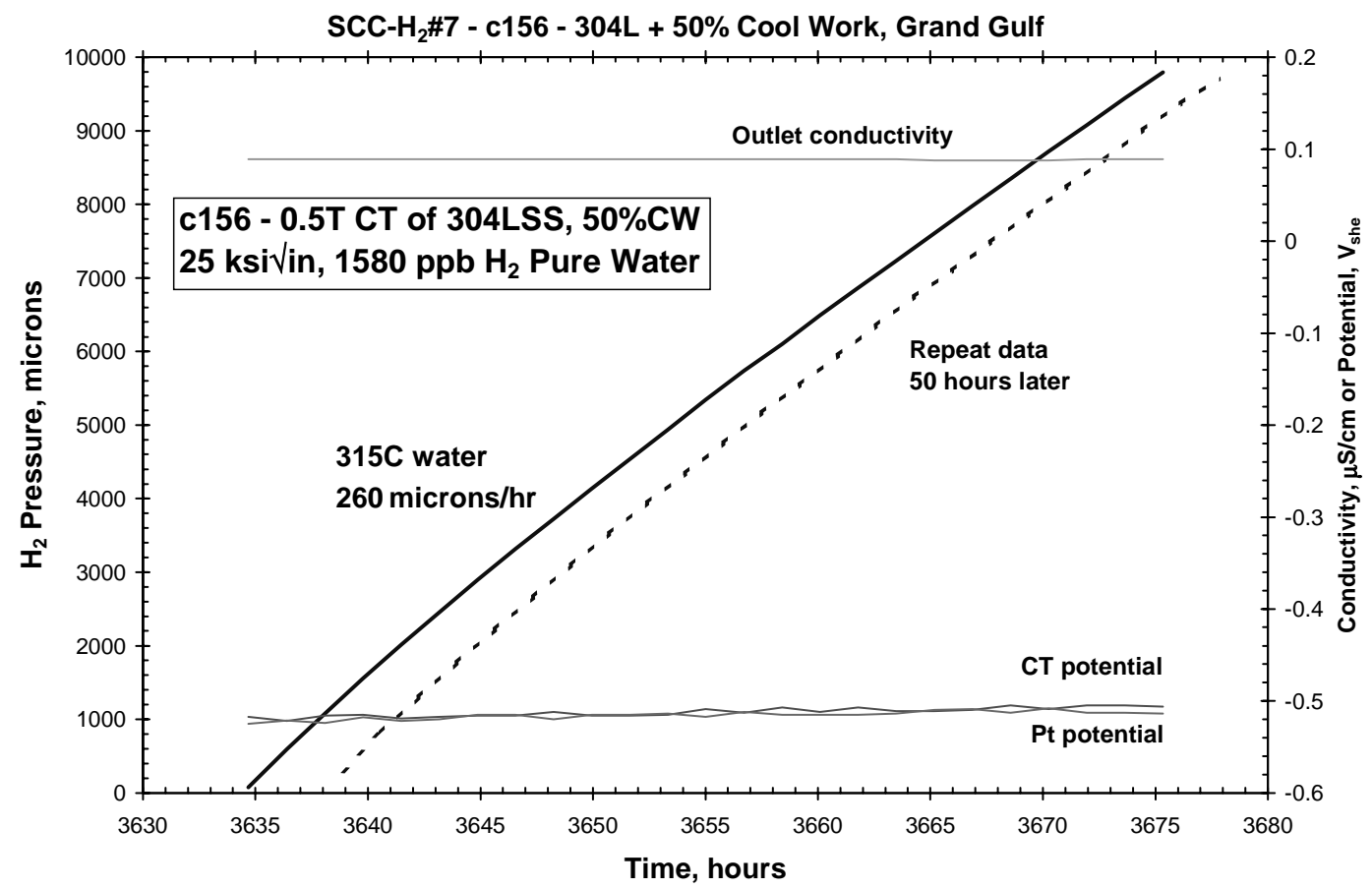

Figure 187. Hydrogen permeation vs. time and coolant $\mathrm{H}_{2}$ fugacity non-sensitized Type 304L $\mathrm{SS}$ in $315^{\circ} \mathrm{C}$ high-purity water with $1580 \mathrm{ppb} \mathrm{H}_{2}(17.7 \mathrm{cc} / \mathrm{kg})$; the fugacity coefficient of $\mathrm{H}_{2}$ changes with temperature. 


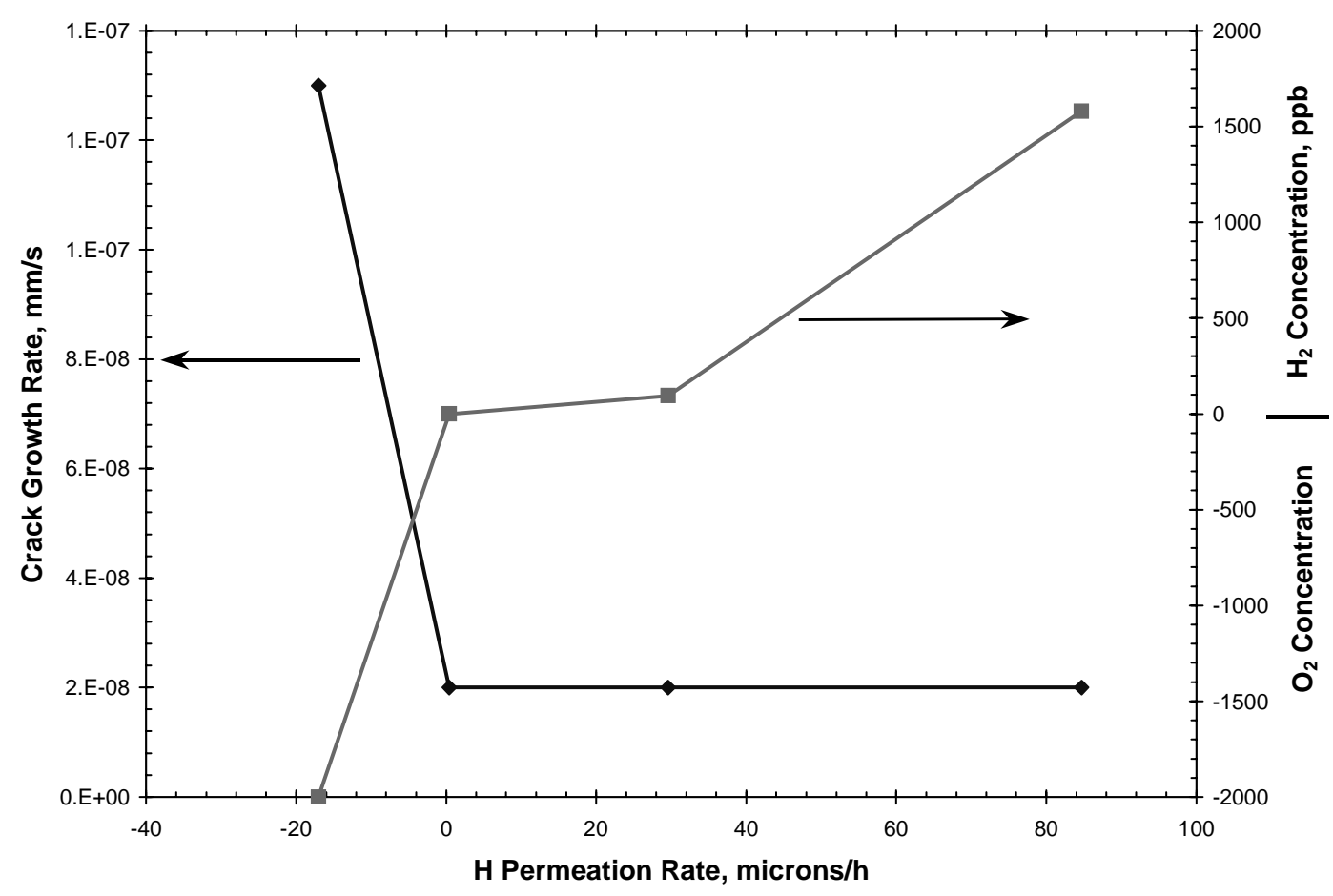

Figure 188. Hydrogen permeation rate (measured as a pressure increase) vs. crack-growth rate and vs. coolant $\mathrm{H}_{2}$ (and $\mathrm{O}_{2}$ ) concentration in non-sensitized Type $304 \mathrm{~L} \mathrm{SS}$ in $288^{\circ} \mathrm{C}$ water.

In turn, the hydrogen-permeation measurements also show a poor (often inverse) correlation between SCC crack-growth rates and permeation rate in Figure 188. Very high hydrogen permeation rates occur as the coolant $\mathrm{H}_{2}$ level is increased, but the growth rate is unchanged whether deaeration (low corrosion potential conditions) is achieved using $\mathrm{N}_{2}, 95 \mathrm{ppb} \mathrm{H}_{2}$, or 1580 ppb $\mathrm{H}_{2}$ - a range which produces more than $200 \mathrm{X}$ change in hydrogen permeation rate at $288^{\circ} \mathrm{C}$. It is also interesting to note that the fairly thick oxides (typically $0.1-0.5 \mu \mathrm{m}$ ) of varying structure that form under high and low potential conditions seem to have little effect on hydrogen permeation, and that permeation both in and out of the tube occurs readily as shown in Figures $185-187$.

These observations on stainless steel are important supplements to the earlier observations on crack-growth rate of alloy 600 (and the recent hydrogen-permeation measurements in alloy $600)^{73}$ because there are important thermodynamic differences between the two materials, notably in deaerated hot water. Because alloy 600 can shift from a regime where its primary constituent can oxidize to $\mathrm{NiO}$ or spinel, to a regime where $\mathrm{Ni}$ metal is stable, the crackgrowth rate of this alloy does not stay constant in the "low corrosion potential" regime as does stainless steel. Under $\mathrm{NiO}$ stability conditions, the growth rates at "low potential" (no dissolved $\mathrm{O}_{2}$ ) - and at high potential - are similar for stainless steel and alloy 600 , but as the $\mathrm{H}_{2}$ fugacity is increased and the transition to Ni metal stability crossed, the crack-growth rate of alloy 600 undergoes a further decrease.

The argument can be made that under Ni metal stability conditions, the corrosion (oxidation) reaction rate on bare metal (slip step offsets) decreases and therefore the $\mathrm{H}_{2}$ (reduction) reaction 
would also be lower. This would reduce the local hydrogen fugacity at the crack tip, and therefore the crack advance by a hydrogen mechanism would also diminish (assuming corrosion is the primary source of $\mathrm{H}_{2}$ ). Of course, $\mathrm{Fe}$ and $\mathrm{Cr}$ are also present, and if we focus on either $\mathrm{Fe}$ (especially for stainless steels) or $\mathrm{Cr}$, very high theoretical $\mathrm{H}_{2}$ fugacities can be calculated if one assumes that the bare metal surface controls the potential. For example, the $\mathrm{Cr}_{2} \mathrm{O}_{3} / \mathrm{Cr}$ equilibrium is $\approx 700 \mathrm{mV}$ below the $288^{\circ} \mathrm{C} \mathrm{H}_{2} / \mathrm{H}_{2} \mathrm{O}$ line at 1 atm. $\mathrm{H}_{2}$, and since the $\mathrm{H}_{2} / \mathrm{H}_{2} \mathrm{O}$ line changes by $56 \mathrm{mV}$ per decade in $\mathrm{H}_{2}$ in $288^{\circ} \mathrm{C}$ water, a $\mathrm{H}_{2}$ fugacity of $700 / 56=12.5$ decades $=$ $3.2 \times 10^{12}$ atm. $\mathrm{H}_{2}$ can be postulated. For $\mathrm{Fe} / \mathrm{Fe}_{3} \mathrm{O}_{4}$, whose equilibrium potential is $\approx 200 \mathrm{mV}$ below the $\mathrm{H}_{2} / \mathrm{H}_{2} \mathrm{O}$ line, the $\mathrm{H}_{2}$ fugacity becomes $200 / 56=3.6$ decades $=4000$ atm. $\mathrm{H}_{2}$. Such equilibrium calculations must be tempered by such balancing kinetics considerations.

However, the best approach to addressing this "corrosion-based $\mathrm{H}_{2}$ " argument is to focus on stainless steels (including materials that are highly cold worked, some of which contain very high levels of martensite), because the change in coolant $\mathrm{H}_{2}$ fugacity over realistic levels will have little impact on the difference in potential between the $\mathrm{H}_{2} / \mathrm{H}_{2} \mathrm{O}$ line and the $\mathrm{Fe} / \mathrm{Fe}_{3} \mathrm{O}_{4}$ equilibrium potential (which remains $>200 \mathrm{mV}$ below the corrosion potential obtained at our highest $\mathrm{H}_{2}$ level of 1 atm. x 0.201 fugacity coefficient in $288^{\circ} \mathrm{C}$ water). This is very different than the nickel alloy situation, where crossing into the regime of Ni metal stability occurs readily as $\mathrm{H}_{2}$ is added, and the possible "corrosion contribution" of $\mathrm{H}_{2}$ diminishes.

It is difficult to argue that such a "corrosion source" of high $\mathrm{H}_{2}$ fugacity controls $\mathrm{SCC}$ in stainless steels, as the high growth rates in $2000 \mathrm{ppb} \mathrm{O}_{2}$ correlate with very low $\mathrm{H}_{2}$ permeation and very low $\mathrm{H}_{2}$ fugacity conditions on the surface (Figures 185 - 188), sufficiently low to reverse the direction of permeation (Figure 186) and yield a projected asymptote (equilibrium $\mathrm{H}_{2}$ fugacity) that is very close to zero. In the low-potential regime where the hydrogen permeation rate does become very large (as the coolant $\mathrm{H}_{2}$ fugacity is increased), there is no discernible change in crack-growth rate (Figure 188), even though the contribution from "corrosion" is essentially unaffected.

These data are also applicable to IASCC of stainless steels, including under conditions where $\mathrm{H}$ is formed by transmutation. The $\mathrm{H}$ concentration in irradiated stainless steels typically saturates in the vicinity of $15-25$ wppm, although the formation of voids (which can occur above $\sim 320^{\circ} \mathrm{C}$ ), correlates with dramatically higher concentrations of $\mathrm{H}$. However, the rate of formation of $\mathrm{H}$ by transmutation (or the rate of proton injection from radiolysis) is many orders of magnitude lower than the permeation rates measured in this study. Note that BWR and PWR

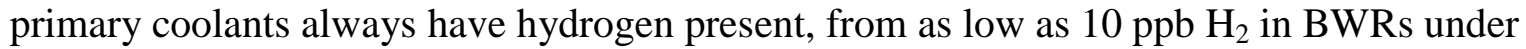
"normal water chemistry", to $50-100 \mathrm{ppb} \mathrm{H}_{2}$ under "hydrogen water chemistry" operation, to about $3000 \mathrm{ppb}$ in PWR primary systems. In turn, these studies have shown that $\mathrm{H}_{2}$ is not readily "trapped" in a void (or tube), but readily dissociates and diffuses. Thus, the fugacity of $\mathrm{H}$ in the metal remains in equilibrium with the coolant $\mathrm{H}_{2}$, except perhaps in the rare case of rapid temperature changes. In turn, it is hard to imagine that the highly elevated (e.g., 50 wppm or 2800 appm) $\mathrm{H}$ concentrations measured in irradiated stainless steel are anything but a reflection of additional "storage" sites in the microstructure (i.e., it is not hydrogen that is microstructurally active). Such $\mathrm{H}$ cannot be attributed to transmutation or corrosion reactions when the permeability of $\mathrm{H}$ from the coolant through the entire structure is orders of magnitude higher. In turn, the effect of lowering the potential by decreasing the dissolved $\mathrm{O}_{2}$ has a pronounced 
beneficial effect on IASCC of even highly irradiated stainless steel, just as in non-irradiated materials.

Most of the hydrogen-effects literature emphasizes a pronounced effect on higher strength Fe and Ni-base alloys tested below $\approx 130^{\circ} \mathrm{C} . .^{57,74,75}$ The importance of martensite in exacerbating hydrogen effects is highlighted in reference, ${ }^{76}$ where the correlation between severe hydrogen embrittlement (that induced highly IG fracture) and martensite content of Type 304 stainless steel was very strong. Clearly, no similar dramatic effect of martensite is noted in our tests. It is certainly possible that some effects of $\mathrm{H}$ on dislocation mobility in metals persist to higher temperatures, although thermally activated dislocation motion increases with temperature. Also, the ubiquitous nature of $\mathrm{H}$ in metals exposed to hot water (most achieve $2-5$ wppm levels within days of exposure) raises the question of whether there is a unique / distinguishable contribution of hydrogen that varies from differences in coolant fugacity that pragmatically vary by $<300 X$ ( $10 \mathrm{ppb}$ to $3000 \mathrm{ppb}$ ) - especially since high hydrogen-permeation rates are recorded even at the lowest fugacity levels. Other findings such as the absence of detrimental effects of applying Pt-group catalysts to the surface, high crack-propagation rates (only) at low temperature of high-strength nickel alloys, ${ }^{77,78}$ and other evidence against a causal role for hydrogen in SCC in hot water is discussed in reference. ${ }^{75}$ However, it should also be noted that there is evidence in the corrosion-fatigue literature that reduced life is observed when high coolant $\mathrm{H}_{2}$ fugacity conditions exist. ${ }^{79}$ This points to some "residual" effect of $\mathrm{H}$ on the reversed slip processes at $\sim 290^{\circ} \mathrm{C}$. 


\section{References}

1. F. A. Garner, Nuclear Materials, Volume Editor B. R. T. Frost, in Materials Science and Technology-A Comprehensive Treatment, Edited by R. W. Cahn, P. Hassen and E. J. Kramer, Vol. 10A (1994) 420.

2. S. M. Bruemmer, E. P. Simonen, P. M. Scott, and P. L. Andresen, G. S. Was, L. E. Rehn and J. L. Nelson, 1999, "Radiation-Induced Material Changes and Susceptibility to Intergranular Failure of Light-Water-Reactor Core Internals," J. Nucl. Mater., 274 (1999) 299-314.

3. G. S. Was, J. T. Busby, T. Allen, E. A. Kenik, A. Jensson, S. M. Bruemmer, J. Gan, D. J. Edwards, P. M. Scott, and P. L. Andresen, J. Nucl. Mater., Vol. 300, No. 2-3, pp. 198-216, 2002.

4. TRIM2000 Program, J. F. Ziegler, J. P. Biersack, IBM Corp. Yorktown, NY.

5. TRIM97 Program, J. F. Ziegler, J. P. Biersack, IBM Corp. Yorktown, NY.

6. D. J. Edwards, E. P. Simonen and S. M. Bruemmer, "Evolution of Fine-Scale Defects in Stainless Steels Neutron-Irradiated at $275^{\circ} \mathrm{C}^{\prime}$, accepted J. Nucl. Mater. 2002.

7. N. Sakaguchi, S. Watanabe, H. Takahashi, Nuclear Instruments and Methods in Physics Research, Section B (Netherlands), vol. 153, no. 1-4, pp. 142-146,1999.

8. T. Kato, H. Takahashi and M. Izumiya, J. Nucl. Mater. 189 (1992):p. 167.

9. R. S. Averback and M. A. Kirk, "Atomic Displacement Processes in Ion-Irradiated Materials, in Surface Alloying by ion, Electron and Laser Beams, eds. L. E. Rehn, S. T. Picraux and H. Wiederisch,, pp. 91-135, American Society for Metals, Metals Park, OH, 1987.

10. T. Okita, N. Sekimura, T. Iwai and F. A. Garner, "Investigation of the Synergistic Influence of Irradiation Temperature and Atomic Displacement rate on the Microstructural Evolution of Ion-Irradiated Model Austenitic Alloy Fe-15Cr-16Ni”, Proc. Tenth Int. Symp. on Environmental Degradation of Materials in Nuclear Power Systems - Water Reactors, NACE, 2001.

11. E. P. Simonen and S. M. Bruemmer, "IASCC: Lattice Defect/Grain Boundary Interactions," J. of Nucl. Mater., 239, (1996) p. 185-193.

12. Ohnuki, S. Yamashita, H. Takahashi and T. Kato, $19^{\text {th }}$ Inter. Sympo., ASTM STP 1366, M. L. Hamilton, AA. S. Kumar, S. T. Rosinski, and M. L. Grossbeck, Eds., American Society for Testing and Materials, West Conshohocken, PA, 2000.

13. L. E. Thomas, "The Stability of $\gamma^{\prime}$ and $\gamma^{\prime}$ in Inconel 706 Under Neutron Irradiation", pp 237-255, in Phase Stability During Irradiation, eds. J. R. Holland, L. K. Mansur and D. I. Potter, The Metallurgical Society of AIME, Warrendale, PA, 1981.

14. L. E. Thomas and S. M. Bruemmer, "Radiation-Induced Microstructural Evolution and Phase Stability in Nickel-Base Alloy 718", Proc. Eighth Int. Symp. on Environmental Degradation of Materials in Nuclear Power Systems - Water Reactors, American Nuclear Society, La Grange Park, IL, 1997.

15. F. Carsughi, H. Derz, G. Pott, W. Sommer and H. Ullmaier, J. Nucl. Mater. 264 (1999) p. 78.

16. D. S. Gelles, "Void Swelling Resistance in Fe-Cr Alloys at $200 \mathrm{dpa}$ " in Microstructure of Irradiated Materials, Materials Research Society Symposium 
Proceedings Vol. 373, I. M. Robertson, L. E. Rehn, S. J. Zinkle and W. J. Phythian, Eds., MRS, Pittsburgh, PA (1995) pp. 69-74.

17. D. S. Gelles , "Gamma Prime Coarsening and Redistribution in Nimonic PE16" in Effects of Radiation on Materials, ASTM STP 725, D. Kramer, H. R. Brager and J. S. Perrin, Eds., ASTM, Philadelphia, PA (1981) pp. 562-582.

18. R. B. Baker, F. E. Bard and J. L. Ethridge, in LMR: A Decade of LMR Progress and Promise, (ANS, La Grange Park, IL., 1991) p. 184.

19. See Fusion Materials Semiannual Progress Reports DOE/ER-0313/1-31, later issues available at www.ms.ornl.gov/programs/fusionmatls/pubs/semiannual.htm.

20. W. Dietz, K. Ehrlich, and J.-J. Huet, Radiation Effects in Breeder Reactor Structural Materials, M. L. Bleiberg and J. W. Bennett, Eds., (AIME, N.Y., 1977) p.13.

21. J.-J. Huet, L. Coheur, A. De Bremaecker, L. De Wilde, J. Gedopt, W. Hendrix and W. Vandermeulen, Nucl. Tech. 70 (1985) 215.

22. J. S. Benjamin, US Patent 3,591,362, 1971.

23. Frontiers of High Temperature Materials, Proceedings of a conference held in New York City on May 18-21, 1981, J. S. Benjamin and R. C. Benn, Eds. (INCO, 1982).

24. Frontiers of High Temperature Materials II, Proceedings of a conference held in London on May 22-25, 1983, J. S. Benjamin, Ed. (INCO, 1985)

25. J. J. Fischer, US Patent 4,075,010, 1978.

26. MA957 INCO Data Sheets.

27. F. H. Froes, J. of Metals, (1989) 25.

28. D. K. Mukhopadhyay, F. H. Froes and D. S. Gelles, J. Nucl. Mater., V 258-263 (1998) 1209.

29. M. L. Hamilton, D. S. Gelles, R. J. Lobsinger, M. M. Paxton and W. F. Brown, PNL13165 (2000).

30. M. L. Hamilton, D. S. Gelles, R. J. Lobsinger, G. D. Johnson, W. F. Brown, M. M. Paxton, R. J. Puigh, C. R. Eiholzer, C. Martinez and M. A. Blotter, PNL-13168 (2000).

31. S. Ukai, M. Harada, H. Okada, M. Inoue, S. Nomura, S. Shikakura, K. Asabe, T. Nishida and M. Fujiwara, J. Nucl. Mater., 204 (1993) 65.

32. S. Ukai, M. Harada, H. Okada, M. Inoue, S. Nomura, S. Shikakura, T. Nishida, M. Fujiwara, and K. Asabe, J. Nucl. Mater., 204 (1993) 74.

33. A. N. Niemi, M. G. McKimpson and D. S. Gelles, in Fusion Reactor Materials Semiannual Progress Report, DOE/ER-0313/6 (1989) 187.

34. A. N. Niemi, M. G. McKimpson and D. S. Gelles, in Fusion Reactor Materials Semiannual Progress Report, DOE/ER-0313/8 (1990) 177.

35. R. Lindau, A. Möslang, M. Schirra, P. Schlossmacker and M. Klimenkov, submitted for inclusion in the Proceedings of ICFRM-10 to be published in J. Nucl. Mater.

36. G.R. Romanoski, L.L. Snead, R.L. Klueh, and D.T. Hoelzer, J. Nucl. Mater., 283-287 (2000) 642.

37. V.V. Sagaradze, V.I. Shalaev, V.L. Arbuzov, B.N. Goshchitskii, Y. Tian, W. Qun, S. Jiguang, J. Nucl. Mater., 295 (2001) 265.

38. D. S. Gelles, J. Nucl. Mater., 233-237 (1996) 293, for a more complete version see Fusion Materials Semiannual Progress Report, DOE/ER-0313/16 (1994) 146.

39. M. B. Toloczko, F. A. Garner and C. R. Eiholzer, J. Nucl. Mater., 258-263 (1998) 1163. 
40. Ph. Van Asbroeck, W. Vanderveulen, M. Snykers and J-J. Huet in Irradiation Embrittlement and Creep in Fuel Cladding and Core Components, (BNES, London, 1972) 179.

41. P. Dubuisson, R. Schill, M-P. Hugon, I. Grislin and J-L. Séran, in Effects of Radiation on Materials: $18^{\text {th }}$ International Symposium, ASTM STP 1325, R. K. Nanstad, M. L. Hamilton, F. A. Garner and A. S. Kumar, Eds., (ASTM, West Conshohocken, PA, 1999) 882.

42. I.-S. Kim, J.D. Hunn, N. Hashimoto, D.L. Larson, P.J. Maziasz, K. Miyahara, and E.H. Lee, J. Nucl. Mater., 280 (2000) 264.

43. J. Saito, T. Suda, S. Yamashita, S. Ohnuki, H. Takahashi, N. Akasaka, M. Nishida, S. Ukai, J. Nucl. Mater., 258-263 (1998) 1264.

44. S. Yamashita, S. Watanabe, S. Ohnuki, H. Takahashi, N. Akasaka, and S. Ukai, J. Nucl. Mater., 283-287 (2000) 647.

45. S. Ukai, S. Mizuta, T. Yoshitake, T. Okuda, S. Hagi, T. Kobayashi, and M. Fujiwara in Jupiter Workshop on Recent Progress in Reduced Activation Ferritic Steels held December 1-2, 1999 (Institute for Advanced Energy, Kyoto University, 2000) 229.

46. S. Ukai, S. Mizuta, T. Yoshitake, T. Okuda, M. Fujiwara, S. Hagi, T. Kobayashi, and M. Fujiwara, J. Nucl. Mater., 283-287 (2000) 702.

47. A. Seeger, Proc. $2^{\text {nd }}$ UN Int. Conf. On Peaceful Uses of Atomic Energy, Geneva, Sept., 1958, Vol. 6, p. 250.

48. G.E. Lucas, J. Nucl. Mater. 206 (1993) 287-305.

49. B.N.Singh, A.J.E. Foreman, and H. Trinkaus, J. Nucl. Mater. (Netherlands), vol. 249, no. 2-3, pp. 103-115, Oct. 1997.

50. H. Trinkaus, H; B.N. Singh, and AJE Foreman, J. Nucl. Mater. (Netherlands), vol. 251, no. 1-3, pp. 173-188, 1997.

51. B.N. Singh, D.J. Edwards, P. Toft, J. Nucl. Mater. (Netherlands), vol. 299, no. 3, pp. 205-218, 2001

52. P.L. Andresen, F.P. Ford, S.M. Murphy, J.M. Perks, "State of Knowledge of Radiation Effects on Environmental Cracking in Light Water Reactor Core Materials", Invited Review Paper, Proc. Fourth International Symposium on Environmental Degradation of Materials in Nuclear Power Systems - Water Reactors, Jekyll Island, NACE, pp. 1-83 to 1-121, 1990.

53. P.L. Andresen, "Irradiation Assisted Stress Corrosion Cracking”, in Book on Stress Corrosion Cracking: Materials Performance and Evaluation, Ed. R.H. Jones, ASM, Materials Park, 1992, p.181-210.

54. T.M. Angeliu, P.L. Andresen, J.A. Sutliff and R.M. Horn, "Intergranular Stress Corrosion Cracking of Unsensitized Stainless Steels in BWR Environments", Proc. Ninth Int. Symp. on Environmental Degradation of Materials in Nuclear Power Systems - Water Reactors, AIME, 1999.

55. T.M. Angeliu, P.L. Andresen, E. Hall, J.A. Sutliff, S. Sitzman, "Strain and Microstructure Characterization of Austenitic Stainless Steel Weld HAZs", Corrosion/2000, Paper 00186, NACE, 2000.

56. P.L. Andresen, T.M. Angeliu, W.R. Catlin, L. M. Young and R.M. Horn, "Effect of Deformation on SCC of Unsensitized Stainless Steel", Corrosion/2000, Paper 00203, NACE, 2000. 
57. P.L. Andresen, T.M. Angeliu, L.M. Young, W.R. Catlin, and R.M. Horn, "Mechanisms and Kinetics of SCC in Stainless Steels", Proc. Tenth Int. Symp. on Environmental Degradation of Materials in Nuclear Power Systems - Water Reactors, NACE, 2001.

58. P.L. Andresen, "Perspective and Direction of Stress Corrosion Cracking in Hot Water", Proc. Tenth Int. Symp. on Environmental Degradation of Materials in Nuclear Power Systems - Water Reactors, NACE, 2001.

59. P.L. Andresen, L.M. Young, W.R. Catlin and R.M. Horn, "Stress Corrosion Crack Growth Rate Behavior of Various Grades of Cold Worked Stainless Steel in High Temperature Water", Corrosion/02, Paper 02511, NACE, 2002.

60. PL Andresen and C.L. Briant, "Environmentally Assisted Cracking of Types 304L / 316L / 316NG Stainless Steel in 288 C Water," Corrosion, Vol. 45, pp. 448-463, 1989.

61. P.L. Andresen and C.L. Briant, "Role of S, P and N Segregation on Intergranular Environmental Cracking of Stainless Steels in High Temperature Water," Proc. 3rd Int. Symp. on Environmental Degradation of Materials in Nuclear Power Systems Water Reactors, TMS-AIME, pp. 371-382, 1988.

62. P.L. Andresen, "Environmentally Assisted Growth Rate Response of Nonsensitized AISI 316 Grade Stainless Steels in High Temperature Water," Corrosion 44, 7, p. 450, 1988.

63. P.L. Andresen, "The Effects of Aqueous Impurities on Intergranular Stress Corrosion Cracking of Sensitized Type 304 Stainless Steel," Final Report NP3384 Contract T115-3, EPRI, 1983. See also, "Innovations in Experimental Techniques for Testing in High Temperature Aqueous Environments," Report No. 81CRD088, GE-CRD, Schenectady, New York, 1981.

64. L.W. Niedrach, "A New Membrane Type $\mathrm{pH}$ Sensor for Use in High Temperature High Pressure Water”, J Electrochem. Soc., 127, p. 2122, 1980.

65. P.L. Andresen, et. al., "In-Reactor Monitoring for Component Life Prediction", Interim Technical Progress Report \#2, EPRI Project RP2006-17, August 1988.

66. P.L. Andresen, "Specific Anion and Corrosion Potential Effects on Environmentally Assisted Cracking in 288C Water", GE CRD Report 93CRD215, December 1993.

67. S. Hettiarachchi, G.P. Wozadlo, T.P. Diaz, P.L. Andresen and R.L Cowan, "The Concept of Noble Metal Addition Technology for IGSCC Mitigation of Nuclear Materials", Proc. Seventh Int. Symp. on Environmental Degradation of Materials in Nuclear Power Systems - Water Reactors, NACE, p.735-746, 1995.

68. P.L. Andresen, "Application of Noble Metal Technology for Mitigation of Stress Corrosion Cracking in BWRs", Proc. Seventh International Symposium on Environmental Degradation of Materials in Nuclear Power Systems - Water Reactors, NACE, p. 563-578, 1995.

69. P.L. Andresen, F.P. Ford, J.P. Higgins, I. Suzuki, M. Koyama, M. Akiyama, T. Okubo, Y. Mishima, S. Hattori, H. Anzai, H. Chujo, Y. Kanazawa, "Life Prediction of Boiling Water Reactor Internals”, Proc., ICONE-4 Conference, ASME, 1996.

70. P.L. Andresen and R.B. Davis, Unpublished SCC growth data on irradiated stainless steel, GE, 1989.

71. T. Karlsen and E. Hauso, Unpublished SCC growth data on irradiated stainless steel, Halden Reactor Project, Norway, 1999. 
72. P.L. Andresen and R.B. Davis, Unpublished SCC growth data on irradiated stainless steel for the Coop. IASCC Research Program, GE, 2000.

73. D.S. Morton, S.A. Attanasio, G.A. Young, P.L. Andresen, and T.M. Angeliu, "The Influence of Dissolved Hydrogen on Nickel Alloy SCC: A Window to Fundamental Insight", Corrosion, Paper 01117, NACE, 2001.

74. P.L. Andresen, T.M. Angeliu and L.M. Young, "Effect of Martensite \& Hydrogen on SCC of Stainless Steels”, Paper \#01228, Corrosion/01, NACE, 2001.

75. C.L. Briant, "Hydrogen Assisted Cracking of Type 304 Stainless Steel", Met Trans A, 10A, p.181, 1979.

76. C.A. Grove and L.D. Petzold, "Mechanism of SCC of Alloy X750 in High Purity Water", J. of Materials for Energy Systems, Vol. 7, No. 2, p.147-162, 1985.

77. L.D. Petzold and C.A. Grove, "Mechanism of SCC of Alloy X750 in High Purity Water", Proc. of Corrosion of Nickel Alloys, Ed. R.C. Scarberry, ASM, 1985, p.165.

78. P.L. Andresen and T.M. Angeliu, "Evaluation of the Role of Hydrogen in SCC in Hot Water", Paper \#97197, Corrosion/97, NACE, 1997.

79. K. Tsutsumi, et al, "Fatigue Life Reduction in PWR Water Environments for Stainless Steel”, PVP Conf. Volume 410-2, ASME, p.23-34, 2000. 